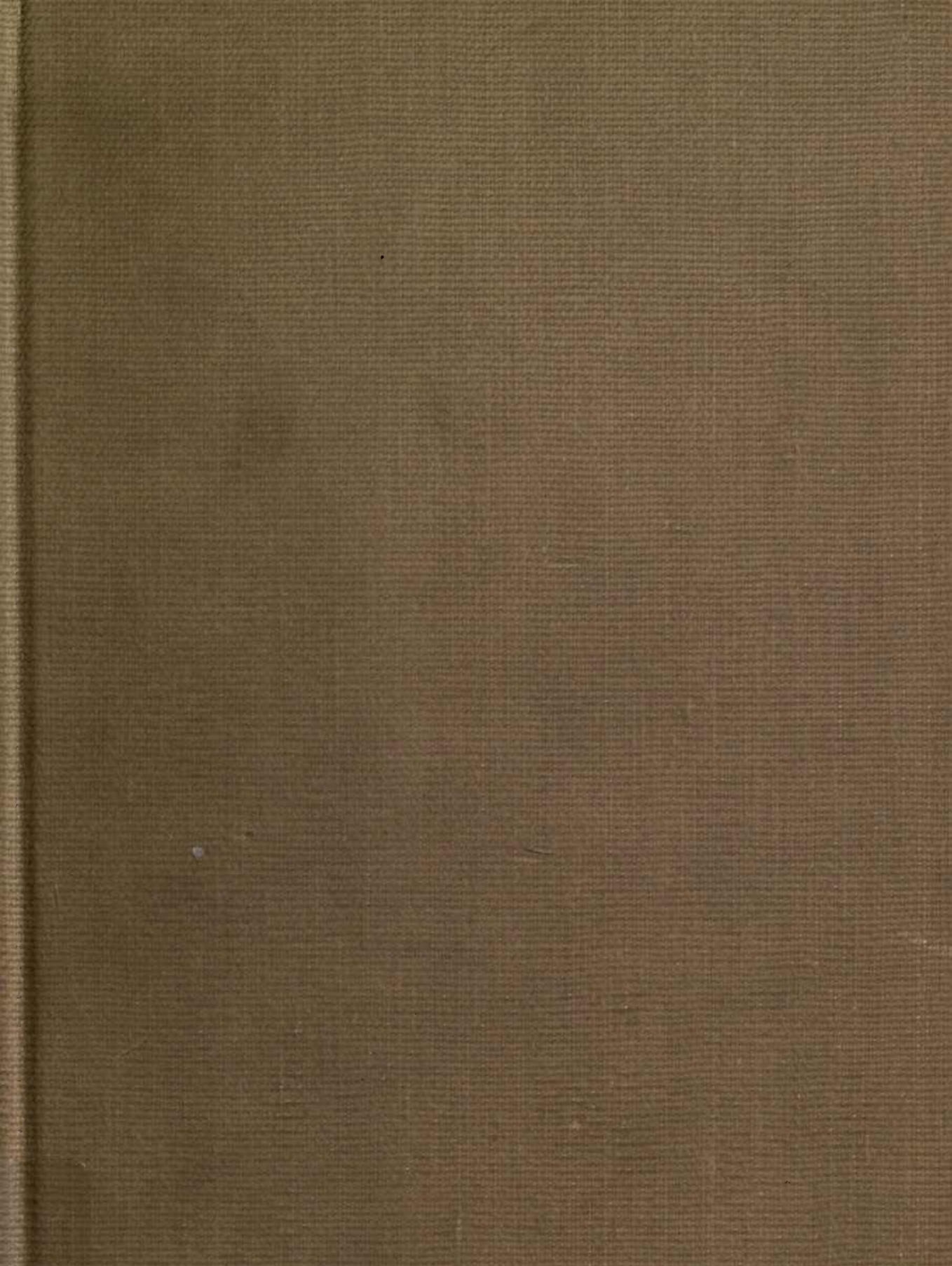





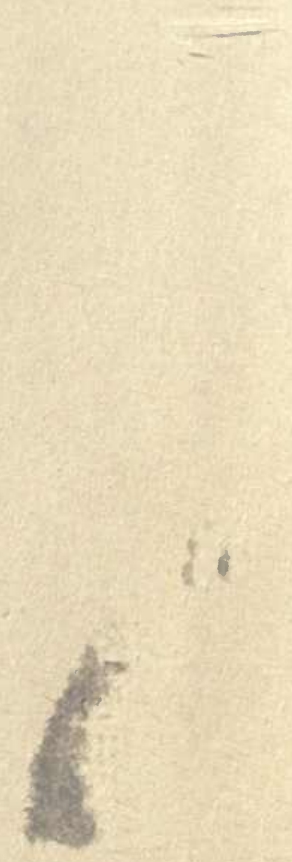
SMITHSONIAN MISCELLANEOUS COLLECTIONS

VOLume 74, Number 1

\section{SMITHSONIAN MATHEMATICAL FORMULAE}

AND

\section{TABLES OF ELLIPTIC FUNCTIONS}

Mathematical Formulae Prepared by

EDWIN P. ADAMS, PH.D.

PROFESSOR OF PHYSICS, PRINCETON UNIVERSITY

Tables of Elliptic Functions Prepared under the Direction of Sir George Greenhill, Bart.

BY

COL. R. L. HIPPISLEY, C.B.

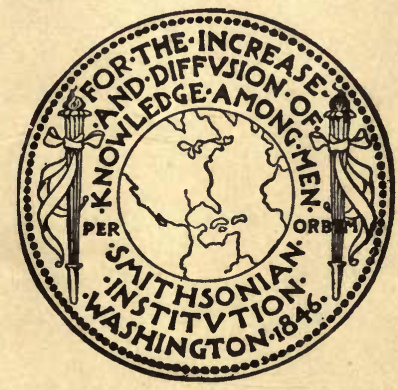

PUBLICATION 2672

CITY OF WASHINGTON

PUBLISHED BY THE SMITHSONIAN INSTITUTION 



\section{ADVERTISEMENT}

The Smithsonian Institution has maintained for many years a group of publications in the nature of handy books of information on geographical, meteorological, physical, and mathematical subjects. These include the Smithsonian Geographical Tables (third edition, reprint, I9I8); the Smithsonian Meteorological Tables (fourth revised edition, I9r8); the Smithsonian Physical Tables (seventh revised edition, I92 I); and the Smithsonian Mathematical Tables: Hyperbolic Functions (second reprint, I92I).

The present volume comprises the most important formulae of many branches of applied mathematics, an illustrated discussion of the methods of mechanical integration, and tables of elliptic functions. The volume has been compiled by Dr. E. P. Adams, of Princeton University. Prof. F. R. Moulton, of the University of Chicago, contributed the section on numerical solution of differential equations. The tables of elliptic functions were prepared by Col. R. L. Hippisley, C. B., under the direction of Sir George Greenhill, Bart., who has contributed the introduction to these tables.

The compiler, Dr. Adams, and the Smithsonian Institution are indebted to many physicists and mathematicians, especially to Dr. H. L. Curtis and colleagues of the Bureau of Standards, for advice, criticism, and coöperation in the preparation of this volume.

May, I922.

Charles D. Walcott, Secretary of the Smithsonian Institution. 


\section{PREFAGE}

The original object of this collection of mathematical formulae was to bring together, compactly, some of the more useful results of mathematical analysis for the benefit of those who regard mathematics as a tool, and not as an end in itself. There are many such results that are difficult to remember, for one who is not constantly using them, and to find them one is obliged to look through a number of books which may not immediately be accessible.

A collection of formulae, to meet the object of the present one, must be largely a matter of individual selection; for this reason this volume is issued in an interleaved edition, so that additions, meeting individual needs, may be made, and be readily available for reference.

It was not originally intended to include any tables of functions in this volume, but merely to give references to such tables. An exception was made, however, in favor of the tables of elliptic functions, calculated, on Sir George Greenhill's new plan, by Colonel Hippisley, which were fortunately secured for this volume, inasmuch as these tables are not otherwise available.

In order to keep the volume within reasonable bounds, no tables of indefinite and definite integrals have been included. For a brief collection, that of the late Professor B. O. Peirce can hardly be improved upon; and the elaborate collection of definite integrals by Bierens de Haan show how inadequate any brief tables of definite integrals would be. A short list of useful tables of this kind, as well as of other volumes, having an object similar to this one, is appended.

Should the plan of this collection meet with favor, it is hoped that suggestions for improving it and making it more generally useful may be received.

To Professor Moulton, for contributing the chapter on the Numerical Integration of Differential Equations, and to Sir George Greenhill, for his Introduction to the Tables of Elliptic Functions, I wish to express my gratitude. And I wish also to record my obligations to the Secretary of the Smithsonian Institution, and to Dr. C. G. Abbot, Assistant Secretary of the Institution, for the way in which they have met all my suggestions with regard to this volume.

E. P. ADAMS

Princeton, New Jersey 
COLLECTIONS OF MATHEMATICAL FORMULAE, ETC.

B: O. PeIrce: A Short Table of Integrals; Boston, I899.

G. Petit Bors: Tables d'Integrales Indefinies, Paris, Igo6.

T. J. I’A. Bromwich: Elementary Integrals, Cambridge, Igri.

D. Bierens de HaAn: Nouvelles Tables d'Integrales Definies, Leiden, I867.

E. JAHNKE and F. EMDE: Funktionentafeln mit Formeln und Kurven, Leipzig, I909.

G. S. CARR: A Synopsis of Elementary Results in Pure and Applied Mathematics, London, 1880.

W. LASKA: Sammlung von Formeln der reinen und angewandten Mathematik, . Braunschweig, I888-1894.

W. Ligowski: Taschenbuch der Mathematik, Berlin, I893.

O. TH. BürkLEN: Formelsammlung und Repetitorium der Mathematik, Berlin, 1922.

F. AUERBACH: Taschenbuch fur Mathematiker und Physiker, I. Jahrgang, I909. Leipzig, I909. 



\section{GONTENTS}

PAGE

SyMbOLS . . . . . . . . . . . . . . . . . . . . viii

I. AlgebrA . . . . . . . . . . . . . . . . . I

II. GeOMETRY . . . . . . . . . . . . . . . . . . . 29

III. TRIgONOMETRY . . . . . . . . . . . . . . . . . . . 6 I

IV. Vector ANAlysis . . . . . . . . . . . . . . . . . . 9I

V. Curvilinear Coördinates . . . . . . . . . . . . . . . 99

VI. Infinite Series. . . . . . . . . . . . . . . . . . . . . IO9

VII. Special Applications of Analysis . . . . . . . . . . . I45

VIII. Differential Equations . . . . . . . . . . . . . . I62

IX. Differential Equations (Continued) . . . . . . . . . . igi

X. Numerical Solution of Differential Equations . . . . . 220

XI. Elliptic Functions . . . . . . . . . . . . . . . . . . . 243

Introduction by Sir George Greenhill, F.R.S. . . . . . . . 245

Tables of Elliptic Functions, by Col. R. L. Hippisley . . . 259

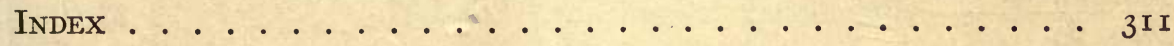




\section{SYMBOLS}

$\log$ logarithm. Whenever used the Naperian iogarithm is understood.

To find the common logarithm to base ro:

$$
\begin{aligned}
& \log _{10} a=0.43429 \ldots \log a . \\
& \log a=2.30259 \ldots \log _{10} a .
\end{aligned}
$$

! Factorial. $n$ ! where $n$ is an integer denotes I.2.3.4.....n Equivalent notation $\llcorner n$

$\neq \quad$ Does not equal.

$>\quad$ Greater than.

$<\quad$ Less than.

$\geqslant \quad$ Greater than, or equal to.

$\leqslant \quad$ Less than, or equal to.

$\left(\begin{array}{l}n \\ k\end{array}\right) \quad$ Binomial coefficient. See 1.51 .

Approaches.

$\left|a_{i k}\right|$ Determinant where $a_{i k}$ is the element in the $i$ th row and $k$ th column, $\frac{\partial\left(u_{1}, u_{2}, \ldots .\right)}{\partial\left(x_{1}, x_{2} \ldots . .\right)}$ Functional determinant. See 1.37.

$|a|$ Absolute value of $a$. If $a$ is a real quantity its numerical value, without regard to sign. If $a$ is a complex quantity, $a=\alpha+i \beta$, $|a|=$ modulus of $a=+\sqrt{\alpha^{2}+\beta^{2}}$.

$i \quad$ The imaginary $=+\sqrt{-\mathrm{I}}$.

$\sum \quad$ Sign of summation, i.e., $\sum_{k=1}^{k=n} a_{k}=a_{1}+a_{2}+a_{3}+\ldots+a_{n}$.

$\prod$ Product, i.e., $\prod_{k=\mathrm{I}}^{k=n}(\mathrm{I}+k x)=(\mathrm{I}+x)(\mathrm{I}+2 x)(\mathrm{I}+3 x) \ldots(\mathrm{I}+n x)$. 


\section{ALGEBRA}

1.00 Algebraic Identities.

I. $a^{n}-b^{n}=(a-b)\left(a^{n-1}+a^{n-2} b+a^{n-3} b^{2}+\ldots \ldots+a b^{n-2}+b^{n-1}\right)$.

2. $a^{n} \pm b^{n}=(a+b)\left(a^{n-1}-a^{n-2} b+a^{n-3} b^{2}-\ldots \ldots \mp a b^{n-2} \pm b^{n-1}\right)$.

\section{$n$ odd: upper sign. \\ $n$ even: lower sign.}

3. $\left(x+a_{1}\right)\left(x+a_{2}\right) \ldots \ldots\left(x+a_{n}\right)=x^{n}+P_{1} x^{n-1}+P_{2} x^{n-2}+\ldots$. $+P_{n-1} x+P_{n}$.

$P_{1}=a_{1}+a_{2}+\ldots \ldots+a_{n}$.

$P_{k}=$ sum of all the products of the $a$ 's taken $k$ at a time. $P_{n}=a_{1} a_{2} a_{3} \ldots a_{n}$.

4. $\left(a^{2}+b^{2}\right)\left(a^{2}+\beta^{2}\right)=(a a \mp b \beta)^{2}+(a \beta \pm b a)^{2}$.

5. $\left(a^{2}-b^{2}\right)\left(a^{2}-\beta^{2}\right)=(a \alpha \pm b \beta)^{2}-(a \beta \pm b a)^{2}$.

6. $\left(a^{2}+b^{2}+c^{2}\right)\left(\alpha^{2}+\beta^{2}+\gamma^{2}\right)=(a a+b \beta+c \gamma)^{2}+(b \gamma-\beta c)^{2}+(c a-\gamma a)^{2}$

$$
+(a \beta-a b)^{2} \text {. }
$$

7. $\left(a^{2}+b^{2}+c^{2}+d^{2}\right)\left(a^{2}+\beta^{2}+\gamma^{2}+\delta^{2}\right)=(a \alpha+b \beta+c \gamma+d \delta)^{2}$

$$
+(a \beta-b a+c \delta-d \gamma)^{2}+(a \gamma-b \delta-c a+d \beta)^{2}+(a \delta+b \gamma-c \beta-d a)^{2} \text {. }
$$

8. $(a c-b d)^{2}+(a d+b c)^{2}=(a c+b d)^{2}+(a d-b c)^{2}$.

9. $(a+b)(b+c)(c+a)=(a+b+c)(a b+b c+c a)-a b c$.

Iо. $(a+b)(b+c)(c+a)=a^{2}(b+c)+b^{2}(c+a)+c^{2}(a+b)+2 a b c$.

II. $(a+b)(b+c)(c+a)=b c(b+c)+c a(c+a)+a b(a+b)+2 a b c$.

2. $3(a+b)(b+c)(c+a)=(a+b+c)^{3}-\left(a^{3}+b^{3}+c^{3}\right)$.

3. $(b-a)(c-a)(c-b)=a^{2}(c-b)+b^{2}(a-c)+c^{2}(b-a)$.

I4. $(b-a)(c-a)(c-b)=a\left(b^{2}-c^{2}\right)+b\left(c^{2}-a^{2}\right)+c\left(a^{2}-b^{2}\right)$.

I5. $(b-a)(c-a)(c-b)=b c(c-b)+c a(a-c)+a b(b-a)$.

16. $(a-b)^{2}+(b-c)^{2}+(c-a)^{2}=2[(a-b)(a-c)+(b-a)(b-c)$

$$
+(c-a)(c-b)] \text {. }
$$

I7. $a^{3}\left(b^{2}-c^{2}\right)+b^{3}\left(c^{2}-a^{2}\right)+c^{3}\left(a^{2}-b^{2}\right)=(a-b)(b-c)(a-c)(a b+b c+c a)$. I8. $(a+b+c)\left(a^{2}+b^{2}+c^{2}\right)=b c(b+c)+c a(c+a)+a b(a+b)+a^{3}+b^{3}+c^{3}$. 9. $(a+b+c)(b c+c a+a b)=a^{2}(b+c)+b^{2}(c+a)+c^{2}(a+b)+3 a b c$. 20. $(b+c-a)(c+a-b)(a+b-c)=a^{2}(b+c)+b^{2}(c+a)+c^{2}(a+b)$ $-\left(a^{3}+b^{3}+c^{3}+2 a b c\right)$. 
2. $(a+b+c)(-a+b+c)(a-b+c)(a+b-c)=2\left(b^{2} c^{2}+c^{2} a^{2}+a^{2} b^{2}\right)$

$$
-\left(a^{4}+b^{4}+c^{4}\right) \text {. }
$$

22. $(a+b+c+d)^{2}+(a+b-c-d)^{2}+(a+c-b-d)^{2}+(a+d-b-c)^{2}$

$$
\begin{aligned}
=4\left(a^{2}+b^{2}+c^{2}+d^{2}\right) . \\
\text { If } A=a \alpha+b \gamma+c \beta \\
B=a \beta+b a+c \gamma \\
C=a \gamma+b \beta+c \alpha
\end{aligned}
$$

23. $(a+b+c)(a+\beta+\gamma)=A+B+C$.

24. $\left[a^{2}+b^{2}+c^{2}-(a b+b c+c a)\right]\left[a^{2}+\beta^{2}+\gamma^{2}-(\alpha \beta+\beta \gamma+\gamma a)\right]$

$$
=A^{2}+B^{2}+C^{2}-(A B+B C+C A) \text {. }
$$

25. $\left(a^{3}+b^{3}+c^{3}-3 a b c\right)\left(a^{3}+\beta^{3}+\gamma^{3}-3 a \beta \gamma\right)=A^{3}+B^{3}+C^{3}-3^{A B C}$.

1.200 The expression

\section{ALGEBRAIC EQUATIONS}

$$
f(x)=a_{0} x^{n}+a_{1} x^{n-1}+a_{2} x^{n-2}+\ldots . .+a_{n-1} x+a_{n}
$$

is an integral rational function, or a polynomial, of the $n$th degree in $x$.

1.201 The equation $f(x)=0$ has $n$ roots which may be real or complex, distinct or repeated.

1.202 If the roots of the equation $f(x)=0$ are $c_{1}, c_{2}, \ldots, c_{n}$,

$$
f(x)=a_{0}\left(x-c_{1}\right)\left(x-c_{2}\right) \ldots\left(x-c_{n}\right)
$$

1.203 Symmetric functions of the roots are expressions giving certain combinations of the roots in terms of the coefficients. Among the more important are :

$$
\begin{aligned}
& c_{1}+c_{2}+\ldots \ldots+c_{n}=-\frac{a_{1}}{a_{0}} \\
& c_{1} c_{2}+c_{1} c_{3}+\ldots+c_{2} c_{3}+c_{2} c_{4}+\ldots \ldots+c_{n-1} c_{n}=\frac{a_{2}}{a_{0}} \\
& c_{1} c_{2} c_{3}+c_{1} c_{2} c_{4}+\ldots+c_{1} c_{3} c_{4}+\ldots \ldots+c_{n-2} c_{n-1} c_{n}=-\frac{a_{3}}{a_{0}} \\
& \ldots \ldots \\
& \ldots \ldots c_{n}=(-1)^{n} \frac{a_{n}}{a_{0}}
\end{aligned}
$$

1.204 Newton's Theorem. If $s_{k}$ denotes the sum of the $k$ th powers of all the roots of $f(x)=0$,

$$
\begin{aligned}
& s_{k}=c_{1}^{k}+c_{2}^{k}+\ldots . . .+c_{n}^{k} \\
& \text { I } a_{1}+s_{1} a_{0}=0 \\
& 2 a_{2}+s_{1} a_{1}+s_{2} a_{0}=0 \\
& 3 a_{3}+s_{1} a_{2}+s_{2} a_{1}+s_{3} a_{0}=0 \\
& 4 a_{4}+s_{1} a_{3}+s_{2} a_{2}+s_{3} a_{1}+s_{4} a_{0}=0 \\
& \ldots \ldots \\
& \ldots \ldots
\end{aligned}
$$


or:

$$
\begin{aligned}
& s_{1}=-\frac{a_{1}}{a_{0}} \\
& s_{2}=-\frac{2 a_{2}}{a_{0}}+\frac{a_{1}^{2}}{a_{0}^{2}} \\
& s_{3}=-\frac{3 a_{3}}{a_{0}}+\frac{3 a_{1} a_{2}}{a_{0}^{2}}-\frac{a_{1}^{3}}{a_{0}^{3}} \\
& s_{4}=-\frac{4 a_{4}}{a_{0}}+\frac{4 a_{1} a_{3}}{a_{0}^{2}}-\frac{4 a_{1}^{2} a_{2}}{a_{0}^{3}}+\frac{2 a_{2}^{2}}{a_{0}^{2}}+\frac{a_{1}^{4}}{a_{0}^{4}} \\
& \ldots . . .
\end{aligned}
$$

1.205 If $S_{k}$ denotes the sum of the reciprocals of the $k$ th powers of all the roots of the equation $f(x)=0$ :

$$
\begin{aligned}
& S_{k}=\frac{\mathrm{I}}{c_{1}{ }^{k}}+\frac{\mathrm{I}}{c_{2}{ }^{k}}+\ldots .+\frac{\mathrm{I}}{c_{n}{ }^{k}} \\
& \mathbf{r} a_{n-1}+S_{1} a_{n}=0 \\
& 2 a_{n-2}+S_{1} a_{n-1}+S_{2} a_{n}=0 \\
& 3 a_{n-3}+S_{1} a_{n-2}+S_{2} a_{n-1}+S_{3} a_{n}=0 \\
& \text { … } \\
& \text {.... } \\
& S_{1}=-\frac{a_{n-1}}{a_{n}} \\
& S_{2}=-\frac{2 a_{n-2}}{a_{n}}+\frac{a^{2}{ }_{n-1}}{a_{n}^{2}} \\
& S_{3}=-\frac{3 a_{n-3}}{a_{n}}+\frac{3 a_{n-1} a_{n-2}}{a_{n}^{2}}-\frac{a^{3}{ }_{n-1}}{a^{3}{ }_{n}} \\
& \text {........ }
\end{aligned}
$$

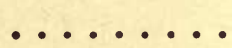

1.220 If $f(x)$ is divided by $x-h$ the result is

$$
f(x)=(x-h) Q+R .
$$

$Q$ is the quotient and $R$ the remainder. This operation may be readily performed as follows:

Write in line the values of $a_{0}, a_{1}, \ldots, a_{n}$. If any power of $x$ is missing write $\circ$ in the corresponding place. Multiply $a_{0}$ by $h$ and place the product in the second line under $a_{1}$; add to $a_{1}$ and place the sum in the third line under $a_{1}$. Multiply this sum by $h$ and place the product in the second line under $a_{2}$; add to $a_{2}$ and place the sum in the third line under $a_{2}$. Continue this series of operations until the third line is full. The last term in the third line is the remainder, $R$. The first term in the third line, which is $a_{0}$, is the coefficient of $x^{n-1}$ in the quotient, $Q$; the second term is the coefficient of $x^{n-2}$, and so on. 
1.221 It follows from 1.220 that $f(h)=R$. This gives a convenient way of evaluating $f(x)$ for $x=h$.

1.222 To express $f(x)$ in the form:

$$
f(x)=A_{0}(x-h)^{n}+A_{1}(x-h)^{n-1}+\ldots .+A_{n-1}(x-h)+A_{n} .
$$

By 1.220 form $f(h)=A_{n}$. Repeat this process with each quotient, and the last term of each line of sums will be a succeeding value of the series of coefficients $A_{n}, A_{n-1}, \ldots . ., A_{0}$.

Example:

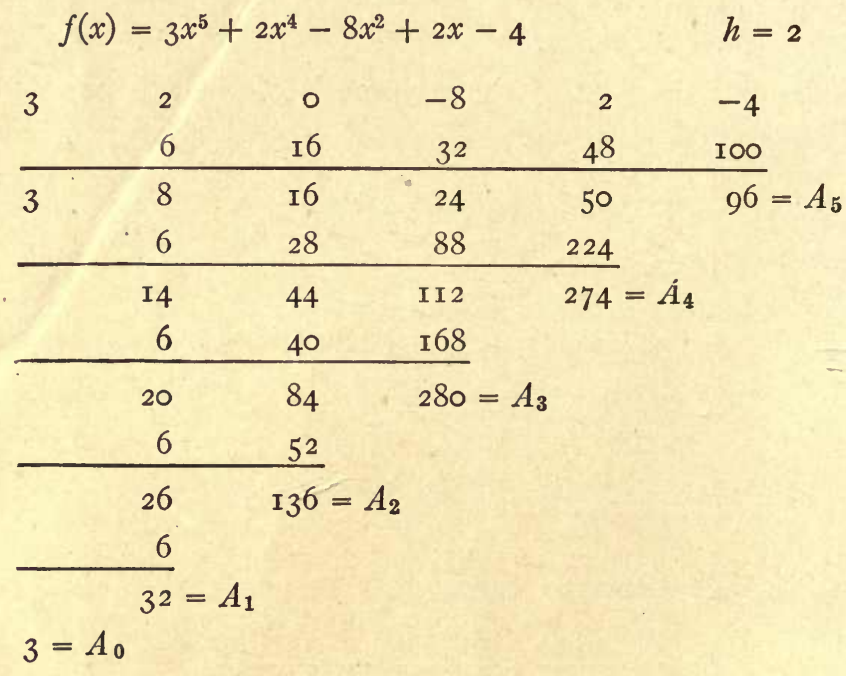

Thus :

$$
\begin{aligned}
Q & =3 x^{4}+8 x^{3}+16 x^{2}+24 x+50 \\
R & =f(2)=96 \\
f(x) & =3(x-2)^{5}+32(x-2)^{4}+136(x-2)^{3}+280(x-2)^{2}+274(x-2)+96
\end{aligned}
$$

1.230 To transform the equation $f(x)=0$ into one whose roots all have their signs changed: Substitute $-x$ for $x$.

1.231 To transform the equation $f(x)=0$ into one whose roots are all multiplied by a constant, $m$ : Substitute $x / m$ for $x$.

1.232 To transform the equation $f(x)=0$ into one whose roots are the reciprocals of the roots of the given equation: Substitute $\mathrm{I} / x$ for $x$ and multiply by $x^{n}$.

1.233 To transform the equation $f(x)=0$ into one whose roots are all increased or diminished by a constant, $h$ : Substitute $x \pm h$ for $x$ in the given equation, 
the upper sign being used if the roots are to be diminished and the lower sign if they are to be increased. The resulting equation will be:

$$
f( \pm h)+x f^{\prime}( \pm h)+\frac{x^{2}}{2 !} f^{\prime \prime}( \pm h)+\frac{x^{3}}{3 !} f^{\prime \prime \prime}( \pm h)+\ldots .=0
$$

where $f^{\prime}(x)$ is the first derivative of $f(x), f^{\prime \prime}(x)$, the second derivative, etc. The resulting equation may also be written:

$$
A_{0} x^{n}+A_{1} x^{n-1}+A_{2} x^{n-2}+\ldots \ldots+A_{n-1} x+A_{n}=0
$$

where the coefficients may be found by the method of 1.222 if the roots are to be diminished. To increase the roots by $h$ change the sign of $h$.

\section{MULTIPLE ROOTS}

1.240 If $c$ is a multiple root of $f(x)=\circ$, of order $m$, i.e.. repeated $m$ times, then

$$
f(x)=(x-c)^{m} Q ; \quad R=0
$$

$c$ is also a multiple root of order $m-\mathbf{I}$ of the first derived equation, $f^{\prime}(x)=0$; of order $m-2$ of the second derived equation, $f^{\prime \prime}(x)=0$, and so on.

1.241 The equation $f(x)=0$ will have no multiple roots if $f(x)$ and $f^{\prime}(x)$ have no common divisor. If $F(x)$ is the greatest common divisor of $f(x)$ and $f^{\prime}(x)$, $f(x) / F(x)=f_{1}(x)$, and $f_{1}(x)$ will have no multiple roots:

1.250 An equation of odd degree, $n$, has at least one real root whose sign is opposite to that of $a_{n}$.

1.251 An equation of even degree, $n$, has one positive and one negative real root if $a_{n}$ is negative.

1.252 The equation $f(x)=0$ has as many real roots between $x=x_{1}$ and $x=x_{2}$ as there are changes of sign in $f(x)$ between $x_{1}$ and $x_{2}$.

1.253 Déscartes' Rule of Signs: No equation can have more positive roots than it has changes of sign from + to - and from - to +, in the terms of $f(x)$. No equation can have more negative roots than there are changes of sign in $f(-x)$. 1.254 If $f(x)=0$ is put in the form

$$
A_{0}(x-h)^{n}+A_{1}(x-h)^{n-1}+\ldots \ldots+A_{n}=0
$$

by 1.222 , and $\dot{A}_{0}, A_{1}, \ldots \ldots, A_{n}$ are all positive, $h$ is an upper limit of the positive roots.

If $f(-x)=0$ is put in a similar form, and the coefficients are all positive, $h$ is a lower limit of the negative roots. 
If $f(\mathrm{I} / x)=0$ is put in a similar form, and the coefficients are all positive, $h$ is a lower limit of the positive roots. And with $f(-\mathrm{I} / x)=0, h$ is an upper limit of the negative roots.

1.255 Sturm's Theorem. Form the functions:

$$
\begin{aligned}
& f(x)=a_{0} x^{n}+a_{1} x^{n-1}+a_{2} x^{n-2}+\ldots+a_{n} \\
& f_{1}(x)=f^{\prime}(x)=n a_{0} x^{n-1}+(n-1) a_{1} x^{n-2}+\ldots+a_{n-1} \\
& f_{2}(x)=-R_{1} \text { in } f(x)=Q_{1} f_{1}(x)+R_{1} \\
& f_{3}(x)=-R_{2} \text { in } f_{1}(x)=Q_{2} f_{2}(x)+R_{2}
\end{aligned}
$$

The number of real roots of $f(x)=0$ between $x=x_{1}$ and $x=x_{2}$ is equal to the number of changes of sign in the series $f(x), f_{1}(x), f_{2}(x), \ldots$ when $x_{1}$ is substituted for $x$ minus the number of changes of sign in the same series when $x_{2}$ is substituted for $x$. In forming the functions $f_{1}, f_{2}, \ldots$ numerical factors may be introduced or suppressed in order to remove fractional coefficients.

Example:

$$
\begin{aligned}
& f(x)=x^{4}-2 x^{3}-3 x^{2}+\text { I0 } x-4 \\
& f_{1}(x)=2 x^{3}-3 x^{2}-3 x+5 \\
& f_{2}(x)=9 x^{2}-27 x+\text { II } \\
& f_{3}(x)=-8 x-3 \\
& f_{4}(x)=- \text { I } 433
\end{aligned}
$$

$\begin{array}{lllllll} & f & f_{1} & f_{2} & f_{3} & f_{4} & \\ x=-\infty & + & - & + & + & - & 3 \text { changes } \\ x=0 & - & + & + & - & - & 2 \text { changes } \\ x=+\infty & + & + & + & - & - & \text { I change }\end{array}$

Therefore there is one positive and one negative real root.

If it can be seen that all the roots of any one of Sturm's functions are imaginary it is unnecessary to calculate any more of them after that one.

If there are any multiple roots of 'the equation $f(x)=0$ the series of Sturm's functions will terminate with $f_{r}, r<n . f_{r}(x)$ is the highest common factor of $f$ and $f_{1}$. In this case the number of real roots of $f(x)=0$ lying between $x=x_{1}$ and $x=x_{2}$, each multiple root counting only once, will be the difference between the number of changes of sign in the series $f, f_{1}, f_{2}, \ldots, f_{r}$ when $x_{1}$ and $x_{2}$ are successively substituted in them.

1.256 Routh's rule for finding the number of roots whose real parts are positive. (Rigid Dynamics, Part II, Art. 297.)

Arrange the coefficients in two rows:

\section{$x^{n}$}

$x^{n-1}$

$$
a_{0}
$$$$
a_{1}
$$

$a_{2}$

$a_{3}$ 
Form a third row by cross-multiplication:
$x^{n-2}$
$\frac{a_{1} a_{2}-a_{0} a_{3}}{a_{1}}$
$\frac{a_{1} a_{4}-a_{0} a_{5}}{a_{1}}$
$\frac{a_{1} a_{6}-a_{0} a_{7}}{a_{1}}$

Form a fourth row by operating on these last two rows by a similar crossmultiplication. Continue this operation until there are no terms left. The number of variations of sign in the first.column gives the number of roots whose real parts are positive.

If there are any equal roots some of the subsidiary functions will vanish. In place of one which vanishes write the differential coefficient of the last one which does not vanish and proceed in the same way. At the left of each row is written the power of $x$ corresponding to the first subsidiary function in that row. This power diminishes by 2 for each succeeding coefficient in the row.

Any row may be multiplied or divided by any positive quantity in order to remove fractions.

\section{DETERMINATION OF THE ROOTS OF AN EQUATION}

1.260 Newton's Method. If a root of the equation $f(x)=0$ is known to lie between $x_{1}$ and $x_{2}$ its value can be found to any desired degree of approximation by Newton's method. This method can be applied to transcendental equations as well as to algebraic equations.

If $b$ is an approximate value of a root,

$$
\begin{aligned}
& b-\frac{f(b)}{f^{\prime}(b)}=c \text { is a second approximation, } \\
& c-\frac{f(c)}{f^{\prime}(c)}=d \text { is a third approximation. }
\end{aligned}
$$

This process may be repeated indefinitely.

1.261 Horner's Method for approximating to the real roots of $f(x)=0$.

Let $p_{1}$ be the first approximation, such that $p_{1}+I>c>p_{1}$, where $c$ is the root sought. The equation can always be transformed into one in which this condition holds by multiplying or dividing the roots by some power of ro by 1.231. Diminish the roots by $p_{1}$ by 1.233. In the transformed equation

$$
A_{0}\left(x-p_{2}\right)^{n}+A_{1}\left(x-p_{1}\right)^{n-1}+\ldots .+A_{n-1}\left(x-p_{1}\right)+A_{n}=0
$$

put

$$
\frac{p_{2}}{\text { IO }}=\frac{A_{n}}{A_{n-1}}
$$

and diminish the roots by $p_{2} /$ Io, yielding a second transformed equation

$$
B_{0}\left(x-p_{1}-\frac{p_{2}}{\mathrm{IO}}\right)^{n}+B_{1}\left(x-p_{1}-\frac{p_{2}}{\mathrm{IO}}\right)^{n-1}+\ldots .+B_{n}=0 .
$$


If $B_{n}$ and $B_{n-1}$ are of the same sign $p_{2}$ was taken too large and must be diminished. Then take

$$
\frac{p_{3}}{100}=\frac{B_{n}}{B_{n-1}}
$$

and continue the operation. The required root will be:

$$
c=p_{1}+\frac{p_{2}}{10}+\frac{p_{3}}{100}+\ldots . .
$$

1.262 Graeffe's Method. This method determines approximate values of all the roots of a numerical equation, complex as well as real. Write the equation of the $n$th degree

$$
f(x)=a_{0} x^{n}-a_{1} x^{n-1}+a_{2} x^{n-2}-\ldots \pm a_{n}=0 .
$$

The product

$$
f(x) \cdot f(-x)=A_{0} x^{2 n}-A_{1} x^{2 n-2}+A_{2} x^{2 n-4}-\ldots . \pm A_{n}=0
$$

contains only even powers of $x$. It is an equation of the $n$th degree in $x^{2}$. The coefficients are determined by

$$
\begin{aligned}
& A_{0}=a_{0}^{2} \\
& A_{1}=a_{1}^{2}-2 a_{0} a_{2} \\
& A_{2}=a_{2}^{2}-2 a_{1} a_{3}+2 a_{0} a_{4} \\
& A_{3}=a_{3}^{2}-2 a_{2} a_{4}+2 a_{1} a_{5}-2 a_{0} a_{6} \\
& A_{4}=a_{4}^{2}-2 a_{3} a_{5}+2 a_{2} a_{6}-2 a_{1} a_{7}+2 a_{0} a_{8} \\
& \ldots \ldots \ldots
\end{aligned}
$$

The roots of the equation

$$
A_{0} y^{n}-A_{1} y^{n-1}+A_{2} y^{n-2}-\ldots . \pm A_{n}=0
$$

are the squares of the roots of the given equation. Continuing this process we get an equation

$$
R_{0} u^{n}-R_{1} u^{n-1}+R_{2} u^{n-2}-\ldots \ldots \pm R_{n}=0
$$

whose roots are the $2^{r}$ th powers of the roots of the given equation. Put $\lambda=2^{r}$. Let the roots of the given equation be $c_{1}, c_{2}, \ldots, c_{n}$. Suppose first that

$$
c_{1}>c_{2}>c_{3}>\ldots \ldots>c_{n}
$$

Then for large values of $\lambda$,

$$
c_{1}{ }^{\lambda}=\frac{R_{1}}{R_{0}}, \quad c_{2}{ }^{\lambda}=\frac{R_{2}}{R_{1}}, \quad \ldots ., \quad c_{n}{ }^{\lambda}=\frac{R_{n}}{R_{n-1}} .
$$

If the roots are real they may be determined by extracting the $\lambda$ th roots of these quantities. Whether they are \pm is determined by taking the sign which approximately satisfies the equation $f(x)=0$.

Suppose next that complex roots enter so that there are equalities among the absolute values of the roots. Suppose that

$$
\begin{gathered}
\left|c_{1}\right| \geqslant\left|c_{2}\right| \geqslant\left|c_{3}\right| \geqslant \ldots \ldots\left|c_{p}\right| ; \quad\left|c_{p}\right|>\left|c_{p+1}\right| ; \\
\left|c_{p+1}\right| \geqslant\left|c_{p+2}\right| \geqslant \ldots \geqslant\left|c_{n}\right|
\end{gathered}
$$






Then if $\lambda$ is large enough so that $c_{p}{ }^{\lambda}$ is large compared to $c_{p+1}{ }^{\lambda}, c_{1}{ }^{\lambda}, c_{2}{ }^{\lambda}, \ldots \ldots$ $c_{p}{ }^{\lambda}$ approximately satisfy the equation:

$$
R_{0} u^{p}-R_{1} u^{p-1}+R_{2} u^{p-2}-\ldots \ldots \pm R_{p}=0
$$

and $c_{p+1}{ }^{\lambda}, c_{p+2}{ }^{\lambda}, \ldots, c_{n}{ }^{\lambda}$ approximately satisfy the equation:

$$
R_{p} u^{n-p}-R_{p+1} u^{n-p-1}+R_{p+2} u^{n-p-2}-\ldots \pm R_{n}=\text { ○. }
$$

Therefore when $\lambda$ is large enough the given equation breaks down into a number of simpler equations. This stage is shown in the process of deriving the successive equations when certain of the coefficients are obtained from those of the preceding equation simply by squaring.

REFERENCES: Encyklopadie der Math. Wiss. I, I, 3a (Runge). BAIRstow: Applied Aerodynamics, pp. 553-560; the solution of a numerical equation of the 8 th degree is given by Graeffe's Method.

1.270 Quadratic Equations.

$$
x^{2}+2 a x+b=0
$$

The roots are:

$$
\begin{aligned}
x_{1} & =-a+\sqrt{a^{2}-b} \\
x_{2} & =-a-\sqrt{a^{2}-b} \\
x_{1}+x_{2} & =-2 a \\
x_{1} x_{2} & =b .
\end{aligned}
$$

If

$a^{2}>b$ roots are real, $a^{2}<b$ roots are complex, $a^{2}=b$ roots are equal.

1.271 Cubic equations.

(I) $x^{3}+a x^{2}+b x+c=0$.

Substitute

where

(2) $x=y-\frac{a}{3}$

(3) $y^{3}-3 p y-2 q=0$

$$
\begin{aligned}
& 3 p=\frac{a^{2}}{3}-b \\
& 2 q=\frac{a b}{3}-\frac{2}{27} a^{3}-c
\end{aligned}
$$

Roots of (3):

If $p>0, q>0, q^{2}>p^{3}$

$$
\cosh \phi=\frac{q}{\sqrt{p^{3}}}
$$




$$
\begin{aligned}
& y_{1}=2 \sqrt{p} \cosh \frac{\phi}{3} \\
& y_{2}=-\frac{y_{1}}{2}+i \sqrt{3 p} \sinh \frac{\phi}{3} \\
& y_{3}=-\frac{y_{1}}{2}-i \sqrt{3 p} \sinh \frac{\phi}{3} .
\end{aligned}
$$

If $p>0, q<0, q^{2}>p^{3}$,

$$
\begin{aligned}
\cosh \phi & =\frac{-q}{\sqrt{p^{3}}} \\
y_{1} & =-2 \sqrt{p} \cosh \frac{\phi}{3} \\
y_{2} & =-\frac{y_{1}}{2}+i \sqrt{3 p} \sinh \frac{\phi}{3} \\
y_{3} & =-\frac{y_{1}}{2}-i \sqrt{3 p} \sinh \frac{\phi}{3} .
\end{aligned}
$$

If $p<0$

$$
\begin{aligned}
\sinh \phi & =\frac{q}{\sqrt{-p^{3}}} \\
y_{1} & =2 \sqrt{-p} \sinh \frac{\phi}{3} \\
y_{2} & =-\frac{y_{1}}{2}+i \sqrt{-3 p} \cosh \frac{\phi}{3} \\
y_{3} & =-\frac{y_{1}}{2}-i \sqrt{-3 p} \cosh \frac{\phi}{3} .
\end{aligned}
$$

If $p>0, q^{2}<p^{3}$

$$
\begin{aligned}
\cos \phi & =\frac{q}{\sqrt{p^{3}}} \\
y_{1} & =2 \sqrt{p} \cos \frac{\phi}{3} \\
y_{2} & =-\frac{y_{1}}{2}+\sqrt{3 p} \sin \frac{\phi}{3} \\
y_{3} & =-\frac{y_{1}}{2}-\sqrt{3 p} \sin \frac{\phi}{3} .
\end{aligned}
$$

1.272 Biquadratic equations.

Substitute

$$
a_{0} x^{4}+a_{1} x^{3}+a_{2} x^{2}+a_{3} x+a_{4}=0 .
$$

$$
\begin{gathered}
x=y-\frac{a_{1}}{a_{0}} \\
y^{4}+\frac{6}{a_{0}^{2}} H y^{2}+\frac{4}{a_{0}^{3}} G y+\frac{I}{a_{0}^{4}} F=0
\end{gathered}
$$




$$
\begin{aligned}
& H=a_{0} a_{2}-a_{1}^{2} \\
& G=a_{0}^{2} a_{3}-3 a_{0} a_{1} a_{2}+2 a_{1}^{3} \\
& F=a_{0}^{3} a_{4}-4 a_{0}^{2} a_{1} a_{3}+6 a_{0} a_{1}^{2} a_{2}-3 a_{1}^{4} \\
& I=a_{0} a_{4}-4 a_{1} a_{3}+3 a_{2}^{2} \\
& F=a_{0}^{2} I-3 H^{2} \\
& J=a_{0} a_{2} a_{4}+2 a_{1} a_{2} a_{3}-a_{0} a_{3}^{2}-a_{1}^{2} a_{4}-a_{2}^{3} \\
& \triangle=I^{3}-27 J^{2}=\text { the discriminant } \\
& G^{2}+{ }_{4} H^{3}=a_{0}^{2}\left(H I-a_{0} J\right) .
\end{aligned}
$$

Nature of the roots of the biquadratic:

$\Delta=0 \quad$ Equal roots are present

Two roots only equal: $I$ and $J$ are not both zero

Three roots are equal: $I=J=0$

Two distinct pairs of equal roots: $G=0 ; \quad a_{0}^{2} I-\mathrm{I}_{2} H^{2}=0$

Four roots equal: $H=I=J=0$.

$\Delta<0$ Two real and two complex roots

$\Delta>0$ Roots are either all real or all complex:

$H<0$ and $a_{0}^{2} I-\mathrm{I}_{2} H^{2}<0$ Roots all real

$H>0$ and $a_{0}^{2} I-\mathrm{I}_{2} H^{2}>0$ Roots all complex.

\section{DETERMINANTS}

1.300 A determinant of the $n$th order, with $n^{2}$ elements, is written:

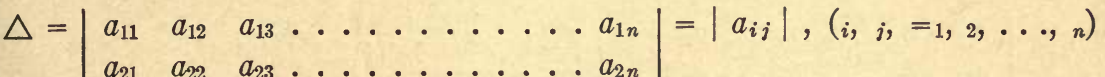

$$
\begin{aligned}
& a_{31} \quad a_{32} \quad a_{33} \ldots \ldots \ldots \ldots a_{3 n} \\
& \ldots \ldots \ldots \ldots \ldots \ldots \\
& \ldots \ldots \ldots \ldots \ldots
\end{aligned}
$$

1.301 A determinant is not changed in value by writing rows for columns and columns for rows.

1.302 If two columns or two rows of a determinant are interchanged the resulting determinant is unchanged in value but is of the opposite sign.

1.303 A determinant vanishes if it has two equal columns or two equal rows.

1.304 If each element of a row or a column is multiplied by the same factor the determinant itself is multiplied by that factor. 
1.305 A determinant is not changed in value if to each element of a row or column is added the corresponding element of another row or column multiplied by a common factor.

1.306 If each element of the $l$ th row or column consists of the sum of two or more terms the determinant splits up into the sum of two or more determinants having for elements of the $l$ th row or column the separate terms of the $l$ th row or column of the given determinant.

1.307 If corresponding elements of two rows or columns of a determinant have a constant ratio the determinant vanishes.

1.308 If the ratio of the differences of corresponding elements in the $p$ th and $q$ th rows or columns to the differences of corresponding elements in the $r$ th and $s$ th rows or columns be constant the determinant vanishes.

1.309 If $p$ rows or columns of a determinant whose elements are rational integral functions of $x$ become equal or proportional when $x=h$, the determinant is divisible by $(x-h)^{p-1}$.

\section{MULTIPLICATION OF DETERMINANTS}

1.320 Two determinants of equal order may be multiplied together by the scheme:

where

$$
\left|a_{i j}\right| \times\left|b_{i j}\right|=\left|c_{i j}\right|
$$

$$
c_{i j}=a_{i 1} b_{j 1}+a_{i 2} b_{j 2}+\ldots \ldots+a_{i n} b_{j n} .
$$

1.321 If the two determinants to be multiplied are of unequal order the one of lower order can be raised to one of equal order by bordering it; i.e.:

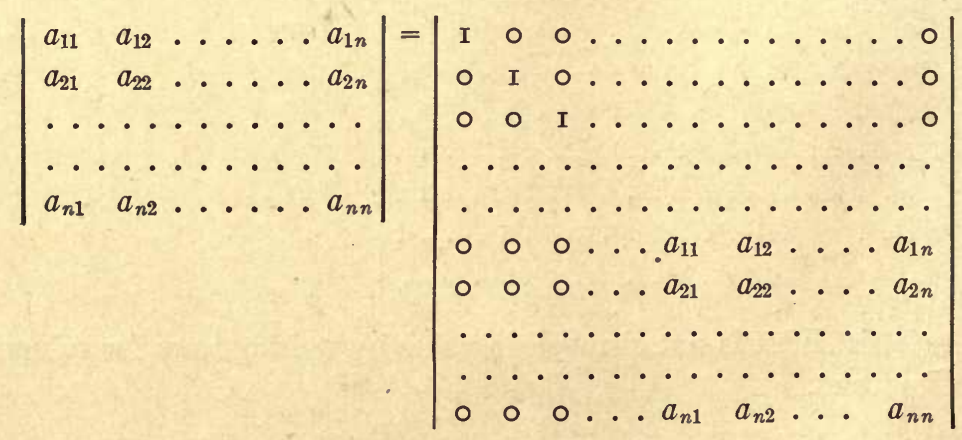

1.322 The product of two determinants may be written:

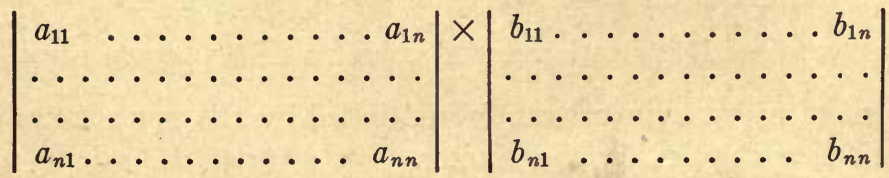




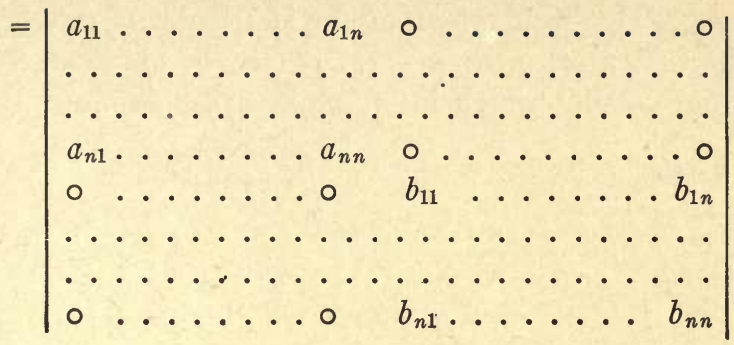

\section{DIFFERENTIATION OF DETERMINANTS}

1.330 If the elements of a determinant, $\Delta$, are functions of a variable, $t$ :

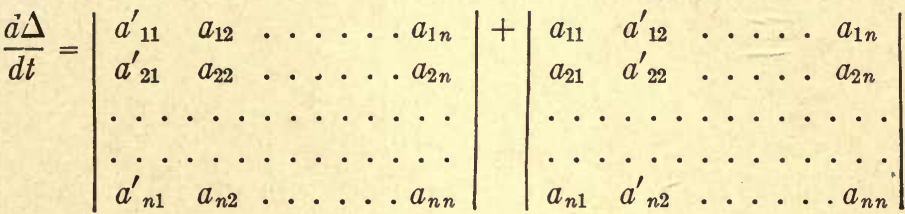

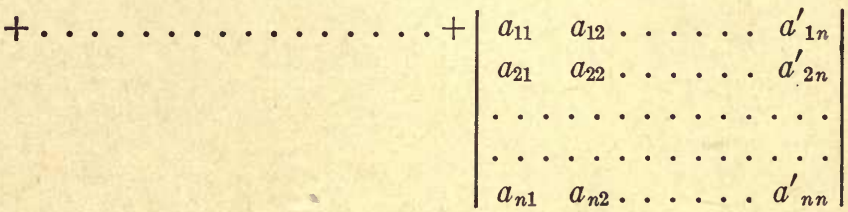

where the accents denote differentiation by $t$.

\section{EXPANSION OF DETERMINANTS}

1.340 The complete expansion of a determinant of the $n$th order contains $n$ ! terms. Each of these terms contains one element from each row and one element from each column. Any term may be obtained from the leading term:

$$
a_{11} a_{22} a_{33} \ldots \ldots a_{n n}
$$

by kecping the first suffixes unchanged and permuting the second suffixes among I, $2,3, \ldots, n$. The sign of any term is determined by the number of inversions from the second suffixes of the leading term, being positive if there is an even number of inversions and negative if there is an odd number of inversions.

1.341 The coefficient of $a_{i j}$ when the determinant $\Delta$ is fully expanded is:

$$
\frac{\partial \Delta}{\partial a_{i} i}=\Delta_{i j}
$$


$\Delta_{i j}$ is the first minor of the determinant $\Delta$ corresponding to $a_{i j}$ and is a determinant of order $n-\mathrm{I}$. It may be obtained from $\Delta$ by crossing out the row and column which intersect in $a_{i j}$, and multiplying by $(-\mathrm{I})^{i+j}$.

1.342

1.343

$$
\begin{aligned}
a_{i 1} \Delta_{j 1}+a_{i 2} \Delta_{j 2}+\ldots \ldots+a_{i n} \Delta_{j n} & =\frac{\circ \text { if } i \neq j}{\Delta \text { if } i=j} \\
a_{1 i} \Delta_{1 j}+a_{2 i} \Delta_{2 j}+\ldots \ldots+a_{n i} \Delta_{n j} & =\frac{\circ \text { if } i \neq j}{\Delta \text { if } i=j}
\end{aligned}
$$

$$
\frac{\partial^{2} \Delta}{\partial a_{i j} \partial a_{k l}}=\frac{\partial \Delta_{k l}}{\partial a_{i j}}=\frac{\partial \Delta_{i j}}{\partial a_{k l}}
$$

is the coefficient of $a_{i j} a_{k l}$ in the complete expansion of the determinant $\Delta$. It may be obtained from $\Delta$, except for sign, by crossing out the rows and columns which intersect in $a_{i j}$ and $a_{k l}$.

1.344

$$
\begin{aligned}
\left|\Delta_{i j}\right| \times\left|a_{i j}\right| & =\Delta^{n} \\
\left|\Delta_{i j}\right| & =\Delta^{n-1} .
\end{aligned}
$$

The determinant $\left|\Delta_{i j}\right|$ is the reciprocal determinant to $\Delta$.

\subsection{5}

$$
\Delta \cdot \frac{\partial^{2} \Delta}{\partial a_{i j} \partial a_{k l}}=\left|\begin{array}{cc}
\Delta_{i j} & \Delta_{i l} \\
\Delta_{k j} & \Delta_{k l}
\end{array}\right|=\frac{\partial \Delta}{\partial a_{i j}} \frac{\partial \Delta}{\partial a_{k l}}-\frac{\partial \Delta}{\partial a_{i l}} \frac{\partial \Delta}{\partial a_{k j}}
$$

1.346

$$
\Delta^{2} \frac{\partial^{3} \Delta}{\partial a_{i j} \partial a_{k l} \partial a_{p q}}=\left|\begin{array}{lll}
\Delta_{i j} & \Delta_{i l} & \Delta_{i q} \\
\Delta_{k j} & \Delta_{k l} & \Delta_{k q} \\
\Delta_{p j} & \Delta_{p l} & \Delta_{p q}
\end{array}\right|
$$

1.347

$$
\frac{\partial^{2} \Delta}{\partial a_{i j} \partial a_{k l}}=-\frac{\partial^{2} \Delta}{\partial a_{i l} \partial a_{k j}}
$$

1.348 If $\Delta=0$,

$$
\frac{\partial \Delta}{\partial a_{i j}} \frac{\partial \Delta}{\partial a_{k l}}=\frac{\partial \Delta}{\partial a_{i l}} \frac{\partial \Delta}{\partial a_{k j}}
$$

1.350 If $a_{i j}=a_{j i}$ the determinant is symmetrical. In a symmetrical determinant

$$
\Delta_{i j}=\Delta_{j i}
$$

1.351 If $a_{i j}=-a_{j i}$ the determinant is a skew determinant. In a skew determinant

$$
\Delta_{i j}=(-1)^{n-1} \Delta_{j i}
$$


1.352 If $a_{i j}=-a_{j i}$, and $a_{i i}=0$, the determinant is a skew symmetrical determinant.

A skew symmetrical determinant of even order is a perfect square.

A skew symmetrical determinant of odd order vanishes.

1.360 A system of linear equations:

$$
\begin{aligned}
& a_{11} x_{1}+a_{12} x_{2}+\ldots \ldots+a_{1 n} x_{n}=k_{1} \\
& a_{21} x_{1}+a_{22} x_{2}+\ldots \ldots+a_{2 n} x_{n}=k_{2} \\
& \ldots \ldots \\
& \ldots \ldots \ldots \\
& a_{n 1} x_{1}+a_{n 2} x_{2}+\ldots \ldots+a_{n n} x_{n}=k_{n}
\end{aligned}
$$

has a solution:

$$
\Delta \cdot x_{i}=k_{1} \Delta_{1 i}+k_{2} \Delta_{2 i}+\ldots \ldots+k_{n} \Delta_{n i}
$$

provided that

$$
\Delta=\left|a_{i j}\right| \neq 0 .
$$

1.361 If $\Delta=0$, but all the first minors are not $\circ$,

$$
\Delta_{s s} \cdot x_{j}=x_{s} \Delta_{s j}+\sum_{r=1}^{n} k_{r} \frac{\partial^{2} \Delta}{\partial a_{s s} \partial a_{r j}} \quad(j=\mathrm{I}, 2, \ldots \ldots n)
$$

where $s$ may be any one of the integers $1,2, \ldots, n$.

1.362 If $k_{1}=k_{2}=\ldots \ldots=k_{n}=0$, the linear equations are homogeneous, and if $\Delta=0$,

$$
\frac{x_{j}}{\Delta_{s j}}=\frac{x_{s}}{\Delta_{s s}} \quad(j=\mathrm{I}, 2, \ldots n) .
$$

1.363 The condition that $n$ linear homogeneous equations in $n$ variables shall be consistent is that the determinant, $\Delta$, shall vanish.

1.364 If there are $n+\mathrm{I}$ linear equations in $n$ variables:

$$
\begin{aligned}
& a_{11} x_{1}+a_{12} x_{2}+\ldots \ldots+a_{1 n} x_{n}=k_{1} \\
& a_{21} x_{1}+a_{22} x_{2}+\ldots \ldots+a_{2 n} x_{n}=k_{2} \\
& a_{n 1} x_{1}+a_{n 2} x_{2}+\cdots \cdots+a_{n n} x_{n}=k_{n} \\
& c_{1} x_{1}+c_{2} x_{2}+\ldots \ldots+c_{n} x_{n}=k_{n+1}
\end{aligned}
$$

the condition that this system shall be consistent is that the determinant:

$$
\left|\begin{array}{ccccccc}
a_{11} & a_{12} & \ldots \ldots \ldots \ldots & \ldots & \ldots & a_{1 n} & k_{1} \\
a_{21} & a_{22} & \ldots & \ldots & \ldots & a_{2 n} & k_{2} \\
\ldots & \ldots & \ldots & \ldots & \ldots & \ldots & \ldots \\
\ldots & \ldots & \ldots & \ldots & \ldots & \ldots & \ldots \\
a_{n 1} & a_{n 2} & \ldots & \ldots & \ldots & a_{n n} & k_{n} \\
c_{1} & c_{2} & \ldots & \ldots & \ldots & c_{n} & k_{n+1}
\end{array}\right|=0
$$


1.370 Functional Determinants.

If

the determinant:

$$
y_{1}, y_{2}, \ldots, y_{n} \text { are } n \text { functions of } x_{1}, x_{2}, \ldots . ., x_{n} \text { : }
$$

$$
y_{k}=f_{k}\left(x_{1}, x_{2}, \ldots ., x_{n}\right)
$$

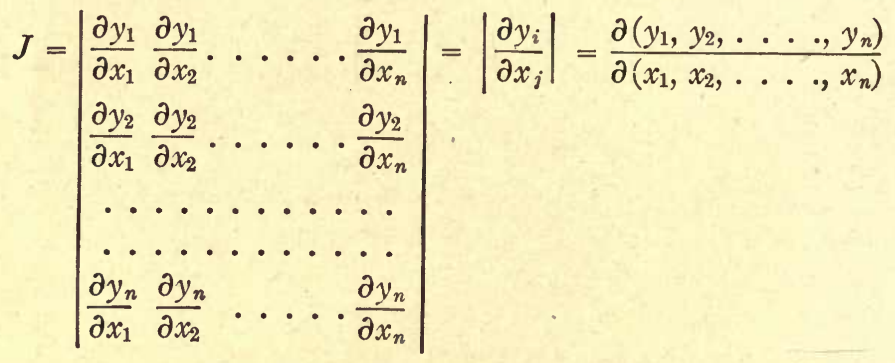

is the Jacobian.

1.371 If $y_{1}, y_{2}, \ldots \ldots \ldots, y_{n}$ are the partial derivatives of a function $F\left(x_{1}, x_{2}, \ldots ., x_{n}\right)$ :

$$
y_{i}=\frac{\partial F}{\partial x_{i}}(i=1,2, \ldots, n)
$$

the symmetrical determinant:

is the Hessian.

$$
H=\left|\frac{\partial^{2} F}{\partial x_{i} \partial x_{j}}\right|=\frac{\partial\left(\frac{\partial F}{\partial x_{1}}, \frac{\partial F}{\partial x_{2}} \ldots . \frac{\partial F}{\partial x_{n}}\right)}{\partial\left(x_{1}, x_{2}, \ldots \ldots, x_{n}\right)}
$$

1.372 If $y_{1}, y_{2}, \ldots \ldots, y_{n}$ are given as implicit functions of $x_{1}, x_{2}, \ldots \ldots$ $x_{n}$ by the $n$ equations:

$$
\begin{aligned}
& F_{1}\left(y_{1}, y_{2}, \ldots \ldots, y_{n}, x_{1}, x_{2}, \ldots \ldots, x_{n}\right)=0 \\
& \ldots \ldots \\
& \dot{F}_{n}\left(y_{1}, y_{2}, \ldots \ldots, y_{n}, x_{1}, x_{2}, \ldots \ldots, x_{n}\right)=0
\end{aligned}
$$

then

$$
\frac{\partial\left(y_{1}, y_{2}, \ldots \ldots, y_{n}\right)}{\partial\left(x_{1}, x_{2}, \ldots, x_{n}\right)}=(-\mathrm{I})^{n} \frac{\partial\left(F_{1}, F_{2}, \ldots, F_{n}\right)}{\partial\left(x_{1}, x_{2}, \ldots, x_{n}\right)} \div \frac{\partial\left(F_{1}, F_{2}, \ldots, F_{n}\right)}{\partial\left(y_{1}, y_{2}, \ldots, y_{n}\right)}
$$

1.373 If the $n$ functions $y_{1}, y_{2}, \ldots \ldots, y_{n}$ are not independent of each other the Jacobian, $J$, vanishes; and if $J=0$ the $n$ functions $y_{1}, y_{2}, \ldots, y_{n}$ are not independent of each other but are connected by a relation

$$
F\left(y_{1}, y_{2}, \ldots \ldots, y_{n}\right)=0
$$


1.374 Covariant property. If the variables $x_{1}, x_{2}, \ldots, x_{n}$ are transformed by a linear substitution:

$$
x_{i}=a_{i 1} \xi_{1}+a_{i 2} \xi_{2}+\ldots \ldots+a_{i n} \xi_{n} \quad(i=1,2, \ldots, n)
$$

and the functions $y_{1}, y_{2}, \ldots \ldots, y_{n}$ of $x_{1}, x_{2}, \ldots \ldots, x_{n}$ become the functions $\eta_{1}, \eta_{2}, \ldots \ldots, \eta_{n}$ of $\xi_{1}, \xi_{2}, \ldots \ldots, \xi_{n}$ :

$$
\begin{gathered}
J^{\prime}=\frac{\partial\left(\eta_{1}, \eta_{2}, \ldots \ldots, \eta_{n}\right)}{\partial\left(\xi_{1}, \xi_{2}, \ldots \ldots, \xi_{n}\right)}=\frac{\partial\left(y_{1}, y_{2}, \ldots \ldots, y_{n}\right)}{\partial\left(x_{1}, x_{2}, \ldots \ldots, x_{n}\right)} \cdot\left|a_{i j}\right| \\
J^{\prime}=J \cdot\left|a_{i j}\right|
\end{gathered}
$$

or

where $\left|a_{i j}\right|$ is the determinant or modulus of the transformation.

For the Hessian,

$$
H^{\prime}=H \cdot\left|a_{i j}\right|^{2}
$$

1.380 To change the variables in a multiple integral:

$$
I=S \ldots \ldots \mathcal{S} F\left(y_{1}, y_{2}, \ldots \ldots, y_{n}\right) d y_{1} d y_{2} \ldots \ldots d y_{n}
$$

to new variables, $x_{1}, x_{2}, \ldots, x_{n}$ when $y_{1}, y_{2}, \ldots, y_{n}$ are given functions of $x_{1}, x_{2}, \ldots \ldots, x_{n}$ :

$$
I=\int \ldots \ldots \int \frac{\partial\left(y_{1}, y_{2}, \ldots \ldots, y_{n}\right)}{\partial\left(x_{1}, x_{2}, \ldots \ldots, x_{n}\right)} F(x) d x_{1} d x_{2} \ldots \ldots d x_{n}
$$

where $F(x)$ is the result of substituting $x_{1}, x_{2}, \ldots, x_{n}$ for $y_{1}, y_{2}, \ldots, y_{n}$ in $F\left(y_{1}, y_{2}, \ldots, y_{n}\right)$.

\section{PERMUTATIONS AND COMBINATIONS}

1.400 Given $n$ different elements. Represent each by a number, $\mathrm{I}, 2,3, \ldots$. . , $n$. The number of permutations of the $n$ different elements is,

$$
{ }_{n} \mathrm{P}_{n}=n \text { ! }
$$

e.g., $n=3$ :

$$
\text { (123), ( } 132),(2 \mathrm{r} 3),(23 \mathrm{r}),(3 \mathrm{r} 2),(32 \mathrm{I})=6=3 \text { ! }
$$

1.401 Given $n$ different elements. The number of permutations in groups of $r(r<n)$, or the number of $r$-permutations, is,

$$
{ }_{n} P_{r}=\frac{n !}{(n-r) !}
$$

e.g., $n=4, r=3$ :

$$
\begin{aligned}
& (\mathrm{I} 23)\left(\mathrm{I}_{32}\right)(\mathrm{I} 24)\left(\mathrm{I}_{42}\right)\left(\mathrm{I}_{34}\right)\left(\mathrm{I}_{43}\right)(234)(243)(23 \mathrm{I})(2 \mathrm{I} 3)(2 \mathrm{I} 4)(24 \mathrm{I})(34 \mathrm{I})(3 \mathrm{I} 4) \\
& (3 \mathrm{I} 2)(32 \mathrm{I})(324)(342)(4 \mathrm{I} 2)(42 \mathrm{I})(43 \mathrm{I})(4 \mathrm{I} 3)(423)(432)=24
\end{aligned}
$$


1.402 Given $n$ different elements. The number of ways they can be divided into $m$ specified groups, with $x_{1}, x_{2}, \ldots, x_{m}$ in each group respectively, $\left(x_{1}+x_{2}+\ldots .+x_{m}\right)=n$ is

$$
\frac{n !}{x_{1} ! x_{2} ! \ldots \ldots x_{m} !}
$$

e.g., $n=6, m=3, x_{1}=2, x_{2}=3, x_{3}=\mathrm{I}$ :
(12) (345) (6)
(I3) (245) (6)
$\times 6=60$
(23) (145) (6)
(24) (135) (6)
(34) (125) (6)
(35) (124) (6)
(45) (123) (6)
(25) (234) (6)
(14) (235) (6)
(15) (234) (6)

1.403 Given $n$ elements of which $x_{1}$ are of one kind, $x_{2}$ of a second kind, ......, $x_{m}$ of an $m$ th kind. The number of permutations is

$$
\begin{gathered}
\frac{n !}{x_{1} ! x_{2} ! \cdots \cdots x_{m} !} \\
x_{1}+x_{2}+\ldots \ldots+x_{m}=n .
\end{gathered}
$$

1.404 Given $n$ different elements. The number of ways they can be permuted among $m$ specified groups, when blank groups are allowed, is

$$
\frac{(m+n-\mathrm{I}) !}{(m-\mathrm{I}) !}
$$

e.g., $n=3, m=2$ :

$$
\begin{aligned}
& (\mathrm{I} 23,0)(\mathrm{I} 32,0)(2 \mathrm{I} 3,0)(23 \mathrm{I}, 0)(3 \mathrm{I} 2,0)(32 \mathrm{I}, \mathrm{O})(\mathrm{I} 2,3)(2 \mathrm{I}, 3)(\mathrm{I} 3,2)(3 \mathrm{I}, 2)(23, \mathrm{I}) \\
& (32, \mathrm{I})(\mathrm{I}, 23)(\mathrm{I}, 32)(2,3 \mathrm{I})(2, \mathrm{I} 3)(3, \mathrm{I} 2)(3,2 \mathrm{I})(\mathrm{O}, \mathrm{I} 23)(\mathrm{O}, 2 \mathrm{I} 3)(\mathrm{O}, \mathrm{I} 32)(0,23 \mathrm{I}) \\
& (0,3 \mathrm{I} 2)(0,32 \mathrm{I})=24
\end{aligned}
$$

1.405 Given $n$ different elements. The number of ways they can be permuted among $m$ specified groups, when blank groups are not allowed, so that each group contains at least one element, is

$$
\frac{n !(n-\mathrm{I}) !}{(n-m) !(m-\mathrm{I}) !}
$$

e.g., $n=3, m=2$ :

$$
(\mathrm{I} 2,3)(2 \mathrm{I}, 3)(\mathrm{I} 3,2)(3 \mathrm{I}, 2)(23, \mathrm{I})(32, \mathrm{I})(\mathrm{I}, 23)(\mathrm{I}, 32)(2,3 \mathrm{I})(2, \mathrm{I} 3)(3, \mathrm{I} 2)(3,2 \mathrm{I})=\mathrm{I} 2
$$

1.406 Given $n$ different elements. The number of ways they can be combined into $m$ specified groups when blank groups are allowed is

$m^{n}$

e.g., $n=3, m=2$ :

$$
(\mathrm{I} 23, \mathrm{O})(\mathrm{I} 2,3)(\mathrm{I} 3,2)(23, \mathrm{I})(\mathrm{I}, 23)(2,3 \mathrm{I})(3, \mathrm{I} 2)(\mathrm{O}, \mathrm{I} 23)=8
$$

1.407 Given $n$ similar elements. The number of ways they can be combined into $m$ different groups when blank groups are allowed is 
e.g., $n=6, m=3$ :

$$
\frac{(n+m-\mathrm{I}) !}{(m-\mathrm{I}) ! n !}
$$

Group I 65544433333222222 I I I I I I O००००००) Group 2 ○IO 20 I $3 \circ 2$ I $4 \circ 3$ I 25044 I 326005 I 423$\}=28$

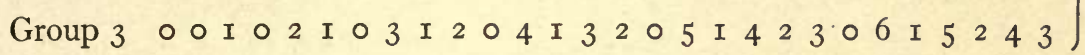

1.408 Given $n$ similar elements. The number of ways they can be combined into $m$ different groups when blank groups are not allowed, so that each group shall contain at least one element, is

$$
\frac{(n-\mathrm{I}) !}{(m-\mathrm{I}) !(n-m) !}
$$

\subsection{1}

\section{BINOMIAL COEFFICIENTS}

I. $\left(\begin{array}{l}n \\ k\end{array}\right)=\frac{n !}{k !(n-k) !}=\left(\begin{array}{c}n \\ n-k\end{array}\right)={ }_{n} C_{k}=\frac{n(n-\mathrm{I})(n-2) \cdots(n-k+\mathrm{I})}{k !}$.

2. $\left(\begin{array}{l}n \\ k\end{array}\right)+\left(\begin{array}{c}n \\ k+\mathrm{I}\end{array}\right)=\left(\begin{array}{l}n+\mathrm{I} \\ k+\mathrm{I}\end{array}\right)$.

3. $\left(\begin{array}{l}n \\ 0\end{array}\right)=\mathrm{I},\left(\begin{array}{l}n \\ \mathrm{I}\end{array}\right)=n,\left(\begin{array}{l}n \\ n\end{array}\right)=\mathrm{I}$.

4. $\left(\begin{array}{c}-n \\ k\end{array}\right)=(-\mathrm{I})^{k}\left(\begin{array}{c}n+k-\mathrm{I} \\ k\end{array}\right)$.

$5 \cdot\left(\begin{array}{l}n \\ k\end{array}\right)=0$ if $n<k$.

6. $\left(\begin{array}{l}k \\ k\end{array}\right)+\left(\begin{array}{c}k+\mathrm{I} \\ k\end{array}\right)+\left(\begin{array}{c}k+2 \\ k\end{array}\right)+\ldots+\left(\begin{array}{l}n \\ k\end{array}\right)=\left(\begin{array}{l}n+\mathrm{I} \\ k+\mathrm{I}\end{array}\right)$.

7. $\mathrm{I}-\left(\begin{array}{c}n \\ \mathrm{I}\end{array}\right)+\left(\begin{array}{c}n \\ 2\end{array}\right)-\ldots+(-\mathrm{I})^{k}\left(\begin{array}{l}n \\ k\end{array}\right)=(-\mathrm{I})^{k}\left(\begin{array}{c}n-\mathrm{I} \\ k\end{array}\right)$.

8. $\left(\begin{array}{l}n \\ k\end{array}\right)+\left(\begin{array}{c}n \\ k-\mathrm{I}\end{array}\right)\left(\begin{array}{l}r \\ \mathrm{I}\end{array}\right)+\left(\begin{array}{c}n \\ k-2\end{array}\right)\left(\begin{array}{l}r \\ 2\end{array}\right)+\ldots .+\left(\begin{array}{l}r \\ k\end{array}\right)=\left(\begin{array}{c}n+r \\ k\end{array}\right)$.

9. $I+\left(\begin{array}{c}n \\ I\end{array}\right)+\left(\begin{array}{c}n \\ 2\end{array}\right)+\ldots .+\left(\begin{array}{l}n \\ n\end{array}\right)=2^{n}$.

Iо. $\mathrm{I}-\left(\begin{array}{c}n \\ \mathrm{I}\end{array}\right)+\left(\begin{array}{c}n \\ 2\end{array}\right)-\ldots+(-\mathrm{I})^{n}\left(\begin{array}{l}n \\ n\end{array}\right)=0$.

II. I $+\left(\begin{array}{c}n \\ 1\end{array}\right)^{2}+\left(\begin{array}{c}n \\ 2\end{array}\right)^{2}+\ldots \ldots+\left(\begin{array}{c}n \\ n\end{array}\right)^{2}=\left(\begin{array}{c}2 n \\ n\end{array}\right)$ 
1.52 Table of Binomial Coefficients.

$$
\left(\begin{array}{l}
n \\
\mathrm{r}
\end{array}\right)=n
$$

$$
\begin{aligned}
& \left(\begin{array}{l}
n \\
I
\end{array}\right)\left(\begin{array}{l}
n \\
2
\end{array}\right)\left(\begin{array}{l}
n \\
3
\end{array}\right)\left(\begin{array}{l}
n \\
4
\end{array}\right)\left(\begin{array}{l}
n \\
5
\end{array}\right)\left(\begin{array}{l}
n \\
6
\end{array}\right)\left(\begin{array}{l}
n \\
7
\end{array}\right)\left(\begin{array}{l}
n \\
8
\end{array}\right)\left(\begin{array}{l}
n \\
9
\end{array}\right)\left(\begin{array}{c}
n \\
\mathrm{IO}
\end{array}\right)\left(\begin{array}{c}
n \\
\mathrm{II}
\end{array}\right)\left(\begin{array}{l}
n \\
\mathrm{I} 2
\end{array}\right) \\
& \begin{array}{ll}
2 & \text { I }
\end{array} \\
& 3 \quad 3 \quad \text { I } \\
& \begin{array}{llll}
4 & 6 & 4 & \text { I }
\end{array} \\
& 5 \text { IO IO } 5 \text { I } \\
& \begin{array}{llllll}
6 & 15 & 20 & 15 & 6 & I
\end{array} \\
& \begin{array}{lllllll}
7 & 21 & 35 & 35 & 21 & 7 & \text { I }
\end{array} \\
& \begin{array}{llllllll}
8 & 28 & 56 & 70 & 56 & 28 & 8 & \text { I }
\end{array} \\
& \begin{array}{lllllllll}
9 & 36 & 84 & \text { I26 } & \text { I26 } & 84 & 36 & 9 & \text { I }
\end{array} \\
& \text { IO } 45 \text { I } 20 \quad 210 \quad 252 \text { 2IO I } 20 \quad 45 \text { IO I } \\
& \text { II } \begin{array}{llllllllll}
55 & \text { I65 } & 330 & 462 & 462 & 330 & \text { I65 } & 55 & \text { II } & \text { I }
\end{array} \\
& \begin{array}{llllllllllll}
\text { I2 } & 66 & 220 & 495 & 792 & 924 & 792 & 495 & 220 & 66 & \text { I2 } & \text { I }
\end{array}
\end{aligned}
$$

1.521 Glaisher, Mess. of Math. 47, p. 97, I918, has given a complete table of binomial coefficients, from $n=2$ to $n=50$, and $k=0$ to $k=n$.

1.61 Resolution into Partial Fractions.

If $F(x)$ and $f(x)$ are two polynomials in $x$ and $f(x)$ is of higher degree than $F(x)$,

$$
\frac{F(x)}{f(x)}=\sum \frac{F(a)}{\phi(a)} \frac{\mathrm{I}}{x-a}+\sum \frac{\mathrm{I}}{(p-\mathrm{I}) !} \frac{d^{p-1}}{d c^{p-1}}\left[\frac{F(c)}{\phi(c)} \frac{\mathrm{I}}{x-c}\right]
$$

where

$$
\begin{aligned}
& \phi(a)=\left[\frac{f(x)}{x-a}\right]_{x=a}, \\
& \phi(c)=\left[\frac{f(x)}{(x-c)^{p}}\right]_{x=c} .
\end{aligned}
$$

The first summation is to be extended for all the simple roots, $a$, of $f(x)$ and the second summation for all the multiple roots, $c$, of order $p$, of $f(x)$.

FINITE DIFFERENCES AND SUMS.

1.811 Definitions.

I. $\Delta f(x)=f(x+h)-f(x)$.

2. $\Delta^{2} f(x)=\Delta f(x+h)-\Delta f(x)$.

$$
=f(x+2 h)-2 f(x+h)+f(x) .
$$


3. $\Delta^{3} f(x)=\Delta^{2} f(x+h)-\Delta^{2} f(x)$.

$$
=f(x+3 h)-3 f(x+2 h)+3 f(x+h)-f(x) .
$$

4: $\Delta^{n} f(x)=f(x+n h)-\frac{n}{\mathrm{I}} f(x+\overline{n-\mathrm{I}} h)+\frac{n(n-\mathrm{I})}{2 !} f(x+\overline{n-2 h})-\ldots$

$$
+(-I)^{n} f(x) \text {. }
$$

\subsection{2}

I. $\Delta[c f(x)]=c \Delta f(x) \quad(c$ a constant $)$.

2. $\Delta\left[f_{1}(x)+f_{2}(x)+\ldots.\right]=\Delta f_{1}(x)+\Delta f_{2}(x)+\ldots$.

3. $\Delta\left[f_{1}(x) \cdot f_{2}(x)\right]=f_{1}(x) \cdot \Delta f_{2}(x)+f_{2}(x+h) \cdot \Delta f_{1}(x)$

$$
=f_{1}(x) \cdot \Delta f_{2}(x)+f_{2}(x) \cdot \Delta f_{1}(x)+\Delta f_{1}(x) \cdot \Delta f_{2}(x) \text {. }
$$

4. $\Delta \frac{f_{1}(x)}{f_{2}(x)}=\frac{f_{2}(x) \cdot \Delta f_{1}(x)-f_{1}(x) \cdot \Delta f_{2}(x)}{f_{2}(x) \cdot f_{2}(x+h)}$.

1.813 The $n$th difference of a polynomial of the $n$th degree is constant. If

$$
\begin{aligned}
f(x) & =a_{0} x_{n}+a_{1} x^{n-1}+\ldots \ldots+a_{n-1} x+a_{n} \\
\Delta^{n} f(x) & =n ! a_{0} h^{n} .
\end{aligned}
$$

\subsection{2}

I. $\frac{\Delta^{m}\{(x-b)(x-b-h)(x-b-2 h) \ldots \ldots(x-b-\overline{n-\mathrm{I} h})\}}{n(n-\mathrm{I})(n-2) \ldots(n-m+\mathrm{I}) h^{m}}$

$$
=(x-b)(x-b-h)(x-b-2 h) \ldots(x-b-\overline{n-m-\mathrm{I} h}) \text {. }
$$

2. $\Delta^{m} \frac{I}{(x+b)(x+b+h)(x+b+2 h) \ldots(x+b+\overline{n-1} h)}$

$$
=(-\mathbf{I})^{m} \frac{n(n+\mathrm{I})(n+2) \ldots(n+m-\mathrm{I}) h^{m}}{(x+b)(x+b+h)(x+b+2 h) \ldots(x+b+\overline{n+m-\mathrm{I} h})} \cdot
$$

3. $\Delta^{m} a^{x}=\left(a^{h}-1\right)^{m} a^{x}$

4. $\Delta \log f(x)=\log \left(1+\frac{\Delta f(x)}{f(x)}\right)$.

5. $\Delta^{m} \sin (c x+d)=\left(2 \sin \frac{c h}{2}\right)^{m} \sin \left(c x+d+m \frac{c h+\pi}{2}\right)$.

6. $\Delta^{m} \cos (c x+d)=\left(2 \sin \frac{c h}{2}\right)^{m} \cos \left(c x+d+m \frac{c h+\pi}{2}\right)$. 
1.83 Newton's Interpolation Formula.

$$
\begin{aligned}
f(x)=f(a) & +\frac{x-a}{h} \Delta f(a)+\frac{(x-a)(x-a-h)}{2 ! h^{2}} \Delta^{2} f(a)+ \\
& +\frac{(x-a)(x-a-h)(x-a-2 h)}{3 ! h^{3}} \Delta^{3} f(a)+\ldots \ldots \\
& +\frac{(x-a)(x-a-h) \ldots \ldots(x-a-\overline{n-1} h)}{n ! h^{n}} \Delta^{n} f(a) \\
& +\frac{(x-a)(x-a-h) \ldots \ldots(x-a-n h)}{n+\mathrm{I} !} f^{n+1)}(\xi)
\end{aligned}
$$

where $\xi$ has a value intermediate between the greatest and least of $a,(a+n h)$, and $x$.

\subsection{1}

$$
\begin{gathered}
f(a+n h)=f(a)+\frac{n}{\mathrm{I} !} \Delta f(a)+\frac{n(n-\mathrm{I})}{2 !} \Delta^{2} f(a)+\frac{n(n-\mathrm{I})(n-2)}{3 !} \Delta^{3} f(a) \\
+\ldots \ldots+n \Delta^{n-1} f(a)+\Delta^{n} f(a) .
\end{gathered}
$$

\subsection{Symbolically}

I. $\Delta=e^{h \frac{\partial}{\partial x}}-\mathrm{I}$

2. $f(a+n h)=(\mathrm{I}+\Delta)^{n} f(a)$

1.833 If $u_{0}=f(a), u_{1}=f(a+h), u_{2}=f(a+2 h), \ldots ., u_{x}=f(a+x h)$,

$$
u_{x}=(\mathrm{I}+\Delta){ }^{x} u_{0}=e^{h x} \frac{\partial}{\partial x} u_{0} .
$$

1.840 The operator inverse to the difference, $\Delta$, is the sum, $\Sigma$.

$$
\Sigma=\Delta^{-1}=\frac{\mathrm{I}}{e^{\lambda \frac{\partial}{\partial x}-I}} .
$$

1.841 If $\Delta F(x)=f(x)$,

$$
\Sigma f(x)=F(x)+C
$$

where $C$ is an arbitrary constant.

\subsection{2}

I. $\Sigma c f(x)=c \Sigma f(x)$.

2. $\Sigma\left[f_{1}(x)+f_{2}(x)+\ldots\right]=\Sigma f_{1}(x)+\Sigma f_{2}(x)+\ldots$

3. $\Sigma\left[f_{1}(x) \cdot \Delta f_{2}(x)\right]=f_{1}(x) \cdot f_{2}(x)-\Sigma\left[f_{2}(x+h) \cdot \Delta f_{1}(x)\right]$. 
1.843 Indefinite Sums.

I. $\Sigma[(x-b)(x-b-h)(x-b-2 h) \ldots(x-b-\overline{n-\mathrm{I}} h)]$

$$
=\frac{\mathrm{I}}{(n+\mathrm{I}) h}(x-b)(x-b-h) \ldots \ldots(x-b-n h)+C .
$$

2.

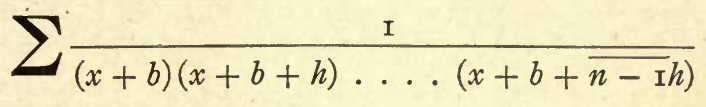

$=-\frac{\mathrm{I}}{(n-\mathrm{I}) h} \frac{\mathrm{I}}{(x+b)(x+b+h) \ldots(x+b+\overline{n-2} h)}+C$.

3. $\sum a^{x}=\frac{a^{x}}{a^{h}-\mathrm{I}}+C$

4. $\sum \cos (c x+d)=\frac{\sin \left(c x-\frac{c h}{2}+d\right)}{2 \sin \frac{c h}{2}}+C$.

5. $\sum \sin (c x+d)=-\frac{\cos \left(c x-\frac{c h}{2}+d\right)}{2 \sin \frac{c h}{2}}+C$.

1.844 If $f(x)$ is a polynomial of degree $n$,

$$
\begin{gathered}
\sum a^{x} f(x)=\frac{a^{x}}{a^{h}-\mathrm{I}}\left\{f(x)-\frac{a^{h}}{a^{h}-\mathrm{I}} \Delta f(x)+\left(\frac{a^{h}}{a^{h}-\mathrm{I}}\right)^{2} \Delta^{2} f(x) \ldots \ldots\right. \\
+\left(\frac{-a^{h}}{a^{h}-\mathrm{I}}\right)^{n} \Delta^{n} f(x)+C
\end{gathered}
$$

1.845 If $f(x)$ is a polynomial of degree $n$,

and

$$
f(x)=a_{0} x^{n}+a_{1} x^{n-1}+\ldots+a_{n-1} x+a_{n},
$$

$$
\begin{aligned}
\Sigma f(x) & =F(x)+C \\
F(x) & =c_{0} x^{n+1}+c_{1} x^{n}+c_{2} x^{n-1}+\ldots \ldots+c_{n} x+c_{n+1}
\end{aligned}
$$

where

$$
\begin{gathered}
(n+\mathrm{I}) h c_{0}=a_{0} \\
\frac{(n+\mathrm{I}) n}{2 !} h^{2} c_{0}+n h c_{1}=a_{1} \\
\frac{(n+\mathrm{I}) n(n-\mathrm{I})}{3 !} h^{3} c_{0}+\frac{n(n-\mathrm{I})}{2 !} h^{2} c_{1}+(n-\mathrm{I}) h c_{2}=a_{2}
\end{gathered}
$$

The coefficient $c_{n+1}$ may be taken arbitrarily. 
1.850 Definite Sums. From the indefinite sum,

$$
\Sigma f(x)=F(x)+C,
$$

a definite sum is obtained by subtraction,

$$
\sum_{a+m h}^{a+n h} f(x)=F(a+n h)-F(a+m h) .
$$

1.851

$$
\begin{aligned}
\sum_{a}^{a+n h} f(x) & =f(a)+f(a+h)+f(a+2 h)+\ldots .+f(a+\overline{n-\mathrm{I} h}) \\
& =F(a+n h)-F(a) .
\end{aligned}
$$

By means of this formula many finite sums may be evaluated.

\subsection{2}

1.853

$$
\begin{aligned}
\sum_{a}^{a+n h}(x-b)(x-b-h)(x-b-2 h) \ldots(x-b \ldots \overline{k-\mathrm{I}} h) \\
= \\
-\frac{(a-b+n h)(a-b+\overline{n-\mathrm{I}} h) \ldots(a-b+\overline{n-k} h)}{(k+\mathrm{I}) h} \\
-\frac{(a-b)(a-b-h) \ldots(a-b-k h)}{(k+\mathrm{I}) h} .
\end{aligned}
$$

$$
\begin{aligned}
\sum_{a}^{a+n h}(x-a)(x-a-h) \ldots(x-a-\overline{k-\mathrm{I}} h) \\
=\frac{n(n-\mathrm{I})(n-2) \ldots(n-k)}{(k+\mathrm{I})} h^{k} .
\end{aligned}
$$

1.854 If $f(x)$ is a polynomial of degree $m$ it can be expressed:

$$
\begin{aligned}
f(x)= & A_{0}+A_{1}(x-a)+A_{2}(x-a)(x-a-h)+\ldots \\
& +A_{m}(x-a)(x-a-h) \ldots(x-a-\overline{m-\mathrm{I}} h), \\
\sum_{a}^{a+n h} f(x)= & A_{0} n+A_{1} \frac{n(n-\mathrm{I})}{2} h+A_{2} \frac{n(n-\mathrm{I})(n-2)}{3} h^{2} \\
& +A_{m} \frac{n(n-\mathrm{I}) \ldots(n-m)}{(m+\mathrm{I})} h^{m} .
\end{aligned}
$$

1.855 If $f(x)$ is a polynomial of degree $(m-1)$ or lower, it can be expressed:

and,

$$
\begin{aligned}
& f(x)=A_{0}+A_{1}(x+m h)+A_{2}(x+m h)(x+\overline{m-\mathrm{I}} h) \\
& +\ldots .+A_{m-1}(x+m h) \ldots(x+2 h) \\
& \sum_{a}^{a+n h} \frac{f(x)}{x(x+h)(x+2 h) \ldots(x+m h)}=\frac{A_{0}}{m h}\left\{\frac{\mathrm{I}}{a(a+h) \ldots(a+\overline{m-\mathrm{x}} h)}\right.
\end{aligned}
$$








$$
\begin{aligned}
&\left.-\frac{\mathrm{I}}{(a+n h) \ldots \ldots(a+\overline{n+m-\mathrm{I} h})}\right\} \\
&+ \frac{A_{1}}{(m-\mathrm{I}) h}\left\{\frac{\mathrm{r}}{a(a+h) \ldots(a+\overline{m-2} h)}-\frac{\mathrm{I}}{(a+n h) \ldots(a+\overline{n+m-2 h})}\right\} \\
&+\ldots \ldots+\frac{A_{m-1}}{h}\left\{\frac{\mathrm{I}}{a}-\frac{\mathrm{I}}{a+n h}\right\} .
\end{aligned}
$$

1.856 If $f(x)$ is a polynomial of degree $m$ it can be expressed:

$$
\begin{gathered}
f(x)=A_{0}+A_{1}(x+m h)+A_{2}(x+m h)(x+\overline{m-1} h)+\ldots \\
+A_{m}(x+m h) \ldots(x+h)
\end{gathered}
$$

and,

$$
\begin{aligned}
\sum_{a}^{a+n h} \frac{f(x)}{x(x+h) \ldots(x+m h)}=\frac{A_{0}}{m h}\left\{\frac{\mathrm{I}}{a(a+h) \ldots(a+\overline{m-\mathrm{I}} h)}\right. & \\
& \left.-\frac{\mathrm{I}}{(a+n h) \ldots(a+\overline{m+n-\mathrm{I} h})}\right\} \\
& +\ldots \ldots+\frac{A_{m-1}}{h}\left\{\frac{\mathrm{I}}{a}-\frac{\mathrm{I}}{a+n h}\right\}+A_{m} \sum_{a}^{a+n h} \frac{\mathrm{I}}{x}
\end{aligned}
$$

where,

$$
\sum_{a}^{a+n h} \frac{\mathrm{I}}{x}=\frac{\mathrm{I}}{a}+\frac{\mathrm{I}}{a+h}+\frac{\mathrm{I}}{a+2 h}+\ldots .+\frac{\mathrm{I}}{a+\overline{n-\mathrm{I} h}} .
$$

1.86 Euler's Summation Formula.

$$
\begin{aligned}
& \sum_{a}^{b} f(x)=\frac{1}{h} \int_{a}^{b} f(z) d z+A_{1}\{f(b)-f(a)\}+A_{2} h\left\{f^{\prime}(b)-f^{\prime}(a)\right\}, \\
& +\ldots+A_{m-1} h^{m-2}\left\{f^{(m-2)}(b)-f^{(m-2)}(a)\right\} \\
& -\int_{0}^{h} \phi_{m}(z) \sum_{x=a}^{x=b} \frac{d^{m} f(x+h-z)}{h d x^{m}} \cdot d z \\
& \phi_{m}(z)=\frac{z^{m}}{m !}+A_{1} \frac{h z^{m-1}}{(m-1) !}+A_{2} \frac{h^{2} z^{m-2}}{(m-2) !}+\ldots .+A_{m-1} h^{m-1} z .
\end{aligned}
$$

$m ! \phi_{m}(z)$, with $h=\mathrm{I}$, is the Bernoullian polynomial.

$A_{1}=-\frac{1}{2}, A_{2 k+1}=0 ;$ the coefficients $A_{2 k}$ are connected with Bernoulli's numbers $(6.902), B_{k}$, by the relation,

$$
\begin{aligned}
& A_{2 k}=(-\mathrm{I})^{k+1} \frac{B_{k}}{(2 k) !} \\
& A_{1}=-\frac{\mathrm{I}}{2}, \quad A_{2}=\frac{\mathrm{I}}{\mathrm{I} 2}, \quad A_{4}=-\frac{\mathrm{I}}{720}, \quad A_{6}=\frac{\mathrm{I}}{30240} \ldots \ldots
\end{aligned}
$$




\subsection{1}

$$
\begin{aligned}
\sum_{a}^{b} f(x) & =\frac{\mathrm{I}}{h} \int_{a}^{b} f(z) d z-\frac{\mathrm{I}}{2}\{f(b)-f(a)\}+\frac{h}{\mathrm{I} 2}\left\{f^{\prime}(b)-f^{\prime}(a)\right\} \\
& -\frac{h^{3}}{720}\left\{f^{\prime \prime \prime}(b)-f^{\prime \prime \prime}(a)\right\}+\frac{h^{5}}{30240}\left\{f^{\mathrm{v}}(b)-f^{\mathrm{v}}(a)\right\}-\ldots \ldots
\end{aligned}
$$

1.862

$$
\sum u_{x}=C+\int u_{x} d x-\frac{\mathrm{I}}{2} u_{x}+\frac{\mathrm{I}}{\mathrm{I} 2} \frac{d u_{x}}{d x}-\frac{\mathrm{I}}{720} \frac{d^{3} u_{x}}{d x^{3}}+\frac{\mathrm{I}}{30240} \frac{d^{5} u_{x}}{d x^{5}}-\ldots \ldots
$$

\section{SPECIAL FINITE SERIES}

1.871 Arithmetical progressions. If $s$ is the sum, $a$ the first term, $\delta$ the common difference, $l$ the last term, and $n$ the number of terms,

$$
\begin{aligned}
s & =a+(a+\delta)+(a+2 \delta)+\ldots[[a+(n-\mathrm{I}) \delta] \\
l & =a+(n-\mathrm{I}) \delta \\
s & =\frac{n}{2}[2 a+(n-\mathrm{I}) \delta] \\
& =\frac{n}{2}(a+l) .
\end{aligned}
$$

1.872 Geometrical progressions.

$$
\begin{aligned}
& s=a+a p+a p^{2}+\ldots \ldots+a p^{n-1} \\
& s=a \frac{p^{n}-\mathrm{I}}{p-\mathrm{I}}
\end{aligned}
$$

If $p<\mathrm{I}, n=\infty, s=\frac{a}{\mathrm{I}-p}$.

1.873 Harmonical progressions. $a, b, c, d, \ldots$ form an harmonical progression if the reciprocals, $\mathrm{I} / a, \mathrm{I} / b, \mathrm{I} / c, \mathrm{I} / d, \ldots$ form an arithmetical progression.

\subsection{4 .}

I

$\sum_{x=\mathrm{I}}^{x=n} x=\frac{n(n+\mathrm{r})}{2}$

2. $\sum_{x=\mathrm{I}}^{x=n} x^{2}=\frac{n(n+\mathrm{I})(2 n+\mathrm{I})}{6}$

$$
\text { 3. } \sum_{x=\mathrm{I}}^{x=n} x^{3}=\left[\frac{n(n+\mathrm{I})}{2}\right]^{2}
$$


1.875 In general,

$\sum_{x=1}^{x=n} x^{k}=\frac{n^{k+1}}{k+\mathrm{I}}+\frac{n^{k}}{2}+\frac{\mathrm{I}}{2}\left(\begin{array}{l}k \\ \mathrm{I}\end{array}\right) B_{1} n^{k-1}-\frac{\mathrm{I}}{4}\left(\begin{array}{l}k \\ 3\end{array}\right) B_{2} n^{k-3}+\frac{\mathrm{I}}{6}\left(\begin{array}{l}k \\ 5\end{array}\right) B_{3} n^{k-5} \ldots$

$B_{1}, B_{2}, B_{3}, \ldots$ are Bernoulli's numbers (6.902), $\left(\begin{array}{l}k \\ h\end{array}\right)$ are the binomial coefficients (1.51); the series ends with the term in $n$ if $k$ is even, and with the term in $n^{2}$ if $k$ is odd.

\subsection{6}

$$
\begin{aligned}
\frac{\mathrm{I}}{\mathrm{I}}+ & \frac{\mathrm{I}}{2}+\frac{\mathrm{I}}{3}+\frac{\mathrm{I}}{4}+\ldots \ldots+\frac{\mathrm{I}}{n}=\gamma+\log n+\frac{\mathrm{I}}{2 n}-\frac{a_{2}}{n(n+\mathrm{I})} \\
& -\frac{a_{3}}{n(n+\mathrm{I})(n+2)}-\cdots
\end{aligned}
$$

$\gamma=$ Euler's constant $=0.5772156649 \cdots$

$$
\begin{aligned}
& a_{2}=\frac{\mathrm{I}}{\mathrm{I} 2} \\
& a_{3}=\frac{\mathrm{I}}{\mathrm{I} 2} \\
& a_{4}=\frac{\mathrm{I} 9}{80} \quad a_{k}=\frac{\mathrm{I}}{k} \int_{0}^{\mathrm{I}} x(\mathrm{I}-x)(2-x) \ldots \ldots(k-\mathrm{I}-x) d x \\
& a_{5}=\frac{9}{20}
\end{aligned}
$$

\subsection{7}

$$
\begin{gathered}
\frac{\mathbf{I}}{\mathbf{I}^{2}}+\frac{\mathbf{I}}{2^{2}}+\frac{\mathrm{I}}{3^{2}}+\ldots+\frac{\mathrm{I}}{n^{2}}=\frac{\pi^{2}}{6}-\frac{b_{1}}{n+\mathrm{I}}-\frac{b_{2}}{(n+\mathrm{I})(n+2)} \\
\frac{b_{3}}{(n+\mathrm{I})(n+2)(n+3)}-\ldots \ldots \\
b_{k}=\frac{(k-\mathrm{I}) !}{k}
\end{gathered}
$$

\subsection{8}

$$
\begin{gathered}
\frac{\mathrm{I}}{\mathrm{I}^{3}}+\frac{\mathrm{I}}{2^{3}}+\frac{\mathrm{I}}{3^{3}}+\ldots+\frac{\mathrm{I}}{n^{3}}=C-\frac{c_{2}}{(n+\mathrm{I})(n+2)} \\
-\frac{c_{3}}{(n+\mathrm{I})(n+2)(n+3)}-\cdots \\
C=\sum_{k=\mathrm{I}}^{\infty} \frac{\mathrm{I}}{k^{3}}=\mathrm{I} .2020569032 \\
c_{k}=\frac{(k-\mathrm{I}) !\left(\frac{\mathrm{I}}{\mathrm{I}}+\frac{\mathrm{I}}{2}+\frac{\mathrm{I}}{3}+\ldots .+\frac{\mathrm{I}}{k-\mathrm{I}}\right) .}{}
\end{gathered}
$$


1.879 Stirling's Formula.

$$
\begin{aligned}
& \log (n !)=\log \sqrt{2 \pi}+\left(n+\frac{\mathrm{r}}{2}\right) \log n-n \\
& +\frac{A_{2}}{n}+\ldots+A_{2 k-2} \frac{(2 k-4) !}{n^{2 k-3}} \\
& +\theta A_{2 k} \frac{(2 k-2) !}{n^{2 k-1}}
\end{aligned}
$$

$\circ<\theta<\mathrm{r}$. The coefficients $A_{k}$ are given in $\mathbf{1 . 8 6}$.

\subsection{8}

I. $\mathrm{I}+\mathrm{I} !+2 \cdot 2 !+3 \cdot 3 !+\ldots+n \cdot n !=(n+\mathrm{r})$ !

2. $\mathrm{I} \cdot 2 \cdot 3+2 \cdot 3 \cdot 4+3 \cdot 4 \cdot 5+\ldots+n(n+\mathrm{I})(n+2)=\frac{\mathrm{I}}{4} n(n+\mathrm{I})(n+2)(n+3)$.

3. $\mathrm{I} \cdot 2 \cdot 3 \ldots+2 \cdot 3 \cdot 4 \ldots(r+\mathrm{I})+\ldots \ldots+n(n+\mathrm{I})(n+2)$

.... $(n+r-\mathrm{r})$

$$
=\frac{n(n+1)(n+2) \ldots(n+r)}{r+\mathbf{I}} .
$$

4. $\mathrm{I} \cdot p+2(p+\mathrm{I})+3(p+2)+\ldots \ldots+n(p+n-\mathrm{I})$

$$
=\frac{\mathrm{r}}{6} n(n+\mathrm{r})(3 p+2 n-2) \text {. }
$$

5. $p \cdot q+(p-\mathrm{I})(q-\mathrm{I})+(\dot{p}-2)(q-2)+\ldots \ldots(p-n)(q-n)$

$$
=\frac{\mathrm{I}}{6} n[6 p q-(n-\mathrm{I})(3 p+3 q-2 n+\mathrm{I})] \text {. }
$$

6. $\mathrm{I}+\frac{b}{a}+\frac{b(b+\mathrm{I})}{a(a+\mathrm{I})}+\ldots+\frac{b(b+\mathrm{I}) \ldots(b+n-\mathrm{I})}{a(a+\mathrm{I}) \ldots(a+n-\mathrm{I})}$.

$$
=\frac{b(b+\mathrm{I}) \ldots(b+n)}{(b+\mathrm{I}-a) a(a+\mathrm{I}) \ldots(a+n-\mathrm{I})}-\frac{a-\mathrm{I}}{b+\mathrm{I}-a} \text {. }
$$




\section{GEOMETRY}

2.00 Transformation of coördinates in a plane.

2.001 Change of origin. Let $x, y$ be a system of rectangular or oblique coördinates with origin at $O$. Referred to $x, y$ the coördinates of the new origin $O^{\prime}$ are $a, b$. Then referred to a parallel system of coördinates with origin at $O^{\prime}$ the coördinates are $x^{\prime}, y^{\prime}$.

$$
\begin{aligned}
& x=x^{\prime}+a \\
& y=y^{\prime}+b .
\end{aligned}
$$

2.002 Origin unchanged. Directions of axes changed. Oblique coördinates. Let $\omega$ be the angle between the $x-y$ axes measured counter-clockwise from the $x$ - to the $y$-axis. Let the $x^{\prime}$-axis make an angle $\alpha$ with the $x$-axis and the $y^{\prime}$-axis an angle. $\beta$ with the $x$-axis. All angles are measured counter-clockwise from the $x$-axis. Then

$$
\begin{aligned}
x \sin \omega & =x^{\prime} \sin (\omega-\alpha)+y^{\prime} \sin (\omega-\beta) \\
y \sin \omega & =x^{\prime} \sin \alpha+y^{\prime} \sin \beta \\
\omega^{\prime} & =\beta-\alpha .
\end{aligned}
$$

2.003 Rectangular axes. Let both new and old axes be rectangular, the new axes being turned through an angle $\theta$ with respect to the old axes. Then $\omega=\frac{\pi}{2}, \alpha=\theta, \beta=\frac{\pi}{2}+\theta$.

$$
\begin{aligned}
& x=x^{\prime} \cos \theta-y^{\prime} \sin \theta \\
& y=x^{\prime} \sin \theta+y^{\prime} \cos \theta
\end{aligned}
$$

2.010 Polar coördinates. Let the $y$-axis make an angle $\omega$ with the $x$-axis and let the $x$-axis be the initial line for a system of polar coördinates $r, \theta$. All angles are measured in a counter-clockwise direction from the $x$-axis.

$$
\begin{aligned}
& x=\frac{r \sin (\omega-\theta)}{\sin \omega} \\
& y=r \frac{\sin \theta}{\sin \omega} .
\end{aligned}
$$

2.011 If the $x, y$ axes are rectangular, $\omega=\frac{\pi}{2}$,

$$
\begin{aligned}
& x=r \cos \theta \\
& y=r \sin \theta .
\end{aligned}
$$


2.020 Transformation of coördinates in three dimensions.

2.021 Change of origin. Let $x, y, z$ be a system of rectangular or oblique coördinates with origin at $O$. Referred to $x, y, z$ the coördinates of the new origin $O^{\prime}$ are $a, b, c$. Then referred to a parallel system of coördinates with origin at $O^{\prime}$ the coördinates are $x^{\prime}, \dot{y}^{\prime}, z^{\prime}$.

$$
\begin{aligned}
& x=x^{\prime}+a \\
& y=y^{\prime}+b \\
& z=z^{\prime}+c
\end{aligned}
$$

2.022 Transformation from one to another rectangular system. Origin unchanged. The two systems are $x, y, z$ and $x^{\prime} y^{\prime} z^{\prime}$.

Referred to $x, y, z$ the direction cosines of $x^{\prime}$ are $l_{1}, m_{1}, n_{1}$ Referred to $x, y, z$ the direction cosines of $y^{\prime}$ are $l_{2}, m_{2}, n_{2}$ Referred to $x, y, z$ the direction cosines of $z^{\prime}$ are $l_{3}, m_{3}, n_{3}$

The two systems are connected by the scheme:

\begin{tabular}{|c|c|c|c|}
\hline & $x^{\prime}$ & $y^{\prime}$ & $z^{\prime}$ \\
\hline$x$ & $l_{1}$ & $l_{2}$ & $l_{3}$ \\
\hline$y$ & $m_{1}$ & $m_{2}$ & $m_{3}$ \\
\hline$z$ & $n_{1}$ & $n_{2}$ & $n_{3}$ \\
\hline
\end{tabular}

$$
\begin{array}{lr}
x=l_{1} x^{\prime}+l_{2} y^{\prime}+l_{3} z^{\prime} & x^{\prime}=l_{1} x+m_{1} y+n_{1} z \\
y=m_{1} x^{\prime}+m_{2} y^{\prime}+m_{3} z^{\prime} & y^{\prime}=l_{2} x+m_{2} y+n_{2} z \\
z=n_{1} x^{\prime}+n_{2} y^{\prime}+n_{3} z^{\prime} & z^{\prime}=l_{3} x+m_{3} y+n_{3} z \\
l_{1}^{2}+m_{1}{ }^{2}+n_{1}^{2}=\mathrm{I} & l_{1}^{2}+l_{2}^{2}+l_{3}^{2}=\mathrm{I} \\
l_{2}^{2}+m_{2}^{2}+n_{2}^{2}=\mathrm{I} & m_{1}^{2}+m_{2}^{2}+m_{3}^{2}=\mathrm{I} \\
l_{3}^{2}+m_{3}^{2}+n_{3}^{2}=\mathrm{I} & n_{1}^{2}+n_{2}^{2}+n_{3}^{2}=\mathrm{I} \\
l_{1} m_{1}+l_{2} m_{2}+l_{3} m_{3}=0 & l_{1} l_{2}+m_{1} m_{2}+n_{1} n_{2}=0 \\
m_{1} n_{1}+m_{2} n_{2}+m_{3} n_{3}=0 & l_{2} l_{3}+m_{2} m_{3}+n_{2} n_{3}=0 \\
n_{1} l_{1}+n_{2} l_{2}+n_{3} l_{3}=0 & l_{3} l_{1}+m_{3} m_{1}+n_{3} n_{1}=0
\end{array}
$$

2.023 If the transformation from one to another rectangular system is a rotation through an angle $\theta$ about an axis which makes angles $\alpha, \beta, \gamma$ with $x, y$, $z$ respectively, 


$$
\frac{\cos ^{2} \alpha}{m_{2}+n_{3}-l_{1}-1}=\frac{\cos ^{2} \beta}{n_{3}+l_{1}-m_{2}-I}=\frac{\cos ^{2} \gamma}{l_{1}+m_{2}-n_{3}-\mathrm{I}}
$$

2.024 Transformation from a rectangular to an oblique system. $x, y, z$ rectangular system: $x^{\prime}, y^{\prime}, z^{\prime}$ oblique system.

$$
\begin{aligned}
& \cos \widehat{x x^{\prime}}=l_{1} \\
& \cos \widehat{y x^{\prime}}=m_{1} \\
& \cos \widehat{z x^{\prime}}=n_{1}
\end{aligned}
$$

$$
\begin{aligned}
& \cos \widehat{x y^{\prime}}=l_{2} \\
& \cos \widehat{y y^{\prime}}=m_{2} \\
& \cos \widehat{z y^{\prime}}=n_{2}
\end{aligned}
$$

$$
\begin{aligned}
& \cos \widehat{x z^{\prime}}=l_{3} \\
& \cos \widehat{y z^{\prime}}=m_{3} \\
& \cos \widehat{z z^{\prime}}=n_{3}
\end{aligned}
$$

$$
\begin{gathered}
x=l_{1} x^{\prime}+l_{2} y^{\prime}+l_{3} z^{\prime} \\
y=m_{1} x^{\prime}+m_{2} y^{\prime}+m_{3} z^{\prime} \\
z=n_{1} x^{\prime}+n_{2} y^{\prime}+n_{3} z^{\prime} \\
\cos \widehat{y^{\prime} z^{\prime}}=l_{2} l_{3}+m_{2} m_{3}+n_{2} n_{3} \\
\cos \widehat{z^{\prime} x^{\prime}}=l_{3} l_{1}+m_{3} m_{1}+n_{3} n_{1} \\
\cos \widehat{x}^{\prime} y^{\prime}=l_{1} l_{2}+m_{1} m_{2}+n_{1} n_{2} \\
l_{1}^{2}+m_{1}^{2}+n_{1}^{2}=\mathbf{I} \\
l_{2}^{2}+m_{2}^{2}+n_{2}^{2}=\mathbf{I} \\
l_{3}^{2}+m_{3}^{2}+n_{3}^{2}=\mathbf{I}
\end{gathered}
$$

2.025 Transformation from one to another oblique system.

$$
\begin{aligned}
& \cos \widehat{x x^{\prime}}=l_{1} \\
& \cos \widehat{y x^{\prime}}=m_{1} \\
& \cos \widehat{z x^{\prime}}=n_{1} \\
& \cos \widehat{x y^{\prime}}=l_{2} \\
& \cos \widehat{y y^{\prime}}=m_{2} \\
& \cos \widehat{z y^{\prime}}=n_{2} \\
& \cos \widehat{x z^{\prime}}=l_{3} \\
& \cos \widehat{y z^{\prime}}=m_{3} \\
& \cos \widehat{z z^{\prime}}=n_{3} \\
& \Delta=\left|\begin{array}{lll}
l_{1} & l_{2} & l_{3} \\
m_{1} m_{2} m_{3} \\
n_{1} & n_{2} & n_{3}
\end{array}\right| \\
& x=l_{1} x^{\prime}+l_{2} y^{\prime}+l_{3} z^{\prime} \\
& y=m_{1} x^{\prime}+m_{2} y^{\prime}+m_{3} z^{\prime} \\
& z=n_{1} x^{\prime}+n_{2} y^{\prime}+n_{3} z^{\prime} \\
& \Delta \cdot x^{\prime}=\left(m_{2} n_{3}-m_{3} n_{2}\right) x+\left(n_{2} l_{3}-n_{3} l_{2}\right) y+\left(l_{2} m_{3}-l_{3} m_{2}\right) z, \\
& \Delta \cdot y^{\prime}=\left(m_{3} n_{1}-m_{1} n_{3}\right) x+\left(n_{3} l_{1}-n_{1} l_{3}\right) y+\left(l_{3} m_{1}-l_{1} m_{3}\right) z \text {, } \\
& \Delta \cdot z^{\prime}=\left(m_{1} n_{2}-m_{2} n_{1}\right) x+\left(n_{1} l_{2}-n_{2} l_{1}\right) y+\left(l_{1} m_{2}-l_{2} m_{1}\right) z \text {. }
\end{aligned}
$$

$l_{1}^{2}+m_{1}^{2}+n_{1}^{2}+2 m_{1} n_{1} \cos \widehat{y z}+2 n_{1} l_{1} \cos \widehat{x x}+2 l_{1} m_{1} \cos \widehat{x y}=\mathrm{I}$, $l_{2}^{2}+m_{2}^{2}+n_{2}^{2}+2 m_{2} n_{2} \cos \widehat{y z}+2 n_{2} l_{2} \cos \widehat{z x}+2 l_{2} m_{2} \cos \widehat{x y}=\mathrm{I}$, $l_{3}^{2}+m_{3}^{2}+n_{3}^{2}+2 m_{3} n_{3} \cos \widehat{y z}+2 n_{3} l_{3} \cos \widehat{z x}+2 l_{3} m_{3} \cos \widehat{x y}=\mathrm{I}$.

$x+y \cos \widehat{x y}+z \cos \widehat{x z}=l_{1} x^{\prime}+l_{2} y^{\prime}+l_{3} z^{\prime}$, $y+x \cos \widehat{x y}+z \cos \widehat{z y}=m_{1} x^{\prime}+m_{2} y^{\prime}+m_{3} z^{\prime}$, $z+x \cos \widehat{x z}+y \cos \widehat{z y}=n_{1} x^{\prime}+n_{2} y^{\prime}+n_{3} z^{\prime}$. 
2.026 Transformation from one to another oblique system.

If $n_{x}, n_{y}, n_{z}$ are the normals to the planes $y z, z x, x y$ and $n_{x}{ }^{\prime}, n_{y}{ }^{\prime}, n_{z}{ }^{\prime}$ the normals to the planes $y^{\prime} z^{\prime}, z^{\prime} x^{\prime}, x^{\prime} y^{\prime}$,

$$
\begin{aligned}
& x \cos \widehat{x n_{x}}=x^{\prime} \cos \widehat{x^{\prime} n_{x}}+y^{\prime} \cos \widehat{y^{\prime} n_{x}}+z^{\prime} \cos \widehat{z^{\prime} n_{x}} \text {. } \\
& y \cos \widehat{y n}_{y}=x^{\prime} \cos \widehat{x}^{\prime} n_{y}+y^{\prime} \cos {\widehat{y^{\prime} n}}_{y}+z^{\prime} \cos \widehat{z^{\prime} n_{y}} \text {. } \\
& z \cos \widehat{z n_{z}}=x^{\prime} \cos \widehat{x}^{\prime} n_{z}+y^{\prime} \cos \widehat{y}^{\prime} n_{z}+z^{\prime} \cos \widehat{z^{\prime} n_{z}} \text {. } \\
& x^{\prime} \cos {\widehat{x} n_{x}^{\prime}}^{\prime}=x \cos \widehat{x n}_{x}^{\prime}+y \cos \widehat{y n}_{x}^{\prime}+z \cos \widehat{z n}_{x}^{\prime} \text {. } \\
& y^{\prime} \cos \widehat{y}^{\prime} n_{y}^{\prime}=x \cos \widehat{x n}_{y}^{\prime}+y \cos \widehat{y n}_{y}^{\prime}+z \cos \widehat{z n}_{y}{ }^{\prime} \text {. } \\
& z^{\prime} \cos {\widehat{z^{\prime} n_{z}^{\prime}}}^{\prime}=x \cos \widehat{x n}_{z}^{\prime}+y \cos \widehat{y n}_{z}^{\prime}+z \cos \widehat{z n}_{z}^{\prime} \text {. }
\end{aligned}
$$

2.030 Transformation from rectangular to spherical polar coördinates.

$r$, the radius vector to a point makes an angle $\theta$ with the $z$-axis, the projection of $r$ on the $x-y$ plane makes an angle $\phi$ with the $x$-axis.

$$
\begin{array}{ll}
x=r \sin \theta \cos \phi & r^{2}=x^{2}+y^{2}+z^{2} \\
y=r \sin \theta \sin \phi & \theta=\cos ^{-1} \frac{z}{\sqrt{x^{2}+y^{2}+z^{2}}} \\
z=r \cos \theta & \phi=\tan ^{-1} \frac{y}{x}
\end{array}
$$

2.031 Transformation from rectangular to cylindrical coördinates.

$\rho$, the perpendicular from the $z$-axis to a point makes an angle $\theta$ with the $x-z$ plane.

$$
\begin{array}{ll}
x=\rho \cos \theta & \rho=\sqrt{x^{2}+y^{2}} \\
y=\rho \sin \theta & \theta=\tan ^{-1} \frac{y}{x} \\
z=z &
\end{array}
$$

2.032 Curvilinear coördinates in general.

\section{See 4.0}

\subsection{Eulerian Angles.}

$O x y z$ and $O x^{\prime} y^{\prime} z^{\prime}$ are two systems of rectangular axes with the same origin $O$. $O K$ is perpendicular to the plane $z O z^{\prime}$ drawn so that if $O z$ is vertical, and the projection of $O z^{\prime}$ perpendicular to $O z$ is directed to the south, then $O K$ is directed to the east.

$$
\text { Angles } \begin{aligned}
z^{\widehat{O} z} & =\theta, \\
\widehat{y O K} & =\phi, \\
y^{\prime} O K & =\psi
\end{aligned}
$$


The direction cosines of the two systems of axes are given by the following scheme :

\begin{tabular}{|c|c|c|c|}
\hline & $x$ & $y$ & $z$ \\
\hline$x^{\prime}$ & $\begin{array}{c}\cos \phi \cos \theta \cos \psi-\sin \phi \sin \psi \\
y^{\prime} \\
z^{\prime}\end{array}$ & $\begin{array}{c}\sin \phi \cos \theta \cos \psi+\cos \phi \sin \psi \\
\cos \phi \sin \theta\end{array}$ & $-\begin{array}{c}\sin \theta \cos \psi \\
\sin \theta \sin \psi \\
\cos \theta\end{array}$ \\
\hline
\end{tabular}

2.050 Trilinear Coördinates.

A point in a plane is defined if its distances from two intersecting lines are given. Let $C A$, $C B$ (Fig. I) be these lines:

$$
P R=p, \quad P S=q, \quad P T=r .
$$

Taking $C A$ and $C B$ as the $x$-, $y$-axes, including an angle $C$,

$$
\begin{aligned}
& x=\frac{p}{\sin C} \\
& y=\frac{q}{\sin C}
\end{aligned}
$$

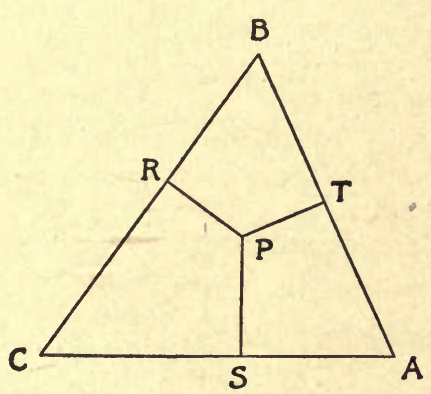

FIG. I

Any curve $f(x, y)=0$ becomes:

$$
f\left(\frac{p}{\sin C}, \frac{q}{\sin C}\right)=O
$$

If $s$ is the area of the triangle $C A B$ (triangle of reference),

$$
\begin{gathered}
2 s=a p+b q+c r, \\
a=B C, \\
b=C A, \\
c=A B,
\end{gathered}
$$

and the equation of a curve may be written in the homogeneous form:

$$
f\left(\frac{2 s p}{(a p+b q+c r) \sin C}, \frac{2 s q}{(a p+b q+c r) \sin C}\right)=0 .
$$

2.060 Quadriplanar Coördinates.

These are the analogue in 3 dimensions of trilinear coördinates in a plane (2.050). 
$x_{1}, x_{2}, x_{3}, x_{4}$ denote the distances of a point $P$ from the four sides of a tetrahedron (the tetrahedron of reference); $l_{1}, m_{1}, n_{1} ; l_{2}, m_{2}, n_{2} ; l_{3}, m_{3}, n_{3}$; and $l_{4}, m_{4}, n_{4}$ the direction cosines of the normals to the planes $x_{1}=0, x_{2}=0, x_{3}=0$, $x_{4}=0$ with respect to a rectangular system of coördinates $x, y, z$; and $d_{1}, d_{2}, d_{3}$, $d_{4}$ the distances of these 4 planes from the origin of coördinates:

$$
\text { (I) }\left\{\begin{array}{l}
x_{1}=l_{1} x+m_{1} y+n_{1} z-d_{1} \\
x_{2}=l_{2} x+m_{2} y+n_{2} z-d_{2} \\
x_{3}=l_{3} x+m_{3} y+n_{3} z-d_{3} \\
x_{4}=l_{4} x+m_{4} y+n_{4} z-d_{4} .
\end{array}\right.
$$

$s_{1}, s_{2}, s_{3}$, and $s_{4}$ are the areas of the 4 faces of the tetrahedron of reference and $V$ its volume:

$$
{ }_{3} V=x_{1} s_{1}+x_{2} s_{2}+x_{3} s_{3}+x_{4} s_{4} .
$$

By means of the first 3 equations of (I) $x, y, z$ are determined:

$$
\begin{aligned}
& x=A_{1} x_{1}+B_{1} x_{2}+C_{1} x_{3}+D_{1}, \\
& y=A_{2} x_{1}+B_{2} x_{2}+C_{2} x_{3}+D_{2}, \\
& z=A_{3} x_{1}+B_{3} x_{2}+C_{3} x_{3}+D_{3} .
\end{aligned}
$$

The equation of any surface,

$$
F(x, y, z)=0,
$$

may be written in the homogeneous form :

$$
\begin{gathered}
F\left\{\left[A_{1} x_{1}+B_{1} x_{2}+C_{1} x_{3}+\frac{D_{1}}{3 V}\left(s_{1} x_{1}+s_{2} x_{2}+s_{3} x_{3}+s_{4} x_{4}\right)\right],\right. \\
{\left[A_{2} x_{1}+B_{2} x_{2}+C_{2} x_{3}+\frac{D_{2}}{3 V}\left(s_{1} x_{1}+s_{2} x_{2}+s_{3} x_{3}+s_{4} x_{4}\right)\right],} \\
\left.\left[A_{3} x_{1}+B_{3} x_{2}+C_{3} x_{3}+\frac{D_{3}}{3 V}\left(s_{1} x_{1}+s_{2} x_{2}+s_{3} x_{3}+s_{4} x_{4}\right)\right]\right\}=0 .
\end{gathered}
$$

2.100 The equation of a line:

\section{PLANE GEOMETRY}

$$
A x+B y+C=0 \text {. }
$$

2.101 If $p$ is the perpendicular from the origin upon the line, and $\alpha$ and $\beta$ the angles $p$ makes with the $x$ - and $y$-axes:

$$
p=x \cos \alpha+y \cos \beta \text {. }
$$

2.102 If $\alpha^{\prime}$ and $\beta^{\prime}$ are the angles the line makes with the $x$-and $y$-axes:

$$
p=y \cos \alpha^{\prime}-x \cos \beta^{\prime} \text {. }
$$

2.103 The equation of a line may be written

$$
y=a x+b \text {. }
$$

$a=$ tangent of angle the line makes with the $x$-axis,

$b=$ intercept of the $y$-axis by the line. 
2.104 The two lines:

intersect at the point:

$$
\begin{gathered}
y=a_{1} x+b_{1} \\
y=a_{2} x+b_{2} \\
x=\frac{b_{2}-b_{1},}{a_{1}-a_{2}} \quad y=\frac{a_{1} b_{2}-a_{2} b_{1}}{a_{1}-a_{2}} .
\end{gathered}
$$

2.105 If $\phi$ is the angle between the two lines 2.104:

$$
\tan \phi= \pm \frac{a_{1}-a_{2}}{\mathrm{I}+a_{1} a_{2}} .
$$

2.106 Equations of two parallel lines:

$$
\left\{\begin{array} { l } 
{ A x + B y + C _ { 1 } = 0 } \\
{ A x + B y + C _ { 2 } = 0 }
\end{array} \quad \text { or } \quad \left\{\begin{array}{l}
y=a x+b_{1} \\
y=a x+b_{2}
\end{array}\right.\right.
$$

2.107 Equations of two perpendicular lines:

$$
\left\{\begin{array} { l } 
{ A x + B y + C _ { 1 } = 0 } \\
{ B x - A y + C _ { 2 } = 0 }
\end{array} \quad \text { or } \quad \left\{\begin{array}{l}
y=a x+b_{1} \\
y=-\frac{x}{a}+b_{2}
\end{array}\right.\right.
$$

2.108 Equation of line through $x_{1}, y_{1}$ and parallel to the line:

$$
\begin{array}{rlrlrl}
A x+B y+C=0 & \text { or } & y & =a x+b, \\
A\left(x-x_{1}\right)+B\left(y-y_{1}\right) & =0 & & \text { or } & y-y_{1} & =a\left(x-x_{1}\right) .
\end{array}
$$

2.109 Equation of line through $x_{1}, y_{1}$ and perpendicular to the line

$$
\begin{aligned}
A x+B y+C & =0 & \text { or } & y & =a x+b, \\
B\left(x-x_{1}\right)-A\left(y-y_{1}\right) & =0 & \text { or } & y-y_{1} & =-\frac{x-x_{1}}{a} .
\end{aligned}
$$

2.110 Equation of line through $x_{1}, y_{1}$ making an angle $\phi$ with the line $y=a x+b$ :

$$
y-y_{1}=\frac{a+\tan \phi}{\mathrm{I}-a \tan \phi}\left(x-x_{1}\right) \text {. }
$$

2.111 Equation of line through the two points, $x_{1}, y_{1}$, and $x_{2}, y_{2}$ :

$$
y-y_{1}=\frac{y_{2}-y_{1}}{x_{2}-x_{1}}\left(x-x_{1}\right) \text {. }
$$

2.112 Perpendicular distance from the point $x_{1}, y_{1}$ to the line

$$
\begin{array}{rlll}
A x+B y+C=0 & \text { or } & y=a x+b, \\
p=\frac{A x_{1}+B y_{1}+C}{\sqrt{A_{2}+B_{2}}} & \text { or } & p=\frac{y_{1}-a x_{1}-b}{\sqrt{1+a^{2}}} .
\end{array}
$$

2.113 Polar equation of the line $y=a x+b$ :

where

$$
r=\frac{b \cos \alpha}{\sin (\theta-\alpha)}
$$

$$
\tan \alpha=a
$$


2.114 If $p$, the perpendicular to the line from the origin, makes an angle $\beta$ with the axis:

$$
p=r \cos (\theta-\beta) \text {. }
$$

2.130 Area of polygon whose vertices are at $x_{1}, y_{1} ; x_{2}, y_{2} ; \ldots \ldots \ldots$ $x_{n}, y_{n}=A$.

$$
2 A=y_{1}\left(x_{n}-x_{2}\right)+y_{2}\left(x_{1}-x_{3}\right)+y_{3}\left(x_{2}-x_{4}\right)+\ldots \ldots+y_{n}\left(x_{n-1}-x_{1}\right) .
$$

\section{PLANE CURVES}

2.200 The equation of a plane curve in rectangular coördinates may be given in the forms:
(a)
(b)
(c)

$$
\begin{aligned}
& y=f(x) . \\
& x=f_{1}(t), y=f_{2}(t) . \text { The parametric form. } \\
& F(x, y)=0 .
\end{aligned}
$$

2.201 If $\tau$ is the angle between the tangent to the curve and the $x$-axis:

(a) $\tan \tau=\frac{d y}{d x}=y^{\prime}$.

(b) $\tan \tau=\frac{\frac{d f_{2}(t)}{d t}}{\frac{d f_{1}(t)}{d t}}$

(c) $\tan \tau=-\frac{\frac{\partial F(x, y)}{\partial x}}{\frac{\partial F(x, y)}{\partial y}}$.

In the following formulas,

$$
y^{\prime}=\frac{d y}{d x}=\tan \tau \text { (2.201). }
$$

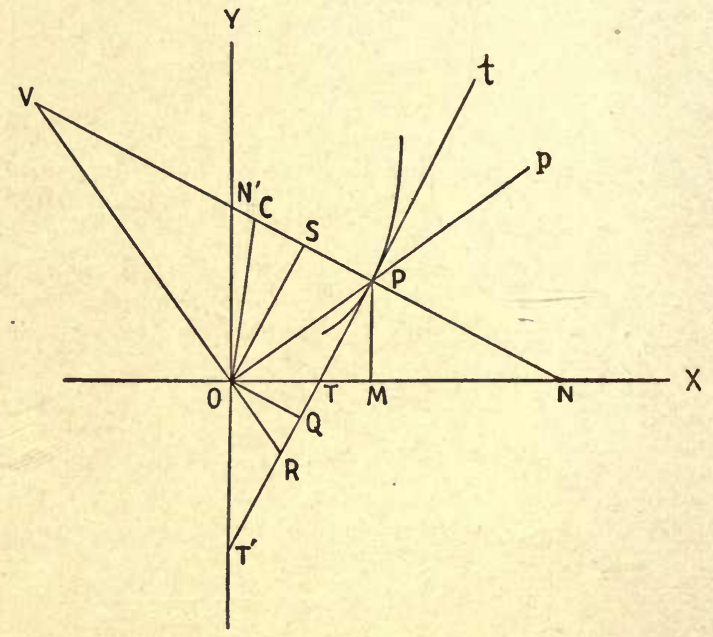

2.202 $O M=x, M P=y$, angle $X T P=\tau$.

FIG. 2

$$
T P=y \csc \tau=\frac{y \sqrt{1+y^{\prime 2}}}{y^{\prime}}=\text { tangent }
$$

$T M=\mathrm{y} \cot \tau=\frac{y}{y^{\prime}}=$ subtangent,

$$
P N=y \sec \tau=y \sqrt{I+y^{\prime 2}}=\text { normal, }
$$

$M N=y \tan \tau=y y^{\prime}=$ subnormal.

2.203 OT $=x-\frac{y}{y^{\prime}}=$ intercept of tangent on $x$-axis, $O T^{\prime}=y-x y^{\prime}=$ intercept of tangent on $y$-axis, $O N=x+y y^{\prime}=$ intercept of normal on $x$-axis, $O N^{\prime}=y+\frac{x}{y^{\prime}}=$ intercept of normal on $y$-axis. 
$2.204 O Q=\frac{y-x y^{\prime}}{\sqrt{I+y^{\prime 2}}}=$ distance of tangent from origin $=P S=$ projection of
radius vector on normal. Coördinates of $Q: \frac{y^{\prime}\left(x y^{\prime}-y\right)}{\mathrm{I}+y^{\prime 2}}, \frac{y-x y^{\prime}}{\mathrm{I}+y^{\prime 2}}$.

2.205 $O S=\frac{x+y y^{\prime}}{\sqrt{1+y^{\prime 2}}}=\begin{aligned} & \text { distance of normal from origin }=P Q=\text { projection of } \\ & \text { radius vector on tangent. }\end{aligned}$ Coördinates of $S: \frac{x+y y^{\prime}}{I+y^{\prime 2}}, \frac{\left(x+y y^{\prime}\right) y^{\prime}}{I+y^{\prime 2}}$.

2.206 $O R=\frac{\sqrt{x^{2}+y^{2}}\left(y-x y^{\prime}\right)}{x+y y^{\prime}}=$ polar subtangent,

$$
\begin{aligned}
& P R=\frac{\left(x^{2}+y^{2}\right) \sqrt{I+y^{\prime 2}}}{x+y y^{\prime}}=\text { polar tangent, } \\
& \text { Coördinates of } R: \frac{y\left(x y^{\prime}-y\right)}{x+y y^{\prime}}, \frac{x\left(y-x y^{\prime}\right)}{x+y y^{\prime}} .
\end{aligned}
$$

2.207 $O V=\frac{\sqrt{x^{2}+y^{2}}\left(x+y y^{\prime}\right)}{y-x y^{\prime}}=$ polar subnormal,

$$
P V=\frac{\left(x^{2}+y^{2}\right) \sqrt{\mathrm{I}+y^{\prime 2}}}{y-x y^{\prime}}=\text { polar normal }
$$

Coördinates of $V: \frac{y\left(x+y y^{\prime}\right)}{y-x y^{\prime}},-\frac{x\left(x+y y^{\prime}\right)}{y-x y^{\prime}}$.

2.210 The equations of the tangent at $x_{1}, y_{1}$ to the curve in the three forms of 2.200 are:
(a)

(c)

$$
\begin{aligned}
& y-y_{1}=f^{\prime}\left(x_{1}\right)\left(x-x_{1}\right) . \\
& \left(y-y_{1}\right) f_{1}^{\prime}\left(t_{1}\right)=\left(x-x_{1}\right) f_{2}^{\prime}\left(t_{1}\right) . \\
& \left(x-x_{1}\right)\left(\frac{\partial F}{\partial x}\right)_{\substack{x=x_{1} \\
y=y_{1}}}+\left(y-y_{1}\right)\left(\frac{\partial F}{\partial y}\right)_{\substack{x=x_{1} \\
y=y_{1}}}=0 .
\end{aligned}
$$

2.211 The equations of the normal at $x_{1}, y_{1}$ to the curve in the three forms of $\mathbf{2 . 2 0 0}$ are:

(a)

(b)

(c)

$$
\begin{aligned}
& f^{\prime}\left(x_{1}\right)\left(y-y_{1}\right)+\left(x-x_{1}\right)=0 . \\
& \left(y-y_{1}\right) f_{2}^{\prime}\left(t_{1}\right)+\left(x-x_{1}\right) f_{1}^{\prime}\left(t_{1}\right)=0 .
\end{aligned}
$$$$
\left(x-x_{1}\right)\left(\frac{\partial F}{\partial y}\right)_{\substack{x=x_{1} \\ y=y_{1}}}=\left(y-y_{1}\right)\left(\frac{\partial F}{\partial x}\right)_{\substack{x=x_{1} \\ y=y_{1}}} \text {. }
$$ 
2.212 The perpendicular from the origin upon the tangent to the curve $F(x, y)=0$ at the point $x, y$ is:

$$
p=\frac{x \frac{\partial F}{\partial x}+y \frac{\partial F}{\partial y}}{\sqrt{\left(\frac{\partial F}{\partial x}\right)^{2}+\left(\frac{\partial F}{\partial y}\right)^{2}}} .
$$

2.213 Concavity and Convexity. If in the neighborhood of a point $P$ a curve lies entirely on one side of the tangent, it is concave or convex upwards according as $y^{\prime \prime}=\frac{d^{2} y}{d x^{2}}$ is positive or negative. The positive direction of the axes are shown in figure 2 .

2.220 Convention as to signs. The positive direction of the normal is related to the positive direction of the tangent as the positive $y$-axis is related to the positive $x$-axis. The angle $\tau$ is measured positively in the counter-clockwise direction from the positive $x$-axis to the positive tangent.

2.221 Radius of curvature $=\rho$; curvature $=\mathrm{I} / \rho$.

$$
\frac{\mathrm{I}}{\rho}=\frac{d \tau}{d s}
$$

where $s$ is the arc drawn from a fixed point of the curve in the direction of the positive tangent.

2.222 Formulas for the radius of curvature of curves given in the three forms of 2.200 .

$$
\begin{gathered}
\rho=\frac{\left\{\mathrm{I}+\left(\frac{d y}{d x}\right)^{2}\right\}^{\frac{3}{2}}}{\frac{d^{2} y}{d x^{2}}}=\frac{\left(\mathrm{I}+y^{\prime 2}\right)^{\frac{3}{2}}}{y^{\prime \prime}} \\
\rho=\frac{\left\{\left(\frac{d x}{d t}\right)^{2}+\left(\frac{d y}{d t}\right)^{2}\right\}^{\frac{3}{2}}}{\frac{d x}{d t} \frac{d^{2} y}{d t^{2}}-\frac{d y}{d t} \frac{d^{2} x}{d t^{2}}}=\frac{\left(\frac{d s}{d t}\right)^{2}}{\left\{\left(\frac{d^{2} x}{d t^{2}}\right)^{2}+\left(\frac{d^{2} y}{d t^{2}}\right)^{2}-\left(\frac{d^{2} s}{d t^{2}}\right)^{2}\right\}^{\frac{1}{2}}}
\end{gathered}
$$

If $s$ is taken as the parameter $t$ :

$$
\begin{gathered}
\frac{\mathrm{I}}{\rho}=\frac{d x}{d s} \frac{d^{2} y}{d s^{2}}-\frac{d y}{d s} \frac{d^{2} x}{d s^{2}}=\left\{\left(\frac{d^{2} x}{d s^{2}}\right)^{2}+\left(\frac{d^{2} y}{d s^{2}}\right)^{2}\right\}^{\frac{1}{2}} \\
\rho=-\frac{\left\{\left(\frac{\partial F}{\partial x}\right)^{2}+\left(\frac{\partial F}{\partial y}\right)^{2}\right\}^{\frac{3}{2}}}{\frac{\partial^{2} F}{\partial x^{2}}\left(\frac{\partial F}{\partial y}\right)^{2}-2 \frac{\partial^{2} F}{\partial x \partial y} \frac{\partial F}{\partial x} \frac{\partial F}{\partial y}+\frac{\partial^{2} F}{\partial y^{2}}\left(\frac{\partial F}{\partial x}\right)^{2}}
\end{gathered}
$$


2.223 The center of curvature is a point $C$ (fig: 2 ) on the normal at $P$ such that $P C=\rho$. If $\rho$ is positive $C$ lies on the positive normal (2.213); if negative, on the negative normal.

2.224 The circle of curvature is a circle with $C$ as center and radius $=\rho$.

2.225 The chord of curvature is the chord of the circle of curvature passing through the origin and the point $P$.

2.226 The coördinates of the center of curvature at the point $x, y$ are $\xi, \eta$ :

$$
\begin{aligned}
& \xi=x-\rho \sin \tau \\
& \eta=y+\rho \cos \tau
\end{aligned}
$$

If $l^{\prime}, m^{\prime}$ are the direction cosines of the positive normal,

$$
\begin{aligned}
& \xi=x+l^{\prime} \rho \\
& \eta=y+m^{\prime} \rho .
\end{aligned}
$$

2.227 If $l, m$ are the direction cosines of the positive tangent and $l^{\prime}, m^{\prime}$ those of the positive normal,

$$
\begin{aligned}
& \frac{d l}{d s}=\frac{l^{\prime}}{\rho}, \frac{d m}{d s}=\frac{m^{\prime}}{\rho} . \\
& l^{\prime}=m, m^{\prime}=-l, \\
& \frac{d l^{\prime}}{d s}=-\frac{l}{\rho}, \frac{d m^{\prime}}{d s}=-\frac{m}{\rho}
\end{aligned}
$$

2.228 If the tangent and normal at $P$ are taken as the $x$ - and $y$-axes, then

$$
\rho=\operatorname{limit}_{x \rightarrow 0} \frac{x^{2}}{2 y}
$$

2.229 Points of Inflexion. For a curve given in the form (a) of 2.200 a point of inflexion is a point at which one at least of $\frac{d^{2} y}{d x^{2}}$ and $\frac{d^{2} x}{d y^{2}}$ exists and is continuous and at which one at least of $\frac{d^{2} y}{d x^{2}}$ and $\frac{d^{2} x}{d y^{2}}$ vanishes and changes sign.

If the curve is given in the form (b) a point of inflexion, $t_{1}$, is a point at which the determinant:

$$
\left|\begin{array}{ll}
f_{1}^{\prime \prime}\left(t_{1}\right) & f_{2}^{\prime \prime}\left(t_{1}\right) \\
f_{1}^{\prime}\left(t_{1}\right) & f_{2}^{\prime}\left(t_{1}\right)
\end{array}\right|
$$

vanishes and changes sign.

2.230 Eliminating $x$ and $y$ between the coördinates of the center of curvature (2.226) and the corresponding equations of the curve (2.200) gives the equation of the evolute of the curve - the locus of the center of curvature. A curve which has a given curve for evolute is called an involute of the given curve. 
2.231 The envelope to a family of curves,

I.

$$
F(x, y, a)=0 \text {, }
$$

where $a$ is a parameter, is obtained by eliminating $a$ between ( $\mathrm{I}$ ) and

2.

$$
\frac{\partial F}{\partial a}=\text { o. }
$$

2.232 If the curve is given in the form,

$$
\begin{array}{ll}
\text { I. } & x=f_{1}(t, a) \\
2 . & y=f_{2}(t, a),
\end{array}
$$

the envelope is obtained by eliminating $t$ and $a$ between (I), (2) and the functional determinant,

$$
\frac{\partial\left(f_{1}, f_{2}\right)}{\partial(t, a)}=\circ \quad(\text { see } 1.370)
$$

2.233 Pedal Curves. The locus of the foot of the perpendicular from a fixed point upon the tangent to a given curve is the pedal of the given curve with reference to the fixed point.

2.240 Asymptotes. The line

$$
y=a x+b
$$

is an asymptote to the curve $y=f(x)$ if

2.241 If the curve is

$$
\begin{aligned}
& a=\operatorname{limit}_{x \rightarrow \infty} f^{\prime}(x) \\
& b=\operatorname{limit}_{x \rightarrow \infty}\left[f(x)-x f^{\prime}(x)\right]
\end{aligned}
$$

$$
x=f_{1}(t), y=f_{2}(t),
$$

and if for a value of $t, t_{1}, f_{1}$ or $f_{2}$ becomes infinite, there will be an asymptote if for that value of $t$ the direction of the tangent to the curve approaches a limit and the distance of the tangent from a fixed point approaches a limit.

2.242 An asymptote may sometimes be determined by expanding the equation of the curve in a series,

$$
y=\sum_{k=0}^{n} a_{k} x^{k}+\sum_{k=\mathrm{I}}^{\infty} \frac{b_{k}}{x^{k}} .
$$

If

$$
\operatorname{limit}_{x \rightarrow \infty} \sum_{k=1}^{\infty} \frac{b_{k}}{x^{k}}=0,
$$

the equation of the asymptote is

$$
y=\sum_{k=0}^{n} a_{k} x^{k}
$$




If of the first degree in $x$, this represents a rectilinear asymptote; if of a higher degree, a curvilinear asymptote.

2.250 Singular Points. If the equation of the curve is $F(x, y)=0$, singular points are those for which

$$
\frac{\partial F}{\partial x}=\frac{\partial F}{\partial y}=0
$$

Put,

$$
\Delta=\frac{\partial^{2} F}{\partial x^{2}} \frac{\partial^{2} F}{\partial y^{2}}-\left(\frac{\partial^{2} F}{\partial x \partial y}\right)^{2}
$$

If $\Delta<0$ the singular point is a double point with two distinct tangents.

$\Delta>0$ the singular point is an isolated point with no real branch of the curve through it.

$\Delta=0$ the singular point is an osculating point, or a cusp. The curve has two branches, with a common tangent, which meet at the singular point. If $\frac{\partial F}{\partial x}, \frac{\partial F}{\partial y}, \frac{\partial^{2} F}{\partial x^{2}}, \frac{\partial^{2} F}{\partial y^{2}}, \frac{\partial^{2} F}{\partial x \partial y}$ simultaneously vanish at a point the singular point is one of higher order.

PLANE CURVES, POLAR COÖRDINATES

2.270 The equation of the curve is given in the form,

$$
r=f(\theta)
$$

In figure $2, O P=r$, angle $X O P=\theta$, angle $X T P=\tau$, angle $p P t=\phi$.

2.271 $\theta$ is measured in the counter-clockwise direction from the initial line, $O X$, and $s$, the arc, is so chosen as to increase with $\theta$. The angle $\phi$ is measured in the counter-clockwise direction from the positive radius vector to the positive tangent. Then,

$$
\tau=\theta+\phi \text {. }
$$

$\tan \phi=\frac{r d \theta}{d r}$

$\sin \phi=\frac{r d \theta}{d s}$

$$
\cos \phi=\frac{d r}{d s}
$$


2.273

$$
\begin{aligned}
& P R=r \sqrt{\mathrm{I}+\left(\frac{r d \theta}{d r}\right)^{2}}=\text { polar tangent } \\
& P V=\sqrt{r^{2}+\left(\frac{d r}{d \theta}\right)^{2}}=\text { polar normal } \\
& O R=r^{2} \frac{d \theta}{d r} \quad=\text { polar subtangent } \\
& O V=\frac{d r}{d \theta} \quad=\text { polar subnormal. }
\end{aligned}
$$

$$
\begin{array}{r}
\tan \tau=\frac{\sin \theta \frac{d r}{d \theta}+r \cos \theta}{\cos \theta \frac{d r}{d \theta}-r \sin \theta} \\
d s=\left\{r^{2}+\left(\frac{d r}{d \theta}\right)^{2}\right\}^{\frac{1}{2}} \dot{d} \theta
\end{array}
$$

2.275 $O Q=\frac{r^{2}}{\sqrt{r^{2}+\left(\frac{d r}{d \theta}\right)^{2}}}=p=$ distance of tangent from origin.

$$
O S=\frac{r \frac{d r}{d \theta}}{\sqrt{r^{2}+\left(\frac{d r}{d \theta}\right)^{2}}}=\text { distance of normal from origin. }
$$

2.276 If $u=\frac{\mathrm{I}}{r}$, the curve $r=f(\theta)$ is concave or convex to the origin according as

$$
u+\frac{d^{2} u}{d \theta^{2}}
$$

is positive or negative. At a point of inflexion this quantity vanishes and changes sign.

2.280 The radius of curvature is,

$$
\rho=\frac{\left\{r^{2}+\left(\frac{d r}{d \theta}\right)^{2}\right\}^{\frac{3}{2}}}{r^{2}+2\left(\frac{d r}{d \theta}\right)^{2}-r \frac{d^{2} r}{d \theta^{2}}}
$$

2.281 If $u=\frac{\mathrm{I}}{r}$ the radius of curvature is

$$
\rho=\frac{\left\{u^{2}+\left(\frac{d u}{d \theta}\right)^{2}\right\}^{\frac{3}{2}}}{u^{3}\left(u+\frac{d^{2} u}{d \theta^{2}}\right)}
$$


2.282 If the equation of the curve is given in the form,

$$
r=f(s)
$$

where $s$ is the arc measured from a fixed point of the curve,

$$
\rho=\frac{r \sqrt{\mathrm{I}-\left(\frac{d r}{d s}\right)^{2}}}{r \frac{d^{2} r}{d s^{2}}+\left(\frac{d r}{d s}\right)^{2}-\mathrm{I}} .
$$

2.283 If $p$ is the perpendicular from the origin upon the tangent to the curve,

$$
\text { I. } \rho=r \frac{d r}{d p} \quad \text { 2. } \rho=p+\frac{d^{2} p}{d \tau^{2}}
$$

2.284 If $u=\frac{\mathrm{I}}{r}$

2.285

$$
\begin{aligned}
& \frac{\mathbf{I}}{p^{2}}=u^{2}+\left(\frac{d u}{d \theta}\right)^{2} \\
& \frac{d^{2} u}{d \theta^{2}}+u=\frac{r^{2}}{p^{3}}\left(\frac{d p}{d r}\right)
\end{aligned}
$$

2.286 Polar coördinates of the center of curvature, $r_{1}, \theta_{1}$ :

$$
\begin{aligned}
r_{1}^{2} & =\frac{r^{2}\left\{\left(\frac{d r}{d \theta}\right)^{2}-r \frac{d^{2} r}{d \theta^{2}}\right\}^{2}+\left(\frac{d r}{d \theta}\right)^{2}\left\{\left(\frac{d r}{d \theta}\right)^{2}+r^{2}\right\}^{2}}{\left\{r^{2}+2\left(\frac{d r}{d \theta}\right)^{2}-r \frac{d^{2} r}{d \theta^{2}}\right\}^{2}} \\
\theta_{1} & =\theta+\chi, \\
\tan \chi & =\frac{\left(\frac{d r}{d \theta}\right)^{3}+r^{2} \frac{d r}{d \theta}}{r\left(\frac{d r}{d \theta}\right)^{2}-r^{2} \frac{d^{2} r}{d \theta^{2}}}
\end{aligned}
$$

2.287 If $2 c$ is the chord of curvature (2.225):

$$
\begin{aligned}
2 c & =2 p \frac{d r}{d p}=2 \rho \frac{p}{r} \\
& =2 \frac{u^{2}+\left(\frac{d u}{d \theta}\right)^{2}}{u^{2}\left(u+\frac{d^{2} u}{d \theta^{2}}\right)} .
\end{aligned}
$$

2.290 Rectilinear Asymptotes. If $r$ approaches $\infty$ as $\theta$ approaches an angle $\alpha$, and if $r(\alpha-\theta)$ approaches a limit, $b$, then the straight line

$$
r \sin (\alpha-\theta)=b
$$

is an asymptote to the curve $r=f(\theta)$. 
2.295 Intrinsic Equation of a plane curve. An intrinsic equation of a plane curve is one giving the radius of curvature, $\rho$, as a function of the arc, $s$,

$$
\rho=f(s)
$$

If $\tau$ is the angle between the $x$-axis and the positive tangent (2.271):

$$
\begin{array}{ll}
d \tau=\frac{d s}{f(s)} & x=x_{0}+\int_{s_{0}}^{s} \cos \tau \cdot d s \\
\tau=\tau_{0}+\int_{s_{0}}^{s} \frac{d s}{f(s)} & y=y_{0}+\int_{s_{0}}^{s} \sin \tau \cdot d s .
\end{array}
$$

\begin{tabular}{|c|c|c|c|c|c|c|}
\hline & \multicolumn{3}{|c|}{$A_{33} \neq 0$} & \multicolumn{3}{|c|}{$A_{33}=O$} \\
\hline \multirow{3}{*}{$A \neq O$} & $A_{33}<O$ & & ${ }_{33}>0$ & \multirow{3}{*}{\multicolumn{2}{|c|}{ Parabola }} & \\
\hline & \multirow{2}{*}{ Hyperbola } & $\begin{aligned} & a_{11} A \\
< & 0\end{aligned}$ & or $\begin{array}{r}a_{22} A \\
>0\end{array}$ & & & \\
\hline & & Ellipse & $\begin{array}{c}\text { Imaginary } \\
\text { Curve }\end{array}$ & & & \\
\hline \multirow[b]{2}{*}{$A=O$} & $A_{33}<O$ & \multicolumn{2}{|c|}{$A_{33}>0$} & $\begin{array}{l}A_{11} \\
<O\end{array}$ & or $\begin{array}{c}A_{22} \\
>O\end{array}$ & $\begin{aligned} A_{11} & =A_{22} \\
& =O\end{aligned}$ \\
\hline & $\begin{array}{l}\text { Pair of } \\
\text { Real } \\
\text { Straight } \\
\text { Lines } \\
\text { Intersect }\end{array}$ & \multicolumn{2}{|c|}{$\begin{array}{c}\text { Pair of Imaginary } \\
\text { Lines }\end{array}$} & $\begin{array}{c}\text { Real } \\
\text { Pair of } \mathrm{I}\end{array}$ & $\begin{array}{l}\text { Imaginary } \\
\text { Parallel Lines }\end{array}$ & $\begin{array}{l}\text { Double } \\
\text { Line }\end{array}$ \\
\hline
\end{tabular}

2.300 The general equation of the second degree:

$$
\begin{gathered}
a_{11} x^{2}+2 a_{12} x y+a_{22} y^{2}+2 a_{13} x+2 a_{23} y+a_{33}=0 \\
A=\left|\begin{array}{lll}
a_{11} & a_{12} & a_{13} \\
a_{21} & a_{22} & a_{23} \\
a_{31} & a_{32} & a_{33}
\end{array}\right| ; a_{h k}=a_{k h} \\
A_{h k}=\text { Minor of } a_{h k} .
\end{gathered}
$$

Criterion giving the nature of the curve:

(Pascal: Repertorium der höheren Mathematik, II, I, p. 228) 
2.400 Parabola (Fig. 3).

$2.401 O$, Vertex; $F$, Focus; ordinate through $D$, Directrix.

Equation of parabola, origin at $O$,

$$
\begin{aligned}
y^{2} & =4 a x \\
x & =O M, y=M P, \\
O F & =O D=a \\
F L & =2 a=\text { semi latus } \\
& \text { rectum. }
\end{aligned}
$$

$F P=D^{\prime} P$.

\section{$2.402 F P=F T=M D$}

$=x+a$.

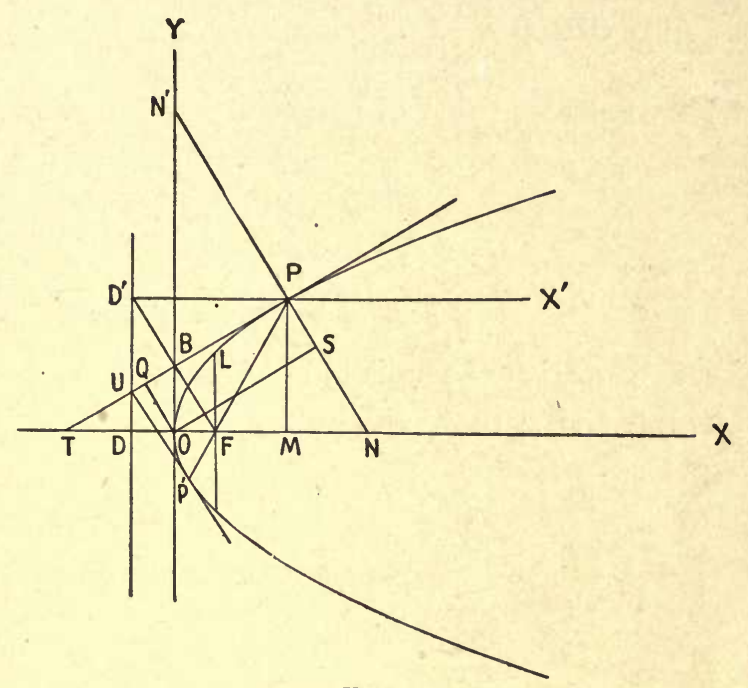

FIG. 3

$$
N P=2 \sqrt{a(a+x)}, T M=2 x, M N=2 a, O N=x+2 a .
$$

$O N^{\prime}=\sqrt{\frac{\bar{x}}{a}}(x+2 a), O Q=x \sqrt{\frac{a}{a+x}}, O S=(x+2 a) \sqrt{\frac{x}{a+x}}$.

$F B$ perpendicular to tangent $T P$.

$$
\begin{aligned}
& F B=\sqrt{a(a+x)}, T P=2 T B=2 \sqrt{x(a+x)} . \\
& \overline{F B}^{2}=F T \times F O=F P \times F O .
\end{aligned}
$$

The tangents $T P$ and $U P^{\prime}$ at the extremities of a focal chord $P F P^{\prime}$ meet on the directrix at $U$ at right angles.

$$
\tau=\text { angle } X T P \text {. }
$$

$$
\tan \tau=\sqrt{\frac{a}{x}}
$$

The tangent at $P$ bisects the angles $F P D^{\prime}$ and $F U D^{\prime}$.

2.403 Radius of curvature:

$$
\rho=\frac{2(x+a)^{\frac{3}{2}}}{\sqrt{a}}=\frac{I}{4} \frac{\overline{N P}^{3}}{a^{2}} .
$$

Coördinates of center of curvature:

$$
\xi=3 x+2 a, \eta=-2 x \sqrt{\frac{x}{a}}
$$

Equation of Evolute:

$$
27 a y^{2}=4(x-2 a)^{3}
$$


2.404 Length of arc of parabola measured from vertex,

$$
s=\sqrt{x(x+a)}+a \log \left(\sqrt{I+\frac{x}{a}}+\sqrt{\frac{x}{a}}\right) .
$$

Area $O P M O=\frac{I}{3} x y$.

2.405 Polar equation of parabola:

$$
\begin{aligned}
& r=F P, \\
& \theta=\text { angle } X F P, \\
& r=\frac{2 a}{\mathrm{I}-\cos \theta} .
\end{aligned}
$$

2.406 Equation of Parabola in terms of $p$, the perpendicular from $F$ upon the tangent, and $r$, the radius vector $F P$ :

$$
\frac{l}{p^{2}}=\frac{2}{r}
$$

$l=$ semi latus rectum.

2.410 Ellipse (Fig. 4).

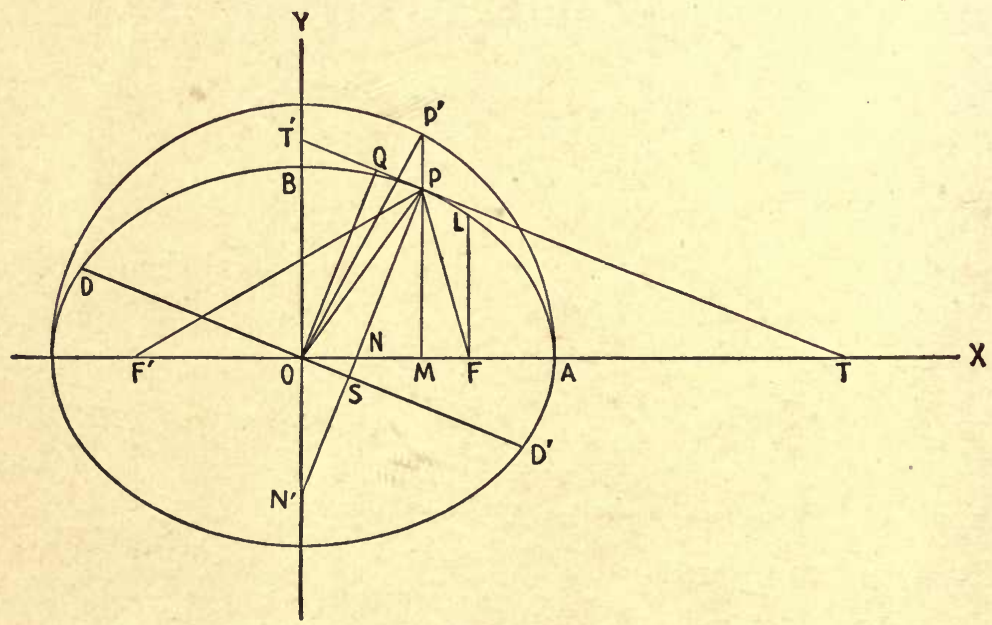

FIG. 4

$2.411 O$, Centre; $F, F^{\prime}$, Foci.

Equation of Ellipse origin at $O$ :

$$
\begin{gathered}
\frac{x^{2}}{a^{2}}+\frac{y^{2}}{b^{2}}=I \\
x=O M, y=M P, a=O A, b=O B .
\end{gathered}
$$


2.412 Parametric Equations of Ellipse,

$$
x=a \cos \phi, \quad y=b \sin \phi .
$$

$\phi=$ angle $X O P^{\prime}$, where $P^{\prime}$ is the point where the ordinate at $P$ meets the eccentric circle, drawn with $O$ as center and radius $a$.

$2.413 \quad O F=O F^{\prime}=e a$

$$
e=\text { eccentricity }=\frac{\sqrt{a^{2}-b^{2}}}{a}
$$

$F L=\frac{b^{2}}{a}=a\left(\mathrm{I}-e^{2}\right)=$ semi latus rectum.

$F^{\prime} P=a+e x, F P=a-e x, F P+F^{\prime} P=2 a$.

$\tau=$ angle $X T T^{\prime}$.

$\tan \tau=-\frac{b x}{a \sqrt{a^{2}-x^{2}}}$.

$$
\begin{aligned}
& N M=\frac{b^{2} x}{a^{2}}, O N=e^{2} x, O T=\frac{a^{2}}{x}, O T^{\prime}=\frac{b^{2}}{y}, M T=\frac{a^{2}-x^{2}}{x} \\
& P T=\frac{\sqrt{a^{2}-x^{2}} \sqrt{a^{2}-e^{2} x^{2}}}{x}, O N^{\prime}=\frac{e^{2} a}{b} \sqrt{a^{2}-x^{2}}, P S=\frac{a b}{\sqrt{a^{2}-e^{2} x^{2}}}, \\
& O S=\frac{e^{2} x \sqrt{a^{2}-x^{2}}}{\sqrt{a^{2}-e^{2} x^{2}}} .
\end{aligned}
$$

2.414 $D D^{\prime}$ parallel to $T^{\prime} T ; D D^{\prime}$ and $P P^{\prime}$ are conjugate diameters:

$$
\begin{aligned}
O D^{2} & =a^{2}-e^{2} x^{2}=F P \times F^{\prime} P . \\
O P^{2}+O D^{2} & =a^{2}+b^{2} . \\
P S \times O D & =a b .
\end{aligned}
$$

Equation of Ellipse referred to conjugate diameters as axes:

$$
\begin{array}{ll}
\frac{x^{2}}{a^{\prime 2}}+\frac{y^{2}}{b^{\prime 2}}=\mathrm{I} & \begin{array}{l}
\alpha=\text { angle } X O P \\
\beta=\text { angle } X O D
\end{array}
\end{array}
$$

$$
\begin{array}{ll}
a^{\prime}=O D^{\prime} & a^{\prime 2}=\frac{a^{2} b^{2}}{a^{2} \sin ^{2} \alpha+b^{2} \cos ^{2} \alpha} \quad \tan \alpha \tan \beta=-\frac{b^{2}}{a^{2}} \\
b^{\prime}=O P & b^{\prime 2}=\frac{a^{2} b^{2}}{a^{2} \sin ^{2} \beta+b^{2} \cos ^{2} \beta}
\end{array}
$$

2.415 Radius of curvature of Ellipse:

$$
\begin{gathered}
\rho=\frac{\left(a^{4} y^{2}+b^{4} x^{2}\right)^{\frac{3}{2}}}{a^{4} b^{4}}=\frac{\left(a^{2}-e^{2} x^{2}\right)^{\frac{3}{2}}}{a b} \\
\text { angle } F P N=\text { angle } F^{\prime} P N=\omega, \\
\tan \omega=\frac{e a y}{b^{2}} \\
\frac{2}{\rho \cos \omega}=\frac{I}{F P}+\frac{I}{F^{\prime} P}
\end{gathered}
$$


Coördinates of center of curvature:

$$
\xi=\frac{e^{2} x^{3}}{a^{2}}, \eta=-\frac{a^{2} e^{2} y^{3}}{b^{4}}
$$

Equation of Evolute of Ellipse,

$$
\left(\frac{a x}{e^{2}}\right)^{3}+\left(\frac{b y}{e^{2}}\right)^{\frac{3}{3}}=\mathrm{r}
$$

2.416 Area of Ellipse, $\pi a b$.

Length of arc of Ellipse,

$$
s=a \int_{0}^{\phi} \sqrt{\mathrm{I}-e^{2} \sin ^{2} \phi} d \phi
$$

2.417 Polar Equation of Ellipse,

$$
\begin{aligned}
r=F^{\prime} P, \theta & =\text { angle } X F^{\prime} P, \\
r & =\frac{a\left(\mathrm{I}-e^{2}\right)}{\mathrm{I}-e \cos \theta}
\end{aligned}
$$

2.418

$$
\begin{aligned}
r=O P, \theta & =\text { angle } X O P, \\
r & =\frac{b}{\sqrt{I-e^{2} \cos ^{2} \theta}}
\end{aligned}
$$

2.419 Equation of Ellipse in terms of $p$, the perpendicular from $F$ upon the tangent at $P$, and $r$, the radius vector $F P$ :

$$
\begin{aligned}
\frac{l}{p^{2}} & =\frac{2}{r}-\frac{I}{a} \\
l & =\text { semi latus rectum. }
\end{aligned}
$$

2.420 Hyperbola (Fig. 5).

$2.421 O$, Center; $F, F^{\prime}$, Foci.

Equation of hyperbola, origin at $O$,

$$
\begin{gathered}
\frac{x^{2}}{a^{2}}-\frac{y^{2}}{b^{2}}=\mathrm{I} \\
x=O M, y=M P, a=O A=O A^{\prime} .
\end{gathered}
$$

2.422 Parametric Equations of hyperbola,

or

$$
\begin{aligned}
& x=a \cosh u, y=b \sinh u . \\
& x=a \sec \phi, y=b \tan \phi .
\end{aligned}
$$

$\phi=$ angle $X O P^{\prime}$, where $P^{\prime}$ is the point where the ordinate at $T$ meets the circle of radius $a$, center $O$. 
$2.423 \quad O F=O F^{\prime}=e a$.

$$
e=\text { eccentricity }=\frac{\sqrt{a^{2}+b^{2}}}{a} .
$$

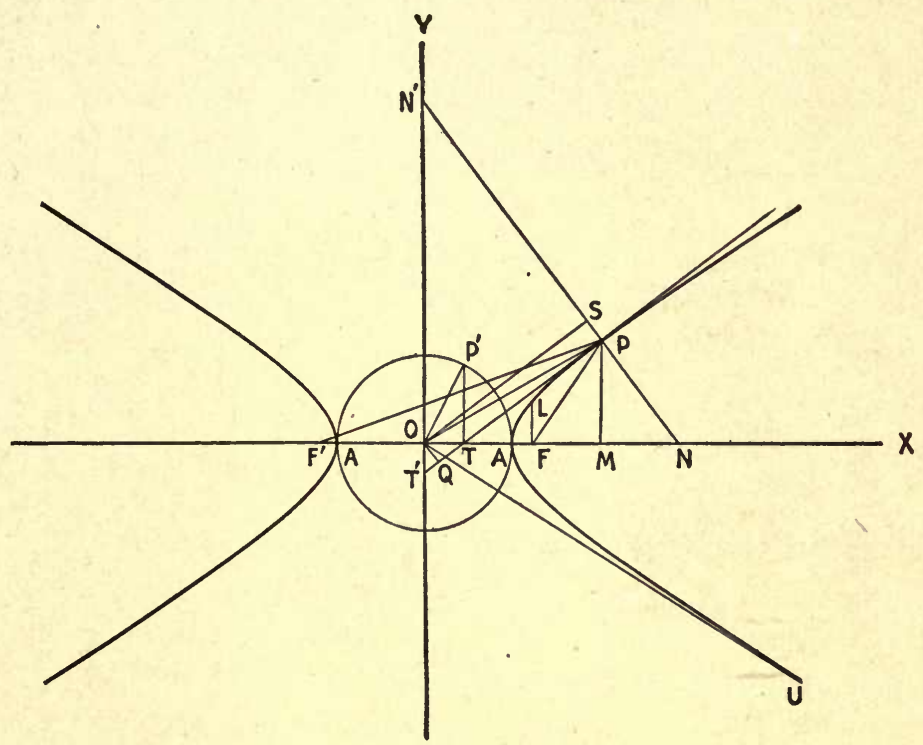

FIG. 5

$F L=\frac{b^{2}}{a}=a\left(e^{2}-\mathrm{I}\right)=$ semi latus rectum.

$F^{\prime} P=e x+a, F P=e x-a, F^{\prime} P-F P=2 a$.

$\boldsymbol{\tau}=$ angle $X T P$.

$\tan \tau=\frac{b x}{a \sqrt{x^{2}-a^{2}}}$

$$
\begin{aligned}
& N M=\frac{b^{2} x}{a^{2}}, O N=e^{2} x, O T=\frac{a^{2}}{x}, O T^{\prime}=\frac{b^{2}}{y}, \\
& M T=\frac{x^{2}-a^{2}}{x}, P T=\frac{\sqrt{x^{2}-a^{2}} \sqrt{e^{2} x^{2}-a^{2}}}{x}, O N^{\prime}=\frac{e^{2} a}{b} \sqrt{x^{2}-a^{2}} \\
& P S=\frac{a b}{\sqrt{e^{2} x^{2}-a^{2}}}, O S=\frac{e^{2} x \sqrt{x^{2}-a^{2}}}{\sqrt{e^{2} x^{2}-a^{2}}} .
\end{aligned}
$$

$$
O U=\text { Asymptote }
$$

$$
\tan X O U=\frac{b}{a} .
$$

$b=$ distance of vertex $A$ from asymptote. 
2.425 Radius of curvature of hyperbola,

$$
\rho=\frac{\left(e^{2} x^{2}-a^{2}\right)^{\frac{3}{2}}}{a b}
$$

$$
\begin{aligned}
\text { angle } F^{\prime} P T & =\text { angle } F P T . \\
\text { angle } F P N & =\omega=\frac{\pi}{2}-F P T . \\
\text { angle } F^{\prime} P N & =\omega^{\prime}=\frac{\pi}{2}+F^{\prime} P T . \\
\tan \omega & =\frac{a e y}{b^{2}} \\
\cos \omega & =\frac{b}{\sqrt{e^{2} x^{2}-a^{2}}} \\
\frac{2}{\rho \cos \omega} & =\frac{\mathrm{I}}{F P}-\frac{\mathrm{I}}{F^{\prime} P}
\end{aligned}
$$

Coördinates of center of curvature,

$$
\xi=\frac{e^{2} x^{3}}{a^{2}}, \eta=-\frac{a^{2} e^{2} y^{3}}{b^{4}}
$$

Equation of Evolute of hyperbola,

$$
\left(\frac{a x}{e^{2}}\right)^{\frac{3}{3}}-\left(\frac{b y}{e^{2}}\right)^{3}=\mathrm{I}
$$

2.426 In a rectangular hyperbola $b=a$; the asymptotes are perpendicular to each other. Equation of rectangular hyperbola with asymptotes as axes and origin at $O$ :

$$
x y=\frac{a^{2}}{2} .
$$

2.427 Length of arc of hyperbola,

$$
s=\frac{b^{2}}{a e} \int_{0}^{\phi} \frac{\sec ^{2} \phi d \phi}{\sqrt{\mathrm{I}-k^{2} \sin ^{2} \phi}}, \quad k=\frac{\mathrm{I}}{e}, \quad \tan \phi=\frac{a e y}{b^{2}} .
$$

2.428 Polar Equation of hyperbola:

$$
\begin{aligned}
& r=F^{\prime} P, \quad \theta=X F^{\prime} P, \quad r=a \frac{e^{2}-\mathrm{I}}{e \cos \theta-\mathrm{I}} \\
& r=O P, \quad \theta=X O P, \quad r^{2}=\frac{b^{2}}{e^{2} \cos ^{2} \theta-\mathrm{I}} .
\end{aligned}
$$

2.429 Equation of right-hand branch of hyperbola in terms of $p$, the perpendicular from $F$ upon the tangent at $P$ and $r$, the radius vector $F P$,

$$
\begin{aligned}
\frac{l}{p^{2}} & =\frac{2}{r}+\frac{I}{a} . \\
l & =\text { semi latus rectum. }
\end{aligned}
$$


2.450 Cycloids and Trochoids.

If a circle of radius $a$ rolls on a straight line as base the extremity of any radius, $a$, describes a cycloid. The rectangular equation of a cycloid is:

$$
\begin{aligned}
& x=a(\phi-\sin \phi), \\
& y=a(I-\cos \phi),
\end{aligned}
$$

where the $x$-axis is the base with the origin at the initial point of contact. $\phi$ is the angle turned through by the moving circle. (Fig. 6.)

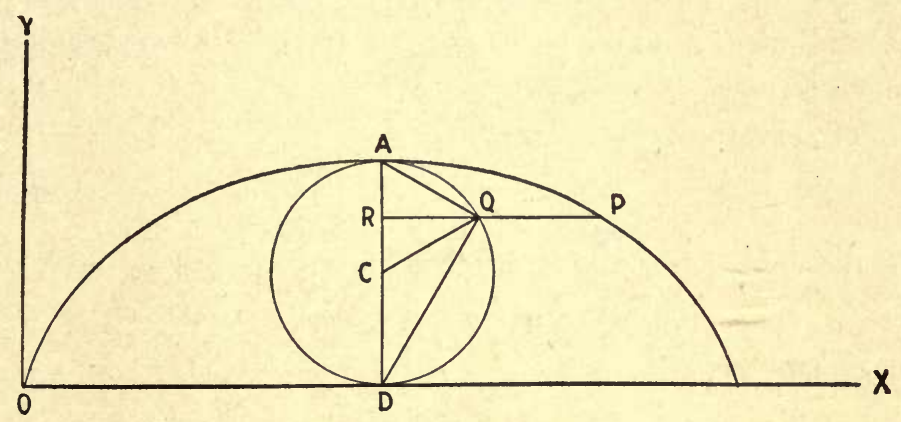

Fig.6

$A=$ vertex of cycloid.

$C=$ center of generating circle, drawn tangent at $A$.

The tangent to the cycloid at $P$ is parallel to the chord $A Q$.

$\operatorname{Arc} A P=2 \times$ chord $A Q$.

The radius of curvature at $P$ is parallel to the chord $Q D$ and equal to $2 \times$ chord $Q D$. $P Q=$ circular $\operatorname{arc} A Q$.

Length of cycloid: $s=8 a ; a=C A$.

Area of cycloid: $S=3 \pi a^{2}$.

2.451 A point on the radius, $b>a$, describes a prolate trochoid. A point, $b<a$, describes a curtate trochoid. The general equation of trochoids and cycloids is

$$
\begin{aligned}
& x=a \phi-(a+d) \sin \phi, \\
& y=(a+d)(\mathrm{I}-\cos \phi), \\
& d=\circ \text { Cycloid, } \\
& d>\circ \text { Prolate trochoid, } \\
& d<0 \text { Curtate trochoid. }
\end{aligned}
$$

Radius of curvature:

$$
\rho=\frac{\left(2 a y+d^{2}\right)^{\frac{3}{2}}}{a y+a d+d^{2}}
$$


2.452 Epi- and Hypocycloids. An epicycloid is described by a point on a circle of radius $a$ that rolls on the convex side o a fixed circle of radius $b$. An hypocycloid is described by a point on a circle of radius $a$ that rolls on the concave side of a fixed circle of radius $b$.

Equations of epi- and hypocycloids.

Upper sign: Epicycloid,

Lower sign: Hypocycloid.

$$
\begin{aligned}
& x=(b \pm a) \cos \phi \mp \cos \frac{b \pm a}{a} \phi, \\
& y=(b \pm a) \sin \phi-a \sin \frac{b \pm a}{a} \phi .
\end{aligned}
$$

The origin is at the center of the fixed circle. The $x$-axis is the line joining the centers of the two circles in the initial position and $\phi$ is the angle turned through by the moving circle.

. Radius of curvature:

$$
\rho=\frac{2 a(b \pm a)}{b \pm 2 a} \sin \frac{a}{2 b} \phi
$$

2.453 In the epicycloid put $b=a$. The curve becomes a Cardioid:

$$
\left(x^{2}+y^{2}\right)^{2}-6 a^{2}\left(x^{2}+y^{2}\right)+8 a^{3} x=3 a^{4} \text {. }
$$

2.454 Catenary. The equation may be written:

I.

$$
\begin{aligned}
& y=\frac{I}{2} a\left(e^{\frac{x}{a}}+e^{-\frac{x}{a}}\right) . \\
& y=a \cosh \frac{x}{a} \\
& x=a \log \frac{y \pm \sqrt{y^{2}-a^{2}}}{a} .
\end{aligned}
$$

The radius of curvature, which is equal to the length of the normal, is:

$$
\rho=a \cosh ^{2} \frac{x}{a}
$$

2.455 Spiral of Archimedes. A point moving uniformly along a line which rotates uniformly about a fixed point describes a spiral of Archimedes. The equation is:

or

$$
r=a \theta
$$

$$
\sqrt{x^{2}+y^{2}}=a \tan ^{-1} \frac{y}{x}
$$

The polar subtangent $=$ polar subnormal $=a$.

Radius of curvature:

2.456 Hyperbolic spiral:

$$
\rho=\frac{r\left(\mathrm{I}+\theta^{2}\right)^{\frac{3}{2}}}{\theta\left(2+\theta^{2}\right)}=\frac{\left(r^{2}+a^{2}\right)^{\frac{3}{2}}}{r^{2}+2 a^{2}} .
$$

$$
r \theta=a .
$$


2.457 Parabolic spiral:

$$
r^{2}=a^{2} \theta
$$

2.458 Logarithmic or equiangular spiral:

$$
\begin{aligned}
& r=a e^{n \theta} \\
& n=\cot \alpha=\text { const., } \\
& \alpha=\text { angle tangent to curve makes with the radius vector. }
\end{aligned}
$$

2.459 Lituus:

$$
r \sqrt{\theta}=a .
$$

2.460 Neoid:

2.461 Cissoid:

$$
r=a+b \theta
$$

$$
\begin{aligned}
\left(x^{2}+y^{2}\right) x & =2 a y^{2}, \\
r & =2 a \tan \theta \sin \theta .
\end{aligned}
$$

2.462 Cassinoid:

$$
\begin{aligned}
\left(x^{2}+y^{2}+a^{2}\right)^{2} & =4 a^{2} x^{2}+b^{4}, \\
r^{4}-2 a^{2} r^{2} \cos 2 \theta & =b^{4}-a^{4} .
\end{aligned}
$$

2.463 Lemniscate ( $b=a$ in Cassinoid):

$$
\begin{aligned}
\left(x^{2}+y^{2}\right)^{2} & =2 a^{2}\left(x^{2}-y^{2}\right), \\
r^{2} & =2 a^{2} \cos 2 \theta .
\end{aligned}
$$

2.464 Conchoid:

2.465 Witch of Agnesi:

$$
x^{2} y^{2}=(b+y)^{2}\left(a^{2}-y^{2}\right) .
$$

$$
x^{2} y=4 a^{2}(2 a-y) \text {. }
$$

2.466 Tractrix:

$$
\begin{aligned}
x & =\frac{1}{2} a \log \frac{a+\sqrt{a^{2}-y^{2}}}{a-\sqrt{a^{2}-y^{2}}}-\sqrt{a^{2}-y^{2}} \\
\frac{d y}{d x} & =-\frac{y}{\sqrt{a^{2}-y^{2}}} \\
\rho & =\frac{a \sqrt{a^{2}-y^{2}}}{y}
\end{aligned}
$$

\section{SOLID GEOMETRY}

2.600 The Plane. The general equation of the plane is:

$$
A x+B y+C z+D=0 \text {. }
$$

2.601 $l, m, n$ are the direction cosines of the normal to the plane and $p$ is the perpendicular distance from the origin upon the plane.

$$
\begin{aligned}
l, m, n & =\frac{A, B, C}{\sqrt{A^{2}+B^{2}+C^{2}}} \\
p & =l x+m y+n z, \\
p & =-\frac{D}{\sqrt{A^{2}+B^{2}+C^{2}}} .
\end{aligned}
$$


2.602 The perpendicular from the point $x_{1}, y_{1}, z_{1}$ upon the plane $A x+B y+$ $C z+D=0$ is:

$$
d=\frac{A x_{1}+B y_{1}+C z_{1}+D}{\sqrt{A^{2}+B^{2}+C^{2}}}
$$

2.603 $\theta$ is the angle between the two planes:

$$
\begin{gathered}
A_{1} x+B_{1} y+C_{1} z+D_{1}=0 \\
A_{2} x+B_{2} y+C_{2} z+D_{2}=0 \\
\cos \theta=\frac{A_{1} A_{2}+B_{1} B_{2}+C_{1} C_{2}}{\sqrt{A_{1}^{2}+B_{1}{ }^{2}+C_{1}^{2}} \sqrt{A_{2}{ }^{2}+B_{2}{ }^{2}+C_{2}{ }^{2}}} .
\end{gathered}
$$

2.604 Equation of the plane passing through the three points $\left(x_{1}, y_{1}, z_{1}\right)\left(x_{2}, y_{2}, z_{2}\right)$ $\left(x_{3}, y_{3}, z_{3}\right)$ :

$$
x\left|\begin{array}{lll}
y_{1} & z_{1} & \mathrm{I} \\
y_{2} & z_{2} & \mathrm{I} \\
y_{3} & z_{3} & \mathrm{I}
\end{array}\right|+y\left|\begin{array}{lll}
z_{1} & x_{1} & \mathrm{I} \\
z_{2} & x_{2} & \mathrm{I} \\
z_{3} & x_{3} & \mathrm{I}
\end{array}\right|+z\left|\begin{array}{lll}
x_{1} & y_{1} & \mathrm{I} \\
x_{2} & y_{2} & \mathrm{I} \\
x_{3} & y_{3} & \mathrm{I}
\end{array}\right|=\left|\begin{array}{lll}
x_{1} & y_{1} & z_{1} \\
x_{2} & y_{2} & z_{2} \\
x_{3} & y_{3} & z_{3}
\end{array}\right|
$$

\section{THE RIGHT LINE}

2.620 The equations of a right line passing through the point $x_{1}, y_{1}, z_{1}$, and whose direction cosines are $l, m, n$ are:

$$
\frac{x-x_{1}}{l}=\frac{y-y_{1}}{m}=\frac{z-z_{1}}{n} .
$$

2.621 $\theta$ is the angle between the two lines whose direction cosines are $l_{1}, m_{1}, n_{1}$ and $l_{2}, m_{2}, n_{2}$ :

$$
\begin{aligned}
& \cos \theta=l_{1} l_{2}+m_{1} m_{2}+n_{1} n_{2}, \\
& \sin ^{2} \theta=\left(l_{1} m_{2}-l_{2} m_{1}\right)^{2}+\left(m_{1} n_{2}-m_{2} n_{1}\right)^{2}+\left(n_{1} l_{2}-n_{2} l_{1}\right)^{2} .
\end{aligned}
$$

2.622 The direction cosines of the normal to the plane defined by the two lines whose direction cosines are $l_{1}, m_{1}, n_{1}$ and $l_{2}, m_{2} n_{2}$ are:

$$
\frac{m_{1} n_{2}-m_{2} n_{1}}{\sin \theta}, \quad \frac{n_{1} l_{2}-n_{2} l_{1}}{\sin \theta}, \quad \frac{l_{1} m_{2}-l_{2} m_{1}}{\sin \theta}
$$

2.623 The shortest distance between the two lines:

is:

$$
\frac{x-x_{1}}{l_{1}}=\frac{y-y_{1}}{m_{1}}=\frac{z-z_{1}}{n_{1}} \text { and } \frac{x-x_{2}}{l_{2}}=\frac{y-y_{2}}{m_{2}}=\frac{z-z_{2}}{n_{2}}
$$

$d=\frac{\left(x_{1}-x_{2}\right)\left(m_{1} n_{2}-m_{2} n_{1}\right)+\left(y_{1}-y_{2}\right)\left(n_{1} l_{2}-n_{2} l_{1}\right)+\left(z_{1}-z_{2}\right)\left(l_{1} m_{2}-l_{2} m_{1}\right)}{\left\{\left(m_{1} n_{2}-m_{2} n_{1}\right)^{2}+\left(n_{1} l_{2}-n_{2} l_{1}\right)^{2}+\left(l_{1} m_{2}-l_{2} m_{1}\right)^{2}\right\}^{\frac{1}{2}}}$

2.624 The direction cosines of the shortest distance between the two lines are:

$$
\frac{\left(m_{1} n_{2}-n_{2} m_{1}\right),\left(n_{1} l_{2}-n_{2} l_{1}\right),\left(l_{1} m_{2}-l_{2} m_{1}\right)}{\left\{\left(m_{1} n_{2}-m_{2} n_{1}\right)^{2}+\left(n_{1} l_{2}-n_{2} l_{1}\right)^{2}+\left(l_{1} m_{2}-l_{2} m_{1}\right)^{2}\right\}^{\frac{1}{2}}}
$$


2.625 The perpendicular distance from the point $x_{2}, y_{2}, z_{2}$ to the line:

is:

$$
\frac{x-x_{1}}{l_{1}}=\frac{y-y_{1}}{m_{1}}=\frac{z-z_{1}}{n_{1}}
$$

$d=\left\{\left(x_{2}-x_{1}\right)^{2}+\left(y_{2}-y_{1}\right)^{2}+\left(z_{2}-z_{1}\right)^{2}\right\}^{\frac{1}{2}}-\left\{l_{1}\left(x_{2}-x_{1}\right)+m_{1}\left(y_{2}-y_{1}\right)+n_{1}\left(z_{3}-z_{1}\right)\right\}$.

2.626 The direction cosines of the line passing through the two points $x_{1}, y_{1}, z_{1}$ and $x_{2}, y_{2}, z_{2}$ are:

$$
\frac{\left(x_{2}-\dot{x}_{1}\right), \quad\left(y_{2}-y_{1}\right), \quad\left(z_{2}-z_{1}\right)}{\left\{\left(x_{2}-x_{1}\right)^{2}+\left(y_{2}-y_{1}\right)^{2}+\left(z_{2}-z_{1}\right)^{2}\right\}^{\frac{1}{2}}} \text {. }
$$

2.627 The two lines:

$$
\begin{aligned}
& x=m_{1} z+p_{1}, \\
& y=n_{1} z+q_{1}, \quad \text { and } \quad y=n_{2} z+q_{2},
\end{aligned}
$$

intersect at a point if,

$$
\left(m_{1}-m_{2}\right)\left(q_{1}-q_{2}\right)-\left(n_{1}-n_{2}\right)\left(p_{1}-p_{2}\right)=0 .
$$

The coördinates of the point of intersection are:

$$
x=\frac{m_{1} p_{2}-m_{2} p_{1}}{m_{1}-m_{2}}, \quad y=\frac{n_{1} q_{2}-n_{2} q_{1}}{n_{1}-n_{2}}, \quad z=\frac{p_{2}-p_{1}}{m_{1}-m_{2}}=\frac{q_{2}-q_{1}}{n_{1}-n_{2}} .
$$

The equation of the plane containing the two lines is then

$$
\left(n_{1}-n_{2}\right)\left(x-m_{1} z-p_{1}\right)=\left(m_{1}-m_{2}\right)\left(y-n_{1} z-q_{1}\right)
$$

\section{SURFACES}

2.640 A single equation in $x, y, z$ represents a surface:

$$
F(x, y, z)=0 \text {. }
$$

2.641 The direction cosines of the normal to the surface are:

$$
l, m, n=\frac{\frac{\partial F}{\partial x}, \quad \frac{\partial F}{\partial y}, \quad \frac{\partial F}{\partial z}}{\left\{\left(\frac{\partial F}{\partial x}\right)^{2}+\left(\frac{\partial F}{\partial y}\right)^{2}+\left(\frac{\partial F}{\partial z}\right)^{2}\right\}^{\frac{1}{2}}}
$$

2.642 The perpendicular from the origin upon the tangent plane at $x, y, z$ is:

$$
p=l x+m y+n z \text {. }
$$

2.643 The two principal radii of curvature of the surface $F(x, y, z)=0$ are given by the two roots of: 
where:

$$
\left|\begin{array}{cccc}
\frac{k}{\rho}+\frac{\partial^{2} F}{\partial x^{2}} & \frac{\partial^{2} F}{\partial x \partial y} & \frac{\partial^{2} F}{\partial x \partial z} & \frac{\partial F}{\partial x} \\
\frac{\partial^{2} F}{\partial x \partial y} & \frac{k}{\rho}+\frac{\partial^{2} F}{\partial y^{2}} & \frac{\partial^{2} F}{\partial y \partial z} & \frac{\partial F}{\partial y} \\
\frac{\partial^{2} F}{\partial x \partial z} & \frac{\partial^{2} F}{\partial y \partial z} & \frac{k}{\rho}+\frac{\partial^{2} F}{\partial z^{2}} & \frac{\partial F}{\partial z} \\
\frac{\partial F}{\partial x} & \frac{\partial F}{\partial y} & \frac{\partial F}{\partial z} & \circ
\end{array}\right|=0,
$$

$$
k^{2}=\left(\frac{\partial F}{\partial x}\right)^{2}+\left(\frac{\partial F}{\partial y}\right)^{2}+\left(\frac{\partial F}{\partial z}\right)^{2}
$$

2.644 The coördinates of each center of curvature are:

$$
\xi=x+\frac{\rho}{k} \frac{\partial F}{\partial x}, \quad \eta=y+\frac{\rho}{k} \frac{\partial F}{\partial y}, \quad \zeta=z+\frac{\rho}{k} \frac{\partial F}{\partial z} .
$$

2.645 The envelope of a family of surfaces:

I.

$$
F(x, y, z, \alpha)=0
$$

is found by eliminating $\alpha$ between (I) and

2.

$$
\frac{\partial F}{\partial \alpha}=0
$$

2.646 The characteristic of a surface is a curve defined by the two equations (I) and (2) in $\mathbf{2 . 6 4 5 .}$

2.647 The envelope of a family of surfaces with two variable parameters, $\alpha, \beta$, is obtained by eliminating $\alpha$ and $\beta$ between:

I.

$$
\begin{aligned}
F(x, y, z, \alpha, \beta) & =0 . \\
\frac{\partial F}{\partial \alpha} & =0 . \\
\frac{\partial F}{\partial \beta} & =0 .
\end{aligned}
$$

3.

2.648 The equations of a surface may be given in the parametric form:

$$
x=f_{1}(u, v), \quad y=f_{2}(u, v), \quad z=f_{3}(u, v) \text {. }
$$

The equation of a tangent plane at $x_{1}, y_{1}, z_{1}$ is:

where

$$
\left(x-x_{1}\right) \frac{\partial\left(f_{2}, f_{3}\right)}{\partial(u, v)}+\left(y-y_{1}\right) \frac{\partial\left(f_{3}, f_{1}\right)}{\partial(u, v)}+\left(z-z_{1}\right) \frac{\partial\left(f_{1}, f_{2}\right)}{\partial(u, v)}=0,
$$

$$
\frac{\partial\left(f_{2}, f_{3}\right)}{\partial(u, v)}=\left|\begin{array}{ll}
\frac{\partial f_{2}}{\partial u} & \frac{\partial f_{2}}{\partial v} \\
\frac{\partial f_{3}}{\partial u} & \frac{\partial f_{3}}{\partial v}
\end{array}\right|, \text { etc. See } 1.370
$$





2.649 The direction cosines to the normal to the surface in the form $\mathbf{2 . 6 4 8}$ are:

$$
l, m, n=\frac{\frac{\partial\left(f_{2}, f_{3}\right)}{\partial(u, v)}, \frac{\partial\left(f_{3}, f_{1}\right)}{\partial(u, v)}, \frac{\partial\left(f_{1}, f_{2}\right)}{\partial(u, v)}}{\left\{\left(\frac{\partial\left(f_{2}, f_{3}\right)}{\partial(u, v)}\right)^{2}+\left(\frac{\partial\left(f_{3}, f_{1}\right)}{\partial(u, v)}\right)^{2}+\left(\frac{\partial\left(f_{1}, f_{2}\right)}{\partial(u, v)}\right)^{2}\right\}^{\frac{1}{2}}} .
$$

2.650 If the equation of the surface is:

$$
z=f(x, y)
$$

the equation of the tangent plane at $x_{1}, y_{1}, z_{1}$ is:

$$
z-z_{1}=\left(\frac{\partial f}{\partial x}\right)_{1}\left(x-x_{1}\right)+\left(\frac{\partial f}{\partial y}\right)_{1}\left(y-y_{1}\right)
$$

2.651 The direction cosines of the normal to the surface in the form $\mathbf{2 . 6 5 0}$ are:

$$
l, m, n=\frac{-\left(\frac{\partial f}{\partial x}\right),-\left(\frac{\partial f}{\partial y}\right),+\mathrm{I}}{\left\{\mathrm{I}+\left(\frac{\partial f}{\partial x}\right)^{2}+\left(\frac{\partial f}{\partial y}\right)^{2}\right\}^{\frac{1}{2}}}
$$

2.652 The two principal radii of curvature of the surface in the form $\mathbf{2 . 6 5 0}$ are given by the two roots of:

$\left(r t-s^{2}\right) \rho^{2}-\left\{\left(\mathrm{I}+q^{2}\right) r-2 p q s+\left(\mathrm{I}+p^{2}\right) t\right\} \sqrt{\mathrm{I}+p^{2}+q^{2}} \rho+\left(\mathrm{I}+p^{2}+q^{2}\right)^{2}=0$, where

$$
p=\frac{\partial f}{\partial x}, \quad q=\frac{\partial f}{\partial y}, \quad r=\frac{\partial^{2} f}{\partial x^{2}}, \quad s=\frac{\partial^{2} f}{\partial x \partial y}, \quad t=\frac{\partial^{2} f}{\partial y^{2}} .
$$

2.653 If $\rho_{1}$ and $\rho_{2}$ are the two principal radii of curvature of a surface, and $\rho$ is the radius of curvature in a plane making an angle $\phi$ with the plane of $\rho_{1}$,

$$
\frac{I}{\rho}=\frac{\cos ^{2} \phi}{\rho_{1}}+\frac{\sin ^{2} \phi}{\rho_{2}} \text {. }
$$

2.654 If $\rho$ and $\rho^{\prime}$ are the radii of curvature in any two mutually perpendicular planes, and $\rho_{1}$ and $\rho_{2}$ the two principal radii of curvature:

$$
\frac{I}{\rho}+\frac{I}{\rho^{\prime}}=\frac{I}{\rho_{1}}+\frac{I}{\rho_{2}}
$$

2.655 Gauss's measure of the curvature of a surface is:

$$
\frac{I}{\rho}=\frac{I}{\rho_{1} \rho_{2}} .
$$

SPACE CURVES

2.670 The equations of a space curve may be given in the forms:

$$
\begin{aligned}
& F_{1}(x, y, z)=0, \quad F_{2}(x, y, z)=0 . \\
& x=f_{1}(t), \quad y=f_{2}(t), \quad z=f_{3}(t) . \\
& y=\phi(x), \quad z=\psi(x) .
\end{aligned}
$$


2.671 The direction cosines of the tangent to a space curve in the form (a) are:

$$
\begin{aligned}
& l=\frac{\frac{\partial F_{1}}{\partial y} \frac{\partial F_{2}}{\partial z}-\frac{\partial F_{1}}{\partial z} \frac{\partial F_{2}}{\partial y}}{T} \\
& m=\frac{\frac{\partial F_{1}}{\partial z} \cdot \frac{\partial F_{2}}{\partial x}-\frac{\partial F_{1}}{\partial x} \frac{\partial F_{2}}{\partial z}}{T} \\
& n=\frac{\frac{\partial F_{1}}{\partial x} \frac{\partial F_{2}}{\partial y}-\frac{\partial F_{1}}{\partial y} \frac{\partial F_{2}}{\partial x}}{T}
\end{aligned}
$$

where $T$ is the positive root of:

$$
\begin{aligned}
T^{2}=\left\{\left(\frac{\partial F_{1}}{\partial x}\right)^{2}+\left(\frac{\partial F_{1}}{\partial y}\right)^{2}+\left(\frac{\partial F_{1}}{\partial z}\right)^{2}\right\}\left\{\left(\frac{\partial F_{2}}{\partial x}\right)^{2}\right. & \left.+\left(\frac{\partial F_{2}}{\partial y}\right)^{2}+\left(\frac{\partial F_{2}}{\partial z}\right)^{2}\right\} \\
& -\left\{\frac{\partial F_{1}}{\partial x} \frac{\partial F_{2}}{\partial x}+\frac{\partial F_{1}}{\partial y} \frac{\partial F_{2}}{\partial y}+\frac{\partial F_{1}}{\partial z} \frac{\partial F_{2}}{\partial z}\right\}^{2} .
\end{aligned}
$$

2.672 The direction cosines of the tangent to a space curve in the form (b) are:

$$
l, m, n=\frac{x^{\prime}, y^{\prime}, z^{\prime}}{\left\{x^{\prime 2}+y^{\prime 2}+z^{\prime 2}\right\}^{\frac{1}{2}}}
$$

where the accents denote differentials with respect to $t$.

2.673 If $s$, the length of arc measured from a fixed point on the curve is the parameter, $t$ :

$$
l, m, n=\frac{d x}{d s}, \frac{d y}{d s}, \frac{d z}{d s} .
$$

2.674 The principal radius of curvature of a space curve in the form (b) is:

$$
\begin{aligned}
\rho & =\frac{\left(x^{\prime 2}+y^{\prime 2}+z^{\prime 2}\right)^{\frac{3}{2}}}{\left\{\left(y^{\prime} z^{\prime \prime}-z^{\prime} y^{\prime \prime}\right)^{2}+\left(z^{\prime} x^{\prime \prime}-x^{\prime} z^{\prime \prime}\right)^{2}+\left(x^{\prime} y^{\prime \prime}-y^{\prime} x^{\prime \prime}\right)^{2}\right\}^{\frac{1}{2}}} \\
& =\frac{s^{\prime 2}}{\left(x^{\prime 2}+y^{\prime \prime 2}+z^{\prime \prime 2}-s^{\prime \prime 2}\right)^{\frac{1}{2}}} \cdot
\end{aligned}
$$

where the double accents denote second differentials with respect to $t$, and $s$, the length of arc, is a function of $t$.

2.675 When $t=s$ :

$$
\frac{I}{\rho}=\left\{\left(\frac{d^{2} x}{d s^{2}}\right)^{2}+\left(\frac{d^{2} y}{d s^{2}}\right)^{2}+\left(\frac{d^{2} z}{d s^{2}}\right)^{2}\right\}^{\frac{1}{2}}
$$

2.676 The direction cosines of the principal normal to the space curve in the form (b) are:

$$
\begin{aligned}
l^{\prime} & =\frac{z^{\prime}\left(z^{\prime} x^{\prime \prime}-x^{\prime} z^{\prime \prime}\right)-y^{\prime}\left(x^{\prime} y^{\prime \prime}-y^{\prime} x^{\prime \prime}\right)}{L}, \\
m^{\prime} & =\frac{x^{\prime}\left(x^{\prime} y^{\prime \prime}-y^{\prime} x^{\prime \prime}\right)-z^{\prime}\left(y^{\prime} z^{\prime \prime}-z^{\prime} y^{\prime \prime}\right)}{L}
\end{aligned}
$$




$$
n^{\prime}=\frac{y^{\prime}\left(y^{\prime} z^{\prime \prime}-z^{\prime} y^{\prime \prime}\right)-x^{\prime}\left(z^{\prime} x^{\prime \prime}-x^{\prime} z^{\prime \prime}\right)}{L}
$$

where

$$
L=\left\{x^{\prime 2}+y^{\prime 2}+z^{\prime 2}\right\}^{\frac{1}{2}}\left\{\left(y^{\prime} z^{\prime \prime}-z^{\prime} y^{\prime \prime}\right)^{2}+\left(z^{\prime} x^{\prime \prime}-x^{\prime} z^{\prime \prime}\right)^{2}+\left(x^{\prime} y^{\prime \prime}-y^{\prime} x^{\prime \prime}\right)^{2}\right\}^{\frac{1}{2}} .
$$

2.677 The direction cosines of the binormal to the curve in the form (b) are:

$$
\begin{aligned}
l^{\prime \prime} & =\frac{y^{\prime} z^{\prime \prime}-z^{\prime} y^{\prime \prime}}{S}, \\
m^{\prime \prime} & =\frac{z^{\prime} x^{\prime \prime}-x^{\prime} z^{\prime \prime}}{S}, \\
n^{\prime \prime} & =\frac{x^{\prime} y^{\prime \prime}-y^{\prime} x^{\prime \prime}}{S},
\end{aligned}
$$

where

$$
S=\left\{\left(y^{\prime} z^{\prime \prime}-z^{\prime} y^{\prime \prime}\right)^{2}+\left(z^{\prime} x^{\prime \prime}-x^{\prime} z^{\prime \prime}\right)^{2}+\left(x^{\prime} y^{\prime \prime}-y^{\prime} x^{\prime \prime}\right)^{2}\right\}^{2} .
$$

2.678 If $s$, the distance measured along the curve from a fixed point on it is the parameter, $t$ :

$$
l^{\prime}=\rho \frac{d^{2} x}{d s^{2}}, m^{\prime}=\rho \frac{d^{2} y}{d s^{2}}, n^{\prime}=\rho \frac{d^{2} z}{d s^{2}},
$$

where $\rho$ is the principal radius of curvature; and

$$
\begin{aligned}
& l^{\prime \prime}=\rho\left(\frac{d y}{d s} \frac{d^{2} z}{d s^{2}}-\frac{d z}{d s} \frac{d^{2} y}{d s^{2}}\right) \\
& m^{\prime \prime}=\rho\left(\frac{d z}{d s} \frac{d^{2} x}{d s^{2}}-\frac{d x}{d s} \frac{d^{2} z}{d s^{2}}\right) \\
& n^{\prime \prime}=\rho\left(\frac{d x}{d s} \frac{d^{2} y}{d s^{2}}-\frac{d y}{d s} \frac{d^{2} x}{d s^{2}}\right)
\end{aligned}
$$

2.679 The radius of torsion, or radius of second curvature of a space curve is:

$$
\begin{aligned}
\tau & =\frac{\left(x^{\prime 2}+y^{\prime 2}+z^{\prime 2}\right)^{\frac{1}{2}}}{\left\{\left(\frac{\partial l^{\prime \prime}}{\partial t}\right)^{2}+\left(\frac{\partial m^{\prime \prime}}{\partial t}\right)^{2}+\left(\frac{\partial n^{\prime \prime}}{\partial t}\right)^{2}\right\}^{\frac{1}{2}}} \\
& =-\frac{\mathrm{I}}{S^{2}}\left|\begin{array}{lll}
x^{\prime} & y^{\prime} & z^{\prime} \\
x^{\prime \prime} & y^{\prime \prime} & z^{\prime \prime} \\
x^{\prime \prime \prime} & y^{\prime \prime \prime} & z^{\prime \prime \prime}
\end{array}\right|,
\end{aligned}
$$

where $S$ is given in $\mathbf{2 . 6 7 7}$.

2.680 When $t=s$ :

$$
\frac{\mathbf{I}}{\boldsymbol{\tau}}=\left\{\left(\frac{\partial l^{\prime \prime}}{\partial s}\right)^{2}+\left(\frac{\partial m^{\prime \prime}}{\partial s}\right)^{2}+\left(\frac{\partial n^{\prime \prime}}{\partial s}\right)^{2}\right\}^{\frac{1}{2}}
$$




$$
=-\rho^{2}\left|\begin{array}{lll}
\frac{d x}{d s} & \frac{d y}{d s} & \frac{d z}{d s} \\
\frac{d^{2} x}{d s^{2}} & \frac{d^{2} y}{d s^{2}} & \frac{d^{2} z}{d s^{2}} \\
\frac{d^{3} x}{d s^{3}} & \frac{d^{3} y}{d s^{3}} & \frac{d^{3} z}{d s^{3}}
\end{array}\right| .
$$

2.681 The direction cosines of the tangent to a space curve in the form (c) are:

$$
l, m, n=\frac{\mathrm{I}, y^{\prime}, z^{\prime}}{\sqrt{\mathrm{I}+y^{\prime 2}+z^{\prime 2}}}
$$

where accents denote differentials with respect to $x$ :

$$
y^{\prime}=\frac{d \phi(x)}{d x}, \quad z^{\prime}=\frac{d \psi(x)}{d x} .
$$

2.682 The principal radius of curvature of a space curve in the form (c) is:

$$
\rho=\left\{\frac{\left(\mathrm{x}+y^{\prime 2}+z^{\prime 2}\right)^{3}}{\left(y^{\prime} z^{\prime \prime}-z^{\prime} y^{\prime \prime}\right)^{2}+y^{\prime \prime 2}+z^{\prime 2}}\right\}^{\frac{1}{2}} .
$$

2.683 The radius of torsion of a space curve in the form (c) is:

$$
\tau=\frac{\left(\mathrm{I}+y^{\prime 2}+z^{\prime 2}\right)^{3}}{\rho^{2}\left(y^{\prime \prime} z^{\prime \prime \prime}-z^{\prime \prime} y^{\prime \prime \prime}\right)}
$$

2.690 The relation between the direction cosines of the tangent, principal normal and binormal to a space curve is:

$$
\left|\begin{array}{lll}
l & m & n \\
l^{\prime} & m^{\prime} & n^{\prime} \\
l^{\prime \prime} & m^{\prime \prime} & n^{\prime \prime}
\end{array}\right|=\mathbf{I} .
$$

2.691 The tangent, principal normal and binormal all being mutually perpendicular the relations of $\mathbf{2 . 0 0}$ hold among their direction cosines. 


\section{TRIGONOMETRY}

$3.00 \tan x=\frac{\sin x}{\cos x}, \sec x=\frac{\mathrm{I}}{\cos x}, \csc x=\frac{\mathrm{I}}{\sin x}, \cot x=\frac{\mathrm{I}}{\tan x}$, $\sec ^{2} x=\mathrm{I}+\tan ^{2} x, \csc ^{2} x=\mathrm{I}+\cot ^{2} x, \sin ^{2} x+\cos ^{2} x=\mathrm{I}$, versin $x=\mathrm{I}-\cos x$, coversin $x=\mathrm{I}-\sin x$, haversin $x=\sin ^{2} \frac{x}{2}$.

$3.01 \sin x=-\sin (-x)=\sqrt{\frac{1-\cos 2 x}{2}},=2 \sqrt{\cos ^{2} \frac{x}{2}-\cos ^{4} \frac{x}{2}}$

$$
\begin{aligned}
& =2 \sin \frac{x}{2} \cos \frac{x}{2}=\frac{\tan x}{\sqrt{I+\tan ^{2} x}}=\frac{2 \tan \frac{x}{2}}{I+\tan ^{2} \frac{x}{2}} \\
& =\frac{I}{\sqrt{I+\cot ^{2} x}}=\frac{I}{\cot \frac{x}{2}-\cot x}=\frac{I}{\tan \frac{x}{2}+\cot x} \\
& =\cot \frac{x}{2} \cdot(I-\cos x)=\tan \frac{x}{2} \cdot(I+\cos x) \\
& =\sin y \cos (x-y)+\cos y \sin (x-y) \\
& =\cos y \sin (x+y)-\sin y \cos (x+y) \\
& =-\frac{1}{2} i\left(e^{i x}-e^{-i x}\right)
\end{aligned}
$$

$3.02 \cos x=\cos (-x)=\sqrt{\frac{1+\cos 2 x}{2}}=I-2 \sin ^{2} \frac{x}{2}$

$$
\begin{aligned}
& =\cos ^{2} \frac{x}{2}-\sin ^{2} \frac{x}{2}=2 \cos ^{2} \frac{x}{2}-I=\frac{I}{\sqrt{I+\tan ^{2} x}} \\
& =\frac{I-\tan ^{2} \frac{x}{2}}{I+\tan ^{2} \frac{x}{2}}=\frac{I}{I+\tan x \tan \frac{x}{2}}=\frac{I}{\tan x \cot \frac{x}{2}-I} \\
& =\frac{\cot \frac{x}{2}-\tan \frac{x}{2}}{\cot \frac{x}{2}+\tan \frac{x}{2}}=\frac{\cot x}{\sqrt{I+\cot ^{2} x}}=\frac{\sin 2 x}{2 \sin x} \\
& =\cos y \cos (x+y)+\sin y \sin (x+y) \\
& =\cos y \cos (x-y)-\sin y \sin (x-y) \\
& =\frac{1}{2}\left(e^{i x}+e^{-i x}\right)
\end{aligned}
$$


$3.03 \tan x=-\tan (-x)=\frac{\sin 2 x}{I+\cos 2 x}=\frac{1-\cos 2 x}{\sin 2 x},=$

$$
\begin{aligned}
& \sqrt{\frac{1-\cos 2 x}{I+\cos 2 x}}=\frac{\sin (x+y)+\sin (x-y)}{\cos (x+y)+\cos (x-y)}, \\
= & \frac{\cos (x-y)-\cos (x+y)}{\sin (x+y)-\sin (x-y)}=\cot x-2 \cot 2 x, \\
= & \frac{\tan \frac{x}{2}}{I-\tan \frac{x}{2}}+\frac{\tan \frac{x}{2}}{I+\tan \frac{x}{2}}=\frac{2 \tan \frac{x}{2}}{I-\tan ^{2} \frac{x}{2}}, \\
= & \frac{I}{I-\tan \frac{x}{2}}-\frac{I}{I+\tan \frac{x}{2}}, \\
= & i \frac{I-e^{2 i x}}{I+e^{2 i x}} .
\end{aligned}
$$

\begin{tabular}{|c|c|c|c|c|c|c|}
\hline & $\sin x=a$ & $\cos x=a$ & $\tan x=a$ & $\cot x=a$ & $\sec x=a$ & $\csc x=a$ \\
\hline $\sin x=$ & $a$ & $\sqrt{\mathrm{I}-a^{2}}$ & $\frac{a}{\sqrt{\mathrm{I}+a^{2}}}$ & $\frac{\mathrm{I}}{\sqrt{\mathrm{I}+a^{2}}}$ & $\frac{\sqrt{a^{2}-\mathrm{I}}}{a}$ & $\frac{\mathrm{I}}{a}$ \\
\hline $\cos x=$ & $\sqrt{\mathrm{I}-a^{2}}$ & $a$ & $\frac{I}{\sqrt{I+a^{2}}}$ & $\frac{a}{\sqrt{I+a^{2}}}$ & $\frac{\mathbf{I}}{a}$ & $\frac{\sqrt{a^{2}-\mathrm{I}}}{a}$ \\
\hline $\tan x=$ & $\frac{a}{\sqrt{\mathrm{I}-a^{2}}}$ & $\frac{\sqrt{\mathrm{I}-a^{2}}}{a}$ & $a$ & $\frac{\mathrm{I}}{a}$ & $\sqrt{a^{2}-\mathrm{I}}$ & $\frac{I}{\sqrt{a^{2}-I}}$ \\
\hline $\cot x=$ & $\frac{\sqrt{\mathrm{I}-a^{2}}}{a}$ & $\frac{a}{\sqrt{\mathrm{I}-a^{2}}}$ & $\frac{\mathbf{I}}{a}$ & $a$ & $\frac{\mathrm{I}}{\sqrt{a^{2}-\mathrm{I}}}$ & $\sqrt{a^{2}-\mathrm{I}}$ \\
\hline $\sec x=$ & $\frac{\mathrm{I}}{\sqrt{\mathrm{I}-a^{2}}}$ & $\frac{\mathbf{I}}{a}$ & $\sqrt{\mathrm{I}+a^{2}}$ & $\frac{\sqrt{\mathrm{I}+a^{2}}}{a}$ & $a$ & $\frac{a}{\sqrt{a^{2}-I}}$ \\
\hline $\csc x$ & $\frac{\mathrm{I}}{a}$ & $\frac{\mathrm{I}}{\sqrt{\mathrm{I}-a^{2}}}$ & $\frac{\sqrt{\mathrm{I}+a^{2}}}{a}$ & $\sqrt{\mathrm{I}+a^{2}}$ & $\frac{a}{\sqrt{a^{2}-1}}$ & $a$ \\
\hline
\end{tabular}

3.04 The values of five trigonometric functions in terms of the sixth are given in the following table. (For signs, see 3.05.)

3.05 The trigonometric functions are periodic, the periods of the $\sin , \cos , \mathrm{sec}$, csc being $2 \pi$, and those of the tan and cot, $\pi$. Their signs may be determined from the following table. In using formulas giving any of the trigonometric 
functions by the root of some quantity, the proper sign may be taken from this table.

\begin{tabular}{|c|c|c|c|c|c|c|c|c|c|}
\hline & $0^{\circ}$ & $\begin{array}{l}\circ-\frac{\pi}{2} \\
\circ-90^{\circ}\end{array}$ & $\begin{array}{l}\frac{\pi}{2} \\
90^{\circ}\end{array}$ & $\begin{array}{c}\frac{\pi}{2}-\pi \\
90^{\circ}-\mathrm{I} 80^{\circ}\end{array}$ & $\begin{array}{c}\pi \\
180^{\circ}\end{array}$ & $\begin{array}{c}\pi-\frac{3}{2} \pi \\
180^{\circ}-270^{\circ}\end{array}$ & $\begin{array}{l}\frac{3}{2} \pi \\
270^{\circ}\end{array}$ & $\begin{array}{c}\frac{3}{2} \pi-2 \pi \\
270^{\circ}-360^{\circ}\end{array}$ & $\begin{array}{l}2 \pi \\
360^{\circ}\end{array}$ \\
\hline $\sin$ & 0 & + & I & + & 0 & - & $-I$ & - & 0 \\
\hline $\cos$ & I & + & 0 & - & $-I$ & - & 0 & + & I \\
\hline $\tan$ & 0 & + & $\pm \infty$ & - & 0 & + & $\pm \infty$ & - & ० \\
\hline $\cot$ & $\mp \infty$ & + & 0 & - & $\mp \infty$ & + & 0 & - & $\mp_{\infty}$ \\
\hline sec & I & + & $\pm \infty$ & - & $-I$ & - & $\pm \infty$ & + & I \\
\hline $\csc$ & $\mp_{\infty}$ & + & I & + & $\pm \infty$ & - & $-I$ & - & $\mp \infty$ \\
\hline
\end{tabular}

3.10 Functions of Half an Angle. (See 3.05 for signs.)

3.101

$$
\begin{aligned}
\sin \frac{I}{2} x & = \pm \sqrt{\frac{I-\cos x}{2}} \\
& =\frac{I}{2}\{ \pm \sqrt{I+\sin x} \mp \sqrt{I-\sin x}\} \\
& = \pm \sqrt{\frac{I}{2}\left(1-\frac{I}{ \pm \sqrt{I+\tan ^{2} x}}\right)}
\end{aligned}
$$

3.102

$$
\begin{aligned}
\cos \frac{I}{2} x & = \pm \sqrt{\frac{1+\cos x}{2}} \\
& =\frac{I}{2}\{ \pm \sqrt{I+\sin x} \pm \sqrt{I-\sin x}\}, \\
& = \pm \sqrt{\frac{I}{2}\left(1+\frac{I}{ \pm \sqrt{I+\tan ^{2} x}}\right)} .
\end{aligned}
$$




$$
\begin{aligned}
& =\frac{\sin x}{\mathrm{I}+\cos x}=\frac{\mathrm{I}-\cos x}{\sin x} \\
& =\frac{ \pm \sqrt{\mathrm{I}+\tan ^{2} x}-\mathrm{I}}{\tan x} .
\end{aligned}
$$

3.11 Functions of the Sum and Difference of Two Angles.

3.111

$$
\begin{aligned}
\sin (x \pm y) & =\sin x \cos y \pm \cos x \sin y, \\
& =\cos x \cos y(\tan x \pm \tan y), \\
& =\frac{\tan x \pm \tan y}{\tan x \mp \tan y} \sin (x \mp y), \\
& =\frac{I}{2}\{\cos (x+y)+\cos (x-y)\}(\tan x \pm \tan y) .
\end{aligned}
$$

3.112

$$
\begin{aligned}
\cos (x \pm y) & =\cos x \cos y \mp \sin x \sin y, \\
& =\cos x \cos y(I \mp \tan x \tan y), \\
& =\frac{\cot x \mp \tan y}{\cot x \pm \tan y} \cos (x \mp y), \\
& =\frac{\cot y \mp \tan x}{\cot y \tan x \mp \mathrm{I}} \sin (x \mp y), \\
& =\cos x \sin y(\cot y \mp \tan x) .
\end{aligned}
$$

3.113

3.114

$$
\begin{aligned}
\tan (x \pm y) & =\frac{\tan x \pm \tan y}{\mathrm{I} \mp \tan x \tan y}, \\
& =\frac{\cot y \pm \cot x}{\cot x \cot y \mp \mathrm{I}} \\
& =\frac{\sin 2 x \pm \sin 2 y}{\cos 2 x+\cos 2 y} \\
& =\sec p q 72{ }^{2} \\
\cot (x \pm y) & =\frac{\cot x \cot y \mp \mathrm{I}}{\cot y \pm \cot x} \\
& =-\frac{\sin 2 x \mp \sin 2 y}{\cos 2 x-\cos 2 y}
\end{aligned}
$$

3.115 The cosine and sine of the sum of any number of angles in terms of the sine and cosine of the angles are given by the real and imaginary parts of $\cos \left(x_{1}+x_{2}+\ldots .+x_{n}\right)+i \sin \left(x_{1}+x_{2}+\ldots .+x_{n}\right)$

$$
=\left(\cos x_{1}+i \sin x_{1}\right)\left(\cos x_{2}+i \sin x_{2}\right) \ldots\left(\cos x_{n}+i \sin x_{n}\right)
$$


3.12 Sums and Differences of Trigonometric Functions.

$$
\begin{aligned}
\sin x \pm \sin y & =2 \sin \frac{1}{2}(x \pm y) \cos \frac{1}{2}(x \mp y), \\
& =(\cos x+\cos y) \tan \frac{1}{2}(x \pm y), \\
& =(\cos y-\cos x) \cot \frac{1}{2}(x \mp y), \\
& =\frac{\tan \frac{1}{2}(x \pm y)}{\tan \frac{1}{2}(x \mp y)}(\sin x \mp \sin y) .
\end{aligned}
$$

3.122

$\cos x+\cos y=2 \cos \frac{1}{2}(x+y) \cos \frac{1}{2}(x-y)$,

$$
\begin{aligned}
& =\frac{\sin x \pm \sin y}{\tan \frac{1}{2}(x \pm y)}, \\
& =\frac{\cot \frac{1}{2}(x+y)}{\tan \frac{1}{2}(x-y)}(\cos y-\cos x) .
\end{aligned}
$$

$$
\begin{aligned}
\cos x-\cos y & =2 \sin \frac{1}{2}(y+x) \sin \frac{1}{2}(y-x) \\
& =-(\sin x \pm \sin y) \tan \frac{1}{2}(x \mp y) .
\end{aligned}
$$

3.124

$\tan x \pm \tan y=\frac{\sin (x \pm y)}{\cos x \cdot \cos y}$.

$$
\begin{aligned}
& =\frac{\sin (x \pm y)}{\sin (x \mp y)}(\tan x \mp \tan y), \\
& =\tan y \tan (x \pm y)(\cot y \mp \tan x), \\
& =\frac{I \mp \tan x \tan y}{\cot (x \pm y)}, \\
& =(I \mp \tan x \tan y) \tan (x \pm y) .
\end{aligned}
$$

3.125

$\cot x \pm \cot y= \pm \frac{\sin (x \pm y)}{\sin x \sin y}$.

\subsection{0}

I.

$\frac{\sin x \pm \sin y}{\cos x+\cos y}=\tan \frac{1}{2}(x \pm y)$.

$\frac{\sin x \pm \sin y}{\cos x-\cos y}=-\cot \frac{1}{2}(x \mp y)$.

$\frac{\sin x+\sin y}{\sin x-\sin y}=\frac{\tan \frac{1}{2}(x+y)}{\tan \frac{1}{2}(x-y)}$. 


\subsection{0}

I.

2.

3.

4.

5.

6.

7.

$$
\begin{aligned}
& \sin ^{2} x+\sin ^{2} y=I-\cos (x+y) \cos (x-y) \\
& \sin ^{2} x-\sin ^{2} y=\cos ^{2} y-\cos ^{2} x \\
& =\sin (x+y) \sin (x-y) \\
& \cos ^{2} x-\sin ^{2} y=\cos (x+y) \cos (x-y) . \\
& \sin ^{2}(x+y)+\sin ^{2}(x-y)=\mathrm{I}-\cos 2 x \cos 2 y . \\
& \sin ^{2}(x+y)-\sin ^{2}(x-y)=\sin 2 x \sin 2 y . \\
& \cos ^{2}(x+y)+\cos ^{2}(x-y)=\mathrm{I}+\cos 2 x \cos 2 y . \\
& \cos ^{2}(x+y)-\cos ^{2}(x-y)=-\sin 2 x \sin 2 y .
\end{aligned}
$$

\subsection{0}

I.

2.

3.

\subsection{0}

I.

2.

3.

4.

5.

6.

7.

8.

9. $\cos n x \cos m x=\frac{1}{2} \cos (n-m) x+\frac{1}{2} \cos (n+m) x$. $\sin n x \sin m x=\frac{1}{2} \cos (n-m) x-\frac{1}{2} \cos (n+m) x$. $\cos n x \sin m x=\frac{1}{2} \sin (n+m) x-\frac{1}{2} \sin (n-m) x$.

\subsection{Sines and Cosines of Multiple Angles.}

$3.171 n$ an even integer:

$\sin n x=n \cos x\left\{\sin x-\frac{\left(n^{2}-2^{2}\right)}{3 !} \sin ^{3} x+\frac{\left(n^{2}-2^{2}\right)\left(n^{2}-4^{2}\right)}{5 !} \sin ^{5} x-\ldots\right\}$ $\cos n x=\mathrm{I}-\frac{n^{2}}{2 !} \sin ^{2} x+\frac{n^{2}\left(n^{2}-2^{2}\right)}{4 !} \sin ^{4} x-\frac{n^{2}\left(n^{2}-2^{2}\right)\left(n^{2}-4^{2}\right)}{6 !} \sin ^{6} x+\ldots$ 
3.172 $n$ an odd integer:

$$
\begin{aligned}
& \sin n x=n\left\{\sin x-\frac{\left(n^{2}-\mathrm{I}^{2}\right)}{3 !} \sin ^{3} x+\frac{\left(n^{2}-\mathrm{I}^{2}\right)\left(n^{2}-3^{2}\right)}{5 !} \sin ^{5} x-\ldots\right\} \cdot \\
& \left.\cos n x=\cos x\left\{\mathrm{I}-\frac{\left(n^{2}-\mathrm{I}^{2}\right)}{2 !} \sin ^{2} x+\frac{\left(n^{2}-\mathrm{I}^{2}\right)\left(n^{2}-3^{2}\right)}{4 !} \sin ^{4} x-\ldots\right\}\right\}
\end{aligned}
$$

3.173 $n$ an even integer:

$\sin n x=(-\mathrm{I})^{\frac{n}{2}-\mathrm{I}} \cos x\left\{2^{n-1} \sin ^{n-1} x-\frac{(n-2)}{\mathrm{I} !} 2^{n-3} \sin ^{n-3} x\right.$

$$
\begin{array}{r}
+\frac{(n-3)(n-4)}{2 !} 2^{n-5} \sin ^{n-5} x-\frac{(n-4)(n-5)(n-6)}{3 !} 2^{n-7} \sin ^{n-7} x \\
+\ldots\} .
\end{array}
$$

$\cos n x=(-1)^{\frac{n}{2}}\left\{2^{n-1} \sin ^{n} x-\frac{n}{1 !} 2^{n-3} \sin ^{n-2} x+\frac{n(n-3)}{2 !} 2^{n-5} \sin ^{n-4} x\right.$

$$
\left.-\frac{n(n-3)(n-5)}{3 !} 2^{n-7} \sin ^{n-6} x+\ldots\right\} \text {. }
$$

3.174 $n$ an odd integer :

$\sin n x=(-\mathrm{I})^{\frac{n-1}{2}}\left\{2^{n-1} \sin ^{n} x-\frac{n}{\mathrm{I} !} 2^{n-3} \sin ^{n-2} x+\frac{n(n-3)}{2 !} 2^{n-5} \sin ^{n-4} x\right.$

$$
\left.-\frac{n(n-3)(n-5)}{3 !} 2^{n-7} \sin ^{n-6} x+\ldots\right\} \text {. }
$$

$\cos n x=(-\mathrm{I})^{\frac{n-\mathrm{I}}{2}} \cos x\left\{2^{n-1} \sin ^{n-1} x-\frac{n-2}{\mathrm{I} !} 2^{n-3} \sin ^{n-3} x\right.$

$$
\begin{array}{r}
+\frac{(n-3)(n-4)}{2 !} 2^{n-5} \sin ^{n-5} x-\frac{(n-4)(n-5)(n-6)}{3 !} 2^{n-7} \sin ^{n-7} x \\
+\ldots \ldots\}
\end{array}
$$

$3.175 n$ any integer:

$\sin n x=\sin x\left\{2^{n-1} \cos ^{n-1} x-\frac{n-2}{\mathrm{I} !} 2^{n-3} \cos ^{n-3} x\right.$

$$
\begin{aligned}
+\frac{(n-3)(n-4)}{2 !} 2^{n-5} \cos ^{n-5} x-\frac{(n-4)(n-5)(n-6)}{3 !} & 2^{n-7} \cos ^{n-7} x \\
& +\ldots \ldots\} .
\end{aligned}
$$

$\cos n x=2^{n-1} \cos ^{n} x-\frac{n}{\mathrm{I} !} 2^{n-3} \cos ^{n-2} x+\frac{n(n-3)}{2 !} 2^{n-5} \cos ^{n-4} x$

$$
-\frac{n(n-4)(n-5)}{3 !} 2^{n-7} \cos ^{n-6} x+\ldots \ldots
$$


3.176

$\sin 2 x=2 \sin x \cos x$.

$$
\begin{aligned}
\sin 3 x & =\sin x\left(3-4 \sin ^{2} x\right) \\
& =\sin x\left(4 \cos ^{2} x-\mathrm{I}\right) . \\
\sin 4 x & =\sin x\left(8 \cos ^{3} x-4 \cos x\right) . \\
\sin 5 x & =\sin x\left(5-20 \sin ^{2} x+\mathrm{I} 6 \sin ^{4} x\right) \\
& =\sin x\left(\mathrm{I} 6 \cos ^{4} x-\mathrm{I} 2 \cos ^{2} x+\mathrm{I}\right) . \\
\sin 6 x & =\sin x\left(32 \cos ^{5} x-32 \cos ^{3} x+6 \cos x\right) .
\end{aligned}
$$

3.177

$$
\begin{aligned}
\cos 2 x & =\cos ^{2} x-\sin ^{2} x \\
& =\mathrm{I}-2 \sin ^{2} x \\
& =2 \cos ^{2} x-\mathrm{I} . \\
\cos 3 x & =\cos x\left(4 \cos ^{2} x-3\right) \\
& =\cos x\left(\mathrm{I}-4 \sin ^{2} x\right) \\
\cos 4 x & =8 \cos ^{4} x-8 \cos ^{2} x+\mathrm{I} . \\
\cos 5 x & =\cos x\left(\mathrm{I} 6 \cos ^{4} x-20 \cos ^{2} x+5\right) \\
& =\cos x\left(\mathrm{I} 6 \sin ^{4} x-\mathrm{I} 2 \sin ^{2} x+\mathrm{I}\right) \\
\cos 6 x & =32 \cos ^{6} x-48 \cos ^{4} x+\mathrm{I} 8 \cos ^{2} x-\mathrm{I} .
\end{aligned}
$$

3.178

$$
\begin{aligned}
& \tan 2 x=\frac{2 \tan x}{1-\tan ^{2} x} \\
& \cot 2 x=\frac{\cot ^{2} x-1}{2 \cot x}
\end{aligned}
$$

\subsection{Integral Powers of Sine and Cosine.}

$3.181 n$ an even integer:

$$
\begin{aligned}
\sin ^{n} x= & \frac{(-I)^{\frac{n}{2}}}{2^{n-1}}\left\{\cos n x-n \cos (n-2) x+\frac{n(n-I)}{2 !} \cos (n-4) x\right. \\
& \left.-\frac{n(n-I)(n-2)}{3 !} \cos (n-6) x+\ldots \ldots+(-I)^{\frac{n}{2}} \frac{1}{2} \frac{n !}{\left(\frac{n}{2}\right) !\left(\frac{n}{2}\right) !}\right\}
\end{aligned}
$$

$\cos ^{n} x=\frac{\mathrm{I}}{2^{n-1}}\left\{\cos n x+n \cos (n-2) x+\frac{n(n-\mathrm{I})}{2 !} \cos (n-4) x\right.$

$$
\left.+\frac{n(n-\mathrm{r})(n-2)}{3 !} \cos (n-6) x+\ldots+\frac{1}{2} \frac{n !}{\left(\frac{n}{2}\right) !\left(\frac{n}{2}\right) !} \cdot\right\}
$$


3.182 $n$ an odd integer :

$\sin ^{n} x=\frac{(-I)^{\frac{n-I}{2}}}{2^{n-1}}\left\{\sin n x-n \sin (n-2) x+\frac{n(n-\mathrm{I})}{2 !} \sin (n-4) x\right.$
$\left.-\frac{n(n-\mathrm{I})(n-2)}{3 !} \sin (n-6) x+\ldots .+(-\mathrm{I})^{\frac{n-\mathrm{I}}{2}} \frac{n !}{\left(\frac{n-\mathrm{I}}{2}\right) !\left(\frac{n+\mathrm{I}}{2}\right) !} \sin x\right\}$.

$\cos ^{n} x=\frac{\mathrm{I}}{2^{n-1}}\left\{\cos n x+n \cos (n-2) x+\frac{n(n-\mathrm{I})}{2 !} \cos (n-4) x\right.$

$\left.+\frac{n(n-\mathrm{I})(n-2)}{3 !} \cos (n-6) x+\ldots \ldots+\frac{n !}{\left(\frac{n-\mathrm{I}}{2}\right) !\left(\frac{n+\mathrm{I}}{2}\right) !} \cos x\right\}$

\subsection{3}

$$
\begin{aligned}
& \sin ^{2} x=\frac{1}{2}(1-\cos 2 x) \\
& \sin ^{3} x=\frac{1}{4}(3 \sin x-\sin 3 x) \\
& \sin ^{4} x=\frac{1}{8}(\cos 4 x-4 \cos 2 x+3) \\
& \sin ^{5} x=\frac{1}{16}(\sin 5 x-5 \sin 3 x+\text { ro } \sin x) . \\
& \sin ^{6} x=-\frac{1}{32}(\cos 6 x-6 \cos 4 x+\text { I } 5 \cos 2 x-\text { I0 }) .
\end{aligned}
$$

\subsection{4}

$$
\begin{aligned}
& \cos ^{2} x=\frac{1}{2}(1+\cos 2 x) \\
& \cos ^{3} x=\frac{1}{4}(3 \cos x+\cos 3 x) \\
& \cos ^{4} x=\frac{1}{8}(3+4 \cos 2 x+\cos 4 x) . \\
& \cos ^{5} x=\frac{1}{16}(\text { 10 } \cos x+5 \cos 3 x+\cos 5 x) . \\
& \cos ^{6} x=\frac{1}{32}(\text { 10 }+ \text { I } 5 \cos 2 x+6 \cos 4 x+\cos 6 x) .
\end{aligned}
$$

\section{INVERSE CIRCULAR FUNCTIONS}

3.20 The inverse circular and logarithmic functions are multiple valued; i.e., if

$$
0<\sin ^{-1} x<\frac{\pi}{2}
$$

the solution of $x=\sin \theta$ is :

$$
\theta=2 n \pi+\sin ^{-1} x,
$$

where $n$ is a positive integer. In the following formulas the cyclic constants are omitted. 


\subsection{1}

$$
\begin{aligned}
\sin ^{-1} x & =-\sin ^{-1}(-x)=\frac{\pi}{2}-\cos ^{-1} x=\cos ^{-1} \sqrt{\mathrm{I}-x^{2}} \\
& =\frac{\pi}{2}-\sin ^{-1} \sqrt{\mathrm{I}-x^{2}}=\frac{\pi}{4}+\frac{\mathrm{I}}{2} \sin ^{-1}\left(2 x^{2}-\mathrm{I}\right) \\
& =\frac{\mathrm{I}}{2} \cos ^{-1}\left(\mathrm{I}-2 x^{2}\right)=\tan ^{-1} \frac{x}{\sqrt{\mathrm{I}-x^{2}}} \\
& =2 \tan ^{-1}\left\{\frac{\mathrm{I}-\sqrt{\mathrm{I}-x^{2}}}{x}\right\}=\frac{\mathrm{I}}{2} \tan ^{-1}\left\{\frac{2 x \sqrt{\mathrm{I}-x^{2}}}{\mathrm{I}-2 x^{2}}\right\} \\
& =\cot ^{-1} \frac{\sqrt{\mathrm{I}-x^{2}}}{x}=\frac{\pi}{2}-i \log \left(x+\sqrt{\left.x^{2}-\mathrm{I}\right)}\right.
\end{aligned}
$$

\subsection{2}

$$
\begin{aligned}
\cos ^{-1} x & =\pi-\cos ^{-1}(-x)=\frac{\pi}{2}-\sin ^{-1} x=\frac{\mathrm{I}}{2} \cos ^{-1}\left(2 x^{2}-\mathrm{I}\right) \\
& =2 \cos ^{-1} \sqrt{\frac{\mathrm{I}+x}{2}}=\sin ^{-1} \sqrt{\mathrm{I}-x^{2}}=\tan ^{-1} \frac{\sqrt{\mathrm{I}-x^{2}}}{x} \\
& =2 \tan ^{-1} \sqrt{\frac{\mathrm{I}-x}{\mathrm{I}+x}}=\frac{\mathrm{I}}{2} \tan ^{-1}\left\{\frac{2 x \sqrt{\mathrm{I}-x^{2}}}{2 x^{2}-\mathrm{I}}\right\}=\cot ^{-1} \frac{x}{\sqrt{\mathrm{I}-x^{2}}} \\
& =i \log \left(x+\sqrt{\left.x^{2}-\mathrm{I}\right)}=\pi-i \log \left(\sqrt{x^{2}-\mathrm{I}}-x\right) .\right.
\end{aligned}
$$

\subsection{3}

$$
\begin{aligned}
\tan ^{-1} x & =-\tan ^{-1}(-x)=\sin ^{-1} \frac{x}{\sqrt{\mathrm{I}+x^{2}}}=\cos ^{-1} \frac{\mathrm{I}}{\sqrt{\mathrm{I}+x^{2}}} \\
& =\frac{\mathrm{I}}{2} \sin ^{-1} \frac{2 x}{\mathrm{I}+x^{2}}=\frac{\pi}{2}-\cot ^{-1} x=\sec ^{-1} \sqrt{\mathrm{I}+x^{2}} \\
& =\frac{\pi}{2}-\tan ^{-1} \frac{\mathrm{I}}{x}=\frac{\mathrm{I}}{2} \cos ^{-1} \frac{\mathrm{I}-x^{2}}{\mathrm{I}+x^{2}} \\
& =2 \cos ^{-1}\left\{\frac{\mathrm{I}+\sqrt{\mathrm{I}+x^{2}}}{2 \sqrt{\mathrm{I}+x^{2}}}\right\}^{\frac{I}{2}}=2 \sin ^{-1}\left\{\frac{\sqrt{\mathrm{I}+x^{2}}-\mathrm{I}}{2 \sqrt{\mathrm{I}+x^{2}}}\right\}^{\frac{1}{2}} \\
& =\frac{\mathrm{I}}{2} \tan ^{-1} \frac{2 x}{\mathrm{I}-x^{2}}=2 \tan ^{-1}\left\{\frac{\sqrt{\mathrm{I}+x^{2}}-\mathrm{I}}{x}\right\} \\
& =-\tan ^{-1} c+\tan ^{-1} \frac{x+c}{\mathrm{I}-c x} \\
& =\frac{\mathrm{I}}{2} i \log \frac{\mathrm{I}-i x}{\mathrm{I}+i x}=\frac{\mathrm{I}}{2} i \log \frac{i+x}{i-x}=-\frac{\mathrm{I}}{2} i \log \frac{\mathrm{I}+i x}{\mathrm{I}-i x} .
\end{aligned}
$$




\subsection{5}

I.

2.

3 .

$$
\begin{aligned}
\sin ^{-1} x \pm \sin ^{-1} y & =\sin ^{-1}\left\{x \sqrt{I-y^{2}} \pm y \sqrt{I-x^{2}}\right\} \\
\cos ^{-1} x \pm \cos ^{-1} y & =\cos ^{-1}\left\{x y \mp \sqrt{\left(I-x^{2}\right)\left(I-y^{2}\right)}\right\} \\
\sin ^{-1} x \pm \cos ^{-1} y & =\sin ^{-1}\left\{x y \pm \sqrt{\left(I-x^{2}\right)\left(I-y^{2}\right)}\right\} \\
& =\cos ^{-1}\left\{y \sqrt{I-x^{2}} \mp x \sqrt{I-y^{2}}\right\} \\
\tan ^{-1} x \pm \tan ^{-1} y & =\tan ^{-1} \frac{x \pm y}{I \mp x y} \\
\tan ^{-1} x \pm \cot ^{-1} y & =\tan ^{-1} \frac{x y \pm I}{y \mp x} \\
& =\cot ^{-1} \frac{y \mp x}{x y \pm I} .
\end{aligned}
$$

\section{HYPERBOLIC FUNCTIONS}

3.30 Formulas for the hyperbolic functions may be obtained from the corresponding formulas for the circular functions by replacing $x$ by $i x$ and using the following relations:

I.

2.

$3 \cdot$

4 .

5 .

6

7

8.

9

IO $\sin i x=\frac{1}{2} i\left(e^{x}-e^{-x}\right)=i \sinh x$.

$\cos i x=\frac{1}{2}\left(e^{x}+e^{-x}\right)=\cosh x$.

$\tan i x=\frac{i\left(e^{2 x}-\mathrm{I}\right)}{e^{2 x}+\mathrm{I}}=i \tanh x$.

$\cot i x=-i \frac{e^{2 x}+\mathrm{I}}{e^{2 x}-\mathrm{I}}=-i \operatorname{coth} x$.

$\sec i x=\frac{2}{e^{x}+e^{-x}}=\operatorname{sech} x$.

$\csc i x=-\frac{2 i}{e^{x}-e^{-x}}=-i \operatorname{csch} x$.

$\sin ^{-1} i x=i \sinh ^{-1} x=i \log \left(x+\sqrt{\mathrm{I}+x^{2}}\right)$.

$\cos ^{-1} i x=-i \cosh ^{-1} x=\frac{\pi}{2}-i \log \left(x+\sqrt{\mathrm{I}+x^{2}}\right)$.

$\tan ^{-1} i x=i \tanh ^{-1} x=i \log \sqrt{\frac{\mathrm{I}+x}{\mathrm{I}-x}}$.

$\cot ^{-1} i x=-i \operatorname{coth}^{-1} x=-i \log \sqrt{\frac{x+I}{x-I}}$. 
3.310 The values of five hyperbolic functions in terms of the sixth are given in the following table:

\begin{tabular}{|c|c|c|c|c|c|c|}
\hline & $\sinh x=$ & $\cosh x=$ & $\tanh x=$ & $\operatorname{coth} x=$ & $\operatorname{sech} x=$ & $\operatorname{csch} x=$ \\
\hline $\sinh x=$ & $a$ & $\sqrt{a^{2}-1}$ & $\frac{a}{\sqrt{I-a^{2}}}$ & $\frac{I}{\sqrt{a^{2}-I}}$ & $\frac{\sqrt{\mathrm{I}-a^{2}}}{a}$ & $\frac{\mathbf{I}}{a}$ \\
\hline $\cosh x=$ & $\sqrt{\mathrm{I}+a^{2}}$ & $a$ & $\frac{I}{\sqrt{I-a^{2}}}$ & $\frac{a}{\sqrt{a^{2}-I}}$ & $\frac{\mathrm{I}}{a}$ & $\frac{\sqrt{\mathrm{x}+a^{2}}}{a}$ \\
\hline $\tanh x=$ & $\frac{a}{\sqrt{I+a^{2}}}$ & $\frac{\sqrt{a^{2}-\mathrm{I}}}{a}$ & $a$ & $\frac{\mathbf{x}}{a}$ & $\sqrt{\mathrm{I}-a^{2}}$ & $\frac{\mathbf{I}}{\sqrt{\mathrm{I}+a^{2}}}$ \\
\hline $\operatorname{coth} x=$ & $\frac{\sqrt{a^{2}+\mathrm{I}}}{a}$ & $\frac{a}{\sqrt{a^{2}-\mathrm{I}}}$ & $\frac{\mathrm{I}}{a}$ & $a$ & $\frac{\mathrm{I}}{\sqrt{\mathrm{I}-a^{2}}}$ & $\sqrt{I+a^{2}}$ \\
\hline $\operatorname{sech} x=$ & $\frac{I}{\sqrt{I+a^{2}}}$ & $\frac{\mathbf{I}}{a}$ & $\sqrt{\mathrm{I}-a^{2}}$ & $\frac{\sqrt{a^{2}-\mathrm{x}}}{a}$ & $a$ & $\frac{a}{\sqrt{I+a^{2}}}$ \\
\hline $\operatorname{csch} x=$ & $\frac{\mathbf{I}}{a}$ & $\frac{\mathrm{I}}{\sqrt{a^{2}-\mathrm{I}}}$ & $\frac{\sqrt{\mathrm{I}-a^{2}}}{a}$ & $\sqrt{a^{2}-I}$ & $\frac{a}{\sqrt{\mathrm{I}-a^{2}}}$ & $a$ \\
\hline
\end{tabular}

3.311 Periodicity of the Hyperbolic Functions.

The functions $\sinh x, \cosh x, \operatorname{sech} x, \operatorname{csch} x$ have an imaginary period $2 \pi i$, e.g. : $\cosh x=\cosh (x+2 \pi i n)$,

where $n$ is any integer. The functions $\tanh x, \operatorname{coth} x$ have an imaginary period $\pi i$.

The values of the hyperbolic functions for the argument $0, \frac{\pi}{2} i, \pi i, \frac{3 \pi i}{2}$, are given in the following table:

\begin{tabular}{|c|c|c|c|c|}
\hline & 0 & $\frac{\pi}{2} i$ & $\pi i$ & $3 \frac{\pi}{2} i$ \\
\hline $\sinh$ & 0 & $i$ & 0 & $-i$ \\
$\cosh$ & $\mathrm{I}$ & 0 & $-\mathrm{I}$ & 0 \\
$\tanh$ & 0 & $\infty \cdot i$ & 0 & $\infty \cdot i$ \\
$\operatorname{coth}$ & $\infty$ & 0 & $\infty$ & 0 \\
$\operatorname{sech}$ & $\mathrm{I}$ & $\infty$ & $-\mathrm{I}$ & $\infty$ \\
$\operatorname{csch}$ & $\infty$ & $-i$ & $\infty$ & $i$ \\
\hline
\end{tabular}




$$
\tan z=\tan (x+i y)=\frac{2 \sin (x+i y)}{2 \cos (x+i y)} \cdot \frac{\cos (x-i y)}{\cos (x-i y)}=\frac{\sin 2 x+\sin 2 i y}{\cos 2 x+\cos 2 i y}
$$

from $p q(65)$ 



I. $\sinh \frac{I}{2} x=\sqrt{\frac{\cosh x-I}{2}}$

2. $\cosh \frac{I}{2} x=\sqrt{\frac{\cosh x+I}{2}}$

$3 \cdot$ $\tanh \frac{\mathbf{I}}{2} x=\frac{\cosh x-\mathrm{I}}{\sinh x}=\frac{\sinh x}{\cosh x+1}=\sqrt{\frac{\cosh x-1}{\cosh x+1}}$

$\sinh (x \pm y)=\sinh x \cosh y \pm \cosh x \sinh y$.

2. $\cosh (x \pm y)=\cosh x \cosh y \pm \sinh x \sinh y$.

3. $\tanh (x \pm y)=\frac{\tanh x \pm \tanh y}{I \pm \tanh x \tanh y}$. $\operatorname{coth}(x \pm y)=\frac{\operatorname{coth} x \operatorname{coth} y \pm \mathrm{I}}{\operatorname{coth} y \pm \operatorname{coth} x}$

\subsection{4}

I.

2.

3.

4 .

5

6

7

8 $\sinh x+\sinh y=2 \sinh \frac{1}{2}(x+y) \cosh \frac{1}{2}(x-y)$. $\sinh x-\sinh y=2 \cosh \frac{1}{2}(x+y) \sinh \frac{1}{2}(x-y)$. $\cosh x+\cosh y=2 \cosh \frac{1}{2}(x+y) \cosh \frac{1}{2}(x-y)$. $\cosh x-\cosh y=2 \sinh \frac{1}{2}(x+y) \sinh \frac{1}{2}(x-y)$. $\tanh x+\tanh y=\frac{\sinh (x+y)}{\cosh x \cosh y}$. $\tanh x-\tanh y=\frac{\sinh (x-y)}{\cosh x \cosh y}$. $\operatorname{coth} x+\operatorname{coth} y=\frac{\sinh (x+y)}{\sinh x \sinh y}$. $\operatorname{coth} x-\operatorname{coth} y=-\frac{\sinh (x-y)}{\sinh x \sinh y}$. 


\subsection{5}

I. $\sinh (x+y)+\sinh (x-y)=2 \sinh x \cosh y$.

2. $\sinh (x+y)-\sinh (x-y)=2 \cosh x \sinh y$.

3. $\cosh (x+y)+\cosh (x-y)=2 \cosh x \cosh y$.

4 . $\cosh (x+y)-\cosh (x-y)=2 \sinh x \sinh y$.

$5 \cdot$ $\tanh \frac{1}{2}(x \pm y)=\frac{\sinh x \pm \sinh y}{\cosh x+\cosh y}$.

6.

$7 \cdot$ $\operatorname{coth} \frac{1}{2}(x \pm y)=\frac{\sinh x \mp \sinh y}{\cosh x-\cosh y}$

8.

$$
\begin{aligned}
& \frac{\tanh x+\tanh y}{\tanh x-\tanh y}=\frac{\sinh (x+y) .}{\sinh (x-y)} \\
& \frac{\operatorname{coth} x+\operatorname{coth} y}{\operatorname{coth} x-\operatorname{coth} y}=-\frac{\sinh (x+y)}{\sinh (x-y)}
\end{aligned}
$$

\subsection{6}

I. $\sinh (x+y)+\cosh (x+y)=(\cosh x+\sinh x)(\cosh y+\sinh y)$.

2. $\quad \sinh (x+y) \sinh (x-y)=\sinh ^{2} x-\sinh ^{2} y$

$$
=\cosh ^{2} x-\cosh ^{2} y \text {. }
$$

3. $\cosh (x+y) \cosh (x-y)=\cosh ^{2} x+\sinh ^{2} y$

$$
=\sinh ^{2} x+\cosh ^{2} y \text {. }
$$

4.

$$
\sinh x+\cosh x=\frac{\mathrm{I}+\tanh \frac{1}{2} x}{\mathrm{I}-\tanh \frac{1}{2} x} .
$$

5

$$
(\sinh x+\cosh x)^{n}=\cosh n x+\sinh n x \text {. }
$$

\subsection{7}

I.

2.

3.

4. $e^{x}=\cosh x+\sinh x$.

$e^{-x}=\cosh x-\sinh x$.

$\sinh x=\frac{1}{2}\left(e^{x}-e^{-x}\right)$.

$\cosh x=\frac{1}{2}\left(e^{x}+e^{-x}\right)$. 


\subsection{8}

I.

$\sinh 2 x=2 \sinh x \cosh x$,

$$
=\frac{2 \tanh x}{\mathrm{I}-\tanh ^{2} x} \text {. }
$$

2.

$\cosh 2 x=\cosh ^{2} x+\sinh ^{2} x=2 \cosh ^{2} x-\mathrm{I}$,

$=\mathrm{I}+2 \sinh ^{2} x$,

$=\frac{I+\tanh ^{2} x}{I-\tanh ^{2} x}$.

$3 \cdot$

$\tanh 2 x=\frac{2 \tanh x}{\mathrm{I}+\tanh ^{2} x}$

4.

$\sinh 3 x=3 \sinh x+4 \sinh ^{3} x$.

5 .

$\cosh 3 x=4 \cosh ^{3} x-3 \cosh x$.

6.

$\tanh 3 x=\frac{3 \tanh x+\tanh ^{3} x}{\mathrm{I}+3 \tanh ^{2} x}$.

3.40 Inverse Hyperbolic Functions.

The hyperbolic functions being periodic, the inverse functions are multiple valued (3.311). In the following formulas the periodic constants are omitted, the principal values only being given.

I.

$$
\begin{aligned}
& \sinh ^{-1} x=\log \left(x+\sqrt{x^{2}+\mathrm{I}}\right)=\cosh ^{-1} \sqrt{x^{2}+\mathrm{I}} . \\
& \cosh ^{-1} x=\log \left(x+\sqrt{x^{2}-\mathrm{I}}\right)=\sinh ^{-1} \sqrt{x^{2}-\mathrm{I}} \\
& \tanh ^{-1} x=\log \sqrt{\frac{\mathrm{I}+x}{\mathrm{I}-x}} \\
& \operatorname{coth}^{-1} x=\log \sqrt{\frac{x+\mathrm{I}}{x-\mathrm{I}}}=\tanh ^{-1} \frac{\mathrm{I}}{x}
\end{aligned}
$$

2.

3 .

4 .

5

$$
\operatorname{sech}^{-1} x=\log \left(\frac{\mathrm{I}}{x}+\sqrt{\frac{\mathrm{I}}{x^{2}}-\mathrm{I}}\right)=\cosh ^{-1} \frac{\mathrm{I}}{x} \text {. }
$$

6

$$
\operatorname{csch}^{-1} x=\log \left(\frac{I}{x}+\sqrt{\frac{I}{x^{2}}+I}\right)=\sinh ^{-1} \frac{I}{x}
$$

\subsection{1}

I.

$$
\sinh ^{-1} x \pm \sinh ^{-1} y=\sinh ^{-1}\left(x \sqrt{I+y^{2}} \pm y \sqrt{I+x^{2}}\right) .
$$

2.

$$
\cosh ^{-1} x \pm \cosh ^{-1} y=\cosh ^{-1}\left(x y \pm \sqrt{\left(x^{2}-\mathrm{I}\right)\left(y^{2}-\mathrm{I}\right)}\right) \text {. }
$$

3.

$$
\tanh ^{-1} x \pm \tanh ^{-1} y=\tanh ^{-1} \frac{x \pm y}{1 \pm x y}
$$




\subsection{2}

I.

$$
\begin{aligned}
\cosh ^{-1} \frac{\mathrm{I}}{2}\left(x+\frac{\mathrm{I}}{x}\right) & =\sinh ^{-1} \frac{\mathrm{I}}{2}\left(x-\frac{\mathrm{I}}{x}\right) \\
& =\tanh ^{-1} \frac{x^{2}-\mathrm{I}}{x^{2}+\mathrm{I}}=2 \tanh ^{-1} \frac{x-\mathrm{I}}{x+\mathrm{I}} \\
& =\log x .
\end{aligned}
$$

2.

$$
\cosh ^{-1} \csc 2 x=-\sinh ^{-1} \cot 2 x=-\tanh ^{-1} \cos 2 x,
$$

$$
=\log \tan x \text {. }
$$

3.

$$
\tanh ^{-1} \tan ^{2}\left(\frac{\pi}{4}+\frac{x}{2}\right)=\frac{I}{\mathrm{I}} \log \csc x .
$$

4.

$$
\tanh ^{-1} \tan ^{2} \frac{x}{2}=\frac{1}{2} \log \sec x \text {. }
$$

3.43 The Gudermannian.

If,

I.

$\cosh x=\sec \theta$.

2. $\sinh x=\tan \theta$.

3.

$$
e^{x}=\sec \theta+\tan \theta=\tan \left(\frac{\pi}{4}+\frac{\theta}{2}\right) .
$$$$
x=\log \tan \left(\frac{\pi}{4}+\frac{\theta}{2}\right) .
$$

4.

$\theta=\operatorname{gd} x$.

\subsection{4}

I.

$\sinh x=\tan \operatorname{gd} x$.

2.

$\cosh x=\sec \operatorname{gd} x$.

3.

$\tanh x=\sin \operatorname{gd} x$.

4.

$$
\tanh \frac{x}{2}=\tan \frac{I}{2} \operatorname{gd} x \text {. }
$$

5.

$$
e^{x}=\frac{I+\sin \operatorname{gd} x}{\cos \operatorname{gd} x}=\frac{I-\cos \left(\frac{\pi}{2}+\operatorname{gd} x\right)}{\sin \left(\frac{\pi}{2}+\operatorname{gd} x\right)}
$$


6. $\tanh ^{-1} \tan x=\frac{1}{2} \operatorname{gd} 2 x$.

7. $\tan ^{-1} \tanh x=\frac{1}{2} \operatorname{gd}^{-1} 2 x$.

3.50

SOLUTION OF OBLIQUE PLANE TRIANGLES

$a, b, c=$ Sides of triangle;

$\alpha, \beta, \gamma=$ angles opposite to $a, b, c$, respectively,

$A=$ area of triangle,

$s=\frac{1}{2}(a+b+c)$.

Given Sought

\section{Formula}

$a, b, c \cdot \alpha$

$$
\begin{aligned}
\sin \frac{\mathrm{I}}{2} \alpha & =\sqrt{\frac{(s-b)(s-c)}{b c}} . \\
\cos \frac{\mathrm{I}}{2} \alpha & =\sqrt{\frac{s(s-a)}{b c}} . \\
\tan \frac{\mathrm{I}}{2} \alpha & =\sqrt{\frac{(s-b)(s-c)}{s(s-a)}} . \\
\cos \alpha & =\frac{c^{2}+b^{2}-a^{2}}{2 b c} .
\end{aligned}
$$

A

$$
A=\sqrt{s(s-a)(s-\bar{b})(s-c)} .
$$

$a, b, \alpha$

$$
\sin \beta=\frac{b \sin \alpha}{a} \text {. }
$$

When $a>b, \beta<\frac{\pi}{2}$ and but one value results. When $b>a$ $\beta$ has two values.

$$
\gamma=180^{\circ}-(\alpha+\beta) \text {. }
$$

$c$

$$
c=\frac{a \sin \gamma}{\sin \alpha} \text {. }
$$

A

$$
A=\frac{1}{2} a b \sin \gamma \text {. }
$$

$a, \alpha, \beta$

$$
b=\frac{a \sin \beta}{\sin \alpha}
$$

$$
\gamma=\mathrm{I} 80^{\circ}-(\alpha+\beta) \text {. }
$$

$$
c=\frac{a \sin \gamma}{\sin \alpha}=\frac{a \sin (\alpha+\beta)}{\sin \alpha} \text {. }
$$


Given Sought

$A$

$a, b, \gamma \quad \alpha$ $\alpha, \beta$

$c$

$A$

Formula

$$
A=\frac{\mathrm{I}}{2} a b \sin \gamma=\frac{\mathrm{I}}{2} a^{2} \frac{\sin \beta \sin \gamma}{\sin \alpha} .
$$$$
\tan \alpha=\frac{a \sin \gamma}{b-a \cos \gamma}
$$

$$
\begin{aligned}
\frac{1}{2}(\alpha+\beta) & =90^{\circ}-\frac{1}{2} \gamma . \\
\tan \frac{\mathrm{I}}{2}(\alpha-\beta) & =\frac{a-b}{a+b} \cot \frac{1}{2} \gamma \\
c & =\left(a^{2}+b^{2}-2 a b \cos \gamma\right)^{\frac{1}{2}} . \\
& =\left\{(a+b)^{2}-4 a b \cos ^{2} \frac{1}{2} \gamma\right\}^{\frac{1}{2}} \\
& =\left\{(a-b)^{2}+4 a b \sin ^{2} \frac{1}{2} \gamma\right\}^{\frac{1}{2}} . \\
& =\frac{a-b}{\cos \phi} \text { where } \tan \phi=2 \sqrt{a b} \frac{\sin \frac{1}{2} \gamma}{a-b} \\
& =\frac{a \sin \gamma}{\sin \alpha} . \\
A & =\frac{1}{2} a b \sin \gamma .
\end{aligned}
$$

\section{SOLUTION OF SPHERICAL TRIANGLES}

3.51 Right-angled spherical triangles.

$a, b, c=$ sides of triangle, $c$ the side opposite $\gamma$, the right angle.

$\alpha, \beta, \gamma=$ angles opposite $a, b, c$, respectively.

3.511 Napier's Rules:

The five parts are $a, b, \operatorname{coc} c, \operatorname{co} \alpha, \operatorname{co} \beta$, where $\operatorname{coc}=\frac{\pi}{2}-c$. The right angle $\gamma$ is omitted.

The sine of the middle part is equal to the product of the tangents of the adjacent parts.

The sine of the middle part is equal to the product of the cosines of opposite parts.

From these rules the following equations follow:

$$
\begin{aligned}
\sin a & =\sin c \sin \alpha, \\
\tan a & =\tan c \cos \beta=\sin b \tan \alpha, \\
\sin b & =\sin c \sin \beta, \\
\tan b & =\tan c \cos \alpha=\sin a \tan \beta, \\
\cos \alpha & =\cos a \sin \beta, \\
\cos \beta & =\cos b \sin \alpha, \\
\cos c & =\cot \alpha \cot \beta=\cos a \cos b .
\end{aligned}
$$


3.52 Oblique-angled spherical triangles.

$a, b, c=$ sides of triangle.

$\alpha, \beta, \gamma=$ angles opposite to $a, b, c$, respectively.

$s=\frac{1}{2}(a+b+c)$,

$\sigma=\frac{1}{2}(\alpha+\beta+\gamma)$,

$\boldsymbol{\epsilon}=\alpha+\beta+\gamma-\mathrm{I} 80=$ spherical excess,

$S=$ surface of triangle on sphere of radius $r$.

Given

Sought

Formula

$a, b, c$

$\alpha$

$\sin ^{2} \frac{1}{2} \alpha=$ haversin $\alpha$,

$$
=\frac{\sin (s-b) \sin (s-c)}{\sin b \sin c}
$$$$
\tan ^{2} \frac{I}{2} \alpha=\frac{\sin (s-b) \sin (s-c)}{\sin s \sin (s-a)} .
$$$$
\cos ^{2} \frac{\mathrm{I}}{2} \alpha=\frac{\sin s \sin (s-a)}{\sin b \sin c} \text {. }
$$

haversin $\alpha=\frac{\text { hav } a-\operatorname{hav}(b-c)}{\sin b \sin c}$.

$\alpha, \beta, \gamma$

$a$

$\sin ^{2} \frac{1}{2} a=$ haversin $a$,

$$
=\frac{-\cos \sigma \cos (\sigma-\alpha)}{\sin \beta \sin \gamma}
$$

$$
\tan ^{2} \frac{\mathrm{I}}{2} a=\frac{-\cos \sigma \cos (\sigma-\alpha)}{\cos (\sigma-\beta) \cos (\sigma-\gamma)} .
$$$$
\cos ^{2} \frac{\mathrm{I}}{2} a=\frac{\cos (\sigma-\beta) \cos (\sigma-\gamma)}{\sin \beta \sin \gamma} \text {. }
$$

$a, c, \alpha$

Ambiguous case.

$$
\sin \gamma=\frac{\sin \alpha \sin c}{\sin a} \text {. }
$$

Two solutions

possible.

$\beta\left\{\begin{aligned} \tan \theta & =\tan \alpha \cos c . \\ \sin (\beta+\theta) & =\sin \theta \tan c \cot a\end{aligned}\right.$

$b\left\{\begin{aligned} \cot \phi & =\tan c \cos \alpha . \\ \sin (b+\phi) & =\frac{\cos a \sin \phi}{\cos c} .\end{aligned}\right.$

$\alpha, \gamma, c$

Ambiguous case.

Two solutions

c $\quad \sin c=\frac{\sin a \sin \gamma}{\sin \alpha}$. possible. 
Given

Sought

Formula

$b\left\{\begin{aligned} \tan \theta & =\tan a \cos \gamma . \\ \sin (b-\theta) & =\cot \alpha \tan \gamma \sin \theta .\end{aligned}\right.$

$b\left\{\begin{aligned} \tan \frac{\mathrm{I}}{2} b & =\frac{\sin \frac{1}{2}(\alpha+\gamma)}{\sin \frac{1}{2}(\alpha-\gamma)} \tan \frac{1}{2}(a-c) \\ & =\frac{\cos \frac{1}{2}(\alpha+\gamma)}{\cos \frac{1}{2}(\alpha-\gamma)} \tan \frac{1}{2}(a+c) .\end{aligned}\right.$

$\beta\left\{\begin{aligned} \cot \phi & =\cos a \tan \gamma \\ \sin \left(\beta^{-}-\phi\right) & =\frac{\cos \alpha \sin \phi}{\cos \gamma} .\end{aligned}\right.$

$\beta\left\{\begin{aligned} \cot \frac{\mathrm{I}}{2} \beta & =\frac{\sin \frac{1}{2}(a+c)}{\sin \frac{1}{2}(a-c)} \tan \frac{1}{2}(\alpha-\gamma) . \\ & =\frac{\cos \frac{1}{2}(a+c)}{\cos \frac{1}{2}(a-c)} \tan \frac{1}{2}(\alpha+\gamma) .\end{aligned}\right.$

$a, b, \gamma$

$c$

$\cos c=\cos a \cos b+\sin a \sin b \cos \gamma$.

$\tan \theta=\tan a \cos \gamma$

$\tan \phi=\tan b \cos \gamma \quad c$

$\cos c=\frac{\cos a \cos (b-\theta)}{\cos \theta}$

$=\frac{\cos b \cos (a-\phi)}{\cos \phi}$.

hav $c=\operatorname{hav}(a-b) .+\sin a \sin b \operatorname{hav} \gamma$

$\alpha$

$\tan \alpha=\frac{\sin \theta \tan \gamma}{\sin (b-\theta)}$.

$\beta$

$\sin \beta=\frac{\sin \gamma \sin b}{\sin c}$.

$=\frac{\sin \alpha \sin b}{\sin a}$.

$\tan \beta=\frac{\sin \phi \tan \gamma}{\sin (a-\phi)}$.

$\alpha, \beta\left\{\begin{array}{l}\tan \frac{\mathrm{I}}{2}(\alpha+\beta)=\frac{\cos \frac{1}{2}(a-b) \cot \frac{1}{2} \gamma}{\cos \frac{1}{2}(a+b)} \\ \tan \frac{\mathrm{I}}{2}(\alpha-\beta)=\frac{\sin \frac{1}{2}(a-b) \cot \frac{1}{2} \gamma}{\sin \frac{1}{2}(a+b)} .\end{array}\right.$

$c, \alpha, \beta$

$\tan \theta=\cos c \tan \alpha$ $\tan \phi=\cos c \tan \beta$ $\gamma$

$\cos \gamma=-\cos \alpha \cos \beta+\sin \alpha \sin \beta \cos c$.

$\cos \gamma=\frac{\cos \alpha \cos (\beta+\theta)}{\cos \theta}$.

$$
=\frac{\cos \beta \cos (\alpha+\phi)}{\cos \phi} \text {. }
$$

$a$ 
Given

$a, b, \gamma$

$$
\begin{aligned}
& \tan b=\frac{\tan c \sin \phi}{\sin (\alpha+\phi)} . \\
& a, b\left\{\begin{aligned}
& b \quad \\
& \tan \frac{1}{2}(a+b)=\frac{\cos \frac{1}{2}(\alpha-\beta) \tan \frac{1}{2} c}{\cos \frac{1}{2}(\alpha+\beta)} \\
& \tan \frac{1}{2}(a-b)=\frac{\sin \frac{1}{2}(\alpha-\beta) \tan \frac{1}{2} c}{\sin \frac{1}{2}(\alpha+\beta)} . \\
& \epsilon \quad \cot \frac{1}{2} \epsilon=\frac{\cot \frac{1}{2} a \cot \frac{1}{2} b+\cos \gamma}{\sin \gamma} . \\
& \epsilon \quad \tan ^{2} \frac{1}{4} \epsilon=\tan \frac{1}{2} s \tan \frac{1}{2}(s-a) \tan \frac{1}{2}(s-b) \\
& \tan \frac{1}{2}(s-c) .
\end{aligned}\right.
\end{aligned}
$$

$\epsilon, \gamma$

$$
S=\frac{\epsilon}{\mathrm{I} 80^{\circ}} \pi r^{2}
$$

\section{FINITE SERIES OF CIRCULAR FUNCTIONS}

3.60 If the sum, $f(r)$, of the finite or infinite series:

$$
f(r)=a_{0}+a_{1} r+a_{2} r^{2}+\ldots \ldots
$$

is known, the sums of the series:

$$
\begin{gathered}
S_{1}=a_{0} \cos x+a_{1} r \cos (x+y)+a_{2} r^{2} \cos (x+2 y)+\ldots \\
S_{2}=a_{0} \sin x+a_{1} r \sin (x+y)+a_{2} r^{2} \sin (x+2 y)+\ldots \\
S_{1}=\frac{1}{2}\left\{e^{i x} f\left(r e^{i y}\right)+e^{-i x} f\left(r e^{-i y}\right)\right\}, \\
S_{2}=-\frac{i}{2}\left\{e^{i x} f\left(r e^{i y}\right)-e^{-i x} f\left(r e^{-i y}\right)\right\} .
\end{gathered}
$$

are:

3.61 Special Finite Series.

I. $\sum_{k=1}^{n} \sin k x=\frac{\sin \frac{n x}{2} \sin \frac{n+1}{2} x}{\sin \frac{x}{2}}$.

2. $\sum_{k=0}^{n} \cos k x=\frac{\cos \frac{n x}{2} \sin \frac{n+\mathrm{I}}{2} x}{\sin \frac{x}{2}}$ 
3. $\sum_{k=1}^{n} \sin ^{2} k x=\frac{n}{2}-\frac{\cos (n+\mathrm{I}) x \cdot \sin n x}{2 \sin x}$.

4. $\sum_{k=0}^{n} \cos ^{2} k x=\frac{n+2}{2}+\frac{\cos (n+\mathrm{I}) x \cdot \sin n x}{2 \sin x}$.

5. $\sum_{k=1}^{n-1} k \sin k x=\frac{\sin n x}{4 \sin ^{2} \frac{x}{2}}-\frac{n \cos \left(\frac{2 n-\mathrm{I}}{2}\right) x}{2 \sin \frac{x}{2}}$.

6. $\sum_{k=1}^{n-\mathrm{I}} k \cos k x=\frac{n \sin \left(\frac{2 n-\mathrm{I}}{2}\right) x}{2 \sin \frac{x}{2}}-\frac{\mathrm{I}-\cos n x}{4 \sin ^{2} \frac{x}{2}}$.

7. $\sum_{k=1}^{n} \sin (2 k-\mathrm{I}) x=\frac{\sin ^{2} n x}{\sin x}$.

8. $\sum_{k=0}^{n} \sin (x+k y)=\frac{\sin \left(x+\frac{n y}{2}\right) \sin \left(\frac{n+\mathrm{I}}{2} y\right)}{\sin \frac{y}{2}}$.

9. $\sum_{k=0}^{n} \cos (x+k y)=\frac{\cos \left(x+\frac{n}{2} y\right) \sin \left(\frac{n+\mathrm{I}}{2} y\right)}{\sin \frac{y}{2}}$.

I0. $\sum_{k=\mathrm{I}}^{n+\mathrm{I}}(-\mathrm{I})^{k-1} \sin (2 k-\mathrm{I}) x=(-\mathrm{I})^{n} \frac{\sin (2 n+2) x}{2 \cos x}$.

II. $\sum_{k=1}^{n}(-\mathrm{I})^{k} \cos k x=-\frac{\mathrm{I}}{2}+(-\mathrm{I})^{n} \frac{\cos \left(\frac{2 n+\mathrm{I}}{2} x\right)}{2 \cos _{2}^{x}}$.

I2. $\sum_{k=\mathrm{I}}^{n-\mathrm{I}} r^{k} \sin k x=\frac{r \sin x\left(\mathrm{I}-r^{n} \cos n x\right)-(\mathrm{I}-r \cos x) r^{n} \sin n x}{\mathrm{I}-2 r \cos x+r^{2}}$.

I3. $\sum_{k=0}^{n-\mathrm{I}} r^{k} \cos k x=\frac{(\mathrm{I}-r \cos x)\left(\mathrm{I}-r^{n} \cos n x\right)+r^{n+1} \sin x \sin n x}{\mathrm{I}-2 r \cos x+r^{2}}$.

I4. $\sum_{k=1}^{n}\left(\frac{1}{2^{k}} \sec \frac{x}{2^{k}}\right)^{2}=\csc ^{2} x-\left(\frac{1}{2^{n}} \csc \frac{x}{2^{n}}\right)^{2}$.

I5. $\sum_{k=1}^{n}\left(2^{k} \sin ^{2} \frac{x}{2^{k}}\right)^{2}=\left(2^{n} \sin \frac{x}{2^{n}}\right)^{2}-\sin ^{2} x$. 
I6. $\sum_{k=0}^{n} \frac{\mathrm{I}}{2^{k}} \tan \frac{x}{2^{k}}=\frac{\mathrm{I}}{2^{n}} \cot \frac{x}{2^{n}}-2 \cot 2 x$.

I7. $\sum_{k=0}^{n-\mathrm{I}} \cos \frac{k^{2} 2 \pi}{n}=\frac{\sqrt{n}}{2}\left(\mathrm{I}+\cos \frac{n \pi}{2}+\sin \frac{n \pi}{2}\right)$.

I8. $\sum_{k=1}^{n-\mathrm{I}} \sin \frac{k^{2} 2 \pi}{n}=\frac{\sqrt{n}}{2}\left(\mathrm{x}+\cos \frac{n \pi}{2}-\sin \frac{n \pi}{2}\right)$.

I9. $\sum_{k=1}^{n-\mathrm{I}} \sin \frac{k \pi}{n}=\cot \frac{\pi}{2 n}$.

20. $\sum_{k=0}^{n} \frac{\mathrm{I}}{2^{2 k}} \tan ^{2} \frac{x}{2^{k}}=\frac{2^{2 n+2}-\mathrm{I}}{3 \cdot 2^{2 n-1}}+4 \cot ^{2} 2 x-\frac{\mathrm{I}}{2^{2 n}} \cot \frac{x}{2^{n}}$.

\subsection{2}

$$
S_{n}=\sum_{k=1}^{n-x} \csc \frac{k \pi}{n}
$$

Watson (Phil. Mag. 3I, p. III, I9I6) has obtained an asymptotic expansion for this sum, and has given the following approximation:

$S_{n}=2 n\left\{0.7329355992 \log _{10}(2 n)-0.180645387 \mathrm{I}\right\}$

$$
-\frac{0.087266}{n}+\frac{0.01035}{n^{3}}-\frac{0.004}{n^{5}}+\frac{0.005}{n^{7}}-\cdots
$$

Values of $S_{n}$ are tabulated by integers from $n=2$ to $n=30$, and from $n=30$ to $n=$ roo at intervals of 5 .

The expansion of

$$
\begin{aligned}
T_{n}= & \sum_{k=1}^{n-1} \csc \left(\frac{k \pi}{n}-\frac{\beta}{2}\right), \\
& -\frac{2 \pi}{n}<\beta<\frac{2 \pi}{n},
\end{aligned}
$$

where

is also obtained. 
3.70 Finite Products.

I. $\sin n x=n \sin x \cos x \prod_{k=1}^{\frac{n}{2}-\mathbf{I}}\left(\mathrm{I}-\frac{\sin ^{2} x}{\sin ^{2} \frac{k \pi}{n}}\right) n$ even.

2. $\cos n x=\prod_{k=\mathrm{r}_{4}}^{\frac{n}{2}}\left(\mathrm{I}-\frac{\sin ^{2} x}{\sin ^{2} \frac{2 k-\mathrm{I}}{2 n} \pi}\right) n$ even.

3. $\sin n x=n \sin x \prod_{k=1}^{\frac{n-1}{2}}\left(\mathrm{I}-\frac{\sin ^{2} x}{\sin ^{2} \frac{k \pi}{n}}\right) n$ odd.

4. $\cos n x=\cos x \prod_{k=1}^{\frac{n-x}{2}}\left(\mathrm{I}-\frac{\sin ^{2} x}{\sin ^{2} \frac{2 k-\mathrm{I}}{2 n} \pi}\right) n$ odd.

5 $\cos n x-\cos n y=2^{n-1} \prod_{k=0}^{n-\mathbf{r}}\left\{\cos x-\cos \left(y+\frac{2 k \pi}{n}\right)\right\}$.

6. $\quad a^{2 n}-2 a^{n} b^{n} \cos n x+b^{2 n}=\prod_{k=0}^{n-1}\left\{a^{2}-2 a b \cos \left(x+\frac{2 k \pi}{n}\right)+b^{2}\right\}$.

$3.800 \tan x=x$.

$$
\text { ROOTS OF TRANSCENDENTAL EQUATIONS }
$$

The first I 7 roots, and the corresponding maxima and minima of $\frac{\sin x}{x}$ are given in the following table (Lommel, Abh. Munch. Akad. (2) I5, I23, I886):

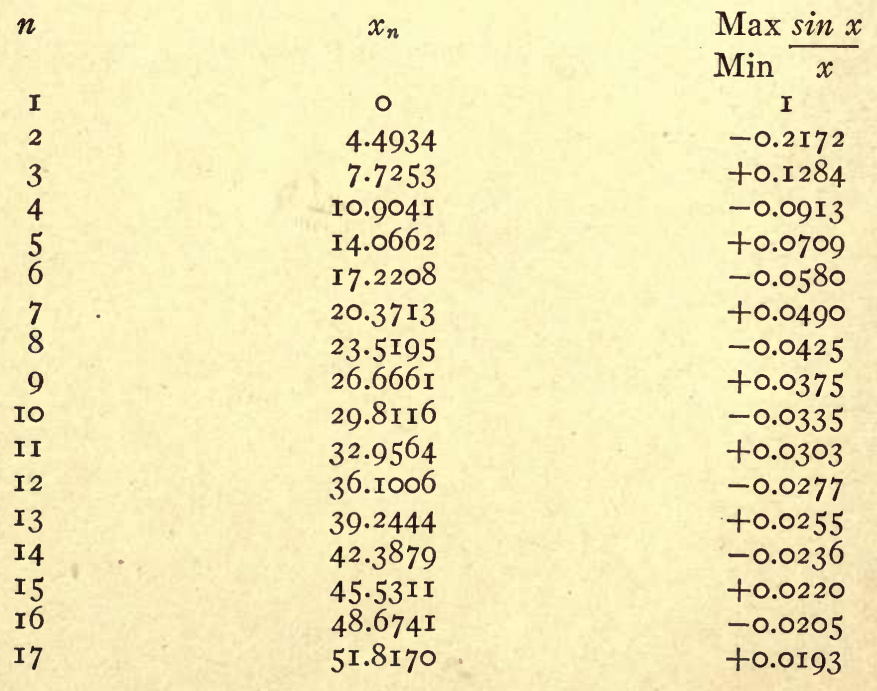




\subsection{1}

The first three roots are:

$$
\tan x=\frac{2 x}{2-x^{2}}
$$

$$
\begin{aligned}
& x_{1}=0, \\
& x_{2}=\operatorname{II} 9.26 \frac{\pi}{180}, \\
& x_{3}=340.35 \frac{\pi}{180} .
\end{aligned}
$$

If $x$ is large

$$
\begin{array}{r}
x_{n}=n \pi-\frac{2}{n \pi}-\frac{16}{3 n^{3} \pi^{3}}+\ldots \\
(\text { Rayleigh, Theory of Sound, II, p. 265.) }
\end{array}
$$

\subsection{2}

The first two roots are:

$$
\tan x=\frac{x^{3}-9 x}{4 x^{2}-9}
$$

$$
\begin{aligned}
& x_{1}=0 \\
& x_{2}=3.3422 .
\end{aligned}
$$

\subsection{3}

The first two roots are:

$$
\tan x=\frac{x}{1-x^{2}}
$$

$$
\begin{aligned}
& x_{1}=0 \\
& x_{2}=2 \cdot 744 \\
& \text { (J. J. Thomson, Recent Researches, p. 373.) }
\end{aligned}
$$

\subsection{4}

The first seven roots are:

$$
\tan x=\frac{3 x}{3-x^{2}}
$$

$$
\begin{aligned}
& x_{1}=0, \\
& x_{2}=\mathrm{r} .8346 \pi, \\
& x_{3}=2.8950 \pi, \\
& x_{4}=3.9225 \pi, \\
& x_{5}=4.9385 \pi, \\
& x_{6}=5.9489 \pi, \\
& x_{7}=6.9563 \pi .
\end{aligned}
$$

(Lamb, London Math. Soc. Proc. I3, I882.)

\subsection{5}

$$
\tan x=\frac{4 x}{4-3 x^{2}}
$$


The first seven roots are:

$$
\begin{aligned}
& x_{1}=0, \\
& x_{2}=0.8 \text { ז } 60 \pi, \\
& x_{3}=1.9285 \pi, \\
& x_{4}=2.9359 \pi, \\
& x_{5}=3.9658 \pi, \\
& x_{6}=4.9728 \pi, \\
& x_{7}=5.9774 \pi .
\end{aligned}
$$

\subsection{6}

The roots are:

$\cos x \cosh x=\mathrm{I}$.

$$
\begin{aligned}
& x_{1}=4.7300408, \\
& x_{2}=7.8532046, \\
& x_{3}=10.9956078, \\
& x_{4}=14.137 \mathrm{I} 655, \\
& x_{5}=\mathrm{I} 7.2787596, \\
& x_{n}=\frac{1}{2}(2 n+\mathrm{I}) \pi n>_{5} .
\end{aligned}
$$

\subsection{7}

(Rayleigh, Theory of Sound, I, p. 278.)

The roots are:

$\cos x \cosh x=-\mathrm{I}$.

\subsection{8}

$$
\begin{aligned}
& x_{1}=\mathrm{I} .875 \mathrm{I0}, \\
& x_{2}=4.694098, \\
& x_{3}=7.854757, \\
& x_{4}=\mathrm{I} 0.99554 \mathrm{I}, \\
& x_{5}=\mathrm{I} 4 . \mathrm{I} 37 \mathrm{I} 68, \\
& x_{6}=\mathrm{I} 7.278759, \\
& x_{n}=\frac{1}{2}(2 n-\mathrm{I}) \pi n>6 . \\
& \mathrm{I}-\left(\mathrm{I}+x^{2}\right) \cos x=0 .
\end{aligned}
$$

The roots are:

$$
\begin{aligned}
& x_{1}=\text { I.102506, } \\
& x_{2}=4.75476 \mathrm{I}, \\
& x_{3}=7.837964, \\
& x_{4}=\mathrm{I} 1.003766, \\
& x_{5}=\mathrm{I} 4.132 \mathrm{I} 85, \\
& x_{6}=\mathrm{I} 7.282097 .
\end{aligned}
$$

(Schlömilch: Ubungsbuch, I, p. 354.)

3.809 The smallest root of is

$$
\theta-\cot \theta=0 \text {, }
$$

$$
\theta=49^{\circ} \mathrm{I} 7^{\prime} 36^{\prime \prime} .5 \text {. }
$$


3.810 The smallest root of is

$$
\begin{gathered}
\theta-\cos \theta=0, \\
\theta=42^{\circ} 20^{\prime} 47^{\prime \prime} \cdot 3 .
\end{gathered}
$$

3.811 The smallest root of

$$
\begin{gathered}
x e^{x}-2=0 \\
x=0.8526
\end{gathered}
$$

3.812 The smallest root of is

$$
\begin{gathered}
\log (1+x)-\frac{3}{4} x=0, \\
x=0.73360
\end{gathered}
$$

\subsection{3}

$$
\tan x-x+\frac{\mathrm{I}}{x}=0
$$

The first roots are:

$$
\begin{aligned}
& x_{1}=4.480, \\
& x_{2}=7.723, \\
& x_{3}=10.90, \\
& x_{4}=14.07 .
\end{aligned}
$$

(Collo, Annalen der Physik, 65, p. 45, I92r.)

\subsection{4}

$$
\cot x+x-\frac{\mathrm{I}}{x}=0
$$

The first roots are:

$$
\begin{aligned}
& x_{1}=0, \\
& x_{2}=2.744, \\
& x_{3}=6 . \mathrm{I} 7, \\
& x_{4}=9.3 \mathrm{I} 7, \\
& x_{5}=\mathrm{I} 2.48, \\
& x_{6}=\mathrm{I} 5.64, \\
& x_{7}=\mathrm{I} 8.80 .
\end{aligned}
$$

(Collo, 1. c.)

\subsection{Special Tables.}

$\sin \theta, \cos \theta:$ The British Association Report for I9r6 contains the following tables:

Table I, p. 6o. $\sin \theta, \cos \theta, \theta$ expressed in radians from $\theta=0$ to $\theta=\mathbf{r} .600$, interval $0.00 \mathrm{I}$, Io decimal places.

Table II, p. 88. $\theta-\sin \theta, \mathrm{I}-\cos \theta, \theta=0.0000 \mathrm{I}$ to $\theta=0.00100$, interval 0.0000 , ro decimal places. 
Table III, p. 9o. $\sin \theta, \cos \theta ; \theta=0.1$ to $\theta=$ ro.o, interval o.I, I 5 decimal places.

J. Peters (Abh. d. K. P. Akad. der Wissen., Berlin, IgII) has given sines and cosines for every sexagesimal second to $2 \mathrm{I}$ places.

hav $\theta, \log _{10}$ hav $\theta$ : Bowditch, American Practical Navigator, five-place tables, $0^{\circ}-180^{\circ}$, for $15^{\prime \prime}$ intervals.

Tables for Solution of Spherical Triangles.

Aquino's Altitude and Azimuth Tables, London, I918. Reprinted in Hydrographic Office Publication, No. 200, Washington, I9r8.

Hyperbolic Functions.

The Smithsonian Mathematical Tables: Hyperbolic Functions, contain the most complete five-place tables of Hyperbolic Functions.

Table I. The common logarithms (base Io) of $\sinh u, \cosh u, \tanh u, \operatorname{coth} u$ :

$$
\begin{aligned}
& u=0.000 \mathrm{I} \text { to } u=0.1000 \text { interval } 0.000 \mathrm{I}, \\
& u=0.00 \mathrm{I} \text { to } u=3.000 \text { interval } 0.00 \mathrm{I} \\
& u=3.00 \text { to } u=6.00 \quad \text { interval } 0.0 \mathrm{I}
\end{aligned}
$$

Table II. $\sinh u, \cosh u, \tanh u, \operatorname{coth} u$. Same ranges and intervals.

Table III. $\sin u, \cos u, \log _{10} \sin u, \log _{10} \cos u$ :

$$
\begin{aligned}
& u=0.000 \mathrm{I} \text { to } u=0.1000 \text { interval } 0.000 \mathrm{I}, \\
& u=0.100 \text { to } u=\mathrm{I} .600 \text { interval } 0.00 \mathrm{I} .
\end{aligned}
$$

Table IV. $\log _{10} e^{u}$ (7 places), $e^{u}$ and $e^{-u}$ ( 7 significant figures):

$$
\begin{aligned}
& u=0.00 \mathrm{I} \text { to } u=2.950 \text { interval } 0.00 \mathrm{I}, \\
& u=3.00 \text { to } u=6.00 \text { interval } 0.0 \mathrm{I}, \\
& u=\mathrm{I} .0 \quad \text { to } u=100 \text { interval I.0 (9-10 figures). }
\end{aligned}
$$

Table V. five-place table of natural logarithms, $\log u$.

$$
\begin{aligned}
& u=\mathrm{r} .0 \text { to } u=1000 \text { interval } \mathrm{r} .0, \\
& u=1000 \text { to } u=10,000 \text { varying intervals. }
\end{aligned}
$$

Table VI. $g d u$ (7 places); $u$ expressed in radians, $u=0.00 \mathrm{I}$ to $u=3.000$, interval $0.00 \mathrm{I}$, and the corresponding angular measure. $u=3.00$ to $u=6.00$, interval 0.01 .

Table VII. $g d^{-1} u$, to $\circ^{\prime}$. or, in terms of $g d u$ in degrees and minutes from $0^{\circ} \mathrm{I}^{\prime}$ to $89^{\circ} 59^{\prime}$.

Table VIII. Table for conversion of radians into angular measure. 




Kennelly: Tables of Complex Hyperbolic and Circular Functions. Cambridge, Harvard University Press, I9I4.

The complex argument, $x+i q=\rho e^{i \delta}$. In the tables this is denoted $\rho \angle \delta$. $\rho=\sqrt{x^{2}+q^{2}}, \tan \delta=q / x$.

Tables I, II, III give the hyperbolic sine, cosine and tangent of $(\rho<\delta)$ expressed as $r \angle \gamma$ :

$$
\begin{array}{ll}
\delta=45^{\circ} \text { to } \delta=90^{\circ} & \text { interval } \mathrm{I}^{\circ} \\
\rho=0.0 \text { I to } \rho=3.0 & \text { interval o.I. }
\end{array}
$$

Tables IV and V give $\frac{\sinh \theta}{\theta}, \frac{\tanh \theta}{\theta}$ expressed as $r \angle \gamma, \theta=\rho \angle \delta$,

$$
\begin{array}{ll}
\rho=\text { O.I to } \rho=3.0 & \text { interval o.I, } \\
\delta=45^{\circ} \text { to } \delta=90^{\circ} & \text { interval I } .
\end{array}
$$

Table VI gives sinh $\left(\rho \angle 45^{\circ}\right), \cosh \left(\rho \angle 45^{\circ}\right), \tanh \left(\rho \angle 45^{\circ}\right)$, coth $\left(\rho \angle 45^{\circ}\right)$, $\operatorname{sech}\left(\rho \angle 45^{\circ}\right), \operatorname{csch}\left(\rho \angle 45^{\circ}\right)$ expressed as $r \angle \gamma$ :

$$
\begin{array}{ll}
\rho=0 \quad \text { to } \rho=6.0 & \text { interval 0.I, } \\
\rho=6.05 \text { to } \rho=20.50 & \text { interval } 0.05 .
\end{array}
$$

Tables VII, VIII and IX give sinh $(x+i q), \cosh (x+i q), \tanh (x+i q)$, expressed as $u+i v$ :

$$
\begin{array}{ll}
x=\circ \text { to } x=3.95 & \text { interval } 0.05, \\
q=\circ \text { to } q=2.0 & \text { interval } 0.05 .
\end{array}
$$

Tables X, XI, XII give sinh $(x+i q), \cosh (x+i q)$, tanh $(x+i q)$ expressed as $r \angle \gamma$ :

$$
\begin{array}{ll}
x=\circ \text { to } x=3.95 & \text { interval } 0.05 \\
q=\circ \text { to } q=2.0 & \text { interval } 0.05
\end{array}
$$

Table XIII gives sinh $(4+i q), \cosh (4+i q), \tanh (4+i q)$ expressed both as $u+i v$ and $r \angle \gamma$ :

$$
q=\circ \text { to } q=2.0 \text { interval } 0.05
$$

Table XIV gives $\frac{e^{x}}{2}$ and $\log _{10} \frac{e^{x}}{2}$.

$$
x=4.00 \text { to } x=\text { I0.00 interval } 0.01 \text {. }
$$

Table XV gives the real hyperbolic functions: $\sinh \theta, \cosh \theta, \tanh \theta, \operatorname{coth} \theta$, $\operatorname{sech} \theta, \operatorname{csch} \theta$.

$$
\begin{aligned}
& \theta=0 \text { to } \theta=2.5 \text { interval o.oI, } \\
& \theta=2.5 \text { to } \theta=7.5 \text { interval o.I. }
\end{aligned}
$$


Pernot and Woods: Logarithms of Hyperbolic Functions to I2 Significant Figures. Berkeley, University of California Press, I9I8.

Table I. $\log _{10} \sinh x$, with the first three differences.

$$
x=.0000 \text { to } x=2 \text { oI } 8 \text { nterval } 0.001 \text {. }
$$

Table II. $\log _{10} \cosh x$.

$$
x=0.000 \text { to } x=2.032 \text { interval } 0.001 .
$$

Table III. $\log _{10} \tanh x$.

$$
x=0.000 \text { to } x=2.018 \text { interval ०.001. }
$$

Table IV. $\log _{10} \frac{\sinh x}{x}$.

$$
x=0.00 \text { to } x=0.506 \text { interval } 0.001 \text {. }
$$

Table V. $\log _{10} \frac{\tanh x}{x}$.

$$
x=0.000 \text { to } x=0.506 \text { interval } 0.00 \mathrm{I} \text {. }
$$

Van Orstrand, Memoirs of the National Academy of Sciences, Vol. XIV, fifth memoir, Washington, I92I.

Tables of $\frac{\mathrm{I}}{n !}, e^{x}, e^{-x}, e^{n \pi}, e^{-n \pi}, e^{ \pm \frac{n \pi}{360}}, \sin x, \cos x$, to $23-62$ decimal places or significant figures. 


\section{VECTOR ANALYSIS}

4.000 A vector A has components along the three rectangular axes, $x, y, z$ : $A_{x}, A_{y}, A_{z}$

$$
\begin{aligned}
& A=\text { length of vector. } \\
& A=\sqrt{A_{z}{ }^{2}+A_{y}{ }^{2}+A_{z}^{2}} .
\end{aligned}
$$

Direction cosines of $\mathrm{A}, \frac{A_{x}}{A}, \frac{A_{y}}{A}, \frac{A_{z}}{A}$.

4.001 Addition of vectors.

$$
\mathbf{A}+\mathbf{B}=\mathbf{C} .
$$

C is a vector with components.

$$
\begin{aligned}
& C_{x}=A_{x}+B_{x} . \\
& C_{y}=A_{y}+B_{y} . \\
& C_{z}=A_{z}+B_{z} .
\end{aligned}
$$

4.002 $\theta=$ angle between $\mathrm{A}$ and $\mathrm{B}$.

$$
\begin{aligned}
C & =\sqrt{A^{2}+B^{2}+{ }^{2 A B \cos \theta} .} \\
\cos \theta & =\frac{A_{x} B_{x}+A_{y} \dot{B}_{y}+A_{z} B_{z}}{A B} .
\end{aligned}
$$

4.003 If $a, b, c$ are any three non-coplanar vectors of unit length, any vector, $R$, may be expressed:

$$
\mathbf{R}=a \mathbf{a}+b \mathbf{b}+c \mathbf{c},
$$

where $a, b, c$ are the lengths of the projections of $\mathbf{R}$ upon $\mathrm{a}, \mathrm{b}, \mathrm{c}$ respectively.

4.004 Scalar product of two vectors:

are equivalent notations.

$$
S A B=(A B)=A B
$$

$$
\mathrm{AB}=A B \cos \widehat{A B} \text {. }
$$

4.005 Vector product of two vectors:

$$
V A B=A \times B=[A B]=C .
$$

C is a vector whose length is

$$
C=A B \sin \widehat{A B}
$$

The direction of $\mathbf{C}$ is perpendicular to both $\mathbf{A}$ and $\mathbf{B}$ such that a right-handed rotation about $\mathbf{C}$ through the angle $\widehat{A B}$ turns $\mathrm{A}$ into $\mathrm{B}$. 
4.006 i, $\mathrm{j}, \mathrm{k}$ are three unit vectors perpendicular to each other. If their directions coincide with the axes $x, y, z$ of a rectangular system of coördinates:

4.007

4.008

$$
\mathbf{A}=A_{x} \mathbf{i}+A_{y} \mathbf{j}+A_{z} \mathbf{k} \text {. }
$$

$$
\begin{aligned}
& \mathrm{ii}=\mathrm{i}^{2}=\mathrm{jj}=\mathrm{j}^{2}=\mathrm{kk}=\mathrm{k}^{2}=\mathrm{I} \\
& \mathrm{ij}=\mathrm{ji}=\mathrm{jk}=\mathrm{kj}=\mathrm{ki}=\mathrm{ik}=\mathrm{o}
\end{aligned}
$$

4.009

$$
\begin{array}{r}
V \mathrm{ij}=-V \mathrm{ji}=\mathbf{k}, \\
V \mathbf{j k}=-V \mathbf{k j}=\mathbf{i}, \\
V \mathbf{k i}=-V \mathbf{i k}=\mathbf{j} .
\end{array}
$$

4.010

$$
\mathbf{A B}=\mathbf{B A}=A B \cos \widehat{A B}=A_{x} B_{x}+A_{y} B_{y}+A_{z} B_{z} \text {. }
$$

$$
\begin{aligned}
& V \mathbf{A B}=-V \mathbf{B A}=\left|\begin{array}{lll}
\mathbf{i} & \mathbf{j} & \mathbf{k} \\
A_{x} & A_{y} & A_{z} \\
B_{x} & B_{y} & B_{z}
\end{array}\right| \\
= & \left(A_{y} B_{z}-A_{z} B_{y}\right) \mathbf{i}+\left(A_{z} B_{x}-A_{x} B_{z}\right) \mathbf{j}+\left(A_{x} B_{y}-A_{y} B_{x}\right) \mathbf{k}
\end{aligned}
$$

4.10 If $\mathbf{A}, \mathbf{B}, \mathbf{C}$, are any three vectors:

$$
\mathbf{A} V \mathbf{B C}=\mathbf{B} V \mathbf{C A}=\mathbf{C} V \mathbf{A B}
$$

$=$ Volume of parallelepipedon having $\mathbf{A}, \mathbf{B}, \mathbf{C}$ as edges

4.11

$$
\left|\begin{array}{lll}
A_{x} & A_{y} & A_{z} \\
B_{x} & B_{y} & B_{z} \\
C_{x} & C_{y} & C_{z}
\end{array}\right|
$$

I. $V \mathbf{A}(\mathbf{B}+\mathbf{C})=V \mathbf{A B}+V \mathbf{A C}$.

2. $V(\mathbf{A}+\mathbf{B})(\mathbf{C}+\mathbf{D})=V \mathbf{A}(\mathbf{C}+\mathbf{D})+V \mathbf{B}(\mathbf{C}+\mathbf{D})$.

3. $V \mathbf{A} V \mathbf{B C}=\mathbf{B} S A C-\mathbf{C} S A B$.

4. $V \mathbf{A} V \mathbf{B C}+V \mathbf{B} V \mathbf{C A}+V \mathbf{C} V \mathbf{A B}=0$.

5. $V \mathrm{AB} \cdot V \mathrm{CD}=\mathrm{AC} \cdot \mathrm{BD}-\mathrm{BC} \cdot \mathrm{AD}$.

6. $V(V \mathbf{A B} \cdot V \mathbf{C D})=\mathbf{C} S(\mathbf{D} V \mathbf{A B})-\mathbf{D} S(\mathbf{C} V \mathbf{A B})$

$$
\begin{aligned}
& =\mathbf{C} S(\mathbf{A} V \mathbf{B D})-\mathbf{D} S(\mathbf{A} V \mathbf{B C}) \\
& =\mathbf{B} S(\mathbf{A} V \mathbf{C D})-\mathbf{A} S(\mathbf{B} V \mathbf{C D}) \\
& =\mathbf{B} S(\mathbf{C} V \mathbf{D A})-\mathbf{A} S(\mathbf{C} V \mathbf{D B}) .
\end{aligned}
$$




$$
\begin{aligned}
d \mathbf{A B} & =\mathbf{A} d \mathbf{B}+\mathbf{B} d \mathbf{A} . \\
d V \mathbf{A B} & =V \mathbf{A} d \mathbf{B}+V d \mathbf{A B} \\
& =V \mathbf{A} d \mathbf{B}-V \mathbf{B} d \mathbf{A} .
\end{aligned}
$$

\subsection{1}

$$
\text { I. } \nabla=\mathrm{i} \frac{\partial}{\partial x}+\mathbf{j} \frac{\partial}{\partial y}+\mathrm{k} \frac{\partial}{\partial z} \text {. }
$$

2. $\nabla \mathbf{A}=\operatorname{div} \mathbf{A}=\frac{\partial A_{x}}{\partial x}+\frac{\partial A_{y}}{\partial y}+\frac{\partial A_{z}}{\partial z}$.

3. $\nabla \phi=\operatorname{grad} \phi=\mathrm{i} \frac{\partial \phi}{\partial x}+\mathrm{j} \frac{\partial \phi}{\partial y}+\mathrm{k} \frac{\partial \phi}{\partial z}$.

4. $V \nabla \mathbf{A}=\operatorname{curl} \mathbf{A}=\operatorname{rot} \mathbf{A}$

$$
\begin{aligned}
= & \left|\begin{array}{ccc}
\mathbf{i} & \mathbf{j} & \mathbf{k} \\
\frac{\partial}{\partial x} & \frac{\partial}{\partial y} & \frac{\partial}{\partial z} \\
A_{x} & A_{y} & A_{z}
\end{array}\right| \\
= & \mathrm{i}\left(\frac{\partial A_{z}}{\partial y}-\frac{\partial A_{y}}{\partial z}\right)+\mathrm{j}\left(\frac{\partial A_{x}}{\partial z}-\frac{\partial A_{z}}{\partial x}\right)+\mathrm{k}\left(\frac{\partial A_{y}}{\partial x}-\frac{\partial A_{x}}{\partial y}\right) .
\end{aligned}
$$

5. $\nabla \nabla=\nabla^{2}=\frac{\partial^{2}}{\partial x^{2}}+\frac{\partial^{2}}{\partial y^{2}}+\frac{\partial^{2}}{\partial z^{2}}$.

\subsection{2}

I. $\operatorname{curl} \operatorname{grad} \phi=\operatorname{curl} \nabla \phi=V \nabla \nabla \phi=0$.

2. $\operatorname{div} \operatorname{grad} \phi=\nabla \nabla \phi=\bar{\nabla}^{2} \phi=\frac{\partial^{2} \phi}{\partial x^{2}}+\frac{\partial^{2} \phi}{\partial y^{2}}+\frac{\partial^{2} \phi}{\partial z^{2}}$.

3. $\operatorname{div} \operatorname{curl} \mathbf{A}=0$.

4. $\operatorname{curl} \operatorname{curl} \mathbf{A}=\operatorname{curl}^{2} \mathbf{A}=\nabla \operatorname{div} \mathbf{A}-\overline{\mathrm{V}}^{2} \mathbf{A}$.

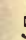
6.$$
\mathbf{A} \nabla=A_{x} \frac{\partial}{\partial x}+A_{y} \frac{\partial}{\partial y}+A_{z} \frac{\partial}{\partial z} \text {. }
$$

$$
\bar{\nabla}^{2} \mathbf{A}=\mathbf{i} \bar{\nabla}^{2} \mathbf{A}_{x}+\mathrm{j} \bar{\nabla}^{2} A_{y}+\mathbf{k} \bar{\nabla}^{2} A_{z} .
$$




\subsection{3}

I. $\nabla \mathbf{A B}=\operatorname{grad} \mathbf{A B}=(\mathbf{A} \nabla) \mathbf{B}+(\mathbf{B} \nabla) \mathbf{A}+V \cdot \mathbf{A} \operatorname{curl} \mathbf{B}+V \cdot \mathbf{B} \operatorname{curl} \mathbf{A}$.

2. $\nabla V \mathbf{A B}=\operatorname{div} V \mathbf{A B}=\mathbf{B} \operatorname{curl} \mathbf{A}-\mathbf{A} \operatorname{curl} \mathbf{B}$.

3. $V \nabla V \mathbf{A B}=(\mathbf{B} \nabla) \mathbf{A}-(\mathbf{A} \nabla) \mathbf{B}+\mathbf{A} \operatorname{div} B-\mathbf{B} \operatorname{div} \mathbf{A}$.

4. $\operatorname{div} \phi \mathbf{A}=\phi \operatorname{div} \mathbf{A}+\mathbf{A} \nabla \phi$.

5. $\operatorname{curl} \phi \mathbf{A}=V \cdot \nabla \phi \mathbf{A}+\phi \operatorname{curl} \mathbf{A}=V \cdot \operatorname{grad} \phi \cdot \mathbf{A}+\phi \operatorname{curl} \mathbf{A}$.

6. $\nabla \mathbf{A}^{2}=2(\mathbf{A} \nabla) \mathbf{A}+2 V \mathbf{A}$ curl $\mathbf{A}$.

7. $\mathbf{C}(\mathbf{A} \nabla) \mathbf{B}=\mathbf{A}(\mathbf{C} \nabla) \mathbf{B}+\mathbf{A} V \mathbf{C}$ curl $\mathbf{B}$.

8. $\quad \mathbf{B} \nabla \mathbf{A}^{2}=2 \mathbf{A}(\mathbf{B} \nabla) \mathbf{A}$.

4.24 $\mathrm{R}$ is a radius vector of length $r$ and $\mathrm{r}$ a unit vector in the direction of $\mathbf{R}$.

I.

$$
\begin{aligned}
& \mathbf{R}=r \mathbf{r}, \\
& r^{2}=x^{2}+y^{2}+z^{2} .
\end{aligned}
$$

I.

$$
\nabla \frac{\mathrm{I}}{r}=-\frac{\mathrm{I}}{r^{3}} \mathbf{R}=-\frac{\mathrm{I}}{r^{2}} \mathbf{r}
$$

2.

$$
\nabla^{2} \frac{\mathrm{I}}{r}=0
$$

3.

$$
\nabla r=\frac{\mathrm{I}}{r} \mathbf{R}=\mathbf{r}=\operatorname{grad} r .
$$

4 .

$$
\bar{\nabla}^{2} r=\frac{2}{r} \text {. }
$$

5 .

$$
V \nabla \mathbf{R}=\operatorname{curl} \mathbf{R}=0 \text {. }
$$

6.

$$
\nabla \mathbf{R}=\operatorname{div} \mathbf{R}=3 .
$$

7 .

$$
\frac{d \phi}{d r}=\mathbf{r} \nabla \phi \text {. }
$$

8.

$$
(\mathbf{R} \nabla) \mathbf{A}=r \frac{d \mathbf{A}}{d r} .
$$

9.

$$
(\mathbf{r} \nabla) \mathbf{A}=\frac{d \mathbf{A}}{d r} .
$$

Io.

$$
(\mathbf{A} \nabla) \mathbf{R}=\mathbf{A} \text {. }
$$

4.30 $d \mathrm{~S}=$ an element of area of a surface regarded as a vector whose direction is that of the positive normal to the surface. $d V=$ an element of volume - a scalar. 
$d \mathbf{s}=$ an element of arc of a curve regarded as a vector whose direction is at of the positive tangent to the curve.

4.31 Gauss's Theorem:

$$
\int \mathcal{S} \mathcal{S} \operatorname{div} \mathbf{A} d V=\int \mathcal{S} \mathbf{A} d \mathbf{S}
$$

4.32 Green's Theorem:

I. $\int \mathcal{S} \int \phi \nabla^{2} \psi d V+\int \mathcal{S} \int \nabla \phi \nabla \psi d V=\int \mathcal{S} \phi \nabla \psi d \mathbf{S}$

2. $\int \mathcal{S} \mathcal{S}\left(\phi \nabla^{2} \psi-\psi \nabla^{2} \phi\right) d V=\int \mathcal{S}(\phi \nabla \psi-\psi \nabla \phi) d \mathbf{S}$.

4.33 Stokes's Theorem:

$$
\iint \operatorname{curl} \mathbf{A} d \mathbf{S}=\int \mathbf{A} d \mathbf{S}
$$

4.40 A polar vector is one whose components, referred to a rectangular system of axes, all change in sign when the three axes are reversed.

t.401 An axial vector is one whose components are unchanged when the axes are reversed.

4.402 The vector product of two polar or of two axial vectors is an axial vector.

4.403 The vector product of a polar and an axial vector is a polar vector.

4.404 The curl of a polar vector is an axial vector and the curl of an axial vector is a polar vector.

4.405 The scalar product of two polar or of two axial vectors is a true scalar, i.e., it keeps its sign if the axes to which the vectors are referred are reversed.

4.406 The scalar product of an axial vector and a polar vector is a pseudo-scalar, i.e., it changes in sign when the axes of reference are reversed.

4.407 The product or quotient of a polar vector and a true scalar is a polar vector; of an axial vector and a true scalar an axial vector; of a polar vector and a pseudo-scalar an axial vector; of an axial vector and a pseudo-scalar a polar vector. 
4.408 The gradient of a true scalar is a polar vector; the gradient of a pseudoscalar is an axial vector.

4.409 The divergence of a polar vector is a true scalar; of an axial vector a pseudo-scalar.

\subsection{Linear Vector Functions.}

4.610 A vector $Q$ is a linear vector function of a vector $\mathbf{R}$ if its components, $Q_{1}, Q_{2}, Q_{3}$, along any three non-coplanar axes are linear functions of the components $R_{1}, R_{2}, R_{3}$ of $\mathrm{R}$ along the same axes.

4.611 Linear Vector Operator. If $\hat{\omega}$ is the linear vector operator,

$$
\mathbf{Q}=\hat{\omega} \mathbf{R} \text {. }
$$

This is equivalent to the three scalar equations,

$$
\begin{aligned}
& Q_{1}=\omega_{11} R_{1}+\omega_{12} R_{2}+\omega_{13} R_{3}, \\
& Q_{2}=\omega_{21} R_{1}+\omega_{22} R_{2}+\omega_{23} R_{3}, \\
& Q_{3}=\omega_{31} R_{1}+\omega_{32} R_{2}+\omega_{33} R_{3} .
\end{aligned}
$$

4.612 If $\mathrm{a}, \mathrm{b}, \mathrm{c}$ are the three non-coplanar unit axes,

$$
\begin{array}{lll}
\omega_{11}=S . \mathrm{a} \hat{\omega} \mathrm{a}, & \omega_{21}=S . \mathrm{b} \hat{\omega} \mathrm{a}, & \omega_{31}=S . c \hat{\omega} \mathrm{a}, \\
\omega_{12}=S . \mathrm{a} \hat{\omega} \mathrm{b}, & \omega_{22}=S . \mathrm{b} \hat{\omega} \mathrm{b}, & \omega_{32}=S . c \hat{\omega} \mathrm{b}, \\
\omega_{13}=S . \mathrm{a} \hat{\omega} \mathrm{c}, & \omega_{23}=S . \mathrm{b} \hat{\omega} \mathrm{c} & \omega_{33}=S . c \hat{\omega} \mathrm{c} .
\end{array}
$$

4.613 The conjugate linear vector operator $\hat{\omega}^{\prime}$ is obtained from $\hat{\omega}$ by replacing $\omega_{h k}$ by $\omega_{k h} ; h, k=\mathrm{I}, 2,3$.

4.614 In the symmetrical, or self-conjugate linear vector operator, denoted by $\omega$,

Hence by 4.612

$$
\omega=\frac{1}{2}\left(\hat{\omega}+\hat{\omega}^{\prime}\right) .
$$

$$
S . \mathrm{a} \omega \mathrm{b}=S . \mathrm{b} \omega \mathrm{a}, \text { etc. }
$$

4.615 The general linear vector function $\hat{\omega} \mathbf{R}$ may always be resolved into the sum of a self-conjugate linear vector function of $\mathbf{R}$ and the vector product of $\mathbf{R}$ by a vector $\mathbf{c :}$

where

$$
\begin{aligned}
\hat{\omega} \mathbf{R} & =\omega \mathbf{R}+V \cdot \mathbf{c R}, \\
\omega & =\frac{1}{2}\left(\hat{\omega}+\hat{\omega}^{\prime}\right),
\end{aligned}
$$

and

$$
\mathbf{c}=\frac{1}{2}\left(\omega_{32}-\omega_{23}\right) \mathbf{i}+\frac{1}{2}\left(\omega_{13}-\omega_{31}\right) \mathbf{j}+\frac{1}{2}\left(\omega_{21}-\omega_{12}\right) \mathbf{k}
$$

if $\mathbf{i}, \mathbf{j}, \mathbf{k}$ are three mutually perpendicular unit vectors.

4.616 The general linear vector operator $\hat{\omega}$ may be determined by three noncoplanar vectors, A, B, C, where, 
and

$$
\begin{aligned}
& \mathbf{A}=a \omega_{11}+b \omega_{12}+c \omega_{13}, \\
& \mathbf{B}=a \omega_{21}+b \omega_{22}+c \omega_{23}, \\
& \mathbf{C}=a \omega_{31}+b \omega_{32}+c \omega_{33}, \\
& \hat{\omega}=a S . \mathbf{A}+b S . \mathbf{B}+\mathbf{c} S . \mathbf{C} .
\end{aligned}
$$

4.617 If $\hat{\omega}$ is the general linear vector operator and $\hat{\omega}^{\prime}$ its conjugate,

$$
\begin{aligned}
\hat{\omega} \mathbf{R} & =\mathbf{R} \hat{\omega}^{\prime}, \\
\hat{\omega}^{\prime} \mathbf{R} & =\mathbf{R} \hat{\omega}
\end{aligned}
$$

4.620 The symmetrical or self-conjugate linear vector operator has three mutually perpendicular axes. If these be taken along $\mathbf{i}, \mathbf{j}, \mathbf{k}$,

$$
\omega=\mathbf{i} S . \omega_{1} \mathbf{i}+\mathbf{j} S \cdot \omega_{2} \mathbf{j}+\mathbf{k} S . \omega_{3} \mathbf{k},
$$

where $\omega_{1}, \omega_{2}, \omega_{3}$ are scalar quantities, the principal values of $\omega$.

4.621 Referred to any system of three mutually perpendicular unit vectors, $\mathrm{a}, \mathrm{b}, \mathrm{c}$, the self-conjugate operator, $\omega$, is determined by the three vectors (4.616):

where

$$
\begin{aligned}
& \mathbf{A}=\omega \mathbf{a}=a \omega_{11}+b \omega_{12}+\mathbf{c} \omega_{13}, \\
& \mathbf{B}=\omega \mathbf{b}=a \omega_{21}+b \omega_{22}+\mathbf{c} \omega_{23}, \\
& \mathbf{C}=\omega \mathbf{c}=a \omega_{31}+b \omega_{32}+\mathbf{c} \omega_{33}
\end{aligned}
$$

$$
\begin{aligned}
\omega_{h \cdot k} & =\omega_{k h}, \\
\omega & =a S . \mathbf{A}+\mathrm{b} S . \mathbf{B}+\mathrm{c} S . \mathbf{C} .
\end{aligned}
$$

4.622 If $n$ is one of the principal values, $\omega_{1}, \omega_{2}, \omega_{3}$, these are given by the roots of the cubic,

$n^{3}-n^{2}(S . \mathbf{A a}+S . \mathbf{B b}+S . \mathbf{C c})+n(S . \mathbf{a} V \mathbf{B C}+S . \mathbf{b} V \mathbf{C A}+\mathbf{S . c} V \mathbf{A} B)$

$$
-S \cdot \mathbf{A} V \mathbf{B C}=0 .
$$

4.623 In transforming from one to another system of rectangular axes the following are invariant:

$$
\begin{aligned}
& S . \mathbf{A a}+S . \mathbf{B b}+S . \mathbf{C C}=\omega_{1}+\omega_{2}+\omega_{3} . \\
& S \mathbf{a} V \mathbf{B C}+S . \mathbf{b} V \mathbf{C A}+S . \mathbf{c} V \mathbf{A B}=\omega_{2} \omega_{3}+\omega_{3} \omega_{1}+\omega_{1} \omega_{2} . \\
& S . \mathbf{A} V \mathbf{B C}=\omega_{1} \omega_{2} \omega_{3} .
\end{aligned}
$$

\subsection{4}

$$
\begin{aligned}
& \omega_{1}+\omega_{2}+\omega_{3}=\omega_{11}+\omega_{22}+\omega_{33} \\
& \omega_{2} \omega_{3}+\omega_{3} \omega_{1}+\omega_{1} \omega_{2}=\omega_{22} \omega_{33}+\omega_{33} \omega_{11}+\omega_{11} \omega_{22}-\omega_{23}^{2}-\omega^{2}{ }_{31}+\omega_{12}^{2} \\
& \omega_{1} \omega_{2} \omega_{3}=\omega_{11} \omega_{22} \omega_{33}+2 \omega_{23} \omega_{31} \omega_{12}-\omega_{11} \omega_{23}^{2}-\omega_{22} \omega_{31}^{2}-\omega_{33} \omega_{12}^{2}
\end{aligned}
$$

4.625 The principal axes of the self-conjugate operator, $\omega$, are those of the quadric:

$$
\omega_{11} x^{2}+\omega_{22} y^{2}+\omega_{33} z^{2}+2 \omega_{23} y z+2 \omega_{31} z x+2 \omega_{12} x y=\text { const. }
$$

where $x, y, z$ are rectangular axes in the direction of $\mathrm{a}, \mathrm{b}, \mathrm{c}$ respectively. 
4.626 Referred to its principal axes the equation of the quadric is,

$$
\omega_{1} x^{2}+\omega_{2} y^{2}+\omega_{3} z^{2}=\text { const. }
$$

4.627 Applying the self-conjugate operator, $\omega$, successively,

$$
\begin{aligned}
\omega \mathrm{R} & =\mathrm{i} \omega_{1} R_{1}+\mathrm{j} \omega_{2} R_{2}+\mathrm{k} \omega_{3} R_{3}, \\
\omega \omega \mathrm{R} & =\omega^{2} \mathrm{R}=\omega_{1}^{2} R_{1}+\mathrm{j} \omega_{2}{ }^{2} R_{2}+\mathrm{k} \omega_{3}{ }^{2} R_{3}, \\
\omega \omega^{2} \mathrm{R} & =\omega^{3} \mathrm{R}=\mathrm{i} \omega_{1}^{3} R_{1}+\mathrm{j} \omega_{2}{ }^{3} R_{2}+\mathrm{k} \omega_{3}{ }^{3} R_{3}, \\
\ldots & \\
\omega^{-1} \mathrm{R} & =\mathrm{i} \frac{R_{1}}{\omega_{1}}+\mathrm{j} \frac{R_{2}}{\omega_{2}}+\mathrm{k} \frac{R_{3}}{\omega_{3}} . \\
\therefore &
\end{aligned}
$$

4.628 Applying a number of self-conjugate operators, $\alpha, \beta, \ldots$. all with the same axes but with different principal values $\left(a_{1} a_{2} a_{3}\right),\left(\beta_{1} \beta_{2} \beta_{3}\right), \ldots \ldots$

$$
\begin{aligned}
a \mathbf{R} & =\mathrm{i} \alpha R_{1}+\mathrm{j} a_{2} R_{2}+\mathrm{k} a_{3} R_{3}, \\
\beta a \mathbf{R} & =a \beta \mathbf{R}=\mathrm{i} a_{1} \beta_{1} R_{1}+\mathrm{j} a_{2} \beta_{2} R_{2}+\mathrm{k} a_{3} \beta_{3} R_{3} .
\end{aligned}
$$

4.629

$$
\begin{aligned}
S . \mathbf{Q} \omega \mathbf{R} & =S . \mathbf{R} \omega Q \\
& =\omega_{1} Q_{1} R_{1}+\omega_{2} Q_{2} R_{2}+\omega_{3} Q_{3} R_{3} .
\end{aligned}
$$




\section{GURVILINEAR GOÖRDINATES}

5.00 Given three surfaces.

I.

$$
\begin{gathered}
\left\{\begin{array}{c}
u=f_{1}(x, y, z), \\
v=f_{2}(x, y, z), \\
w=f_{3}(x, y, z) .
\end{array}\right. \\
\left\{\begin{array}{c}
x=\phi_{1}(u, v, w), \\
y=\phi_{2}(u, v ; w), \\
z=\phi_{3}(u, v, w):
\end{array}\right. \\
\left\{\begin{array}{l}
\frac{\mathrm{I}}{h_{1}{ }^{2}}=\left(\frac{\partial \phi_{1}}{\partial u}\right)^{2}+\left(\frac{\partial \phi_{2}}{\partial u}\right)^{2}+\left(\frac{\partial \phi_{3}}{\partial u}\right)^{2}, \\
\frac{\mathbf{I}}{h_{2}{ }^{2}=}\left(\frac{\partial \phi_{1}}{\partial v}\right)^{2}+\left(\frac{\partial \phi_{2}}{\partial v}\right)^{2}+\left(\frac{\partial \phi_{3}}{\partial v}\right)^{2} \\
\frac{\mathbf{I}}{h_{3}{ }^{2}=}\left(\frac{\partial \phi_{1}}{\partial w}\right)^{2}+\left(\frac{\partial \phi_{2}}{\partial w}\right)^{2}+\left(\frac{\partial \phi_{3}}{\partial w}\right)^{2} . \\
g_{1}=\frac{\partial \phi_{1}}{\partial v} \frac{\partial \phi_{1}}{\partial w}+\frac{\partial \phi_{2}}{\partial v} \frac{\partial \phi_{2}}{\partial w}+\frac{\partial \phi_{3}}{\partial v} \frac{\partial \phi_{3}}{\partial w}, \\
g_{2}=\frac{\partial \phi_{1}}{\partial w} \frac{\partial \phi_{1}}{\partial u}+\frac{\partial \phi_{2}}{\partial w} \frac{\partial \phi_{2}}{\partial u}+\frac{\partial \phi_{3}}{\partial w} \frac{\partial \phi_{3}}{\partial u}, \\
g_{3}=\frac{\partial \phi_{1}}{\partial u} \frac{\partial \phi_{1}}{\partial v}+\frac{\partial \phi_{2}}{\partial u} \frac{\partial \phi_{2}}{\partial v}+\frac{\partial \phi_{3}}{\partial u} \frac{\partial \phi_{3}}{\partial v}
\end{array}\right.
\end{gathered}
$$

5.01 The linear element of arc, $d s$, is given by:

$$
d s^{2}=d x^{2}+d y^{2}+d z^{2}=\frac{d u^{2}}{h_{1}{ }^{2}}+\frac{d v^{2}}{h_{2}{ }^{2}}+\frac{d w^{2}}{h_{3}{ }^{2}}+2 g_{1} d v d w+2 g_{2} d w d u+2 g_{3} d u d v
$$

5.02 The surface elements, areas of parallelograms on the three surfaces, are:

$$
\begin{aligned}
& d S_{u}=\frac{d v d w}{h_{2} h_{3}} \sqrt{\mathrm{I}-h_{2}^{2} h_{3}^{2} g_{1}^{2}} \\
& d S_{v}=\frac{d w d u}{h_{3} h_{1}} \sqrt{\mathrm{I}-h_{3}^{2} h_{1}^{2} g_{2}^{2}} \\
& d S_{w}=\frac{d u d v}{h_{1} h_{2}} \sqrt{\mathrm{I}-h_{1}^{2} h_{2}^{2} g_{3}{ }^{2}}
\end{aligned}
$$


5.03 The volume of an elementary parallelepipedon is:

$$
d \tau=\frac{d u d v d w}{h_{1} h_{2} h_{3}}\left\{\mathrm{I}-h_{1}{ }^{2} h_{2}{ }^{2} g_{3}{ }^{2}-h_{2}{ }^{2} h_{3}{ }^{2} g_{1}{ }^{2}-h_{3}{ }^{2} h_{1}{ }^{2} g_{2}{ }^{2}+h_{1}{ }^{2} h_{2}{ }^{2} h_{3}{ }^{2} g_{1} g_{2} g_{3}\right\}^{\frac{1}{3}}
$$

5.04 $\omega_{1}, \omega_{2}, \omega_{3}$ are the angles between the normals to the surface $f_{2}, f_{3} ; f_{3}, f_{1}$; $f_{1}, f_{2}$ respectively:

$$
\begin{aligned}
& \cos \omega_{1}=h_{2} h_{3} g_{1}, \\
& \cos \omega_{2}=h_{3} h_{1} g_{2}, \\
& \cos \omega_{3}=h_{1} h_{2} g_{3} .
\end{aligned}
$$

5.05 Orthogonal Curvilinear Coördinates.

$$
\begin{aligned}
g_{1} & =g_{2}=g_{3}=0 \\
d s^{2} & =\frac{d u^{2}}{h_{1}^{2}}+\frac{d v^{2}}{h_{2}^{2}}+\frac{d w^{2}}{h_{3}^{2}} \\
d S_{u} & =\frac{d v d w}{h_{2} h_{3}}, d S_{v}=\frac{d w d u}{h_{3} h_{1}}, d S_{w}=\frac{d u d v}{h_{1} h_{2}} \\
d \tau & =\frac{d u d v d w}{h_{1} h_{2} h_{3}}
\end{aligned}
$$

$5.06 h_{1}^{2}, h_{2}^{2}, h_{3}^{2}$ are given by $5.00(3)$ and also by:

$$
\begin{aligned}
& h_{1}^{2}=\left(\frac{\partial f_{1}}{\partial x}\right)^{2}+\left(\frac{\partial f_{1}}{\partial y}\right)^{2}+\left(\frac{\partial f_{1}}{\partial z}\right)^{2} \\
& h_{2}^{2}=\left(\frac{\partial f_{2}}{\partial x}\right)^{2}+\left(\frac{\partial f_{2}}{\partial y}\right)^{2}+\left(\frac{\partial f_{2}}{\partial z}\right)^{2} \\
& h_{3}{ }^{2}=\left(\frac{\partial f_{3}}{\partial x}\right)^{2}+\left(\frac{\partial f_{3}}{\partial y}\right)^{2}+\left(\frac{\partial f_{3}}{\partial z}\right)^{2} .
\end{aligned}
$$


5.07 A vector, A, will have three components in the directions of the rormals to the orthogonal surfaces $u, v, w$ :

$$
A=\sqrt{A_{u}{ }^{2}+A_{v}{ }^{2}+A_{w^{2}}} \text {. }
$$

5.08

I. $\operatorname{div} \mathbf{A}=h_{1} h_{2} h_{3}\left\{\frac{\partial}{\partial u}\left(\frac{A_{u}}{h_{2} h_{3}}\right)+\frac{\partial}{\partial v}\left(\frac{A_{v}}{h_{3} h_{1}}\right)+\frac{\partial}{\partial w}\left(\frac{A_{w}}{h_{1} h_{2}}\right)\right\}$

2. $\bar{\nabla}^{2}=h_{1} h_{2} h_{3}\left\{\frac{\partial}{\partial u}\left(\frac{h_{1}}{h_{2} h_{3}} \frac{\partial}{\partial u}\right)+\frac{\partial}{\partial v}\left(\frac{h_{2}}{h_{3} h_{1}} \frac{\partial}{\partial v}\right)+\frac{\partial}{\partial w}\left(\frac{h_{3}}{h_{1} h_{2}} \frac{\partial}{\partial w}\right)\right\}$.

$3 \cdot$

$$
\left\{\begin{array}{l}
\operatorname{curl}_{u} \mathbf{A}=h_{2} h_{3}\left\{\frac{\partial}{\partial v}\left(\frac{A_{w}}{h_{3}}\right)-\frac{\partial}{\partial w}\left(\frac{A_{v}}{h_{2}}\right)\right\}, \\
\operatorname{curl}_{v} \mathbf{A}=h_{3} h_{1}\left\{\frac{\partial}{\partial w}\left(\frac{A_{u}}{h_{1}}\right)-\frac{\partial}{\partial u}\left(\frac{A_{w}}{h_{3}}\right)\right\}, \\
\operatorname{curl}_{w} \mathbf{A}=h_{1} h_{2}\left\{\frac{\partial}{\partial u}\left(\frac{A_{v}}{h_{2}}\right)-\frac{\partial}{\partial v}\left(\frac{A_{u}}{h_{1}}\right)\right\} .
\end{array}\right.
$$

5.09 The gradient of a scalar function, $\psi$, has three components in the directions of the normals to the three orthogonal surfaces:

$$
h_{1} \frac{\partial \psi}{\partial u}, h_{2} \frac{\partial \psi}{\partial v}, h_{3} \frac{\partial \psi}{\partial w} .
$$

5.20 Spherical Polar Coördinates.

I.

$$
\begin{aligned}
& \left\{\begin{array}{r}
u=r, \\
v=\theta \\
w=\phi .
\end{array}\right. \\
& \left\{\begin{array}{l}
x=r \sin \theta \cos \phi . \\
y=r \sin \theta \sin \phi, \\
z=r \cos \theta .
\end{array}\right. \\
& h_{1}=\mathrm{I}, h_{2}=\frac{\mathrm{T}}{r}, h_{3}=\frac{\mathrm{I}}{r \sin \theta} \text {. } \\
& \left\{\begin{array}{l}
d S_{r}=r^{2} \sin \theta d \theta d \phi, \\
d S_{\theta}=r \sin \theta d r d \phi, \\
d S_{\phi}=r d r d \theta .
\end{array}\right.
\end{aligned}
$$

5

6 $d \tau=r^{2} \sin \theta d r d \theta d \phi$.

6. $\operatorname{div} \mathbf{A}=\frac{\mathrm{I}}{r^{2} \sin \theta}\left\{\sin \theta \frac{\partial}{\partial r}\left(r^{2} A_{r}\right)+r \frac{\partial}{\partial \theta}\left(\sin \theta A_{\theta}\right)+r \frac{\partial A_{\phi}}{\partial \phi}\right\}$.

7. $\bar{\nabla}^{2}=\frac{I}{r^{2} \sin \theta}\left\{\sin \theta \frac{\partial}{\partial r}\left(r^{2} \frac{\partial}{\partial r}\right)+\frac{\partial}{\partial \theta}\left(\sin \theta \frac{\partial}{\partial \theta}\right)+\frac{I}{\sin \theta} \frac{\partial^{2}}{\partial \phi^{2}}\right\}$. 
8.

$$
\left\{\begin{array}{l}
\operatorname{curl}_{r} \mathbf{A}=\frac{\mathrm{I}}{r \sin \theta}\left\{\frac{\partial}{\partial \theta}\left(\sin \theta A_{\phi}\right)-\frac{\partial A_{\phi}}{\partial \phi}\right\}, \\
\operatorname{curl}_{\theta} \mathbf{A}=\frac{\mathrm{I}}{r \sin \theta}\left\{\frac{\partial A_{r}}{\partial \phi}-\sin \theta \frac{\partial\left(r A_{\phi}\right)}{\partial r}\right\}, \\
\operatorname{curl}_{\phi} \mathbf{A}=\frac{\mathrm{I}}{r}\left\{\frac{\partial}{\partial r}\left(r A_{\theta}\right)-\frac{\partial A_{r}}{\partial \theta}\right\} .
\end{array}\right.
$$

5.21 Cylindrical Coördinates.

I.

2.$$
\left\{\begin{array}{l}
u=\rho, \\
v=\theta, \\
w=z .
\end{array}\right.
$$$$
\left\{\begin{array}{l}
x=\rho \cos \theta \\
y=\rho \sin \theta, \\
z=z .
\end{array}\right.
$$

3.

$$
h_{1}=\mathrm{I}, \quad h_{2}=\frac{\mathrm{I}}{\rho}, \quad h_{3}=\mathrm{I} .
$$

4 .

$$
\left\{\begin{aligned}
d S_{r} & =\rho d \theta d z \\
d S_{\theta} & =d z d \rho \\
d S_{z} & =\rho d \rho d \theta \\
d \tau & =\rho d \rho d \theta d z .
\end{aligned}\right.
$$

5 .

6.

$$
\operatorname{div} \mathbf{A}=\frac{\mathbf{I}}{\rho}\left\{\frac{\partial}{\partial \rho}\left(\rho A_{\rho}\right)+\frac{\partial A_{\theta}}{\partial \theta}+\rho \frac{\partial A_{z}}{\partial z}\right\} .
$$

7 .

$$
\begin{aligned}
& \bar{\nabla}^{2}=\frac{\mathrm{I}}{\rho}\left\{\frac{\partial}{\partial \rho}\left(\rho \frac{\partial}{\partial \rho}\right)+\frac{\mathrm{I}}{\rho} \frac{\partial^{2}}{\partial \theta^{2}}+\rho \frac{\partial^{2}}{\partial z^{2}}\right\} \\
& \left\{\begin{array}{l}
\operatorname{curl}_{\rho} \mathbf{A}=\frac{\mathrm{I}}{\rho} \frac{\partial A_{z}}{\partial \theta}-\frac{\partial A_{\theta}}{\partial z} \\
\operatorname{curl}_{\theta} \mathbf{A}=\frac{\partial A_{\rho}}{\partial z}-\frac{\partial A_{z}}{\partial \rho} . \\
\operatorname{curl}_{z} \mathbf{A}=\frac{\mathrm{I}}{\rho}\left\{\frac{\partial}{\partial \rho}\left(\rho A_{\theta}\right)-\frac{\partial A_{\rho}}{\partial \theta}\right\}
\end{array}\right.
\end{aligned}
$$

5.22 Ellipsoidal Coördinates.

$u, v, w$ are the three roots of the equation:

I.

$$
\begin{gathered}
\frac{x^{2}}{a^{2}+\theta}+\frac{v^{2}}{b^{2}+\theta}+\frac{z^{2}}{c^{2}+\theta}=\mathrm{I} . \\
a>b>c, \quad u>v>w .
\end{gathered}
$$

$\theta=u: \quad$ Ellipsoid.

$\theta=v$ : Hyperboloid of one sheet.

$\theta=w:$ Hyperboloid of two sheets. 
2.

$$
\begin{aligned}
& \left\{\begin{array}{l}
x^{2}=\frac{\left(a^{2}+u\right)\left(a^{2}+v\right)\left(a^{2}+w\right)}{\left(a^{2}-b^{2}\right)\left(a^{2}-c^{2}\right)}, \\
y^{2}=-\frac{\left(b^{2}+u\right)\left(b^{2}+v\right)\left(b^{2}+w\right)}{\left(b^{2}-c^{2}\right)\left(a^{2}-b^{2}\right)}, \\
z^{2}=\frac{\left(c^{2}+u\right)\left(c^{2}+v\right)\left(c^{2}+w\right)}{\left(a^{2}-c^{2}\right)\left(b^{2}-c^{2}\right)} .
\end{array}\right. \\
& \left\{\begin{array}{l}
h_{1}{ }^{2}=\frac{4\left(a^{2}+u\right)\left(b^{2}+u\right)\left(c^{2}+u\right)}{(u-v)(u-w)}, \\
h_{2}{ }^{2}=\frac{4\left(a^{2}+v\right)\left(b^{2}+v\right)\left(c^{2}+v\right)}{(v-w)(v-u)}, \\
h_{3}{ }^{2}=\frac{4\left(a^{2}+w\right)\left(b^{2}+w\right)\left(c^{2}+w\right)}{(w-u)(w-v)} .
\end{array}\right.
\end{aligned}
$$

4. $\operatorname{div} \mathbf{A}=2 \frac{\sqrt{\left(a^{2}+u\right)\left(b^{2}+u\right)\left(c^{2}+u\right)}}{(u-v)(u-w)} \frac{\partial}{\partial u}\left(\sqrt{(u-v)(u-w)} A_{u}\right)$

$$
\begin{array}{r}
+2 \frac{\sqrt{\left(a^{2}+v\right)\left(b^{2}+v\right)\left(c^{2}+v\right)}}{(v-w)(u-v)} \frac{\partial}{\partial v}\left(\sqrt{(w-v)(u-v)} A_{v}\right) \\
+2 \frac{\sqrt{\left(a^{2}+w\right)\left(b^{2}+w\right)\left(c^{2}+w\right)}}{(u-w)(v-w)} \frac{\partial}{\partial w}\left(\sqrt{(u-w)(v-w)} A_{w}\right) .
\end{array}
$$

5. $\bar{\nabla}^{2}=4 \frac{\sqrt{\left(a^{2}+u\right)\left(b^{2}+u\right)\left(c^{2}+u\right)}}{(u-v)} \frac{\partial}{\partial u}\left(\sqrt{\left(a^{2}+u\right)\left(b^{2}+u\right)\left(c^{2}+u\right)} \frac{\partial}{\partial u}\right)$

$$
\begin{aligned}
& +4 \frac{\sqrt{\left(a^{2}+v\right)(2+v)\left(b c^{2}+v\right)}}{(u-v)(v-w)} \frac{\partial}{\partial v}\left(\sqrt{\left(a^{2}+v\right)\left(b^{2}+v\right)\left(c^{2}+v\right)} \frac{\partial}{\partial v}\right) \\
& +4 \frac{\sqrt{\left(a^{2}+w\right)\left(b^{2}+w\right)\left(c^{2}+w\right)}}{\partial(a-w)(v-w)}\left(\sqrt{\left(a^{2}+w\right)\left(b^{2}+w\right)\left(c^{2}+w\right)} \frac{\partial}{\delta w}\right) .
\end{aligned}
$$

6.

$$
\left\{\begin{array}{r}
\operatorname{curl}_{u} \mathbf{A}=\frac{2}{v-w}\left\{\sqrt{\frac{\left(a^{2}+v\right)\left(b^{2}+v\right)\left(c^{2}+v\right)}{u-v}} \frac{\partial}{\partial v}\left(\sqrt{w-v} A_{w}\right)\right. \\
-\sqrt{\frac{\left(a^{2}+w\right)\left(b^{2}+w\right)\left(c^{2}+w\right)}{u-w}} \frac{\partial}{\partial w}\left(\sqrt{v-w} A_{v}\right\} .
\end{array}\right.
$$

$\operatorname{curl}_{v} \mathbf{A}=\frac{2}{u-w}\left\{\sqrt{\frac{\left(a^{2}+w\right)\left(b^{2}+w\right)\left(c^{2}+w\right)}{v-w}} \frac{\partial}{\partial w}\left(\sqrt{u-w} A_{u}\right)\right.$

$$
\left.-\sqrt{\frac{\left(a^{2}+u\right)\left(b^{2}+u\right)\left(c^{2}+u\right)}{v-u}} \frac{\partial}{\partial u}\left(\sqrt{w-u} A_{w}\right)\right\}
$$

$\operatorname{curl}_{w} \mathbf{A}=\frac{2}{u-v}\left\{\sqrt{\frac{\left(a^{2}+u\right)\left(b^{2}+u\right)\left(c^{2}+u\right)}{w-u}} \frac{\partial}{\partial u}\left(\sqrt{v-u} A_{v}\right)\right.$

$$
\left.-\sqrt{\frac{\left(a^{2}+v\right)\left(b^{2}+v\right)\left(c^{2}+v\right)}{w-v}} \frac{\partial}{\partial v}\left(\sqrt{u-v} A_{u}\right)\right\} \text {. }
$$




\subsection{Conical Coördinates.}

The three orthogonal surfaces are: the spheres, I.

$$
x^{2}+y^{2}+z^{2}=u^{2} \text {, }
$$

the two cones:

2.

3.

$$
\begin{gathered}
\frac{x^{2}}{v^{2}}+\frac{y^{2}}{v^{2}-b^{2}}+\frac{z^{2}}{v^{2}-c^{2}}=0 . \\
\frac{x^{2}}{w^{2}}+\frac{y^{2}}{w^{2}-b^{2}}+\frac{z^{2}}{w^{2}-c^{2}}=0 . \\
c^{2}>v^{2}>b^{2}>w^{2} \\
\left\{\begin{array}{l}
x^{2}=\frac{u^{2} v^{2} w^{2}}{b^{2} c^{2}} \\
y^{2}=\frac{u^{2}\left(v^{2}-b^{2}\right)\left(w^{2}-b^{2}\right)}{b^{2}\left(b^{2}-c^{2}\right)} \\
z^{2}=\frac{u^{2}\left(v^{2}-c^{2}\right)\left(w^{2}-c^{2}\right)}{c^{2}\left(c^{2}-b^{2}\right)}
\end{array}\right.
\end{gathered}
$$

4.

5 .

$$
h_{1}=\mathrm{I}, \quad h_{2}^{2}=\frac{\left(v^{2}-b^{2}\right)\left(c^{2}-v^{2}\right)}{u^{2}\left(v^{2}-w^{2}\right)}, \quad h_{3}^{2}=\frac{\left(b^{2}-w^{2}\right)\left(c^{2}-w^{2}\right)}{u^{2}\left(v^{2}-w^{2}\right)} .
$$

6. $\operatorname{div} \mathbf{A}=\frac{\mathbf{I}}{u^{2}} \frac{\partial}{\partial u}\left(u^{2} A_{u}\right)+\frac{\sqrt{\left(v^{2}-b^{2}\right)\left(c^{2}-v^{2}\right)}}{u\left(v^{2}-w^{2}\right)} \frac{\partial}{\partial v}\left(\sqrt{v^{2}-w^{2}} A_{v}\right.$

$$
+\frac{\sqrt{\left(b^{2}-w^{2}\right)\left(c^{2}-w^{2}\right)}}{u\left(v^{2}-w^{2}\right)} \frac{\partial}{\partial w}\left(\sqrt{v^{2}-w^{2}} A_{w}\right) .
$$

7. $\bar{\nabla}^{2}=\frac{\mathbf{I}}{u^{2}} \frac{\partial}{\partial u}\left(u^{2} \frac{\partial}{\partial u}\right)+\frac{\sqrt{\left(v^{2}-b^{2}\right)\left(c^{2}-v^{2}\right)}}{u^{2}\left(v^{2}-w^{2}\right)} \frac{\partial}{\partial v}\left(\sqrt{\left(v^{2}-b^{2}\right)\left(c^{2}-v^{2}\right)} \frac{\partial}{\partial v}\right)$.

$$
+\frac{\sqrt{\left(b^{2}-w^{2}\right)\left(c^{2}-w^{2}\right)}}{u^{2}\left(v^{2}-w^{2}\right)} \frac{\partial}{\partial w}\left(\sqrt{\left(b^{2}-w^{2}\right)\left(c^{2}-w^{2}\right)} \frac{\partial}{\partial w}\right) .
$$

8.

$\int \operatorname{curl}_{u} \mathbf{A}=\frac{\mathbf{I}}{u\left(v^{2}-w^{2}\right)}\left\{\sqrt{\left(v^{2}-b^{2}\right)\left(c^{2}-v^{2}\right)} \frac{\partial}{\partial v}\left(\sqrt{v^{2}-w^{2}} A_{w}\right)\right.$

$$
\left.-\sqrt{\left(b^{2}-w^{2}\right)\left(c^{2}-w^{2}\right)} \frac{\partial}{\partial w}\left(\sqrt{v^{2}-w^{2}} A_{v}\right)\right\}
$$

$\left.\operatorname{curl}_{v} \mathbf{A}=\frac{\sqrt{\left(b^{2}-w^{2}\right)\left(c^{2}-w^{2}\right)}}{u \sqrt{v^{2}-w^{2}}} \frac{\partial A_{u}}{\partial w}-\frac{\mathrm{I}}{u} \frac{\partial}{\partial u}\left(u A_{w}\right)\right\}$,

$\operatorname{curl}_{w} \mathbf{A}=\frac{\mathbf{I}}{u} \frac{\partial}{\partial u}\left(u A_{v}\right)-\frac{\sqrt{\left(v^{2}-b^{2}\right)\left(c^{2}-v^{2}\right)}}{u \sqrt{v^{2}-w^{2}}} \frac{\partial A_{u}}{\partial v} ;$

5.30 Elliptic Cylinder Coördinates.

The three orthogonal surfaces are:

I. The elliptic cylinders:

$$
\frac{x^{2}}{c^{2} u^{2}}+\frac{y^{2}}{c^{2}\left(u^{2}-\mathrm{I}\right)}=\mathrm{I} \text {. }
$$





2. The hyperbolic cylinders:

$$
\frac{x^{2}}{c^{2} v^{2}}-\frac{y^{2}}{c^{2}\left(\mathrm{I}-v^{2}\right)}=\mathrm{I} .
$$

3. The planes:

$$
z=w \text {. }
$$

$2 c$ is the distance between the foci of the confocal ellipses and hyperbolas:

4.

5 .

6.

$$
\begin{aligned}
x & =c u v . \\
y & =c \sqrt{u^{2}-\mathrm{I}} \sqrt{\mathrm{I}-v^{2}} . \\
\frac{\mathrm{I}}{h_{1}^{2}} & =\frac{\mathrm{I}}{h_{2}^{2}}=c^{2}\left(u^{2}-v^{2}\right), \quad h_{3}=\mathrm{I} .
\end{aligned}
$$

7. $\operatorname{div} \mathbf{A}=\frac{\mathrm{I}}{c\left(u^{2}-v^{2}\right)}\left\{\frac{\partial}{\partial u}\left(\sqrt{u^{2}-v^{2}} A_{u}\right)+\frac{\partial}{\partial v}\left(\sqrt{u^{2}-v^{2}} A_{v}\right)\right\}+\frac{\partial A_{z}}{\partial z}$.

8. $\quad \bar{\nabla}^{2}=\frac{\mathrm{I}}{c^{2}\left(u^{2}-v^{2}\right)}\left(\frac{\partial^{2}}{\partial u^{2}}+\frac{\partial^{2}}{\partial v^{2}}\right)+\frac{\partial^{2}}{\partial z^{2}}$.

9. $\left\{\begin{array}{l}\operatorname{curl}_{u} \mathbf{A}=\frac{\mathrm{I}}{c \sqrt{u^{2}-v^{2}}} \frac{\partial A_{z}}{\partial v}-\frac{\partial A_{v}}{\partial z}, \\ \operatorname{curl}_{v} \mathbf{A}=\frac{\partial A_{u}}{\partial z}-\frac{\mathrm{I}}{c \sqrt{u^{2}-v^{2}}} \frac{\partial A_{z}}{\partial u} \\ \operatorname{curl}_{z} \mathbf{A}=\frac{\mathrm{I}}{c\left(u^{2}-v^{2}\right)}\left\{\frac{\partial}{\partial u}\left(\sqrt{u^{2}-v^{2}} A_{v}\right)-\frac{\partial}{\partial v}\left(\sqrt{u^{2}-v^{2}} A_{u}\right)\right\} .\end{array}\right.$

5.31 Parabolic Cylinder Coördinates.

The three orthogonal surfaces are the two parabolic cylinders:

x.

2.

And the planes:

3 .

4.

5 .

6.

7. $\operatorname{div} \mathbf{A}=\frac{\sqrt{u v}}{u+v}\left\{\frac{\partial}{\partial u}\left(\sqrt{\frac{u+v}{v}} A_{u}\right)+\frac{\partial}{\partial v}\left(\sqrt{\frac{u+v}{u}} A_{v}\right)\right\}+\frac{\partial A_{z}}{\partial z}$.

8. $\bar{\nabla}^{2}=\frac{\sqrt{u v}}{u+v}\left\{\frac{\partial}{\partial u}\left(\frac{u}{v} \frac{\partial}{\partial u}\right)+\frac{\partial}{\partial v}\left(\frac{v}{u} \frac{\partial}{\partial v}\right)\right\}+\frac{\partial^{2}}{\partial z^{2}}$.

$$
\begin{aligned}
& y^{2}=4 c u x+4 c^{2} u^{2} . \\
& y^{2}=-4 c v x+4 c^{2} v^{2} .
\end{aligned}
$$

$$
\begin{aligned}
& z=w . \\
& x=c(v-u) . \\
& y=2 c \sqrt{u v} .
\end{aligned}
$$

$$
\frac{\mathrm{I}}{h_{1}^{2}}=\frac{u+v}{u}, \frac{\mathrm{I}}{h_{2}^{2}}=\frac{u+v}{v}, \quad h_{3}=\mathrm{I} .
$$


9. $\left\{\begin{array}{l}\operatorname{curl}_{u} \mathrm{~A}=\sqrt{\frac{v}{u+v}} \frac{\partial A_{z}}{\partial v}-\frac{v}{u+v} \frac{\partial A_{v}}{\partial z}, \\ \operatorname{curl}_{v} \mathrm{~A}=\frac{u}{u+v} \frac{\partial A_{u}}{\partial z}-\sqrt{\frac{u}{u+v}} \frac{\partial A_{z}}{\partial u}, \\ \operatorname{curl}_{z} \mathrm{~A}=\frac{\sqrt{u v}}{u+v}\left\{\frac{\partial}{\partial u}\left(\sqrt{\frac{v}{u+v}} A_{v}\right)-\frac{\partial}{\partial v}\left(\sqrt{\frac{u}{u+v}} A_{u}\right)\right\} .\end{array}\right.$

5.40 Helical Coördinates. (Nicholson, Phil. Mag. 19, 77, r910.)

A cylinder of any cross-section is wound on a circular cylinder in the form of a helix of angle $\alpha$. $a$ = radius of circular cylinder on which the central line of the normal cross-sections of the helical cylinder lies. The z-axis is along the axis of the cylinder of radius $a$.

$u=\rho$ and $v=\phi$ are the polar coördinates in the plane of any normal section of the helical cylinder. $\phi$ is measured from a line perpendicular to $z$ and to the tangent to the cylinder.

$w=\theta=$ the twist in a plane perpendicular to $z$ of the radius in that plane measured from a line parallel to the $x$-axis:

I.

$$
\left\{\begin{array}{l}
x=(a+\rho \cos \phi) \cos \theta+\rho \sin \alpha \sin \theta \sin \phi, \\
y=(a+\rho \cos \phi) \sin \theta-\rho \sin \alpha \cos \theta \sin \phi, \\
z=a \theta \tan \alpha+\rho \cos \alpha \sin \phi .
\end{array}\right.
$$

2.

$$
\left\{\begin{array}{l}
h_{1}=\mathrm{I}, \quad h_{2}=\frac{\mathrm{I}}{\rho}, \\
h_{3}^{2}=\frac{\mathrm{I}}{a^{2} \sec ^{2} \alpha+2 a \rho \cos \phi+\rho^{2}\left(\cos ^{2} \phi+\sin ^{2} \alpha \sin ^{2} \phi\right)} .
\end{array}\right.
$$

5.50 Surfaces of Revolution.

$z$-axis $=$ axis of revolution.

$\rho, \theta=$ polar coördinates in any plane perpendicular to z-axis.

I.

$$
\begin{aligned}
d s^{2} & =d z^{2}+d \rho^{2}+\rho^{2} d \theta^{2} \\
& =\frac{d u^{2}}{h_{1}{ }^{2}}+\frac{d v^{2}}{h_{2}{ }^{2}}+\frac{d w^{2}}{h_{3}{ }^{2}} .
\end{aligned}
$$

In any meridian plane, $z, \rho$, determine $u, v$, from:

2.

$$
\begin{aligned}
f(z+i \rho) & =u+i v . \\
w & =\theta .
\end{aligned}
$$

3.

Then $u, v, \theta$ will form a system of orthogonal curvilinear coördinates. 
5.51 Spheroidal Coördinates (Prolate Spheroids):

I.

$$
\begin{gathered}
z+i \rho=c \cosh (u+i v) . \\
\left\{\begin{array}{l}
z=c \cosh u \cos v \\
\rho=c \sinh u \sin v .
\end{array}\right.
\end{gathered}
$$

The three orthogonal surfaces are the ellipsoids and hyperboloids of revolution, and the planes, $\theta$ :

3 .

$$
\left\{\begin{array}{l}
\frac{z^{2}}{c^{2} \cosh ^{2} u}+\frac{\rho^{2}}{c^{2} \sinh ^{2} u}=\mathrm{I} \\
\frac{z^{2}}{c^{2} \cos ^{2} v}-\frac{\rho^{2}}{c^{2} \sin ^{2} v}=\mathrm{I}
\end{array}\right.
$$

With $\cos u=\lambda, \cos v=\mu$ :

4 .

$$
\left\{\begin{array}{l}
z=c \lambda \mu \\
\rho=c \sqrt{\left(\lambda^{2}-\mathrm{I}\right)\left(\mathrm{I}-\mu^{2}\right)} .
\end{array}\right.
$$

5. $\quad h_{1}^{2}=\frac{\lambda^{2}-I}{c^{2}\left(\lambda^{2}-\mu^{2}\right)}, \quad h_{2}^{2}=\frac{I-\mu^{2}}{c^{2}\left(\lambda^{2}-\mu^{2}\right)}, \quad h_{3}^{2}=\frac{I}{c^{2}\left(\lambda^{2}-I\right)\left(I-\mu^{2}\right)}$.

5.52 Spheroidal Coördinates (Oblate Spheroids):

I.

2.

3.

4. $\quad h_{1}^{2}=\frac{I-\mu^{2}}{c^{2}\left(\lambda^{2}-\mu^{2}\right)}, \quad h_{2}^{2}=\frac{\lambda^{2}-I}{c^{2}\left(\lambda^{2}-\mu^{2}\right)}, \quad h_{3}^{2}=\frac{I}{c^{2}\left(\lambda^{2}-I\right)\left(I-\mu^{2}\right)}$.$$
\cosh u=\lambda, \quad \cos v=\mu \text {. }
$$

$$
\begin{aligned}
\rho+i z & =c \cosh (u+i v) . \\
z & =c \sinh u \sin v . \\
\rho & =c \cosh u \cos v .
\end{aligned}
$$

\subsection{Parabolic Coördinates:}

I.

$$
\begin{gathered}
z+i \rho=c(u+i v)^{2} . \\
\left\{\begin{array}{l}
z=c\left(u^{2}-v^{2}\right), \\
\rho=2 c u v .
\end{array}\right.
\end{gathered}
$$$$
u^{2}=\lambda, \quad v^{2}=\mu .
$$

3.

With curvilinear coördinates, $\lambda, \mu, \theta$ : 
4 .

$$
h_{1}=\frac{I}{c} \sqrt{\frac{\lambda}{\lambda+\mu}}, \quad h_{2}=\frac{I}{c} \sqrt{\frac{\mu}{\lambda+\mu}}, \quad h_{3}=\frac{I}{2 c \sqrt{\lambda \mu}} .
$$

\subsection{Toroidal Coördinates:}

I.

$$
\begin{aligned}
u+i v & =\log \frac{z+a+i \rho}{z-a+i \rho}, \\
\rho & =\frac{a \sinh u}{\cosh u-\cos v} . \\
z & =\frac{a \sin v}{\cosh u-\cos v} .
\end{aligned}
$$

2.

$3 \cdot$

$$
h_{1}=h_{2}=\frac{\cosh u-\cos v}{a}, \quad h_{3}=\frac{\cosh u-\cos v}{a \sinh u} .
$$

The three orthogonal surfaces are:

(a) Anchor rings, whose axial circles have radii,

$$
a \text { coth } u \text {, }
$$

and whose cross-sections are circles of radii,

$$
a \operatorname{csch} u \text {; }
$$

(b) Spheres, whose centers are on the axis of revolution at distances,

$$
\pm a \cot v
$$

from the origin, whose radii are,

$$
a \csc v \text {, }
$$

and which accordingly have a common circle,

$$
\rho=a, z=0
$$

(c) Planes through the axis,

$$
w=\theta=\text { const. }
$$




\section{INFINITE · SERIES}

6.00 An infinite series:

$$
\sum_{n=1}^{\infty} u_{n}=u_{1}+u_{2}+u_{3}+\ldots
$$

is absolutely convergent if the series formed of the moduli of its terms:

is convergent.

$$
\left|u_{1}\right|+\left|u_{2}\right|+\left|u_{2}\right|+\ldots .
$$

A series which is convergent, but whose moduli do not form a convergent series, is conditionally convergent.

\section{TESTS FOR CONVERGENCE}

6.011 Comparison test. The series $\Sigma u_{n}$ is absolutely convergent if $\left|u_{n}\right|$ is less than $C\left|v_{n}\right|$ where $C$ is a number independent of $n$, and $v_{n}$ is the $n$th term of another series which is known to be absolutely convergent.

6.012 Cauchy's test. If

$$
\operatorname{Limit}_{n \rightarrow \infty}\left|u_{n}\right|^{\frac{1}{n}}<\mathrm{I}
$$

the series $\Sigma u_{n}$ is absolutely convergent.

6.013 D'Alembert's test. If for all values of $n$ greater than some fixed value, $r$, the ratio $\left|\frac{u_{n+1}}{u_{n}}\right|$ is less than $\rho$, where $\rho$ is a positive number less than unity and independent of $n$, the series $\Sigma u_{n}$ is absolutely convergent.

6.014 Cauchy's integral test. Let $f(x)$ be a steadily decreasing positive function such that,

$$
f(n) \geqslant a_{n} \text {. }
$$

Then the positive term series $\Sigma a_{n}$ is convergent if, .

is convergent.

$$
\int_{m}^{\infty} f(x) d x
$$

6.015 Raabe's test. The positive term series $\Sigma a_{n}$ is convergent if,

It is divergent if,

$$
n\left(\frac{a_{n}}{a_{n+1}}-\mathrm{I}\right) \geqslant l \quad \text { where } l>\mathrm{I} \text {. }
$$

$$
n\left(\frac{a_{n}}{a_{n+1}}-\mathrm{I}\right) \leqslant \mathrm{I} .
$$


6.020 Alternating series. A series of real terms, alternately positive and negative, is convergent if $a_{n+1} \leqslant a_{n}$ and

$$
\operatorname{limit}_{n \rightarrow \infty} a_{n}=0 \text {. }
$$

In such a series the sum of the first $s$ terms differs from the sum of the series by a quantity less than the numerical value of the $(s+\mathrm{I}) s t$ term.

6.025 If $\underset{n \rightarrow \infty}{\operatorname{limit}}\left|\frac{u_{n+1}}{u_{n}}\right|=$ I, the series $\Sigma u_{n}$ will be absolutely convergent if there is a positive number $c$, independent of $n$, such that,

$$
\operatorname{limit}_{n \rightarrow \infty} n\left\{\left|\frac{u_{n+1}}{u_{n}}\right|-\mathrm{I}\right\}=-\mathrm{I}-c \text {. }
$$

6.030 The sum of an absolutely convergent series is not affected by changing the order in which the terms occur.

6.031 Two absolutely convergent series,

$$
\begin{aligned}
& S=u_{1}+u_{2}+u_{3}+\ldots \ldots \\
& T=v_{1}+v_{2}+v_{3}+\ldots \ldots
\end{aligned}
$$

may be multiplied together, and the sum of the products of their terms, written in any order, is $S T$,

$$
S T=u_{1} v_{1}+u_{2} v_{1}+u_{1} v_{2}+\ldots \ldots
$$

6.032 An absolutely convergent power series may be differentiated or integrated term by term and the resulting series will be absolutely convergent and equal to the differential or integral of the sum of the given series.

6.040 Uniform Convergence. An infinite series of functions of $x$,

$$
S(x)=u_{1}(x)+u_{2}(x)+u_{3}(x)+\ldots \ldots .
$$

is uniformly convergent within a certain region of the variable $x$ if a finite number, $N$, can be found such that for all values of $n \geqslant N$ the absolute value of the remainder, $\left|R_{n}\right|$ after $n$ terms is less than an assigned arbitrary small quantity $e$ at all points within the given range.

Example. The series,

$$
\sum_{n=0}^{\infty} \frac{x^{2}}{\left(1+x^{2}\right)^{n}},
$$

is absolutely convergent for all real values of $x$. Its sum is $\mathrm{I}+x^{2}$ if $x$ is not zero. If $x$ is zero the sum is zero. The series is non-uniformly convergent in the neighborhood of $x=0$. 
6.041 A uniformly convergent series is not necessarily absolutely convergent, nor is an absolutely convergent series necessarily uniformly convergent.

6.042 A sufficient, though not necessary, test for uniform convergence is as follows:

If for all values of $x$ within a certain region the moduli of the terms of the series,

$$
S=u_{1}(x)+u_{2}(x)+\ldots . .
$$

are less than the corresponding terms of a convergent series of positive terms,

$$
T=M_{1}+M_{2}+M_{3}+\ldots .
$$

where $M_{n}$ is independent of $x$, then the series $S$ is uniformly convergent in the given region.

6.043 A power series is uniformly convergent at all points within its circle of convergence.

6.044 A uniformly convergent series,

$$
S=u_{1}(x)+u_{2}(x)+\ldots . .
$$

may be integrated term by term, and,

$$
\int S d x=\sum_{n=1}^{\infty} \int u_{n}(x) d x
$$

6.045 A uniformly convergent series,

$$
S=u_{1}(x)+u_{2}(x)+\ldots .
$$

may be differentiated term by term, and if the resulting series is uniformly convergent,

$$
\frac{d}{d x} S=\sum_{n=1}^{\infty} \frac{d}{d x} u_{n}(x) .
$$

6.100 Taylor's theorem.

$$
f(x+h)=f(x)+\frac{h}{\mathrm{I} !} f^{\prime}(x)+\frac{h^{2}}{2 !} f^{\prime \prime}(x)+\ldots .+\frac{h^{n}}{n !} f^{(n)}(x)+R_{n}
$$

6.101 Lagrange's form for the remainder:

$$
R_{n}=f^{(n+1)}(x+\theta h) \cdot \frac{h^{n+1}}{(n+\mathrm{I}) !} ; 0<\theta<\mathrm{I} .
$$

6.102 Cauchy's form for the remainder:

$$
R_{n}=f^{(n+\mathrm{I})}(x+\theta h) \frac{h^{n+1}(\mathrm{r}-\theta)^{n}}{n !} ; 0<\theta<\mathrm{I} .
$$


6.103

$$
\begin{gathered}
f(x)=f(h)+f^{\prime}(h) \cdot \frac{x-h}{\mathrm{I} !}+f^{\prime \prime}(h) \cdot \frac{(x-h)^{2}}{2 !}+\ldots+f^{(n)}(h) \frac{(x-h)^{n}}{n !}+R_{n} \\
R_{n}=f^{(n+\mathrm{I})}\{h+\theta(x-h)\} \frac{(x-h)^{n+1}}{(n+\mathrm{I}) !} \quad 0<\theta<\mathrm{I} .
\end{gathered}
$$

6.104 Maclaurin's theorem:

$$
\begin{aligned}
f(x)=f(\mathrm{o})+f^{\prime}(\mathrm{o}) & \frac{x}{\mathrm{I} !}+f^{\prime \prime}(\mathrm{o}) \frac{x^{2}}{2 !}+\ldots+f^{(n)}(\mathrm{o}) \frac{x^{n}}{n !}+R_{n} \\
& R_{n}=f^{(n+\mathrm{I})}(\theta x) \frac{x^{n+1}}{(n+\mathrm{I}) !}(\mathrm{I}-\theta)^{n} ; 0<\theta<\mathrm{I} .
\end{aligned}
$$

6.105 Lagrange's theorem. Given:

$$
y=z+x \phi(y) \text {. }
$$

The expansion of $f(y)$ in powers of $x$ is:

$$
\begin{aligned}
f(y)=f(z)+x \phi(z) f^{\prime}(z)+\frac{x^{2}}{2 !} \frac{d}{d z}\left[\{\phi(z)\}^{2} f^{\prime}(z)\right] & \\
& +\ldots \ldots+\frac{x^{n}}{n !} \frac{d^{n-1}}{d z^{n-1}}\left[\{\phi(z)\}^{n} f^{\prime}(z)\right]+\ldots
\end{aligned}
$$

\section{SYMBOLIC REPRESENTATION OF INFINITE SERIES}

6.150 The infinite series:

may be written:

$$
f(x)=\mathbf{I}+a_{1} x+\frac{\mathbf{I}}{2 !} a_{2} x^{2}+\frac{\mathbf{I}}{3 !} a_{3} x^{3}+\ldots \ldots+\frac{\mathbf{I}}{k !} a_{k} x^{k}+\ldots \ldots
$$

$$
f(x)=e^{a x},
$$

where $a^{k}$ is interpreted as equivalent to $a_{k}$.

6.151 The infinite series, written without factorials,

$$
f(x)=\mathrm{I}+a_{1} x+a_{2} x^{2}+\ldots \ldots+a_{k} x^{k}+\ldots \ldots
$$

may be written:

$$
f(x)=\frac{1}{\mathrm{I}-a x},
$$

where $a^{k}$ is interpreted as equivalent to $a_{k}$.

6.152 Symbolic form of Taylor's theorem:

$$
f(x+h)=e^{h \frac{\partial}{\partial x}} f(x) .
$$

6.153 Taylor's theorem for functions of many variables:

$$
\begin{aligned}
& f\left(x_{1}+h_{1}, x_{2}+h_{2}, \ldots .\right)=e^{h_{1} \frac{\partial}{\partial x_{1}}+h_{2} \frac{\partial}{\partial x_{2}}+\ldots} \\
& =f\left(x_{1}, x_{2}, \ldots\right)+h_{1} \frac{\partial f}{\partial x_{1}}+h_{2} \frac{\partial f}{\partial x_{2}}+\ldots \\
& \left.+\frac{h_{1}^{2}}{2 !} \frac{\partial^{2} f}{\partial x_{1}^{2}}+\frac{2}{2 !} h_{1} h_{2} \frac{\partial^{2} f}{\partial x_{1} \partial x_{2}}+\frac{h_{2}^{2}}{2 !} \frac{\partial^{2} f}{\partial x_{2}^{2}}+\ldots\right) \\
& +\ldots
\end{aligned}
$$


TRANSFORMATION OF INFINITE SERIES

Series which converge slowly may often be transformed to more rapidly converging series by the following methods.

6.20 Euler's transformation formula:

where:

$$
\begin{aligned}
S & =a_{0}+a_{1} x+a_{2} x^{2}+\ldots \ldots \ldots \\
& =\frac{\mathrm{I}}{\mathrm{I}-x} a_{0}+\frac{\mathrm{I}}{\mathrm{I}-x} \sum_{k=\mathrm{I}}^{\infty}\left(\frac{x}{\mathrm{I}-x}\right)^{k} \Delta^{k} a_{0}, \\
\Delta a_{0} & =a_{1}-a_{0},
\end{aligned}
$$$$
\Delta^{2} a_{0}=\Delta a_{1}-\Delta a_{0}=a_{2}-2 a_{1}+a_{0},
$$$$
\Delta^{3} a_{0}=\Delta^{2} a_{1}-\Delta^{2} a_{0}=a_{3}-3 a_{2}+3 a_{1}-a_{0},
$$

$$
\Delta^{k} a_{n}=\sum_{m=0}^{k}(-\mathrm{I})^{m}\left(\begin{array}{l}
k \\
m
\end{array}\right) a_{k+n-m} .
$$

The second series may converge more rapidly than the first.

Example $\mathbf{~}$.

$$
\begin{aligned}
& S=\sum_{k=0}^{\infty}(-\mathrm{I})^{k} \frac{\mathrm{I}}{2 k+\mathrm{I}} \\
& x=-\mathrm{I}, \quad a_{k}=\frac{\mathrm{I}}{2 k+\mathrm{I}}, \\
& S=\frac{\mathrm{I}}{2} \sum_{k=0}^{\infty} \frac{k !}{\mathrm{I} \cdot 3 \cdot 5 \cdots(2 k+\mathrm{I})} .
\end{aligned}
$$

Example 2.

$$
\begin{aligned}
& S=\sum_{k=0}^{\infty}(-\mathrm{I})^{k} \frac{\mathrm{I}}{k+\mathrm{I}}=\log 2, \\
& x=-\mathrm{I}, \quad a_{k}=\frac{\mathrm{I}}{k+\mathrm{I}} . \\
& S=\sum_{k=\mathrm{I}}^{\infty} \frac{\mathrm{I}}{k 2^{k}}
\end{aligned}
$$

6.21 Markoff's transformation formula. (Differenzenrechnung, p. I80.)

$$
\sum_{k=0}^{n} a_{k} x^{k}-\left(\frac{x}{\mathrm{I}-x}\right)^{m} \sum_{k=0}^{n} x^{k} \Delta^{m} a_{k}=\sum_{k=0}^{m} \frac{x^{k}}{(\mathrm{I}-x)^{k+1}} \Delta^{k} a_{0}-\sum_{k=0}^{m} \frac{x^{k+n}}{(\mathrm{I}-x)^{k+1}} \Delta^{k} a_{n} \text {. }
$$


6.22 Kummer's transformation.

$A_{0}, A_{1}, A_{2}, \ldots$ is a sequence of positive numbers such that

and

$$
\lambda_{m}=A_{m}-A_{m+1} \frac{a_{m+1}}{a_{m}}
$$

$$
\operatorname{Limit}_{m \rightarrow \infty} \lambda_{m},
$$

approaches a definite positive value. Usually this limit can be taken as unity. If not, it is only necessary to divide $A_{m}$ by this limit:

Then:

$$
\alpha=\operatorname{Limit}_{m \rightarrow \infty} A_{m} a_{m} .
$$

Example I.

$$
\sum_{m=n}^{\infty} a_{m}=\left(A_{n} a_{n}-\alpha\right)+\sum_{m=n}^{\infty}\left(\mathrm{r}-\lambda_{m}\right) a_{m}
$$

$$
\begin{aligned}
S & =\sum_{m=\mathrm{I}}^{\infty} \frac{\mathrm{I}}{m^{2}}, \\
A_{m} & =m, \quad \lambda_{m}=\frac{m}{m+\mathrm{I}}, \quad \begin{array}{l}
\text { Limit } \\
m \rightarrow \infty
\end{array} \lambda_{m}=\mathrm{I}, \\
\alpha & =0 \\
\sum_{m=\mathrm{I}}^{\infty} \frac{\mathrm{I}}{m^{2}} & =\mathrm{I}+\sum_{m=1}^{\infty} \frac{\mathrm{I}}{(m+\mathrm{I}) m^{2}} .
\end{aligned}
$$

Applying the transformation to the series on the right:

$$
\begin{gathered}
A_{m}=\frac{m}{2}, \quad \lambda_{m}=\frac{m}{m+2}, \quad \alpha=0 \\
\sum_{m=\mathrm{I}}^{\infty} \frac{\mathrm{I}}{m^{2}}=\mathrm{I}+\frac{\mathrm{I}}{2^{2}}+2 \sum_{m=\mathrm{x}}^{\infty} \frac{\mathrm{I}}{m^{2}(m+\mathrm{I})(m+2)} .
\end{gathered}
$$

Applying the transformation $n$ times:

$$
\sum_{m=n+1}^{\infty} \frac{\mathrm{I}}{m^{2}}=n ! \sum_{m=1}^{\infty} \frac{\mathrm{I}}{m^{2}(m+\mathrm{I})(m+2) \ldots(m+n)}
$$

Example 2.

$$
\begin{aligned}
S & =\sum_{m=1}^{\infty}(-\mathrm{I})^{m-1} \frac{\mathrm{I}}{2 m-\mathrm{I}} \\
A_{m} & =\frac{\mathrm{I}}{2}, \quad \lambda_{m}=\frac{2 m}{2 m+\mathrm{I}}, \quad \alpha=0 \\
S & =\frac{\mathrm{I}}{2}+\sum_{m=\mathrm{I}}^{\infty}(-\mathrm{I})^{m-1} \frac{\mathrm{I}}{4 m^{2}-\mathrm{I}}
\end{aligned}
$$


Applying the transformation again, with:

$$
\begin{aligned}
& A_{m}=\frac{\mathrm{I}}{2} \frac{2 m+\mathrm{I}}{2 m-\mathrm{I}}, \quad \lambda_{m}=\frac{4 m^{2}+\mathrm{I}}{4 m^{2}-\mathrm{I}}, \quad \alpha=0, \\
& S=\mathrm{I}-2 \sum_{m=\mathrm{I}}^{\infty}(-\mathrm{I})^{m-1} \frac{\mathrm{I}}{\left(4 m^{2}-\mathrm{I}\right)^{2}} .
\end{aligned}
$$

Applying the transformation again, with:

$$
\begin{aligned}
A_{m} & =\frac{\mathrm{I}}{2} \frac{2 m+\mathrm{I}}{2 m-3}, \quad \lambda_{m}=\frac{4 m^{2}+3}{4 m^{2}-9}, \quad \alpha=0 \\
S & =\frac{4}{3}+24 \sum_{n=\mathrm{I}}^{\infty}(-\mathrm{I})^{m-1} \frac{\mathrm{I}}{\left(4 m^{2}-\mathrm{I}\right)^{2}\left(4 m^{2}-9\right)} .
\end{aligned}
$$

Example 3 .

$$
\begin{gathered}
S=\sum_{m=\mathrm{I}}^{\infty}(-\mathrm{I})^{m-1} \frac{\mathrm{I}}{(2 m-\mathrm{I})^{2}}, \\
A_{m}=\frac{2 m-\mathrm{I}}{2(2 m-3)}, \quad \lambda_{m}=\frac{4 m^{2}-4 m+\mathrm{I}}{(2 m-3)(2 m+\mathrm{I})}, \quad \alpha=0, \\
S=\frac{5}{6}+4 \sum_{m=\mathrm{I}}^{\infty}(-\mathrm{I})^{m-1} \frac{\mathrm{I}}{(2 m-\mathrm{I})(2 m+3)(2 m+\mathrm{I})^{2}} .
\end{gathered}
$$

6.23 Leclert's modification of Kummer's transformation. With the same notation as in 6.22 and,

$$
\begin{gathered}
\operatorname{Limit}_{m \rightarrow \infty} \lambda_{m}=\omega \\
\sum_{n=0}^{\infty} a_{n}=a_{0}+\frac{A_{1} a_{1}}{\lambda_{1}}-\frac{\alpha}{\omega}+\sum_{m=1}^{\infty}\left(\frac{\mathrm{I}}{\lambda_{m+1}}-\frac{\mathrm{I}}{\lambda_{m}}\right) A_{m+1} a_{m+1} .
\end{gathered}
$$

Example I.

$$
\begin{gathered}
S=\sum_{n=\mathrm{I}}^{\infty}(-\mathrm{I})^{n-1} \frac{\mathrm{I}}{2 n-\mathrm{I}} \\
a_{0}=0, \quad A_{m}=\mathrm{I}, \quad \omega=2, \quad \alpha=0, \quad \lambda_{m}=\frac{4 m}{2 m+\mathrm{I}} \\
S=\frac{3}{4}+\frac{\mathrm{I}}{4} \sum_{m=\mathrm{I}}^{\infty}(-\mathrm{I})^{m-1} \frac{\mathrm{I}}{m(2 m+\mathrm{I})(m+\mathrm{I})}
\end{gathered}
$$


Applying the transformation to the series on the right, with:

$$
\begin{gathered}
a_{0}=0, \quad A_{m}=\frac{2 m+\mathrm{I}}{m-\mathrm{I}}, \quad \lambda_{m}=\frac{(2 m+\mathrm{I})^{2}}{(m-\mathrm{I})(m+2)}, \quad \omega=4, \quad \alpha=0, \\
S=\frac{\mathrm{I} 9}{24}+\frac{9}{2} \sum_{m=\mathrm{I}}^{\infty}(-\mathrm{I})^{m} \frac{\mathrm{I}}{m(m+2)(2 m+\mathrm{I})^{2}(2 m+3)^{2}} .
\end{gathered}
$$

6.26 Reversion of series. The power series:

$$
z=x-b_{1} x^{2}-b_{2} x^{3}-b_{3} x^{4}-\ldots \ldots
$$

may be reversed, yielding:

where:

$$
x=z+c_{1} z^{2}+c_{2} z^{3}+c_{3} z^{4}+\ldots \ldots
$$

$c_{1}=b_{1}$,

$c_{2}=b_{2}+2 b_{1}^{2}$,

$c_{3}=b_{3}+5 b_{1} b_{2}+5 b_{1}^{3}$,

$c_{4}=b_{4}+6 b_{1} b_{3}+3 b_{2}^{2}+2 \mathrm{r} b_{1}^{2} b_{2}+\mathrm{I} 4 b_{1}^{4}$

$c_{5}=b_{5}+7\left(b_{1} b_{4}+b_{2} b_{3}\right)+28\left(b_{1}^{2} b_{3}+b_{1} b_{2}^{2}\right)+8{ }_{4} b_{1}{ }^{3} b_{2}+42 b_{1}{ }^{5}$,

$c_{6}=b_{6}+4\left(2 b_{1} b_{5}+2 b_{2} b_{4}+b_{3}^{2}\right)+\mathrm{I} 2\left(3 b_{1}^{2} b_{4}+6 b_{1} b_{2} b_{3}+b_{2}^{3}\right)$

$+60\left(2 b_{1}^{3} b_{3}+3 b_{1}^{2} b_{2}^{2}\right)+330 b_{1}^{4} b_{2}+\mathrm{I}_{32} b_{1}{ }^{6}$

$c_{7}=b_{7}+9\left(b_{1} b_{6}+b_{2} b_{5}+b_{3} b_{4}\right)+45\left(b_{1}^{2} b_{5}+b_{1} b_{3}^{2}+b_{2}^{2} b_{3}+2 b_{1} b_{2} b_{4}\right)$

$+16_{5}\left(b_{1}{ }^{3} b_{4}+b_{1} b_{2}{ }^{3}+3 b_{1}{ }^{2} b_{2} b_{3}\right)+495\left(b_{1}{ }^{4} b_{3}+2 b_{1}{ }^{3} b_{2}{ }^{2}\right)$

$+\mathrm{I} 287 b_{1}{ }^{5} b_{2}+429 b_{1} .^{7}$

Van Orstrand (Phil. Mag. I9, 366, I9ro) gives the coefficients of the reversed series up to $c_{12}$.

6.30 Binomial series.

$$
\begin{aligned}
& (\mathrm{I}+x)^{n}=\mathrm{I}+\frac{n}{\mathrm{I}} x+\frac{n(n-\mathrm{I})}{2 !} x^{2}+\frac{n(n-\mathrm{I})(n-2)}{3 !} x^{3}+\ldots \\
& +\frac{n !}{(n-k) ! k !} x^{k}+\ldots \ldots=\mathrm{I}+\left(\begin{array}{c}
n \\
\mathrm{I}
\end{array}\right) x+\left(\begin{array}{c}
n \\
2
\end{array}\right) x^{2}+\left(\begin{array}{l}
n \\
3
\end{array}\right) x^{3}+\ldots \ldots\left(\begin{array}{l}
n \\
k
\end{array}\right) x^{k}+\ldots
\end{aligned}
$$


6.31 Convergence of the binomial series.

The series converges absolutely for $|x|<$ I and diverges for $|x|>$ I. When $x=\mathrm{I}$, the series converges for $n>-\mathrm{I}$ and diverges for $n \leqslant-\mathrm{I}$. It is absolutely convergent only for $n>0$.

When $x=-\mathrm{I}$ it is absolutely convergent for $n>0$, and divergent for $n<0$.

6.32 Special cases of the binomial series.

$$
(a+b)^{n}=a^{n}\left(\mathrm{I}+\frac{b}{a}\right)^{n}=b^{n}\left(\mathrm{I}+\frac{a}{b}\right)^{n} \text {. }
$$

If $\left|\frac{b}{a}\right|<\mathrm{I}$ put $x=\frac{b}{a}$ in 6.30 ; if $\left|\frac{b}{a}\right|>$ I put $x=\frac{a}{b}$ in 6.30 .

\subsection{3}

I. $(\mathrm{I}+x)^{\frac{n}{m}}=\mathrm{I}+\frac{n}{m} x-\frac{n(m-n)}{2 ! m^{2}} x^{2}+\frac{n(m-n)(2 m-n)}{3 ! m^{3}} x^{3}-$

$$
\ldots+(-\mathrm{I})^{k} \frac{n(m-n)(2 m-n) \ldots[(k-\mathrm{I}) m-n]}{k ! m^{k}} x^{k}
$$

2. $(\mathrm{I}+x)^{-1}=\mathrm{I}-x+x^{2}-x^{3}+x^{4}-\ldots$.

3. $(\mathrm{I}+x)^{-2}=\mathrm{I}-2 x+3 x^{2}-4 x^{3}+5 x^{4}-\ldots$.

4. $\sqrt{\mathrm{I}+x}=\mathrm{I}+\frac{\mathrm{I}}{2} x-\frac{\mathrm{I} \cdot \mathrm{I}}{2 \cdot 4} x^{2}+\frac{\mathrm{I} \cdot \mathrm{I} \cdot 3}{2 \cdot 4 \cdot 6} x^{3}-\frac{\mathrm{I} \cdot \mathrm{I} \cdot 3 \cdot 5}{2 \cdot 4 \cdot 6 \cdot 8} x^{4}+\ldots$

5. $\frac{I}{\sqrt{I+x}}=I-\frac{I}{2} x+\frac{I \cdot 3}{2 \cdot 4} x^{2}-\frac{I \cdot 3 \cdot 5}{2 \cdot 4 \cdot 6} x^{3}+\frac{I \cdot 3 \cdot 5 \cdot 7}{2 \cdot 4 \cdot 6 \cdot 8} x^{4} \ldots$

6. $(\mathrm{I}+x)^{\frac{1}{3}}=\mathrm{I}+\frac{\mathrm{I}}{3} x-\frac{\mathrm{I} \cdot 2}{3 \cdot 6} x^{2}+\frac{\mathrm{I} \cdot 2 \cdot 5}{3 \cdot 6 \cdot 9} x^{3}-\frac{\mathrm{I} \cdot 2 \cdot 5 \cdot 8}{3 \cdot 6 \cdot 9 \cdot \mathrm{I} 2} x^{4}+\ldots$.

7. $(I+x)^{-3}=I-\frac{I}{3} x+\frac{I \cdot 4}{3 \cdot 6} x^{2}-\frac{I \cdot 4 \cdot 7}{3 \cdot 6 \cdot 9} x^{3}+\frac{I \cdot 4 \cdot 7 \cdot \text { IO }}{3 \cdot 6 \cdot 9 \cdot \text { I } 2} x^{4}-\ldots$

8. $(I+x)^{\frac{3}{2}}=I+\frac{3}{2} x+\frac{3 \cdot I}{2 \cdot 4} x^{2}-\frac{3 \cdot I \cdot I}{2 \cdot 4 \cdot 6} x^{3}+\frac{3 \cdot I \cdot I \cdot 3}{2 \cdot 4 \cdot 6 \cdot 8} x^{4}-\frac{3 \cdot I \cdot I \cdot 3 \cdot 5}{2 \cdot 4 \cdot 6 \cdot 8 \cdot I 0} x^{5}+\ldots$

9. $(\mathrm{I}+x)-^{\frac{3}{2}}=\mathrm{I}-\frac{3}{2} x+\frac{3 \cdot 5}{2 \cdot 4} x^{2}-\frac{3 \cdot 5 \cdot 7}{2 \cdot 4 \cdot 6} x^{3}+\ldots$.

Iо. $(1+x)^{\frac{1}{4}}=\mathrm{I}+\frac{1}{4} x-\frac{3}{3^{2}} x^{2}+\frac{7}{128} x^{3}-\frac{77}{2048} x^{4}+\ldots$

II. $(\mathrm{I}+x)^{-\frac{1}{4}}=\mathrm{I}-\frac{\mathrm{I}}{4} x+\frac{5}{32} x^{2}-\frac{\text { I } 5}{\text { I } 28} x^{3}+\frac{\text { I95 }}{2048} x^{4}-\ldots$

I2. $(I-L x)^{\frac{1}{3}}=\mathrm{I}+\frac{\mathrm{I}}{5} x-\frac{2}{25} x^{2}+\frac{6}{\mathrm{I} 25} x^{3}-\frac{2 \mathrm{I}}{625} x^{4}+\ldots$. 
I3. $(\mathrm{I}+x)^{-\frac{1}{3}}=\mathrm{I}-\frac{\mathrm{I}}{5} x+\frac{3}{25} x^{2}-\frac{\text { I I }}{\text { I } 25} x^{3}+\frac{44}{625} x^{4}-\ldots$

I4. $(\mathrm{I}+x)^{\frac{1}{6}}=\mathrm{I}+\frac{\mathrm{I}}{6} x-\frac{5}{72} x^{2}+\frac{55}{\mathrm{I} 296} x^{3}-\frac{935}{3 \text { I I04 }} x^{4}+\ldots$

I5. $(\mathrm{I}+x)^{-\frac{1}{6}}=\mathrm{I}-\frac{\mathrm{I}}{6} x+\frac{7}{72} x^{2}-\frac{9 \mathrm{I}}{\mathrm{I} 296} x^{3}+\frac{\mathrm{I} 729}{3 \mathrm{IIO4}} x^{4} \ldots .$.

\subsection{0}

I. $\frac{x}{\mathrm{I}-x}=\frac{x}{\mathrm{I}+x}+\frac{2 x^{2}}{\mathrm{I}+x^{2}}+\frac{4 x^{4}}{\mathrm{I}+x^{4}}+\frac{8 x^{8}}{\mathrm{I}+x^{8}}+\ldots$

2. $\frac{x}{\mathrm{I}-x}=\frac{x}{\mathrm{I}-x^{2}}+\frac{x^{2}}{\mathrm{I}-x^{4}}+\frac{x^{4}}{\mathrm{I}-x^{8}}+\ldots$.

3. $\frac{\mathrm{I}}{x-\mathrm{I}}=\frac{\mathrm{I}}{x+\mathrm{I}}+\frac{2}{x^{2}+\mathrm{I}}+\frac{4}{x^{4}+\mathrm{I}}+\ldots$.

\subsection{1}

I. $\{\mathrm{I}+\sqrt{\mathrm{I}+x}\}^{n}=2^{n}\left\{\mathrm{I}+n\left(\frac{x}{4}\right)+\frac{n(n-3)}{2 !}\left(\frac{x}{4}\right)^{2}\right.$ $\left.+\frac{n(n-4)(n-5)}{3 !}\left(\frac{x}{4}\right)^{3}+\ldots\right\}$.

$n$ may be any real number.

$$
\begin{aligned}
\left(x+\sqrt{\mathrm{I}+x^{2}}\right)^{n} & =\mathrm{I}+\frac{n^{2}}{2 !} x^{2}+\frac{n^{2}\left(n^{2}-2^{2}\right)}{4 !} x^{4}+\frac{n^{2}\left(n^{2}-2^{2}\right)\left(n^{2}-4^{2}\right)}{6 !} x^{6}+\ldots \\
+\frac{n}{\mathrm{I} !} & x+\frac{n\left(n^{2}-\mathrm{I}^{2}\right)}{3 !} x^{3}+\frac{n\left(n^{2}-\mathrm{I}^{2}\right)\left(n^{2}-3^{2}\right)}{5 !} x^{5}+\ldots \quad\left[x^{2}<\mathrm{I}\right]
\end{aligned}
$$

6.352 If $a$ is a positive integer:

$$
\frac{\mathrm{I}}{a}+\frac{\mathrm{I}}{a(a+\mathrm{I})} x+\frac{\mathrm{I}}{a(a+\mathrm{I})(a+2)} x^{2}+\ldots . . .=\frac{(a-\mathrm{I}) !}{x^{a}}\left\{e^{x}-\sum_{n=0}^{a-\mathrm{I}} \frac{x^{n}}{n !}\right\} .
$$

6.353 If $a$ and $b$ are positive integers, and $a<b$ :

$\frac{a}{b}+\frac{a(a+\mathrm{I})}{b(b+\mathrm{I})} x+\frac{a(a+\mathrm{I})(a+2)}{b(b+\mathrm{I})(b+2)} x^{2}+\ldots$

$$
\begin{gathered}
=(b-a)\left(\begin{array}{c}
b-\mathrm{I} \\
a-\mathrm{I}
\end{array}\right)\left\{\frac{(-\mathrm{I})^{b-a} \log (\mathrm{I}-x)}{x^{b}}(\mathrm{I}-x)^{b-a-1}\right. \\
\left.+\frac{\mathrm{I}}{x^{a}} \sum_{k=\mathrm{I}}^{b-a}(-\mathrm{I})^{k}\left(\begin{array}{c}
b-a-\mathrm{I} \\
k-\mathrm{I}
\end{array}\right) \sum_{n=\mathrm{I}}^{a+k-\mathrm{I}} \frac{x^{n-k}}{n}\right\} .
\end{gathered}
$$

(Schwatt, Phil. Mag. 31, 75, I916) 
POLYNOMIAL SERIES

6.360

$$
\begin{aligned}
& \frac{b_{0}+b_{1} x+b_{2} x^{2}+b_{3} x^{3}+\ldots .}{a_{0}+a_{1} x+a_{2} x^{2}+a_{3} x^{3}+\ldots}=\frac{\mathrm{I}}{a_{0}}\left(c_{0}+c_{1} x+c_{2} x^{2}+\ldots\right) \text {, } \\
& c_{0}-b_{0}=0 \\
& c_{1}+\frac{c_{0} a_{1}}{a_{0}}-b_{1}=0 \\
& c_{2}+\frac{c_{1} a_{1}}{a_{0}}+\frac{c_{0} a_{2}}{a_{0}}-b_{2}=0, \\
& c_{3}+\frac{c_{2} a_{1}}{a_{0}}+\frac{c_{1} a_{2}}{a_{0}}+\frac{c_{0} a_{3}}{a_{0}}-b_{3}=\text { o. } \\
& \text { … }
\end{aligned}
$$

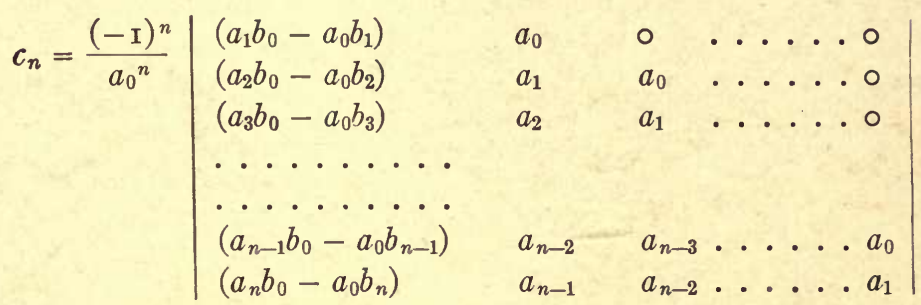

6.361

$$
\left(a_{0}+a_{1} x+a_{2} x^{2}+\ldots .\right)^{n}=c_{0}+c_{1} x+c_{2} x^{2}+\ldots .
$$

$$
\begin{aligned}
c_{0} & =a_{0}^{n}, \\
a_{0} c_{1} & =n a_{1} c_{0} \\
2 a_{0} c_{2} & =(n-\mathrm{I}) a_{1} c_{1}+2 n a_{2} c_{0}, \\
3 a_{0} c_{3} & =(n-2) a_{1} c_{2}+(2 n-\mathrm{I}) a_{2} c_{1}+3 n a_{3} c_{0} .
\end{aligned}
$$$$
\cdots \cdot \cdot
$$

\subsection{2}

$$
\begin{aligned}
& y=a_{1} x+a_{2} x^{2}+a_{3} x^{3}+\ldots \\
& b_{1} y+b_{2} y^{2}+b_{3} y^{3}+\ldots \\
& c_{1}=a_{1} b_{1} \\
& c_{2}=a_{2} b_{1}+a_{1}^{2} b_{2}, \\
& c_{3}=a_{3} b_{1}+2 a_{1} a_{2} b_{2}+a_{1}^{3} b_{3} \\
& c_{4}=a_{4} b_{1}+a_{2}^{2} b_{2}+2 a_{1} a_{3} b_{2}+3 a_{1}^{2} a_{2} b_{3}+a_{1}^{4} b_{4} .
\end{aligned}
$$

\subsection{3}

$$
\begin{aligned}
& e^{a_{1} x+a_{2} x^{2}+a_{3} x^{3}}+\ldots=. \mathrm{I}+c_{1} x+c_{2} x^{2}+\ldots \\
& c_{1}=a_{1}, \\
& c_{2}=a_{2}+\frac{I}{2} a_{1}^{2}
\end{aligned}
$$




$$
\begin{aligned}
& c_{3}=a_{3}+a_{1} a_{2}+\frac{I}{6} a_{1}^{3}, \\
& c_{4}=a_{4}+a_{1} a_{3}+\frac{I}{2} a_{2}^{2}+\frac{I}{2} a_{2} a_{1}^{2}+\frac{I}{24} a_{1}^{4} .
\end{aligned}
$$

6.364

$$
\begin{aligned}
\log \left(\mathrm{I}+a_{1} x+a_{2} x^{2}\right. & \left.+a_{3} x^{3}+\ldots\right)=c_{1} x+c_{2} x^{2}+c_{3} x^{3}+\ldots \\
a_{1} & =c_{1} \\
2 a_{2} & =a_{1} c_{1}+2 c_{2} \\
3 a_{3} & =a_{2} c_{1}+2 a_{1} c_{2}+3 c_{3} \\
4 a_{4} & =a_{3} c_{1}+2 a_{2} c_{2}+3 a_{3} c_{3}+4 a_{4} . \\
\cdots & = \\
c_{1} & =a_{1}, \\
c_{2} & =a_{2}-\frac{\mathrm{I}}{2} c_{1} a_{1}, \\
c_{3} & =a_{3}-\frac{\mathrm{I}}{3} c_{1} a_{2}-\frac{2}{3} c_{2} a_{1}, \\
c_{4} & =a_{4}-\frac{\mathrm{I}}{4} c_{1} a_{3}-\frac{2}{4} c_{2} a_{2}-\frac{3}{4} c_{3} a_{1} .
\end{aligned}
$$

\subsection{5}

$$
\begin{aligned}
y & =a_{1} x+a_{2} x^{2}+a_{3} x^{3}+\ldots \\
z & =b_{1} x+b_{2} x^{2}+b_{3} x^{3}+\ldots \\
y z & =c_{2} x^{2}+c_{3} x^{3}+c_{4} x^{4}+\ldots \\
c_{2} & =a_{1} b_{1} \\
c_{3} & =a_{1} b_{2}+a_{2} b_{1} \\
c_{4} & =a_{1} b_{3}+a_{2} b_{2}+a_{3} b_{1} \\
\cdots & \cdot \\
c_{k} & =a_{1} b_{k-1}+a_{2} b_{k-2}+a_{3} b_{k-3}+\ldots a_{k-1} b_{1}
\end{aligned}
$$

6.37. The Multinomial Theorem.

The general term in the expansion of

$$
\left(a_{0}+a_{1} x+a_{2} x^{2}+a_{3} x^{3}+\ldots\right)^{n}
$$

where $n$ is positive or negative, integral or fractional, is,

$$
\frac{n(n-\mathrm{I})(n-2) \ldots(p+\mathrm{I})}{c_{1} ! c_{2} ! c_{3} ! \ldots} a_{0}{ }^{p} a_{1}^{c_{1}} a_{2}{ }^{c_{2}} a_{3}{ }^{c_{3}} \ldots x^{c_{1}+2 c_{2}+3 c_{3}+} \ldots
$$

where

$$
p+c_{1}+c_{2}+c_{3}+\ldots \ldots=n .
$$

$c_{1}, c_{2}, c_{3}, \ldots$ are positive integers.

If $n$ is a positive integer, and hence $p$ also, the general term in the expansion may be written, 



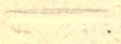




(3)

$$
\frac{n !}{p ! c_{1} ! c_{2} ! \ldots} a_{0}^{p} a_{1}^{c_{1}} a_{2}^{c_{2}} a_{3}^{c_{3}} \ldots x^{c_{1}+2 c_{2}+3 c_{3}+} \ldots
$$

The coefficient of $x^{k}$ ( $k$ an integer) in the expansion of (I) is found by taking the sum of all the terms (2) or (3) for the different combinations of $p, c_{1}, c_{2}$, $c_{3}, \ldots$ whicin satisfy

$$
\begin{aligned}
& c_{1}+2 c_{2}+3 c_{3}+\ldots .=k, \\
& p+c_{1}+c_{2}+c_{3}+\ldots=n .
\end{aligned}
$$

cf. 6.361 .

In the following series the coefficients $B_{n}$ are Bernoulli's numbers (6.902) and the coefficients $E_{n}$, Euler's numbers (6.903).

\subsection{0}

I. $\sin x=x-\frac{x^{3}}{3 !}+\frac{x^{5}}{5 !}-\frac{x^{7}}{7 !}+\ldots=\sum_{n=0}^{\infty}(-\mathrm{I})^{n} \frac{x^{2 n+1}}{(2 n+\mathrm{I}) !}$

2. $\cos x=\mathrm{I}-\frac{x^{2}}{2 !}+\frac{x^{4}}{4 !}-\frac{x^{6}}{6 !}+\ldots=\sum_{n=0}^{\infty}(-\mathrm{I})^{n} \frac{x^{2 n}}{(2 n) !}$

3. $\tan x=x+\frac{1}{3} x^{3}+\frac{2}{15} x^{5}+\frac{17}{315} x^{7}+\frac{62}{2835} x^{9}+\ldots$.

$$
=\sum_{n=1}^{\infty} \frac{2^{2 n}\left(2^{2 n}-\mathrm{I}\right)}{(2 n) !} B_{n} x^{2 n-1} \quad\left[x^{2}<\frac{\pi^{2}}{4}\right] .
$$

4. $\cot x=\frac{1}{x}-\frac{x}{3}-\frac{1}{45} x^{3}-\frac{2}{945} x^{5}-\frac{1}{4725} x^{7}-\ldots \ldots$

$$
=\frac{\mathrm{I}}{x}-\sum_{n=1}^{\infty} \frac{2^{2 n} B_{n}}{(2 n) !} x^{2 n-1}
$$

5. $\sec x=\mathrm{I}+\frac{\mathrm{I}}{2 !} x^{2}+\frac{5}{4 !} x^{4}+\frac{6 \mathrm{I}}{6^{1}} x^{6}+.=\sum_{n=0}^{\infty} \frac{E_{n}}{(2 n) !} x^{2 n}$ $\left[x^{2}<\frac{\pi^{2}}{4}\right]$

6. $\csc x=\frac{\mathrm{I}}{x}+\frac{\mathrm{I}}{3 !} x+\frac{7}{3 \cdot 5 !} x^{3}+\frac{3 \mathrm{I}}{3 \cdot 7 !} x^{5}+\ldots$

$$
=\frac{\mathrm{I}}{x}+\sum_{n=0}^{\infty} \frac{2\left(2^{2 n+1}-\mathrm{I}\right)}{(2 n+2) !} B_{n+1} x^{2 n+1} \quad\left[x^{2}<\pi^{2}\right] .
$$

\subsection{1}

I. $\sin ^{-1} x=x+\frac{I}{2 \cdot 3} x^{3}+\frac{I \cdot 3}{2 \cdot 4 \cdot 5} x^{5}+\frac{I \cdot 3 \cdot 5}{2 \cdot 4 \cdot 6 \cdot 7} x^{7}+\ldots$

$$
=\frac{\pi}{2}-\cos ^{-1} x=\sum_{n=0}^{\infty} \frac{(2 n) !}{2^{2 n}(n !)^{2}(2 n+1)} x^{2 n+1} \text {. }
$$


2. $\tan ^{-1} x=x-\frac{I}{3} x^{3}+\frac{I}{5} x^{5}-\frac{I}{7} x^{7}+\ldots . \quad$ (Gregory's Series) $\quad\left[x^{2} \leqslant \mathrm{I}\right]$

$$
=\frac{\pi}{2}-\cot ^{-1} x=\sum_{n=0}^{\infty}(-\mathrm{I})^{n} \frac{x^{2 n+1}}{2 n+\mathrm{I}} \text {. }
$$

3. $\tan ^{-1} x=\frac{x}{\mathrm{I}+x^{2}}\left\{\mathrm{I}+\frac{2}{3} \frac{x^{2}}{\mathrm{I}+x^{2}}+\frac{2 \cdot 4}{3 \cdot 5}\left(\frac{x^{2}}{\mathrm{I}+x^{2}}\right)^{2}+\ldots\right\}$

$$
=\frac{x}{\mathrm{I}+x^{2}} \sum_{n=0}^{\infty} \frac{2^{2 n}(n !)^{2}}{(2 n+\mathrm{I}) !}\left(\frac{x^{2}}{\mathrm{I}+x^{2}}\right)^{n}
$$

4. $\tan ^{-1} x=\frac{\pi}{2}-\frac{\mathrm{I}}{x}+\frac{\mathrm{I}}{3 x^{3}}-\frac{\mathrm{I}}{5 x^{5}}+\frac{\mathrm{I}}{7 x^{7}}-\ldots$

$$
=\frac{\pi}{2}-\sum_{n=0}^{\infty}(-\mathrm{I})^{n} \frac{\mathrm{I}}{(2 n+\mathrm{I}) x^{2 n+1}} \quad\left[x^{2} \geqslant \mathrm{I}\right] .
$$

5. $\sec ^{-1} x=\frac{\pi}{2}-\frac{\mathrm{I}}{x}-\frac{\mathrm{I}}{2 \cdot 3} \frac{\mathrm{I}}{x^{3}}-\frac{\mathrm{I} \cdot 3}{2 \cdot 4 \cdot 5} \frac{\mathrm{I}}{x^{5}}+\frac{\mathrm{I} \cdot 3 \cdot 5}{2 \cdot 4 \cdot 6 \cdot 7} \frac{\mathrm{I}}{x^{7}}-\ldots$

$$
=\frac{\pi}{2}-\csc ^{-1} x=\frac{\pi}{2}-\sum_{n=0}^{\infty} \frac{(2 n) !}{2^{2 n}(n !)^{2}(2 n+1)} x^{-2 n-1}
$$

\subsection{2}

I. $\left(\sin ^{-1} x\right)^{2}=x^{2}+\frac{2}{3} \frac{x^{4}}{2}+\frac{2 \cdot 4}{3 \cdot 5} \frac{x^{6}}{3}+\frac{2 \cdot 4 \cdot 6}{3 \cdot 5 \cdot 7} \frac{x^{8}}{4}+\ldots$

$$
=\sum_{n=0}^{\infty} \frac{2^{2 n}(n !)^{2}}{(2 n+\mathrm{I}) !(n+\mathrm{I})} x^{2 n+2}
$$

2. $\left(\sin ^{-1} x\right)^{3}=x^{3}+\frac{3 !}{5 !} 3^{2}\left(\mathrm{I}+\frac{\mathrm{I}}{3^{2}}\right) x^{5}+\frac{3 !}{7 !} 3^{2} 5^{2}\left(\mathrm{I}+\frac{\mathrm{I}}{3^{2}}+\frac{\mathrm{I}}{5^{2}}\right) x^{7}+\ldots\left[x^{2} \leqslant \mathrm{I}\right]$.

3. $\left(\tan ^{-1} x\right)^{p}=p ! \sum_{k_{0}=\mathrm{I}}^{\infty}(-\mathrm{I})^{k_{\circ}-\mathrm{r}} \frac{x^{2 k_{\mathrm{o}}+p-2}}{2 k_{0}+p-2} \prod_{a=1}^{p-\mathrm{I}}\left(\sum_{k_{a}=\mathrm{I}}^{k_{a}-\mathrm{I}} \frac{\mathrm{I}}{2 k_{a}+p-a-2}\right)$.

(Schwatt, Phil. Mag. 31, p. 490, I916).

4. $\sqrt{1-x^{2}} \sin ^{-1} x=x-\frac{x^{3}}{3}+\frac{2}{3 \cdot 5} x^{5}-\frac{2 \cdot 4}{3 \cdot 5 \cdot 7} x^{7}+\ldots$

$$
=x+\sum_{n=1}^{\infty}(-\mathrm{I})^{n} \frac{2^{2 n-2}[(n-\mathrm{I}) !]^{2}}{(2 n-\mathrm{I}) !(2 n+\mathrm{I})} x^{2 n+1} \quad\left[x^{2}<\mathrm{I}\right] .
$$

5. $\frac{\sin ^{-1} x}{\sqrt{1-x^{2}}}=x+\frac{2}{3} x^{3}+\frac{2 \cdot 4}{3 \cdot 5} x^{5}+\frac{2 \cdot 4 \cdot 6}{3 \cdot 5 \cdot 7} x^{7}+\ldots$

$$
=\sum_{n=0}^{\infty} \frac{2^{2 n}(n !)^{2}}{(2 n+1) !} x^{2 n+1}
$$




\subsection{3}

I. $\log \sin x=\log x-\left\{\frac{\mathrm{I}}{6} x^{2}+\frac{\mathrm{I}}{\mathrm{I} 80} x^{4}+\frac{\mathrm{I}}{2835} x^{6}+\ldots\right\}$

$$
=\log x-\sum_{n=1}^{\infty} \frac{2^{2 n-1}}{n(2 n) !} B_{n} x^{2 n}
$$

2. $\log \cos x=-\frac{\mathrm{I}}{2} x^{2}-\frac{\mathrm{I}}{\mathrm{I} 2} x^{4}-\frac{\mathrm{I}}{45} x^{6}-\frac{\mathrm{I} 7}{2520} x^{8}-\ldots$

$$
=-\sum_{n=1}^{\infty} \frac{2^{2 n-1}\left(2^{2 n}-\mathrm{I}\right) B_{n}}{n(2 n) !} x^{2 n}
$$

3. $\log \tan x=\log x+\frac{1}{3} x^{2}+\frac{7}{90} x^{4}+\frac{62}{2835} x^{6}+\frac{\text { I } 27}{18900} x^{8}+\ldots$.

$$
=\log x+\sum_{n=\mathrm{I}}^{\infty} \frac{\left(2^{2 n-1}-\mathrm{I}\right) 2^{2 n}}{n(2 n) !} B_{n} x^{2 n} \quad\left[x^{2}<\frac{\pi^{2}}{4}\right] .
$$

4. $\log \cos x=-\frac{I}{2}\left\{\sin ^{2} x+\frac{I}{2} \sin ^{4} x+\frac{I}{3} \sin ^{6} x+\ldots\right\}$

$$
=-\frac{\mathrm{I}}{2} \sum_{n=\mathrm{I}}^{\infty} \frac{\mathrm{I}}{n} \sin ^{2 n} x \text {. }
$$

\subsection{4}

I. $\log (\mathrm{I}+x)=x-\frac{\mathrm{I}}{2} x^{2}+\frac{\mathrm{I}}{3} x^{3}-\frac{\mathrm{I}}{4} x^{4}+\ldots$

$$
=\sum_{n=1}^{\infty}(-\mathrm{I})^{n+1} \frac{x^{n}}{n}
$$

$\{\log (\mathrm{I}+x)\}^{p} \quad$ see 7.369 .

2. $\log \left(x+\sqrt{I+x^{2}}\right)=x-\frac{I \cdot I}{2 \cdot 3} x^{3}+\frac{I \cdot I \cdot 3}{2 \cdot 4 \cdot 5} x^{5}-\frac{I \cdot I \cdot 3 \cdot 5}{2 \cdot 4 \cdot 6 \cdot 7} x^{7}+\ldots$

$$
=x+\sum_{n=\mathrm{I}}^{\infty}(-\mathrm{I})^{n} \frac{(2 n-\mathrm{I}) ! x^{2 n+1}}{2^{2 n-1} n !(n-\mathrm{I}) !(2 n+\mathrm{I})} \quad[-\mathrm{I} \leqslant x \leqslant \mathrm{I}] .
$$

3. $\log \left(\mathrm{I}+\sqrt{\mathrm{I}+x^{2}}\right)=\log 2+\frac{\mathrm{I} \cdot \mathrm{I}}{2 \cdot 2} x^{2}-\frac{\mathrm{I} \cdot \mathrm{I} \cdot 3}{2 \cdot 4 \cdot 4} x^{4}+\frac{\mathrm{I} \cdot \mathrm{I} \cdot 3 \cdot 5}{2 \cdot 4 \cdot 6 \cdot 6} x^{6}-\ldots$

$$
=\log 2-\sum_{n=1}^{\infty}(-\mathrm{I})^{n} \frac{(2 n-\mathrm{I}) !}{2^{2 n-1} n !(n-\mathrm{I}) !} \frac{x^{2 n}}{2 n} \quad\left[x^{2} \leqslant \mathrm{I}\right] .
$$


4. $\log \left(\mathrm{I}+\sqrt{\mathrm{I}+x^{2}}\right)=\log x+\frac{\mathrm{I}}{x}-\frac{\mathrm{I} \cdot \mathrm{I}}{2 \cdot 3} \frac{\mathrm{I}}{x^{3}}+\frac{\mathrm{I} \cdot \mathrm{I} \cdot 3}{2 \cdot 4 \cdot 5} \frac{\mathrm{I}}{x^{5}}-\ldots$

$$
=\log x+\frac{\mathrm{I}}{x}+\sum_{n=\mathrm{I}}^{\infty}(-\mathrm{I})^{n} \frac{(2 n-\mathrm{I}) !}{2^{2 n-1} n !(n-\mathrm{I}) !} \frac{x^{-2 n-1}}{(2 n+\mathrm{I})} \quad\left[x^{2} \geqslant \mathrm{I}\right] .
$$

5. $\log x=(x-\mathrm{I})-\frac{\mathrm{I}}{2}(x-\mathrm{I})^{2}+\frac{\mathrm{I}}{3}(x-\mathrm{I})^{3}-\ldots$

$$
=\sum_{n=1}^{\infty}(-\mathrm{I})^{n+1} \frac{(x-\mathrm{I})^{n}}{n}
$$

6. $\log x=\frac{x-\mathrm{I}}{x}+\frac{\mathrm{I}}{2}\left(\frac{x-\mathrm{I}}{x}\right)^{2}+\frac{\mathrm{I}}{3}\left(\frac{x-\mathrm{I}}{x}\right)^{3}+\ldots$

$$
=\sum_{n=\mathrm{I}}^{\infty} \frac{\mathrm{I}}{n}\left(\frac{x-\mathrm{I}}{x}\right)^{n}
$$

7. $\log x=2\left\{\frac{x-\mathrm{I}}{x+\mathrm{I}}+\frac{\mathrm{I}}{3}\left(\frac{x-\mathrm{I}}{x+\mathrm{I}}\right)^{3}+\frac{\mathrm{I}}{5}\left(\frac{x-\mathrm{I}}{x+\mathrm{I}}\right)^{5}+\ldots\right\}$

$$
=2 \sum_{n=0}^{\infty} \frac{\mathrm{I}}{2 n+\mathrm{I}}\left(\frac{x-\mathrm{I}}{x+\mathrm{I}}\right)^{2 n+1}
$$

8. $\log \frac{\mathrm{I}+x}{\mathrm{I}-x}=2\left\{x+\frac{\mathrm{I}}{3} x^{3}+\frac{\mathrm{I}}{5} x^{5}+\ldots.\right\}$

$$
=2 \sum_{n=0}^{\infty} \frac{\mathrm{I}}{2 n+\mathrm{I}} x^{2 n+1}
$$

9. $\log \frac{x+\mathrm{I}}{x-\mathrm{I}}=2\left\{\frac{\mathrm{I}}{x}+\frac{\mathrm{I}}{3} \frac{\mathrm{I}}{x^{3}}+\frac{\mathrm{I}}{5} \frac{\mathrm{I}}{x^{5}}+\ldots\right\}$

$$
=2 \sum_{n=0}^{\infty} \frac{\mathrm{I}}{(2 n+\mathrm{I}) x^{2 n+1}}
$$

I0. $\sqrt{I+x^{2}} \log \left(x+\sqrt{\left.I+x^{2}\right)}=x+\frac{I}{3} x^{3}-\frac{I \cdot 2}{3 \cdot 5} x^{5}+\frac{I \cdot 2 \cdot 4}{3 \cdot 5 \cdot 7} x^{7}-\ldots\right.$

$$
=x-\sum_{n=\mathrm{I}}^{\infty}(-\mathrm{I})^{n} \frac{(n-\mathrm{I}) ! 2^{2 n-1} n !}{(2 n+\mathrm{I}) !} x^{2 n+1} \quad\left[x^{2}<\mathrm{I}\right] .
$$

II. $\frac{\log \left(x+\sqrt{1+x^{2}}\right)}{\sqrt{1+x^{2}}}=x-\frac{2}{3} x^{3}+\frac{2 \cdot 4}{3 \cdot 5} x^{5}-\frac{2 \cdot 4 \cdot 6}{3 \cdot 5 \cdot 7} x^{7}+\ldots$

$$
=\sum_{n=0}^{\infty}(-\mathrm{I})^{n} \frac{2^{2 n}(n !)^{2}}{(2 n+\mathrm{I}) !} x^{2 n+1} \quad\left[x^{2}<\mathrm{I}\right]
$$

I2. $\left\{\log \left(x+\sqrt{I+x^{2}}\right)\right\}^{2}=\frac{x^{2}}{I}-\frac{2}{3} \frac{x^{4}}{2}+\frac{2 \cdot 4}{3 \cdot 5} \frac{x^{6}}{3}-\ldots$.

$$
=\sum_{n=\mathrm{I}}^{\infty}(-\mathrm{I})^{n-1} \frac{2^{2 n-2}(n-\mathrm{I}) !(n-\mathrm{I}) !}{(2 n-\mathrm{I}) !} \frac{x^{2 n}}{n} \cdot\left[x^{2}<\mathrm{I}\right]
$$


3. $\frac{1}{2}\{\log (I+x)\}^{2}=\frac{I}{2} s_{1} x^{2}-\frac{I}{3} s_{2} x^{3}+\frac{I}{4} s_{3} x^{4}-\ldots$

where $s_{n}=\frac{\mathrm{I}}{\mathrm{I}}+\frac{\mathrm{I}}{2}+\frac{\mathrm{I}}{3}+\ldots \frac{\mathrm{I}}{n}$

(See 1.876).

I4. $\frac{\mathrm{I}}{6}\{\log (\mathrm{I}+x)\}^{3}=\frac{\mathrm{I}}{3} \cdot \frac{\mathrm{I}}{2} s_{1} x^{3}-\frac{\mathrm{I}}{4}\left(\frac{\mathrm{I}}{2} s_{1}+\frac{\mathrm{I}}{3} s_{2}\right) x^{4}$

$$
+\frac{\mathrm{I}}{5}\left(\frac{\mathrm{I}}{2} s_{1}+\frac{\mathrm{I}}{3} s_{2}+\frac{\mathrm{I}}{4} s_{3}\right) x^{5}-\ldots\left[x^{2}<\mathrm{I}\right]
$$

I5. $\frac{\log (\mathrm{I}+x)}{(\mathrm{I}+x)^{n}}=x-n(n+\mathrm{I})\left(\frac{\mathrm{I}}{n}+\frac{\mathrm{I}}{n+\mathrm{I}}\right) \frac{x^{2}}{2 !}$

$$
+n(n+\mathrm{I})(n+2)\left(\frac{\mathrm{I}}{n}+\frac{\mathrm{I}}{n+\mathrm{I}}+\frac{\mathrm{I}}{n+2}\right) \frac{x^{3}}{3 !}-\ldots\left[x^{2}<\mathrm{I}\right] .
$$

\subsection{5 (See 6.705.)}

I. $\frac{3}{4 x}-\frac{\mathrm{I}}{2 x^{2}}+\frac{(\mathrm{I}-x)^{2}}{2 x^{3}} \log \frac{\mathrm{I}}{\mathrm{I}-x}=\frac{\mathrm{I}}{\mathrm{I} \cdot 2 \cdot 3}+\frac{x}{2 \cdot 3 \cdot 4}+\frac{x^{2}}{3 \cdot 4 \cdot 5}+\ldots\left[x^{2}<\mathrm{I}\right]$

2. $\frac{\mathrm{I}}{4 x}\left\{\frac{\mathrm{I}+x}{\sqrt{x}} \log \frac{\mathrm{I}+\sqrt{x}}{\mathrm{I}-\sqrt{x}}+2 \log (\mathrm{I}-x)-2\right\}=\frac{\mathrm{I}}{\mathrm{I} \cdot 2 \cdot 3}+\frac{x}{3 \cdot 4 \cdot 5}$ $+\frac{x^{2}}{5 \cdot 6 \cdot 7}+\ldots \quad[0<x<\mathrm{I}]$.

3. $\frac{\mathrm{I}}{2 x}\left\{\mathrm{I}-\log (\mathrm{I}+x)-\frac{\mathrm{I}-x}{\sqrt{x}} \tan ^{-1} x\right\}=\frac{\mathrm{I}}{\mathrm{I} \cdot 2 \cdot 3}-\frac{x}{3 \cdot 4 \cdot 5}$

$$
+\frac{x^{2}}{5 \cdot 6 \cdot 7}-\ldots \quad[0<x \leqslant \mathrm{I}]
$$

\subsection{5}

$-\log (\mathrm{I}+x) \cdot \log (\mathrm{I}-x)=x^{2}+\left(\mathrm{I}-\frac{\mathrm{I}}{2}+\frac{\mathrm{I}}{3}\right) \frac{x^{4}}{2}$

$$
+\left(\mathrm{I}-\frac{\mathrm{I}}{2}+\frac{\mathrm{I}}{3}-\frac{\mathrm{I}}{4}+\frac{\mathrm{I}}{5}\right) \frac{x^{6}}{3}+\ldots \ldots \quad\left[x^{2}<\mathrm{I}\right] .
$$

2. $\frac{\mathrm{I}}{2} \tan ^{-1} x \cdot \log \frac{\mathrm{I}+x}{\mathrm{I}-x}=x^{2}+\left(\mathrm{I}-\frac{\mathrm{I}}{3}+\frac{\mathrm{I}}{5}\right) \frac{x^{6}}{3}+\left(\mathrm{I}-\frac{\mathrm{I}}{3}+\frac{\mathrm{I}}{5}-\frac{\mathrm{I}}{7}+\frac{\mathrm{I}}{9}\right) \frac{x^{10}}{5}$

3. $\frac{\mathrm{I}}{2} \tan ^{-1} x \cdot \log \left(\mathrm{I}+x^{2}\right)=\left(\mathrm{I}+\frac{\mathrm{I}}{2}\right) \frac{x^{3}}{3}-\left(\mathrm{I}+\frac{\mathrm{I}}{2}+\frac{\mathrm{I}}{3}+\frac{\mathrm{I}}{4}\right) \frac{x^{5}}{5}+\ldots \quad\left[x^{2}<\mathrm{I}\right]$.

\subsection{6}

I. $\cos \left\{k \log \left(x+\sqrt{\mathrm{I}+x^{2}}\right)\right\}=\mathrm{I}-\frac{k^{2}}{2 !} \aleph^{2}+\frac{k^{2}\left(k^{2}+2^{2}\right)}{4 !} x^{4}$

$$
-\frac{k^{2}\left(k^{2}+2^{2}\right)\left(k^{2}+4^{2}\right)}{6 !} x^{6}+\ldots \ldots \quad x^{2}<\mathrm{r} .
$$


$k$ may be any real number.

2. $\sin \left\{k \log \left(x+\sqrt{\mathrm{I}+x^{2}}\right)\right\}=\frac{k}{\mathrm{I} !} x-\frac{k^{2}\left(k^{2}+\mathrm{I}^{2}\right)}{3 !} x^{3}$

\subsection{7}

$$
+\frac{k^{2}\left(k^{2}+\mathrm{I}^{2}\right)\left(k^{2}+3^{2}\right)}{5 !} x^{5}-\ldots
$$

$$
\frac{\mathrm{I}}{\mathrm{I}-2 x \cos \alpha+x^{2}}=\mathrm{I}+\sum_{n=\mathrm{I}}^{\infty} A_{n} x^{n}
$$

where,

$$
\begin{aligned}
A_{2 n} & =(-\mathrm{I})^{n} \sum_{k=0}^{n}(-\mathrm{I})^{k}\left(\frac{n+k}{2 k}\right)(2 \cos \alpha)^{2 k} \\
A_{2 n+1} & =(-\mathrm{I})^{n} \sum_{k=0}^{n}(-\mathrm{I})^{k}\left(\frac{n+k+\mathrm{I}}{2 k+\mathrm{I}}\right)(2 \cos \alpha)^{2 k+1}
\end{aligned}
$$

\subsection{0}

I. $e^{x}=\mathrm{I}+x+\frac{x^{2}}{2 !}+\frac{x^{3}}{3 !}+\ldots . .=\sum_{n=0}^{\infty} \frac{x^{n}}{n !}$

2. $a^{x}=\mathrm{I}+x \log a+\frac{(x \log a)^{2}}{2 !}+\frac{(x \log a)^{3}}{3 !}+\ldots$

3. $e^{e^{x}}=e\left(\mathrm{I}+x+\frac{2}{2 !} x^{2}+\frac{5}{3 !} x^{3}+\frac{\mathrm{I} 5}{4 !} x^{4}+\ldots\right)$.

4. $e^{\sin x}=\mathrm{I}+x+\frac{x^{2}}{2 !}-\frac{3 x^{4}}{4 !}-\frac{8 x^{5}}{5 !}+\frac{3 x^{6}}{6 !}+\frac{56 x^{7}}{7 !}+\ldots$

5. $e^{\cos x}=e\left(\mathrm{I}-\frac{x^{2}}{2 !}+\frac{4 x^{4}}{4 !}-\frac{3 \mathrm{I} x^{6}}{6 !}+\ldots\right)$

6. $e^{\tan x}=\mathrm{I}+x+\frac{x^{2}}{2 !}+\frac{3 x^{3}}{3 !}+\frac{9 x^{4}}{4 !}+\frac{37 x^{5}}{5 !}+\ldots$

7. $e^{\sin ^{-1} x}=\mathrm{I}+x+\frac{x^{2}}{2 !}+\frac{2 x^{3}}{3 !}+\frac{5 x^{4}}{4 !}+\ldots$.

8. $e^{\tan ^{-1} x}=\mathrm{I}+x+\frac{x^{2}}{2}-\frac{x^{3}}{6}+\frac{7 x^{4}}{24}-\ldots$

\subsection{0}

I. $\sinh x=x+\frac{x^{3}}{3 !}+\frac{x^{5}}{5 !}+\frac{x^{7}}{7 !}+\ldots=\sum_{n=0}^{\infty} \frac{x^{2 n+1}}{(2 n+\mathrm{I}) !}$ 
2. $\cosh x=\mathrm{I}+\frac{x^{2}}{2 !}+\frac{x^{4}}{4 !}+\frac{x^{6}}{6 !}+\ldots=\sum_{n=0}^{\infty} \frac{x^{2 n}}{(2 n) !}$ $\left[x^{2}<\infty\right]$

3. $\tanh x=x-\frac{\mathrm{I}}{3} x^{3}+\frac{2}{\mathrm{I} 5} x^{5}-\frac{\mathrm{I} 7}{3 \mathrm{I} 5} x^{7}+\ldots$

$$
=\sum_{n=\mathrm{I}}^{\infty}(-\mathrm{I})^{n-1} \frac{2^{2 n}\left(2^{2 n}-\mathrm{I}\right)}{(2 n) !} B_{n} x^{2 n-1} \quad\left[x^{2}<\frac{\pi^{2}}{4}\right] .
$$

4. $x \operatorname{coth} x=\mathrm{I}+\frac{\mathrm{I}}{3} x^{2}-\frac{\mathrm{I}}{45} x^{4}+\frac{2}{945} x^{6}-\ldots$

$$
=\mathrm{I}+\sum_{n=\mathrm{I}}^{\infty}(-\mathrm{I})^{n-1} \frac{2^{2 n} B_{n}}{(2 n) !} x^{2 n}
$$

5. $\operatorname{sech} x=\mathrm{I}-\frac{\mathrm{I}}{2} x^{2}+\frac{5}{24} x^{4}-\frac{6 \mathrm{I}}{720} x^{6}+\ldots=\mathrm{I}+\sum_{n=\mathrm{I}}^{\infty}(-\mathrm{I})^{n} \frac{E_{n}}{(2 n) !} x^{2 n} \quad\left[x^{2}<\frac{\pi}{4}\right]$. 6. $x \operatorname{csch} x=\mathrm{I}-\frac{\mathrm{I}}{6} x^{2}+\frac{7}{360} x^{4}-\frac{3 \mathrm{I}}{\mathrm{I} 5 \mathrm{I} 20} x^{6}+\ldots$

$$
=\mathrm{I}+\sum_{n=\mathrm{I}}^{\infty}(-\mathrm{I})^{n} \frac{2\left(2^{2 n-1}-\mathrm{I}\right)}{(2 n) !} B_{n} x^{2 n} \quad\left[x^{2}<\pi^{2}\right] .
$$

\subsection{5}

I. $\cosh x \cos x=\mathrm{I}-\frac{2^{2}}{4 !} x^{4}+\frac{2^{4}}{8 !} x^{8}-\frac{2^{6}}{\mathrm{I} 2 !} x^{12}+\ldots$

2. $\sinh x \sin x=\frac{2^{2}}{2 !} x^{2}-\frac{2^{4}}{6 !} x^{6}+\frac{2^{6}}{10 !} x^{10}-\ldots \ldots$

\subsection{6}

I.

$$
e^{x \cos \theta} \cos (x \sin \theta)=\sum_{n=0}^{\infty} \frac{x^{n} \cos n \theta}{n !}
$$$$
\left[x^{2}<\mathrm{I}\right]
$$$$
e^{x \cos \theta} \sin (x \sin \theta)=\sum_{n=1}^{\infty} \frac{x^{n} \sin n \theta}{n !}
$$$$
\left[x^{2}<\mathrm{I}\right]
$$

2 .

$$
\sum_{n=0}^{\infty} \frac{x^{2 n} \cos 2 n \theta}{(2 n) !}
$$

$\left[x^{2}<\mathrm{I}\right]$

$\left[x^{2}<\mathrm{I}\right]$

$\left[x^{2}<\mathrm{I}\right]$

$\left[x^{2}<\mathrm{I}\right]$ 


\subsection{0}

I. $\sinh ^{-1} x=x-\frac{I}{2 \cdot 3} x^{3}+\frac{I \cdot 3}{2 \cdot 4 \cdot 5} x^{5}-\ldots$

$$
=\sum_{n=0}^{\infty}(-\mathrm{I})^{n} \frac{(2 n) !}{2^{2 n}(n !)^{2}(2 n+\mathrm{I})} x^{2 n+1}
$$

2. $\sinh ^{-1} x=\log 2 x+\frac{I}{2} \frac{I}{2 x^{2}}-\frac{I \cdot 3}{2 \cdot 4} \frac{I}{4 x^{4}}+\ldots$

$$
=\log 2 x+\sum_{n=0}^{\infty}(-I)^{n} \frac{(2 n) !}{2^{2 n}(n !)^{2} 2 n} x^{-2 n}
$$

3. $\cosh ^{-1} x=\log 2 x-\frac{I}{2} \frac{I}{2 x^{2}}-\frac{I \cdot 3}{2 \cdot 4} \frac{I}{4 x^{4}}-\ldots$

$$
=\log 2 x-\sum_{n=0}^{\infty} \frac{(2 n) !}{2^{2 n}(n !)^{2} 2 n} x^{-2 n}
$$

4. $\tanh ^{-1} x=x+\frac{\mathrm{I}}{3} x^{3}+\frac{\mathrm{I}}{5} x^{5}+\frac{\mathrm{I}}{7} x^{7}+\ldots=\sum_{n=0}^{\infty} \frac{x^{2 n+1}}{2 n+\mathrm{I}}$

5. $\sinh ^{-1} \frac{\mathrm{I}}{x}=\frac{\mathrm{I}}{x}-\frac{\mathrm{I}}{2} \frac{\mathrm{I}}{3 x^{3}}+\frac{\mathrm{I} \cdot 3}{2 \cdot 4} \frac{\mathrm{I}}{5 x^{5}} \ldots$.

$$
=\operatorname{csch}^{-1} x=\sum_{n=0}^{\infty}(-\mathrm{I})^{n} \frac{(2 n) !}{2^{2 n}(n !)^{2}(2 n+\mathrm{I})} x^{-2 n-1} \quad\left[x^{2}>\mathrm{I}\right]
$$

6. $\cosh ^{-1} \frac{I}{x}=\log \frac{2}{x}-\frac{I}{2} \frac{x^{2}}{2}-\frac{I \cdot 3}{2 \cdot 4} \frac{x^{4}}{4}-\ldots$

$$
=\operatorname{sech}^{-1} x=\log \frac{2}{x}-\sum_{n=0}^{\infty} \frac{(2 n) !}{2^{2 n}(n !)^{2} 2 n} x^{2 n}
$$

7. $\sinh ^{-1} \frac{I}{x}=\log \frac{2}{x}+\frac{I}{2} \frac{x^{2}}{2}-\frac{I \cdot 3}{2 \cdot 4} \frac{x^{4}}{4}+\ldots$.

$$
=\operatorname{csch}^{-1} x=\log \frac{2}{x}+\sum_{n=0}^{\infty}(-\mathrm{I})^{n} \frac{(2 n) !}{2^{2 n}(n !)^{2} 2 n} x^{2 n} \quad\left[x^{2}<\mathrm{I}\right] \text {. }
$$

8. $\tanh ^{-1} \frac{I}{x}=\frac{I}{x}+\frac{I}{3 x^{3}}+\frac{I}{5 x^{5}}+\ldots$

$$
=\operatorname{coth}^{-1} x=\sum_{n=0}^{\infty} \frac{x^{-2 n-1}}{2 n+I}
$$




\subsection{0}

I.

$$
\begin{aligned}
& \frac{\mathrm{I} \cdot}{2 \sinh x}=\sum_{n=0}^{\infty} e^{-x(2 n+\mathrm{r})} . \\
& \frac{\mathrm{I}}{2 \cosh x}=\sum_{n=0}^{\infty}(-\mathrm{I})^{n} e^{-x(2 n+\mathrm{r})}
\end{aligned}
$$

3. $\frac{\mathrm{I}}{2}(\tanh x-\mathrm{I})=\sum_{n=\mathrm{I}}^{\infty}(-\mathrm{I})^{n} e^{-2 n x}$.

4. $-\frac{I}{2} \log \tanh \frac{x}{2}=\sum_{n=0}^{\infty} \frac{I}{2 n+I} e^{-x(2 n+I) .}$

\subsection{1}

$$
\frac{\mathrm{I}}{2}+\sum_{n=\mathrm{I}}^{\infty} e^{-(n x)^{2}}=\frac{\sqrt{\pi}}{x}\left\{\frac{\mathrm{I}}{2}+\sum_{n=\mathrm{I}}^{\infty} e^{-\left(\frac{n \pi}{x}\right)^{2}}\right\}
$$

By means of this formula a slowly converging series may be transformed into a rapidly converging series.

\subsection{5}

I. $\tan x=2 x\left\{\frac{\mathrm{I}}{\left(\frac{\pi}{2}\right)^{2}-x^{2}}+\frac{\mathrm{I}}{\left(\frac{3 \pi}{2}\right)^{2}-x^{2}}+\frac{\mathrm{I}}{\left(\frac{5 \pi}{2}\right)^{2}-x^{2}}+\ldots\right\}$

$$
=\sum_{n=1}^{\infty} \frac{8 x}{(2 n-1)^{2} \pi^{2}-4 x^{2}}
$$

2. $\cot x=\frac{I}{x}-\frac{2 x}{\pi^{2}-x^{2}}-\frac{2 x}{(2 \pi)^{2}-x^{2}}-\frac{2 x}{(3 \pi)^{2}-x^{2}}-\ldots=\frac{I}{x}-\sum_{n=\mathbf{I}}^{\infty} \frac{2 x}{n^{2} \pi^{2}-x^{2}}$. 3. $\sec x=\frac{\pi}{\left(\frac{\pi}{2}\right)^{2}-x^{2}}-\frac{3 \pi}{\left(\frac{3 \pi}{2}\right)^{2}-x^{2}}+\frac{5 \pi}{\left(\frac{5 \pi}{2}\right)^{2}-x^{2}}-\ldots$

$$
=\sum_{n=1}^{\infty}(-I)^{n-1} \frac{4(2 n-I) \pi}{(2 n-I)^{2} \pi^{2}-4 x^{2}} \text {. }
$$

4. $\csc x=\frac{1}{x}+\frac{2 x}{\pi^{2}-\lambda^{2}}-\frac{2 x}{(2 \pi)^{2}-x^{2}}+\frac{2 x}{(3 \pi)^{2}-x^{2}}-\ldots$

$$
=\frac{\mathrm{I}}{x}+\sum_{n=\mathrm{r}}^{\infty}(-\mathrm{I})^{n-1} \frac{2 x}{n^{2} \pi^{2}-x^{2}} .
$$

By replacing $x$ by $i x$ the corresponding series for the hyperbolic functions may be written. 


\subsection{0}

I. $\sin x=x \prod_{n=\mathrm{I}}^{\infty}\left(\mathrm{I}-\frac{x^{2}}{n^{2} \pi^{2}}\right)$.

2. $\sinh x=x \prod_{n=I}^{\infty}\left(1+\frac{x^{2}}{n^{2} \pi^{2}}\right)$.

3. $\cos x=\prod_{n=0}^{\infty}\left(\mathrm{I}-\frac{4 x^{2}}{(2 n+\mathrm{I})^{2} \pi^{2}}\right)$.

4. $\cosh x=\prod_{n=0}^{\infty}\left(\mathrm{I}+\frac{4 x^{2}}{(2 n+\mathrm{I})^{2} \pi^{2}}\right)$.

\subsection{1}

I. $\frac{\sin x}{x}=\prod_{n=1}^{\infty} \cos \frac{x}{2^{n}}$.

\subsection{2}

I. $\frac{\mathrm{I}}{\mathrm{I}-x} \quad=\prod_{n=0}^{\infty}\left(\mathrm{I}+x^{2 n}\right)$.

\subsection{3}

I. $\cosh x-\cos y=2\left(\mathrm{I}+\frac{x^{2}}{y^{2}}\right) \sin ^{2} \frac{y}{2} \prod_{n=1}^{\infty}\left(\mathrm{I}+\frac{x^{2}}{(2 n \pi+y)^{2}}\right)\left(\mathrm{I}+\frac{x^{2}}{(2 n \pi-y)^{2}}\right)$.

2. $\cos x-\cos y=2\left(I-\frac{x^{2}}{y^{2}}\right) \sin ^{2} \frac{y}{2} \prod_{n=1}^{\infty}\left(I-\frac{x^{2}}{(2 n \pi+y)^{2}}\right)\left(I-\frac{x^{2}}{(2 n \pi-y)^{2}}\right)$.

6.55 The convergent infinite series:

$$
\mathrm{I}+u_{1}+u_{2}+\ldots .=\mathrm{I}+\sum_{n=1}^{\infty} u_{n}
$$


may be transformed into the infinite product

$$
\begin{aligned}
& \left(I+v_{1}\right)\left(I+v_{2}\right)\left(I+v_{3}\right) \ldots \ldots \\
& =\prod_{n=I}^{\infty}\left(I+v_{n}\right),
\end{aligned}
$$

where

$$
v_{n}=\frac{u_{n}}{I+u_{1}+u_{2}+\ldots+u_{n-1}}
$$

6.600 The Gamma Function:

$$
\Gamma(z)=\frac{\mathrm{I}}{z} \prod_{n=\mathrm{I}}^{\infty} \frac{\left(\mathrm{I}+\frac{\mathrm{I}}{n}\right)^{z}}{\mathrm{I}+\frac{z}{n}},
$$

$z$ may have any real or complex value, except $0,-1,-2,-3, \ldots \ldots$

6.601

$$
\frac{I}{\Gamma(z)}=z e^{\gamma z} \prod_{n=1}^{\infty}\left(\mathrm{I}+\frac{z}{n}\right) e^{-\frac{z}{n}} .
$$

6.602

$$
\begin{aligned}
\gamma & =\operatorname{Limit}_{m \rightarrow \infty}\left\{\mathrm{I}+\frac{\mathrm{I}}{2}+\frac{\mathrm{I}}{3}+\ldots .+\frac{\mathrm{I}}{m}-\log m\right\} \\
& =\int_{0}^{\infty}\left\{\frac{e^{-t}}{\mathrm{I}-e^{-t}}-\frac{e^{-t}}{t}\right\} d t=0.5772 \mathrm{I} 57 \ldots
\end{aligned}
$$

6.603

$$
\begin{aligned}
\Gamma(z+1) & =z \Gamma(z) \\
\Gamma(z) \Gamma(1-z) & =\frac{\pi}{\sin \pi z} .
\end{aligned}
$$

6.604 For $z$ real and positive $=x$ :

$$
\Gamma(x)=\int_{0}^{\infty} e^{-t} t^{x-1} d t
$$

$\log \Gamma(\mathrm{I}+x)=\left(x+\frac{\mathrm{I}}{2}\right) \log x-x+\frac{\mathrm{I}}{2} \log 2 \pi+\int_{0}^{\infty}\left\{\frac{\mathrm{I}}{e^{t}-\mathrm{I}}-\frac{\mathrm{I}}{t}+\frac{\mathrm{I}}{2}\right\} e^{-x t} \frac{d t}{t}$

6.605 If $z=n$, a positive integer:

$$
\begin{aligned}
\Gamma(n) & =(n-\mathrm{I}) ! \\
\Gamma\left(n+\frac{\mathrm{I}}{2}\right) & =\frac{\mathrm{I} \cdot 3 \cdot 5 \cdots(2 n-\mathrm{I})}{2^{n}} \sqrt{\pi} \\
\Gamma\left(\frac{1}{2}\right) & =\sqrt{\pi}
\end{aligned}
$$


6.606 The Beta Function. If $x$ and $y$ are real and positive:

$$
\begin{aligned}
\mathrm{B}(x, y) & =\mathrm{B}(y, x)=\frac{\Gamma(x) \Gamma(y)}{\Gamma(x+y)}, \\
\mathrm{B}(x, y) & =\int_{0}^{1} t^{x-1}(\mathrm{I}-t)^{y-1} d t \\
\mathrm{~B}(x+\mathrm{r}, y) & =\frac{x}{x+y} \mathrm{~B}(x, y), \\
\mathrm{B}(x, \mathrm{I}-x) & =\frac{\pi}{\sin \pi x} .
\end{aligned}
$$

6.610 For $x$ real and positive:

6.611

$$
\psi(x)=\frac{\Gamma^{\prime}(x)}{\Gamma(x)}=-\gamma-\sum_{n=0}^{\infty}\left(\frac{\mathrm{I}}{x+n}-\frac{\mathrm{I}}{n+\mathrm{I}}\right) .
$$

6.612

$$
\begin{aligned}
& \psi(x+\mathrm{I})=\frac{\mathrm{r}}{x}+\psi(x), \\
& \psi(\mathrm{I}-x)=\psi(x)+\pi \cot \pi x .
\end{aligned}
$$

$$
\begin{aligned}
& \psi\left(\frac{1}{2}\right)=-\gamma-2 \log 2 \\
& \psi(I)=-\gamma \\
& \psi(2)=I-\gamma \\
& \psi(3)=I+\frac{I}{2}-\gamma \\
& \psi(4)=I+\frac{I}{2}+\frac{I}{3}-\gamma
\end{aligned}
$$

\subsection{3}

$$
\begin{aligned}
\psi(x) & =\int_{0}^{\infty}\left\{\frac{e^{-t}}{t}-\frac{e^{-t x}}{\mathrm{I}-e^{-t}}\right\} d t \\
& =-\gamma+\int_{0}^{1} \frac{\mathrm{I}-t^{x-1}}{\mathrm{I}-t} d t
\end{aligned}
$$


6.620

6.621

$$
\begin{aligned}
\beta(x) & =\sum_{n=0}^{\infty} \frac{(-\mathrm{I})^{n}}{x+n} \\
& =\frac{I}{2}\left\{\psi\left(\frac{x+\mathrm{I}}{2}\right)-\psi\left(\frac{x}{2}\right)\right\} .
\end{aligned}
$$

6.622

$$
\begin{aligned}
& \beta(x+\mathrm{I})+\beta(x)=\frac{\mathrm{I}}{x} \\
& \beta(x)+\beta(\mathrm{I}-x)=\frac{\pi}{\sin \pi x}
\end{aligned}
$$

$$
\begin{aligned}
& \beta(\mathrm{I})=\log 2, \\
& \beta\left(\frac{\mathrm{I}}{2}\right)=\frac{\pi}{2} .
\end{aligned}
$$

6.630 Gauss's II Function:

I. $\Pi(k, z)=k^{2} \prod_{n=\mathrm{x}}^{k} \frac{n}{z+n}$.

2. $\Pi(k, z+\mathrm{I})=\Pi(k, z) \cdot \frac{\mathrm{I}+z}{\mathrm{I}+\frac{\mathrm{I}+z}{k}}$.

3. $\Pi(z)={ }_{k \rightarrow \infty}^{\text {Limit }} \Pi(k, z)$.

4. $\Pi(z)=\Gamma(z+1)$.

5. $\Pi(-z) \Pi(z-\mathrm{I})=\pi \csc \pi z$.

6. $\Pi\left(\frac{I}{2}\right)=\frac{I}{2} \sqrt{\pi}$

6.631 If $z$ is an integer, $n$,

$$
\Pi(n)=n !
$$

DEFINITE INTEGRALS EXPRESSED AS INFINITE SERIES

6.700

$$
\begin{aligned}
\int_{0}^{x} e^{-x^{2}} d x & =\sum_{k=0}^{\infty} \frac{(-\mathrm{I}) k}{k !(2 k+\mathrm{I})} x^{2 k+1} \\
& =e^{-x^{2}} \sum_{k=0}^{\infty} \frac{2^{k} x^{2 k+1}}{\mathrm{I} \cdot 3 \cdot 5 \cdots(2 k+\mathrm{I})}
\end{aligned}
$$


Darling (Quarterly Journal, 49, p. 36, 1920) has obtained an approximation to this integral:

Fresnel's Integrals:

$$
\frac{\sqrt{\pi}}{2}-\frac{2}{\sqrt{\pi}} \tan ^{-1}\left\{e^{\sqrt{\pi}}\left(\mathrm{I}+x^{2} e^{-\sqrt{\pi}}\right)^{2}\right\}^{-x}
$$

$$
\begin{aligned}
6.701 \int_{0}^{x} \cos \left(x^{2}\right) d x & =\sum_{k=0}^{\infty} \frac{(-\mathrm{I})^{k}}{(2 k) !(4 k+\mathrm{I})} x^{4 k+1} \\
& =\cos \left(x^{2}\right) \sum_{k_{i}=0}^{\infty}(-\mathrm{I})^{k} \frac{2^{2 k} x^{4 k+1}}{\mathrm{I} \cdot 3 \cdot 5 \cdots(4 k+\mathrm{I})} \\
& +\sin \left(x^{2}\right) \sum_{k=0}^{\infty}(-\mathrm{I})^{k} \frac{2^{2 k+1} x^{4 k+3}}{\mathrm{I} \cdot 3 \cdot 5 \cdots(4 k+3)}
\end{aligned}
$$

$6.702 \int_{0}^{x} \sin \left(x^{2}\right) d x=\sum_{k=0}^{\infty} \frac{(-\mathrm{I})^{k}}{(2 k+\mathrm{I}) !(4 k+3)} x^{4 k+3}$

$$
\begin{aligned}
& =\sin \left(x^{2}\right) \sum_{k^{\prime}=0}^{\infty}(-\mathrm{I})^{k} \frac{2^{2 k}}{\mathrm{I} \cdot 3 \cdot 5 \cdots(4 k+\mathrm{I})} x^{4 k+1} \\
& -\cos \left(x^{2}\right) \sum_{k=0}^{\infty}(-\mathrm{I})^{k} \frac{2^{2 k+1} x^{4 k+3}}{\mathrm{I} \cdot 3 \cdot 5 \cdots(4 k+3)} \cdot
\end{aligned}
$$

$6.703 \int_{0}^{1} \frac{t^{a-1}}{\mathrm{I}+t^{b}} d t=\sum_{n=0}^{\infty}(-\mathrm{I})^{n} \frac{\mathrm{I}}{a+n b}$

$6.704 \frac{\mathrm{I}}{(k-\mathrm{I}) !} \int_{0}^{1} \frac{t^{a-1}(\mathrm{I}-t)^{k-1}}{\mathrm{I}-x t^{b}} d t$

$$
=\sum_{n=0}^{\infty} \frac{x^{n}}{(a+n b)(a+n b+\mathrm{I})(a+n b+2) \ldots(a+n b+k-\mathrm{I})}
$$

(Special cases, 6.445 and 6.923).
6.705

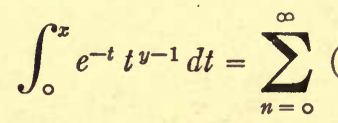

$$
(-\mathrm{I})^{n} \frac{x^{n+y}}{n !(n+y)}=e^{-x} \sum_{n=0}^{\infty}
$$$$
\frac{x^{n+y}}{y(y+1) \cdots(y+n)}
$$

6.706 If the sum of the series,

is known, then

$$
f(x)=\sum_{n=0}^{\infty} c_{n} x^{n}
$$

$$
\begin{aligned}
\sum_{n=0}^{\infty} \frac{c_{n} x^{n}}{(a+n b)(a+n b+\mathrm{I})(a+n b+2) \ldots} & \\
& =\frac{\mathrm{I}}{(k-\mathrm{I}) !} \int_{0}^{1} t^{a-1}(\mathrm{I}-t)^{k-1} f\left(x t^{b}\right) d t .
\end{aligned}
$$


$6.707 \int_{0}^{\infty} f(x) \sum_{n=\mathrm{I}}^{\infty} \frac{\mathrm{I}}{n} \sin n x \cdot d x=\frac{\mathrm{I}}{2} \int_{0}^{2 \pi}(\pi-t) \sum_{n=0}^{\infty} f(t+2 n \pi) \cdot d t$.

Example I. $\quad f(x)=e^{-k x}$

$[k>0]$.

I. $\quad \frac{\mathbf{I}}{k}+2 k \sum_{n=\mathbf{I}}^{\infty} \frac{\mathbf{I}}{k^{2}+n^{2}}=\pi \frac{e^{k \pi}+e^{-k \pi}}{e^{k \pi}-e^{-k \pi}}$.

Replacing $k$ by $\frac{k}{2}$, and subtracting,

$$
\frac{\mathrm{I}}{k}+2 k \sum_{n=\mathbf{I}}^{\infty}(-\mathrm{I})^{n} \frac{\mathrm{I}}{k^{2}+n^{2}}=\frac{2 \pi}{e^{k \pi}-e^{-k \pi}} .
$$

Example 2. With $f(x)=e^{-\lambda x} \cos \mu x$ and $e^{-\lambda x} \sin \mu x$.

3. $\frac{\lambda}{\lambda^{2}+\mu^{2}}+\sum_{n=1}^{\infty}\left\{\frac{\lambda}{\lambda^{2}+(n-\mu)^{2}}+\frac{\lambda}{\lambda^{2}+(n+\mu)^{2}}\right\}=\frac{\pi \sinh 2 \lambda \pi}{\cosh 2 \lambda \pi-\cos 2 \mu \pi}$.

4. $\frac{\mu}{\lambda^{2}+\mu^{2}}-\sum_{n=1}^{\infty}\left\{\frac{n-\mu}{\lambda^{2}+(n-\mu)^{2}}+\frac{n+\mu}{\lambda^{2}+(n+\mu)^{2}}\right\}=\frac{\pi \sin 2 \mu \pi}{\cosh 2 \lambda \pi-\cos 2 \mu \pi}$.

6.709 If the sum of the series,

is known, then

$$
f(x)=\sum_{n=0}^{\infty} a_{n} x^{n}
$$

$a_{0}+a_{1} y+a_{2} y(y+\mathrm{I})+a_{3} y(y+\mathrm{I})(y+2)+\ldots \ldots=\frac{\int_{0}^{\infty} e^{-t} t^{y-1} f(t) d t}{\Gamma(y)}$.

6.710. The complete elliptic integral of the first kind:

$$
\begin{aligned}
K & =\int_{0}^{\mathrm{I}} \frac{d x}{\sqrt{\left(\mathrm{I}-x^{2}\right)\left(\mathrm{I}-k^{2} x^{2}\right)}}=\int_{0}^{\frac{\pi}{2}} \frac{d \theta}{\sqrt{\mathrm{I}-k^{2} \sin ^{2} \theta}} \\
& =\frac{\pi}{2}\left\{\mathrm{I}+\left(\frac{\mathrm{I}}{2}\right)^{2} k^{2}+\left(\frac{\mathrm{I} \cdot 3}{2 \cdot 4}\right)^{2} k^{4}+\ldots .\right\} \\
& =\frac{\pi}{2}\left\{\mathrm{I}+\sum_{n=\mathrm{I}}^{\infty}\left(\frac{\mathrm{I} \cdot 3 \cdot 5 \ldots(2 n-\mathrm{I})}{2 \cdot 4 \cdot 6 \ldots 2 n}\right)^{2} k^{2 n}\right\}
\end{aligned}
$$

If

$$
\begin{aligned}
k^{\prime} & =\frac{\mathrm{I}-\sqrt{\mathrm{I}-k^{2}}}{\mathrm{I}+\sqrt{\mathrm{I}-k^{2}}} \\
K & =\frac{\pi\left(\mathrm{I}+k^{\prime}\right)}{2}\left\{\mathrm{I}+\left(\frac{\mathrm{I}}{2}\right) 2 k^{\prime 2}+\left(\frac{\mathrm{I} \cdot 3}{2 \cdot 4}\right)^{2} k^{\prime 4}+\ldots\right\} \\
& =\frac{\pi\left(\mathrm{I}+k^{\prime}\right)}{2}\left\{\mathrm{I}+\sum_{n=\mathrm{I}}^{\infty}\left(\frac{\mathrm{I} \cdot 3 \cdot 5 \ldots(2 n-\mathrm{I})}{2 \cdot 4 \cdot 6 \ldots 2 n}\right)^{2} k^{\prime 2 n}\right\} .
\end{aligned}
$$


6.711 The complete elliptic integral of the second kind:

$$
\begin{aligned}
E & =\int^{\frac{\pi}{2}} \sqrt{\mathrm{I}-k^{2} \sin ^{2} \theta} d \theta . \\
E & =\frac{\pi}{2}\left\{\mathrm{I}-\left(\frac{\mathrm{I}}{2}\right)^{2} \frac{k^{2}}{\mathrm{I}}-\left(\frac{\mathrm{I} \cdot 3}{2 \cdot 4}\right)^{2} \frac{k^{4}}{3} \ldots \ldots\right\} . \\
& =\frac{\pi}{2}\left\{\mathrm{I}-\sum_{n=\mathrm{I}}^{\infty}\left(\frac{\mathrm{I} \cdot 3 \cdot 5 \ldots(2 n-\mathrm{I})}{2 \cdot 4 \cdot 6 \ldots .2 n}\right)^{2} \frac{k^{2 n}}{2 n-\mathrm{I}} .\right. \\
\text { If } \quad & k^{\prime}=\frac{\mathrm{I}-\sqrt{\mathrm{I}-k^{2}}}{\mathrm{I}+\sqrt{\mathrm{I}-k^{2}} \cdot} \\
E= & \frac{\pi\left(\mathrm{I}-k^{\prime}\right)}{2}\left\{\mathrm{I}+5\left(\frac{\mathrm{I}}{2}\right)^{2} k^{\prime 2}+9\left(\frac{\mathrm{I} \cdot 3}{2 \cdot 4}\right)^{2} k^{\prime 4}+\ldots\right\} \\
= & \frac{\pi\left(\mathrm{I}-k^{\prime}\right)}{2}\left\{\mathrm{I}+\sum_{n=1}^{\infty}(4 n+\mathrm{I})\left(\frac{\mathrm{I} \cdot 3 \cdot 5 \cdots(2 n-\mathrm{I})}{2 \cdot 4 \cdot 6 \ldots 2 n}\right)^{2} k^{\prime 2 n}\right\} \\
= & \frac{\pi}{2\left(\mathrm{I}+k^{\prime}\right)}\left\{\mathrm{I}+\left(\frac{\mathrm{I}}{2}\right)^{2} k^{\prime 2}+\left(\frac{\mathrm{I}}{2 \cdot 4}\right)^{2} k^{\prime 4}+\left(\frac{\mathrm{I} \cdot 3}{2 \cdot 4 \cdot 6}\right)^{2} k^{\prime 6}+\ldots\right\} \\
= & \frac{\pi}{2\left(\mathrm{I}+k^{\prime}\right)}\left\{\mathrm{I}+k^{\prime 2}\left[\frac{\mathrm{I}}{4}+\sum_{n=1}^{\infty}\left(\frac{\mathrm{I} \cdot 3 \cdot \ldots(2 n-\mathrm{I})}{2 \cdot 4 \cdot 6 \ldots(2 n+2)}\right)^{2} k^{\prime 2 n}\right]\right\} .
\end{aligned}
$$

\section{FOURIER'S SERIES}

6.800 If $f(x)$ is uniformly convergent in the interval:

$$
-c<x<+c
$$

$f(x)=\frac{\mathrm{I}}{2} b_{0}+b_{1} \cos \frac{\pi x}{c}+b_{2} \cos \frac{2 \pi x}{c}+b_{3} \cos \frac{3 \pi x}{c}+\ldots$

$$
\begin{aligned}
& +a_{1} \sin \frac{\pi x}{c}+a_{2} \sin \frac{2 \pi x}{c}+a_{3} \sin \frac{3 \pi x}{c}+\ldots \\
b_{m}= & \frac{I}{c} \int_{-c}^{+c} f(x) \cos \frac{m \pi x}{c} d x \\
a_{m}= & \frac{I}{c} \int_{-c}^{+c} f(x) \sin \frac{m \pi x}{c} d x .
\end{aligned}
$$

6.801 If $f(x)$ is uniformly convergent in the interval:

$$
0<x<c
$$

$$
\begin{aligned}
f(x)=\frac{1}{2} b_{0}+b_{1} \cos \frac{2 \pi x}{c}+b_{2} \cos \frac{4 x \pi}{c}+b_{3} \cos \frac{6 \pi x}{c}+\ldots \\
+a_{1} \sin \frac{2 \pi x}{c}+a_{2} \sin \frac{4 \pi x}{c}+a_{3} \sin \frac{6 \pi x}{c}+\ldots . \\
b_{m}=\frac{2}{c} \int_{0}^{c} f(x) \cos \frac{2 m \pi x}{c} d x \\
a_{m}=\frac{2}{c} \int_{0}^{c} f(x) \sin \frac{2 m \pi x}{c} d x .
\end{aligned}
$$





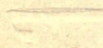




6.802 Special Developments in Fourier's Series.

$$
\begin{aligned}
& f(x)=a \text { from } x=k c \text { to } x=\left(k+\frac{\mathrm{I}}{2}\right) c \\
& f(x)=-a \text { from } x=\left(k+\frac{\mathrm{I}}{2}\right) c \text { to } x=(k+\mathrm{I}) c,
\end{aligned}
$$

where $k$ is any integer, including $\circ$.

$$
f(x)=\frac{4 a}{\pi} \sum_{n=\mathrm{I}}^{\infty} \frac{\mathrm{I}}{2 n-\mathrm{I}} \sin \frac{2(2 n-\mathrm{I}) \pi}{c} x .
$$

6.803

$$
\begin{aligned}
f(x) & =m x, & & -\frac{c}{4} \leqslant x \leqslant+\frac{c}{4} \\
& =-m\left(x-\frac{c}{2}\right), & & \frac{c}{4} \leqslant x \leqslant \frac{3 c}{4} \\
& =m(x-c), & & \frac{3 c}{4} \leqslant x \leqslant \frac{5 c}{4} \\
& =-m\left(x-\frac{3 c}{2}\right), & & \frac{5 c}{4} \leqslant x \leqslant \frac{7 c}{4}
\end{aligned}
$$

$$
f(x)=\frac{2 m c}{\pi^{2}} \sum_{n=I}^{\infty}(-\mathrm{I})^{n-1} \frac{\mathrm{I}}{(2 n-\mathrm{I})^{2}} \sin \frac{2(2 n-\mathrm{I}) \pi}{c} x
$$

6.804

$$
\begin{aligned}
& f(x)=m x, \\
& -\frac{c}{2}<x<+\frac{c}{2} \\
& =m(x-c), \quad+\frac{c}{2}<x<\frac{3 c}{2}, \\
& f(x)=\frac{c m}{\pi} \sum_{n=1}^{\infty} \frac{(-\mathrm{I})^{n-1}}{n} \sin \frac{2 n \pi x}{c} . \\
& f(x)=-a, \quad-5^{b} \leqslant x \leqslant-3 b, \\
& =\frac{a}{b}(x+2 b), \quad-3 b \leqslant x \leqslant-b, \\
& =a, \quad-b \leqslant x \leqslant+b \text {, } \\
& =-\frac{a}{b}(x-2 b), \quad b \leqslant x \leqslant 3 b, \\
& =-a, \quad 3 b \leqslant x \leqslant 5 b . \\
& f(x)=\frac{8 \sqrt{2} a}{\pi^{2}}\left\{\cos \frac{\pi x}{4^{b}}-\frac{\mathrm{I}}{3^{2}} \cos \frac{3 \pi x}{4 b}-\frac{\mathrm{I}}{5^{2}} \cos \frac{7 \pi x}{4 b}+\frac{\mathrm{I}}{7^{2}} \cos \frac{7 \pi x}{4 b}\right. \\
& +\ldots\}
\end{aligned}
$$$$
6.805
$$ 
6.806

$$
\begin{array}{rlrl}
f(x) & =\frac{b}{l} x+b, & -l \leqslant x \leqslant 0, \\
& =-\frac{b}{l} x+b, \quad 0 \leqslant x \leqslant l . \\
f(x) & =\frac{8 b}{\pi^{2}} \sum_{n=0}^{\infty} \frac{\mathrm{I}}{(2 n+\mathrm{I})^{2}} \cos (2 n+\mathrm{I}) \frac{\pi x}{2 l} .
\end{array}
$$

6.807

$$
\begin{array}{rlrl}
f(x) & =\frac{a}{b} x, & 0 \leqslant x \leqslant b, \\
& =-\frac{a}{l-b} x+\frac{a l}{l-b}, \quad b \leqslant x \leqslant l, \\
f(x) & =\frac{2 a l^{2}}{\pi^{2} b(l-b)} \sum_{n=1}^{\infty} \frac{\mathrm{I}}{n^{2}} \sin \frac{n \pi b}{l} \sin \frac{n \pi x}{l} .
\end{array}
$$

$6.810 x=2 \sum_{n=1}^{\infty} \frac{(-1)^{n-1}}{n} \sin n x$

$[-\pi<x<\pi]$

$6.811 \cos a x=\frac{2}{\pi} \sin a \pi\left\{\frac{\mathrm{I}}{2 a}+a \sum_{n=\mathrm{I}}^{\infty} \frac{(-\mathrm{I})^{n-1}}{n^{2}-a^{2}} \cos n x\right\}$

$[-\pi<x<\pi]$

$6.812 \sin a x=\frac{2}{\pi} \sin a \pi \sum_{n=\mathrm{I}}^{\infty} \frac{(-\mathrm{I})^{n-1}}{n^{2}-a^{2}} n \sin n x$

$[-\pi<x<\pi]$

$6.813 \frac{\pi-x}{2}=\sum_{n=1}^{\infty} \frac{\sin n x}{n}$

$[0<x<2 \pi]$

$6.814 \frac{\mathrm{I}}{2} \log \frac{\mathrm{I}}{2(\mathrm{I}-\cos x)}=\sum_{n=1}^{\infty} \frac{\cos n x}{n}$

$6.815 \frac{\pi^{2}}{6}-\frac{\pi x}{2}+\frac{x^{2}}{4}=\sum_{n=1}^{\infty} \frac{\cos n x}{n^{2}}$

$[0<x<2 \pi]$

$6.816 \frac{\pi^{2} x}{6}-\frac{\pi x^{2}}{4}+\frac{x^{3}}{\mathrm{I} 2}=\sum_{n=1}^{\infty} \frac{\sin n x}{n^{3}}$

$[0<x<2 \pi]$

$6.817 \frac{\pi^{4}}{90}-\frac{\pi^{2} x^{2}}{12}+\frac{\pi x^{3}}{12}-\frac{x^{4}}{48}=\sum_{n=1}^{\infty} \frac{\cos n x}{n^{4}}$

$[0<x<2 \pi]$.

$6.818 \frac{\pi^{4} x}{90}-\frac{\pi^{2} x^{3}}{36}+\frac{\pi x^{4}}{48}-\frac{x^{5}}{240}=\sum_{n=1}^{\infty} \frac{\sin n x}{n^{5}}$ 
$6.820 x^{2}=\frac{c^{2}}{3}-\frac{4 c^{2}}{\pi^{2}} \sum_{n=1}^{\infty} \frac{(-\mathrm{I})^{n-1}}{n^{2}} \cos \frac{n \pi x}{c}$ $[-c \leqslant x \leqslant c]$

$6.821 \frac{e^{x}}{e^{c}-e^{-c}}=\frac{\mathrm{I}}{2 c}-c \sum_{n=\mathrm{I}}^{\infty}(-\mathrm{I})^{n-1} \frac{\mathrm{I}}{(n \pi)^{2}+c^{2}} \cos \frac{n \pi x}{c}$

$$
+\pi \sum_{n=1}^{\infty}(-\mathrm{I})^{n-1} \frac{\mathrm{I}}{(n \pi)^{2}+c^{2}} \sin \frac{n \pi x}{c} \quad[-c<x<c]
$$

$6.822 e^{c x}=\frac{2 c}{\pi}\left(e^{c \pi}-\mathrm{I}\right)\left\{\frac{\mathrm{I}}{2 c^{2}}-\sum_{n=\mathrm{I}}^{\infty}(-\mathrm{I})^{n-1} \frac{\mathrm{I}}{c^{2}+n^{2}} \cos n x\right\} \quad[0<x<\pi]$.

$6.823 \cos 2 x-\left(\frac{\pi}{2}-x\right) \sin 2 x+\sin ^{2} x \log \left(4 \sin ^{2} x\right)=\sum_{n=1}^{\infty} \frac{\cos 2(n+\mathrm{I}) x}{n(n+\mathrm{I})}$

$6.824 \sin 2 x-(\pi-2 x) \sin ^{2} x-\sin x \cos x \log \left(4 \sin ^{2} x\right)$

$[0 \leqslant x \leqslant \pi]$.

$$
=\sum_{n=\mathbf{x}}^{\infty} \frac{\sin 2(n+\mathrm{I}) x}{n(n+\mathrm{I})}[0 \leqslant x \leqslant \pi] \text {. }
$$

$6.825 \frac{\mathrm{I}}{2}-\frac{\pi}{4} \sin x=\sum_{n=\mathrm{I}}^{\infty} \frac{\cos 2 n x}{(2 n-\mathrm{I})(2 n+\mathrm{I})}$

$\left[0 \leqslant x \leqslant \frac{\pi}{2}\right]$

$6.830 \frac{r \sin x}{I-2 r \cos x+r^{2}}=\sum_{n=I}^{\infty} r^{n} \sin n x$

$\left[r^{2}<\mathrm{I}\right]$

$6.831 \tan ^{-1} \frac{r \sin x}{\mathrm{I}-r \cos x}=\sum_{n=\mathrm{I}}^{\infty} \frac{\mathrm{I}}{n} r^{n} \sin n x$

$[r<\mathrm{I}]$

$6.832 \frac{\mathrm{I}}{2} \tan ^{-1} \frac{2 r \sin x}{\mathrm{I}-r^{2}}=\sum_{n=1}^{\infty} \frac{r^{2 n-1}}{2 n-\mathrm{I}} \sin (2 n-\mathrm{I}) x$

$\left[r^{2}<\mathrm{I}\right]$

$6.833 \frac{\mathrm{I}-r \cos x}{\mathrm{I}-2 r \cos x+r^{2}}=\sum_{n=0}^{\infty} r^{n} \cos n x$

$\left[r^{2}<\mathrm{I}\right]$

$6.834 \operatorname{og} \frac{\mathrm{I}}{\sqrt{\mathrm{I}-2 r \cos x+r^{2}}}=\sum_{n=\mathrm{I}}^{\infty} \frac{\mathrm{I}}{n} r^{n} \cos n x$ 
$6.835 \frac{\mathrm{I}}{2} \tan ^{-1} \frac{2 r \cos x}{\mathrm{I}-r^{2}}=\sum_{n=\mathrm{I}}^{\infty}(-\mathrm{I})^{n-1} \frac{r^{2 n-1}}{2 n-\mathrm{I}} \cos (2 n-\mathrm{I}) x$ $\left[r^{2}<\mathrm{I}\right]$

6.900

\author{
NUMERICAL SERIES
}

$$
\begin{array}{ll}
S_{n}=\frac{\mathrm{I}}{\mathrm{I}^{n}}+\frac{\mathrm{I}}{2^{n}}+\frac{\mathrm{I}}{3^{n}}+\frac{\mathrm{I}}{4^{n}}+\ldots=\sum_{k=\mathrm{I}}^{\infty} \frac{\mathrm{I}}{k^{n}}, \\
S_{1}=\infty & S_{6}=\frac{\pi^{6}}{945}=\mathrm{I} .0 \mathrm{I} 73430620, \\
S_{2}=\frac{\pi^{2}}{6}=\mathrm{I} .6449340668 & S_{7}=\frac{\pi^{7}}{2995.286}=\mathrm{I} .0083492774 \\
S_{3}=\frac{\pi^{3}}{25.79436}=\mathrm{I} .2020569032 & S_{8}=\frac{\pi^{8}}{945^{\circ}}=\mathrm{I} .0040773562, \\
S_{4}=\frac{\pi^{4}}{90}=\mathrm{I} .0823232337 & S_{9}=\frac{\pi^{9}}{29749.35}=\mathrm{r} .0020083928, \\
S_{5}=\frac{\pi^{5}}{295 . \mathrm{I} 2 \mathrm{I} 5}=\mathrm{I} .036927755 \mathrm{I} & S_{10}=\mathrm{r} .000994575 \mathrm{I}, \\
S_{11}=\mathrm{I} .000494 \mathrm{I} 886 .
\end{array}
$$

6.901

$$
\begin{aligned}
& u_{n}=\mathrm{I}-\frac{\mathrm{I}}{3^{n}}+\frac{\mathrm{I}}{5^{n}}-\frac{\mathrm{I}}{7^{n}}+\ldots \ldots=\sum_{k=0}^{\infty}(-\mathrm{I})^{k-1} \frac{\mathrm{I}}{(2 k+\mathrm{I})^{n}} \\
& u_{1}=\frac{\pi}{4} \\
& u_{2}=0.9 \mathrm{I} 59656 \ldots \\
& u_{4}=0.98894455 \ldots \\
& u_{6}=0.998685^{22} \ldots
\end{aligned}
$$

A table of $u_{n}$ from $n=\mathrm{I}$ to $n=38$ to $\mathrm{I} 8$ decimal places is given by Glaisher, Messenger of Mathematics, 42, p. 49, I9r3.

6.902 Bernoulli's Numbers.

$$
\begin{aligned}
& \text { I. } \frac{2^{2 n-1} \pi^{2 n}}{(2 n) !} B_{n}=\frac{\mathrm{I}}{\mathrm{I}^{2 n}}+\frac{\mathrm{I}}{2^{2 n}}+\frac{\mathrm{I}}{3^{2 n}}+\frac{\mathrm{I}}{4^{2 n}}+\ldots=\sum_{k=\mathrm{I}}^{\infty} \frac{\mathrm{I}}{k^{2 n}} \text {. } \\
& \text { 2. } \frac{\left(2^{2 n}-\mathrm{I}\right) \pi^{2 n}}{2(2 n) !} B_{n}=\frac{\mathrm{I}}{\mathrm{I}^{2 n}}+\frac{\mathrm{I}}{3^{2 n}}+\frac{\mathrm{I}}{5^{2 n}}+\frac{\mathrm{I}}{7^{2 n}}+\ldots=\sum_{k=0}^{\infty} \frac{\mathrm{I}}{(2 k+\mathrm{I})^{2 n}} \text {. } \\
& \text { 3. } \frac{\left(2^{2 n-1}-\mathrm{I}\right) \pi^{2 n}}{(2 n) !} B_{n}=\frac{\mathrm{I}}{\mathrm{I}^{2 n}}-\frac{\mathrm{I}}{2^{2 n}}+\frac{\mathrm{I}}{3^{2 n}}-\frac{\mathrm{I}}{4^{2 n}}+\ldots .=\sum_{k=\mathrm{I}}^{\infty}(-\mathrm{I})^{n-1} \frac{\mathrm{I}}{k^{2 n}} \text {. } \\
& \begin{array}{ll}
B_{1}=\frac{I}{6}, & B_{3}=\frac{I}{42} \\
B_{2}=\frac{I}{30}, & B_{4}=\frac{I}{30}
\end{array}
\end{aligned}
$$




$$
\begin{aligned}
B_{5} & =\frac{5}{66}, & B_{8} & =\frac{3617}{5 \mathrm{IO}}, \\
B_{6} & =\frac{69 \mathrm{I}}{2730} & B_{9} & =\frac{43867}{798}, \\
B_{7} & =\frac{7}{6}, & B_{10} & =\frac{\mathrm{I} 746 \mathrm{II}}{330} .
\end{aligned}
$$

6.903 Euler's Numbers

$$
\begin{array}{rlrl}
\frac{\pi^{2 n+1}}{2^{2 n+2}(2 n) !} E_{n}=\mathrm{I}-\frac{\mathrm{I}}{3^{2 n+1}} & +\frac{\mathrm{I}}{5^{2 n+1}}-\frac{\mathrm{I}}{7^{2 n+1}}+\ldots=\sum_{k=\mathrm{I}}^{\infty}(-\mathrm{I})^{k-1} \frac{\mathrm{I}}{(2 k-\mathrm{I})^{2 n+1}} . \\
E_{1} & =\mathrm{I}, & E_{4} & =\mathrm{I} 385 \\
E_{2} & =5, & E_{5} & =5052 \mathrm{I} \\
E_{3} & =6 \mathrm{I}, & E_{6} & =2702765 .
\end{array}
$$

6.904

$E_{n}-\frac{2 n(2 n-\mathrm{I})}{2 !} E_{n-1}+\frac{2 n(2 n-\mathrm{I})(2 n-2)(2 n-3)}{4 !} E_{n-2}-\ldots$

\subsection{5}$$
-\ldots \ldots+(-1)^{n}=0 \text {. }
$$

$$
\begin{aligned}
& \frac{2^{2 n}\left(2^{2 n}-\mathrm{I}\right)}{2 n} B_{n}=(2 n-\mathrm{I}) E_{n-1}-\frac{(2 n-\mathrm{I})(2 n-2)(2 n-3)}{3 !} E_{n-2} \\
& +\frac{(2 n-\mathrm{I})(2 n-2)(2 n-3)(2 n-4)(2 n-5)}{5 !} E_{n-3}-\ldots \ldots+(-\mathrm{I})^{n-1} .
\end{aligned}
$$

\subsection{0}

$$
S_{r}=\sum_{n=1}^{\infty} \frac{n^{r}}{n !}
$$

$$
\begin{array}{ll}
S_{1}=e, & S_{5}=52 e, \\
S_{2}=2 e, & S_{6}=203 e, \\
S_{3}=5 e, & S_{7}=877 e, \\
S_{4}=\mathrm{I}_{5} e, & S_{8}=4 \mathrm{I} 40 e .
\end{array}
$$

\subsection{1}

$$
S_{r}=\sum_{n=1}^{\infty} \frac{\mathrm{I}}{\left(4 n^{2}-\mathrm{I}\right)^{r}} .
$$

$$
\begin{array}{ll}
S_{1}=\frac{I}{2}, & S_{3}=\frac{32-3 \pi^{2}}{64}, \\
S_{2}=\frac{\pi^{2}-8}{I 6}, & S_{4}=\frac{\pi^{4}+30 \pi^{2}-384}{768} .
\end{array}
$$




\subsection{2}

I. $\log 2=\sum_{n=1}^{\infty} \frac{\mathrm{I}}{n \cdot 2^{n}}$.

2. $\frac{\pi^{2}}{\mathrm{I} 2}-\frac{\mathrm{I}}{2}(\log 2)^{2}=\sum_{n=\mathrm{I}}^{\infty} \frac{\mathrm{I}}{n^{2} 2^{n}}$.

\subsection{3}

I. $2 \log 2-\mathrm{I}=\sum_{n=\mathrm{I}}^{\infty} \frac{\mathrm{I}}{n\left(4 n^{2}-\mathrm{I}\right)}$.

2. $\frac{3}{2}(\log 3-\mathrm{I})=\sum_{n=1}^{\infty} \frac{\mathrm{I}}{n\left(9 n^{2}-\mathrm{I}\right)}$.

3. $-3+\frac{3}{2} \log 3+2 \log 2=\sum_{n=1}^{\infty} \frac{\mathrm{I}}{n\left(36 n^{2}-\mathrm{I}\right)}$.

\subsection{4}

$$
S_{r}=\sum_{n=1}^{\infty}\left(\frac{\mathrm{I} \cdot 3 \cdot 5 \ldots \ldots(2 n-\mathrm{I})}{2 \cdot 4 \cdot 6 \ldots \ldots 2 n}\right)^{2} \frac{\mathrm{I}}{2 n+r},
$$

$S_{0}=2 \log 2-\frac{4}{\pi} u_{2}$

$S_{1}=\frac{4}{\pi} u_{2}-\mathrm{I}$,

$S_{2}=\frac{2}{\pi}-{ }_{2}$,

$S_{3}=\frac{\mathrm{I}}{2 \pi}\left(2 u_{2}+\mathrm{I}\right)-\frac{\mathrm{I}}{3}$,

$S_{4}=\frac{\text { IO }}{9 \pi}-\frac{I}{4}$

$S_{5}=\frac{\mathrm{I}}{32 \pi}\left(\mathrm{I} 8 u_{2}+\mathrm{I} 3\right)-\frac{\mathrm{I}}{5}$,

$S_{6_{s}}=\frac{178}{225 \pi}-\frac{I}{6}$

$S_{7}=\frac{\mathrm{I}}{\mathrm{I} 28 \pi}\left(50 u_{2}+43\right)-\frac{\mathrm{I}}{7}$

When $r$ is a negative even integer the value $n=\frac{r}{2}$ is to be excluded in the summation.

\subsection{5}

I. $A_{n}=\frac{\mathrm{I} \cdot 3 \cdot 5 \ldots(2 n-\mathrm{I})}{2 \cdot 4 \cdot 6 \ldots 2 n}=\frac{(2 n-\mathrm{I}) !}{2^{2 n-1} n !(n-\mathrm{I}) !}$.

2. $\mathrm{I}-\frac{\pi}{4}=\sum_{n=1}^{\infty} A_{n} \frac{\mathrm{I}}{4 n^{2}-\mathrm{I}}$.

$$
\begin{aligned}
& S_{-1}=\mathrm{I}-\frac{2}{\pi} \\
& S_{-2}=\frac{\mathrm{I}}{2} \log 2+\frac{\mathrm{I}}{4}-\frac{\mathrm{I}}{2 \pi}\left(2 u_{2}+\mathrm{I}\right) \\
& S_{-3}=\frac{\mathrm{I}}{3}-\frac{\mathrm{IO}}{9 \pi} \\
& S_{-4}=\frac{9}{32} \log 2+\frac{\mathrm{II}}{\mathrm{I} 28}-\frac{\mathrm{I}}{32 \pi}\left(\mathrm{I} 8 u_{2}+\mathrm{I} 3\right) \\
& S_{-5}=\frac{\mathrm{I}}{5}-\frac{\mathrm{I} 78}{225 \pi} \\
& S_{-6}=\frac{25}{\mathrm{I} 28} \log 2+\frac{7 \mathrm{I}}{\mathrm{I} 536}-\frac{\mathrm{I}}{\mathrm{I} 28 \pi}\left(50 u_{2}+43\right)
\end{aligned}
$$


3. $\frac{\pi}{2}-\mathbf{I}=\sum_{n=1}^{\infty} A_{n} \frac{\mathrm{I}}{2 n+\mathrm{I}}$.

4. $\log (\mathrm{I}+\sqrt{2})-\mathrm{I}=\sum_{n=\mathrm{I}}^{\infty}(-\mathrm{I})^{n} A_{n} \frac{\mathrm{I}}{2 n+\mathrm{I}}$.

5. $\frac{I}{2}=\sum_{n=1}^{\infty} A_{n^{2}} \frac{4 n+\mathrm{I}}{(2 n-\mathrm{I})(2 n+2)}$.

6. $\frac{2}{\pi}-\frac{\mathrm{I}}{2}=\sum_{n=1}^{\infty}(-\mathrm{I})^{n+1} A_{n}{ }^{3} \frac{4 n+\mathrm{I}}{(2 n-\mathrm{I})(2 n+2)}$.

7. $\frac{2}{\pi}-\mathrm{I}=\sum_{n=\mathrm{I}}^{\infty}(-\mathrm{I})^{n} A_{n}^{3}(4 n+\mathrm{I})$.

8. $\frac{\mathrm{I}}{2}-\frac{4}{\pi^{2}}=\sum_{n=1}^{\infty} A_{n} \frac{4 n+\mathrm{I}}{(2 n-\mathrm{I})(2 n+2)}$

\subsection{6}

If $m$ is an integer, and $n=m$ is excluded from the summation:

I. $-\frac{3}{4 m^{2}}=\sum_{n=1}^{\infty} \frac{\mathrm{I}}{m^{2}-n^{2}}$.

2. $\frac{3}{4 m^{2}}=\sum_{n=\mathrm{I}}^{\infty} \frac{(-\mathrm{I})^{n-1}}{m^{2}-n^{2}} \cdot \quad(m$ even $)$

\subsection{7}

I. $\mathrm{I}=\sum_{n=2}^{\infty} \frac{n-\mathrm{I}}{n !}$

2. $\frac{\mathrm{I}}{2}=\sum_{n=\mathrm{I}}^{\infty} \frac{\mathrm{I}}{4 n^{2}-\mathrm{I}}$

3. $2 \log 2=\sum_{n=1}^{\infty} \frac{\mathrm{I} 2 n^{2}-\mathrm{I}}{n\left(4 n^{2}-\mathrm{I}\right)^{2}}$.

$6.918 \quad \frac{2}{\sqrt{3}} \log \frac{I+\sqrt{3}}{\sqrt{2}}=I+\sum_{n=1}^{\infty}(-I)^{n} \frac{2 \cdot 4 \cdot 6 \ldots \ldots 2 n}{3 \cdot 5 \cdot 7 \cdots(2 n+I)} \frac{I}{2^{n}}$.

6.919

$$
\frac{\mathrm{I}}{2}(\mathrm{I}-\log 2)=\sum_{n=\mathrm{I}}^{\infty}\left\{n \log \left(\frac{2 n+\mathrm{I}}{2 n-\mathrm{I}}\right)-\mathrm{I}\right\} \text {. }
$$

\subsection{0}

I. $e=\mathrm{I}+\frac{\mathrm{I}}{\mathrm{I} !}+\frac{\mathrm{I}}{2 !}+\frac{\mathrm{I}}{3 !}+\ldots=2.7 \mathrm{I} 828$ 
2. $\frac{\mathrm{I}}{e}=\mathrm{I}-\frac{\mathrm{I}}{\mathrm{I} !}+\frac{\mathrm{I}}{2 !}-\frac{\mathrm{I}}{3 !}-\ldots=0.36788$

3. $\frac{\mathrm{I}}{2}\left(e+\frac{\mathrm{I}}{e}\right)=\mathrm{I}+\frac{\mathrm{I}}{2 !}+\frac{\mathrm{I}}{4 !}+\ldots .=\mathrm{I} .54308$.

4. $\frac{\mathrm{I}}{2}\left(e-\frac{\mathrm{I}}{e}\right)=\mathrm{I}+\frac{\mathrm{I}}{3 !}+\frac{\mathrm{I}}{5 !}+\ldots . \mathrm{I}$. I $7520 \mathrm{I}$.

5. $\cos I=I-\frac{I}{2 !}+\frac{I}{4 !}-\ldots .=0.54030$.

6. $\sin I=I-\frac{I}{3 !}+\frac{I}{5 !}-\ldots=0.84 I 47$

\subsection{1}

I. $\frac{4}{5}=I-\frac{I}{2^{2}}+\frac{I}{2^{4}}-\frac{I}{2^{6}}+\ldots$.

2. $\frac{9}{\mathrm{IO}}=\mathrm{I}-\frac{\mathrm{I}}{3^{2}}+\frac{\mathrm{I}}{3^{4}}-\frac{\mathrm{I}}{3^{6}}+\ldots$.

3. $\frac{I 6}{I 7}=I-\frac{I}{4^{2}}+\frac{I}{4^{4}}-\frac{I}{4^{6}}+\ldots$.

4. $\frac{25}{26}=I-\frac{I}{5^{2}}+\frac{I}{5^{4}}-\frac{I}{5^{6}}+\ldots$.

$6.922 \quad \frac{\left(2^{\frac{1}{4}}-\mathrm{I}\right) \Gamma\left(\frac{1}{4}\right)}{2^{12} \pi^{\frac{3}{4}}}=e^{-\pi}+e^{-9 \pi}+e^{-25 \pi}+\ldots ; \Gamma\left(\frac{1}{4}\right)=3.6256 \ldots$

6.923 (Special cases of 6.705):
I. $\frac{I}{I \cdot 2 \cdot 3}+\frac{I}{3 \cdot 4 \cdot 5}+\frac{I}{5 \cdot 6 \cdot 7}+\ldots \cdot$
$=\log 2-\frac{I}{2}$.
2. $\frac{I}{I \cdot 2 \cdot 3}-\frac{I}{3 \cdot 4 \cdot 5}+\frac{I}{5 \cdot 6 \cdot 7}-\ldots$
$=\frac{I}{2}(I-\log 2)$.
3. $\frac{I}{2 \cdot 3 \cdot 4}+\frac{I}{4 \cdot 5 \cdot 6}+\frac{I}{6 \cdot 7 \cdot 8}+\ldots$
$=\frac{3}{4}-\log 2$.
4. $\frac{I}{2 \cdot 3 \cdot 4}-\frac{I}{4 \cdot 5 \cdot 6}+\frac{I}{6 \cdot 7 \cdot 8}-\ldots$
$=\frac{I}{4}(\pi-3)$.
5. $\frac{I}{I \cdot 2 \cdot 3}+\frac{I}{4 \cdot 5 \cdot 6}+\frac{I}{7 \cdot 8 \cdot 9}+\ldots$
$=\frac{I}{4}\left(\frac{\pi}{\sqrt{3}}-\log 3\right)$.

6. $\frac{I}{2 \cdot 3 \cdot 4}+\frac{I}{6 \cdot 7 \cdot 8}+\frac{I}{I O \cdot I I \cdot I 2}+\ldots=\frac{\pi}{8}-\frac{I}{2} \log 2$.

7. $\frac{I}{I \cdot 2 \cdot 3 \cdot 4}+\frac{I}{4 \cdot 5 \cdot 6 \cdot 7}+\frac{I}{7 \cdot 8 \cdot 9 \cdot 10}+\ldots=\frac{I}{6}\left(I+\frac{\pi}{2 \sqrt{3}}\right)-\frac{I}{4} \log 3$. 


\section{SPECIAL APPLICATIONS OF ANALYSIS.}

\subsection{Indeterminate Forms.}

$7.101 \frac{\circ}{\circ}$. If $\frac{f(x)}{F(x)}$ assumes the indeterminate value $\frac{\circ}{\circ}$ for $x=a$, the true value of the quotient may be found by replacing $f(x)$ and $F(x)$ by their developments in series, if valid for $x=a$.

Example:

Therefore,

$$
\begin{gathered}
{\left[\frac{\sin ^{2} x}{1-\cos x}\right]_{x=0} ;} \\
\frac{\sin ^{2} x}{\mathrm{I}-\cos x}=\frac{\left(x-\frac{x^{3}}{3 !}+\ldots\right)^{2}}{\frac{x^{2}}{2 !}-\frac{x^{4}}{4 !}+\ldots}=\frac{\left(\mathrm{I}-\frac{x^{2}}{3 !}+\ldots\right)^{2}}{\frac{\mathrm{I}}{2 !}-\frac{x^{2}}{4 !}+\ldots}
\end{gathered}
$$

$$
\left[\frac{\sin ^{2} x}{I-\cos x}\right]_{x=0}=2 \text {. }
$$

7.102 L'Hospital's Rule. If $f(a+h)$ and $F(a+h)$ can be developed by Taylor's Theorem (6.100) then the true value of $\frac{f(x)}{F(x)}$ for $x=a$ is,

$$
\frac{f^{\prime}(a)}{F^{\prime}(a)}
$$

provided that this has a definite value ( 0 , finite, or infinite). If the ratio of the first derivatives is still indeterminate, the true value may be found by taking that of the ratio of the first one of the higher derivatives that is definite.

7.103 The true value of $\frac{f(x)}{F(x)}$ for $x=a$ is the limit, for $h=0$, of

$$
\frac{q !}{p !} h^{p-q} \frac{f^{(p)}(a)}{F^{(q)}(a)}
$$

where $f^{(p)}(a)$ and $F^{(a)}(a)$ are the first of the higher derivatives of $f(x)$ and $F(x)$ that do not vanish for $x=a$. The true value of $\frac{f(x)}{F(x)}$ for $x=a$ is $\circ$ if $p>q, \infty$ if $p<q$, and equal to $\frac{f^{(p)}(a)}{F^{(p)}(a)}$ if $p=q$. 
Example:

$$
\begin{aligned}
& {\left[\frac{\sinh x-x \cosh x}{\sin x-x \cos x}\right]_{x=0}=\left[\frac{-x \sinh x}{x \sin x}\right]_{x=0}} \\
& =\left[-\frac{\sinh x}{\sin x}\right]_{x=0}=\left[-\frac{\cosh x}{\cos x}\right]_{x=0}=-\mathrm{I} .
\end{aligned}
$$

7.104 Failure of L'Hospital's Rule. In certain cases this rule fails to determine the true value of an expression for the reason that all the higher derivatives vanish at the limit. In such cases the true value may often be found by factoring the given expression, or resolving into partial fractions (1.61).

Example:

$$
\left[\frac{\sqrt{x^{2}-a^{2}}}{\sqrt{x-a}}\right]_{x=a}=[\sqrt{x+a}]_{x=a}=\sqrt{2 a} .
$$

7.105 In applying L'Hospital's Rule, if any of the successive quotients contains a factor which can be evaluated at once its determinate value may be substituted.

Example:

$$
\begin{aligned}
{\left[\frac{(\mathrm{I}-x) e^{x}-\mathrm{I}}{\tan ^{2} x}\right]_{x=0} } & =\left[\frac{-x e^{x}}{2 \tan x \sec _{1}^{2} x}\right]_{x=0} \\
{\left[\frac{x}{\tan x}\right]_{x=0} } & =\mathbf{I} .
\end{aligned}
$$

Hence the given function is,

$$
\left[-\frac{e^{x}}{2 \sec ^{2} x}\right]_{x=0}=-\frac{\mathbf{I}}{2}
$$

7.106 If the given function can be separated into factors each of which is indeterminate, the factors may be evaluated separately.

Example:

$$
\left[\frac{\left(e^{x}-\mathrm{I}\right) \tan ^{2} x}{x^{3}}\right]_{x=0}=\left[\left(\frac{\tan x}{x}\right)^{2} \frac{e^{x}-\mathrm{I}}{x}\right]_{x=0}=\mathbf{I} .
$$

$7.110 \frac{\infty}{\infty}$. If, for $x=a, \frac{f(x)}{F(x)}$ takes the form $\frac{\infty}{\infty}$, this quotient may be written:

$$
\frac{\frac{I}{F(x)}}{\frac{I}{f(x)}}
$$

which takes the form $\frac{0}{\circ}$ for $x=a$ and the preceding sections will apply to it.

7.111 L'Hospital's Rule (7.102) may be applied directly to indeterminate forms $\frac{\infty}{\infty}$, if the expansion by Taylor's Theorem is valid. 
Example:

$$
\left[\frac{x}{e^{x}}\right]_{x=\infty}=\left[\frac{\mathrm{I}}{e^{x}}\right]_{x=\infty}=0 .
$$

7.112 If $f(x)$ and $x$ approach $\infty$ together, and if $f(x+1)-f(x)$ approaches a definite limit, then,

$$
\operatorname{Limit}_{x \rightarrow \infty}\left[\frac{f(x)}{x}\right]=\operatorname{Limit}_{x \rightarrow \infty}[f(x+\mathrm{I})-f(x)]
$$

$7.120 \circ \times \infty$. If, for $x=a, f(x) \times F(x)$ takes the form $\circ \times \infty$, this product may be written,

$$
\frac{\frac{f(x)}{I}}{\frac{I}{F(x)}}
$$

which takes the form $\frac{0}{0}$ (7.101).

7.130 $\infty-\infty$. If, $\underset{x \rightarrow a}{\text { Limit }} f(x)=\infty$ and $\underset{x \rightarrow \infty}{\operatorname{Limit}} F(x)=\infty$, .

$$
f(x)-F(x)=f(x)\left\{\mathbf{I}-\frac{F(x)}{f(x)}\right\}
$$

If $\operatorname{Limit}_{x \rightarrow \infty} \frac{F(x)}{f(x)}$ is different from unity the true value of $f(x)-F(x)$ for $x=a$ is $\infty$. If $\operatorname{Limit}_{x \rightarrow \infty} \frac{F(x)}{f(x)}=+\mathrm{I}$, the expression has the indeterminate form $\infty \times \circ$ which may be treated by 7.120 .

7.140 I $\infty, 0^{0}, \infty^{0}$. If $\{F(x)\}^{(f x)}$ is indeterminate in any of these forms for $x=a$, its true value may be found by finding the true value of the logarithm of the given expression.

Example:

$$
\begin{gathered}
{\left[\left(\frac{\mathrm{I}}{x}\right)^{\tan x}\right]_{x \rightarrow 0} .} \\
\left(\frac{\mathrm{I}}{x}\right)^{\tan x}=y ; \quad \log y=-\tan x \cdot \log x
\end{gathered}
$$


$[\tan x \cdot \log x]_{x=0}=\left[\frac{\log x}{\cot x}\right]_{x=0}=\left[\frac{\frac{1}{x}}{\csc ^{2} x}\right]_{x=0}=\left[\frac{\sin x}{x} \cdot \sin x\right]_{x=0}=0$.

Hence,

$$
\left[\left(\frac{\mathbf{I}}{x}\right)^{\tan x}\right]_{x=0}=\mathbf{I} \text {. }
$$

7.141 If $f(x)$ and $x$ approach $\infty$ together, and $\frac{f(x+\mathrm{I})}{f(x)}$ approaches a definite limit, then,

$$
\operatorname{Limit}_{x \rightarrow \infty}\left[\{f(x)\}^{\frac{1}{x}}\right]=\operatorname{Limit}_{x \rightarrow \infty} \frac{f(x+1)}{f(x)} .
$$

7.150 Differential Coefficients of the form $\frac{\circ}{\circ}$. In determining the differential coefficient $\frac{d y}{d x}$ from an equation $f(x, y)=0$, by means of the formula,

$$
\frac{d y}{d x}=-\frac{\frac{\partial f}{\partial f}}{\frac{\partial f}{\partial y}}
$$

it may happen that for a pair of values, $x=a, y=b$, satisfying $f(x, y)=0$, $\frac{d y}{d x}$ takes the form $\frac{0}{\circ}$.

Writing $\frac{d y}{d x}=y^{\prime}$, and applying 7.102 to the quotient (I), a quadratic equation is obtained for determining $y^{\prime}$, giving, in general, two different determinate values. If $y^{\prime}$ is still indeterminate, apply $\mathbf{7 . 1 0 2}$ again, giving a cubic equation for determining $y^{\prime}$. This process may be continued until determinate values result.

Example:

$$
\begin{aligned}
f(x, y) & =\left(x^{2}+y^{2}\right)^{2}-c^{2} x y=0 \\
y^{\prime} & =-\frac{4 x\left(x^{2}+y^{2}\right)-c^{2} y}{4 y\left(x^{2}+y^{2}\right)-c^{2} x}
\end{aligned}
$$

For $x=0, y=0, y^{\prime}$ takes the value $\frac{0}{0}$.

Applying 7.102,

$$
-y^{\prime}=\frac{\mathrm{I} 2 x^{2}+4 y^{2}+\left(8 x y-c^{2}\right) y^{\prime}}{4 y^{\prime}\left(x^{2}+3 y^{2}\right)+8 x y-c^{2}}
$$

Solving this quadratic equation in $y^{\prime}$, the two determinate values, $y^{\prime}=0, y^{\prime}=\infty$, result for $x=0, y=0$. 
7.17 Special Indeterminate Forms and Limiting Values. In the following the notation $[f(x)]_{a}$ means the limit approached by $f(x)$ as $x$ approaches $a$ as a limit.

\subsection{1}

I. $\left[\left(\mathrm{I}+\frac{c}{x}\right)^{x}\right]_{\infty}=e^{c} \quad(c$ a constant $)$.

2. $[\sqrt{x+c}-\sqrt{x}]_{\infty}=0$.

3. $[\sqrt{x(x+c)}-x]_{\infty}=\frac{c}{2}$.

4. $\left[\sqrt{\left(x+c_{1}\right)\left(x+c_{2}\right)}-x\right]_{\infty}=\frac{1}{2}\left(c_{1}+c_{2}\right)$.

5. $\left[\sqrt[n]{\left(x+c_{1}\right)\left(x+c_{2}\right) \ldots\left(x+c_{n}\right)}-x\right]_{\infty}=\frac{\mathrm{I}}{n}\left(c_{1}+c_{2}+\ldots c_{n}\right)$.

6. $\left[\frac{\log \left(c_{1}+c_{2} e^{x}\right)}{x}\right]_{\infty}=\mathrm{I}$.

7. $\left[\log \left(c_{1}+c_{2} e^{x}\right) \cdot \log \left(\mathrm{I}+\frac{\mathrm{I}}{x}\right)\right]_{\infty}=\mathrm{I}$.

8. $\left[\left(\frac{\log x}{x}\right)^{\frac{1}{x}}\right]_{\infty}=\mathrm{I}$.

9. $\left[\frac{x}{(\log x)^{m}}\right]_{\infty}=\infty$.

I0. $\left[\frac{a^{x}}{x^{m}}\right]_{\infty}=\infty \quad(a>\mathrm{r})$.

II. $\left[\frac{a^{x}}{x !}\right]_{\infty}=0 \quad(x$ a positive integer $)$.

I2. $\left[x^{\frac{1}{x}}\right]_{\infty}=\mathrm{I}$.

I3. $\left[\frac{\log x}{x}\right]_{\infty}=0$.

I4. $\left[\left(a+b c^{x}\right)^{\frac{1}{x}}\right]_{\infty}=c \quad(c>1)$.

I5. $\left[\left(\frac{1}{a+b e^{x}}\right)^{\frac{c}{x}}\right]_{\infty}=e^{-c}$.

I6. $\left[\frac{x}{\alpha+\beta x^{2}} \cdot \log \left(a+b e^{x}\right)\right]_{\infty}=\frac{\mathbf{I}}{\beta}$.

I7. $\left[\left(a+b x^{m}\right)^{\frac{1}{\alpha+\beta \log x}}\right]_{\infty}=e^{\frac{m}{\beta}} \quad(m>0)$. 
If MATHEMATICAL FORMULA AND ELLIPTIC FUNCTIONS

7.172

I. $\left[x \sin \frac{c}{x}\right]_{\infty}=c$.

7. $\left[\frac{\cot \frac{c}{x}}{x}\right]_{\infty}=\frac{\mathrm{I}}{c}$

2. $\left[x\left(\mathrm{I}-\cos \frac{c}{x}\right)\right]_{\infty}=0$.

8. $\left[\sin \frac{c}{x} \cdot \log \left(a+b e^{x}\right)\right]_{\infty}=c$.

3. $\left[x^{2}\left(\mathrm{I}-\cos \frac{c}{x}\right)\right]_{\infty}=\frac{c^{2}}{2}$.

9. $\left[\left(\cos \sqrt{\frac{2 c}{x}}\right)^{x}\right]_{\infty}=e^{-c .}$

4. $\left[\left(\cos \frac{c}{x}\right)^{x}\right]_{\infty}=\mathrm{I}$.

IO. $\left[\left(\mathrm{I}+a \tan \frac{c}{x}\right)^{x}\right]_{\infty}=e^{a c}$.

5. $\left[\left(\cos \frac{c}{x}\right)^{x^{2}}\right]_{\infty}=e^{-\frac{c^{2}}{2}}$.

II. $\left[\left(\cos \frac{c}{x}+a \sin \frac{c}{x}\right)^{x}\right]_{\infty}=e^{a c}$.

6. $\left[\left(\frac{\sin \frac{c}{x}}{\frac{c}{x}}\right)^{x}\right]_{\infty}=\mathrm{I}$.

7.173

I. $\left[\frac{\sin x}{x}\right]_{0}=\mathbf{I}$.

4. $\left[\sin ^{-1} x \cdot \cot x\right]_{0}=\mathrm{I}$.

2. $\left[\frac{\tan x}{x}\right]_{0}=\mathrm{I}$.

5. $\left[\left\{\tan \left(\frac{\pi}{4}+\frac{x}{2}\right)\right\}^{\cot x}\right]_{0}=e$.

3. $\left[\left(\frac{\sin n x}{x}\right)^{m}\right]_{0}=n^{m}$.

7.174

I. $\left[x^{x}\right]_{0}=\mathrm{I}$.

7. $\left[\frac{e^{x}-\mathrm{I}}{x}\right]_{0}=\mathrm{I}$.

2. $\left[x^{\frac{\mathrm{I}}{a+b \log x}}\right]_{0}=e^{\frac{\mathrm{I}}{b}}$

8. $\left[x^{m} \log x\right]_{0}=0 \quad(m>0)$.

3. $\left[x^{\frac{1}{\log \left(e^{x}-1\right)}}\right]_{0}=e$.

9. $\left[\frac{e^{x}-e^{-x}-2 x}{\left(e^{x}-\mathrm{I}\right)^{3}}\right]_{0}=\frac{\mathrm{I}}{3}$.

4. $\left[x^{m} \log \frac{\mathrm{I}}{x}\right]_{0}=0 \quad(m \geqslant \mathrm{I})$.

Io. $\left[x e^{\stackrel{\mathrm{I}}{\bar{x}}}\right]_{0}^{\bullet}=\infty$.

5. $[\log \cos x \cdot \cot x]_{0}=0$.

II. $\left[\frac{e^{x}-e^{-x}}{\log (1+x)}\right]_{0}=2$.

6. $\left[\log \tan \left(\frac{\pi}{4}+\frac{x}{2}\right) \cdot \cot x\right]_{0}=\mathrm{I}$

I2. $\left[\frac{\log \tan 2 x}{\log \tan x}\right]_{0}=$ I. 


\subsection{5}

I. $\left[x^{\frac{\mathrm{I}}{1-x}}\right]_{1}=\frac{\mathrm{I}}{e}$.

2. $[(\pi-2 x) \tan x]_{\frac{\pi}{2}}=2$.

3. $\left[\log \left(2-\frac{x}{c}\right) \cdot \tan \frac{\pi x}{2 c}\right]_{c}=\frac{2}{\pi}$.

4. $\left[\left(e^{c}-e^{x}\right) \tan \frac{\pi x}{2 c}\right]_{c^{\circ}}=\frac{2 c}{\pi} e^{c}$.
5. $\left[\cos ^{-1} \frac{x}{c} \cdot \tan \frac{\pi x}{2 c}\right]_{c}=\infty$

6. $\left[\left(a+b e^{\tan x}\right)^{\pi-2 x}\right]_{\frac{\pi}{2}}=e^{2}$.

7. $\left[\left(2-\frac{2 x}{\pi}\right)^{\tan x}\right]_{\frac{\pi}{2}}=e^{\frac{2}{\pi}}$

8. $\left[(\tan x)^{\tan 2 x}\right]_{\frac{\pi}{4}}=\frac{\mathrm{I}}{e}$.

7.18 Limiting Values of Sums.

I. $\operatorname{Limit}_{n \rightarrow \infty}\left(\frac{\mathrm{I}^{k}+2^{k}+3^{k}+\ldots \ldots+n^{k}}{n^{k+1}}\right)=\frac{\mathrm{I}}{k+\mathrm{I}}$ if $k>-\mathbf{I}$. $\infty$ if $k<-$ I.

2. $\operatorname{Limit}_{n \rightarrow \infty}\left(\frac{\mathrm{I}}{n a}+\frac{\mathrm{I}}{n a+b}+\frac{\mathrm{I}}{n a+2 b}+\ldots+\frac{\mathrm{I}}{n a+(n-\mathrm{I}) b}\right)$ $=\frac{\log (a+b)-\log a}{b}(a, b>0)$.

3. $\operatorname{Limit}_{n \rightarrow \infty}\left(\frac{n-\mathrm{I}^{2}}{\mathrm{I} \cdot 2 \cdot(n+\mathrm{I})}+\frac{n-2^{2}}{2 \cdot 3 \cdot(n+2)}+\frac{n-3^{2}}{3 \cdot 4 \cdot(n+3)}+\ldots\right.$

$$
\left.+\frac{\left(n-n^{2}\right.}{n \cdot(n+\mathrm{I}) \cdot(n+n)}\right)=\mathrm{I}-\log 2 .
$$

4. $\operatorname{Limit}_{n \rightarrow \infty}\left[\left(a+b \frac{\sqrt{\mathbf{I}}}{n}\right)^{2}+\left(a^{2}+b \frac{\sqrt{2}}{n}\right)^{2}+\left(a^{3}+b \frac{\sqrt{3}}{n}\right)^{2}+\ldots \ldots\right.$

$$
\left.+\left(a^{n}+b \frac{\sqrt{n}}{n}\right)^{2}\right]=\frac{a^{2}}{\mathrm{I}-a^{2}}+\frac{b^{2}}{2}
$$

if $a$ is a positive proper fraction.

5. $\operatorname{Limit}_{n \rightarrow \infty}\left[\sqrt{a+\frac{b}{n}}+\sqrt{a^{2}+\frac{b}{n}}+\sqrt{a^{3}+\frac{b}{n}}+\ldots+\sqrt{a^{n}+\frac{b}{n}}\right]=\infty$, if $b>0$ and $a$ is a positive proper fraction.

6. $\operatorname{Limit}_{n \rightarrow \infty}\left[\sqrt{a+\frac{b}{I \cdot n}}+\sqrt{a^{2}+\frac{b}{2 \cdot n}}+\sqrt{a^{3}+\frac{b}{3 \cdot n}}+\ldots+\sqrt{a^{n}+\frac{b}{n \cdot n}}\right]$

$$
=\frac{\sqrt{a}}{\mathrm{I}-\sqrt{a}}+2 \sqrt{b}
$$

if $b>0$ and $a$ is a positive proper fraction.

7. $\operatorname{Limit}_{n \rightarrow \infty}\left[I+\frac{I}{2}+\frac{I}{3}+\ldots+\frac{I}{n}-\log n\right]=\gamma=0.5772157 \ldots$ 
7.19 Limiting Values of Products.

I. $\operatorname{Limit}_{n \rightarrow \infty}\left[\left(\mathrm{I}+\frac{c}{n}\right)\left(\mathrm{I}+\frac{c}{n+\mathrm{I}}\right)\left(\mathrm{I}+\frac{c}{n+2}\right) \ldots\left(\mathrm{I}+\frac{c}{2 n-\mathrm{I}}\right)\right]=2^{c}$, if $c>0$.

2. $\operatorname{Limit}_{n \rightarrow \infty}\left[\left(\mathrm{I}+\frac{c}{n a}\right)\left(\mathrm{I}+\frac{c}{n a+b}\right)\left(\mathrm{I}+\frac{c}{n a+2 b}\right) \ldots\left(\mathrm{I}+\frac{c}{n a+(n-\mathrm{I}) b}\right)\right]$

if $a, b, c$ are all positive.

$$
=\left(\mathbf{I}+\frac{b}{a}\right)^{c}
$$

3. $\operatorname{Limit}_{n \rightarrow \infty}\left[\frac{\{m(m+I)(m+2) \ldots \ldots(m+n-I)\}^{\frac{I}{n}}}{m+\frac{1}{2}(n-I)}\right]=\frac{2}{e}$, if $m>0$.

4. $\operatorname{Limit}_{n \rightarrow}\left[\left(\mathrm{I}+\frac{2 c}{n^{2}}\right)\left(\mathrm{I}+\frac{4 c}{n^{2}}\right)\left(\mathrm{I}+\frac{6 c}{n^{2}}\right) \ldots\left(\mathrm{I}+\frac{2 n c}{n^{2}}\right)\right]=e^{c}$.

7.20 Maxima and Minima.

7.201 Functions of One Variable. $y=f(x)$ is a maximum or minimum for the values of $x$ satisfying the equation, $f^{\prime}(x)=\frac{\partial f(x)}{\partial x}=0$,

provided that $f^{\prime}(x)$ is continuous for these values of $x$.

7.202 If, for $x=a, f^{\prime}(a)=0$,

Example:

$$
\begin{aligned}
& y=f(a) \text { is a maximum if } f^{\prime \prime}(a)<0 \\
& y=f(a) \text { is a minimum if } f^{\prime \prime}(a)>0
\end{aligned}
$$

$$
\begin{aligned}
y & =\frac{x}{x^{2}+\alpha x+\beta}, \quad \beta>0, \\
f^{\prime}(x) & =\frac{-x^{2}+\beta}{\left(x^{2}+\alpha x+\beta\right)^{2}} \\
f^{\prime}(x) & =0 \text { when } x= \pm \sqrt{\beta} \\
f^{\prime \prime}(x) & =\frac{2 x^{3}-6 \beta x-2 \alpha \beta}{\left(x^{2}+\alpha x+\beta\right)^{3}}
\end{aligned}
$$

For $x=+\sqrt{\beta}, f^{\prime \prime}(x)=\frac{-2}{\sqrt{\beta}} \frac{I}{(2 \sqrt{\beta}+\alpha)^{2}}$ Maximum, 






$$
\text { For } \begin{aligned}
x=-\sqrt{\beta}, f^{\prime \prime}(x) & =\frac{+2}{\sqrt{\beta}} \frac{\mathrm{I}}{(2 \sqrt{\bar{\beta}}-\alpha)^{2}} \text { Minimum, } \\
y_{\max } & =\frac{\mathrm{I}}{\alpha+2 \sqrt{\beta}}, \\
y_{\min } & =\frac{\mathrm{I}}{\alpha-2 \sqrt{\beta}} .
\end{aligned}
$$

7.203 If for $x=a, f^{\prime}(a)=0$ and $f^{\prime \prime}(a)=0$, in order to determine whether $y=f(a)$ is a maximum or minimum it is necessary to form the higher differential coefficients, until one of even order is found which does not vanish for $x=a$. $y=f(a)$ is a maximum or minimum according as the first of the differential coefficients, $f^{\prime \prime}(a), f^{\text {iv }}(a), f^{\text {vi }}(a), \ldots \ldots$ of even order which does not vanish is negative or positive.

7.210 Functions of Two Variables. $F(x, y)$ is a maximum or minimum for the pair of values of $x$ and $y$ that satisfy the equations,

and for which

$$
\frac{\partial F}{\partial x}=\circ, \frac{\partial F}{\partial y}=\circ \text {, }
$$

$$
\left(\frac{\partial^{2} F}{\partial x \partial y}\right)^{2}-\frac{\partial^{2} F}{\partial x^{2}} \frac{\partial^{2} F}{\partial y^{2}}<0
$$

If both $\frac{\partial^{2} F}{\partial x^{2}}$ and $\frac{\partial^{2} F}{\partial y^{2}}$ are negative for this pair of values of $x$ and $y, F(x, y)$ is a maximum. If they are both positive $F(x, y)$ is a minimum.

7.220 Functions of $n$ Variables. For the maximum or minimum of a function of $n$ variables, $F\left(x_{1}, x_{2} \ldots \ldots, x_{n}\right)$, it is necessary that the first derivatives, $\frac{\partial F}{\partial x_{1}}, \frac{\partial F}{\partial x_{2}}, \ldots \ldots, \frac{\partial F}{\partial x_{n}}$ all vanish; and that the lowest order of the higher derivatives which do not all vanish be an even number. If this number be 2 the necessary condition for a minimum is that all of the determinants,

where

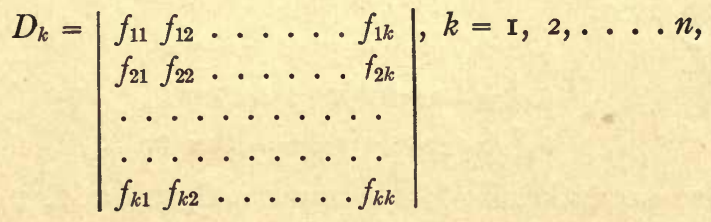

$$
f_{i j}=\frac{\partial^{2} F}{\partial x_{i} \partial x_{j}}
$$


shall be positive. For a maximum the determinants must be alternately negative and positive, beginning with $D_{1}=\frac{\partial^{2} F}{\partial x_{1}^{2}}$ negative.

7.230 Maxima and Minima with Conditions. If $F\left(x_{1}, x_{2}, \ldots \ldots, x_{n}\right)$ is to be made a maximum or minimum subject to the conditions,

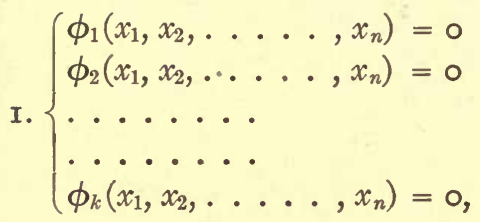

where $k<n$, the necessary conditions are,

2.

$$
\frac{\partial F}{\partial x_{i}}+\sum_{j=\mathrm{I}}^{k} \lambda_{j} \frac{\partial \phi_{j}}{\partial x_{i}}=0 \quad i=\mathrm{I}, 2, \ldots . n,
$$

where the $\lambda$ 's are $k$ undetermined multipliers. The $n$ equations (2) together with the $k$ equations of condition (I) furnish $k+n$ equations to determine the $k+n$ quantities, $x_{1}, x_{2}, \ldots \ldots, x_{n}, \lambda_{1}, \lambda_{2}, \ldots \ldots, \lambda_{k}$.

Example:

To find the axes of the ellipsoid, referred to its center as origin,

$$
a_{11} x^{2}+a_{22} y^{2}+a_{33} z^{2}+2 a_{12} x y+2 a_{23} y z+2 a_{13} x z=\mathrm{I} .
$$

Denoting the radius vector to the surface by $r$, and its direction-cosines by $l, m, n$, so that $x=l r, y=m r, z=n r$, it is necessary to find the maxima and minima of

$$
r^{2}=\frac{\mathrm{I}}{a_{11} l^{2}+a_{22} m^{2}+a_{33} n^{2}+2 a_{12} l m+2 a_{23} m+2 a_{13} l n n},
$$

subject to the condition

$$
\phi(l, m, n)=l^{2}+m^{2}+n^{2}-\mathrm{I}=0 .
$$

This is the same as finding the minima and maxima of

$$
F(l, m, n)=a_{11} l^{2}+a_{22} m^{2}+a_{33} n^{2}+2 a_{12} l m+2 a_{23} m n+2 a_{13} l n .
$$

Equation (2) gives:

$$
\begin{aligned}
& \left(a_{11}+\lambda\right) l+a_{12} m+a_{13} n=0 \\
& a_{12} l+\left(a_{22}+\lambda\right) m+a_{23} n=0 \\
& a_{13} l+a_{23} m+\left(a_{33}+\lambda\right) n=0
\end{aligned}
$$

Multiplying these 3 equations by $l, m, n$ respectively and adding,

$$
\lambda=-\frac{\mathrm{I}}{r^{2}}
$$


Then by (I. 1.363) the 3 values of $r$ are given by the 3 roots of

$\left|\begin{array}{lll}a_{11}-\frac{\mathrm{I}}{r^{2}} & a_{12} & a_{13} \\ a_{12} & a_{22}-\frac{\mathrm{I}}{r^{2}} & a_{23} \\ a_{13} & a_{23} & a_{33}-\frac{\mathrm{I}}{r^{2}}\end{array}\right|=0$.

7.30 Derivatives.

7.31 First Derivatives.
I. $\frac{d x^{n}}{d x^{n}}=n x^{n-1}$.
4. $\frac{d x^{x}}{d x}=x^{x}(\mathrm{I}+\log x)$.
2. $\frac{d a^{x}}{d x}=a^{x} \log a$.
5. $\frac{d \log _{a} x}{d x}=\frac{\mathrm{I}}{x \log a}=\frac{\log _{a} e}{x}$.
3. $\frac{d e^{x}}{d x}=e^{x}$.
6. $\frac{d \log x}{d x}=\frac{\mathrm{I}}{x}$.
7. $\frac{d x^{\log x}}{d x}=2 x^{\log x-1} \log x$.
8. $\frac{d(\log x)^{x}}{d x}=(\log x)^{x-1}\{\mathrm{I}+\log x \cdot \log \log x\}$.
9. $\frac{d\left(\frac{x}{e}\right)^{x}}{d x}=\left(\frac{x}{e}\right)^{x} \log x$

I0. $\frac{d \sin x}{d x}=\cos x$.

II. $\frac{d \cos x}{d x}=-\sin x$

I2. $\frac{d \tan x}{d x}=\sec ^{2} x$.

I3. $\frac{d \cot x}{d x}=-\csc ^{2} x$

I4. $\frac{d \sec x}{d x}=\sec ^{2} x \cdot \sin x$.
I5. $\frac{d \csc x}{d x}=-\csc ^{2} x \cdot \cos x$

16. $\frac{d \sin ^{-1} x}{d x}=-\frac{d \cos ^{-1} x}{d x}=\frac{\mathrm{I}}{\sqrt{\mathrm{I}-x^{2}}}$.

I7. $\frac{d \tan ^{-1} x}{d x}=-\frac{d \cot ^{-1} x}{d x}=\frac{\mathrm{I}}{\mathrm{I}+x^{2}}$.

18. $\frac{d \sec ^{-1} x}{d x}=-\frac{d \csc ^{-1} x}{d x}=\frac{\mathrm{I}}{x \sqrt{x^{2}-\mathrm{I}}}$.

I9. $\frac{d \sinh x}{d x}=\cosh x$.

20. $\frac{d \cosh x}{d x}=\sinh x$. 
2I. $\frac{d \tanh x}{d x}=\operatorname{sech}^{2} x$.

22. $\frac{d \operatorname{coth} x}{d x}=-\operatorname{csch}^{2} x$.

23. $\frac{d \operatorname{sech} x}{d x}=-\operatorname{sech} x \cdot \tanh x$.

24. $\frac{d \operatorname{csch} x}{d x}=-\operatorname{csch} x \cdot \operatorname{coth} x$.

25. $\frac{d \sinh ^{-1} x}{d x}=\frac{\mathrm{I}}{\sqrt{x^{2}+\mathrm{I}}}$.

26. $\frac{d \cosh ^{-1} x}{d x}=\frac{\mathrm{I}}{\sqrt{x^{2}-1}}$.
27. $\frac{d \tanh ^{-1} x}{d x}=\frac{d \operatorname{coth}^{-1} x}{d x}-\frac{\mathrm{I}}{\mathrm{I}-x^{2}}$.

28. $\frac{d \operatorname{sech}^{-1} x}{d x}=-\frac{\mathrm{I}}{x \sqrt{\mathrm{I}-x^{2}}}$.

29. $\frac{d \operatorname{csch}^{-1} x}{d x}=-\frac{\mathrm{I}}{x \sqrt{\mathrm{I}+x^{2}}}$.

30. $\frac{d g d x}{d x}=\operatorname{sech} x$.

3I. $\frac{d g d^{-1} x}{d x}=\sec x$.

\subsection{2}

I. $\frac{d\left(y_{1} y_{2} y_{3} \ldots y_{n}\right)}{d x}=y_{1} y_{2} \ldots y_{n}\left(\frac{\mathrm{I}}{y_{1}} \frac{d y_{1}}{d x}+\frac{\mathrm{I}}{y_{2}} \frac{d y_{2}}{d x}+\ldots+\frac{\mathrm{I}}{y_{n}} \frac{d y_{n}}{d x}\right)$.

$\frac{d\left(\frac{u}{v}\right)}{d x}=\frac{v \frac{d u}{d x}-u \frac{d v}{d x}}{v^{2}}$

4. $\frac{d e^{u}}{d x}=e^{u} \frac{d u}{d x}$.

3. $\frac{d a^{u}}{d x}=a^{u} \frac{d u}{d x} \log a$.

5. $\frac{d f(u)}{d x}=\frac{d f(u)}{d u} \cdot \frac{d u}{d x}$

7.33 Derivative of a Definite Integral.

I. $\frac{d}{d a} \int_{\psi(a)}^{\phi(a)} f(x, a) d x=f(\phi(a), a) \frac{d \phi(a)}{d a}-f(\psi(a), a) \frac{d \psi(a)}{d a}+\int_{\psi(a)}^{\phi(a)} \frac{d}{d a} f(x, a) d x$
2. $\frac{d}{d a} \int_{b}^{a} f(x) d x=f(a)$
3. $\frac{d}{d b} \int_{b}^{a} f(x) d x=-f(b)$.

\subsection{Higher Derivatives.}


7.351 Leibnitz's Theorem. If $u$ and $v$ are functions of $x$,

$\frac{d^{n}(u v)}{d x^{n}}=u \frac{d^{n} v}{d x^{n}}+\frac{n}{\mathrm{I} !} \frac{d u}{d x} \frac{d^{n-1} v}{d x^{n-1}}+\frac{n(n-\mathrm{I})}{2 !} \frac{d^{2} u}{d x^{2}} \frac{d^{n-2} v}{d x^{n-2}}$

$$
+\frac{n(n-\mathrm{I})(n-2)}{3 !} \frac{d^{3} u}{d x^{3}} \frac{d^{n-3} v}{d x^{n-3}}+\ldots \ldots+v \frac{d^{n} u}{d x^{n}} .
$$

7.352 Symbolically,

$$
\frac{d^{n}(u v)}{d x^{n}}=(u+v)^{(n)},
$$

where

7.353

$$
u^{0}=u, \quad v^{0}=v .
$$

$$
\frac{d^{n} e^{a x} u}{d x^{n}}=e^{a x}\left(a+\frac{d}{d x}\right)^{n} u .
$$

7.354 If $\phi\left(\frac{d}{d x}\right)$ is a polynomial in $\frac{d}{d x}$,

$$
\phi\left(\frac{d}{d x}\right) e^{a x} u=e^{a x} \phi\left(a+\frac{d}{d x}\right) u .
$$

7.355 Euler's Theorem. If $u$ is a homogeneous function of the $n$th degree of $r$ variables, $x_{1}, x_{2}, \ldots x_{r}$

$$
\left(x_{1} \frac{\partial}{\partial x_{1}}+x_{2} \frac{\partial}{\partial x_{2}}+\ldots .+x_{r} \frac{\partial}{\partial x_{r}}\right)^{m} u=n^{m} u,
$$

where $m$ may be any integer, including 0 .

7.36 Derivatives of Functions of Functions.

7.361 If $f(x)=F(y)$, and $y=\phi(x)$,

I. $\frac{d^{n}}{d x^{n}} f(x)=\frac{U_{1}}{I !} F^{\prime}(y)+\frac{U_{2}}{2 !} F^{\prime \prime}(y)+\frac{U_{3}}{3 !} F^{\prime \prime \prime}(y)+\ldots+\frac{U_{n}}{n !} F^{(n)}(y)$, where

2. $U_{k}=\frac{\partial^{n}}{\partial x^{n}} y^{k}-\frac{k}{\mathrm{I} !} y \frac{\partial^{n}}{\partial x^{n}} y^{k-1}+\frac{k(k-\mathrm{I})}{2 !} y^{2} \frac{\partial^{n}}{\partial x^{n}} y^{k-2}-\ldots \ldots$

\subsection{2}

I. $(-\mathrm{I})^{n} \frac{d^{n}}{d x^{n}} F\left(\frac{\mathrm{I}}{x}\right)=\frac{\mathrm{I}}{x^{2 n}} F^{(n)}\left(\frac{\mathrm{I}}{x}\right)+\frac{n-\mathrm{I}}{x^{2 n-1}} \frac{n}{\mathrm{I} !} F^{(n-1)}\left(\frac{\mathrm{I}}{x}\right)$

$$
+\frac{(n-\mathrm{I})(n-2)}{x^{2 n-2}} \cdot \frac{n(n-\mathrm{I})}{2 !} F^{(n-2)}\left(\frac{\mathrm{I}}{x}\right)+\ldots \ldots
$$

2. $(-\mathrm{I})^{n} \frac{d^{n}}{d x^{n}} e^{\frac{a}{x}}=\frac{\mathrm{I}}{x^{n}} e^{\frac{a}{x}}\left\{\left(\frac{a}{x}\right)^{n}+(n-\mathrm{I}) \frac{n}{\mathrm{I} !}\left(\frac{a}{x}\right)^{n-1}\right.$

$$
\begin{gathered}
+(n-\mathrm{I})(n-2) \frac{n(n-\mathrm{I})}{2 !}\left(\frac{a}{x}\right)^{n-2} \\
\left.+(n-\mathrm{I})(n-2)(n-3) \frac{n(n-\mathrm{I})(n-2)}{3 !}\left(\frac{a}{x}\right)^{n-3}+\ldots\right\}
\end{gathered}
$$




\subsection{3}

$$
\begin{aligned}
& \text { I. } \begin{array}{r}
\frac{d^{n}}{d x^{n}} F\left(x^{2}\right)=(2 x)^{n} F^{(n)}\left(x^{2}\right)+\frac{n(n-\mathrm{I})}{\mathrm{I} !}(2 x)^{n-2} F^{(n-1)}\left(x^{2}\right) \\
\quad+\frac{n(n-\mathrm{I})(n-2)(n-3)}{2 !}(2 x)^{n-4} F^{(n-2)}\left(x^{2}\right) \\
+\frac{n(n-\mathrm{I})(n-2)(n-3)(n-4)(n-5)}{3 !}(2 x)^{n-6} F^{(n-3)}\left(x^{2}\right)+\ldots \\
\text { 2. } \frac{d^{n}}{d x^{n}} e^{a x^{2}}=(2 a x)^{n} e^{a x^{2}}\left\{\mathrm{I}+\frac{n(n-\mathrm{I})}{\mathrm{I} !\left(4 a x^{2}\right)}+\frac{n(n-\mathrm{I})(n-2)(n-3)}{2 !\left(4 a x^{2}\right)^{2}}\right. \\
\left.\quad+\frac{n(n-\mathrm{I})(n-2)(n-3)(n-4)(n-5)}{3 !\left(4 a x^{2}\right)^{3}}+\ldots\right\}
\end{array}
\end{aligned}
$$

3. $\frac{d^{n}}{d x^{n}}\left(\mathrm{I}+a x^{2}\right)^{\mu}$

$$
\begin{aligned}
=\frac{\mu(\mu-\mathrm{I})(\mu-2) \ldots(\mu-n+\mathrm{I})(2 a x)^{n}}{\left(\mathrm{I}+a x^{2}\right)^{n-\mu}}\left\{\mathrm{I}+\frac{n(n-\mathrm{I})}{\mathrm{I} \cdot(\mu-n+\mathrm{I})} \frac{\left(\mathrm{I}+a x^{2}\right)}{4 a x^{2}}\right. \\
\left.+\frac{n(n-\mathrm{I})(n-2)(n-3)}{2 !(\mu-n+\mathrm{I})(\mu-n+2)}\left(\frac{\mathrm{I}+a x^{2}}{4 a x^{2}}\right)^{2}+\ldots .\right\} .
\end{aligned}
$$

4. $\frac{d^{m-1}}{d x^{m-1}}\left(\mathrm{I}-x^{2}\right)^{m-\frac{1}{2}}=(-\mathrm{I})^{m-1} \frac{\mathrm{I} \cdot 3 \cdot 5 \cdots(2 m-\mathrm{I})}{m} \sin \left(m \cos ^{-1} x\right)$.

\subsection{4}

I. $\begin{aligned} \frac{d^{n^{0}}}{d x^{n}} F(\sqrt{x})=\frac{F^{(n)}(\sqrt{x})}{(2 \sqrt{x})^{n}}-\frac{n(n-\mathrm{I})}{\mathrm{I} !} \frac{F^{(n-1)}(\sqrt{x})}{(2 \sqrt{x})^{n+1}} & \\ & +\frac{(n+\mathrm{I}) n(n-\mathrm{I})(n-2)}{2 !} \frac{F^{(n-2)}(\sqrt{x})}{(2 \sqrt{x})^{n+2}}-\ldots .\end{aligned}$

2. $\frac{d^{n}}{d x^{n}}(\mathrm{I}+a \sqrt{x})^{2 n-1}=\frac{\mathrm{I} \cdot 3 \cdot 5 \cdots(2 n-\mathrm{I})}{2^{n}} \frac{a}{\sqrt{x}}\left(a^{2}-\frac{\mathrm{I}}{x}\right)^{n-1}$.

\subsection{5}

I. $\frac{d^{n}}{d x^{n}} F\left(e^{x}\right)=\frac{E_{1}}{\mathrm{I} !} e^{x} F^{\prime}\left(e^{x}\right)+\frac{E_{2}}{2 !} e^{2 x} F^{\prime \prime}\left(e^{x}\right)+\frac{E_{3}}{3 !} e^{3 x} F^{\prime \prime \prime}\left(e^{x}\right)+\ldots$ where

2 .

$$
E_{k}=k^{n}-\frac{k}{\mathrm{I} !}(k-\mathrm{I})^{n}+\frac{k(k-\mathrm{I})}{2 !}(k-2)^{n}-\ldots
$$

3. $\frac{d^{n}}{d x^{n}} \frac{\mathrm{I}}{\mathrm{I}+e^{2 x}}=-E_{1} e^{x} \frac{\sin \left(2 \tan ^{-1} e^{-x}\right)}{\sqrt{\left(\mathrm{I}+e^{2 x}\right)^{2}}}+E_{2} e^{2 x} \frac{\sin \left(3 \tan ^{-1} e^{-x}\right)}{\sqrt{\left(\mathrm{I}+e^{2 x}\right)^{3}}}$

$$
-E_{3} e^{3 x} \frac{\sin \left(4 \tan ^{-1} e^{-x}\right)}{\sqrt{\left(\mathrm{I}+e^{2 x}\right)^{4}}}+\ldots
$$

4. $\frac{d^{n}}{d x^{n}} \frac{e^{x}}{\mathrm{I}+e^{2 x}}=-E_{1} e^{x} \frac{\cos \left(2 \tan ^{-1} e^{-x}\right)}{\sqrt{\left(\mathrm{I}+e^{2 x}\right)^{2}}}+E_{2} e^{2 x} \frac{\cos \left(3 \tan ^{-1} e^{-x}\right)}{\sqrt{\left(\mathrm{I}+e^{2 x}\right)^{3}}}$

$$
-E_{3} e^{3 x} \frac{\cos \left(4 \tan ^{-1} e^{-x}\right)}{\sqrt{\left(1+e^{2 x}\right)^{4}}}+\ldots
$$




\subsection{6}

I. $\frac{d^{n}}{d x^{n}} F(\log x)=\frac{\text { I }}{x^{n}}\left\{\stackrel{n}{C_{0}} F^{(n)}(\log x)-\stackrel{n}{C_{1}} F^{(n-1)}(\log x)+\stackrel{n}{C_{2}} F^{(n-2)}(\log x)-\ldots\right\}$ $\stackrel{n}{C}_{0}=\mathrm{I}$

$\stackrel{n}{C_{1}}=\mathrm{I}+2+3+\ldots \ldots+(n-\mathrm{I})$

$$
=\frac{n(n-\mathrm{I})}{2}
$$

$\stackrel{n}{C}_{2}=\mathrm{I} \cdot 2+\mathrm{I} \cdot 3+\mathrm{I} \cdot 4+\ldots \ldots+\mathrm{I} \cdot(n-\mathrm{I})$

$+2 \cdot 3+2 \cdot 4+\ldots \ldots+2 \cdot(n-. . \mathrm{I})$

$+3 \cdot 4+\ldots \ldots+3 \cdot(n-1)$

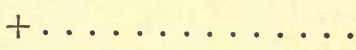

$$
+(n-2)(n-\mathrm{I})=\frac{n(n-\mathrm{I})(n-2)(3 n-\mathrm{I})}{24} .
$$

2. $\stackrel{n+\mathrm{I}}{C_{k}}=\stackrel{n}{C_{k}}+n \stackrel{n}{C}_{k-1}$.

3. $\stackrel{-n}{C}_{k}=\stackrel{-(n-1)}{C_{k}}+n \stackrel{-n}{C}_{k-1}$.

\begin{tabular}{|c|c|c|c|c|c|c|c|c|c|c|c|c|c|}
\hline$n=$ & -4 & -3 & -2 & $-I$ & $+\mathbf{I}$ & +2 & +3 & +4 & +5 & +6 & +7 & +8 & +9 \\
\hline$C_{0}=$ & I & $\mathbf{I}$ & I & I & $\mathbf{I}$ & I & I & I & I & I & I & I & I \\
\hline$C_{1}=$ & IO & 6 & 3 & I & $\ldots$ & I & 3 & 6 & IO & I5 & $2 \mathrm{I}$ & 28 & 36 \\
\hline$C_{2}=$ & 65 & 25 & 7 & I & $\ldots$ & $\ldots$ & 2 & I I & 35 & 85 & I75 & 322 & 546 \\
\hline$C_{3}=$ & 350 & 90 & I5 & I & $\ldots$ & & & 6 & 50 & 225 & 735 & I960 & $453^{6}$ \\
\hline$C_{4}=$ & I 70I & $30 I$ & $3 I$ & I & $\cdots$ & & & & 24 & 274 & I6 24 & 6769 & 22449 \\
\hline$C_{5}=$ & 7770 & 966 & 63 & I & $\ldots$ & & $\cdots$ & & $\ldots$ & I 20 & I 764 & I3I32 & 67284 \\
\hline$C_{6}=$ & 34105 & 3025 & I 27 & I & $\ldots$ & $\ldots$ & $\ldots$ & $\ldots$ & $\cdots$ & $\ldots$ & 720 & I 3068 & II 8 I 24 \\
\hline$C_{7}=$ & $\mathrm{I} 4575^{\circ}$ & 9330 & 225 & I & $\ldots$ & & $\cdots$ & & $\cdots$ & $\ldots$ & $\ldots$ & 5040 & IOg 584 \\
\hline$C_{8}=$ & 6 I I $50 \mathrm{I}$ & $2850 \mathrm{I}$ & 5 II & I & & & & & & & & & 40320 \\
\hline
\end{tabular}

$$
\begin{aligned}
& \stackrel{n}{C_{0}}=\mathrm{I} \quad \stackrel{k}{C_{k}}=\text { o, } \\
& \stackrel{-n}{C_{0}}=\mathrm{I} \quad \stackrel{-\mathrm{I}}{C}_{k}=\mathrm{I}, \\
& \stackrel{2}{C_{1}}=\mathrm{I} \quad \stackrel{3}{C}_{1}=3 \quad \stackrel{4}{C}_{1}=6, \\
& \bar{C}_{1}^{2}=3 \quad \bar{C}_{1}^{3}=6 \quad \bar{C}_{1}^{4}=\text { Io, } \\
& \stackrel{3}{C}_{2}=2 \quad \stackrel{4}{C}_{2}=\text { II , } \\
& \bar{C}_{2}^{2}=7 \quad \bar{C}_{2}^{3}=25 \quad \bar{C}_{2}^{-4}=65, \\
& \stackrel{4}{C}_{3}=6 \text {. } \\
& \bar{C}_{3}^{2}=I_{5} \quad \bar{C}_{3}^{3}=90 \quad \bar{C}_{3}^{4}=35 \text { o. }
\end{aligned}
$$

\subsection{Table of $\stackrel{n}{C}_{k}$.}




\subsection{8}

I. $\frac{d^{n}}{d x^{n}}(\log x)^{p}=\frac{(-\mathrm{r})^{n-1}}{x^{n}}\left\{\stackrel{n}{C}_{n-1} p(\log x)^{p-1}-\stackrel{n}{C}_{n-2} p(p-\mathrm{I})(\log x)^{p-2}\right.$

$$
\left.+\stackrel{n}{C}_{n-3} p(p-1)(p-2)(\log x)^{p-3}-\ldots\right\}
$$

where $p$ is a positive integer. If $n<p$ there are $n$ terms in the series. If $n \geqslant p$,

2. $\begin{aligned} & \frac{d^{n}}{d x^{n}}(\log x)^{p}=\frac{(-\mathrm{I})^{n-1}}{x^{n}}\left\{\stackrel{n}{C}_{n-1} p(\log x)^{p-1}-\stackrel{n}{C}_{n-2} p(p-\mathrm{I})(\log x)^{p-2}\right. \\ &\left.+\ldots \ldots+(-\mathrm{I})^{p+1} \stackrel{n}{C}_{n-p} p(p-\mathrm{I})(p-2) \ldots . \mathrm{I}\right\}\end{aligned}$

$7.369\{\log (\mathrm{I}+x)\}^{p}=\stackrel{p}{C}_{0} x^{p}-\stackrel{p+1}{C}_{1} \frac{x^{p+1}}{p+\mathrm{I}}+\stackrel{p+2}{C_{2}} \frac{x^{p+2}}{(p+\mathrm{I})(p+2)} \ldots \ldots$

$$
-\mathrm{I}<x<+\mathrm{r} \text {. }
$$

7.37 Derivatives of Powers of Functions. If $y=\phi(x)$.

I. $\frac{d^{n}}{d x^{n}} y^{p}=p\left(\begin{array}{c}n-p \\ n\end{array}\right)\left\{-\left(\begin{array}{c}n \\ \mathrm{I}\end{array}\right) \frac{\mathrm{I}}{p-\mathrm{I}} y^{p-1} \frac{d^{n} y}{d x^{n}}+\left(\begin{array}{c}n \\ 2\end{array}\right) \frac{\mathrm{I}}{p-2} y^{p-2} \frac{d^{n} y^{2}}{d x^{n}}-\ldots \ldots\right\}$.

2. $\frac{d^{n}}{d x^{n}} \log y=\left(\begin{array}{l}n \\ \mathrm{I}\end{array}\right) \frac{\mathrm{I}}{\mathrm{I} \cdot y} \frac{d^{n} y}{d x^{n}}-\left(\begin{array}{c}n \\ 2\end{array}\right) \frac{\mathrm{I}}{2 \cdot y^{2}} \frac{d^{n} y^{2}}{d x^{n}}+\left(\begin{array}{l}n \\ 3\end{array}\right) \frac{\mathrm{I}}{3 \cdot y^{3}} \frac{d^{n} y^{3}}{d x^{n}}-\ldots \ldots$

\subsection{8}

I. $\frac{d^{n}(a+b x)^{m}}{d x^{n}}=m(m-\mathrm{I})(m-2) \ldots \ldots(m-[n-\mathrm{I}]) b^{n}(a+b x)^{m-n}$.

2. $\frac{d^{n}(a+b x)^{-1}}{d x^{n}}=(-\mathrm{I})^{n} \frac{n ! b^{n}}{(a+b x)^{n+1}}$.

3. $\frac{d^{n}(a+b x)^{-\frac{1}{2}}}{d x^{n}}=(-\mathrm{I})^{n} \frac{\mathrm{I} \cdot 3 \cdot 5 \ldots(2 n-\mathrm{I})}{2^{n}(a+b x)^{n+\frac{1}{2}}} b^{n}$.

4. $\frac{d^{n} \log (a+b x)}{d x^{n}}=(-\mathrm{I})^{n-1} \frac{(n-\mathrm{I}) ! b^{n}}{(a+b x)^{n}}$.

5. $\frac{d^{n} e^{a x}}{d x^{n}}=a^{n} e^{a x}$.

6. $\frac{d^{n} \sin x}{d x^{n}}=\sin \left(\frac{1}{2} n \pi+x\right)$.

7. $\frac{d^{n} \cos x}{d x^{n}}=\cos \left(\frac{1}{2} n \pi+x\right)$. 
8. $\frac{d^{n}}{d x^{n}}\left(\frac{\log x}{x}\right)=(-\mathrm{I})^{n} \frac{n !}{x^{n+1}}\left\{\log x-\left(\frac{\mathrm{I}}{\mathrm{I}}+\frac{\mathrm{I}}{2}+\frac{\mathrm{I}}{3}+\ldots \ldots+\frac{\mathrm{I}}{n}\right)\right\}$.

9. $\frac{d^{n+1}}{d x^{n+1}} \sin ^{-1} x=\frac{\mathrm{I} \cdot 3 \cdot 5 \ldots(2 n-\mathrm{I})}{2^{n}(\mathrm{I}-x)^{n} \sqrt{\mathrm{I}-x^{2}}}\left\{\mathrm{I}-\frac{\mathrm{I}}{2 n-\mathrm{I}}\left(\begin{array}{l}n \\ \mathrm{I}\end{array}\right) \frac{\mathrm{I}-x}{\mathrm{I}+x}\right\}$

$$
+\frac{\mathrm{I} \cdot 3}{(2 n-\mathrm{I})(2 n-3)}\left(\begin{array}{c}
n \\
2
\end{array}\right)\left(\frac{\mathrm{I}-x}{\mathrm{I}+x}\right)^{2}-\frac{\mathrm{I} \cdot 3 \cdot 5}{(2 n-\mathrm{I})(2 n-3)(2 n-5)}\left(\begin{array}{l}
n \\
3
\end{array}\right)\left(\frac{\mathrm{I}-x}{\mathrm{I}+x}\right)^{3}
$$

I0. $\frac{d^{n}}{d x^{n}}\left(\tan ^{-1} x\right)=(-\mathrm{I})^{n-1} \frac{(n-\mathrm{I}) !}{\left(\mathrm{I}+x^{2}\right)^{\frac{n}{2}}} \sin \left(n \tan ^{-1} \frac{\mathrm{I}}{x}\right)$

7.39 Derivatives of Implicit Functions.

7.391 If $y$ is a function of $x$, and $f(x, y)=0$.

I. $\frac{d y}{d x}=-\frac{\frac{\partial}{\partial x}}{\frac{\partial f}{\partial y}}$

$\frac{d^{2} y}{d x^{2}}=-\frac{\left(\frac{\partial f}{\partial y}\right)^{2} \frac{\partial^{2} f}{\partial x^{2}}-2 \frac{\partial f}{\partial x} \frac{\partial f}{\partial y} \frac{\partial^{2} f}{\partial x \partial y}+\left(\frac{\partial f}{\partial x}\right)^{2} \frac{\partial^{2} f}{\partial y^{2}}}{\left(\frac{\partial f}{\partial y}\right)^{3}}$

7.392 If $z$ is a function of $x$ and $y$, and $f(x, y, z)=0$.

$\frac{\partial z}{\partial x}=-\frac{\frac{\partial f}{\partial x}}{\frac{\partial f}{\partial z}} ; \quad \frac{\partial z}{\partial y}=-\frac{\frac{\partial f}{\partial y}}{\frac{\partial f}{\partial z}}$

$\frac{\partial^{2} z}{\partial x^{2}}=-\frac{\left(\frac{\partial f}{\partial z}\right)^{2} \frac{\partial^{2} f}{\partial x^{2}}-2 \frac{\partial f}{\partial x} \frac{\partial f}{\partial x} \frac{d^{2} f}{\partial x \partial z}+\left(\frac{\partial f}{\partial x}\right)^{2} \frac{\partial^{2} f}{\partial z^{2}}}{\left(\frac{\partial f}{\partial z}\right)^{3}}$

3. $\frac{\partial^{2} z}{\partial y^{2}}=-\frac{\left(\frac{\partial f}{\partial z}\right)^{2} \frac{\partial^{2} f}{\partial y^{2}}-2 \frac{\partial f}{\partial z} \frac{\partial f}{\partial y} \frac{\partial^{2} f}{\partial y \partial z}+\left(\frac{\partial f}{\partial y}\right)^{2} \frac{\partial^{2} f}{\partial z^{2}}}{\left(\frac{\partial f}{\partial z}\right)^{3}}$

$\frac{\partial^{2} z}{\partial x \partial y}=-\frac{\left(\frac{\partial f}{\partial z}\right)^{2} \frac{\partial^{2} f}{\partial x \partial y}-\frac{\partial f}{\partial z}\left(\frac{\partial f}{\partial x} \frac{\partial^{2} f}{\partial y \partial z}+\frac{\partial f}{\partial y} \frac{\partial^{2} f}{\partial x \partial z}\right)+\frac{\partial f}{\partial x} \frac{\partial f}{\partial y} \frac{\partial^{2} f}{\partial z^{2}}}{\left(\frac{\partial f}{\partial z}\right)^{3}}$ 


\section{DIFFERENTIAL EQUATIONS.}

8.000 Ordinary differential equations of the first order. General form:

$$
\frac{d y}{d x}=f(x, y)
$$

8.001 Variables are separable. $f(x, y)$ is of, or can be reduced to, the form:

$$
f(x, y)=-\frac{X}{Y}
$$

where $X$, is a function of $x$ alone and $Y$ is a function of $y$ alone. The solution is:

$$
\int X d x+\int Y d y=C
$$

8.002 Linear equations of the form:

Solution:

$$
\frac{d y}{d x}+P(x) y=Q(x)
$$

$$
y=e^{-\int P(x) d x}\left\{\int Q(x) e^{-\int P(x) d x} d x+C\right\}
$$

8.003 Equations of the form:

Solution:

$$
\frac{d y}{d x}+P(x) y=y^{n} Q(x)
$$

$$
\frac{\mathrm{I}}{y^{n-1}} e^{-(n-\mathrm{I}) \boldsymbol{S} P(x) d x}+(n-\mathrm{I}) \int Q(x) e^{-(n-\mathrm{I}) \boldsymbol{S} P(x) d x} d x=C .
$$

8.010 Homogeneous equations of the form:

$$
\frac{d y}{d x}=-\frac{P(x, y)}{Q(x, y)}
$$

where $P(x, y)$ and $Q(x, y)$ are homogeneous functions of $x$ and $y$ of the same degree. The change of variable:

gives the solution:

$$
y=v x
$$

$$
\int \frac{d v}{\frac{P(\mathrm{I}, v)}{Q(\mathrm{I}, v)}+v}+\log x=C \text {. }
$$


8.011 Equations of the form:

$$
\frac{d y}{d x}=\frac{a^{\prime} x+b^{\prime} y+c^{\prime}}{a x+b y+c}
$$

If $a b^{\prime}-a^{\prime} b \neq 0$, the substitution

where

$$
\begin{gathered}
x=x^{\prime}+p, \quad y=y^{\prime}+q, \\
a p+b q+c=0, \\
a^{\prime} p+b^{\prime} q+c^{\prime}=0,
\end{gathered}
$$

renders the equation homogeneous, and it may be solved by 8.010 .

If $a b^{\prime}-a^{\prime} b=0$ and $b^{\prime} \neq 0$, the change of variables to either $x$ and $z$ or $y$ and $z$ by means of

$$
z=a x+b y
$$

will make the variables separable (8.001).

8.020 Exact differential equations. The equation,

is exact $1 \mathrm{I}$,

$$
P(x, y) d x+Q(x, y) d y=0 \text {, }
$$

$$
\frac{\partial Q}{\partial x}=\frac{\partial P}{\partial y} \text {. }
$$

The solution is:

$$
\begin{aligned}
& \int P(x, y) d x+\int\left\{Q(x, y)-\frac{\partial}{\partial y} \int P(x, y) d x\right\} d y=C, \\
& \int Q(x, y) d y+\int\left\{P(x, y)-\frac{\partial}{\partial x} \int Q(x, y) d y\right\} d x=C
\end{aligned}
$$

8.030 Integrating factors. $v(x, y)$ is an integrating factor of

if

$$
\begin{gathered}
P(x, y) d x+Q(x, y) d y=0, \\
\frac{\partial}{\partial x}(v Q)=\frac{\partial}{\partial y}(v P) .
\end{gathered}
$$

8.031 If one only of the functions $P x+Q y$ and $P x-Q y$ is equal to 0 , the reciprocal of the other is an integrating factor of the differential equation.

8.032 Homogeneous equations. If neither $P x+Q y$ nor $P x-Q y$ is equal to o, $\frac{\mathrm{I}}{P x+Q y}$ is an integrating factor of the equation if it is homogeneous. 
8.033 An equation of the form,

$$
P(x, y) y d x+Q(x, y) x d y=0,
$$

has an integrating factor:

\subsection{If}

$$
\frac{\mathrm{I}}{x P-y Q} \text {. }
$$

$$
\frac{\frac{\partial P}{\partial y}-\frac{\partial Q}{\partial x}}{Q}=F(x)
$$

is a function of $x$ only, an integrating factor is

$$
e^{\int F(x) d x} \text {. }
$$

\subsection{If}

$$
\frac{\frac{\partial Q}{\partial x}-\frac{\partial P}{\partial y}}{P}=F(y)
$$

is a function of $y$ only, an integrating factor is

\subsection{If}

$$
e^{\int F(y) d y} \text {. }
$$

$$
\frac{\frac{\partial P}{\partial y}-\frac{\partial Q}{\partial x}}{Q y-P x}=F(x y)
$$

is a function of the product $x y$ only, an integrating factor is

$$
e^{f F(x y) d(x y)} \text {. }
$$

8.037 If

$$
\frac{x^{2}\left(\frac{\partial Q}{\partial x}-\frac{\partial P}{\partial y}\right)}{P x+Q y}=F\left(\frac{y}{x}\right)
$$

is a function of the quotient $\frac{y}{x}$ only, an integrating factor is

$$
e^{\int F}\left(\frac{y}{x}\right) d\left(\frac{y}{x}\right) \text {. }
$$

8.040. Ordinary differential equations of the first order and of degree higher than the first.

Write:

General form of equation:

$$
\frac{d y}{d x}=p
$$

$$
f(x, y, p)=0
$$


8.041 The equation can be solved as an algebraic equation in $p$. It can be written

$$
\left(p-R_{1}\right)\left(p-R_{2}\right) \ldots \ldots\left(p-R_{n}\right)=0 .
$$

The differential equations:

$$
\begin{aligned}
& p=R_{1}(x, y), \\
& p=R_{2}(x, y),
\end{aligned}
$$

may be solved by the previous methods. Write the solutions:

$$
f_{1}(x, y, c)=0 ; f_{2}(x, y, c)=0 ; \ldots \ldots
$$

where $c$ is the same arbitrary constant in each. The solution of the given differential equation is:

$$
f_{1}(x, y, c) f_{2}(x, y, c) \ldots \ldots f_{n}(x, y, c)=0 .
$$

8.042 The equation can be solved for $y$ :

I.

Differentiate with respect to $x$ :

$$
y=f(x, p) \text {. }
$$

2.

$$
p=\psi\left(x, p, \frac{d p}{d x}\right)
$$

It may be possible to integrate (2) regarded as an equation in the two variables $x, p$, giving a solution

3.

$$
\phi(x, p, c)=0 .
$$

If $p$ is eliminated between (I) and (3) the result will be the solution of the given equation.

8.043 The equation can be solved for $x$ :

I.

$$
x=f(y, p) \text {. }
$$

Differentiate with respect to $y$ :

$$
\frac{\mathrm{I}}{p}=\psi\left(y, p, \frac{d p}{d y}\right)
$$

If a solution of (2) can be found:

$$
\phi(y, p, c)=0 .
$$

Eliminate $p$ between ( $\mathrm{r}$ ) and (3) and the result will be the solution of the given equation.

8.044 The equation does not contain $x$ :

It may be solved for $p$, giving,

$$
f(y, p)=0 \text {. }
$$

$$
\frac{d y}{d x}=F(y),
$$

which can be integrated. 
8.045 The equation does not contain $y$ :

$$
f(x, p)=0 .
$$

It may be solved for $p$, giving,

which can be integrated.

$$
\frac{d y}{d x}=F(x),
$$

It may be solved for $x$, giving,

which may be solved by 8.043 .

$$
x=F(p),
$$

8.050 Equations homogeneous in $x$ and $y$.

General form:

$$
F\left(p, \frac{y}{x}\right)=0 .
$$

(a) Solve for $p$ and proceed as in $\mathbf{8 . 0 0 1}$

(b) Solve for $\frac{y}{x}$ :

$$
y=x f(p) .
$$

Differentiate with respect to $x$ :

$$
\frac{d x}{x}=\frac{f^{\prime}(p) d p}{p-f(p)},
$$

which may be integrated.

8.060 Clairaut's differential equation:

I.

the solution is:

$$
y=p x+f(p),
$$$$
y=c x+f(c) .
$$

The singular solution is obtained by eliminating $p$ between (I) and 2.

$$
x+f^{\prime}(p)=0 .
$$

8.061 The equation

I.

$$
y=x f(p)+\phi(p)
$$

The solution is that of the linear equation of the first order:

2.

$$
\frac{d x}{d p}-\frac{f^{\prime}(p)}{p-f(p)} x=\frac{\phi^{\prime}(p)}{p-f(p)}
$$

which may be solved by 8.002 . Eliminating $p$ between (I) and the solution of (2) gives the solution of the given equation. 
8.062 The equation:

$$
x \phi(p)+y \psi(p)=\chi(p)
$$

may be reduced to 8.061 by dividing by $\psi(p)$.

DIFFERENTIAL EQUATIONS OF AN ORDER HIGHER THAN THE FIRST

8.100 Linear equations with constant coefficients. General form:

$$
\frac{d^{n} y}{d x^{n}}+a_{1} \frac{d^{n-1} y}{d x^{n-1}}+a_{2} \frac{d^{n-2} y}{d x^{n-2}}+\ldots+a_{n} y=V(x)
$$

The complete solution consists of the sum of

(a) The complementary function, obtained by solving the equation with $V(x)=0$, and containing $n$ arbitrary constants, and

(b) The particular integral, with no arbitrary constants.

8.101 The complementary function. Assume $y=e^{\lambda x}$. The equation for determining $\lambda$ is:

$$
\lambda^{n}+a_{1} \lambda^{n-1}+a_{2} \lambda^{n-2}+\ldots \ldots+a_{n}=0 .
$$

8.102 If the roots of $\mathbf{8 . 1 0 1}$ are all real and distinct the complementary function is:

$$
y=c_{1} e^{\lambda_{1} x}+c_{2} e^{\lambda_{2} x}+\ldots .+c_{n} e^{\lambda_{n} x} .
$$

8.103 For a pair of complex roots:

$$
\mu \pm i \nu
$$

the corresponding terms in the complementary function are:

$$
e^{\mu x}(A \cos \nu x+B \cos \nu x)=C e^{\mu x} \cos (\nu x-\theta)=C e^{\mu x} \sin (\nu x+\theta),
$$
where

$$
C=\sqrt{A^{2}+B^{2}}, \quad \tan \theta=\frac{B}{A}
$$

8.104 If there are $r$ equal real roots the terms in the complementary function corresponding to them are:

$$
e^{\lambda x}\left(A_{1}+A_{2} x+A_{3} x^{2}+\ldots .+A_{r} x^{r-1}\right),
$$

where $\lambda$ is the repeated root, and $A_{1}, A_{2}, \ldots, A_{r}$ are the $r$ arbitrary constants.

8.105 If there are $m$ equal pairs of complex roots the terms in the complementary function corresponding to them are:

$$
\begin{aligned}
& e^{\mu x}\left\{\left(A_{1}+A_{2} x+A_{3} x^{2}+\ldots+A_{m} x^{m-1}\right) \cos \nu x\right. \\
& \left.+\left(B_{1}+B_{2} x+B_{3} x^{2}+\ldots \ldots+B_{m} x^{m-1}\right) \sin \nu x\right\} \\
= & e^{\mu x}\left\{C_{1} \cos \left(\nu x-\theta_{1}\right)+C_{2} x \cos \left(\nu x-\theta_{2}\right)+\ldots \ldots+C_{m} x^{m-1} \cos \left(\nu x-\theta_{m}\right)\right\} \\
= & e^{\mu x}\left\{C_{1} \sin \left(\nu x+\theta_{1}\right)+C_{2} x \sin \left(\nu x+\theta_{2}\right)+\ldots \ldots+C_{m} x^{m-1} \sin \left(\nu x+\theta_{m}\right)\right\}
\end{aligned}
$$


where $\lambda \pm i \mu$ is the repeated root and

$$
\begin{aligned}
C_{k} & =\sqrt{A_{k}^{2}+B_{k}^{2}}, \\
\tan \theta_{k} & =\frac{B_{k}}{A_{k}} .
\end{aligned}
$$

The particular integral.

8.110 The operator $D$ stands for $\frac{\partial}{\partial x}, D^{2}$ for $\frac{\partial^{2}}{\partial x^{2}}, \ldots \ldots \ldots$

The differential equation 8.100 may be written:

$$
\begin{gathered}
\left(D^{n}+a_{1} D^{n-1}+a_{2} D^{n-2}+\ldots \ldots+a_{n}\right) y=f(D) y=V(x) \\
y=\frac{V(x)}{f(D)}, \\
f(D)=\left(D-\lambda_{1}\right)\left(D-\lambda_{2}\right) \ldots \ldots\left(D-\lambda_{n}\right),
\end{gathered}
$$

where $\lambda_{1}, \lambda_{2}, \ldots \ldots, \lambda_{n}$ are determined as in 8.101. The particular integral is:

$$
y=e^{\lambda_{1} x} \int e^{\left(\lambda_{2}-\lambda_{1}\right) x} d x \int e e^{\left(\lambda_{3}-\lambda_{2}\right) x} d x \ldots \ldots \int e^{-\lambda_{n}(x)} V(x) d x .
$$

8.111 $\frac{\mathrm{I}}{f(D)}$ may be resolved into partial fractions:

$$
\frac{\mathrm{r}}{f(D)}=\frac{N_{1}}{D-\lambda_{1}}+\frac{N_{2}}{D-\lambda_{2}}+\cdots+\frac{N_{n}}{D-\lambda_{n}} .
$$

The particular integral is:

$$
\begin{aligned}
y=N_{1} e^{\lambda_{1} x} \int e^{-\lambda_{1} x} V(x) d x+N_{2} e^{\lambda_{2} x} \int e^{-\lambda_{2} x} V(x) d x+\ldots & \\
& +N_{n} e^{\lambda_{n} x} \int e^{-\lambda_{n} x} V(x) d x .
\end{aligned}
$$

THE PARTICULAR INTEGRAL IN SPECIAL CASES

8.120 $V(x)=$ const. $=c$,

$$
y=\frac{c}{a_{n}} .
$$

8.121 $V(x)$ is a rational integral function of $x$ of the $m$ th degree. Expand $\frac{\mathrm{I}}{f(D)}$ in ascending powers of $D$, ending with $D^{m}$. Apply the operators $D, D^{2}$, ....., $D^{m}$ to each term of $V(x)$ separately and the particular integral will be the sum of the results of these operations. 


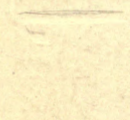




\subsection{2}

$$
\begin{aligned}
V(x) & =c e^{k x}, \\
y & =\frac{c}{f(k)} e^{k x},
\end{aligned}
$$

unless $k$ is a root of $f(D)=0$. If $k$ is a multiple root of order $r$ of $f(D)=0$

where

$$
y=\frac{c x^{r} e^{k x}}{r ! \psi(k)}
$$

$$
\begin{aligned}
& f(D)=(D-k)^{r} \psi(D) . \\
& V(x)=c \cos (k x+\alpha) .
\end{aligned}
$$

\subsection{3}

If $i k$ is not a root of $f(D)=0$ the particular integral is the real part of

$$
\frac{c}{f(i k)} e^{i(k x+\alpha)} \text {. }
$$

If $i k$ is a multiple root of order $r$ of $f(D)=0$ the particular integral is the real part of

$$
\frac{c x^{r} e^{i(k x+\alpha)}}{f^{(r)}(i k)},
$$

where $f^{(r)}(i k)$ is obtained by taking the $r$ th derivative of $f(D)$ with respect to $D$, and substituting $i k$ for $D$.

8.124

$$
V(x)=c \sin (k x+\alpha) .
$$

If $i k$ is not a root of $f(D)=0$ the particular integral is the real part of

$$
\frac{-i c e^{i(k x+\alpha)}}{f(i k)} \text {. }
$$

If $i k$ is a multiple root of order $r$ of $f(D)=0$ the particular integral is the real part of

$$
\frac{-i c x^{r} e^{i(k x+\alpha)}}{f^{(r)}(i k)} \text {. }
$$

\subsection{5}

$$
V(x)=c e^{k x} \cdot X,
$$

where $X$ is any function of $x$.

$$
y=c e^{k x} \frac{\mathrm{I}}{f(D+k)} X .
$$

If $X$ is a rational integral function of $x$ this may be evaluated by the method of 8.121.

\subsection{6}

$$
V(x)=c \cos (k x+\alpha) \cdot X,
$$

where $X$ is any function of $x$. The particular integral is the real part of

$$
c e^{i(k x+\alpha)} \frac{\mathrm{I}}{f(D+i k)} X .
$$

8.127

$$
V(x)=c \sin (k x+\alpha) \cdot X .
$$

The particular integral is the real part of

$$
-i c e^{i(k x+\alpha)} \frac{\mathrm{I}}{f(D+i k)} X \text {. }
$$




\subsection{8}

$$
V(x)=c e^{\beta x} \cos (k x+\alpha) .
$$

If $(\beta+i k)$ is not a root of $f(D)=0$ the particular integral is the real part of

$$
c e^{i(k x+\alpha)} \frac{\mathrm{I}}{f(\beta+i k)} e^{\beta x} \text {. }
$$

If $(\beta+i k)$ is a multiple root of order $r$ of $f(D)=0$ the particular integral is the real part of

$$
\frac{c e^{i(k x+\alpha)} x^{r} e^{\beta x}}{f^{(r)}(\beta+i k)}
$$

where $f^{(r)}(\beta+i k)$ is formed as in 8.123.

8.129

$$
V=c e^{\beta x} \sin (k x+\alpha) \text {. }
$$

If $(\beta+i k)$ is not a root of $f(D)=0$ the particular integral is the real part of

$$
\frac{-i c e^{i(k x+\alpha)} e^{\beta x}}{f(\beta+i k)} \text {. }
$$

If $(\beta+i k)$ is a multiple root of order $r$ of $f(D)=0$ the particular integral is the real part of

$$
\frac{-i c e^{i(k x+\alpha)} x^{r} e^{\beta x}}{f^{(r)}(\beta+i k)}
$$

8.130

$$
V(x)=x^{m} X,
$$

where $X$ is any function of $x$.

$y=x^{m} \frac{\mathrm{I}}{f(D)} X+m x^{m-1}\left\{\frac{d}{d D} \frac{\mathrm{I}}{f(D)}\right\} X+\frac{m(m-\mathrm{I})}{2 !} x^{m-2}\left\{\frac{d^{2}}{d D^{2}} \frac{\mathrm{I}}{f(D)}\right\} X+\ldots \ldots$

The series must be extended to the $(m+\mathbf{r})$ th term.

8.200 Homogeneous linear equations. General form:

$$
x^{n} \frac{d^{n} y}{d x^{n}}+a_{1} x^{n-1} \frac{d^{n-1} y}{d x^{n-1}}+\ldots .+a_{n-1} x \frac{d y}{d x}+a_{n} y=\cdot V(x) .
$$

Denote the operator:

$$
\begin{gathered}
x \frac{d}{d x}=\theta \\
x^{m} \frac{d^{m}}{d x^{m}}=\theta(\theta-\mathrm{I})(\theta-2) \ldots(\theta-m+\mathrm{I}) .
\end{gathered}
$$

The differential equation may be written:

$$
F(\theta) \cdot y=V(x)
$$

The complete solution is the sum of the complementary function, obtained by solving the equation with $V(x)=0$, and the particular integral. 
8.201 The complementary function.

$$
y=c_{1} x^{\lambda_{1}}+c_{2} x^{\lambda_{2}}+\ldots .+c_{n} x^{\lambda_{n}}
$$

where $\lambda_{1}, \lambda_{2}, \ldots, \lambda_{n}$ are the $n$ roots of

if the roots are all distinct.

$$
F(\lambda)=0
$$

If $\lambda_{k}$ is a multiple root of order $r$, the corresponding terms in the complementary function are:

$$
x^{\lambda_{k}}\left\{b_{1}+b_{2} \log x+b_{3}(\log x)^{2}+\ldots+b_{r}(\log x)^{r-1}\right\} .
$$

If $\lambda=\mu \pm i \nu$ is a pair of complex roots, of order $r$, the corresponding terms in the complementary function are:

$$
\begin{aligned}
x^{\mu}\left\{\left[A_{1}\right.\right. & \left.+A_{2} \log x+A_{3}(\log x)^{2}+\ldots+A_{r}(\log x)^{r-1}\right] \cos (\nu \log x) \\
& \left.+\left[B_{1}+B_{2} \log x+B_{3}(\log x)^{2}+\ldots .+B_{r}(\log x)^{r-1}\right] \sin (\nu \log x)\right\} .
\end{aligned}
$$

8.202 The particular integral.

If

$$
\begin{gathered}
F(\theta)=\left(\theta-\lambda_{1}\right)\left(\theta-\lambda_{2}\right) \ldots \ldots\left(\theta-\lambda_{n}\right) \\
y=x^{\lambda_{1}} \int x^{\lambda_{2}-\lambda_{1}-1} d x \int x^{\lambda_{3}-\lambda_{2}-1} d x \ldots . \int x^{\lambda_{n-\lambda_{n-1}-1}} V(x) d x
\end{gathered}
$$

8.203 The operator $\frac{I}{F(\theta)}$ may be resolved into partial fractions:

$$
\begin{aligned}
\frac{\mathrm{I}}{F(\theta)}=\frac{N_{1}}{\theta-\lambda_{1}}+\frac{N_{2}}{\theta-\lambda_{2}}+\ldots & \ldots+\frac{N_{n}}{\theta-\lambda_{n}} \\
y=N_{1} x^{\lambda_{1}} \int x^{-\lambda_{1}-1} V(x) d x & +N_{2} x^{\lambda_{2}} \int x^{-\lambda_{2}-1} V(x) d x \\
& \ldots \ldots+N_{n} x^{\lambda_{n}} \int x^{-\lambda_{n}-1} V(x) d x
\end{aligned}
$$

The particular integral in special cases.

8.210

$$
\begin{aligned}
V(x) & =c x^{k}, \\
y & =\frac{c}{F(k)} x^{k},
\end{aligned}
$$

unless $k$ is a root of $F(\theta)=0$.

If $k$ is a multiple root of order $r$ of $F(\theta)=0$.

$$
y=\frac{c(\log x)^{r}}{F^{(r)}(k)}
$$

where $F^{(r)}(k)$ is obtained by taking the $r$ th derivative of $F(\theta)$ with respect to $\theta$ and after differentiation substituting $k$ for $\theta$. 
8.211

$V(x)=c x^{k} X$,

where $X$ is any function of $x$.

8.220 The differential equation:

$$
(a+b x)^{n} \frac{d^{n} y}{d x^{n}}+(a+b x)^{n-1} a_{1} \frac{d^{n-1} y}{d x^{n-1}}+\ldots .+(a+b x) a_{n-1} \frac{d y}{d x}+a_{n} y=V(x),
$$

may be reduced to the homogeneous linear equation (8.200) by the change of variable

$$
z=a+b x .
$$

It may be reduced to a linear equation with constant coefficients by the change of variable:

$$
e^{z}=a+b x .
$$

8.230 The general linear equation. General form:

$$
P_{0} \frac{d^{n} y}{d x^{n}}+P_{1} \frac{d^{n-1} y}{d x^{n-1}}+\ldots .+P_{n-1} \frac{d y}{d x}+P_{n}=V,
$$

where $P_{0}, P_{1}, \ldots \ldots, P_{n}, V$ are functions of $x$ only.

The complete solution is the sum of:

(a) The complementary function, which is the general solution of the equation with $V=0$, and containing $n$ arbitrary constants, and

(b) The particular integral.

8.231 Complementary Function. If $y_{1}, y_{2}, \ldots, y_{n}$ are $n$ independent solutions of $\mathbf{8 . 2 3 0}$ with $V=0$, the complementary function is

$$
y=c_{1} y_{1}+c_{2} y_{2}+\cdots \cdots+c_{n} y_{n} .
$$

The conditions that $y_{1}, y_{2}^{\prime}, \ldots \ldots, y_{n}$ be $n$ independent solutions is that the determinant $\Delta \neq 0$.

When $\Delta \neq 0$ :

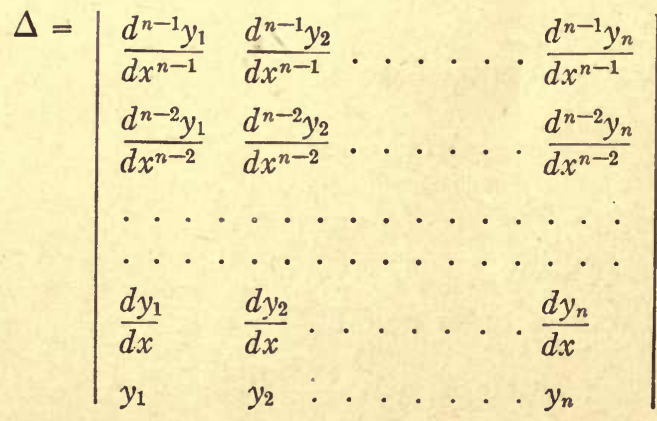

$$
\Delta=C e^{-\int \frac{P_{1}}{P_{0}} d x}
$$


8.232 The particular integral. If $\Delta_{k}$ is the minor of $\frac{d^{n-1} y_{k}}{d x^{n-1}}$ in $\Delta$, the particular integral is:

$$
y=y_{1} \int \frac{V \Delta_{1}}{P_{0} \Delta} d x+y_{2} \int \frac{V \Delta_{2}}{P_{0} \Delta} d x+\ldots+y_{n} \int \frac{V \Delta_{n}}{P_{0} \Delta} d x .
$$

8.233 If $y_{1}$ is one integral of the equation 8.230 with $v=0$, the substitution

$$
y=u y_{1}, \quad v=\frac{d u}{d x}
$$

will result in a linear equation of order $n-\mathrm{I}$.

8.234 If $y_{1}, y_{2}, \ldots \ldots, y_{n-1}$ are $n-\mathrm{I}$ independent integrals of 8.230 with $V=0$ the complete solution is:

where $\Delta$ is the determinant:

$$
y=\sum_{k=\mathrm{I}}^{n-\mathrm{I}} y c_{k k}+c_{n} \sum_{k=\mathrm{r}}^{n-\mathrm{I}} y_{k} \int \frac{\Delta_{k}}{\Delta^{2}} e^{-\int \frac{P_{1}}{P_{0}} d x} d x
$$

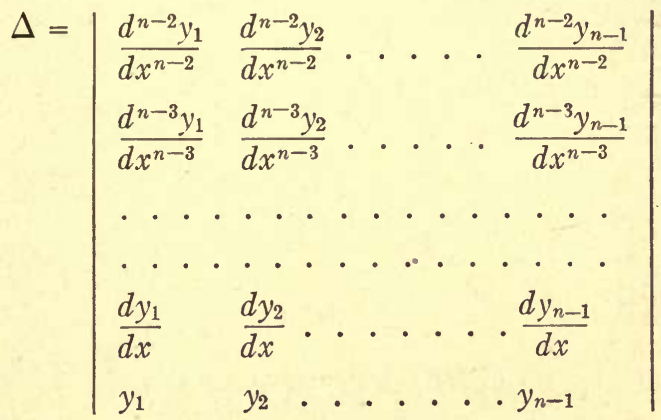

and $\Delta_{k}$ is the minor of $\frac{d^{n-2} y_{k}}{d x^{n-2}}$ in $\Delta$.

\section{SYMBOLIC METHODS}

8.240 Denote the operators:

$$
\begin{aligned}
\frac{d}{d x} & =D \\
x \frac{d}{d x} & =\theta
\end{aligned}
$$

8.241 If $X$ is a function of $x$ :

I.

$$
(D-m)^{-1} X=e^{m x} \int e^{-m x} X d x \text {. }
$$

2. $(D-m)^{-1} \circ=c e^{m x}$. 
8.242 If $F(D)$ is a polynomial in $D$,

I.

2.

3.

8.243 If $F(\theta)$ is a polynomial in $\theta$,

I.

2 .

3 .

8.244

$$
\begin{aligned}
F(D) e^{m x} & =e^{m x} F(m) . \\
F(D) e^{m x} X & =e^{m x} F(D+m) X . \\
e^{m x} F(D) X & =F(D-m) e^{m x} X .
\end{aligned}
$$

INTEGRATION IN SERIES

8.250 If a linear differential equation can be expressed in the symbolic form:

$$
\left[x^{m} F(\theta)+f(\theta)\right] y=0,
$$

where $F(\theta)$ and $f(\theta)$ are polynomials in $\theta$, the substitution,

leads to the equations,

$$
y=\sum_{n=0}^{\infty} a_{n} x^{\rho+n m},
$$

$$
\begin{aligned}
a_{0} f(\rho) & =\mathrm{o}, \\
a_{0} F(\rho)+a_{1} f(\rho+m) & =0, \\
a_{1} F(\rho+m)+a_{2} f(\rho+2 m) & =0, \\
a_{2} F(\rho+2 m)+a_{3} f(\rho+3 m) & =0 . \\
\ldots &
\end{aligned}
$$

8.251 The equation

$$
f(\rho)=0,
$$

is the "indicial equation." If it is satisfied $a_{0}$ may be chosen arbitrarily, and the other coefficients are then determined.

8.252 An equation:

$$
\left[F(\theta)+\phi(\theta) \frac{d^{m}}{d x^{m}}\right] y=0,
$$

may be reduced to the form 8.250 , where,

$$
f(\theta)=\phi(\theta-m) \theta(\theta-\mathrm{I})(\theta-2) \ldots \ldots(\theta-m+\mathrm{I}) .
$$

If the degree of the polynomial $f$ is greater than that of $F$ the series always converges; if the degree of $f$ is less than that of $F$ the series always diverges. 


\subsection{0}

ORDINARY DIFFERENTIAL EQUATIONS OF SPECIAL TYPES

where $X$ is a function of $x$ only.

$$
\frac{d^{n} y}{d x^{n}}=X
$$

$$
y=\frac{\mathrm{I}}{(n-\mathrm{I}) !} \int_{0}^{x}(x-t)^{n-1} T d t+c_{1} x^{n-1}+c_{2} x^{n-2}+\ldots+c_{n-1} x+c_{n},
$$

where $T$ is the same function of $t$ that $X$ is of $x$.

\subsection{1}

$$
\frac{d^{2} y}{d x^{2}}=Y
$$

where $Y$ is a function of $y$ only.

If

the solution is:

$$
\psi(y)=2 \int Y d y
$$

$$
\int \frac{d y}{\left\{\psi(y)+c_{1}\right\}^{\frac{1}{2}}}=x+c_{2}
$$

\subsection{2}

Put

$$
\begin{gathered}
\frac{d^{n} y}{d x^{n}}=F\left(\frac{d^{n-1} y}{d x^{n-1}}\right) . \\
\frac{d^{n-1} y}{d x^{n-1}}=Y ; \quad \frac{d Y}{d x}=F(Y), \\
x+c_{1}=\int \frac{d Y}{F(Y)}=\psi(Y), \\
Y=\phi\left(x+c_{1}\right), \\
\frac{d^{n-1} y}{d x^{n-1}}=\phi\left(x+c_{1}\right),
\end{gathered}
$$

and this equation may be solved by 8.300 .

Or the equation can be solved:

$$
y=\int \frac{d Y}{F(Y)} \int \frac{d Y}{F(Y)} \cdots \cdots \int \frac{Y d Y}{F(Y)}
$$

where the integration is to be carried out from right to left and an arbitrary constant added after each integration. Eliminating $Y$ between this result and gives the solution.

$$
Y=\phi\left(x+c_{1}\right)
$$

$$
\frac{d^{n} y}{d x^{n}}=F\left(\frac{d^{n-2} y}{d x^{n-2}}\right)
$$


Put

$$
\begin{aligned}
\frac{d^{n-2} y}{d x^{n-2}} & =Y, \\
\frac{d^{2} Y}{d x^{2}} & =F(Y),
\end{aligned}
$$

which may be solved by $\mathbf{8 . 3 0 1}$. If the solution can be expressed:

$$
Y=\phi(x),
$$

$n-2$ integrations will solve the given differential equation.

Or putting

$$
\begin{gathered}
\psi(y)=2 \int Y d y, \\
y=\int \frac{d Y}{\left\{c_{1}+\psi(Y)\right\}^{\frac{1}{2}}} \int \frac{d Y}{\left\{c_{1}+\psi(Y)\right\}^{\frac{1}{2}}} \ldots \ldots \int \frac{Y d Y}{\left\{c_{1}+\psi(Y)\right\}^{\frac{1}{2}}},
\end{gathered}
$$

where the integration is to be carried out from right to left and an arbitrary constant added after each integration. The solution of the given differential equation is obtained by elimination between this result and

$$
Y=\phi(x) .
$$

8.304 Differential equations of the second order in which the independent variable does not appear. General type:

Put

$$
F\left(y, \frac{d y}{d x}, \frac{d^{2} y}{d x^{2}}\right)=0
$$

$$
p=\frac{d y}{d x}, \quad p \frac{d p}{d y}=\frac{d^{2} y}{d x^{2}} .
$$

A differential equation of the first order results:

$$
F\left(y, p, p \frac{d p}{d y}\right)=0 \text {. }
$$

If the solution of this equation is:

$$
p=f(y)
$$

the solution of the given equation is,

$$
x+c_{2}=\int \frac{d y}{f(y)} .
$$

8.305 Differential equations of the second order in which the dependent variable does not appear. General type:

Put

$$
F\left(x, \frac{d y}{d x}, \frac{d^{2} y}{d x^{2}}\right)=0 .
$$

$$
p=\frac{d y}{d x}, \quad \frac{d p}{d x}=\frac{d^{2} y}{d x^{2}} .
$$


A differential equation of the first order results:

$$
F\left(x, p, \frac{d p}{d x}\right)=0
$$

If the solution of this equation is:

$$
p=f(x),
$$

the solution of the given equation is:

$$
y=c_{2}+\int f(x) d x .
$$

8.306 Equations of an order higher than the second in which either the independent or the dependent variable does not appear. The substitution:

$$
\frac{d y}{d x}=p,
$$

as in 8.304 and 8.305 will result in an equation of an order less by unity than the given equation.

8.307 Homogeneous differential equations. If $y$ is assumed to be of dimensions $n, x$ of dimensions I, $\frac{d y}{d x}$ of dimensions $(n-\mathrm{I}), \frac{d^{2} y}{d x^{2}}$ of dimensions $(n-2)$, ..... then if every term has the same dimensions the equation is homogeneous. If the independent variable is changed to $\theta$ and the dependent variable changed to $z$ by the relations,

$$
x=e^{\theta}, \quad y=z e^{n \theta},
$$

the resulting equation will be one in which the independent variable does not appear and its order can be lowered by unity by $\mathbf{8 . 3 0 6}$.

If $y, \frac{d y}{d x}, \frac{d^{2} y}{d x^{2}}, \ldots$ are assumed all to be of the same dimensions, and the equation is homogeneous, the substitution:

$$
y=e^{\int u d x},
$$

will result in an equation in $u$ and $x$ of an order less by unity than the given equation.

8.310 Exact differential equations. A linear differential equation:

$$
P_{n} \frac{d^{n} y}{d x^{n}}+P_{n-1} \frac{d^{n-1} y}{d x^{n-1}}+\ldots+P_{1} \frac{d y}{d x}+P_{0}=P,
$$

where $P, P_{0}, P_{1}, \ldots \ldots P_{n}$ are functions of $x$ is exact if:

$$
P_{0}-\frac{d P_{1}}{d x}+\frac{d^{2} P_{2}}{d x^{2}}-\ldots \ldots+(-\mathrm{I})^{n} \frac{d^{n} P_{n}}{d x^{n}}=\text { o. }
$$


The first integral is:

where,

$$
Q_{n} \frac{d^{n-1}}{d x^{n-1}}+Q_{n-1} \frac{d^{n-2} y}{d x^{n-2}}+\ldots+Q_{1} y=\int P d x+c_{1},
$$

$$
\begin{aligned}
Q_{n} & =P_{n}, \\
Q_{n-1} & =P_{n-1}-\frac{d P_{n}}{d x}, \\
Q_{n-2} & =P_{n-2}-\frac{d P_{n-1}}{d x}+\frac{d^{2} P_{n}}{d x^{2}}, \\
\ldots & \\
\ldots & \\
Q_{1} & =P_{1}-\frac{d P_{2}}{d x}+\frac{d^{2} P_{3}}{d x^{2}}-\ldots+(-\mathrm{I})^{n-1} \frac{d^{n-1} P_{n}}{d x^{n-1}} .
\end{aligned}
$$

If the first integral is an exact differential equation the process may be continued as long as the coefficients of each successive integral satisfy the condition of integrability.

8.311 Non-linear differential equations. A non-linear differential equation of the $n$th order:

$$
V\left(\frac{d^{n} y}{d x^{n}}, \frac{d^{n-1} y}{d x^{n-1}}, \ldots \ldots, \frac{d y}{d x}, y, x\right)=0
$$

to be exact must contain $\frac{d^{n} y}{d x^{n}}$ in the first degree only. Put

$$
\frac{d^{n-1} y}{d x^{n-1}}=p, \quad \frac{d^{n} y}{d x^{n}}=\frac{d p}{d x} .
$$

Integrate the equation on the assumption that $p$ is the only variable and $\frac{d p}{d x}$ its differential coefficient. Let the result be $V_{1}$. In $V d x-d V_{1}, \frac{d^{n-1} y}{d x^{n-1}}$ is the highest differential coefficient and it occurs in the first degree only. Repeat this process as often as may be necessary and the first integral of the exact differential equation will be

$$
V_{1}+V_{2}+\ldots \ldots=c .
$$

If this process breaks down owing to the appearance of the highest differential coefficient in a higher degree than the first the given differential equation was not exact. 
8.312 General condition for an exact differential equation. Write:

$$
\frac{d y}{d x}=y^{\prime} \quad \frac{d^{2} y}{d x^{2}}=y^{\prime \prime} \ldots \ldots \quad \frac{d^{n} y}{d x^{n}}=y^{(n)} .
$$

In order that the differential equation:

$$
V\left(x, y, y^{\prime}, y^{\prime \prime}, \ldots \ldots, y^{(n)}\right)=0,
$$

be exact it is necessary and sufficient that

$$
\frac{\partial V}{\partial y}-\frac{\partial}{\partial x}\left(\frac{\partial V}{\partial y^{\prime}}\right)+\frac{\partial^{2}}{\partial x^{2}}\left(\frac{\partial V}{\partial y^{\prime \prime}}\right)-\ldots .+(-\mathrm{I})^{n} \frac{\partial^{n}}{\partial x^{n}}\left(\frac{\partial V}{\partial y^{(n)}}\right)=0 .
$$

8.400 Linear differential equations of the second order. General form:

$$
\frac{d^{2} y}{d x^{2}}+P \frac{d y}{d x}+Q y=R
$$

where $P, Q, R$ are, in general, functions of $x$.

8.401 If a solution of the equation with $R=0$ :

$$
y=w
$$

can be found, the complete solution of the given differential equation is:

$$
y=c_{2} w+c_{1} w \int e^{-\int P d x} \frac{d x}{w^{2}}+w \int e^{-\int P d x} \frac{d x}{w^{2}} \int w e^{\mathcal{S} P d x} d x .
$$

8.402 The general linear differential equation of the second order may be reduced to the form:

where:

$$
\begin{aligned}
\frac{d^{2} v}{d x^{2}}+I v & =R e^{\frac{1}{2} \int P d x}, \\
y & =v e^{-\frac{1}{2} \int P d x} \\
I & =Q-\frac{\mathrm{I}}{2} \frac{d P}{d x}-\frac{\mathrm{I}}{4} P^{2} .
\end{aligned}
$$

8.403 The differential equation:

$$
\frac{d^{2} y}{d x^{2}}+P \frac{d y}{d x}+Q y=0
$$

by the change of independent variable to

$$
z=\int e^{-\int P d x} d x
$$

becomes:

$$
\frac{d^{2} y}{d z^{2}}+Q e^{2 \int P d x} y=0 \text {. }
$$

By the change of independent variable.

it becomes:

$$
\begin{aligned}
& d z=Q e^{\int P d x} d x \\
& Q e^{2} \quad P d x=\frac{\mathrm{I}}{U(z)},
\end{aligned}
$$

$$
\frac{d}{d z}\left\{\frac{\mathrm{I}}{U} \frac{d y}{d z}\right\}+y=0
$$


8.404 Resolution of the operator. The differential equation:

$$
u \frac{d^{2} y}{d x^{2}}+v \frac{d y}{d x}+w y=0,
$$

may sometimes be solved by resolving the operator,

into the product,

$$
u \frac{d^{2}}{d x^{2}}+v \frac{d}{d x}+w
$$

$$
\left(p \frac{d}{d x}+q\right)\left(r \frac{d}{d x}+s\right)
$$

The solution of the differential equation reduces to the solution of

$$
r \frac{d y}{d x}+s y=c_{1} e^{-\int \frac{q}{p} d x}
$$

The equations for determining $p, r, q, s$ are:

$$
\begin{aligned}
p r & =u, \\
q r+p s+p \frac{d r}{d x} & =v \\
q s+p \frac{d s}{d x} & =w .
\end{aligned}
$$

8.410 Variation of parameters. The complete solution of the differential equation:

is

$$
\frac{d^{2} y}{d x^{2}}+P \frac{d y}{d x}+Q y=R
$$

$$
y=c_{1} f_{2}(x)+c_{2} f_{1}(x)+\frac{I}{C} \iint^{x} R(\xi) e^{\int^{\xi} P d x}\left\{f_{2}(x) f_{1}(\xi)-f_{1}(x) f_{2}(\xi)\right\} d \xi,
$$

where $f_{1}(x)$ and $f_{2}(x)$ are two particular solutions of the differential equation with $R=0$, and are therefore connected by the relation

$$
f_{1} \frac{d f_{2}}{d x}-f_{2} \frac{d f_{1}}{d x}=C e^{-P d x} .
$$

$C$ is an absolute constant depending upon the forms of $f_{1}$ and $f_{2}$ and may be taken as unity.

8.500 The differential equation:

$$
\left(a_{2}+b_{2} x\right) \frac{d^{2} y}{d x^{2}}+\left(a_{1}+b_{1} x\right) \frac{d y}{d x}+\left(a_{0}+b_{0} x\right) y=0 .
$$

8.501 Let

$$
D=\left(a_{0} b_{1}-a_{1} b_{0}\right)\left(a_{1} b_{2}-a_{2} b_{1}\right)-\left(a_{0} b_{2}-a_{2} b_{0}\right)^{2}
$$


Special cases.

8.502 $b_{2}=b_{1}=b_{0}=$ o.

The solution is:

where:

$$
y_{1}=c_{1} e^{\lambda_{1} x}+c_{2} e^{\lambda_{2} x}
$$

8.503 $D=\circ, b_{2}=\circ$,

$$
\frac{\lambda_{1}}{\lambda_{2}}=\frac{-a_{1} \pm \sqrt{a_{1}^{2}-4 a_{0} a_{2}}}{2 a_{2}}
$$

where:

$$
y=e^{\lambda x}\left\{c_{1}+c_{2} \int e^{-(k+2 \lambda) x-m x^{2}} d x\right\}
$$

8.504 $D=\circ, b_{2} \neq$ ०:

$$
k=\frac{a_{1}}{a_{2}} \quad m=\frac{b_{1}}{2 a_{2}} \quad \lambda=-\frac{b_{0}}{b_{1}} .
$$

$$
y=e^{\lambda x}\left\{c_{1}+c_{2} \int e^{-(k+2 \lambda) x}\left(a_{2}+b_{2} x\right)^{m} d x\right\},
$$

where

$$
k=\frac{b_{1}}{\bar{b}_{2}} \quad m=\frac{a_{2} b_{1}-a_{1} b_{2}}{b_{2}{ }^{3}},
$$

and $\lambda$ is the common root of:

$$
\begin{aligned}
& a_{2} \lambda^{2}+a_{1} \lambda+a_{0}=0, \\
& b_{2} \lambda^{2}+b_{1} \lambda+b_{0}=0 .
\end{aligned}
$$

8.505 $D \neq \circ, b_{2}=b_{1}=\circ$. If $\eta=f(\xi)$ is the complete solution of:

where

$$
\begin{aligned}
\frac{d^{2} \eta}{d \xi^{2}}+\xi \eta & =\circ, \\
y & =e^{\lambda} x_{f}\left(\frac{\alpha+\beta x}{\beta^{3}}\right),
\end{aligned}
$$

$$
\alpha=\frac{4 a_{0} a_{2}-a_{1}^{2}}{4 a_{2}^{2}} \quad \beta=\frac{b_{0}}{a_{2}} \quad \lambda=-\frac{a_{1}}{2 a_{2}} .
$$

8.510 The differential equation 8.500 under the condition $D \neq \circ$ can always be reduced to the form:

$$
\xi \frac{d^{2} \phi}{d \xi^{2}}+(p+q+\xi) \frac{d \phi}{d \xi}+p \phi=0 .
$$

8.511 Denote the complete solution of 8.510:

$8.512 b_{2}=b_{1}=\circ 0$ :

$$
\phi=F\{\xi\} .
$$

where:

$$
\begin{gathered}
y=e^{\lambda x+(\mu+\nu x)_{2}^{\frac{3}{2}}} F\left\{2(\mu+\nu x)^{\frac{3}{2}}\right\}, \\
\lambda=-\frac{a_{1}}{2 a_{2}} \quad \mu=\frac{a_{1}{ }^{2}-4 a_{0} a_{2}}{4 a_{2}^{2}}\left(\frac{4 a_{2}^{2}}{9 b_{0}^{2}}\right)^{3}, \\
\nu=-\left(\frac{4 b_{0}}{9 a_{2}}\right)^{3}, \\
p=q=\frac{\mathrm{I}}{6} .
\end{gathered}
$$


$8.513 b_{2}=0, b_{1} \neq 0$ :

where:

$$
y=e^{\lambda x} F\left\{\frac{\left(\alpha_{1}+\beta_{1} x\right)^{2}}{2 \beta_{1}}\right\}
$$

$$
\begin{aligned}
\lambda & =-\frac{b_{0}}{b_{1}} \quad \alpha_{1}=\frac{a_{1} b_{1}-2 a_{2} b_{0}}{a_{2} b_{1}}, \beta_{1}=\frac{b_{1}}{a_{2}}, \\
p & =\frac{a_{2} b_{0}^{2}-a_{1} b_{0} b_{1}+a_{0} b_{1}^{2}}{2 b_{1}^{3}} \\
q & =\frac{I}{2}-p .
\end{aligned}
$$

$8.514 b_{2} \neq \circ, b_{0}=\frac{b_{1}^{2}}{4 b_{2}}:$

where:

$$
y=e^{\lambda x+\sqrt{\mu+\nu x}} F\{2 \sqrt{\mu+\nu x}\},
$$

$$
\begin{aligned}
\lambda & =-\frac{b_{1}}{2 b_{2}}, \quad \mu=-a_{2} \frac{4 a_{0} b_{2}^{2}-2 a_{1} b_{1} b_{2}+a_{2} b_{1}^{2}}{b_{2}{ }^{2}}, \\
\nu & =-\frac{4 a_{0} b_{2}^{2}-2 a_{1} b_{1} b_{2}+a_{2} b_{1}^{2}}{b_{2}{ }^{3}} \\
p & =q=\frac{a_{1} b_{2}-a_{2} b_{1}}{b_{2}{ }^{2}}-\frac{\mathbf{I}}{2}
\end{aligned}
$$

$8.515 b_{2} \neq 0, b_{0} \neq \frac{b_{1}^{2}}{4 b_{2}}:$

$$
y=e^{\lambda x} F\left\{\frac{\beta_{1}\left(\alpha_{2}+\beta_{2} x\right)}{\beta_{2}^{2}}\right\},
$$

where $\alpha_{2}=a_{2}, \beta_{2}=b_{2}, \beta_{1}=2 b_{2} \lambda+b_{1}$ and $\lambda$ is one of the roots of $b_{2} \lambda^{2}+b_{1} \lambda+b_{0}=$ o.

$$
p=\frac{a_{2} \lambda^{2}+a_{1} \lambda+a_{0}}{2 b_{2} \lambda+b_{1}}, \quad q=\frac{a_{1} b_{2}-a_{2} b_{1}}{b_{2}^{2}}-p
$$

8.520 The solution of $\mathbf{8 . 5 1 0}$ will be denoted:

I.

2.

3.

4.

5 .

6.

7.

$$
\phi=F(p, q, \xi) \text {. }
$$

$$
\begin{aligned}
& F(p, q, \xi)=e^{-\xi} F(q, p,-\xi) . \\
& F(p, q,-\xi)=e^{\xi} F(q, p, \xi)
\end{aligned}
$$$$
F(q, p, \xi)=e^{-\xi} F(p, q,-\xi) \text {. }
$$$$
F(p, q, \xi)=\xi^{1-p-q} F(\mathrm{I}-q, \mathrm{I}-p, \xi) \text {. }
$$$$
F(-p,-q, \xi)=\xi^{1+p+q} F(\mathrm{I}+q, \mathrm{I}+p, \xi) \text {. }
$$$$
F(p+m, q, \xi)=\frac{d^{m}}{d \xi^{m}} F(p, q, \xi) \text {. }
$$$$
F(p, q+n, \xi)=(-\mathrm{I})^{n} e^{-\xi} \frac{d^{n}}{d \xi^{n}}\left\{e^{\xi} F(p, q, \xi)\right\}
$$ 
8.521 The function $F(p, q, \xi)$ can always be found if it is known for positive proper fractional values of $p$ and $q$.

8.522 $p$ and $q$ positive improper fractions:

$$
p=m+r, \quad q=n+s
$$

where $m$ and $n$ are positive integers and $r$ and $s$ positive proper fractions.

$$
F(m+r, n+s, \xi)=(-\mathrm{I})^{n} \frac{d^{m}}{d \xi^{m}}\left[e^{-\xi} \frac{d^{n}}{d \xi^{n}}\left\{e^{\xi} F(r, s, \xi)\right\}\right] .
$$

8.523 $p$ and $q$ both negative:

$$
p=-(m-\mathrm{I}+r) \quad q=-(n-\mathrm{I}+s),
$$

$F(-m+\mathrm{I}-r,-n+\mathrm{I}-s, \xi)=(-\mathrm{I})^{m} \xi^{m+n+r+s-1} \frac{d^{n}}{d \xi^{n}}\left[e^{-\xi} \frac{d^{m}}{d \xi^{m}}\left\{e^{\xi} F(s, r, \xi)\right\}\right]$.

8.524 $p$ positive, $q$ negative:

$$
\begin{gathered}
p=m+r, \quad q=-n+s \\
F(m+r,-n+s, \xi)=\frac{d^{m}}{d \xi^{m}}\left[\xi^{n+1-r-s} \frac{d^{n}}{d \xi^{n}} F(\mathrm{I}-s, \mathrm{I}-r, \xi)\right] .
\end{gathered}
$$

8.525 $p$ negative, $q$ positive:

$$
p=-m+r, q=n+s,
$$

$F(-m+r, n+s, \xi)=(-\mathrm{I})^{m+n} e^{-\xi} \frac{d^{n}}{d \xi^{n}}\left[\xi^{m+1-r-8} \frac{d^{m}}{d \xi^{m}}\left\{e^{\xi} F(\mathrm{I}-s, \mathrm{I}-r, \xi)\right\}\right]$

8.530 If either $p$ or $q$ is zero the relation $D=0$ is satisfied and the complete solution of the differential equation is given in 8.502, 3 .

8.531 If $p=m$, a positive integer:

$\phi=F(m, q, \xi)=c_{1} \frac{d^{m-1}}{d \xi^{m-1}}\left[\xi^{-q} e^{-\xi} \int \xi^{q-1} e^{\xi} d \xi\right]+c_{2} \frac{d^{m-1}}{d \xi^{m-1}}\left[\xi^{-q} e^{-\xi}\right]$.

8.532 If $p=m$, a positive integer and both $q$ and $\xi$ are positive:

$\phi=F(m, q, \xi)=c_{1} \int_{0}^{\mathrm{x}} u^{m-1}(\mathrm{I}-u)^{q-1} e^{-\xi u} d u+c_{2} e^{-\xi} \int^{\infty}(\mathrm{I}+u)^{m-1} u^{q-1} e^{-\xi u} d u$.

8.533 If $q=n$, a positive integer:

$\phi=F(p, n, \xi)=c_{1} e^{-\xi} \frac{d^{n-1}}{d \xi^{n-1}}\left[\xi^{-p} e^{\xi} \int \xi^{p-1} e^{-\xi} d \xi\right]+c_{2} e^{-\xi} \frac{d^{n-1}}{d \xi^{n-1}}\left[\xi^{-p} e^{\xi}\right]$

8.534 If $q=n$, a positive integer and both $p$ and $\xi$ are positive:

$\phi=F(p, n, \xi)=c_{1} \int_{0}^{\mathrm{I}} u^{p-1}(\mathrm{I}-u)^{n-1} e^{-\xi u} d u+c_{2} e^{-\xi} \int_{0}^{\infty}(\mathrm{I}+u)^{p-1} u^{n-1} e^{-\xi u} d u$. 
8.540 The general solution of equation 8.510 may be written:

$$
\begin{aligned}
& \phi=F(p, q, \xi)=c_{1} M+c_{2} N, \\
& M=\int_{0}^{I} u^{p-1}(\mathrm{I}-u)^{q-1} e^{-\xi u} d u \\
& N=\int_{0}^{\infty}(\mathrm{I}+u)^{p-1} u^{q-1} e^{-\xi(1+u)} d u \\
& M=\frac{\Gamma(p) \Gamma(q)}{\Gamma(s)}\left\{\mathrm{I}-\frac{p}{s} \frac{\xi}{\mathrm{I} !}+\frac{p(p+\mathrm{I})}{s(s+\mathrm{I})} \frac{\xi^{2}}{2 !}-\frac{p(p+\mathrm{I})(p+2)}{s(s+\mathrm{I})(s+2)} \frac{\xi^{3}}{3 !}+\ldots .\right\} \\
& s=p+q, \\
& N=\frac{\Gamma(q) e^{-\xi}}{\xi^{q}}\left\{\mathrm{I}+\frac{(p-\mathrm{I}) q}{\mathrm{I} ! \xi}+\frac{(p-\mathrm{I})(p-2) q(q+\mathrm{I})}{2 ! \xi}+\ldots \ldots\right. \\
& +\frac{(p-\mathrm{I})(p-2) \ldots \ldots(p-\overline{n-\mathrm{I}})(q)(q+\mathrm{I}) \ldots \ldots(q+n-2)}{(n-\mathrm{I}) ! \xi^{n-1}} \\
& \left.+\frac{\rho(p-\mathrm{I})(p-2) \ldots(p-n) q(q+\mathrm{I})(q+2) \ldots(q+n-\mathrm{I})}{n ! \xi^{n}}\right\}
\end{aligned}
$$

where $\circ<\rho<\mathrm{I}$ and the real part of $\xi$ is positive.

THE COMPLETE SOLUTION OF EQUATION 8.510 IN SPECIAL CASES

$8.550 p>0, q>0$, real part of $\xi>0$ :

$$
F(p, q, \xi)=c_{1} \int_{0}^{\mathrm{I}} u^{p-1}(\mathrm{I}-u)^{q-1} e^{-\xi u} d u+c_{2} e^{-\xi} \int_{0}^{\infty}(\mathrm{I}+u)^{p-1} u^{q-1} e^{-\xi u} d u .
$$

$8.551 p>0, q>0, \xi<0$ :

$F(p, q, \xi)=c_{1} \int_{0}^{\mathrm{I}} u^{p-1}(\mathrm{I}-u)^{q-1} e^{-\xi u} d u+c_{2} \int^{\infty} u^{p-1}(\mathrm{I}+u)^{q-1} e^{\xi u} d u$.

$8.552 p<0, q<0, \xi>0$ :

$$
F(p, q, \xi)=\xi^{1-p-q}\left\{c_{1} \int_{0}^{\mathrm{I}}(\mathrm{I}-u)^{-p} u^{-q} e^{-\xi u} d u+c_{2} e^{-\xi} \int_{0}^{\infty} u^{-p}(\mathrm{I}+u)^{-q} e^{-\xi u} d u\right\}
$$

$8.553 p<0, q<0, \xi<0$ :

$$
F(p, q, \xi)=\xi^{1-p-q}\left\{c_{1} \int_{0}^{1}(\mathrm{I}-u)^{-p} u^{-q} e^{-\xi u} d u+c_{2} \int_{0}^{\infty}(\mathrm{I}+u)^{-p} u^{-q} e^{+\xi u} d u\right\}
$$

\section{$8.554 p>0, q<0$}

$p=\dot{m}+r$, where $m$ is a positive integer and $r$ a proper fraction.

$$
F(m+r, q, \xi)^{\prime}=\frac{d^{m}}{d \xi^{m}}\left\{\xi^{1-r-q} F(\mathbf{I}-r, \mathbf{I}-q, \xi)\right\},
$$






$\xi>0: \quad F(\mathrm{I}-r, \mathrm{I}-q, \xi)=c_{1} \int_{0}^{\mathrm{I}} u^{-r}(\mathrm{I}-u)^{-q} e^{-\xi u} d u$

$\xi<0: \quad F(\mathbf{I}-r, \mathbf{I}-q, \xi)=c_{1} \int_{0}^{x} u^{-r}(\mathbf{I}-u)^{-q} e^{-\xi u} d u$

$$
+c_{2} e^{-\xi} \int_{0}^{\infty}(\mathrm{I}+u)-u^{-q} e^{-\xi u} d u
$$

$8.555 p<0, q>0$,

$$
+c_{2} \int_{0}^{\infty} u \rightarrow(1+u)^{-q} e^{\xi u} d u .
$$

$q=n+s$, where $n$ is a positive integer and $s$ a proper fraction.

$$
F(p, n+s, \xi)=e^{-\xi} \frac{d^{n}}{d \xi^{n}}\left\{e^{\xi} \xi^{1-p-s} F(\mathrm{I}-s, \mathrm{I}-p, \xi)\right\},
$$

$\xi>0: \quad F(\mathrm{I}-s, \mathrm{I}-p, \xi)=c_{1} \int_{0}^{x} u^{-s}(\mathrm{I}-u)^{-p} e^{-\xi u} d u$

$$
+c_{2} e^{-\xi} \int_{0}^{\infty}(\mathrm{I}+u)^{-8} u^{-p} e^{-\xi u} d u
$$

$\xi<0: \quad F(\mathrm{I}-s, \mathrm{I}-p, \xi)=c_{1} \int_{0}^{\mathrm{I}} u^{-s}(\mathrm{I}-u)^{-p} e^{-\xi} d u$

$$
+c_{2} \int_{0}^{\infty} u^{-8}(1+u)^{-p_{\epsilon} \xi u} d u \text {. }
$$

8.556 $\xi$ pure imaginary:

$p=r, q=s$, where $r$ and $s$ are positive proper fractions.

$r+s \neq \mathrm{I}:$

$r+s=\mathrm{I}:$

$$
\begin{aligned}
F(r, s, \xi)=c_{1} \int_{0}^{\mathrm{x}} u^{r-1}(\mathrm{I}-u)^{8-1} e^{-\xi u} d u & \\
& +c_{2} \xi^{1-r-s} \int_{0}^{\mathrm{x}} u^{-8}(\mathrm{I}-u)^{-r} e^{-\xi u} d u .
\end{aligned}
$$

$$
\begin{aligned}
F(r, s, \xi)=c_{1} \int_{0}^{\mathrm{x}} & u^{r-1}(\mathrm{I}-u)^{s-1} e^{-\xi u} d u \\
& +c_{2} \int_{0}^{\mathrm{x}} u^{r-1}(\mathrm{I}-u)^{s-1} e^{-\xi u} \log \{\xi u(\mathrm{I}-u)\} d u .
\end{aligned}
$$

8.600 The differential equation:

$$
x \frac{d^{2} y}{d x^{2}}+(\gamma-x) \frac{d y}{d x}-\alpha y=0
$$

is satisfied by the confluent hypergeometric function. The complete solution is:

$$
y=c_{1} M(\alpha, \gamma, x)+c_{2} x^{1-\gamma} M(\alpha-\gamma+\mathrm{I}, 2-\gamma, x)=\bar{M}(\alpha, \gamma, x),
$$


where

$$
M(\alpha, \gamma, x)=\mathrm{I}+\frac{\alpha}{\gamma} \frac{x}{\mathrm{I}}+\frac{\alpha(\alpha+\mathrm{I})}{\gamma(\gamma+\mathrm{I})} \frac{x^{2}}{2 !}+\frac{\alpha(\alpha+\mathrm{I})(\alpha+2)}{\gamma(\gamma+\mathrm{I})(\gamma+2)} \frac{x^{3}}{3 !}+\ldots \ldots
$$

The series is absolutely and uniformly convergent for all real and complex values of $\alpha, \gamma, x$, except when $\gamma$ is a negative integer or zero.

When $\gamma$ is a positive integer the complete solution of the differential equation is:

$$
\begin{aligned}
y & =\left\{c_{1}+c_{2} \log x\right\} M(\alpha, \gamma, x)+c_{2}\left\{\frac{a x}{\gamma}\left(\frac{\mathrm{I}}{\alpha}-\frac{\mathrm{I}}{\gamma}-\mathrm{I}\right)\right. \\
& +\frac{\alpha(\alpha+\mathrm{I})}{\gamma(\gamma+\mathrm{I})} \frac{x^{2}}{2 !}\left(\frac{\mathrm{I}}{\alpha}+\frac{\mathrm{I}}{\alpha+\mathrm{I}}-\frac{\mathrm{I}}{\gamma}-\frac{\mathrm{I}}{\gamma+\mathrm{I}}-\mathrm{I}-\frac{\mathrm{I}}{2}\right) \\
& +\frac{\alpha(\alpha+\mathrm{I})(\alpha+2)}{\gamma(\gamma+\mathrm{I})(\gamma+2)} \frac{x^{3}}{3 !}\left(\frac{\mathrm{I}}{\alpha}+\frac{\mathrm{I}}{\alpha+\mathrm{I}}+\frac{\mathrm{I}}{\alpha+2}-\frac{\mathrm{I}}{\gamma}-\frac{\mathrm{I}}{\gamma+\mathrm{I}}-\frac{\mathrm{I}}{\gamma+2}-\mathrm{I}-\frac{\mathrm{I}}{2}-\frac{\mathrm{I}}{3}\right) \\
& +\ldots . .
\end{aligned}
$$

8.601 For large values of $x$ the following asymptotic expansion may be used: $M(\alpha, \gamma, x)$

$$
\begin{aligned}
& =\frac{\Gamma(\gamma)}{\Gamma(\gamma-\alpha)}(-x)-\alpha\left\{\mathrm{I}-\frac{\alpha(\alpha-\gamma+\mathrm{I})}{\mathrm{I}} \frac{\mathrm{I}}{x}+\frac{\alpha(\alpha+\mathrm{I})(\alpha-\gamma+\mathrm{I})(\alpha-\gamma+2)}{2 !} \frac{\mathrm{I}}{x^{2}} \cdots\right\} \\
& +\frac{\Gamma(\gamma)}{\Gamma(\alpha)} e^{x} x^{\alpha-\gamma}\left\{\mathrm{I}+\frac{(\mathrm{I}-\alpha)(\gamma-\alpha)}{\mathrm{I}} \frac{\mathrm{I}}{x}+\frac{(\mathrm{I}-\alpha)(2-\alpha)(\gamma-\alpha)(\gamma-\alpha+\mathrm{I})}{2 !} \frac{\mathrm{I}}{x^{2}}+\cdots\right\}
\end{aligned}
$$

\subsection{1}

I. $M(\alpha, \gamma, x)=e^{x} M(\gamma-\alpha, \gamma,-x)$.

2. $x^{1-\gamma} M(\alpha-\gamma+\mathrm{I}, 2-\gamma, x)=e^{x} x^{1-\gamma} M(1-\alpha, 2-\gamma,-x)$.

3. $\frac{x}{\gamma} M(\alpha+\mathrm{I}, \gamma+\mathrm{I}, x)=M(\alpha+\mathrm{I}, \gamma, x)-M(\alpha, \gamma, x)$.

4. $\alpha M(\alpha+\mathrm{I}, \gamma+\mathrm{I}, x)=(\alpha-\gamma) M(\alpha, \gamma+\mathrm{I}, x)+\gamma M(\alpha, \gamma, x)$.

5. $(\alpha+x) M(\alpha+\mathrm{I}, \gamma+\mathrm{I}, x)=(\alpha-\gamma) M(\alpha, \gamma+\mathrm{I}, x)+\gamma M(\alpha+\mathrm{I}, \gamma, x)$.

6. $\alpha \gamma M(\alpha+\mathrm{I}, \gamma, x)=\gamma(\alpha+x) M(\alpha, \gamma, x)-x(\gamma-\alpha) M(\alpha, \gamma+\mathrm{I}, x)$.

7. $\alpha M(\alpha+\mathrm{I}, \gamma, x)=(x+2 \alpha-\gamma) M(\alpha, \gamma, x)+(\gamma-\alpha) M(\alpha-\mathrm{I}, \gamma, x)$.

8. $\frac{\gamma-\alpha}{\gamma} x M(\alpha, \gamma+\mathrm{I}, x)=(x+\gamma-\mathrm{I}) M(\alpha, \gamma, x)+(\mathrm{I}-\gamma) M(\alpha, \gamma-\mathrm{I}, x)$.

\subsection{2}

เ. $\frac{d}{d x} M(\alpha, \gamma, x)=\frac{\alpha}{\gamma} M(\alpha+\mathrm{I}, \gamma+\mathrm{I}, x)$

2. $(\mathrm{I}-\alpha) \int_{0}^{x} M(\alpha, \gamma, x) d x=(\mathrm{I}-\gamma) M(\alpha-\mathrm{I}, \gamma-\mathrm{I}, x)+(\gamma-\mathrm{I})$. 
SPECIAL DIFFERENTIAL EQUATIONS AND THEIR SOLUTIONS IN TERMS OF $\bar{M}(\alpha, \gamma, x)$ 8.630

8.631

$$
\begin{gathered}
\frac{d^{2} y}{d x^{2}}+2(p+q x) \frac{d y}{d x}+\left\{4 \alpha q+p^{2}-q^{2} m^{2}+2 q x(p+q m)\right\} y=0, \\
y=e^{-(p+q m) x} \bar{M}\left(\alpha, \frac{\mathrm{I}}{2} ;-q(x-m)^{2}\right) .
\end{gathered}
$$

$$
\begin{gathered}
\frac{d^{2} y}{d x^{2}}+\left(2 p+\frac{\gamma}{x}\right) \frac{d y}{d x}+\left\{p^{2}-t^{2}+\frac{\mathrm{I}}{x}(\gamma p+\gamma t-2 \alpha t)\right\} y=0 \\
y=e^{-(p+t) x} \bar{M}(\alpha, \gamma, 2 t x) .
\end{gathered}
$$

\subsection{2}

\subsection{3}

$$
\begin{gathered}
\frac{d^{2} y}{d x^{2}}+2(p+q x) \frac{d y}{d x}+\left\{q+c(\mathrm{I}-4 \alpha)+(p+q x)^{2}-c^{2}(x-m)^{2}\right\} y=0 \\
y=e^{-p x-\frac{1}{2} q x^{2}-\frac{1}{2} c(x-m)^{2}} \bar{M}\left(\alpha, \frac{\mathrm{I}}{2}, c(x-m)^{2}\right)
\end{gathered}
$$

$\frac{d^{2} y}{d x^{2}}+\left(2 p+\frac{q}{x}\right) \frac{d y}{d x}+\left\{p^{2}-\imath^{2}+\frac{\mathrm{I}}{x}(p q+\gamma t-2 \alpha t)+\frac{\mathrm{I}}{4 x^{2}}(\gamma-q)(2-q-\gamma)\right\} y=0$,

\subsection{4}

$$
y=e^{-(p+t) x} x^{\frac{\gamma-q}{2}} \bar{M}(\alpha, \gamma, 2 t x) .
$$

$\frac{d^{2} y}{d x^{2}}+\left\{\frac{2 \gamma-I}{x}+2 \alpha+2(b-c) x\right\} \frac{d y}{d x}$

$$
+\left\{\frac{\alpha(2 \gamma-\mathrm{I})}{x}+\left(a^{2}+2 b \gamma-4 \alpha c\right)+2 a(b-c) x+b(b-2 c) x^{2}\right\} y=0,
$$

\subsection{5}

$$
y=e^{-a x-\frac{1}{2} b x^{2}} \bar{M}\left(\alpha, \gamma, c x^{2}\right) .
$$

$$
\begin{aligned}
& \frac{d^{2} y}{d x^{2}}+\frac{\mathrm{I}}{x}\left(2 p x^{r}+q r-r+\mathrm{I}\right) \frac{d y}{d x} \\
& +\frac{\mathrm{I}}{x^{2}}\left\{\left(p^{2}-t^{2}\right) x^{2 r}+r(p q+\gamma t-2 \alpha t) x^{r}+\frac{\mathrm{I}}{4} r^{2}(\gamma-q)(2-q-\gamma)\right\} y=0 \\
& y=e^{-\frac{(p+t)}{r} x^{r}} x^{\frac{r}{2}(\gamma-q)} \bar{M}\left(\alpha, \gamma, \frac{2 t x^{r}}{r}\right)
\end{aligned}
$$

8.640 Tables and graphs of the function $M(\alpha, \gamma, x)$ are given by Webb and Airey (Phil. Mag. 36, p. I29, I918) for getting approximate numerical solu- 
tions of any of these differential equations. The range in $x$ is $\mathrm{I}$ to Io; in $\alpha,+0.5$ to +4.0 and -0.5 to -3.0 ; in $\gamma$, I to 7 . For negative values of $x$ the equations of $\mathbf{8 . 6 1}$ may be used.

\section{SPECIAL DIFFERENTIAL EQUATIONS}

8.700

$$
\frac{d^{2} y}{d x^{2}}+n^{2} y=X(x)
$$

where $X(x)$ is any function of $x$. The complete solution is:

$$
y=c_{1} e^{n x}+c_{2} e^{-n x}+\frac{I}{n} \int^{x} X(\xi) \sinh n(x-\xi) d \xi .
$$

8.701

$$
\frac{d^{2} y}{d x^{2}}+\kappa \frac{d y}{d x}+n^{2} y=X(x) .
$$

The complete solution, satisfying the conditions:

$$
\begin{array}{ll}
x=0 & y=y_{0}, \\
x=0 & \frac{d y}{d x}=y_{0}{ }^{\prime},
\end{array}
$$

$y=e^{-\frac{1}{2} \kappa x}\left\{y_{0}^{\prime} \frac{\sin n^{\prime} x}{n^{\prime}}+y_{0}\left(\cos n^{\prime} x+\frac{\kappa}{2 n^{\prime}} \sin n^{\prime} x\right)\right\}$

where

$$
+\frac{\mathrm{I}}{n^{\prime}} \int_{0}^{x} e^{-\frac{1}{2} \kappa(x-\xi)} \sin n^{\prime}(x-\xi) X(\xi) d \xi
$$

$$
n^{\prime}=\sqrt{n^{2}-\frac{\kappa^{2}}{4}}
$$

8.702

$$
\begin{gathered}
\frac{d^{2} y}{d x^{2}}+f(x) \frac{d y}{d x}+g(x)\left(\frac{d y}{d x}\right)^{2}=0, \\
y=\int \frac{e^{-\int f(x) d x} d x}{\int e^{-\int f(x) d x} g(x) d x+c_{1}}+c_{2} .
\end{gathered}
$$

8.703

$$
\begin{gathered}
\frac{d^{2} y}{d x^{2}}+f(y)\left(\frac{d y}{d x}\right)^{2}+g(y)=0, \\
x= \pm \int \frac{e^{\int f(y) d y} d y}{\left\{c_{1}-2 \int e^{2 \int f(y) d y} g(y) d y\right\}^{\frac{1}{2}}}+c_{2} .
\end{gathered}
$$

8.704

$$
\begin{gathered}
\frac{d^{2} y}{d x^{2}}+f(y) \frac{d y}{d x}+g(y)\left(\frac{d y}{d x}\right)^{2}=0, \\
x=\int \frac{e^{\int o(y) d y} d y}{c_{1}-\int e^{\int o(y) d y} f(y) d y}+c_{2} .
\end{gathered}
$$




\subsection{5}

$$
\begin{gathered}
\frac{d^{2} y}{d x^{2}}+f(x) \frac{d y}{d x}+g(y)\left(\frac{d y}{d x}\right)^{2}=0, \\
\int_{e^{-}}{ }^{i(y) d y} d y=c_{1} \int e^{-\iint(x) d x} d x+c_{2} .
\end{gathered}
$$

\subsection{6}

$$
\begin{aligned}
& \frac{d^{2} y}{d x^{2}}+(a+b x) \frac{d y}{d x}+a b x y=0 . \\
& y=e^{-a x}\left\{c_{1}+c_{2} \int e^{a x-\frac{1}{2} b x^{2}} d x\right\}
\end{aligned}
$$

\subsection{7}

\subsection{8}

$$
\begin{aligned}
& x \frac{d^{2} y}{d x^{2}}+(a+b x) \frac{d y}{d x}+a b y=0, \\
& y=e^{-b x}\left\{c_{1}+c \int x^{-a} e^{b x} d x\right\}
\end{aligned}
$$

$$
\frac{d^{2} y}{d x^{2}}+\frac{a}{x} \frac{d y}{d x}+\frac{b}{x^{2}} y=0 .
$$

I. $(a-\mathrm{I})^{2}>4 b ; \quad \lambda=\frac{\mathrm{I}}{2} \sqrt{(a-\mathrm{I})^{2}-4^{b}}$

$$
y=x^{-\frac{a-1}{2}}\left\{c_{1} x+c_{2} x^{-\lambda}\right\} .
$$

2. $(a-\mathrm{I})^{2}<4 b ; \quad \lambda=\frac{\mathrm{I}}{2} \sqrt{4 b-(a-\mathrm{I})^{2}}$

$$
y=x^{-\frac{a-1}{2}}\left\{c_{1} \cos (\lambda \log x)+c_{2} \sin (\lambda \log x)\right\} .
$$

3. $(a-\mathrm{I})^{2}=4 b$

$$
y=x^{-\frac{a-1}{2}}\left(c_{1}+c_{2} \log x\right)
$$

\subsection{9}

$$
\frac{d^{2} y}{d x^{2}}+2 b x \frac{d y}{d x}+\left(a+b^{2} x^{2} y=0 .\right.
$$

I. $a<b, \quad \lambda=\sqrt{b-a}$,

$$
y=e^{-\frac{b x^{2}}{2}}\left(c_{1} e^{\lambda x}+c_{2} e^{-\lambda x}\right) .
$$

2. $a>b, \quad \lambda=\sqrt{a-b}$,

$$
y=e^{-\frac{b x^{2}}{2}}\left(c_{1} \cos \lambda x+c_{2} \sin \lambda x\right)
$$

\subsection{0}

$$
\begin{gathered}
f(x) \frac{d^{2} y}{d x^{2}}-(a+b x) \frac{d y}{d x}+b y=0, \\
\int \frac{a+b x}{f(x)} d x=X \\
y=c_{1}(a+b x)+c_{2}\left\{e^{X}-(a+b x) \int \frac{\mathrm{I}}{f(x)} e^{X} d x\right\} .
\end{gathered}
$$




\subsection{1}

8.712

$$
\begin{gathered}
\left(a^{2}-x^{2}\right) \frac{d^{2} y}{d x^{2}}+2(\mu-\mathrm{I}) x \frac{d y}{d x}-\mu(\mu-\mathrm{I}) y=0, \\
y=(a+x)_{\mu}\left\{c_{1}+c_{2} \int \frac{(a-x)^{\mu-1}}{(a+x)^{\mu+1}} d x\right\} .
\end{gathered}
$$

8.713

$$
\begin{gathered}
\frac{d^{2} y}{d x^{2}}+\frac{2}{x} \frac{d y}{d x}+\mu^{2} y=\frac{a}{x}, \\
y=\frac{I}{x}\left\{1 \cos \mu x+c_{2} \sin \mu x+\frac{a}{\mu^{2}}\right\} .
\end{gathered}
$$

$$
\frac{d^{4} y}{d x^{4}}+2 d \frac{d^{3} y}{d x^{3}}+c \frac{d^{2} y}{d x^{2}}+2 b \frac{d y}{d x}+a y=0,
$$

$y=c_{1} e^{-\rho_{1} x}\left\{\rho_{1} \sin \left(\omega_{1} x+\alpha_{1}\right)+\omega_{1} \cos \left(\omega_{1} x+\alpha_{1}\right)\right\}$

where:

$$
+c_{2} e^{-\rho_{2} x}\left\{\rho_{2} \sin \left(\omega_{2} x+\alpha_{2}\right)+\omega_{2} \cos \left(\omega_{2} x+\alpha_{2}\right)\right\},
$$

$$
\begin{aligned}
4 \omega_{1}^{2} & =z+c-2 d^{2}+2 \sqrt{z^{2}-4 a}-2 d \sqrt{z-c+d^{2}}, \\
4 \omega_{2}^{2} & =z+c-2 d^{2}-2 \sqrt{z^{2}-4 a}+2 d \sqrt{z-c+d^{2}} \\
2 \rho_{1} & =d+\sqrt{z-c+d^{2}} \\
2 \rho_{2} & =d-\sqrt{z-c+d^{2}}
\end{aligned}
$$

and $z$ is a root of

$$
z^{3}-c z^{2}-4(a-b d) z+4\left(a c-a d^{2}-b^{2}\right)=0 .
$$

(Kiebitz, Ann. d. Physik, 40, p. I38, I9I3) 


\section{DIFFERENTIAL EQUATIONS (continued)}

9.00 Legendre's Equation:

$$
\left(\mathrm{I}-x^{2}\right) \frac{d^{2} y}{d x^{2}}-2 x \frac{d y}{d x}+n(n+\mathrm{I}) y=0 .
$$

9.001 If $n$ is a positive integer one solution is the Legendre polynomial, or Zonal Harmonic, $P_{n}(x)$ :

$P_{n}(x)=\frac{(2 n) !}{2^{n}(n !)^{2}}\left\{x^{n}-\frac{n(n-\mathrm{I})}{2(2 n-\mathrm{I})} x^{n-2}+\frac{n(n-\mathrm{I})(n-2)(n-3)}{2 \cdot 4 \cdot(2 n-\mathrm{I})(2 n-3)} x^{n-4}-\ldots\right\}$.

9.002 If $n$ is even the last term in the finite series in the brackets is:

$$
(-I)^{\frac{n}{2}} \frac{(n !)^{3}}{\left(\frac{n}{2}\right)^{2}(2 n) !}
$$

9.003 If $n$ is odd the last term in the brackets is:

$$
(-I)^{\frac{n-I}{2}} \frac{(n !)^{2}(n-I) !}{\left(\left[\frac{1}{2}(n-I)\right] !\right)^{2}(2 n-I) !} x \text {. }
$$

9.010 If $n$ is a positive integer a second solution of Legendre's Equation is the infinite series:

$$
\begin{aligned}
Q_{n}(x)=\frac{2^{n}(n !)^{2}}{(2 n+\mathrm{I}) !}\left\{x^{-(n+1)}\right. & +\frac{(n+\mathrm{I})(n+2)}{2(2 n+3)} x^{-(n+3)} \\
& \left.+\frac{(n+\mathrm{I})(n+2)(n+3)(n+4)}{2 \cdot 4 \cdot(2 n+3)(2 n+5)} x^{-(n+5)}+\ldots .\right\}
\end{aligned}
$$

\subsection{1}

$P_{2 n}(\cos \theta)=(-\mathrm{I})^{n} \frac{(2 n) !}{2^{2 n}(n !)^{2}}\left\{\sin ^{2 n} \theta-\frac{(2 n)^{2}}{2 !} \sin ^{2 n-2} \theta \cos ^{2} \theta\right.$

\subsection{2}

$$
\left.+\ldots+(-I)^{n} \frac{(2 n)^{2}(2 n-2)^{2} \cdots 4^{2} 2^{2}}{(2 n) !} \cos ^{2 n} \theta\right\} .
$$

$$
\begin{array}{r}
P_{2 n+1}(\cos \theta)=(-\mathrm{I})^{n} \frac{(2 n+\mathrm{I}) !}{2^{2 n}(n !)^{2}}\left\{\sin ^{2 n} \theta \cos \theta-\frac{(2 n)^{2}}{3 !} \sin ^{2 n-2} \theta \cos ^{3} \theta\right. \\
\left.+\ldots+(-\mathrm{I})^{n} \frac{(2 n)^{2}(2 n-2)^{2} \cdots 4^{2} 2^{2}}{(2 n+\mathrm{I}) !} \cos ^{2 n+1} \theta\right\}
\end{array}
$$

(Brodetsky: Mess. of Math. 42, p. 65, I9I2) 
9.02 Recurrence formulae for $P_{n}(x)$ :

$$
\text { I. }
$$

$$
(n+\mathrm{I}) P_{n+1}+n P_{n-1}=(2 n+\mathrm{I}) x P_{n} \text {. }
$$

2.

$$
(2 n+\mathrm{I}) P_{n}=\frac{d P_{n+1}}{d x}-\frac{d P_{n-1}}{d x} \text {. }
$$

3.

$$
(n+\mathrm{I}) P_{n}=\frac{d P_{n+1}}{d x}-x \frac{d P_{n}}{d x} .
$$

4.

$$
n P_{n}=x \frac{d P_{n}}{d x}-\frac{d P_{n-1}}{d x} \text {. }
$$

5.

$$
\left(\mathrm{I}-x^{2}\right) \frac{d P_{n}}{d x}=(n+\mathrm{I})\left(x P_{n}-P_{n+1}\right) \text {. }
$$

6.

$$
\left(\mathrm{I}-x^{2}\right) \frac{d P_{n}}{d x}=n\left(P_{n-1}-x P_{n}\right) \text {. }
$$

7.

$$
(2 n+\mathrm{I})\left(\mathrm{I}-x^{2}\right) \frac{d P_{n}}{d x}=n(n+\mathrm{I})\left(P_{n-1}-P_{n+1}\right) .
$$

9.028 Recurrence formulae for $Q_{n}(x)$. These are the same as those for $P_{n}(x)$.

9.030 Special Values.

$$
\begin{aligned}
& P_{0}(x)=\mathrm{I} \\
& P_{1}(x)=x \\
& P_{2}(x)=\frac{1}{2}\left(3 x^{2}-\mathrm{I}\right) \\
& P_{3}(x)=\frac{1}{2}\left(5 x^{3}-3 x\right) \\
& P_{4}(x)=\frac{1}{8}\left(35 x^{4}-30 x^{2}+3\right) \\
& P_{5}(x)=\frac{1}{8}\left(63 x^{5}-70 x^{3}+\mathrm{I} 5 x\right) \\
& P_{6}(x)=\frac{1}{16}\left(23 \mathrm{I} x^{6}-3 \mathrm{I} 5 x^{4}+\mathrm{IO} 5 x^{2}-5\right) \\
& P_{7}(x)=\frac{1}{16}\left(429 x^{7}-693 x^{5}+3 \mathrm{I} 5 x^{3}-35 x\right), \\
& P_{8}(x)=\frac{1}{128}\left(6435 x^{8}-\mathrm{I} 20 \mathrm{I} 2 x^{6}+6930 x^{4}-\mathrm{I} 260 x^{2}+35\right)
\end{aligned}
$$

9.031

$$
\begin{aligned}
& Q_{0}(x)=\frac{\mathrm{I}}{2} \log \frac{x+\mathrm{I}}{x-\mathrm{I}} \\
& Q_{1}(x)=\frac{\mathrm{I}}{2} x \log \frac{x+\mathrm{I}}{x-\mathrm{I}}-\mathrm{I} \\
& Q_{2}(x)=\frac{\mathrm{I}}{2} P_{2}(x) \log \frac{x+\mathrm{I}}{x-\mathrm{I}}-\frac{3}{2} x \\
& Q_{3}(x)=\frac{\mathrm{I}}{2} P_{3}(x) \log \frac{x+\mathrm{I}}{x-\mathrm{I}}-\frac{5}{2} x^{2}+\frac{2}{3}
\end{aligned}
$$


9.032

$$
\begin{aligned}
P_{2 n}(\mathrm{O}) & =(-\mathrm{I})^{n} \frac{\mathrm{I} \cdot 3 \cdot 5 \cdots(2 n-\mathrm{I})}{2 \cdot 4 \cdot 6 \ldots 2 n}, \\
P_{2 n+1}(\mathrm{O}) & =\mathrm{O}, \\
P_{n}(\mathrm{I}) & =\mathrm{I}, \\
P_{n}(-x) & =(-\mathrm{I})^{n} P_{n}(x) .
\end{aligned}
$$

9.033 If $z=r \cos \theta$ :

$$
\begin{aligned}
\frac{\partial P_{n}(\cos \theta)}{\partial z}=\frac{n+\mathrm{I}}{r}\left\{P_{1}(\cos \theta) P_{n}(\cos \theta)-P_{n+1}(\cos \theta)\right\} \\
=\frac{n(n+\mathrm{I})}{(2 n+\mathrm{I}) r}\left\{P_{n-1}(\cos \theta)-P_{n+1}(\cos \theta)\right\} .
\end{aligned}
$$

9.034 Rodrigues' Formula:

$$
P_{n}(x)=\frac{\mathrm{I}}{2^{n} n !} \frac{d^{n}}{d x^{n}}\left(x^{2}-\mathrm{I}\right)^{n} .
$$

9.035 If $z=r \cos \theta$ :

$$
P_{n}(\cos \theta)=\frac{(-\mathrm{I})^{n}}{n !} r^{n+1} \frac{\partial^{n}}{\partial z^{n}}\left(\frac{\mathrm{I}}{r}\right) .
$$

9.036 If $m \leqslant n:$

where:

$$
P_{m}(x) P_{n}(x)=\sum_{k=0}^{m} \frac{A_{m-k} A_{k} A_{n-k}}{A_{n+m-k}}\left(\frac{2 n+2 m-4 k+\mathrm{I}}{2 n+2 m-2 k+\mathrm{I}}\right) P_{n+m-2 k}(x),
$$

$$
A_{r}=\frac{\mathrm{I} \cdot 3 \cdot 5 \ldots(2 r-\mathrm{I})}{r !} .
$$

\section{MEHLER'S INTEGRALS}

9.040 For all values of $n$ :

$$
P_{n}(\cos \theta)=\frac{2}{\pi} \int_{0}^{\theta} \frac{\cos \left(n+\frac{1}{2}\right) \phi d \phi}{\sqrt{2(\cos \phi-\cos \theta)}} .
$$

9.041 If $n$ is a positive integer:

$$
P_{n}(\cos \theta)=\frac{2}{\pi} \int^{\pi} \frac{\sin \left(n+\frac{1}{2}\right) \phi d \phi}{\sqrt{2(\cos \theta-\cos \phi)}} .
$$

\subsection{2}

LAPLACE'S INTEGRALS, FOR ALL VALUES OF $n$

9.043

$$
P_{n}(x)=\frac{I}{\pi} \int_{0}^{\pi}\left\{x+\sqrt{x^{2}-I} \cos \phi\right\}^{n} d \phi
$$

$$
Q_{n}(x)=\int^{\infty} \frac{d \phi}{\left\{x+\sqrt{x^{2}-I} \cosh \phi\right\}^{n+1}} .
$$


9.044 INTEGRAL PROPERTIES

\subsection{5}

$$
\begin{aligned}
\int_{-1}^{+1} P_{m}(x) P_{n}(x) d x & =\text { o if } m \neq n \\
& =\frac{2}{2 n+1} \text { if } m=n .
\end{aligned}
$$

$$
\begin{aligned}
(m-n)(m+n+\mathrm{I}) & \int_{x}^{\mathrm{I}} P_{m}(x) P_{n}(x) d x \\
& =\frac{1}{2}\left\{P_{m}\left[(n+\mathrm{I}) P_{n+1}-n P_{n-1}\right]-P_{n}\left[(m+\mathrm{I}) P_{m+1}-m P_{m-1}\right]\right\} .
\end{aligned}
$$

9.046

$$
\begin{aligned}
(2 n+\mathrm{I}) \int^{\mathrm{I}} P_{n}^{2}(x) d x=\mathrm{I}-x P_{n}{ }^{2}-2 x\left(P_{1}^{2}\right. & \left.+P_{2}^{2}+\ldots+P_{n-1}^{2}\right) \\
& +2\left(P_{1} P_{2}+P_{2} P_{3}+\ldots .+P_{n-1} P_{n}\right)
\end{aligned}
$$

EXPANSIONS IN LEGENDRE FUNCTIONS

9.050 Neumann's expansion:

$$
\begin{aligned}
f(x) & =\sum_{n=0}^{\infty} a_{n} P_{n}(x), \\
a_{n} & =\left(n+\frac{1}{2}\right) \int_{-\mathrm{I}}^{+\mathrm{I}} f(x) P_{n}(x) d x, \\
& =\frac{n+\frac{1}{2}}{2^{n} n !} \int_{-\mathrm{I}}^{+\mathrm{I}} f^{(n)}(x) \cdot\left(\mathrm{I}-x^{2}\right)^{n} d x .
\end{aligned}
$$

9.051 Any polynomial in $x$ may be expressed as a series of Legendre's polynomials. If $f_{n}(x)$ is a polynomial of degree $n$ :

$$
\begin{aligned}
f_{n}(x) & =\sum_{k=0}^{n} a_{k} P_{k}(x) \\
a_{k} & =\frac{2 k+\mathrm{I}}{2} \int_{-\mathrm{I}}^{+\mathrm{I}} f_{n}(x) P_{k}(x) d x .
\end{aligned}
$$

\section{SPECIAL EXPANSIONS IN LEGENDRE FUNCTIONS}

9.060 For all positive real values of $n$ :

I. $\cos n \theta=-\frac{\mathrm{I}+\cos n \pi}{2\left(n^{2}-\mathrm{I}\right)}\left\{P_{0}(\cos \theta)+\frac{5 n^{2}}{\left(n^{2}-3^{2}\right)} P_{2}(\cos \theta)\right.$

$$
\begin{aligned}
& \left.+\frac{9 n^{2}\left(n^{2}-2^{2}\right)}{\left(n^{2}-3^{2}\right)\left(n^{2}-5^{2}\right)} P_{4}(\cos \theta)+\ldots\right\}-\frac{\mathrm{I}-\cos n \pi}{2\left(n^{2}-2^{2}\right)}\left\{{ }_{3} P_{1}(\cos \theta)\right. \\
& \left.+\frac{7\left(n^{2}-\mathrm{I}^{2}\right)}{\left(n^{2}-4^{2}\right)} P_{3}(\cos \theta)+\frac{\mathrm{II}\left(n^{2}-\mathrm{I}^{2}\right)\left(n^{2}-3^{2}\right)}{\left(n^{2}-4^{2}\right)\left(n^{2}-6^{2}\right)} P_{5}(\cos \theta)+\ldots\right\} .
\end{aligned}
$$


2. $\sin n \theta=-\frac{\mathrm{I}}{2} \frac{\sin n \pi}{\left(n^{2}-\mathrm{I}\right)}\left\{P_{0}(\cos \theta)+\frac{5 n^{2}}{\left(n^{2}-3^{2}\right)} P_{2}(\cos \theta)\right.$

$$
\begin{aligned}
& \left.+\frac{9 n^{2}\left(n^{2}-2^{2}\right)}{\left(n^{2}-3^{2}\right)\left(n^{2}-5^{2}\right)} P_{4}(\cos \theta)+\ldots .\right\}+\frac{\mathrm{I}}{2} \frac{\sin n \pi}{\left(n^{2}-2^{2}\right)}\left\{{ }_{3} P_{1}(\cos \theta)\right. \\
& \left.+\frac{7\left(n^{2}-\mathrm{I}^{2}\right)}{\left(n^{2}-4^{2}\right)} P_{3}(\cos \theta)+\frac{\mathrm{II}\left(n^{2}-\mathrm{I}^{2}\right)\left(n^{2}-3^{2}\right)}{\left(n^{2}-4^{2}\right)\left(n^{2}-6^{2}\right)} P_{5}(\cos \theta)+\ldots\right\} .
\end{aligned}
$$

9.061 If $n$ is a positive integer:

I. $\cos n \theta=\frac{\mathrm{I}}{2} \frac{2 \cdot 4 \cdot 6 \ldots 2 n}{3 \cdot 5 \cdot 7 \ldots(2 n+\mathrm{I})}\left\{(2 n+\mathrm{I}) P_{n}(\cos \theta)\right.$

$$
\begin{array}{r}
+(2 n-3) \frac{\left[n^{2}-(n+\mathrm{I})^{2}\right]}{\left[n^{2}-(n-2)^{2}\right]} P_{n-2}(\cos \theta) \\
\left.+(2 n-7) \frac{\left[n^{2}-(n+1)^{2}\right]\left[n^{2}-(n-\mathrm{I})^{2}\right]}{\left[n^{2}-(n-2)^{2}\right]\left[n^{2}-(n-4)^{2}\right]} P_{n-4}(\cos \theta)+\ldots\right\} .
\end{array}
$$

2. $\sin n \theta=\frac{\pi}{4} \frac{\mathrm{I} \cdot 3 \cdot 5 \ldots(2 n-3)}{2 \cdot 4 \cdot 6 \ldots(2 n-2)}\left\{(2 n-\mathrm{I}) P_{n-1}(\cos \theta)\right.$

$$
+(2 n+3) \frac{\left[n^{2}-(n-1)^{2}\right]}{\left[n^{2}-(n+2)^{2}\right]} P_{n+1}(\cos \theta)
$$

$$
\left.+(2 n+7) \frac{\left[n^{2}-(n-\mathrm{I})^{2}\right]\left[n^{2}-(n+\mathrm{r})^{2}\right]}{\left[n^{2}-(n+2)^{2}\right]\left[n^{2}-(n+4)^{2}\right]} P_{n+3}(\cos \theta)+\ldots\right\} \text {. }
$$

\subsection{2}

I. $\quad \theta=\frac{\pi}{2}-\frac{\pi}{2} \sum_{n=\mathrm{I}}^{\infty} \frac{(4 n-\mathrm{I})}{(2 n-\mathrm{I})^{2}}\left(\frac{\mathrm{I} \cdot 3 \cdot 5 \cdots(2 n-\mathrm{I})}{2 \cdot 4 \cdot 6 \ldots 2 n}\right)^{2} P_{2 n-1}(\cos \theta)$.

2. $\sin \theta=\frac{\pi}{4}-\frac{\pi}{2} \sum_{n=I}^{\infty} \frac{(4 n+I)}{(2 n-I)(2 n+2)}\left(\frac{I \cdot 3 \cdot 5 \cdots(2 n-I)}{2 \cdot 4 \cdot 6 \ldots 2 n}\right)^{2} P_{2 n}(\cos \theta)$

3. $\cot \theta=\frac{\pi}{2} \sum_{n=1}^{\infty} \frac{2 n(4 n-\mathrm{I})}{(2 n-\mathrm{I})}\left(\frac{\mathrm{I} \cdot 3 \cdot 5 \cdots(2 n-\mathrm{I})}{2 \cdot 4 \cdot 6 \ldots 2 n}\right)^{2} P_{2 n-1}(\cos \theta)$.

4. $\csc \theta=\frac{\pi}{2}+\frac{\pi}{2} \sum_{n=I}^{\infty}(4 n+\mathrm{I})\left(\frac{\mathrm{I} \cdot 3 \cdot 5 \ldots(2 n-\mathrm{I})}{2 \cdot 4 \cdot 6 \ldots 2 n}\right)^{2} P_{2 n}(\cos \theta)$

\subsection{3}

I. $\log \frac{\mathrm{I}+\sin \frac{\theta}{2}}{\sin \frac{\theta}{2}}=\mathrm{I}+\sum_{n=\mathrm{I}}^{\infty} \frac{\mathrm{I}}{n+\mathrm{I}} P_{n}(\cos \theta)$.

2. $\log \frac{\tan \frac{1}{4}(\pi-\theta)}{\frac{1}{2} \sin \theta}=-\log \sin \frac{\theta}{2}-\log \left(\mathrm{I}+\sin \frac{\theta}{2}\right)=\sum_{n=\mathrm{I}}^{\infty} \frac{\mathrm{I}}{n} P_{n}(\cos \theta)$.

9.064 $K(k)$ and $E(k)$ denote the complete elliptic integrals of the first and second kinds, and $k=\sin \theta$ :

I. $K(k)=\frac{\pi^{2}}{4}+\frac{\pi^{2}}{4} \sum_{n=I}^{\infty}(-I)^{n}(4 n+I)\left(\frac{I \cdot 3 \cdot 5 \cdots(2 n-I)}{2 \cdot 4 \cdot 6 \ldots 2 n}\right)^{3} P_{2 n}(\cos \theta)$. 
2. $E(k)=\frac{\pi^{2}}{8}+\frac{\pi^{2}}{4} \sum_{n=\mathrm{I}}^{\infty}(-\mathrm{I})^{n+1} \frac{(4 n+\mathrm{I})}{(2 n-\mathrm{I})(2 n+2)}\left(\frac{\mathrm{I} \cdot 3 \cdot 5 \ldots(2 n-\mathrm{I})}{2 \cdot 4 \cdot 6 \ldots 2 n}\right)^{3} P_{2 n}(\cos \theta)$. (Hargreaves, Mess. of Math. 26, p. 89, I897)

9.070 The differential equation:

$$
\left(\mathrm{I}-x^{2}\right) \frac{d^{2} y}{d x^{2}}-2 x \frac{d y}{d x}+\left\{n(n+\mathrm{I})-\frac{m^{2}}{\mathrm{I}-x^{2}}\right\} y=0 .
$$

If $m$ is a positive integer, and $-\mathrm{I}>x>+\mathrm{I}$, two solutions of this differential equation are the associated Legendre functions

$$
\begin{aligned}
& P_{n}^{m}(x)=\left(\mathrm{I}-x^{2}\right)^{\frac{m}{2}} \frac{d^{m} P_{n}(x)}{d x^{m}}, \\
& Q_{n}^{m}(x)=\left(\mathrm{I}-x^{2}\right)^{\frac{m}{2}} \frac{d^{m} Q_{n}(x)}{d x^{m}} .
\end{aligned}
$$

9.071 If $n, m, r$ are positive integers, and $n>m, r>m$ :

$$
\begin{aligned}
\int_{-\mathrm{I}}^{+\mathrm{I}} P_{n}^{m}(x) P_{r}^{m}(x) d x & =0 \text { if } r \neq n, \\
& =\frac{2}{2 n+\mathrm{I}} \frac{(n+m) !}{(n-m) !} \text { if } r=n .
\end{aligned}
$$

9.100 Bessel's Differential Equation:

9.101 One solution is:

$$
\frac{d^{2} y}{d x^{2}}+\frac{\mathrm{I}}{x} \frac{d y}{d x}+\left(\mathrm{I}-\frac{\nu^{2}}{x^{2}}\right) y=0 .
$$

$$
y=J_{\nu}(x)=\sum_{k=0}^{\infty}(-\mathrm{I})^{k} \frac{x^{\nu+2 k}}{2^{\nu+2 k} k ! \Gamma(\nu+k+\mathrm{I})} .
$$

9.102 A second independent solution when $\nu$ is not an integer is:

9.103 If $\nu=n$, an integer:

$$
y=J_{-\nu}(x) \text {. }
$$

$$
J_{-n}(x)=(-\mathrm{I})^{n} J_{n}(x) .
$$

9.104 A second independent solution when $\nu=n$, an integer, is:

$$
\begin{aligned}
\pi Y_{n}(x)={ }_{2} J_{n}(x) & \cdot \log \frac{x}{2}-\sum_{k=0}^{n-\mathrm{x}} \frac{(n-k-\mathrm{I}) !}{k !}\left(\frac{x}{2}\right)^{2 k-n} \\
& -\sum_{k=0}^{\infty}(-\mathrm{I})^{k} \frac{\mathrm{I}}{k !(k+n) !}\left(\frac{x}{2}\right)^{n+2 k}\{\psi(k+\mathrm{I})+\psi(k+n+\mathrm{I})\}
\end{aligned}
$$


9.105 For all values of $\nu$, whether integral or not:

$$
\begin{aligned}
& Y_{\nu}(x)=\frac{\mathrm{I}}{\sin \nu \pi}\left(\cos \nu \pi J_{\nu}(x)-J_{-\nu}(x)\right), \\
& J_{-\nu}(x)=\cos \nu \pi J_{\nu}(x)-\sin \nu \pi Y_{\nu}(x) \\
& Y_{-\nu}(x)=\sin \nu \pi J_{\nu}(x)+\cos \nu \pi Y_{\nu}(x) .
\end{aligned}
$$

9.106 For $\nu=n$, an integer:

$$
Y_{-n}(x)=(-\mathbf{I})^{n} Y_{n}(x) .
$$

9.107 Cylinder Functions of the third kind, solutions of Bessel's differential equation:

I.

2.

3 .

4 .

$$
\begin{aligned}
H_{\nu}^{\mathrm{I}}(x) & =J_{\nu}(x)+i Y_{\nu}(x) . \\
H_{\nu}^{\mathrm{II}}(x) & =J_{\nu}(x)-i Y_{\nu}(x) . \\
H_{-\nu}^{\mathrm{I}}(x) & =e^{\nu \pi i} H_{\nu}^{\mathrm{I}}(x) . \\
H_{-\nu}^{\mathrm{II}}(x) & =e^{-\nu \pi i} H_{\nu}^{\mathrm{II}}(x) .
\end{aligned}
$$

9.110 Recurrence formulae satisfied by the functions $J_{\nu}, Y_{\nu}, H_{\nu}^{\mathrm{I}}, H_{\nu}^{\mathrm{II}}, C_{\nu}$ represents any one of these functions.

I.

$$
\begin{aligned}
& C_{\nu-1}(x)-C_{\nu+1}(x)=2 \frac{d}{d x} C_{\nu}(x) . \\
& C_{-1}(x)+C_{\nu+1}(x)=\frac{2 \nu}{x} C_{\nu}(x) . \\
& \frac{d}{d x} C_{\nu}(x)=C_{\nu-1}(x)-\frac{\nu}{x} C_{\nu}(x) . \\
& \frac{d}{d x} C(x)=\frac{\nu}{x} C_{\nu}(x)-C_{\nu+1}(x) . \\
& \frac{d}{d x}\left\{x^{\nu} C_{\nu(x)}\right\}=x^{\nu} C_{\nu-1}(x) . \\
& \frac{d^{2} C_{\nu}(x)}{d x^{2}}=\frac{\mathrm{I}}{4}\left\{C_{\nu+2}(x)+C_{\nu-2}(x)-{ }_{2} C_{\nu}(x)\right\}
\end{aligned}
$$

\subsection{1}
I. $J_{\nu}(x) \frac{d Y_{\nu}(x)}{d x}-Y_{\nu}(x) \frac{d J_{\nu}(x)}{d x}=\frac{2}{\pi x}$.
2. $J_{\nu+1}(x) Y_{\nu}(x)-J_{\nu}(x) Y_{\nu+1}(x)=\frac{2}{\pi x}$

\subsection{0} ASYMPTOTIC EXPANSIONS FOR LARGE VALUES OF $x$

I. $J_{\nu}(x)=\sqrt{\frac{2}{\pi x}}\left\{P(x) \cos \left(x-\frac{2 \nu+\mathrm{I}}{4} \pi\right)-Q_{\nu}(x) \sin \left(x-\frac{2 \nu+\mathrm{I}}{4} \pi\right)\right\}$, 2. $Y_{\nu}(x)=\sqrt{\frac{2}{\pi x}}\left\{P_{\nu}(x) \sin \left(x-\frac{2 \nu+\mathrm{I}}{4} \pi\right)+Q_{\nu}(x) \cos \left(x-\frac{2 \nu+\mathrm{I}}{4} \pi\right)\right\}$, 
3. $H_{\nu}^{\mathrm{I}}(x)=e^{i\left(x-\frac{2 \nu+\mathrm{r}}{4} \pi\right)} \sqrt{\frac{2}{\pi x}}\left\{P_{\nu}(x)+i Q_{\nu}(x)\right\}$,

4. $H_{\nu}^{\mathrm{II}}(x)=e^{-i\left(x-\frac{2 \nu+\mathrm{r}}{4} \pi\right)} \sqrt{\frac{2}{\pi x}}\left\{P_{\nu}(x)-i Q_{\nu}(x)\right\}$,

where

$P_{\nu}(x)=\mathrm{I}+\sum_{k=\mathrm{I}}^{\infty}(-\mathrm{I})^{k} \frac{\left(4 \nu^{2}-\mathrm{I}^{2}\right)\left(4 \nu^{2}-3^{2}\right) \ldots \ldots\left(4 \nu^{2}-\overline{4 k}-\overline{\mathrm{I}}^{2}\right)}{(2 k) ! 2^{6 k} x^{2 k}}$,

$Q_{\nu}(x)=\sum_{k=\mathrm{I}}^{\infty}(-\mathrm{I})^{k+1} \frac{\left(4 \nu^{2}-\mathrm{I}^{2}\right)\left(4 \nu^{2}-3^{2}\right) \ldots \ldots\left(4 \nu^{2}-\overline{4 k-3}^{2}\right)}{(2 k-\mathrm{I}) ! 2^{6 k-3} x^{2 k-1}}$.

\section{SPECIAL VALUES}

\subsection{0}

I. $J_{0}(x)=\mathrm{I}-\frac{\mathrm{I}}{(\mathrm{I} !)^{2}}\left(\frac{x}{2}\right)^{2}+\frac{\mathrm{I}}{(2 !)^{2}}\left(\frac{x}{2}\right)^{4}-\frac{\mathrm{I}}{(3 !)^{2}}\left(\frac{x}{2}\right)^{6}+\ldots$.

2. $J_{1}(x)=-\frac{d J_{0}(x)}{d x}=\frac{x}{2}\left\{\mathrm{I}-\frac{\mathrm{I}}{\mathrm{I} ! 2 !}\left(\frac{x}{2}\right)^{2}+\frac{\mathrm{I}}{2 ! 3 !}\left(\frac{x}{2}\right)^{4}-\frac{\mathrm{I}}{3 ! 4 !}\left(\frac{x}{2}\right)^{6}+\ldots\right\}$.

3. $\frac{\pi}{2} Y_{0}(x)=\left(\log \frac{x}{2}+\gamma\right) J_{0}(x)+\left(\frac{x}{2}\right)^{2}-\frac{\mathrm{I}}{(2 !)^{2}}\left(\mathrm{I}+\frac{\mathrm{I}}{2}\right)\left(\frac{x}{2}\right)^{4}$

$$
+\frac{I}{(3 !)^{2}}\left(I+\frac{I}{2}+\frac{I}{3}\right)\left(\frac{x}{2}\right)^{6}-\ldots
$$

$$
=\left(\log \frac{x}{2}+\gamma\right) J_{0}(x)+4\left\{\frac{\mathrm{I}}{2} J_{2}(x)-\frac{\mathrm{I}}{4} J_{4}(x)+\frac{\mathrm{I}}{6} J_{6}(x)-\ldots\right\} .
$$

4. $\frac{\pi}{2} Y_{1}(x)=\left(\log \frac{x}{2}+\gamma\right) J_{1}(x)-\frac{\mathrm{I}}{x} J_{0}(x)-\frac{x}{2}\left\{\mathrm{I}-\frac{\mathrm{I}}{\mathrm{I} ! 2 !}\left(\mathrm{I}+\frac{\mathrm{I}}{2}\right)\left(\frac{x}{2}\right)^{2}\right.$

$$
\begin{aligned}
& \left.+\frac{I}{2 ! 3 !}\left(I+\frac{I}{2}+\frac{I}{3}\right)\left(\frac{x}{2}\right)^{4}-\ldots .\right\} \\
& =\left(\log \frac{x}{2}+\gamma\right) J_{1}(x)-\frac{\mathrm{I}}{x} J_{0}(x)+\frac{3}{\mathrm{I} \cdot 2} J_{3}(x)-\frac{5}{2 \cdot 3} J_{5}(x) \\
& \gamma=0.5772157 \quad(6.602) \text {. } \\
& +\frac{7}{3 \cdot 4} J_{7}(x)-\ldots \ldots
\end{aligned}
$$

9.131 Limiting values for $x=0$ :

$$
\begin{aligned}
J_{0}(x) & =\mathrm{I} \\
J_{1}(x) & =0 \\
Y_{0}(x) & =\frac{2}{\pi}\left(\log \frac{x}{2}+\gamma\right) \\
Y_{1}(x) & =-\frac{2}{\pi x}
\end{aligned}
$$


9.132 Limiting values for $x=\infty$ :

$$
\begin{array}{ll}
J_{0}(x)=\frac{\cos \left(x-\frac{\pi}{4}\right)}{\sqrt{\frac{\pi x}{2}}}, & Y_{0}(x)=\frac{\sin \left(x-\frac{\pi}{4}\right)}{\sqrt{\frac{\pi x}{2}}}, \\
J_{1}(x)=\frac{\sin \left(x-\frac{\pi}{4}\right)}{\sqrt{\frac{\pi x}{2}}}, & Y_{1}(x)=-\frac{\cos \left(x-\frac{\pi}{4}\right)}{\sqrt{\frac{\pi x}{2}}} .
\end{array}
$$

9.140 Bessel's Addition Formula:

$$
J_{\nu}(x+h)=\left(\frac{x+h}{x}\right)^{\nu} \sum_{k=0}^{\infty}(-\mathrm{I})^{k} \frac{h^{k}}{k !}\left(\frac{2 x+h}{2 x}\right)^{k} J_{\nu+k}(x) .
$$

9.141 Multiplication formula:

$$
J_{\nu}(\alpha x)=\alpha^{\nu} \sum_{k=0}^{\infty} \frac{\left(\mathrm{I}-\alpha^{2}\right)^{k}}{k !}\left(\frac{x}{2}\right)^{k} J_{\nu+k}(x) .
$$

9.142

$$
J_{\nu}(\alpha x) J_{\mu}(\beta x)=\sum_{k=0}^{\infty}(-\mathrm{I})^{k} A_{k}\left(\frac{x}{2}\right)^{\mu+\nu+2 k}
$$

where

$$
A_{k}=\sum_{s=0}^{k} \frac{\alpha^{2 k-2 s} \beta^{2 s}}{s !(k-s) ! \Gamma(\nu+k-s+\mathrm{I}) \Gamma(\mu+s+\mathrm{I})} .
$$

\subsection{3}

$$
J_{\nu}(x) J_{\mu}(x)=\sum_{k=0}^{\infty} \frac{(-\mathrm{I})^{k}}{\Gamma(\nu+k+\mathrm{I}) \Gamma(\mu+k+\mathrm{I})}\left(\begin{array}{c}
\mu+\nu+2 k \\
k
\end{array}\right)\left(\frac{x}{2}\right)^{\mu+\nu+2 k} .
$$

DEFINITE INTEGRAL EXPRESSIONS FOR BESSEL'S FUNCTIONS

9.150

$$
J_{\nu}(x)=\frac{2\left(\frac{x}{2}\right)^{\nu}}{\sqrt{\pi} \Gamma\left(\nu+\frac{I}{2}\right)} \int_{4}^{\frac{\pi}{2}} \cos (x \sin \phi) \cos ^{2 \nu} \phi \cdot d \phi .
$$

9.151

$$
J_{\nu}(x)=\frac{2\left(\frac{x}{2}\right)}{\sqrt{\pi} \Gamma\left(\nu+\frac{I}{2}\right)} \int_{0}^{\pi} \cos (x \cos \phi) \sin ^{2 \nu} \phi \cdot d \phi .
$$


9.152

If $n$ is an integer:

$$
J_{\nu}(x)=\frac{\left(\frac{x}{2}\right)^{\nu}}{\sqrt{\pi} \Gamma\left(\nu+\frac{\mathrm{I}}{2}\right)} \int_{0}^{\pi} e^{i x \cos \phi} \sin ^{2 \nu} \phi \cdot d \phi .
$$

\subsection{3}

9.154

$$
J_{2 n}(x)=\frac{I}{\pi} \int_{0}^{\pi} \cos (x \sin \phi) \cos (2 n \phi) d \phi=\frac{2}{\pi} \int_{0}^{\frac{\pi}{2}} .
$$

9.155

$$
J_{2 n}(x)=\frac{(-\mathrm{I})^{n}}{\pi} \int_{0}^{\pi} \cos (x \cos \phi) \cos (2 n \phi) d \phi=\frac{2(-\mathrm{I})^{n}}{\pi} \int_{0}^{\frac{\pi}{2}} .
$$

9.156

$$
J_{2 n+1}(x)=\frac{\mathrm{I}}{\pi} \int_{0}^{\pi} \sin (x \sin \phi) \sin (2 n+\mathrm{I}) \phi d \phi=\frac{2}{\pi} \int_{0}^{\frac{\pi}{2}} .
$$

9.157

$$
J_{2 n+1}(x)=\frac{(-\mathrm{I})^{n}}{\pi} \int_{0}^{\pi} \sin (x \cos \phi) \cos (2 n+\mathrm{I}) \phi d \phi=\frac{2(-\mathrm{I})^{n}}{\pi} \int_{0}^{\frac{\pi}{2}} .
$$

$$
J_{n}(x)=\frac{\mathrm{I}}{2 \pi} \int_{-\pi}^{+\pi} e^{-i n \phi+i x \sin \phi} d \phi=\frac{\mathrm{I}}{2 \pi} \int_{0}^{2 \pi} e^{-i n \phi+i x \sin \phi} d \phi .
$$

INTEGRAL PROPERTIES

9.160 If $C_{\nu}(\mu x)$ is any one of the particular integrals:

$$
J_{\nu}(\mu x), Y_{\nu}(\mu x), H_{\nu}^{\mathrm{I}}(\mu x), H_{\nu}^{\mathrm{II}}(\mu x),
$$

of the differential equation:

$$
\begin{gathered}
\frac{d^{2} y}{d x^{2}}+\frac{\mathrm{I}}{x} \frac{d y}{d x}+\left(\mu^{2}-\frac{\nu^{2}}{x^{2}}\right) y=0 \\
\int_{a}^{b} C_{\nu}\left(\mu_{k} x\right) C_{\nu}\left(\mu_{l} x\right) x d x \\
=\frac{\mathrm{I}}{\mu_{k}{ }^{2}-\mu_{l}{ }^{2}}\left[x\left\{\mu_{l} C_{\nu}\left(\mu_{k} x\right) C_{\nu}{ }^{\prime}\left(\mu_{l} x\right)-\mu_{k} C_{\nu}\left(\mu_{l} x\right) C_{\nu}{ }^{\prime}\left(\mu_{k} x\right)\right\}\right]_{a}^{b} ; \mu_{k} \neq \mu_{l} .
\end{gathered}
$$

9.161 If $\mu_{k}$ and $\mu_{l}$ are two different roots of

$$
C_{\nu}(\mu b)=0,
$$

$\int_{a}^{b} C_{\nu}\left(\mu_{k} x\right) C_{v}\left(\mu_{l} x\right) x d x=\frac{a}{\mu_{k}^{2}-\mu_{l}{ }^{2}}\left\{\mu_{k} C_{v}\left(\mu_{l} a\right) C_{v}{ }^{\prime}\left(\mu_{k} a\right)-\mu_{l} C_{\nu}\left(\mu_{k} a\right) C_{v}{ }^{\prime}\left(\mu_{l} a\right)\right\}$.

9.162 If $\mu_{k}$ and $\mu_{l}$ are two different roots of

and

$$
a \frac{C_{\nu}{ }^{\prime}(\mu a)}{C_{v}(\mu a)}=p \mu+q \frac{\mathrm{I}}{\mu}
$$

If $\mu_{k}=\mu_{l}$ :

$$
\begin{gathered}
C_{\nu}(\mu b)=0, \\
\int^{b} C_{\nu}\left(\mu_{k} x\right) C_{v}\left(\mu_{l} x\right) x d x=p C_{v}\left(\mu_{k} a\right) C_{\nu}\left(\mu_{l} a\right) .
\end{gathered}
$$

$$
\int^{b} C_{\nu}\left(\mu_{k} x\right) C_{\nu}\left(\mu_{l} x\right) x d x=\frac{I}{2}\left\{b^{2} C_{\nu}{ }^{2}\left(\mu_{k} b\right)-a^{2} C_{\nu}{ }^{2}\left(\mu_{k} a\right)-\left(a^{2}-\frac{\nu^{2}}{\mu_{k}^{2}}\right) C_{\nu}{ }^{2}\left(\mu_{k} a\right)\right\} .
$$








\section{EXPANSIONS IN BESSEL'S FUNCTIONS}

9.170 Schlömilch's Expansion. Any function $f(x)$ which has a continuous differential coefficient for all values of $x$ in the closed range $(0, \pi)$ may be expanded in the series:

where

$$
f(x)=a_{0}+\sum_{k=1} a_{k} J_{0}(k x)
$$

9.171

$$
\begin{aligned}
& a_{0}=f(0)+\frac{\mathrm{I}}{\pi} \int_{0}^{\pi} u \int_{0}^{\frac{\pi}{2}} f^{\prime}(u \sin \theta) d \theta d u, \\
& a_{k}=\frac{2}{\pi} \int_{0}^{\pi} u \cos k u \int_{0}^{\frac{\pi}{2}} f^{\prime}(u \sin \theta) d \theta d u .
\end{aligned}
$$

where

$$
f(x)=a_{0} x^{n}+\sum_{k=1}^{\infty} a_{k} J_{n}\left(\alpha_{k} x\right) \quad 0<x<\mathrm{r},
$$

$$
\begin{aligned}
J_{n+1}\left(\alpha_{k}\right) & =0, \\
a_{0} & =2(n+\mathrm{I}) \int^{\mathrm{I}} f(x) x^{n+1} d x, \\
a_{k} & =\frac{2}{\left[J_{n}\left(\alpha_{k}\right)\right]^{2}} \int_{0}^{\mathrm{I}} x f(x) J_{n}\left(\alpha_{k} x\right) d x .
\end{aligned}
$$

(Bridgman, Phil. Mag. I6, p. 947, I908)

9.172

$$
f(x)=\sum_{k=\mathbf{I}}^{\infty} A_{k} J_{0}\left(\mu_{k} x\right) \quad a<x<b,
$$

where:

$$
a \frac{J_{0}^{\prime}\left(\mu_{k} a\right)}{J_{0}\left(\mu_{k} a\right)}=p \mu_{k}+\frac{q}{\mu_{k}}
$$

and

$$
\begin{gathered}
J_{0}\left(\mu_{k} b\right)=0 \\
A_{k}=2 \frac{\int_{a}^{b} x f(x) J_{0}\left(\mu_{k} x\right) d x-p f(a) J_{0}\left(\mu_{k} a\right)}{b^{2} J_{0}^{\prime 2}\left(\mu_{k} b\right)-a^{2} J_{0}^{\prime 2}\left(\mu_{k} a\right)-\left(a^{2}+2 p\right) J_{0}^{2}\left(\mu_{k} a\right)} .
\end{gathered}
$$

(Stephenson, Phil. Mag. I4, p. 547, I907)

\subsection{0}

I. $\sin x=2 \sum_{k=0}^{\infty}(-\mathrm{I})^{k} J_{2 k+1}(x)$,

2. $\cos x=J_{0}(x)+2 \sum_{k=1}^{\infty}(-\mathrm{I})^{k} J_{2 k}(x)$. 


\subsection{1}

I. $\cos (x \sin \theta)=J_{0}(x)+2 \sum_{k=I}^{\infty} J_{2 k}(x) \cos 2 k \theta$,

2. $\sin (x \sin \theta)=2 \sum_{k=0}^{\infty} J_{2 k+1}(x) \sin (2 k+\mathrm{I}) \theta$.

\subsection{2}

I. $\left(\frac{x}{2}\right)^{n}=\sum_{k=0}^{\infty} \frac{(n+2 k)(n+k-\mathrm{I}) !}{k !} J_{n+2 k}(x)$,

2. $\sqrt{\frac{2 x}{\pi}}=\sum_{k=0}^{\infty} \frac{(4 k+\mathrm{I})(2 k) !}{2^{2 k}(k !)^{2}} J_{2 k+\frac{1}{2}}(x)$.

\subsection{3}

$$
\begin{aligned}
\frac{d J_{\nu}(x)}{d \nu} & =\left\{\log \frac{x}{2}-\psi(\nu+\mathrm{I})\right\} J(x)+\sum_{k=1}^{\infty}(-\mathrm{I})^{k-1} \frac{\nu+2 k}{k(\nu+k)} J_{\nu+2 k}(x) \\
& =J_{\nu}(x) \log \frac{x}{2}-\sum_{k=0}^{\infty}(-\mathrm{I})^{k} \frac{\psi(\nu+k+\mathrm{I})}{k ! \Gamma(\nu+k+\mathrm{I})}\left(\frac{x}{2}\right)^{\nu+2 k} .
\end{aligned}
$$

9.200 The differential equation:

$$
\frac{d^{2} y}{d x^{2}}+\frac{2}{x} \frac{d y}{d x}+\left(\mu^{2}-\frac{n(n+\mathrm{I})}{x^{2}}\right) y=0
$$

with the substitution:

becomes:

$$
z=y \sqrt{x}, \quad \mu x=\rho
$$

$$
\frac{d^{2} z}{d \rho^{2}}+\frac{\mathrm{I}}{\rho} \frac{d z}{d \rho}+\left(\mathrm{I}-\frac{\left(n+\frac{1}{2}\right)^{2}}{\rho^{2}}\right) z=0
$$

which is Bessel's equation of order $n+\frac{\mathrm{I}}{2}$.

9.201 Two independent solutions are:

$$
\begin{aligned}
& z=J_{n+\frac{1}{2}}(\rho) . \\
& z=J_{-n-\frac{1}{2}}(\rho) .
\end{aligned}
$$

The former remains finite for $\rho=0$; the latter becomes infinite for $\rho=0$. 
9.202 Special values.

$$
\begin{aligned}
& J_{\frac{1}{2}(x)}=\sqrt{\frac{2}{\pi x}} \sin x, \\
& J(x)=\sqrt{\frac{2}{\pi x}}\left(\frac{\sin x}{x}-\cos x\right), \\
& J_{\frac{5}{2}}(x)=\sqrt{\frac{2}{\pi x}}\left\{\left(\frac{3}{x^{2}}-\mathrm{I}\right) \sin x-\frac{3}{x} \cos x\right\}, \\
& J_{\frac{5}{2}}(x)=\sqrt{\frac{2}{\pi x}}\left\{\left(\frac{15}{x^{3}}-\frac{6}{x}\right) \sin x-\left(\frac{15}{x^{2}}-\mathrm{I}\right) \cos x\right\}, \\
& J_{\frac{9}{2}}(x)=\sqrt{\frac{2}{\pi x}}\left\{\left(\frac{105}{x^{4}}-\frac{45}{x^{2}}+\mathrm{I}\right) \sin x-\left(\frac{\text { I05 }}{x^{3}}-\frac{\text { I0 }}{x}\right) \cos x\right\} .
\end{aligned}
$$

9.203

$$
\begin{aligned}
& J_{-\frac{1}{2}}(x)=\sqrt{\frac{2}{\pi x}} \cos x \\
& J_{-\frac{3}{2}}(x)=-\sqrt{\frac{2}{\pi x}}\left(\sin x+\frac{\cos x}{x}\right), \\
& J_{-\frac{1}{2}}(x)=\sqrt{\frac{2}{\pi x}}\left\{\frac{3}{x} \sin x+\left(\frac{3}{x^{2}}-\mathrm{I}\right) \cos x\right\}, \\
& J_{-\frac{7}{2}}(x)=-\sqrt{\frac{2}{\pi x}}\left\{\left(\frac{15}{x^{2}}-\mathrm{I}\right) \sin x+\left(\frac{15}{x^{3}}-\frac{6}{x}\right) \cos x\right\}, \\
& J_{-\frac{9}{2}}(x)=\sqrt{\frac{2}{\pi x}}\left\{\left(\frac{105}{x^{3}}-\frac{\text { IO }}{x}\right) \sin x+\left(\frac{\text { IO5 }}{x^{4}}-\frac{45}{x^{2}}+\mathrm{I}\right) \cos x\right\} .
\end{aligned}
$$

\subsection{4}

$$
\begin{aligned}
& H_{\frac{1}{2}}^{\mathrm{I}}(x)=-i \sqrt{\frac{2}{\pi x}} e^{i x}, \\
& H_{\frac{3}{2}}^{\mathrm{I}}(x)=-\sqrt{\frac{2}{\pi x}} e^{i x}\left(\mathrm{I}+\frac{i}{x}\right), \\
& H_{\frac{5}{2}}^{\mathrm{I}}(x)=-\sqrt{\frac{2}{\pi x}} e^{i x}\left\{\frac{3}{x}+i\left(\frac{3}{x^{2}}-\mathrm{I}\right)\right\} .
\end{aligned}
$$

\subsection{5}

$$
\begin{aligned}
& H_{\frac{1}{2}}^{\mathrm{II}}(x)=i \sqrt{\frac{2}{\pi x}} e^{-i x} \\
& H_{\frac{3}{2}}^{\mathrm{II}}(x)=-\sqrt{\frac{2}{\pi x}} e^{-i x}\left(\mathrm{I}-\frac{i}{x}\right) \\
& H_{\frac{1}{2}}^{\mathrm{II}}(x)=-\sqrt{\frac{2}{\pi x}} e^{-i x}\left\{\frac{3}{x}-i\left(\frac{3}{x^{2}}-\mathrm{I}\right)\right\}
\end{aligned}
$$


9.210 The differential equation:

$$
\frac{d^{2} y}{d x^{2}}+\frac{\mathrm{I}}{x} \frac{d y}{d x}-\left(\mathrm{I}+\frac{\nu^{2}}{x^{2}}\right) y=0
$$

with the substitution,

becomes Bessel's equation.

$$
x=i z
$$

9.211 Two independent solutions of 9.210 are:

$$
\begin{aligned}
& I_{\nu}(x)=i^{-\nu} J_{\nu}(i x), \\
& K^{\nu}(x)=e^{\frac{\nu+\mathrm{I}}{2} \pi i} \frac{\pi}{2} H_{\nu}^{\mathrm{I}}(i x) .
\end{aligned}
$$

9.212 If $\nu=n$, an integer:

$$
\begin{aligned}
I_{n}(x) & =\sum_{k=0}^{\infty} \frac{\mathrm{I}}{k !(n+k) !}\left(\frac{x}{2}\right)^{n+2 k}, \\
K_{n}(x) & =i^{n+1} \frac{\pi}{2} H_{n}^{I}(x) .
\end{aligned}
$$

9.213

$$
\begin{aligned}
& I_{\nu}(x)=\frac{\mathrm{I}}{\sqrt{\pi} \Gamma\left(\nu+\frac{1}{2}\right)}\left(\frac{x}{2}\right)^{\nu} \int_{0}^{\pi} \cosh (x \cos \phi) \sin ^{2 \nu} \phi d \phi, \\
& K_{\nu}(x)=\frac{\sqrt{\pi}}{\Gamma\left(\nu+\frac{1}{2}\right)}\left(\frac{x}{2}\right)^{\nu} \int^{\infty} \sinh ^{2 \nu} \phi e^{-x \cosh \phi} d \phi .
\end{aligned}
$$

9.214 If $x$ is large, to a first approximation:

$$
\begin{aligned}
I_{n}(x) & =(2 \pi x \cosh \beta)^{-\frac{1}{2}} e^{x(\cosh \beta-\beta \sinh \beta),} \\
K_{n}(x) & =\pi(2 \pi x \cosh \beta)^{-\frac{1}{2}} e^{-x(\cosh \beta-\beta \sinh \beta),} \\
n & =x \sinh \beta .
\end{aligned}
$$

9.215 Ber and Bei Functions.

$$
\begin{gathered}
\text { ber } x+i \text { bei } x=I(x \sqrt{i}) \\
\text { ber } x-i \text { bei } x=I_{0}(i x \sqrt{i}) \\
\text { ber } x=\mathrm{I}-\frac{\mathrm{I}}{(2 !)^{2}}\left(\frac{x}{2}\right)^{4}+\frac{\mathrm{I}}{(4 !)^{2}}\left(\frac{x}{2}\right)^{8}-\ldots \\
\text { bei } x=\left(\frac{x}{2}\right)^{2}-\frac{\mathrm{I}}{(3 !)^{2}}\left(\frac{x}{2}\right)^{6}+\frac{\mathrm{I}}{(5 !)^{2}}\left(\frac{x}{2}\right)^{10} \ldots
\end{gathered}
$$


9.216 Ker and Kei Functions:

$$
\begin{aligned}
& \operatorname{ker} x+i \text { kei } x=K_{0}(x \sqrt{i}), \\
& \operatorname{ker} x-i \text { kei } x=K_{0}(i x \sqrt{i}),
\end{aligned}
$$

ker $x=\left(\log \frac{2}{x}-\gamma\right)$ ber $x+\frac{\pi}{4}$ bei $x-\frac{\mathrm{I}}{(2 !)^{2}}\left(\mathrm{I}+\frac{\mathrm{I}}{2}\right)\left(\frac{x}{2}\right)^{4}$

$$
+\frac{I}{(4 !)^{2}}\left(I+\frac{I}{2}+\frac{I}{3}+\frac{I}{4}\right)\left(\frac{x}{2}\right)^{8}-\ldots
$$

kei $x=\left(\log \frac{2}{x}-\gamma\right)$ bei $x-\frac{\pi}{4}$ ber $x+\left(\frac{x}{2}\right)^{2}-\frac{\mathrm{I}}{(3 !)^{2}}\left(\mathrm{I}+\frac{\mathrm{I}}{2}+\frac{\mathrm{I}}{3}\right)\left(\frac{x}{2}\right)^{6}+\ldots$

9.220 The Bessel-Clifford Differential-Equation:

With the substitution:

$$
x \frac{d^{2} y}{d x^{2}}+(\nu+\mathrm{I}) \frac{d y}{d x}+y=0 .
$$

$$
z=x^{\nu / 2} y \quad u=2 \sqrt{x}
$$

the differential equation reduces to Bessel's equation.

9.221 Two independent solutions of $\mathbf{9 . 2 2 0}$ are:

$$
\begin{aligned}
& C_{\nu}(x)=x^{-\frac{\nu}{2}} J_{\nu}(2 \sqrt{x})=\sum_{k=0}^{\infty}(-\mathrm{I})^{k} \frac{x^{k}}{k ! \Gamma(\nu+k+\mathrm{I})}, \\
& D_{\nu}(x)=x^{-\frac{\nu}{2}} Y_{\nu}(2 \sqrt{x}) .
\end{aligned}
$$

9.222

$$
\begin{aligned}
& C_{\nu+1}(x)=-\frac{d}{d x} C_{\nu}(x) \\
& x C_{\nu+2}(x)=(\nu+\mathrm{I}) C_{\nu+1}(x)-C_{\nu}(x)
\end{aligned}
$$

9.223 If $\nu=n$, an integer:

$$
\begin{aligned}
& C_{n}(x)=(-\mathrm{I})^{n} \frac{d^{n}}{d x^{n}} C_{0}(x), \\
& C_{0}(x)=\sum_{k=0}^{\infty}(-\mathrm{I})^{k} \frac{x^{k}}{(k !)^{2}}
\end{aligned}
$$

9.224 Changing the sign of $\nu$, the corresponding solution of:

$$
\begin{gathered}
x \frac{d^{2} y}{d x^{2}}-(\nu-\mathrm{I}) \frac{d y}{d x}+y=0, \\
y=x^{\nu} C_{\nu}(x) .
\end{gathered}
$$


9.225 If $\nu$ is half an odd integer:

$$
\begin{aligned}
& C_{\frac{1}{2}}(x)=\frac{\sin (2 \sqrt{x}+\epsilon)}{2 \sqrt{x}}, \\
& C_{\frac{3}{2}}(x)=-\frac{d}{d x} C_{\frac{1}{2}}(x)=\frac{\sin (2 \sqrt{x}+\epsilon)}{4 x^{\frac{3}{2}}}-\frac{\cos (2 \sqrt{x}+\epsilon)}{2 x}, \\
& C_{\frac{5}{2}}(x)=-\frac{d}{d x} C_{\frac{3}{2}}(x)=\frac{3-4 x}{8 x^{\frac{5}{2}}} \sin (2 \sqrt{x}+\epsilon)-\frac{3 \cos (2 \sqrt{x}+\epsilon)}{4 x^{2}}, \\
& \ldots \ldots \\
& \ldots \ldots \\
& C_{-\frac{1}{2}}(x)=-\cos (2 \sqrt{x}+\epsilon), \\
& C_{-\frac{3}{2}}(x)=x^{\frac{3}{2}} C_{\frac{3}{2}}(x), \\
& C_{-\frac{5}{2}}(x)=x^{\frac{5}{2}} C_{\frac{5}{2}}(x) . \\
& \ldots
\end{aligned}
$$

$\epsilon$ is arbitrary so as to give a second arbitrary constant.

9.226 For $x$ negative, the solution of the equation:

$$
x \frac{d^{2} y}{d x^{2}}+( \pm \nu+\mathrm{I}) \frac{d y}{d x}-y=0,
$$

when $\nu$ is half an odd integer, is obtained from the values in $\mathbf{9 . 2 2 5}$ by changi sin and $\cos$ to $\sinh$ and cosh respectively.

\subsection{7}

$$
\begin{aligned}
& (m+n+\mathrm{I}) \int C_{m+1}(x) C_{n+1}(x) d x=-x C_{m+1}(x) C_{n+1}(x)-C_{m}(x) C_{n}(x), \\
& (m+n+\mathrm{I}) \int x^{m+n} C_{m}(x) C_{n}(x) d x=x^{m+n+1}\left\{x C_{m+1}(x) C_{n+1}(x)+C_{m}(x) C_{n}(x)\right)
\end{aligned}
$$

\subsection{8}

I.

$$
\int_{0}^{\pi} C_{-\frac{1}{2}}\left(x \cos ^{2} \phi\right) d \phi=\pi C_{0}(x) .
$$

2.

$$
\int_{0}^{\pi} C_{\frac{1}{2}}\left(x \cos ^{2} \phi\right) d \phi=\pi C_{1}(x)
$$

3.

$$
\int_{0}^{\pi} C_{0}\left(x \sin ^{2} \phi\right) \sin \phi d \phi=C_{\frac{1}{2}}(x) .
$$

4.

$$
\int_{0}^{\pi} C_{1}\left(x \sin ^{2} \phi\right) \sin ^{3} \phi d \phi=C_{\frac{2}{2}}(x) .
$$

$$
\int_{0}^{\pi} C_{1}\left(x \sin ^{2} \phi\right) \sin \phi d \phi=\frac{1-\cos 2 \sqrt{x}}{x} .
$$


9.229 Many differential equations can be solved in a simpler form by the use of the $C_{n}$ functions than by the use of Bessel's functions.

(Greenhill, Phil. Mag. 38, p. 501, r919)

9.240 The differential equation:

$$
\frac{d^{2} y}{d x^{2}}+\frac{2(n+\mathrm{r})}{x} \frac{d y}{d x}+y=0
$$

with the change of variable:

becomes Bessel's equation 9.200.

$$
y=z x^{-n-\frac{1}{2}}
$$

9.241 Solutions of 9.240 are:

I.

2.

3 .

4.

$$
\begin{aligned}
& y=x^{-n-\frac{1}{2}} J_{n+\frac{1}{2}}(x) . \\
& y=x^{-n-\frac{1}{2}} \quad Y_{n+\frac{1}{2}}(x) . \\
& y=x^{-n-\frac{1}{2}} H_{n^{+\frac{1}{2}}}^{\mathrm{I}}(x) . \\
& y=x^{-n-\frac{1}{2}} H_{n^{+\frac{1}{2}}}^{\mathrm{II}}(x) .
\end{aligned}
$$

9.242 The change of variable:

$$
x=2 \sqrt{z},
$$

transforms equation $\mathbf{9 . 2 4 0}$ into the Bessel-Clifford differential equation $\mathbf{9 . 2 2 0}$. This leads to a general solution of 9.240:

$$
y=C_{n+\frac{2}{2}}\left(\frac{x^{2}}{4}\right) \text {. }
$$

When $n$ is an integer the equations of 9.225 may be employed.

$$
\begin{aligned}
& C_{1}\left(\frac{x^{2}}{4}\right)=\frac{\sin (x+\epsilon)}{x}, \\
& C_{\frac{3}{2}}\left(\frac{x^{2}}{4}\right)=\frac{2 \sin (x+\epsilon)}{x^{3}}-\frac{\cos (x+\epsilon)}{x} .
\end{aligned}
$$

9.243 The solution of

$$
\frac{d^{2} y}{d x^{2}}+\frac{2(n+\mathrm{I})}{x} \frac{d y}{d x}-y=0,
$$

may be obtained from 9.242 by writing $\sinh$ and $\cosh$ for $\sin$ and $\cos$ respectively.

9.244 The differential equation 9.240 is also satisfied by the two independent functions (when $n$ is an integer):

$\psi_{n}(x)=\left(-\frac{1}{x} \frac{d}{d x}\right)^{n} \frac{\sin x}{x}$

$$
=\frac{\mathrm{I}}{\mathrm{I} \cdot 3 \cdot 5 \cdots(2 n+\mathrm{I})} \sum_{k=0}^{\infty}(-\mathrm{I})^{k} \frac{x^{2 k}}{2^{k} k !(2 n+3) \ldots \ldots(2 n+2 k+\mathrm{I})},
$$




$$
\begin{aligned}
\Psi_{n}(x) & =\left(-\frac{\mathrm{I}}{x} \frac{d}{d x}\right)^{n} \frac{\cos x}{x} \\
& =\frac{\mathrm{I} \cdot 3 \cdot 5 \cdots(2 n-\mathrm{I})}{x^{2 n+1}} \sum_{k=0}^{\infty}(-\mathrm{I})^{k} \frac{x^{2 k}}{2^{k} k !(\mathrm{I}-2 n)(3-2 n) \ldots(2 k-2 n-}
\end{aligned}
$$

9.245 The general solution of $\mathbf{9 . 2 4 0}$ may be written:

$$
y=\left(\frac{\mathrm{I}}{x} \frac{d}{d x}\right)^{n} \frac{A e^{i x}+B e^{-i x}}{x} .
$$

9.246 Another particular solution of $\mathbf{9 . 2 4 0}$ is:

$$
\begin{gathered}
y=f_{n}(x)=\left(-\frac{\mathrm{I}}{x} \frac{d}{d x}\right)^{n} \frac{e^{-i x}}{x}=\Psi_{n}(x)-i \psi_{n}(x), \\
f_{n}(x)=\frac{i^{n} e^{-i x}}{x^{n+1}}\left\{\mathrm{I}+\frac{n(n+\mathrm{I})}{2 i x}+\frac{(n-\mathrm{I}) n(n+\mathrm{I})(n+2)}{2 \cdot 4 \cdot(i x)^{2}}+\ldots \ldots\right. \\
+\frac{\mathrm{I} \cdot 2 \cdot 3 \ldots \ldots 2 n}{2 \cdot 4 \cdot 6 \ldots 2 n(i x)^{n}}
\end{gathered}
$$

9.247 The functions $\psi_{n}(x), \Psi_{n}(x), f_{n}(x)$ satisfy the same recurrence formula

$$
\begin{gathered}
\frac{d \psi_{n}(x)}{d x}=-x \psi_{n+1}(x), \\
x \frac{d \psi_{n}(x)}{d x}+(2 n+\mathrm{I}) \psi_{n}(x)=\psi_{n-1}(x) .
\end{gathered}
$$

9.260 The differential equation:

$$
\frac{d^{2} y}{d x^{2}}-\frac{n(n+\mathrm{I})}{x^{2}} y+y=0
$$

with the change of variable:

$$
y=u \sqrt{x}
$$

is transformed into Bessel's equation of order $n+\frac{x}{2}$.

9.261 Solutions of $\mathbf{9 . 2 6 0}$ are:

I.

$$
S_{n}(x)=\sqrt{\frac{\pi x}{2}} J_{n+\frac{1}{2}}(x)=x^{n+1}\left(-\frac{\mathrm{I}}{x} \frac{d}{d x}\right)^{n} \frac{\sin x}{x} .
$$

2.

$$
C_{n}(x)=(-\mathrm{I})^{n} \sqrt{\frac{\pi x}{2}} J_{-n-\frac{1}{2}}(x)=x^{n+1}\left(-\frac{\mathrm{I}}{x} \frac{d}{d x}\right)^{n} \frac{\cos x}{x} .
$$

3.

$$
E_{n}(x)=C_{n}(x)-i S_{n}(x)=x^{n+1}\left(-\frac{\mathrm{I}}{x} \frac{d}{d x}\right)^{n} \frac{e^{-i x}}{x}
$$

9.262 The functions $S_{n}(x), C_{n}(x), E_{n}(x)$ satisfy the same recurrence formula

$$
\text { I. } \frac{d S_{n}(x)}{d x}=\frac{n+\mathrm{I}}{x} S_{n}(x)-S_{n+1}(x)
$$



2. $\frac{d S_{n}(x)}{d x}=S_{n-1}(x)-\frac{n}{x} S_{n}(x)$.
3. $S_{n+1}(x)=\frac{2 n+\mathrm{I}}{x} S_{n}(x)-S_{n-1}(x)$.

9.30 The hyperg-bmetric differential equation:

$$
x(\mathrm{I}-x) \frac{d^{2} y}{d x^{2}}+\{\gamma-(\alpha+\beta+\mathrm{I}) x\} \frac{d y}{d x}-\alpha \beta y=0 .
$$

9.31 The equation 9.30 is satisfied by the hypergeometric series:

$F(\alpha, \beta, \gamma, x)=\mathrm{I}+\frac{\alpha}{\mathrm{I}} \frac{\beta}{\gamma} x+\frac{\alpha(\alpha+\mathrm{I})}{\mathrm{I} \cdot 2} \frac{\beta(\beta+\mathrm{I})}{\gamma(\gamma+\mathrm{I})} x^{2}$

$$
+\frac{\alpha(\alpha+\mathrm{I})(\alpha+2)}{\mathrm{I} \cdot 2 \cdot 3} \frac{\beta(\beta+\mathrm{I})(\beta+2)}{\gamma(\gamma+\mathrm{I})(\gamma+2)} x^{3}+\ldots \ldots
$$

The series converges absolutely when $x<\mathrm{I}$ and diverges when $x>\mathrm{I}$. When $x=+\mathrm{I}$ it converges only when $\alpha+\beta-\gamma<0$, and then absolutely. When $x=-\mathrm{I}$ it converges only when $\alpha+\beta-\gamma-\mathrm{I}<\mathrm{O}$, and absolutely if $\alpha+\beta-\gamma<0$.

\subsection{2}

$$
\begin{aligned}
\frac{d}{d x} F(\alpha, \beta, \gamma, x) & =\frac{\alpha \beta}{\gamma} F(\alpha+\mathrm{I}, \beta+\mathrm{I}, \gamma+\mathrm{I}, x) \\
F(\alpha, \beta, \gamma, \mathrm{I}) & =\frac{\Gamma(\gamma) \Gamma(\gamma-\alpha-\beta)}{\Gamma(\gamma-\alpha) \Gamma(\gamma-\beta)}
\end{aligned}
$$

9.33 Representation of various functions by hypergeometric series.

$$
\begin{aligned}
(\mathrm{I}+x)^{n} & =F(-n, \beta, \beta,-x), \\
\log (\mathrm{I}+x) & =x F(\mathrm{I}, \mathrm{I}, 2,-x), \\
e^{x} & =\operatorname{Limit}_{\beta=\infty} F\left(\mathrm{I}, \beta, \mathrm{I}, \frac{x}{\beta}\right),
\end{aligned}
$$




$$
\begin{aligned}
(\mathrm{I}+x)^{n}+(\mathrm{I}-x)^{n} & =2 F\left(-\frac{n}{2},-\frac{n}{2}+\frac{\mathrm{I}}{2}, \frac{\mathrm{I}}{2}, x^{2}\right), \\
\log \frac{\mathrm{I}+x}{\mathrm{I}-x} & =2 x F\left(\frac{\mathrm{I}}{2}, \mathrm{I}, \frac{3}{2}, x^{2}\right), \\
\cos n x & =F\left(\frac{n}{2},-\frac{n}{2}, \frac{\mathrm{I}}{2}, \sin ^{2} x\right), \\
\sin n x & =n \sin x F\left(\frac{n+\mathrm{I}}{2}, \frac{\mathrm{I}-n}{2}, \frac{3}{2}, \sin ^{2} x\right), \\
\cosh x & =\alpha=\frac{\operatorname{Limit}}{\beta}=\infty\left(\alpha, \beta, \frac{\mathrm{I}}{2}, \frac{x^{2}}{4 \alpha \beta}\right), \\
\sin ^{-1} x & =x F\left(\frac{\mathrm{I}}{2}, \frac{\mathrm{I}}{2}, \frac{3}{2}, x^{2}\right), \\
\tan ^{-1} x & =x F\left(\frac{\mathrm{I}}{2}, \mathrm{I}, \frac{3}{2},-x^{2}\right), \\
P_{n}(x) & =F\left(-n, n+\mathrm{I}, \mathrm{I}, \frac{\mathrm{I}-x}{2}\right), \\
Q_{n}(x) & =\frac{\sqrt{\pi} \Gamma(n+\mathrm{I})}{2^{n+1} \Gamma\left(n+\frac{3}{2}\right)} \frac{\mathrm{I}}{x^{n+1}} F\left(\frac{n+\mathrm{I}}{2}, \frac{n+2}{2}, n+\frac{3}{2}, \frac{\mathrm{I}}{x^{2}}\right) .
\end{aligned}
$$

9.4 Heaviside's Operational Methods of Solving Partial Differential Equations.

9.41 The partial differential equation,

$$
a \frac{\partial^{2} u}{\partial x^{2}}=\frac{\partial u}{\partial t},
$$

where $a$ is a constant, may be solved by Heaviside's operational method.

Writing $\frac{\partial}{\partial t}=p$, and $\frac{p}{a}=q^{2}$, the equation becomes,

$$
\frac{\partial^{2} u}{\partial x^{2}}=q^{2} u
$$

whose complete solution is $u=e^{q x} A+e^{-q x} B$, where $A$ and $B$ are integration constants to be determined by the boundary conditions. In many applications the solution $u=e^{-q x} B$, only, is required: and the boundary conditions will lead to $u=e^{-q x} f(q) u_{0}$, where $u_{0}$ is a constant. If $e^{-q x} f(q)$ be expanded in an infinite power series in $q$, and the integral and fractional, positive and negative powers of $p$ be interpreted as in $\mathbf{9 . 4 2}$, the resulting series will be a solution of the differential equation, satisfying the boundary conditions, and reducing to $u=0$ at $t=0$. The expansion of $e^{-q x} f(q)$ may be carried out in two or more ways, leading to series suitable for numerical calculation under different conditions. 
9.42 Fractional Differentiation and Integration.

In the following expressions, I stands for a function of $t$ which is zero up to $t=0$, and equal to $\mathrm{I}$ for $t>0$.

\subsection{1}

$$
\begin{aligned}
& p^{\frac{1}{2}} \mathrm{I}=\frac{\mathrm{I}}{\sqrt{\pi t}} \\
& p^{\frac{3}{2}} \mathrm{I}=\frac{\mathrm{I}}{2 t \sqrt{\pi t}} \\
& p^{\frac{5}{2}} \mathrm{I}=\frac{3}{2^{2} t^{2} \sqrt{\pi t}}
\end{aligned}
$$$$
p^{\frac{2 n+1}{2}} \mathrm{I}=(-\mathrm{I})^{n} \frac{\mathrm{I} \cdot 3 \cdot 5 \cdots(2 n-\mathrm{I})}{2^{n} t^{n} \sqrt{\pi t}}
$$

\subsection{2}

$$
\begin{array}{ll}
p \mathrm{I}=0 & \\
p^{2} \mathrm{I}=0 & p^{n} \mathrm{I}=0 \\
p^{3} \mathrm{I}=0 &
\end{array}
$$

\subsection{3}

$$
\begin{aligned}
& p^{-\frac{1}{2}}=2 \sqrt{\frac{t}{\pi}} \\
& p^{-\frac{3}{2}}=\frac{2^{2} t}{3} \sqrt{\frac{t}{\pi}} \\
& p^{-\frac{5}{2}}=\frac{2^{3} t^{2}}{3 \cdot 5} \sqrt{\frac{t}{\pi}}
\end{aligned}
$$$$
p^{-\frac{2 n+1}{2}} \mathrm{I}=\frac{2^{2 n-1} t^{n}}{\mathrm{I} \cdot 3 \cdot 5 \cdots(2 n+\mathrm{I})} \sqrt{\frac{\bar{t}}{\pi}}
$$

\subsection{4}

$$
\frac{\mathrm{I}}{p^{\nu}}=\frac{t^{\nu}}{\Gamma(\mathrm{I}+\nu)}
$$

where $\nu$ may have any real value, except a negative integer. (Conjectural.)

\subsection{5}

$$
\begin{aligned}
& \frac{p}{p-a} I=e^{a t} \\
& \frac{I}{p-a} I=\frac{I}{a}\left(e^{a t}-I\right)
\end{aligned}
$$

9.426 With $p=a q^{2}$,

$$
\begin{aligned}
& q^{2 n+1} \mathrm{I}=(-\mathrm{I})^{n} \frac{\mathrm{I} \cdot 3 \cdot 5 \cdots(2 n-\mathrm{I})}{(2 a t)^{n} \sqrt{\pi a t}} \\
& q^{-2 n} \mathrm{I}=\frac{(a t)^{n}}{n !}
\end{aligned}
$$


9.427

$$
q e^{-q x} \mathrm{I}=\frac{\mathrm{I}}{\sqrt{\pi a t}} e^{-\frac{x^{2}}{4 a t}}
$$

9.428 If $z=\frac{x}{2 \sqrt{a t}}$

$$
\begin{aligned}
e^{-q x} & =\frac{2}{\sqrt{\pi}} \int_{z}^{\infty} e^{-v 2} d v \\
\frac{\mathrm{I}}{q} e^{-q x} & =\frac{x}{\sqrt{\pi}} \int_{z}^{\infty} e^{-v^{2}} \frac{d v}{v^{2}} .
\end{aligned}
$$

9.43 Many examples of the use of this method are given by Heaviside: Electromagnetic Theory, Vol. II. Bromwich, Proceedings Cambridge Philosophical Society, XX, p. 4II, I92I, has justified its application by the method of contour integration and applied it to the solution of a problem in the conduction of heat.

9.431 Herlitz, Arkiv for Matematik, Astronomi och Fysik, XIV, I9I9, has shown that the same methods may be applied to the more general partial differential equations of the type,

$$
\sum_{\alpha, \beta} A_{\alpha, \beta}(x) \frac{\partial^{\alpha+\beta}(u)}{\partial x^{\alpha} \partial t^{\beta}}=0,
$$

and the relations of $\mathbf{9 . 4 2}$ are valid.

9.44 Heaviside's Expansion Theorem.

The operational solution of the differential equation of $\mathbf{9 . 4 1}$, or the more general equation, 9.431, satisfying the given boundary conditions, may be written in the form,

$$
u=\frac{F(p)}{\Delta(p)} u_{0}
$$

where $F(p)$ and $\Delta(p)$ are known functions of $p=\frac{\partial}{\partial t}$. Then Heaviside's Expansion Theorem is:

$$
u=u_{0}\left\{\frac{F(0)}{\Delta(0)}+\sum \frac{F(\alpha)}{\alpha \Delta^{\prime}(\alpha)} e^{\alpha t}\right\}
$$

where $\alpha$ is any root, except $o$, of $\Delta(p)=0, \Delta^{\prime}(p)$ denotes the first derivative of $\Delta(p)$ with respect to $p$, and the summation is to be taken over all the roots of $\Delta(p)=0$. This solution reduces to $u=0$ at $t=0$.

Many applications of this expansion theorem are given by Heaviside, Electromagnetic Theory, II, and III; Electrical Papers, Vol. II. Herlitz, 9.431, has also applied this expansion theorem to the solution of the problem of the distribution of magnetic induction in cylinders and plates.

9.45 Bromwich's Expansion Theorem. Bromwich has extended Heaviside's Expansion Theorem as follows. If the operational solution of the partial differential equation of $\mathbf{9 . 4 1}$, obtained to satisfy the boundary conditions, is

$$
u=\frac{F(p)}{\Delta(p)}(G t)
$$


where $G$ is a constant, then the solution of the differential equation is

$$
u=G\left\{N_{0} t+N_{1}+\sum \frac{F(\alpha)}{\alpha^{2} \Delta^{\prime}(\alpha)} e^{\alpha t}\right\}
$$

where $N_{0}$ and $N_{1}$ are defined by the expansion,

$$
\frac{F(p)}{\Delta(p)}=N_{0}+N_{1} p+N_{2} p^{2}+\ldots
$$

$\alpha$ is any root of $\Delta(p)=0, \Delta^{\prime}(p)$ is the first derivative of $\Delta(p)$ with respect to $p$, and the summation is over all the roots, $\alpha$. This solution reduces to $u=0$ at $t=0$. Phil. Mag. 37, p. 407, I9I9; Proceedings London Mathematical Society, I5, p. 40r, I9I6.

9.9 References to Bessel Functions.

Nielsen: Handbuch der Theorie der Cylinder Funktionen.

Leipzig, 1904.

The notation and definitions given by Nielsen have been adopted in the present collection of formulae. The only difference is that Nielsen uses an upper index, $J^{n}(x)$, to denote the order, where the more usual custom of writing $J_{n}(x)$ is here employed. In place of $H_{1}{ }^{n}$ and $H_{2}{ }^{n}$ used by Nielsen for the cylinder functions of the third kind, $H_{n}{ }^{\mathrm{I}}$ and $H_{n}{ }^{\mathrm{II}}$ are employed in this collection.

Gray and Mathews: Treatise on Bessel Functions.

London, I895. ${ }^{1}$

The Bessel Function of the second kind, $Y_{n}(x)$, employed by Gray and Mathews is the function

$$
\frac{\pi}{2} Y_{n}(x)+(\log 2-\gamma) J_{n}(x)
$$

of Nielsen.

Schafheitlin: Die Theorie der Besselschen Funktionen.

Leipzig, 1908.

Schafheitlin defines the function of the second kind, $Y_{n}(x)$, in the same way as Nielsen, except that its sign is changed.

Note. A Treatise on the Theory of Bessel Functions, by G. N. Watson, Cambridge University Press, 1922, has been brought out while this volume is in press. This Treatise gives by far the most complete account of the theory and properties of Bessel Functions that exists, and should become the standard work on the subject with respect to notation. A particularly valuable feature is the Collection of Tables of Bessel Functions at the end of the volume and the Bibliography, giving references to all the important works on the subject.

9.91 Tables of Legendre, Bessel and allied functions.

$P_{n}(x) \quad$ (9.001).

1 A second edition of Gray and Mathews' Treatise, prepared by A. Gray and T. M. MacRobert, has been published $(1922)$ while this volume is in press. The notation of the first
edition has been altered in some respects. 
B. A. Report, 1879 , pp. 54-57. Integral values of $n$ from I to 7 ; from $x=0.0 \mathrm{I}$ to $x=\mathrm{I} .00$, interval $0.0 \mathrm{I}$, $\mathrm{I} 6$ decimal places.

Jahnke and Emde: Funktionentafeln, p. 83; same to 4 decimal places.

$P_{n}(\cos \theta)$

Phil. Trans. Roy. Soc. London, 203, p. I00, I904. Integral values of $n$ from I to 20 , from $\theta=0$ to $\theta=90$, interval 5,7 decimal places.

Phil. Mag. 32, p. 5 1 2, I89I. Integral values of $n$ from I to $7, \theta=0$ to $\theta=90$, interval I; 4 decimal places. Reproduced in Jahnke and Emde, p. 85 .

Tallquist, Acta Soc. Sc. Fennicae, Helsingfors, 33, pp. I-8. Integral values of $n$ from I to $8 ; \theta=0$ to $\theta=90$, interval I, Io decimal places.

Airey, Proc. Roy. Soc. London, 96, p. I, I9r9. Tables by means of which zonal harmonics of high order may be calculated.

Lodge, Phil. Trans. Roy. Soc. London, 203, I904, p. 87. Integral values of $n$ from I to $20 ; \theta=0$ to $\theta=90$, interval 5,7 decimal places. Reprinted in Rayleigh, Collected Works, Volume V, p. I62.

$\frac{\partial P_{n}(\cos \theta)}{\partial \theta}$.

Farr, Proc. Roy. Soc. London, 64, 199, I899. Integral values of $n$ from I to 7 ; $\theta=\circ$ to $\theta=90$, interval I, 4 decimal places. Reproduced in Jahnke and Emde, p. 88 .

$J_{0}(x), J_{1}(x)$ (9.101).

Meissel's tables, $x=0.01$ to $x=\mathbf{I} 5.50$, interval 0.01 , to 12 decimal places, are given in Table I of Gray and Mathews' Treatise on Bessel's Functions.

Aldis, Proc. Roy. Soc. London 66, 40, I900. $x=$ 0.I to $x=6.0$, interval O.I, 2 I decimal places.

Jahnke and Emde, Funktionentafeln, Table III. $x=0.0 \mathrm{I}$ to $x=\mathrm{I} 5.50$, interval $0.0 \mathrm{I}, 4$ decimal places.

$J_{n}(x)$ (9.101).

Gray and Mathews, Table II. Integral values of $n$ from $n=0$ to $n=60$; integral values of $x$ from $x=\mathrm{I}$ to $x=24$, I 8 decimal places.

Jahnke and Emde, Table XXIII, same, to 4 significant figures.

B. A. Report, I9I 5, p. $29 ; n=0$ to $n=\mathrm{I} 3$.

$$
\begin{array}{ll}
x=0.2 \text { to } x=6.0 & \text { interval } 0.2 \quad 6 \text { decimal places, } \\
x=6.0 \text { to } x=\mathrm{r} 6.0 & \text { interval } 0.5 \quad \text { Io decimal places. }
\end{array}
$$

Hague, Proc. London Physical Soc. 29, 21 I, I916-I 7, gives graphs of $J_{n}(x)$ for integral values of $n$ from o to I 2 , and $n=18, x$ ranging from $\circ$ to I 7 .

$-\frac{\pi}{2} Y_{0}(x)=G_{0}(x) ; \quad-\frac{\pi}{2} Y_{1}(x)=G_{1}(x)$.

B. A. Report, I9I3, pp. I16-130. $x=0.01$ to $x=16.0$, interval $0.01,7$ decimal places. 
B. A. Report, I9I5, $x=6.5$ to $x=15.5$, interval 0.5 , io decimal places.

Aldis, Proc. Roy. Soc. London, 66, 40, I900: $x=0.1$ to $x=6.0$. Interval O.I, 2 I decimal places.

Jahnke and Emde, Tables VII and VIII, functions denoted $\mathrm{K}_{0}(x)$ and $\mathrm{K}_{1}(x)$, $x=0 . \mathrm{I}$ to $x=6.0$, interval o.I; $x=0.0 \mathrm{I}$ to $x=0.99$, interval $0.0 \mathrm{I} ; x=\mathrm{r}$.० to $x=\mathrm{I0.3}$, interval $0 . \mathrm{I} ; 4$ decimal places.

$-\frac{\pi}{2} Y_{n}(x)=G_{n}(x)$

B. A. Report, I914, p. 83. Integral values of $n$ from $\circ$ to I3. $x=0.01$ to $x=6.0$, interval $\mathrm{O} . \mathrm{I} ; x=6.0$ to $x=\mathrm{I} 6.0$, interval $0.5 ; 5$ decimal places.

$$
\begin{aligned}
& \frac{\pi}{2} Y_{0}(x)+(\log 2-\gamma) J_{0}(x) \\
& \frac{\pi}{2} Y_{1}(x)+(\log 2-\gamma) J_{1}(x)
\end{aligned}
$$

Denoted $Y_{0}(x)$ and $Y_{1}(x)$ respectively in the tables.

B. A. Report, I9I4, p. $76, x=0.02$ to $x=15.50$, interval $0.02,6$ decimal places.

B. A. Report, I9I5, p. $33, x=$ o.I to $x=6 . \dot{0}$, interval o.I; $x=6.0$ to $x=\mathrm{I} 5 \cdot 5$, interval 0.5 , 10 decimal places.

Jahnke and Emde, Table VI, $x=0.0 \mathrm{I}$ to $x=\mathrm{I} .00$, interval $0.0 \mathrm{I} ; x=\mathrm{I} .0$ to $x=$ Io.2, interval o.I, 4 decimal places.

$Y_{0}(x), Y_{1}(x)$.

Denoted $N_{0}(x)$ and $N_{1}(x)$ respectively.

Jahnke and Emde, Table IX, $x=$ o.I to $x=$ I0.2, interval o.I, 4 decimal places.

$\frac{\pi}{2} Y_{n}(x)+(\log 2-\gamma) J_{n}(x)$

Denoted $Y_{n}(x)$ in tables.

B. A. Report, I9I5. Integral values of $n$ from I to I3. $x=0.2$ to $x=6.0$, interval $0.2 ; x=6.0$ to $x=15.5$, interval $0.5,6$ decimal places.

$J_{n+\frac{1}{2}}(x)$.

Jahnke and Emde, Table II. Integral values of $n$ from $n=0$ to $n=6$, and $n=-\mathrm{I}$ to $n=-7 ; x=\circ$ to $x=5 \circ$, interval $\mathrm{I} .0,4$ figures.

$J_{\frac{1}{3}}(x), J_{-\frac{1}{3}}(x)$.

Watson, Proc. Roy. Soc. London, 94, 204, 1918.

$$
\begin{aligned}
& x=0.05 \text { to } x=2.00 \text { interval } 0.05, \\
& x=2.0 \text { to } x=8.0 \text { interval } 0.2,
\end{aligned}
$$

4 decimal places.

$J_{\alpha}(\alpha), J_{\alpha-1}(\alpha)$

$-\frac{\pi}{2} Y_{\alpha}(\alpha),-\frac{\pi}{2} Y_{\alpha-1}(\alpha)$

Denoted $G_{\alpha}(\alpha)$ and $G_{\alpha-1}(\alpha)$ respectively. 


$$
\begin{aligned}
& \frac{\pi}{2} Y_{\alpha}(\alpha)+(\log 2-\gamma) J_{\alpha}(\alpha) \\
& \frac{\pi}{2} Y_{\alpha-1}(\alpha)+(\log 2-\gamma) J_{\alpha-1}(\alpha)
\end{aligned}
$$$$
\text { Denoted }-Y_{\alpha}(\alpha) \text { and }-Y_{\alpha-1}(\alpha)
$$

Tables of these six functions are given in the B. A. Report, I916, as follows:

$\begin{array}{crr}\text { From } \alpha & \text { to } \alpha & \text { Interval } \\ \text { I } & 50 & \text { I } \\ 50 & 100 & 5 \\ 100 & 200 & \text { I0 } \\ 200 & 400 & 20 \\ 400 & 1000 & 50 \\ 1000 & 2000 & 100 \\ 2000 & 5000 & 500 \\ 5000 & 20000 & 1000 \\ 20000 & 30000 & 10000 \\ 100,000 & & \\ 500,000 & & \\ I, 000,000 & & \end{array}$

$$
I_{0}(x), I_{1}(x) \text { (9.211). }
$$

Aldis, Proc. Roy. Soc. London, 64, pp. 218-223, I899; $x=0.1$ to $x=6.0$, interval ०.I; $x=6.0$ to $x=$ II.०, interval I.०, 2 I decimal places.

Jahnke and Emde, Tables XI and XII, 4 places:

$$
\begin{aligned}
& x=0.0 \mathrm{I} \text { to } x=5 . \mathrm{I0} \text { interval } 0.0 \mathrm{I} \text {, } \\
& x=5.10 \text { to } x=6.0 \quad \text { interval o.I, } \\
& x=6.0 \text { to } x=\text { I } 1.0 \quad \text { interval } \mathbf{\text { r.o. }}
\end{aligned}
$$

\section{$\mathrm{I}_{0}(x) \quad(9.211)$.}

B. A. Report, $\mathrm{I} 896 ; x=0.00 \mathrm{I}$ to $x=5.100$, interval $0.00 \mathrm{I}, 9$ decimal places.

\section{$\mathrm{I}_{1}(x)$ (9.211).}

B. A. Report, $\mathrm{I} 893 ; x=0.00 \mathrm{I}$ to $x=5.100$, interval $0.00 \mathrm{I}, 9$ decimal places.

Gray and Mathews, Table V, $x=0.0 \mathrm{I}$ to $x=5 . \mathrm{I0}$, interval $0.0 \mathrm{I}, 9$ decimal places.

\section{$\mathrm{I}_{n}(x) \quad(9.211)$.}

B. A. Report, I889, pp. $28-32$; integral values of $n$ from $\circ$ to II,$x=0.2$ to $x=6.0$, interval $\circ>2$, I 2 decimal places. These tables are reproduced in Gray and Mathews, Table VI.

Jahnke and Emde, Table XXIV; same ranges, to 4 places.

$$
\begin{array}{ll}
J_{0}(x \sqrt{i}) & =X-i Y, \\
\sqrt{2} J_{1}(x \sqrt{i}) & =X_{1}+i Y_{1}
\end{array}
$$






Aldis, Proc. Roy. Soc. London, 66, I42, I900; $x=$ 0.I to $x=6.0$, interval O.I, 2 I decimal places.

Jahnke and Emde, Tables XV and XVI, same range, to 4 places. $J_{0}(x \sqrt{i})$.

Gray and Mathews, Table IV; $x=0.2$ to $x=6.0$, interval $0.2,9$ decimal places.

$Y_{0}(x \sqrt{i}) \quad(9.104)$

Denoted $N_{0}(x \sqrt{i})$ in table.

$H_{0}^{\mathrm{I}}(x \sqrt{i}), H_{1}^{\mathrm{I}}(x \sqrt{i})$.

Jahnke and Emde, Tables XVII and XVIII; $x=0.2$ to $x=6.0$, interval $0.2,4^{-7}$ figures.

$$
\begin{aligned}
\frac{i \pi}{2} H_{0}^{\mathrm{I}}(i x) & =K_{0}(x) \\
-\frac{\pi}{2} H_{0}^{\mathrm{I}}(i x) & =K_{1}(x)
\end{aligned}
$$

Aldis, Proc. Roy. Soc. London, 64, 219-223, I899; $x=0$ o.I to $x=12.0$, interval O.I, 2I decimal places.

Jahnke and Emde, Table XIV; same, to 4 places.

$i H_{0}^{\mathrm{I}}(i x),-H_{0}^{\mathrm{I}}(i x) \quad(9.107)$.

Jahnke and Emde, Table XIII; $x=0 . \mathrm{I} 2$ to $x=6.0$, interval $0.2,4$ figures. ber $x$, $\operatorname{ber}^{\prime} x$, bei $x$, bei' $x$,

B. A. Report, I9I $2 ; x=0.1$ to $x=$ I0.०, interval O.I, 9 decimal places.

Jahnke and Emde, Table XX; $x=0.5$ to $x=6.0$, interval 0.5 , and $x=8$, Io, $15,20,4$ decimal places.

$\operatorname{ker} x, \operatorname{ker}^{\prime} x$, kei $x, \operatorname{kei}^{\prime} x$,

B. A. Report, I9I5; $x=0.1$ to $x=$ I0.0, interval 0.I, 7-10 decimal places. $\operatorname{ber}^{2} x+\operatorname{bei}^{2} x$ $\operatorname{ber}^{\prime 2} x+$ bei $^{\prime 2} x$,

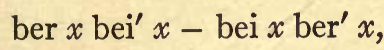
ber $x \operatorname{ber}^{\prime} x+$ bei $x$ bei $^{\prime} x$, and the corresponding ker and kei functions.

B. A. Report, I9I6; $x=0.2$ to $x=$ I0.0, interval 0.2 , decimal places. $S_{n}(x), S_{n}^{\prime}(x), \log S_{n}(x), \log S_{n}^{\prime}(x)$, $C_{n}(x), C^{\prime}{ }_{n}(x), \log C_{n}(x), \log C_{n}^{\prime}(x), \quad$ (9.261). $E_{n}(x), E_{n}^{\prime}(x), \log E_{n}(x), \log E_{n}^{\prime}(x)$,

B. A. Report, I916; integral values of $n$ from $\circ$ to Io, $x=$ I.I to $x=$ I.9, interval o.I, 7 decimal places. 


$$
\begin{aligned}
& G(x)=-\sqrt{2} \Pi\left(\frac{\mathrm{I}}{4}\right) x^{-\frac{1}{2}} J_{\frac{1}{4}}\left(\frac{x}{2}\right)=-\frac{\mathrm{I}}{0.780 \mathrm{I} 2} x^{-\frac{1}{2}} J_{\frac{1}{4}}\left(\frac{x}{2}\right) \\
& D(x)=\frac{\mathrm{I}}{\sqrt{2}} \Pi\left(-\frac{\mathrm{I}}{4}\right) x^{\frac{1}{2}} J_{-\frac{1}{2}}\left(\frac{x}{2}\right)=\frac{\mathrm{I}}{\mathrm{I} . \mathrm{I} 5407} x^{\frac{1}{2}} J_{-\frac{1}{2}}\left(\frac{x}{2}\right)
\end{aligned}
$$

Table I of Jahnke and Emde gives these two functions to 3 decimal places for $x=0.2$ to $x=8.0$, interval 0.2 , and $x=8.0$ to $x=\mathrm{I} 2.0$, interval I.o.

Roots of $J_{0}(x)=$ o.

Airey, Phil. Mag. 36, p. 24I, I9r8: First 40 roots $(\rho)$ with corresponding values of $J_{1}(\rho), 7$ decimal places.

Jahnke and Emde, Table IV, same, to 4 decimal places.

Roots of $J_{1}(x)=0$.

Gray and Mathews, Table III, first 50 roots, with corresponding values of $J_{0}(x)$, I6 decimal places.

Airey, Phil. Mag. 36, p. 24I: First 40 roots $(r)$ with corresponding values of $J_{0}(r), 7$ decimal places.

Jahnke and Emde, Table IV, same, to 4 decimal places.

Roots of $J_{n}(x)=0$.

B. A. Report, I9I7, first Io roots, to 6 figures, for the following integral values of $n$ : 0-10, I5, 20, 30, 40, 50, 75, 100, 200, 300, 400, 500, 750, 1000 .

Jahnke and Emde, Table XXII, first 9 roots, 3 decimal places, integral values of $n$ 0-9.

Roots of:

$(\log 2-\gamma) J_{n}(x)+\frac{\pi}{2} Y_{n}(x)=0$.

Denoted $Y_{n}(x)=0$ in table.

Airey: Proc. London Phys. Soc. 23, p. 219, I910-11. First 40 roots for $n=0,1,2,5$ decimal places.

Jahnke and Emde, Table X, first 4 roots for $n=0$, I. $E$ decimal places.

Roots of:

$Y_{0}(x)=0$,

$Y_{1}(x)=0$.

Denoted $N_{0}(x)$ and $N_{1}(x)$ in tables.

Airey: 1. c. First to roots, 5 decimal places.

Roots of:

$$
\begin{array}{crr}
J_{0}(x) \pm(\log 2-\gamma) J_{0}(x)+\frac{\pi}{2} Y_{0}(x)=\circ . & \text { Denoted } & J_{0}(x) \pm Y_{0}(x)=\circ \\
J_{1}(x)+(\log 2-\gamma) J_{1}(x)+\frac{\pi}{2} \cdot Y_{1}(x)=\circ . & \text { Denoted } & J_{1}(x)+Y_{1}(x)=\circ \\
J_{0}(x)-2(\log 2-\gamma) J_{0}(x)+\frac{\pi}{2} Y_{0}(x)=\circ . & \text { Denoted } & J_{0}(x)-2 V_{0}(x)=\circ \\
\operatorname{IO}_{0}(x) \pm(\log 2-\gamma) J_{0}(x)+\frac{\pi}{2} Y_{0}(x)=0 . & \text { Denoted } & \operatorname{Iо} J_{0}(x) \pm Y_{0}(x)=0
\end{array}
$$


Airey, 1. c. First to roots, 5 decimal places. Roots of

$$
\frac{J_{n+1}(x)}{J_{n}(x)}+\frac{I_{n+1}(x)}{I_{n}(x)}=0 .
$$

Airey, 1. c. First Io roots: $n=0,4$ decimal places, $n=1,2,3,3$ decimal places.

Jahnke and Emde, Table XXV, first 5 roots for $n=0,3$ for $n=\mathrm{I}, 2$ for $n=2: 4$ figures.

Airey, 1. c. gives roots of some other equations involving Bessel's functions connected with the vibration of circular plates.

Roots of:

$$
J_{\nu}(x) Y_{\nu}(x)=J_{\nu}(k x) Y_{\nu}(k x) .
$$

Jahnke and Emde, Table XXVI, first 6 roots, 4 decimal places, for $\nu=0, \mathrm{I} / 2, \mathrm{I}, 3 / 2,2,5 / 2: k=\mathrm{I} .2, \mathrm{I} .5,2.0$.

Table XXVIII, first ront, multiplied by $(k-\mathrm{I})$ for $k=\mathrm{I}, \mathrm{I} .2, \mathrm{I} .5,2-\mathrm{II}$, I9, 39, $\infty: \nu$ same as above.

Table XXIX, first 4 roots, multiplied by $(k-\mathrm{I})$ for certain irrational values of $k$, and $\nu=0, \mathrm{I}$. 


\title{
X. NUMERICAL SOLUTION OF DIFFERENTIAL EQUATIONS
}

\author{
By F. R. Moulton, Ph.D., \\ Professor of Astronomy, University of Chicago; \\ Research Associate of the Carnegie Institution of Washington.
}

\section{INTRODUCTION}

Differential equations are usually first encountered in the final chapter of a book on integral calculus. The methods which are there given for solving them are essentially the same as those employed in the calculus. Similar methods are used in the first special work on the subject. That is, numerous types of differential equations are given in which the variables can be separated by suitable devices; little or nothing is said about the existence of solutions of other types, or about methods of finding the solutions. The false impression is often left that only exceptionally can differential equations be solved. Whatever satisfaction there may be in learning that some problems in geometry and physics lead to standard forms of differential equations is more than counterbalanced by the discovery that most practical problems do not lead to such forms.

10.01 The point of view adopted here and the methods which are developed can be best understood by considering first some simpler and better known mathematical theories. Suppose

I.

$$
F(x)=x^{n}+a_{1} x^{n-1}+\ldots \ldots+a_{n-1} x+a_{n}=0
$$

is a polynomial equation in $x$ having real coefficients $a_{1}, a_{2}, \ldots, a_{n}$. If $n$ is I, 2, 3, or 4 the values of $x$ which satisfy the equation can be expressed as explicit functions of the coefficients. If $n$ is greater than 4 , formulas for the solution can not in general be written down. Nevertheless, it is possible to prove that $n$ solutions exist and that at least one of them is real if $n$ is odd. If the coefficients are given numbers, there are straightforward, though somewhat laborious, methods of finding the solutions. That is, even though general formulas for the solutions are not known, yet it is possible both to prove the existence of the solutions and also to find them in any special numerical case.

10.02 Consider as another illustration the definite integral

I.

$$
I=\int_{a}^{b} f(x) d x
$$

where $f(x)$ is continuous for $a \leqslant x \leqslant b$. If $F(x)$ is such a function that

$$
\frac{d F}{d x}=f(x)
$$


then $I=F(b)-F(a)$. But suppose no $F(x)$ can be found satisfying (2). It is nevertheless possible to prove that the integral $I$ exists, and if the value of $(x)$ is given for every value of $x$ in the interval $a \leqslant x \leqslant b$, it is possible to find the numerical value of $I$ with any desired degree of approximation. That is, it is not necessary that the primitive of the integrand of a definite integral be known in order to prove the existence of the integral, or even to find its value in any particular example.

10.03 The facts are analogous in the case of differential equations. Those having numerical coefficients and prescribed initial conditions can be solved regardless of whether or not their variables can be separated. They need to satisfy only mild conditions which are always fulfilled in physical problems. It is with a sense of relief that one finds he can solve, numerically, any particular problem which can be expressed in terms of differential equations.

10.04 This chapter will contain an account of a method of solving ordinary differential equations which is applicable to a broad class including all those which arise in physical problems. A large amount of experience has shown that the method is very convenient in practice. It must be understood that there is for it an underlying logical basis, involving refinements of modern analysis, which fully justifies the procedure. In other words, it can be proved that the process is capable of furnishing the solution with any desired degree of accuracy. The proofs of these facts belong to the domain of pure analysis and will not be given here.

10.10 Simpson's Method of Computing Definite Integrals. The method of solving differential equations which will be given later involves the computation of definite integrals by a special process which will be developed in this and the following sections.

Let $t$ be the variable of integration, and consider the definite integral

$$
\text { I. } \quad F=\int_{a}^{b} f(t) d t \text {. }
$$

This integral can be interpreted as the area between the $t$-axis and the curve $y=f(t)$ and bounded by the ordinates $t=a$ and $t=b$, figure $\mathrm{I}$.

Let $t_{0}=a, t_{n}=b, y_{i}=f\left(t_{i}\right)$, and divide the interval $a \leqslant t \leqslant b$ up into $n$ equal parts, each of length $h=$

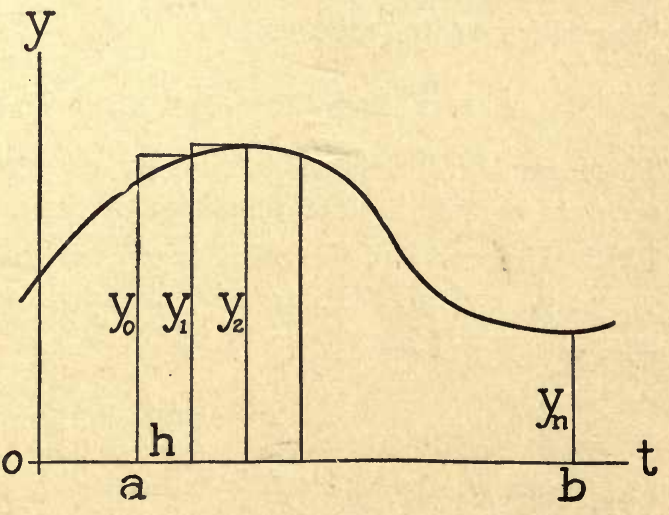

FIG. I $(b-a) / n$. Then an approximate value of $F$ is 2

$$
F_{0}=h\left(y_{1}+y_{2}+\ldots+y_{n}\right) \text {. }
$$

This is the sum of rectangles whose ordinates, figure $\mathrm{I}$, are $y_{1}, y_{2}, \ldots, y_{n}$. 10.11 A more nearly exact value can be obtained for the first two intervals, for example, by putting a curve of the second degree through the three points 
$y_{0}, y_{1}, y_{2}$, and finding the area between the $t$-axis and this curve and bounded by the ordinates $t_{0}$ and $t_{2}$. The equation of the curve is

I.

$$
y=a_{0}+a_{1}\left(t-t_{0}\right)+a_{2}\left(t-t_{0}\right)^{2},
$$

where the coefficients $a_{0}, a_{1}$, and $a_{2}$ are determined by the conditions that $y$ shall equal $y_{0}, y_{1}$, and $y_{2}$ at $t$ equal to $t_{0}, t_{1}$ and $t_{2}$ respectively; or

2.

$$
\left\{\begin{array}{l}
y_{0}=a_{0}, \\
y_{1}=a_{0}+a_{1}\left(t_{1}-t_{0}\right)+a_{2}\left(t_{1}-t_{0}\right)^{2}, \\
y_{2}=a_{0}+a_{1}\left(t_{2}-t_{0}\right)+a_{2}\left(t_{2}-t_{0}\right)^{2} .
\end{array}\right.
$$

It follows from these equations and $t_{2}-t_{1}=t_{1}-t_{0}=h$ that

3.

$$
\left\{\begin{array}{l}
a_{0}=y_{0}, \\
a_{1}=-\frac{\mathrm{I}}{2 h}\left(3 y_{0}-4 y_{1}+y_{2}\right), \\
a_{2}=\frac{\mathrm{I}}{2 h^{2}}\left(y_{0}-2 y_{1}+y_{2}\right) .
\end{array}\right.
$$

The definite integral $\int_{t_{0}}^{t_{2}} y d t$ is approximately

$$
I=\int_{t_{0}}^{t_{2}}\left[a_{0}+a_{1}\left(t-t_{0}\right)+a_{2}\left(t-t_{0}\right)^{2}\right] d t=2 h\left[a_{0}+a_{1} h+\frac{4}{3} a_{2} h^{2}\right],
$$

which becomes as a consequence of (3)

4.

$$
I=\frac{h}{3}\left(y_{0}+4 y_{1}+y_{2}\right) \text {. }
$$

10.12 The value of the integral over the next two intervals, or from $t_{2}$ to $t_{4}$, can be computed in the same way. If $n$ is even, the approximate value of the integral from $t_{0}$ to $t_{n}$ is therefore

$$
F_{1}=\frac{h}{3}\left[y_{0}+4 y_{1}+2 y_{2}+4 y_{3}+2 y_{4}+\ldots \ldots+4 y_{n-1}+y_{n}\right] .
$$

This formula, which is due to Simpson, gives results which are usually remarkably accurate considering the simplicity of the arithmetical operations.

10.13 If a curve of the third degree had been passed through the four points $y_{0}, y_{1}, y_{2}$, and $y_{3}$, the integral corresponding to (4), but over the first three intervals, would have been found to be

$$
I=\frac{3 h}{8}\left[y_{0}+3 y_{1}+3 y_{2}+y_{3}\right]
$$

10.20 Digression on Difference Functions. For later work it will be necessary to have some properties of the successive differences of the values of a function for equally spaced values of its argument.

As before, let $y_{i}$ be the value of $f(t)$ for $t=t_{i}$. Then let 


$$
\begin{aligned}
& \Delta_{1} y_{1}=y_{1}-y_{0} \\
& \Delta_{1} y_{2}=y_{2}-y_{1}, \\
& \because \cdots \cdots \\
& \Delta_{1} y_{n}=y_{n}-y_{n-1},
\end{aligned}
$$

These are the first differences of the values of the function $y$ for successive values of $t$. All the successive intervals for $t$ are supposed to be equal.

10.21 In a similar way the second differences are defined by

$$
\begin{aligned}
\Delta_{2} y_{2} & =\Delta_{1} y_{2}-\Delta_{1} y_{1}, \\
\Delta_{2} y_{3} & =\Delta_{1} y_{3}-\Delta_{1} y_{2}, \\
\ddot{\Delta_{2} y_{n}} & =\ddot{\Delta_{1} y_{n}}-\dot{\Delta}_{1} y_{n-1},
\end{aligned}
$$

10.22 In a similar way third differences are defined by

$$
\begin{aligned}
& \Delta_{3} y_{3}=\Delta_{2} y_{3}-\Delta_{2} y_{2}, \\
& \Delta_{3} y_{4}=\Delta_{2} y_{4}-\Delta_{2} y_{3}, \\
& \because \cdots \cdots \\
& \Delta_{3} y_{n}=\Delta_{2} y_{n}-\Delta_{2} y_{n-1},
\end{aligned}
$$

and obviously the process can be repeated as many times as may be desired. 10.23 The table of successive differences can be formed conveniently from the tabular values of the function and can be arranged in a table as follows:

TABLE I

\begin{tabular}{|c|c|c|c|}
\hline$y$ & $\Delta_{1} y$ & $\Delta_{2} y$ & $\Delta_{3} y$ \\
\cline { 3 - 4 }$y_{0}$ & & & \\
$y_{1}$ & $\Delta_{1} y_{1}$ & & \\
$y_{2}$ & $\Delta_{1} y_{2}$ & $\Delta_{2} y_{2}$ & \\
$y_{3}$ & $\Delta_{1} y_{3}$ & $\Delta_{2} y_{3}$ & $\Delta_{3} y_{3}$ \\
$\ldots \ldots \ldots \ldots$ & $\ldots \ldots \ldots \ldots \ldots \ldots \ldots$ & $\ldots \ldots \ldots \ldots \ldots$ \\
\hline
\end{tabular}

In this table the numbers in each column are subtracted from those immediately below them and the remainders are placed in the next column to the right on the same line as the minuends. Variations from this precise arrangement could be, and indeed often have been, adopted.

10.24 A very important advantage of a table of differences is that it is almost sure to reveal any errors that may have been committed in computing the $y_{i}$. If a single $y_{i}$ has an error $\epsilon$, it follows from 10.20 that the first difference $\Delta_{1} y_{i}$ will contain the error $+\epsilon$ and $\Delta_{1} y_{i+1}$ will contain the error $-\epsilon$. But the second differences $\Delta_{2} y_{i}, \Delta_{2} y_{i+1}$, and $\Delta_{2} y_{i+2}$ will contain the respective errors $+\epsilon,-2 \epsilon$, $+\epsilon$. Similarly, the third differences $\Delta_{3} y_{i}, \Delta_{3} y_{i+1}, \Delta_{3} y_{i+2}$, and $\Delta_{3} y_{i+3}$ will contain the respective errors $+\epsilon,-3 \epsilon,+3 \epsilon,-\epsilon$. An error in a single $y_{i}$ affects $j+I$ differences of order $j$, and the coefficients of the error are the binomial coefficients with alternating signs. The algebraic sums of the errors in the affected 
numbers in the various difference columns are zero. Now in such functions as ordinarily occur in practice the numerical values of the differences, if the intervals are not too great, decrease with rapidity and run smoothly. If an error is present, however, the differences of higher order become very irregular. 10.25 As an illustration, consider the function $y=\sin t$ for $t$ equal to $10^{\circ}$, I $5^{\circ}, \ldots .$. The following table gives the function and its successive differences, expressed in terms of units of the fourth decimal: ${ }^{1}$

TABLE II

\begin{tabular}{|c|c|c|c|c|}
\hline$t$ & $\sin t$ & $\Delta_{1} \sin t$ & $\Delta_{2} \sin t$ & $\Delta_{3} \sin t$ \\
\cline { 1 - 3 } I0 $^{\circ}$ & I 736 & & & \\
I5 & 2588 & 852 & & \\
20 & 3420 & 832 & -20 & \\
25 & 4226 & 806 & -26 & -6 \\
30 & 5000 & 774 & -32 & -6 \\
35 & 5736 & 736 & -38 & -6 \\
40 & 6428 & 692 & -44 & -6 \\
45 & 7071 & 643 & -49 & -5 \\
50 & 7660 & 589 & -54 & -4 \\
55 & 8191 & 531 & -58 & -4 \\
60 & 8660 & 469 & -62 & -4 \\
65 & 9063 & 403 & -66 & -3 \\
70 & 9397 & 334 & -69 & \\
\hline
\end{tabular}

Suppose, however, that an error of two units had been made in determining the sine of $45^{\circ}$ and that 7073 had been taken in place of $707 \mathrm{I}$. Then the part of the table adjacent to this number would have been the following:

TABLE III

\begin{tabular}{|c|c|c|c|c|}
\hline$t$ & $\sin t$ & $\Delta_{1} \sin$ & $\Delta_{2} \sin t$ & $\Delta_{3} \sin t$ \\
\cline { 2 - 4 } $25^{\circ}$ & 4226 & & & \\
30 & 5000 & 774 & & \\
35 & 5736 & 736 & -38 & -6 \\
40 & 6428 & 692 & -44 & -3 \\
45 & 7073 & 645 & -47 & - I I \\
50 & 7660 & 587 & -58 & -2 \\
55 & 8191 & 531 & -62 & -4 \\
60 & 8660 & 469 & -66 & -6 \\
65 & 9063 & 403 & & \\
\hline
\end{tabular}

The irregularity in the numbers of the last column shows the existence of an error, and, in fact, indicates its location. In the third differences four numbers

${ }^{1}$ Often it is not necessary to carry along the decimal and zeros to the left of the first significant figure. 
will be affected by an error in the value of the function. The erroneous numbers in the last column are clearly the second, third, fourth, and fifth. The algebraic sum of these four numbers equals the sum of the four correct numbers, or - I8. Their average is -4.5 . Hence the central numbers are probably -5 and -4 . Since the errors in these numbers are $-3 \epsilon$ and $+3 \epsilon$, it follows that $\epsilon$ is probably +2 . The errors in the second and fifth numbers are $+\epsilon$ and $-\epsilon$ respectively. On making these corrections and working back to the first column, it is found that 7073 should be replaced by $707 \mathrm{I}$.

10.30 Computation of Definite Integrals by Use of Difference Functions.

Suppose the values of $f(t)$ are known for $t=t_{n-2}, t_{n-1}, t_{n}$, and $t_{n+1}$. Suppose it is desired to find the integral

I.

$$
I_{n}=\int_{t_{n}}^{t_{n+1}} f(t) d t
$$

The coefficients $b_{0}, b_{1}, b_{2}$, and $b_{3}$ of the polynomial can be determined, as above, so that the function

2.

$$
y=b_{0}+b_{1}\left(t-t_{n}\right)+b_{2}\left(t-t_{n}\right)^{2}+b_{3}\left(t-t_{n}\right)^{3}
$$

shall take the same values as $f(t)$ for $t=t_{n-2}, t_{n-1}, t_{n}$, and $t_{n+1}$.

With this approximation to the function $f(t)$, the integral becomes (since $\left.t_{n+1}-t_{n}=h\right)$

3 .

$$
\begin{aligned}
I_{n} & =\int_{t_{n}}^{t_{n+1}}\left[b_{0}+b_{1}\left(t-t_{n}\right)+b_{2}\left(t-t_{n}\right)^{2}+b_{3}\left(t-t_{n}\right)^{3}\right] d t \\
& =h\left[b_{0}+\frac{\mathrm{I}}{2} b_{1} h+\frac{\mathrm{I}}{3} b_{2} h^{2}+\frac{\mathrm{I}}{4} b_{3} h^{3}\right] .
\end{aligned}
$$

The coefficients $b_{0}, b_{1}, b_{2}$, and $b_{3}$ will now be expressed in terms of $y_{n+1}, \Delta_{1} y_{n+1}$, $\Delta_{2} y_{n+1}$, and $\Delta_{3} y_{n+1}$. It follows from (2) that

4 .

$$
\left\{\begin{array}{l}
y_{n-2}=b_{0}-2 b_{1} h+4 b_{2} h^{2}-8 b_{3} h^{3} \\
y_{n-1}=b_{0}-b_{1} h+b_{2} h^{2}-b_{3} h^{3} \\
y_{n}=b_{0} \\
y_{n+1}=b_{0}+b_{1} h+b_{2} h^{2}+b_{3} h^{3}
\end{array}\right.
$$

Then it follows from the rules for determining the difference functions that

5 .

$$
\left\{\begin{array}{l}
\Delta_{1} y_{n-1}=b_{1} h-3 b_{2} h^{2}+7 b_{3} h^{3} \\
\Delta_{1} y_{n}=b_{1} h-b_{2} h^{2}+b_{3} h^{3} \\
\Delta_{1} y_{n+1}=b_{1} h+b_{2} h^{2}+b_{3} h^{3}
\end{array}\right.
$$

6.

$$
\left\{\begin{array}{l}
\Delta_{2} y_{n}=2 b_{2} h^{2}-6 b_{3} h^{3} \\
\Delta_{2} y_{n+1}=2 b_{2} h^{2}
\end{array}\right.
$$


It follows from the last equations of these four sets of equations that

8.

$$
\left\{\begin{array}{l}
b_{0}=y_{n+1}-\Delta_{1} y_{n+1} \\
b_{1} h=\Delta_{1} y_{n+1}-\frac{I}{2} \Delta_{2} y_{n+1}-\frac{\overline{6}}{6} \Delta_{3} y_{n+1} \\
b_{2} h^{2}=\frac{I}{2} \Delta_{2} y_{n+1} \\
b_{3} h^{3}=\frac{I}{6} \Delta_{3} y_{n+1}
\end{array}\right.
$$

Therefore the integral (3) becomes

9.

$$
I_{n}=h\left[y_{n+1}-\frac{\mathrm{I}}{2} \Delta_{1} y_{n+1}-\frac{\mathrm{I}}{\mathrm{I} 2} \Delta_{2} y_{n+1}-\frac{\mathrm{I}}{24} \Delta_{3} y_{n+1}-\ldots . .\right.
$$

The coefficients of the higher order terms $\Delta_{4} y_{n+1}$ and $\Delta_{5} y_{n+1}$ are $-\frac{\text { I9 }}{720}$ and $\frac{\mathrm{I}}{48}$ respectively.

10.31 Obviously, if it were desired, the integral from $t_{n-2}$ to $t_{n-1}$, or over any other part of this interval, could be computed by the same methods. For example, the integral from $t_{n-1}$ to $t_{n}$ is

$$
\begin{aligned}
I_{n-1} & =\int_{t_{n-1}}^{t_{n}} f(t) d t \\
& =h\left[y_{n+1}-\frac{3}{2} \Delta_{1} y_{n+1}+\frac{5}{\mathrm{I} 2} \Delta_{2} y_{n+1}+\frac{\mathrm{I}}{24} \Delta_{3} y_{n+1}+\ldots \ldots\right]
\end{aligned}
$$

\section{NUMERICAL ILLUSTRATIONS}

10.32 Consider first the application of Simpson's method. Suppose it is required to find

$$
I=\int_{25^{\circ}}^{55^{\circ}} \sin t d t=-[\cos t]_{25^{\circ}}^{55^{\circ}}=0.3327
$$

On applying 10.12 with the numbers taken from Table $I$, it is found that

$$
I_{1}=\frac{5^{\circ}}{3}[.4226+2.0000+\mathrm{I} . \mathrm{I} 472+2.57 \mathrm{I} 2+\mathrm{I} .4 \mathrm{I} 42+3.0640+.8 \mathrm{I} 9 \mathrm{I}]
$$

which becomes, on reducing $5^{\circ}$ to radians,

$$
I_{1}=0.3327
$$

agreeing to four places with the correct result.

10.33 On applying 10.11 (4) and omitting alternate entries in Table II, it is found that

$$
I=\int_{25^{\circ}}^{245^{\circ}} \sin t d t=\frac{10^{\circ}}{3}[.4226+2.2944+.707 \mathrm{I}]=0.1992
$$

which is also correct to four places. These formulas could hardly be surpassed in ease and convenience of application. 
10.34 Now consider the application of 10.30 (9). As it stands it furnishes the integral over the single interval $t_{n}$ to $t_{n+1}$. If it is desired to find the integral from $t_{n}$ to $t_{n+m}$, the formula for doing so is obviously the sum of $m$ formulas such as (9), the value of the subscript going from $n+\mathrm{I}$ to $n+m+\mathrm{I}$, or

$$
\begin{aligned}
& I_{n}, m=h\left[\left(y_{n+1}+\ldots \ldots+y_{n+m+1}\right)-\frac{\mathrm{I}}{2}\left(\Delta_{1} y_{n+1}+\ldots \ldots+\Delta_{1} y_{n+m+1}\right)\right. \\
& \left.-\frac{\mathrm{I}}{\mathrm{I} 2}\left(\Delta_{2} y_{n+1}+\ldots .+\Delta_{2} y_{n+m+1}\right)-\frac{\mathrm{I}}{24}\left(\Delta_{3} y_{n+1}+\ldots \ldots+\Delta_{3} y_{n+m+1}\right)+\ldots\right] .
\end{aligned}
$$

On applying this formula to the numbers of Table $\mathrm{I}$, it is found that

$$
\begin{aligned}
I=\int_{25^{\circ}}^{.55^{\circ}} \sin t d t=5^{\circ}[(.5000 & +.5736+.6428+.707 \mathrm{I}+.7660+.8 \mathrm{I} 9 \mathrm{I}) \\
& -\frac{\mathrm{I}}{2}(.0774+.0736+.0692+.0643+.0589+.053 \mathrm{I}) \\
& +\frac{\mathrm{I}}{\mathrm{I} 2}(.0032+.0038+.0044+.0049+.0054+.0058) \\
& \left.+\frac{\mathrm{I}}{24}(.0006+.0006+.0006+.0005+.0005+.0004)\right] \\
& =0.3327,
\end{aligned}
$$

agreeing to four places with the exact value. When a table of differences is at hand covering the desired range this method involves the simplest numerical operations. It must be noted, however, that some of the required differences necessitate a knowledge of the value of the function for earlier values of the argument than the lower limit of the integral.

10.40 Reduced Form of the Differential Equations. Differential equations which arise from physical problems usually involve second derivatives. For example, the differential equation satisfied by the motion of a vibrating tuning fork has the form

$$
\frac{d^{2} x}{d t^{2}}=-k x
$$

where $k$ is a constant depending on the tuning fork.

10.41 The differential equations for the motion of a body subject to gravity and a retardation which is proportional to its velocity are

$$
\left\{\begin{array}{l}
\frac{d^{2} x}{d t^{2}}=-c \frac{d x}{d t} \\
\frac{d^{2} y}{d t^{2}}=-c \frac{d y}{d t}-g
\end{array}\right.
$$

where $c$ is a constant depending on the resisting medium and the mass and shape of the body, while $g$ is the acceleration of gravity. 
10.42 The differential equations for the motion of a body moving subject to the law of gravitation are

$$
\left\{\begin{array}{l}
\frac{d^{2} x}{d t^{2}}=-k^{2} \frac{x}{r^{3}}, \\
\frac{d^{2} y}{d t^{2}}=-k^{2} \frac{y}{r^{3}}, \\
\frac{d^{2} z}{d t^{2}}=-k^{2} \frac{z}{r^{3}}, \\
r^{2}=x^{2}+y^{2}+z^{2}
\end{array}\right.
$$

10.43 These examples illustrate sufficiently the types of differential equations which arise in practical problems. The number of the equations depends on the problem and may be small or great. In the problem of three bodies there are nine equations. The equations are usually not independent as is illustrated in 10.42, where each equation involves all three variables $x, y$, and $z$ through $r$. On the other hand, equations $\mathbf{1 0 . 4 1}$ are mutually independent for the first does not involve $y$ or its derivatives and the second does not involve $x$ or its derivatives. The right members may involve $x, y$, and $z$ as is the case in 10.42 , or they may involve the first derivatives, as is the case in 10.41, or they may involve both the coördinates and their first derivatives. In some problems they also involve the independent variable $t$.

10.44 Hence physical problems usually lead to differential equations which are included in the form

$$
\left\{\begin{array}{l}
\frac{d^{2} x}{d t^{2}}=f\left(x, y, \frac{d x}{d t}, \frac{d y}{d t}, t\right), \\
\frac{d^{2} y}{d t^{2}}=g\left(x, y, \frac{d x}{d t}, \frac{d y}{d t}, t\right),
\end{array}\right.
$$

where $f$ and $g$ are functions of the indicated arguments. Of course, the number of equations may be greater than two.

10.45 If we let

$$
x^{\prime}=\frac{d x}{d t}, \quad y^{\prime}=\frac{d y}{d t},
$$

equations 10.44 can be written in the form

$$
\left\{\begin{array}{l}
\frac{d x}{d t}=x^{\prime} \\
\frac{d x^{\prime}}{d t}=f\left(x, y, x^{\prime}, y^{\prime}, t\right) \\
\frac{d y}{d t}=y^{\prime} \\
\frac{d y^{\prime}}{d t}=g\left(x, y, x^{\prime}, y^{\prime}, t\right)
\end{array}\right.
$$


10.46 If we let $x=x_{1}, x^{\prime}=x_{2}, y=x_{3}, y^{\prime}=x_{4}, \ldots \ldots$ equations 10.45 are included in the form

$$
\left\{\begin{array}{l}
\frac{d x_{1}}{d t}=f_{1}\left(x_{1}, x_{2}, \ldots \ldots, x_{n}, t\right), \\
\ldots \ldots \ldots \ldots \\
\ldots \ldots \ldots \ldots \\
\frac{d x_{n}}{d t}=f_{n}\left(x_{1}, x_{2}, \ldots \ldots, x_{n}, t\right) .
\end{array}\right.
$$

This is the final standard form to which it will be supposed the differential equations are reduced.

10.50 Definition of a Solution of Differential Equations. For simplicity in writing, suppose the differential equations are two in number and write them in the form

I.

$$
\left\{\begin{array}{l}
\frac{d x}{d t}=f(x, y, t), \\
\frac{d y}{d t}=g(x, y, t),
\end{array}\right.
$$

where $f$ and $g$ are known functions of their arguments. Suppose $x=a, y=b$ at $t=0$. Then

2 .

$$
\left\{\begin{array}{l}
x=\phi(t) \\
y=\psi(t)
\end{array}\right.
$$

is the solution of (I) satisfying these initial conditions if $\phi$ and $\psi$ are such functions that

3.

$$
\begin{aligned}
\phi(0) & =a, \\
\psi(0) & =b, \\
\frac{d \phi}{d t} & =f(\phi, \psi, t), \\
\frac{d \psi}{d t} & =g(\phi, \psi, t),
\end{aligned}
$$

the last two equations being satisfied for all $\circ \leqslant t \leqslant T$, where $T$ is a positive constant, the largest value of $t$ for which the solution is determined. It is not necessary that $\phi$ and $\psi$ be given by any formulas - it is sufficient that they have the properties defined by (3). Solutions always exist, though it will not •be proved here, if $f$ and $g$ are continuous functions of $t$ and have derivatives with respect to both $x$ and $y$.

10.51 Geometrical Interpretation of a Solution of Differential Equations.. Geometrical interpretations of definite integrals have been of great value not only in leading to an understanding of their real meaning but also in suggesting 
practical means of obtaining their numerical values. The same things are true in the case of differential equations.

For simplicity in the geometrical representation, consider a single equation

I.

$$
\frac{d x}{d t}=f(x, t),
$$

where $x=a$ at $t=0$. Suppose the solution is

2.

$$
x=\phi(t),
$$

Equation (2) defines a curve whose coördinates are $x$ and $t$. Suppose it is represented by figure 2. The value of the tangent to the curve at every point on it

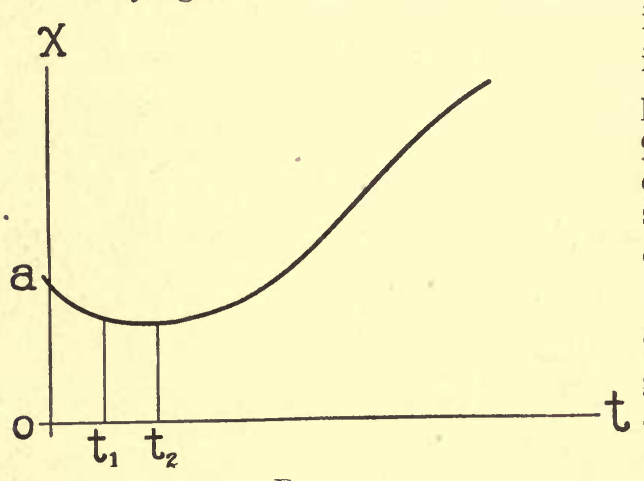

FIG. 2

is given by equation (I), for there is, corresponding to each point, a pair of values of $x$ and $t$ which gives $\frac{d x}{d t}$, the value of the tangent, when substituted in the right member of equation ( $\mathrm{I}$ ).

Consider the initial point on the curve, viz. $x=a, t=0$. The tangent at this point is $f(a, 0)$. The curve lies close to the tangent for a short distance from the initial point. Hence an approximate value of $x$ at $t=t_{1}, t_{1}$ being small, is the ordinate of the point where the tangent at $a$ intersects the line $t=t_{1}$, or

$$
x_{1}=f(a, 0) t_{1}+f(a, 0) \text { ? }
$$

The tangent at $x_{1}, t_{1}$ is defined by ( $\mathrm{I}$ ), and a new step in the solution can be made in the same way. Obviously the process can be continued as long as $x$ and $t$ have values for which the right member of (I) is defined. And the same process can be applied when there are any number of equations. While the steps of this process can be taken so short that it will give the solution with any desired degree of accuracy, it is not the most convenient process that may be employed. It is the one, however, which makes clearest to the intuitions the nature of the solution.

10.6 Outline of the Method of Solution. Consider equations 10.50 (I) and their solution (2). The problem is to find functions $\phi$ and $\psi$ having the properties (2). If we integrate the last two equations of $\mathbf{1 0 . 5 0}(3)$ we shall have

I.

$$
\left\{\begin{array}{l}
\phi=a+\int_{0}^{t} f(\phi, \psi, t) d t \\
\psi=b+\int_{0}^{t} g(\phi, \psi, t) d t
\end{array}\right.
$$

The difficulty arises from the fact that $\phi$ and $\psi$ are not known in advance and the integrals on the right can not be formed. Since $\phi$ and $\psi$ are the solution values of $x$ and $y$, we may replace them by the latter in order to preserve the original notation, and we have 
2.

$$
\left\{\begin{array}{l}
x=a+\int_{0}^{t} f(x, y, t) d t \\
y=b+\int_{0}^{t} g(x, y, t) d t .
\end{array}\right.
$$

If $x$ and $y$ do not change rapidly in numerical value, then $f(x, y, t)$ and $g(x, y, t)$ will not in general change rapidly, and a first approximation to the values of $x$ and $y$ satisfying equations (2) is

3.

$$
\left\{\begin{array}{l}
x_{1}=a+\int_{0}^{t} f(a, b, t) d t \\
y_{1}=b+\int_{0}^{t} g(a, b, t) d t
\end{array}\right.
$$

at least for values of $t$ near zero. Since $a$ and $b$ are constants, the integrands in (3) are known and the integrals can be computed. If the primitives can not be found the integrals can be computed by the methods of 10.1 or 10.3 .

After a first approximation has been found a second approximation is given by

4 .

$$
\left\{\begin{array}{l}
x_{2}=a+\int_{0}^{t} f\left(x_{1}, y_{1}, t\right) d t \\
y_{2}=b+\int_{0}^{t} g\left(x_{1}, y_{1}, t\right) d t .
\end{array}\right.
$$

The integrands are again known functions of $t$ because $x_{1}$ and $y_{1}$ were determined as functions of $t$ by equations (3). Consequently $x_{2}$ and $y_{2}$ can be computed. The process can evidently be repeated as many times as is desired. The $n$th approximation is

5 .

$$
\left\{\begin{array}{l}
x_{n}=a+\int_{0}^{t} f\left(x_{n-1}, y_{n-1}, t\right) d t \\
y_{n}=b+\int_{0}^{t} g\left(x_{n-1}, y_{n-1}, t\right) d t .
\end{array}\right.
$$

There is no difficulty in carrying out the process, but the question arises whether it converges to the solution. The answer, first established by Picard, is that, as $n$ increases, $x_{n}$ and $y_{n}$ tend toward the solution for all values of $t$ for which all the approximations belong to those values of $x, y$, and $t$ for which $f$ and $g$ have the properties of continuity with respect to $t$ and differentiability with respect to $x$ and $y$. If, for example, $f=\frac{\sin x}{x^{2}}$ and the value of $x_{n}$ tends towards zero for $t=T$, then the solution can not be extended beyond $t=T$.

It is found in practice that the longer the interval over which the integration is extended in the successive approximations, the greater the number of approximations which must be made in order to obtain a given degree of accuracy. In fact, it is preferable to take first a relatively short interval and to find the solution over this interval with the required accuracy, and then to continue from the end values of this interval over a new interval. This is what is done in actual work. The details of the most convenient methods of doing it will be explained in the succeeding sections. 
10.7 The Step-by-Step Construction of the Solution. Suppose the differential equations are

I

$$
\left\{\begin{array}{l}
\frac{d x}{d t}=f(x, y, t), \\
\frac{d y}{d t}=g(x, y, t),
\end{array}\right.
$$

with the initial conditions $x=a, y=b$ at $t=0$. It is more difficult to start a solution than it is to continue one after the first few steps have been made. Therefore, it will be supposed in this section that the solution is well under way, and it will be shown how to continue it. Then the method of starting a solution will be explained in the next section, and the whole process will be illustrated numerically in the following one.

Suppose the values of $x$ and $y$ have been found for $t=t_{1}, t_{2}, \ldots, t_{n}$. Let them be respectively $x_{1}, y_{1} ; x_{2}, y_{2} ; \ldots ; x_{n}, y_{n}$, care bejing taken not to confuse the subscripts with those used in section 10.6 in a different sense. Suppose the intervals $t_{2}-t_{1}, t_{3}-t_{2}, \ldots, t_{n}-t_{n-1}$ are all equal to $h$ and that it is desired to find the values of $x$ and $y$ at $t_{n+1}$, where $t_{n+1}-t_{n}=h$.

It follows from this notation and equations (2) of $\mathbf{1 0 . 6}$ that the desired quantities are

2.

$$
\left\{\begin{array}{l}
x_{n+1}=x_{n}+\int_{t_{n}}^{t_{n+1}} f(x, y, t) d t, \\
y_{n+1}=y_{n}+\int_{t_{n}}^{t_{n}+\mathbf{r}} g(x, y, t) d t .
\end{array}\right.
$$

The values of $x$ and $y$ in the integrands are of course unknown. They can be found by successive approximations, and if the interval is short, as is supposed, the necessary approximations will be few in number.

A fortunate circumstance makes it possible to reduce the number of approximations. The values of $x$ and $y$ are known at $t=t_{n}, t_{n-1}, t_{n-2}, \ldots$ From these values it is possible to determine in advance, by extrapolation, very close approximations to $x$ and $y$ for $t=t_{n+1}$. The corresponding values of $f$ and $g$ can be computed because these functions are given in terms of $x, y$, and $t$. They are also given for $t=t_{n}, t_{n-1}, \ldots \ldots$ Consequently, curves for $f$ and $g$ agreeing with their values at $t=t_{n+1}, t_{n}, t_{n-1}, \ldots$. can be constructed and the integrals (2) can be computed by the methods of 10.1 and $\mathbf{1 0 . 3}$.

The method of extrapolating valuès of $x_{n+1}$ and $y_{n+1}$ must be given. Since the method is the same for both, consider only the former. Since, by hypothesis, $x$ is known for $t=t_{n}, t_{n-1}, t_{n-2}, \ldots$ the values of $x_{n}, \Delta_{1} x_{n}, \Delta_{2} x_{n}$, and $\Delta_{3} x_{n}$ are known. If the interval $h$ is not too large the value of $\Delta_{3} x_{n+1}$ is very nearly equal to $\Delta_{3} x_{n}$. As an approximation $\Delta_{3} x_{n+1}$ may be taken equal to $\Delta_{3} x_{n}$, or perhaps a closer value may be determined from the way the third differences 




$\Delta_{3} x_{n-3}, \Delta_{3} x_{n-2}, \Delta_{3} x_{n-1}$, and $\Delta_{3} x_{n}$ vary. For example, in Table II it is easy to see that $\Delta_{3} \sin 75^{\circ}$ is almost certainly -3 . It follows from $10.20,1,2$ that

3 .

$$
\left\{\begin{array}{l}
\Delta_{2} x_{n+1}=\Delta_{3} x_{n+1}+\Delta_{2} x_{n}, \\
\Delta_{1} x_{n+1}=\Delta_{2} x_{n+1}+\Delta_{1} x_{n} \\
x_{n+1}=\Delta_{1} x_{n+1}+x_{n} .
\end{array}\right.
$$

After the adopted value of $\Delta_{3} x_{n+1}$ has been written in its column the successive entries to the left can be written down by simple additions to the respective numbers on the line of $t_{n}$. For example, it is found from Table II that $\Delta_{2} \sin 75^{\circ}=-72, \Delta_{1} \sin 75^{\circ}=262, \sin 75^{\circ}=9659$. This is, indeed, the correct value of $\sin 75^{\circ}$ to four places.

Now having extrapolated approximate values of $x_{n+1}$ and $y_{n+1}$ it remains to compute $f$ and $g$ for $x=x_{n+1}, y=y_{n+1}, t=t_{n+1}$. The next step is to pass curves through the values of $f$ and $g$ for $t=t_{n+1}, t_{n}, t_{n-1}, \ldots$ and to compute the integrals (2). This is the precise problem that was solved in 10.30 , the only difference being that in that section the integrand was designated by $y$. On applying equation 10.30 (9) to the computation of the integrals (2), the latter give

4.

$$
\left\{\begin{array}{l}
x_{n+1}=x_{n}+h\left[f_{n+1}-\frac{\mathrm{I}}{2} \Delta_{1} f_{n+1}-\frac{\mathrm{I}}{\mathrm{I} 2} \Delta_{2} f_{n+1}-\frac{\mathrm{I}}{24} \Delta_{3} f_{n+1} \ldots\right], \\
y_{n+1}=y_{n}+h\left[g_{n+1}-\frac{\mathrm{I}}{2} \Delta_{1} g_{n+1}-\frac{\mathrm{I}}{\mathrm{I} 2} \Delta_{2} g_{n+1}-\frac{\mathrm{I}}{24} \Delta_{3} g_{n+1} \ldots\right]
\end{array}\right.
$$

where

5 .

$$
\left\{\begin{array}{l}
f_{n+1}=f\left(x_{n+1}, y_{n+1}, t_{n+1}\right), \\
g_{n+1}=g\left(x_{n+1}, y_{n+1}, t_{n+1}\right)
\end{array} .\right.
$$

The right members of (4) are known and therefore $x_{n+1}$ and $y_{n+1}$ are determined.

It will be recalled that $f_{n+1}$ and $g_{n+1}$ were computed from extrapolated values of $x_{n+1}$ and $y_{n+1}$, and hence are subject to some error. They should now be recomputed with the values of $x_{n+1}$ and $y_{n+1}$ furnished by (4). Then more nearly correct values of the entire right members of (4) are at hand and the values of $x_{n+1}$ and $y_{n+1}$ should be corrected if necessary. If the interval $h$ is small it will not generally be necessary to correct $x_{n+1}$ and $y_{n+1}$. But if they require corrections, then new values of $f_{n+1}$ and $g_{n+1}$ should be computed. In practice it is advisable to take the interval $h$ so small that one correction to $f_{n+1}$ and $g_{n+1}$ is sufficient.

After $x_{n+1}$ and $y_{n+1}$ have been obtained, values of $x$ and $y$ at $t_{n+2}$ can be found in precisely the same manner, and the process can be continued to $t=t_{n+3}, t_{n+4}$, ..... If the higher differences become large and irregular it is advisable to interpolate values at the mid-intervals of the last two steps and to continue with an interval half as great. On the other hand, if the higher differences become very small it is advisable to proceed with an interval twice as great as that used in the earlier part of the computation.

The foregoing, expressed in words, seems rather complicated. As a matter of fact, it goes very simply in practice, as will be shown in section 10.9 . 
10.8 The Start of the Construction of the Solution. Suppose the differential equations are again

I.

$$
\left\{\begin{array}{l}
\frac{d x}{d t}=f(x, y, t), \\
\frac{d y}{d t}=g(x, y, t),
\end{array}\right.
$$

with the initial conditions $x=a, y=b$ at $t=0$. Only the initial values of $x$ and $y$ are known. But it follows from (I) that the rates of change of $x$ and $y$ at $t=0$ are $f(a, b, o)$ and $g(a, b, o)$ respectively. Consequently, first approximations to values of $x$ and $y$ at $t=t_{1}=h$ are

2.

$$
\left\{\begin{array}{l}
x_{1}^{(1)}=a+h f(a, b, \circ), \\
y_{1}^{(1)}=b+h g(a, b, 0) .
\end{array}\right.
$$

Now it follows from (I) that the rates of change of $x$ and $y$ at $x=x_{1}, y=y_{1}$, $\ell=t_{1}$ are approximately $f\left(x_{1}{ }^{(1)}, y_{1}{ }^{(1)}, t_{1}\right)$ and $g\left(x_{1}{ }^{(1)}, y_{1}{ }^{(1)}, t_{1}\right)$. These rates will be different from those at the beginning, and the average rates of change for the first interval will be nearly the average of the rates at the beginning and at the end of the interval. Therefore closer approximations than those given in (2) to the values of $x$ and $y$ at $t=t_{1}$ are

3.

$$
\left\{\begin{array}{l}
x_{1}^{(2)}=a+\frac{1}{2} h\left[f(a, b, 0)+f\left(x_{1}^{(1)}, y_{1}^{(1)}, t_{1}\right)\right], \\
y_{1}^{(2)}=b+\frac{1}{2} h\left[g(a, b, 0)+g\left(x_{1}^{(1)}, y_{1}^{(1)}, t_{1}\right)\right] .
\end{array}\right.
$$

The process could be repeated on the first interval, but it is not advisable when the interval is taken as short as it should be.

The rates of change at the beginning of the second interval are approximately $f\left(x_{1}{ }^{(2)}, y_{1}{ }^{(2)}, t_{1}\right)$ and $g\left(x_{1}{ }^{(2)}, y_{1}{ }^{(2)}, t_{1}\right)$ respectively. Consequently, first approximations to the values of $x$ and $y$ at $t=t_{2}$, where $t_{2}-t_{1}=h$, are

4 .

$$
\left\{\begin{array}{l}
x_{2}^{(1)}=x_{1}^{(2)}+h f\left(x_{1}^{(2)}, y_{1}^{(2)}, t_{1}\right) \\
y_{2}{ }^{(1)}=y_{1}^{(2)}+h g\left(x_{1}^{(2)}, y_{1}^{(2)}, t_{1}\right) .
\end{array}\right.
$$

With these values of $x$ and $y$ approximate values of $f_{2}$ and $g_{2}$ are computed. Since $f_{0}, g_{0} ; f_{1}, g_{1}$ are known; it follows that $\Delta_{1} f_{2}, \Delta_{1} g_{2} ; \Delta_{2} f_{2}$, and $\Delta_{2} g_{2}$ are also known. Hence equations (4) of $\mathbf{1 0 . 7}$, for $n+\mathrm{I}=2$, can be used, with the exception of the last terms in the right members, for the computation of $x_{2}$ and $y_{2}$.

At this stage of work $x_{0}=a, y_{0}=b ; x_{1}, y_{1} ; x_{2}, y_{2}$ are known, the first pair exactly and the last two pairs with considerable approximation. After $f_{2}$ and $g_{2}$ have been computed, $x_{1}$ and $y_{1}$ can be corrected by 10.31 for $n=\mathrm{I}$. Then approximate values of $x_{3}$ and $y_{3}$ can be extrapolated by the method explained in the preceding section, after which approximate values of $f_{3}$ and $g_{3}$ can be computed. With these values and the corresponding difference functions, $x_{2}$ and $y_{2}$ can be corrected by using 10.31. Then after correcting all the corresponding differences of all the functions, the solution is fully started and proceeds by the method given in the preceding section.

10.9 Numerical Illustration. In this section a numerical problem will be treated which will illustrate both the steps which must be taken and also the method of 
arranging the work. A convenient arrangement of the computation which preserves a complete record of all the numerical work is very important.

Suppose the differential equation is

I.

$$
\left\{\begin{array}{c}
\frac{d^{2} x}{d t^{2}}=-\left(\mathrm{I}+\kappa^{2}\right) x+2 \kappa^{2} x^{3} \\
x=0, \frac{d x}{d t}=\mathrm{I} \text { at } t=0 .
\end{array}\right.
$$

The problem of the motion of a simple pendulum takes this form when expressed in suitable variables. This problem is chosen here because it has an actual physical interpretation, because it can be integrated otherwise so as to express $t$ in terms of $x$, and because it will illustrate sufficiently the processes which have been explained.

Equation (I) will first be integrated so as to express $t$ in terms of $x$. On multiplying both sides of (I) by $2 \frac{d x}{d t}$ and integrating, it is found that the integral which satisfies the initial conditions is

2.

$$
\left(\frac{d x}{d t}\right)^{2}=\left(1-x^{2}\right)\left(1-\kappa^{2} x^{2}\right)
$$

On separating the variables this equation gives

3.

$$
t=\int_{0}^{x} \frac{d x}{\sqrt{\left(1-x^{2}\right)\left(1-\kappa^{2} x^{2}\right)}} .
$$

Suppose $\kappa^{2}<\mathrm{I}$ and that the upper limit $x$ does not exceed unity. Then

$$
\frac{\mathrm{I}}{\sqrt{\mathrm{I}-\kappa^{2} x^{2}}}=\mathrm{I}+\frac{\mathrm{I}}{2} \kappa^{2} x^{2}+\frac{3}{8} \kappa^{4} x^{4}+\frac{5}{\mathrm{I} 6} \kappa^{6} x^{6}+\ldots
$$

where the right member is a converging series. On substituting (4) into (3) and integrating, it is found that

5. $t=\sin ^{-1} x+\frac{1}{4}\left[-x \sqrt{\mathrm{I}-x^{2}}+\sin ^{-1} x\right] \kappa^{2}+\frac{3}{8}\left[-x^{3} \sqrt{\mathrm{I}-x^{2}}-\frac{3}{4} x\left(\mathrm{I}-x^{2}\right)^{\frac{2}{2}}\right.$

When $x=\mathrm{I}$ this integral becomes

$$
\left.\left.+\frac{3}{8} x \sqrt{1-x^{2}}+\frac{3}{8} \sin ^{-1} x\right] \kappa^{4}+\ldots \ldots\right] \text {. }
$$

6.

$$
T=\frac{\pi}{2}\left[\mathrm{I}+\left(\frac{\mathrm{I}}{2}\right)^{2} \kappa^{2}+\left(\frac{\mathrm{I} \cdot 3}{2 \cdot 4}\right)^{2} \kappa^{4}+\left(\frac{\mathrm{I} \cdot 3 \cdot 5}{2 \cdot 4 \cdot 6}\right)^{2} \kappa^{6}+\ldots\right] .
$$

Equation (5) gives $t$ for any value of $x$ between $-\mathrm{I}$ and $+\mathrm{I}$. But the problem is to determine $x$ in terms of $t$. Of course, if a table is constructẹd giving $t$ for many values of $x$, it may be used inversely to obtain the value of $x$ corresponding to any value of $t$. The labor involved is very great. When $\kappa^{2}$ is given numerically it is simpler to compute the integral (3) by the method of 10.1 or 10.3 .

In mathematical terms, $t$ is an elliptical integral of $x$ of the first kind, and the inverse function, that is, $x$ as a function of $t$, is the sine-amplitude function, which has the real period $4 T$. 
Suppose $\kappa^{2}=\frac{I}{2}$ and let $y=\frac{d x}{d t}$. Then equation (I) is equivalent to the two equations

$7 \cdot$

$$
\left\{\begin{array}{l}
\frac{d x}{d t}=y \\
\frac{d y}{d t}=-\frac{3}{2} x+x^{3}
\end{array}\right.
$$

which are of the form 10.50 (I), where

8.

$$
\left\{\begin{array}{l}
f=y, \\
g=-\frac{3}{2} x+x^{3},
\end{array}\right.
$$

and $x=0, y=\mathrm{I}$ at $t=0$.

The first step is to determine the interval which is to be used in the start of the solution. No general rule can be given. The larger $f_{0}$ and $g_{0}$ the smaller must the interval be taken. A fairly good rule is in general to take $h$ so small that $h f_{0}$ and $h g_{0}$ shall not be greater than rooo times the permissible error in the results. In the present instance we may take $h=0$.I.

First approximations to $x$ and $y$ at $t=0.1$ are found from the initial conditions and equations 10.8 (2) to be

$9 \cdot$

$$
\left\{\begin{array}{l}
x_{1}^{(1)}=0+\frac{I}{10} I=0.1000 \\
y_{1}^{(1)}=I+\frac{I}{10} 0=I .0000
\end{array}\right.
$$

It follows from (8) and these values of $x_{1}$ and $y_{1}$ that

Io.

$$
\left\{\begin{array}{l}
f\left(x_{1}{ }^{(1)}, y_{1}^{(1)}, t_{1}\right)=\text { I.0000, } \\
g\left(x_{1}^{(1)}, y_{1}^{(1)}, t_{1}\right)=-0.1490 .
\end{array}\right.
$$

Hence the more nearly correct values of $x_{1}$ and $y_{1}$, which are given by $10.8(3)$, are

II.

$$
\left\{\begin{array}{l}
x_{1}{ }^{(2)}=0+\frac{0 . I}{2}[\mathrm{I} .0000+\mathrm{I} .0000]=0.1000 \\
y_{1}{ }^{(2)}=\mathrm{I}+\frac{0 . \mathrm{I}}{2}[0.0000-0.1490]=0.9925 .
\end{array}\right.
$$

Since in this particular problem $x=\int y d t$, it is not necessary to compute both $f$ and $g$ by the exact process explained in section 10.8, for after $y$ has been determined $x$ is given by the integral. It follows from (7), (8), (Io), and (II) that a first approximation to the value of $y$ at $t=t_{2}=0.2$ is

I2.

$$
y_{2}^{(1)}=.0925-\frac{1}{10} \cdot 1490=.9776 .
$$

With the values of $y$ at $0, . I, .2$ given by the initial conditions and in equations (9) and (12), the first trial $y$-table is constructed as follows: 
First Trial $y$-Table

\begin{tabular}{|c|c|c|c|}
\hline$t$ & $y$ & $\Delta_{1} y$ & $\Delta_{2} y$ \\
\hline 0 & $\mathrm{I} .0000$ & & \\
. $\mathrm{I}$ & .9925 & -.0075 & \\
.2 & .9776 & -.0149 & -.0074 \\
\hline
\end{tabular}

Since $y=f$ it now follows from the first equations of (II) and 10.7 (4) for $n=\mathrm{I}$ that an approximate value of $x_{2}$ is

I3.

$$
x_{2}{ }^{(1)}=0.1000+\frac{\mathrm{I}}{\mathrm{IO}}\left[.9776+\frac{\mathrm{I}}{2} .0 \mathrm{I} 49+\frac{\mathrm{I}}{\mathrm{I} 2} .0074\right]=. \mathrm{I} 986 .
$$

With this value of $x_{2}$ it is found from the second of (8) that $g_{2}=.290 \mathrm{I}$. Then the first trial $g$-table constructed from the values of $g$ at $t=0,0.1,0.2$, is:

First Trial $g$-Table

\begin{tabular}{|c|r|c|c|}
\hline \multicolumn{1}{c|}{$t$} & \multicolumn{1}{c|}{$g$} & $\Delta_{1} g$ & $\Delta_{2} g$ \\
\cline { 2 - 4 } 0 & .0000 & & \\
\cline { 2 - 3 }. $\mathrm{I}$ &.$- \mathrm{I} 490$ &.$- \mathrm{I} 490$ & \\
.2 & $-.290 \mathrm{I}$ &.$- \mathrm{I} 4 \mathrm{II}$ & +.0079 \\
\hline
\end{tabular}

Then the second equation of 10.7 (4) gives for $n=\mathrm{I}$ the more nearly correct value of $y_{2}$,

I4.

$$
y_{2}=.9925+\frac{\mathrm{I}}{\mathrm{IO}}\left[-.290 \mathrm{I}+\frac{\mathrm{I}}{\mathrm{I} 2} \cdot \mathrm{I} 4 \mathrm{II}-\frac{\mathrm{I}}{\mathrm{I} 2} \cdot 0079\right]=.9705 .
$$

This value of $y_{2}$ should replace the last entry in the first trial $y$-table. When this is done it is found that $\Delta_{1} y_{2}=-.0220, \Delta_{2} y_{2}=-.0145$. Then the first equation of 10.7 (4) gives

15 .

$$
x_{2}=.1000+\frac{I}{\mathrm{IO}}\left[.9705+\frac{\mathrm{I}}{2} .0220+\frac{\mathrm{I}}{\mathrm{I} 2} .0145\right]=.1983 \text {. }
$$

The computation is now well started although $x_{1}, y_{1}, x_{2}$, and $y_{2}$ are still subject to slight errors. The values of $x_{1}$ and $y_{1}$ can be corrected by applying 10.31 for $n=\mathrm{I}$. It is necessary first to compute a more nearly correct value of $g_{2}$ by using the value of $x_{2}$ given in (I5). The result is $g_{2}=-.2896, \Delta_{1} g_{2}=-.1406$, $\Delta_{2} g_{2}=+.0084$. Then the second equation of 10.7 (4) gives

I6.

$$
y_{2}=.9925+\frac{\mathrm{I}}{\mathrm{IO}}\left[-.2896+\frac{\mathrm{I}}{2} \cdot \mathrm{I} 406-\frac{\mathrm{I}}{\mathrm{I} 2} \cdot 0084\right]=.9705
$$

agreeing with (I4). This value of $y_{2}$ is therefore essentially correct. An application of 10.31 then gives

I7.

$$
x_{1}=.0000+\frac{I}{10}\left[.9705+\frac{3}{2} .0220-\frac{5}{12} .0145\right]=.0997
$$


after which it is found that $g_{1}=-$.I486, $\Delta_{1} g_{1}=-$.I 486 . Now the first trial $y$-table can be corrected by using the value of $y_{2}$ given in (14). The result is:

Second Trial $y$-Table

\begin{tabular}{|c|c|c|c|}
\hline \multicolumn{1}{c|}{$t$} & $y$ & $\Delta_{1} y$ & $\Delta_{2} y$ \\
\cline { 1 - 4 } . & $\mathrm{I} .0000$ & & \\
.2 & .9925 & -.0075 & \\
.2 & .9705 & -.0220 & $-.0 \mathrm{I} 45$ \\
\hline
\end{tabular}

In order to correct $x_{2}$ and $y_{2}$ by the same method, which is the most convenient one to follow, it is necessary first to obtain approximate values of $g_{3}$ and $y_{3}$. The trial $g$-table can be corrected by computing $g$ with the values of $x$ given by (I 7 ) and (I5). Then the line for $g_{3}$ can be extrapolated. The results are:

Second Trial $g$-Table

\begin{tabular}{|c|c|c|c|}
\hline$t$ & $g$ & $\Delta_{1} g$ & $\Delta_{2} g$ \\
\hline 0 & .0000 & & \\
. $\mathrm{I}$ &.$- \mathrm{I} 486$ &.$- \mathrm{I} 486$ & \\
.2 & -.2896 & -.1410 & +.0076 \\
.3 & -.4230 &.$- \mathrm{I} 334$ & +.0076 \\
\hline
\end{tabular}

Then the second equation of 10.7 (4) gives for $n=2$,

I8.

$$
y_{3}=.9705+\frac{I}{I 0}\left[-.4230+\frac{I}{2} \cdot I 334-\frac{I}{I 2} .0076\right]=.9348
$$

When this is added to the second trial $y$-table, it is found that

I9.

$$
y_{3}=.9348, \Delta_{1} y_{3}=-.0357, \Delta_{2} y_{3}=-.0137, \Delta_{3} y_{3}=+.0008 \text {. }
$$

Now $x_{2}$ and $y_{2}$ can be corrected by applying 10.31 to these numbers and those in the last line of the second trial $g$-table. The results are

20.

$$
\left\{\begin{array}{l}
x_{2}=.0997+\frac{\mathrm{I}}{\mathrm{IO}}\left[.9348+\frac{3}{2} .0357-\frac{5}{\mathrm{I} 2} .0 \mathrm{I} 37+\frac{\mathrm{I}}{24} .0008\right]=. \mathrm{I} 980 \\
y_{2}=.9925+\frac{\mathrm{I}}{\mathrm{IO}}\left[-.4230+\frac{3}{2} \cdot \mathrm{I} 334+\frac{5}{\mathrm{I} 2} .0076\right]=.9705 .
\end{array}\right.
$$

The preliminary work is finished and $x$ and $y$ have been determined for $t=0$, .1 , and .2 with an error of probably not more than one unit in the last place. As the process is read over it may seem somewhat complicated, but this is largely because on the printed page preliminary values of the unknown quantities can hot be erased and replaced by more nearly correct ones. As a matter of fact, the 
first steps are very simple and can be carried out in practice in a few minutes if the chosen time-interval is not too great.

The problem now reduces to simple routine. There are an $x$-table, a $y$-table (which in this problem serves also as an $f$-table), a $g$-table, and a schedule for computing $g$. It is advisable to use large sheets so that all the computations except the schedule for computing $g$ can be kept side by side on the same sheet. The process consists of six steps: (I) Extrapolate a value of $g_{n+1}$ and its differences in the $g$-table; (2) compute $y_{n+1}$ by the second equation of 10.7 (4); (3) enter the result in the $y$-table and write down the differences; (4) use these results to compute $x_{n+1}$ by the first equation of $10.7(4)$; (5) 'with this value of $x_{n+1}$ compute $g_{n+1}$ by the $g$-computation schedule; and (6) correct the extrapolated value of $g_{n+1}$ in the $g$-table.

Usually the correction to $g_{n+1}$ will not be great enough to require a sensible correction to $y_{n+1}$. But if a correction is required, it should, of course, be made. It follows from the integration formulas 10.7 (4) and the way that the difference functions are formed that an error $\epsilon$ in $g_{n+1}$ produces the error $\frac{3}{8} h \epsilon$ in $y_{n+1}$, and the corresponding error in $x_{n+1}$ is $\frac{9}{64} h^{2} \epsilon$. It is never advisable to use so large a value of $h$ that the error in $x_{n+1}$ is appreciable. On the other hand, if the differences in the $g$-table and the $y$-table become so small that the second differences are insensible the interval may be doubled.

The following tables show the results of the computations in this problem reduced from five to four places.

Final $x$-Table

\begin{tabular}{|c|c|c|c|c|}
\hline$t$ & $x$ & $\Delta_{1} x$ & $\Delta_{2} x$ & $\Delta_{3} x$ \\
\hline 0 & .0000 & & & \\
\hline.$I$ & .0997 & .0997 & & 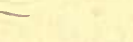 \\
\hline .2 & . I980 & .0983 & -.0014 & \\
\hline .3 & .2934 & .0954 & -.0029 & -.0015 \\
\hline .4 & .3847 & .0913 & $-.004 \mathrm{I}$ & -.0012 \\
\hline .5 & .4708 & $.086 \mathrm{I}$ & -.0052 & $-.001 \mathrm{I}$ \\
\hline .6 & .5508 & .0800 & $-.006 \mathrm{I}$ & -.0009 \\
\hline .7 & .6243 & .0735 & -.0065 & -.0004 \\
\hline .8 & .6909 & .0666 & -.0069 & -.0004 \\
\hline .9 & .7505 & .0596 & -.0070 & -.0001 \\
\hline I. 0 & .8030 & .0525 & -.0071 & -.0001 \\
\hline I. I & .8486 & $.045^{6}$ & -.0069 & +.0002 \\
\hline I. 2 & .8877 & $.039 I$ & -.0065 & +.0004 \\
\hline I. 3 & .9205 & .0328 & -.0063 & +.0002 \\
\hline I. 4 & .9472 & .0267 & $-.006 \mathrm{I}$ & +.0002 \\
\hline I. 5 & .9682 & .0210 & -.0057 & +.0004 \\
\hline I. 6 & .9837 & .0155 & -.0055 & +.0002 \\
\hline I. 7 & .9940 & .0103 & -.0052 & +.0003 \\
\hline I. 8 & .9993 & .0053 & -.0050 & +.0002 \\
\hline I. 9 & .9995 & .0002 & $-.005 \mathrm{I}$ & -.0001 \\
\hline
\end{tabular}


Final $y$-Table

\begin{tabular}{|c|c|c|c|c|}
\hline$t$ & $y$ & $\Delta_{1} y$ & $\Delta_{2} y$ & $\Delta_{3} y$ \\
\hline & & & & \\
\cline { 2 - 4 } .1 & .0000 & & & \\
.2 & .9925 & -.0075 & & \\
.3 & .9352 & -.0220 & -.0145 & +.0012 \\
.4 & .8882 & -.0470 & -.0117 & +.0016 \\
.5 & .8320 & -.0562 & -.0092 & +.0025 \\
.6 & .7687 & -.0633 & -.0071 & +.0019 \\
.7 & .7009 & -.0678 & -.0045 & +.0016 \\
.8 & .6308 & -.0701 & -.0023 & +.0022 \\
.9 & .5602 & -.0706 & -.0005 & +.0008 \\
I.0 & .4906 & -.0696 & +.0010 & +.0015 \\
I.I & .4231 & -.0675 & +.0021 & +.0011 \\
I.2 & .3584 & -.0647 & +.0028 & +.0007 \\
I.3 & .2968 & -.0616 & +.0031 & +.0003 \\
I.4 & .2382 & -.0586 & +.0030 & -.0001 \\
I.5 & .1824 & -.0558 & +.0028 & -.0002 \\
I.6 & .1290 & -.0534 & +.0024 & -.0004 \\
I.7 & .0775 & -.0515 & +.0019 & -.0005 \\
I.8 & .0271 & -.0504 & +.0011 & -.0008 \\
I.9 & -.0230 & -.0501 & +.0003 & -.0008 \\
\hline
\end{tabular}

Final $g$-Schedule

\begin{tabular}{|c|c|c|c|c|c|c|c|c|c|}
\hline$t$ &.$I$ & .2 & $\cdot 3$ & .4 & .5 & .6 & .7 & .8 & .9 \\
\hline $\log x$ & 8.9989 & 9.2967 & 9.4675 & $9.5^{85} \mathrm{I}$ & 9.6728 & 9.7410 & 9.7954 & 9.8394 & 9.8753 \\
\hline $\log x^{3}$ & 6.9967 & $7.890 \mathrm{I}$ & 8.4025 & 8.7553 & 9.0184 & 9.2230 & 9.3862 & 9.5182 & 9.6259 \\
\hline $3 x$ & .2992 & $.594 \mathrm{I}$ & .8802 & I.I 54 I & I.4I 24 & I. 6524 & I. 8729 & 2.0727 & 2.2515 \\
\hline$-\frac{3}{2} x$ & -.1496 & -.2970 & -.4401 & -.5770 & -.7062 & -.8262 & -.9365 & -1.0364 & -1.1257 \\
\hline$x^{3}$ & .0010 & .0077 & .0252 & .0569 & .1044 & .1671 & .2434 & .3298 & .4227 \\
\hline$g$ & -.1486 & -.2893 & -.4149 & -.5201 & -.6018 & $-.659 \mathrm{I}$ & $-.693 I$ & -.7066 & -.7030 \\
\hline
\end{tabular}


Final $g$-Table

\begin{tabular}{|c|c|c|c|c|}
\hline$t$ & $g$ & $\Delta_{1} g$ & $\Delta_{2} g$ & $\Delta_{3} g$ \\
\cline { 1 - 2 } 0 & .0000 & & & \\
.$I$ & -.1486 & -.1486 & & \\
.2 & -.2893 & -.1407 & +.0079 & \\
.3 & -.4149 & -.1256 & +.0151 & +.0072 \\
.4 & -.5201 & -.1052 & +.0204 & +.0053 \\
.5 & -.6018 & -.0817 & +.0235 & +.0031 \\
.6 & -.6591 & -.0573 & +.0244 & +.0009 \\
.7 & -.6931 & -.0340 & +.0233 & -.0011 \\
.8 & -.7066 & -.0135 & +.0205 & -.0028 \\
.9 & -.7030 & +.0036 & +.0171 & -.0034 \\
I.0 & -.6867 & +.0163 & +.0127 & -.0044 \\
I.I & -.6618 & +.0249 & +.0086 & -.0041 \\
I.2 & -.6320 & +.0298 & +.0049 & -.0037 \\
I.3 & -.6008 & +.0312 & +.0014 & -.0035 \\
I.4 & -.5710 & +.0298 & -.0014 & -.0028 \\
I.5 & -.5447 & +.0263 & -.0035 & -.0021 \\
I.6 & -.5236 & +.0211 & -.0052 & -.0017 \\
I.7 & -.5088 & +.0148 & -.0063 & -.0011 \\
I.8 & -.5011 & +.0077 & -.0071 & -.0008 \\
I.9 & -.5008 & +.0003 & -.0074 & -.0003 \\
\hline
\end{tabular}

Final $g$-Schedule - Continued

\begin{tabular}{|c|c|c|c|c|c|c|c|c|c|}
\hline 1.0 & I.I & I. 2 & I. 3 & I. 4 & I. 5 & I. 6 & I.7 & I. 8 & I.9 \\
\hline 9.9047 & 9.9287 & 9.9483 & 9.9640 & 9.9764 & 9.9860 & 9.9929 & 9.9974 & 9.9997 & 9.9998 \\
\hline $9.7 \mathrm{I} 4 \mathrm{I}$ & $9.786 \mathrm{r}$ & 9.8449 & 9.8920 & 9.9292 & 9.9580 & 9.9787 & 9.9922 & $9.999 \mathrm{I}$ & 9.9994 \\
\hline 2.4090 & $2.545^{8}$ & $2.663 \mathrm{I}$ & $2.76 \times 5$ & 2.8416 & 2.9046 & $2.95 \mathrm{II}$ & 2.9820 & 2.9979 & 2.9985 \\
\hline$-\mathrm{I} .2045$ & -1.2729 & - I.3316 & -1.3807 & -1.4208 & $-1.45^{23}$ & -1.4756 & -1.4910 & $-\mathrm{I} .4989$ & $-\mathrm{I} .499^{2}$ \\
\hline .5178 & $6 \operatorname{lin}$ & 6996 & .7799 & .8498 & .9076 & .9520 & .9822 & .9978 & .9984 \\
\hline-.6867 & -.6618 & -.6320 & -.6008 & -.5710 & -.5447 & -.5236 & -.5088 & -.5011 & -.5008 \\
\hline
\end{tabular}


As has been remarked, large sheets should be used so that the $x, y$, and $g$-tables can be put side by side on one sheet. Then the $t$-column need be written but once for these three tables. The $g$-schedule, which is of a different type, should be on a separate sheet.

The differential equation (I) has an integral which becomes for $\kappa^{2}=\frac{I}{2}$ and $\frac{d x}{d t}=y$.

$2 I$.

$$
y^{2}+\frac{3}{2} x^{2}-\frac{1}{4} x^{4}=\mathrm{I},
$$

and which may be used to check the computation because it must be satisfied at every step. It is found on trial that (2I) is satisfied to within one unit in the fourth place by the results given in the foregoing tables for every value of $t$.

The value of $t$ for which $x=\mathrm{I}$ and $y=0$ is given by (6). When $\kappa^{2}=\frac{1}{2}$ it is found that $T=\mathrm{r} .854 \mathrm{I}$. It is found from the final $x$-table by interpolation based on first and second differences that $x$ rises to its maximum unity for almost exactly this value of $t$; and, similarly, that $y$ vanishes for this value of $t$. 


\section{ELLIPTIC FUNCTIONS}

By Sir George Greenhill, F. R. S. 



\section{INTRODUCTION TO THE TABLES OF ELLIPTIC FUNCTIONS}

\section{By Sir George Greenhill}

In the integral calculus, $\int \frac{d x}{\sqrt{X}}$, and more generally, $\int \frac{M+N \sqrt{X}}{P+Q \sqrt{X}} d x$, where $M, N, P, Q$ are rational algebraical functions of $x$, can always be expressed by the elementary functions of analysis, the algebraical, circular, logarithmic or hyperbolic, so long as the degree of $X$ does not exceed the second. But when $X$ is of the third or fourth degree, new functions are required, called elliptic functions, because encountered first in the attempt at the rectification of an ellipse by means of an integral.

To express an elliptic integral numerically, when required in an actual question of geometry, mechanics, or physics and electricity, the integral must be normalised to a standard form invented by Legendre before the Tables can be employed; and these Tables of the Elliptic Functions have been calculated as an extension of the usual tables of the logarithmic and circular functions of trigonometry. The reduction to a standard form of any assigned elliptic integral that arises is carried out in the procedure described in detail in a treatise on the elliptic functions.

11.1. Legendre's Standard Elliptic Integral of the First Kind (E. I. I) is

$$
F(\varphi, k)=\int_{0}^{\phi} \frac{d \phi}{\sqrt{I-\kappa^{2} \sin ^{2} \phi}}=\int_{0}^{x} \frac{d x}{\sqrt{\left(I-x^{2}\right)\left(I-\kappa^{2} x^{2}\right)}}=u,
$$

defining $\phi$ as the amplitude of $u$, to the modulus $\kappa$, with the notation,

$$
\begin{aligned}
& \phi=\operatorname{am} u \\
& x=\sin \phi=\sin \operatorname{am} u
\end{aligned}
$$

abbreviated by Gudermann to,

$$
\begin{aligned}
x & =\operatorname{sn} u \\
\cos \phi & =\operatorname{cn} u \\
\Delta \phi & =\sqrt{ }\left(\mathrm{I}-\kappa^{2} \sin ^{2} \phi\right)=\Delta \operatorname{am} u=\operatorname{dn} u,
\end{aligned}
$$

and sn $u, \mathrm{cn} u, \operatorname{dn} u$ are the three elliptic functions. Their differentiations are,

$$
\begin{aligned}
\frac{d \phi}{d u} & =\Delta \phi & & \text { or } \frac{d \operatorname{am} u}{d u}=\operatorname{dn} u \\
\frac{d \sin \phi}{d u} & =\cos \phi \cdot \Delta \phi & & \text { or } \frac{d \operatorname{sn} u}{d u}=\operatorname{cn} u \operatorname{dn} u
\end{aligned}
$$




$$
\begin{aligned}
& \frac{d \cos \phi}{d u}=-\sin \phi \Delta \phi \quad \text { or } \frac{d \operatorname{cn} u}{d u}=-\operatorname{sn} u \operatorname{dn} u \\
& \frac{d \Delta \phi}{d u}=-\kappa^{2} \sin \phi \cos \phi \text { or } \frac{d \operatorname{dn} u}{d u}=-\kappa^{2} \operatorname{sn} u \operatorname{cn} u
\end{aligned}
$$

11.11. The complete integral over the quadrant, $0<\phi<\frac{\pi}{2}, 0<x<$ I, defines the (quarter) period, $K$,

$$
K=F \frac{\pi}{2}=\int_{0}^{\frac{1}{3} \pi} \frac{d \phi}{\Delta \phi}
$$

making

$$
\begin{aligned}
& \text { sn } K=\text { I } \\
& \text { cn } K=\circ \\
& \operatorname{dn} K=\kappa^{\prime} .
\end{aligned}
$$

$\kappa^{\prime}$ is the comodulus to $\kappa, \kappa^{2}+\kappa^{\prime 2}=\mathrm{I}$, and the coperiod, $K^{\prime}$, is,

$$
K^{\prime}=\int_{0}^{\frac{\pi}{2}} \frac{d \phi}{\sqrt{\left(I-\kappa^{\prime 2} \sin ^{2} \phi\right)}}
$$

11.12.

$$
\begin{gathered}
\operatorname{sn}^{2} u+\mathrm{cn}^{2} u=\mathrm{I} \\
\operatorname{cn}^{2} u+\kappa^{2} \operatorname{sn}^{2} u=\mathrm{I} \\
\operatorname{dn}^{2} u-\kappa^{2} \mathrm{cn}^{2} u=\kappa^{\prime 2} \\
\text { sn } \circ=0, \quad \text { cn } \circ=d, \quad \operatorname{dno}=\mathrm{I} . \\
\text { sn } K=\mathrm{I}, \quad \text { cn } K=0, \quad \operatorname{dn} K=\kappa^{\prime} .
\end{gathered}
$$

11.13. Legendre has calculated for every degree of $\theta$, the modular angle, $\kappa=\sin \theta$, the value of $F \phi$ for every degree in the quadrant of the amplitude $\phi$, and tabulated them in his Table IX, Fonctions elliptiques, t. II, $90 \times 90=8 \mathrm{I} 00$ entries.

But in this new arrangement of the Table, we take $u=F \phi$ as the independent variable of equal steps, and divide it into 90 degrees of a quadrant $K$, putting

$$
u=e K=\frac{r^{\circ}}{90^{\circ}} K, \quad r^{\circ}=90^{\circ} e .
$$

As in the ordinary trigonometrical tables, the degrees of $r$ run down the left of the page from $0^{\circ}$ to $45^{\circ}$, and rise up again on the right from $45^{\circ}$ to $90^{\circ}$. Then columns II, III, X, XI are the equivalent of Legendre's Table of $F \phi$ and $\phi$, but rearranged so that $F \phi$ proceeds by equal increments $I^{\circ}$ in $r^{\circ}$, and the increments in $\phi$ are unequal, whereas Legendre took equal increments of $\phi$ giving unequal increments in $u={ }^{\prime} F \phi$.

The reason of this rearrangement was the great advance made in elliptic function theory when Abel pointed out that $F \phi$ was of the nature of an inverse function, as it would be in a degenerate circular integral with zero modular angle. On Abel's recommendation, the notation is reversed, and $\phi$ is to be 
considered a function of $u$, denoted already by $\phi=$ am $u$, instead of looking at $u$, in Legendre's manner, as a function, $F \phi$, of $\phi$. . Jacobi adopted the idea in his Fundamenta nova, and employs the elliptic functions

$$
\sin \phi=\sin \mathrm{am} u, \quad \cos \phi=\cos \mathrm{am} u, \quad \Delta \phi=\Delta \mathrm{am} u,
$$

single-valued, uniform, periodic functions of the argument $u$, with (quarter) period $K$, as $\phi$ grows from $\circ$ to $\frac{1}{2} \pi$. Gudermann abbreviated this notation to the one employed usually today.

11.2. The E. I. I is encountered in its simplest form, not as the elliptic arc, but in the expression of the time in the pendulum motion of finite oscillation, unrestricted to the small invisible motion of elementary treatment.

The compound pendulum, as of a clock, is replaced by its two equivalent particles, one at $O$ in the centre of suspension, and the other at the centre of oscillation, $P$; the particles are adjusted so as to have the same total weight as the pendulum, the same centre of gravity at $G$, and the same moment of inertia about $G$ or $O$; the two particles, if rigidly connected, are then the kinetic equivalent of the compound pendulum and move in the same way in the same field of force (Maxwell, Matter and Motion, CXXI).

Putting $O P=l$, called the simple equivalent pendulum length, and $P$ starting from rest at $B$, in Figure $\mathbf{I}$, the particle $P$ will move in the circular arc $B A B^{\prime}$ as if sliding down a smooth curve; and $P$ will acquire the same velocity as if it fell vertically $K P=N D$; this is all the dynamical theory required.

$(\text { velocity of } P)^{2}=2 g \cdot K P$,

$(\text { velocity of } N)^{2}=2 g \cdot N D \cdot \sin ^{2} A O P$ $=2 g \cdot N D \cdot \frac{N P^{2}}{O P^{2}}=\frac{g^{2}}{l^{2}} \cdot N D \cdot N A \cdot N E$, and with $A D=h, A N=y, N D$ $=h-y, A E=2 l, N E=2 l-y$, $\left(\frac{d y}{d t}\right)^{2}=\frac{2 g}{l^{2}}\left(h y-y^{2}\right)(2 l-y)=\frac{2 g}{l^{2}} Y$, where $Y$ is a cubic in $y$. Then $t$ is given by an elliptic integral of the form

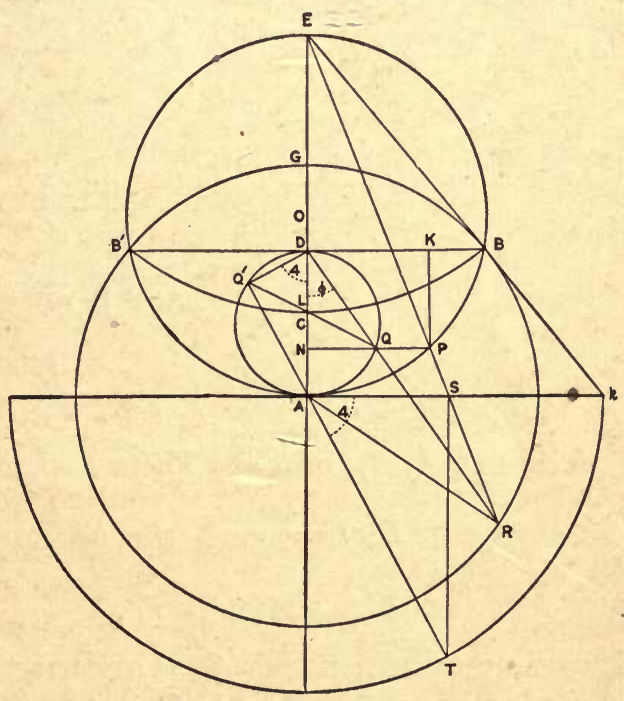

FIG. I $\int \frac{d y}{\sqrt{Y}}$. This integral is normalised to Legendre's standard form of his E. I. I by putting $y=h \sin ^{2} \phi$, making $A O Q=\phi, h-y=h \cos ^{2} \phi$, $2 l-y=2 l\left(I-\kappa^{2} \sin ^{2} \phi\right)$,

$$
\kappa^{2}=\frac{h}{2 l}=\frac{A D}{A E}=\sin ^{2} A E B .
$$

$\kappa$ is called the modulus, $A E B$ the modular angle which Legendre denoted by $\theta ; \sqrt{\left(\mathrm{I}-\kappa^{2} \sin ^{2} \phi\right)}$ he denoted by $\Delta \phi$. 
With $g=\ln ^{2}$, and reckoning the time $t$ from $A$, this makes

$$
n t=\int_{0}^{\phi} \frac{d \phi}{\Delta \phi}=F \phi
$$

in Legendre's notation. Then the angle $\phi$ is called the amplitude of $n t$, to be denoted am $n t$, the particle $P$ starting up from $A$ at time $t=0$; and with $u=n t$,

$$
\begin{aligned}
\text { sn } u & =\frac{A P}{A B}=\frac{A Q}{A D} & \operatorname{sn}^{2} u & =\frac{A N}{A D} \\
\text { cn } u & =\frac{D Q}{A D} & \mathrm{cn}^{2} u & =\frac{P K}{A D} \\
\operatorname{dn} u & =\frac{E P}{E A} & \operatorname{dn}^{2} u & =\frac{N E}{A E}
\end{aligned}
$$

Velocity of $P=n \cdot A B \cdot \mathrm{cn} u=\sqrt{B P \cdot P B^{\prime}}$, with an oscillation beat of $T$ seconds in $u=e K, e=2 t / T$.

11.21. The numerical values of $\mathrm{sn}, \mathrm{cn}, \mathrm{dn}, \operatorname{tn}(u, \kappa)$ are taken from a table to modulus $\kappa=\sin$ (modular angle, $\theta$ ) by means of the functions $\mathrm{Dr}, \mathrm{Ar}, \mathrm{Br}$, $\mathrm{Cr}$, in columns V, VI, VII, VIII, by the quotients,

$$
\begin{aligned}
\sqrt{\kappa^{\prime}} \operatorname{sn} e K & =\frac{A}{D} \\
\operatorname{cn} e K & =\frac{B}{D} \\
\frac{\operatorname{dn} e K}{\sqrt{\kappa^{\prime}}} & =\frac{C}{D} \\
\sqrt{\kappa^{\prime}} \operatorname{tn} e K & =\frac{A}{B} \\
r^{\circ} & =90^{\circ} e \\
u & =e K .
\end{aligned}
$$

These $D, A, B, C$ are the Theta Functions of Jacobi, normalised, defined by

$$
\begin{array}{ll}
D(r)=\frac{\Theta u}{\Theta o}, & A(r)=\frac{H u}{H K}, \\
B(r)=A\left(90^{\circ}-r\right) & C(r)=D\left(90^{\circ}-r\right) .
\end{array}
$$

They were calculated from the Fourier series of angles proceeding by multiples of $r^{\circ}$, and powers of $q$ as coefficients, defined by

$$
\begin{gathered}
q=e^{-\pi \frac{k^{\prime}}{k}} \\
\Theta u=\mathrm{I}-2 q \cos 2 r+2 q^{4} \cos 4 r-2 q^{9} \cos 6 r+\ldots \\
H u=2 q^{\frac{1}{1}} \sin r-2 q^{9} \sin 3^{r}+2 q^{25} \sin 5^{r}-\ldots
\end{gathered}
$$

11.3. The Elliptic Integral of the Second Kind (E. I. II) arose first historically in the rectification of the ellipse, hence the name. With $B O P=\phi$ in Figure 2, the minor eccentric angle of $P$, and $s$ the $\operatorname{arc} B P$ from $B$ to $P$ at $x=a \sin \phi$, $y=b \cos \phi$ 






$$
\frac{d s}{d \phi}=\sqrt{a^{2} \cos ^{2} \phi+b^{2} \sin ^{2} \phi}=a \Delta(\phi, \kappa),
$$

to the modulus $\kappa$, the eccentricity of the ellipse. Then $s=a \dot{E} \phi$, where $\int_{0}^{\phi} \Delta \phi \cdot d \phi$ is denoted by $E \phi$ in Legendre's notation of his standard E. I. II; it is tabulated in his Table IX alongside of $F \phi$ for every degree of the modular angle $\theta$, and to every degree in the quadrant of the amplitude $\phi$.

But it is not possible to make the inversion and express $\phi$ as a single-valued function of $E \phi$.

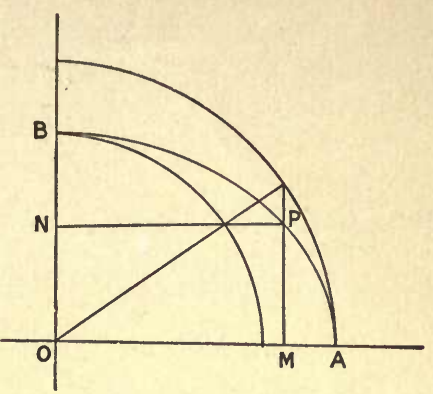

FIG. 2

11.31. The E. I. II, E $\phi$, arises also in the expression of the time, $t$, in the oscillation of a particle, $P$, on the arc of a parabola, as $F \phi$ was required on the arc

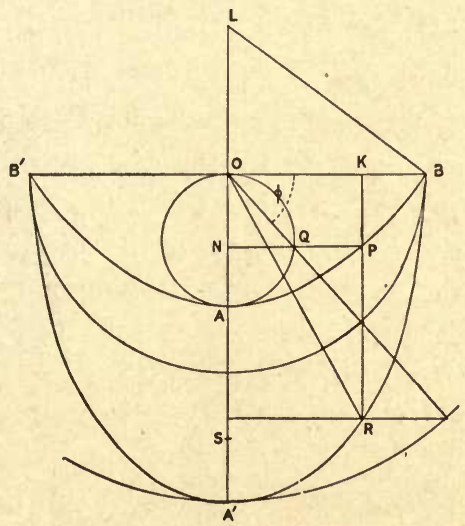
of a circle. Starting from $B$ along the parabola $B A B^{\prime}$, Figure 3 , and with $A O=h, O B=b$, $B O Q=\phi, A N=y=h \cos ^{2} \phi, N P=x=b \cos$ $\phi$ and with $O S=2 h=b \tan \alpha, O A^{\prime}=S B$ $=b \sec \alpha$, the parabola cutting the horizontal at $B$ at an angle $\alpha$, the modular angle, $B R A^{\prime} B^{\prime}$ is a semi-ellipse, with focus at $S$, and eccentricity $\kappa=\sin \alpha$.

$$
\begin{aligned}
& (\text { Velocity of } P)^{2}=\left(\frac{d x}{d t}\right)^{2}+\left(\frac{d y}{d t}\right)^{2} \\
& =\left(b^{2} \cos ^{2} \phi+4 h^{2} \sin ^{2} \phi \cos ^{2} \phi\right)\left(\frac{d \phi}{d t}\right)^{2}
\end{aligned}
$$

FIG. 3

$$
\begin{aligned}
& =a^{2}\left(\mathrm{I}-\sin ^{2} \alpha \sin ^{2} \phi\right) \cos ^{2} \phi\left(\frac{d \phi}{d t}\right)^{2}=2 g y=2 g h \cos ^{2} \phi \\
& =V^{2} \cos ^{2} \phi
\end{aligned}
$$

if $V$ denotes the velocity of $P$ at $A$, and $O A^{\prime}=a$. Then with $s$ the elliptic $\operatorname{arc} B R$,

$$
V \frac{d t}{d \phi}=a \Delta \phi=a \frac{d s}{d \phi}, V t=s
$$

and so the point $R$ moves round the ellipse with constant velocity $V$, and accompanies the point $P$ on the same vertical, oscillating on the parabola from $B$ to $B^{\prime}$.

In the analogous case of the circular pendulum, the time $t$ would be given by the arc of an Elastica, in Kirchhoff's Kinetic Analogue, and this can be placed as a bow on Figure I, with the cord along $A E$ and vertex at $B$.

Legendre has shown also how in the oscillation of $R$ on the semi-ellipse $B R B^{\prime}$ in a gravity field the time $t$ is expressible by elliptic integrals, two of the first and two of the second kind, to complementary modulus (Fonctions elliptiques, I, p. I83). 
11.32. In these tables, $E \phi$ is replaced by the columns IV, IX, of $E(r)$ and $G(r)=E(9 \circ-r)$, defined, in Jacobi's notation, by

$$
\begin{aligned}
& E(r)=\text { zn } e K=E \phi-e E \\
& G(r)=\text { zn }(\mathrm{I}-e) K, \quad r=90 e .
\end{aligned}
$$

This is the periodic part of $E \phi$ after the secular term $e E=\frac{E}{K} u$ has been set aside, $E$ denoting the complete E. I. II,

$$
E=E \frac{1}{2} \pi=\int^{\frac{1}{2} \pi} \Delta \phi \cdot d \phi .
$$

The function $z$ n $u$, or $Z u$ in Jacobi's notation, or $E(r)$ in our notation, is calculated from the series,

$$
E r=Z u=\frac{\pi}{K} \sum_{m=1}^{\infty} \frac{\sin 2 m r}{\sinh m \pi \frac{K^{\prime}}{K}}=\frac{2 \pi}{K} \sum_{m=1}^{\infty}\left(q^{m}+q^{3 m}+q^{5 m}+\ldots\right) \sin 2 m r
$$

This completes the explanation of the twelve columns of the tables.

\subsection{The Double Periodicity of the Elliptic Functions.}

This can be visualised in pendulum motion if gravity is supposed reversed suddenly at $B$ (Figure $I$ ) the end of a swing; as if by the addition of a weight to bring the centre of gravity above $O$, or by the movement of a weight, as in the metronome. The point $P$ then oscillates on the arc $B E B^{\prime}$, and beats the elliptic function to the complementary modulus $\kappa^{\prime}$, as if in imaginary time, to imaginary argument $n t i=f K^{\prime} i$ : and it reaches $P^{\prime}$ on $A X$ produced, where $\tan A E P^{\prime}$ $=\tan A E B \cdot \mathrm{cn}\left(n t^{\prime} i, \kappa\right)$, or $\tan E A P^{\prime}=\tan E A B \cdot \mathrm{cn}\left(n t^{\prime}, \kappa^{\prime}\right)$; or with $\mathrm{nt}^{\prime}=v$, $D R^{\prime}=D B \cdot \mathrm{cn}\left(i v, \kappa^{\prime}\right), D R=D B \cdot \mathrm{cn}\left(v, \kappa^{\prime}\right)$, with $D R \cdot D R^{\prime}=D B^{2}, E P^{\prime}$ crossing $D B$ in $R^{\prime}$.

$$
\begin{aligned}
\operatorname{cn}(i v, \kappa) & =\frac{\mathrm{I}}{\operatorname{cn}\left(v, \kappa^{\prime}\right)} \\
\operatorname{sn}(i v, \kappa) & =\frac{i \operatorname{sn}\left(v, \kappa^{\prime}\right)}{\operatorname{cn}\left(v, \kappa^{\prime}\right)}=i \operatorname{tn}\left(v, \kappa^{\prime}\right) \\
\operatorname{dn}(i v, \kappa) & =\frac{\operatorname{dn}\left(v, \kappa^{\prime}\right)}{\operatorname{cn}\left(v, \kappa^{\prime}\right)}=\frac{I}{\operatorname{sn}\left(K^{\prime}-v, \kappa^{\prime}\right)}
\end{aligned}
$$

where $K^{\prime}$ denotes the complementary (quarter) period to comodulus $\kappa^{\prime}$.

If $m, m^{\prime}$ are any integers, positive or negative, including $\circ$,

$$
\begin{array}{ll}
\operatorname{sn}\left(u+4 m K+2 m^{\prime} i K^{\prime}\right) & =\operatorname{sn} u \\
\operatorname{cn}\left[u+4 m K+2 m^{\prime}\left(K+i K^{\prime}\right)\right] & =\operatorname{cn} u \\
\operatorname{dn}\left(u+2 m K+4 m^{\prime} i K^{\prime}\right) & =\operatorname{dn} u
\end{array}
$$

11.41. The Addition Theorem of the Elliptic Functions.

$$
\begin{aligned}
& \operatorname{sn}(u \pm v)=\frac{\operatorname{sn} u \operatorname{cn} v \operatorname{dn} v \pm \operatorname{sn} v \operatorname{cn} u \operatorname{dn} u}{I-\kappa^{2} \operatorname{sn}^{2} u \operatorname{sn}^{2} v} \\
& \text { cn }(v \pm u)=\frac{\operatorname{cn} u \operatorname{cn} v \mp \operatorname{sn} u \operatorname{dn} u \operatorname{sn} v \operatorname{dn} v}{I-\kappa^{2} \operatorname{sn}^{2} u \operatorname{sn}^{2} v} \\
& \operatorname{dn}(v \pm u)=\frac{\operatorname{dn} u \operatorname{dn} v \mp \kappa^{2} \operatorname{sn} u \operatorname{cn} u \operatorname{sn} v \operatorname{cn} v}{I-\kappa^{2} \operatorname{sn}^{2} u \operatorname{sn}^{2} v}
\end{aligned}
$$


11.42. Coamplitude Formulas, with $v= \pm K$,

$$
\begin{aligned}
& \operatorname{sn}(K-u)=\frac{\operatorname{cn} u}{\operatorname{dn} u}=\operatorname{sn}(K+u) \\
& \text { cn }(K-u)=\frac{\kappa^{\prime} \operatorname{sn} u}{\operatorname{dn} u} \\
& \operatorname{dn}(K-u)=\frac{\kappa^{\prime}}{\operatorname{dn} u}=\operatorname{dn}(K+u) \\
& \operatorname{tn}(K-u)=\frac{\mathrm{I}}{\kappa^{\prime} \operatorname{tn} u}
\end{aligned}
$$$$
\operatorname{cn}(K+u)=-\frac{\kappa^{\prime} \operatorname{sn} u}{\operatorname{dn} u}
$$$$
\operatorname{tn}(K+u)=-\frac{}{\kappa^{\prime} \operatorname{tn} u}
$$

11.43. Legendre's Addition Formula for his E. I. II,

$$
E \phi=\mathcal{S} \phi \phi \cdot d \phi=\int \mathrm{dn}^{2} u \cdot d u, \quad \phi=\mathcal{S} \operatorname{dn} u \cdot d u=\operatorname{am} u .
$$

$E \phi+E \psi-E \sigma=\kappa^{2} \sin \phi \sin \psi \sin \sigma, \psi=\operatorname{am} v, \sigma=\operatorname{am}(v+u)$

or, in Jacobi's notation,

$$
\text { zn } u+\operatorname{zn} v-\operatorname{zn}(u+v)=\kappa^{2} \operatorname{sn} u \operatorname{sn} v \operatorname{sn}(v+u),
$$

the secular part cancelling.

Another form of the Addition Theorem for Legendre's E. I. II,

$$
E \sigma-E \theta-2 E \psi=\frac{-2 \kappa^{2} \sin \psi \cos \psi \Delta \psi \sin ^{2} \phi}{I-\kappa^{2} \sin ^{2} \phi \sin ^{2} \psi}, \theta=\operatorname{am}(v-u)
$$

or, in Jacobi's notation,

$$
\mathrm{zn}(v+u)+\mathrm{zn}(v-u)-2 \mathrm{zn} v=\frac{-2 \kappa^{2} \operatorname{sn} v \operatorname{cn} v \operatorname{dn} v \mathrm{sn}^{2} u}{\mathrm{I}-\kappa^{2} \operatorname{sn}^{2} u \operatorname{sn}^{2} v} .
$$

11.5. The Elliptic Integral of the Third Kind (E. I. III) is given by the next integration with respect to $u$, and introduces Jacobi's Theta Function, $\theta u$, defined by,

$$
\begin{aligned}
& \frac{d \log \theta u}{d u}=Z u=\operatorname{zn} u \\
& \frac{\theta u}{\theta_{o}}=\exp \cdot \int_{0} \mathrm{zn} u \cdot d u .
\end{aligned}
$$

Integrating then with respect to $u$,

$\log \theta(v+u)-\log \theta(v-u)-2 u \operatorname{zn} v=\int_{0}^{-2 \kappa^{2} \operatorname{sn} v \operatorname{cn} v \operatorname{dn} v \operatorname{sn}^{2} u} \frac{1-\kappa^{2} \operatorname{sn}^{2} u \operatorname{sn}^{2} v}{I} d u$,

and this integral is Jacobi's standard form of the E. I. III, and is denoted by $-2 \Pi(u, v)$; thus,

$$
\Pi(u, v)=\int \frac{\kappa^{2} \operatorname{sn} v \operatorname{cn} v \operatorname{dn} v \operatorname{sn}^{2} u}{\mathrm{I}-\kappa^{2} \operatorname{sn}^{2} u \operatorname{sn}^{2} v} d u=u \operatorname{zn} v+\frac{1}{2} \log \frac{\Theta(v-u)}{\Theta(v+u)} .
$$

Jacobi's Eta Function, $\mathrm{H} v$, is defined by

and then

$$
\frac{\mathrm{H} v}{\Theta v}=\sqrt{\kappa} \operatorname{sn} v
$$

$$
\frac{d \log \mathrm{H} v}{d v}=\frac{\mathrm{cn} v \mathrm{dn} v}{\mathrm{sn} v}+\mathrm{zn} v, \text { denoted by zs } v
$$


so that

$$
\begin{aligned}
\int \frac{\frac{\operatorname{cn} v \operatorname{dn} v}{\operatorname{sn} v} d u}{\mathrm{I}-\kappa^{2} \operatorname{sn}^{2} u \operatorname{sn}^{2} v} & =u \frac{\operatorname{cn} v \operatorname{dn} v}{\operatorname{sn} v}+\Pi(u, v) \\
& =u \operatorname{zs} v+\frac{I}{2} \log \frac{\Theta(v-u)}{\Theta(v+u)} \\
& =\frac{\mathrm{I}}{2} \log \frac{\Theta(v-u)}{\Theta(v+u)} e^{2 u \cdot z \mathrm{~s} v}
\end{aligned}
$$

This gives Legendre's standard E. I. III,

$$
\int \frac{M}{\mathrm{I}+n \sin ^{2} \phi} \frac{d \phi}{\Delta \phi}
$$

where we put $n=-\kappa^{2} \operatorname{sn}^{2} v=-\kappa^{2} \sin ^{2} \psi$,

$$
M^{2}=-\left(\mathrm{I}+\frac{\kappa^{2}}{n}\right)(\mathrm{I}+n)=\frac{\cos ^{2} \psi \Delta^{2} \psi}{\sin ^{2} \psi}=\frac{\operatorname{cn}^{2} v \operatorname{dn}^{2} v}{\operatorname{sn}^{2} v}
$$

the normalising multiplier, $M$.

The E. I. III arises in the dynamics of the gyroscope, top, spherical pendulum, and in Poinsot's herpolhode. It can be visualized in the solid angle of a slant cone, or in the perimeter of the reciprocal cone, a sphero-conic, or in the magnetic potential of the circular base.

11.51. We arrive here at the definitions of the functions in the tables. Jacobi's $\Theta u$ and $\mathrm{H} u$ are normalised by the divisors $\theta_{o}$ and $\mathrm{H} K$, and with $r=90 e$,

$$
D(r) \text { denotes } \frac{\theta e K}{\Theta K}, \quad A(r) \text { denotes } \frac{\mathrm{H} e K}{\mathrm{H} K}
$$

while $B(r)=A(90-r), C(r)=D(9 \circ-r)$, and $B(0)=A(90)=D(0)=C(90)$ $=\mathrm{I}, \mathrm{C}(\mathrm{0})=D(90)=\frac{\mathrm{I}}{\sqrt{\kappa}}$.

Then in the former definitions,

$$
\begin{aligned}
& \frac{A(r)}{D(r)}=\frac{A(90)}{D(90)} \text { sn } u=\sqrt{\kappa^{\prime}} \operatorname{sn} e K \\
& \frac{B(r)}{D(r)}=\frac{B(0)}{D(0)} \text { cn } u=\operatorname{cn} e K \\
& \frac{C(r)}{D(r)}=\frac{C(0)}{D(0)} \operatorname{dn} u=\frac{\operatorname{dn} e K}{\sqrt{\kappa^{\prime}}} .
\end{aligned}
$$

Then, with $u=e K, v=f K, r=90 e, s=9 \circ f$,

$$
\begin{aligned}
(u, v) & =e K \operatorname{zn} f K+\frac{\mathrm{I}}{2} \log \frac{\Theta(f-e) K}{\Theta(f+e) K} \\
& =e K E(s)+\frac{\mathrm{I}}{2} \log \frac{D(s-r)}{D(s+r)} \\
\text { zn } f K & =E(s), \quad \text { zn }(\mathrm{I}-f) K=E(90-s)=G(s) .
\end{aligned}
$$


The Jacobian multiplication relations of his theta functions can then be rewritten

$$
\begin{aligned}
& D(r+s) D(r-s)=D^{2} r D^{2} s-\tan ^{2} \theta A^{2} r A^{2} s \\
& A(r+s) A(r-s)=A^{2} r D^{2} s-D^{2} r A^{2} s \\
& B(r+s) B(r-s)=B^{2} r B^{2} s-A^{2} r A^{2} s
\end{aligned}
$$

But unfortunately for the physical applications the number $s$ proves usually to be imaginary or complex, and Jacobi's expression is useless; Legendre calls this the circular form of the E. I. III, the logarithmic or hyperbolic form corresponding to real $s$. However, the complete E. I. III between the limits $\circ<\phi<\frac{1}{2} \pi$, or $\circ<u<K, \circ<e<$ I, can always be expressed by the E. I. I and II, as Legendre pointed out.

11.6. The standard forms are given above to which an elliptic integral must be reduced when the result is required in a numerical form taken from the Tables. But in a practical problem the integral arises in a general algebraical form, and theory shows that the result can always be made, by a suitable substitution, to depend on three differential elements, of the I, II, III kind,

$$
\begin{aligned}
& \text { I } \frac{d s}{\sqrt{S}} \\
& \text { II }(s-a) \frac{d s}{\sqrt{S}} \\
& \text { III } \frac{\text { I }}{(s-\sigma)} \frac{d s}{\sqrt{S}}
\end{aligned}
$$

where $S$ is a cubic in the variable $s$ which may be written, when resolved into three factors,

$$
\dot{S}=4 \cdot s-s_{1} \cdot s-s_{2} \cdot s-s_{3}
$$

in the sequence $\propto>s_{1}>s_{2}>s_{3}>-\propto$, and normalised to a standard form of zero degree these differential elements are

$$
\begin{gathered}
\text { I } \frac{\sqrt{s_{1}-s_{3}} d s}{\sqrt{S}} \\
\text { II } \frac{s-a}{\sqrt{s_{1}-s_{3}}} \frac{d s}{\sqrt{S}} \\
\text { III } \frac{\frac{1}{2} \sqrt{\Sigma}}{s-\sigma} \frac{d s}{\sqrt{S}}
\end{gathered}
$$

$\Sigma$ denoting the value of $S$ when $s=\sigma$.

The relative positions of $s$ and $\sigma$ in the intervals of the sequence require preliminary consideration before introducing the Elliptic Functions and their notation. 
11.7. For the E. I. I and its representation in a tabular form with

$$
\begin{aligned}
\kappa^{2}=\frac{s_{2}-s_{3}}{s_{1}-s_{3}}, & \kappa^{\prime 2}=\frac{s_{1}-s_{2}}{s_{1}-s_{3}} \\
K=\int_{s_{1}, s_{3}}^{\infty, s_{2}} \frac{\sqrt{s_{1}-s_{3}} d s}{\sqrt{S}}, & K^{\prime}=\int_{s_{2},-\infty}^{s_{1}, s_{3}} \frac{\sqrt{s_{1}-s_{3}} d s}{\sqrt{-S}},
\end{aligned}
$$

and utilizing the inverse notation, then in the first interval of the sequence,

$$
\begin{gathered}
\quad \propto>s>s_{1} \\
e K=\int_{s}^{\infty} \frac{\sqrt{s_{1}-s_{3}} d s}{\sqrt{S}}=\mathrm{sn}^{-1} \sqrt{\frac{s_{1}-s_{3}}{s-s_{3}}}=\mathrm{cn}^{-1} \sqrt{\frac{s-s_{1}}{s-s_{3}}}=\mathrm{dn}^{-1} \sqrt{\frac{s-s_{2}}{s-s_{3}}} \\
(\mathrm{I}-e) K=\int_{s_{1}}^{s} \frac{\sqrt{s_{1}-s_{3}} d s}{\sqrt{S}}=\mathrm{sn}^{-1} \sqrt{\frac{s-s_{1}}{s-s_{2}}}=\mathrm{cn}^{-1} \sqrt{\frac{s_{1}-s_{2}}{s-s_{2}}}=\mathrm{dn}^{-1} \sqrt{\frac{s_{1}-s_{2} \cdot s-s_{3}}{s_{1}-s_{3} \cdot s-s_{2}}}
\end{gathered}
$$

indicating the substitutions,

$$
\frac{s_{1}-s_{3}}{s-s_{3}}=\sin ^{2} \phi=\operatorname{sn}^{2} e K, \quad \frac{s-s_{1}}{s-s_{2}}=\sin ^{2} \psi=\operatorname{sn}^{2}(\mathrm{I}-e) K .
$$

In the next interval $S$ is negative, and the comodulus $\kappa^{\prime}$ is required.

$$
\begin{gathered}
s_{1}>s>s_{2} \\
f K^{\prime}=\int \frac{s_{1} \frac{\sqrt{s_{1}-s_{3}}}{\sqrt{-S}}=\mathrm{sn}^{-1} \sqrt{\frac{s_{1}-s}{s_{1}-s_{2}}}=\mathrm{cn}^{-1} \sqrt{\frac{s-s_{2}}{s_{1}-s_{2}}}=\mathrm{dn}^{-1} \sqrt{\frac{s-s_{3}}{s_{1}-s_{3}}}}{(\mathrm{I}-f) K^{\prime}=\int_{s_{2}} \frac{\sqrt{s_{1}-s_{3}} d s}{\sqrt{-S}}=\mathrm{sn}^{-1} \sqrt{\frac{s_{1}-s_{3} \cdot s-s_{2}}{s_{1}-s_{2} \cdot s-s_{3}}}=\mathrm{cn}^{-1} \sqrt{\frac{s_{2}-s_{3} \cdot s_{1}-s}{s_{1}-s_{2} \cdot s-s_{1}}}} \\
=\mathrm{dn}^{-1} \sqrt{\frac{s_{2}-s_{3}}{s-s_{3}}}
\end{gathered}
$$

$S$ is positive again in the next interval, and the modulus is $\kappa$.

$$
\begin{aligned}
& s_{2}>s>s_{3} \\
& (\mathrm{I}-e) K=\int_{s}^{s_{2}} \frac{\sqrt{s_{1}-s_{3}} d s}{\sqrt{S}}=\mathrm{sn}^{-1} \sqrt{\frac{s_{1}-s_{3} \cdot s_{2}-s}{s_{2}-s_{3} \cdot s_{1}-s}}=\mathrm{cn}^{-1} \sqrt{\frac{s_{1}-s_{2} \cdot s-s_{3}}{s_{2}-s_{3} \cdot s_{1}-s}} \\
& =\mathrm{dn}^{-1} \sqrt{\frac{s_{1}-s_{2}}{s_{1}-s}} \\
& e K=\int_{s_{3}}^{s} \frac{\sqrt{s_{1}-s_{3}} d s}{\sqrt{S}}=\mathrm{sn}^{-1} \sqrt{\frac{s-s_{3}}{s_{2}-s_{3}}}=\mathrm{cn}^{-1} \sqrt{\frac{s_{2}-s}{s_{2}-s_{3}}}=\mathrm{dn}^{-1} \sqrt{\frac{s_{1}-s}{s_{1}-s_{3}}}
\end{aligned}
$$

indicating the substitutions,

$$
\begin{gathered}
\frac{s_{1}-s_{2}}{s_{1}-s}=\Delta^{2} \psi=\operatorname{dn}^{2}(I-e) K, \quad \frac{s-s_{3}}{s_{2}-s_{3}}=\sin ^{2} \phi=\operatorname{sn}^{2} e K \\
s=s_{2} \sin ^{2} \phi+s_{3} \cos ^{2} \phi .
\end{gathered}
$$


$S$ is negative again in the last interval, and the modulus $\kappa^{\prime}$.

$$
\begin{gathered}
s_{3}>s>-\infty \\
(\mathrm{I}-f) K^{\prime}=\int_{s}^{s_{3}} \frac{\sqrt{s_{1}-s_{3}} d s}{\sqrt{-S}}=\mathrm{sn}^{-1} \sqrt{\frac{s_{3}-s}{s_{2}-s}}=\mathrm{cn}^{-1} \sqrt{\frac{s_{2}-s_{3}}{s_{2}-s}}=\mathrm{dn}^{-1} \sqrt{\frac{s_{2}-s_{3} \cdot s_{1}-s}{s_{1}-s_{3} \cdot s_{2}-s}} \\
f K^{\prime}=\int_{-\infty}^{s} \frac{\sqrt{s_{1}-s_{3}} d s}{\sqrt{-S}}=\mathrm{sn}^{-1} \sqrt{\frac{s_{1}-s_{3}}{s_{1}-s}}=\mathrm{cn}^{-1} \sqrt{\frac{s_{3}-s}{s_{1}-s}}=\mathrm{dn}^{-1} \sqrt{\frac{s_{2}-s}{s_{1}-s}}
\end{gathered}
$$

11.8. For the notation of the E. I. II and the various reductions, take the treatment given in the Trans. Am. Math. Soc., I907, vol. 8, p. 450. The Jacobian Zeta Function and the Er, Gr of the Tables, are defined by the standard integral

$$
\int_{s_{3}}^{s} \frac{s_{1}-s}{\sqrt{s_{1}-s_{3}}} \frac{d s}{\sqrt{S}}=\int_{0}^{{ }^{\phi} \phi} \Delta \phi \cdot d \phi=E \phi=\int_{0}^{e} \mathrm{dn}^{2}(e K) \cdot d(e K)=E \text { am } e K=e H+\mathrm{zn} e K,
$$

or,

$$
\int_{s_{2}}^{\sigma} \frac{\sigma-s_{3}}{\sqrt{s_{1}-s_{3}}} \frac{d \sigma}{\sqrt{-\Sigma}}=\int_{0}^{f} \operatorname{dn}^{2}\left(f K^{\prime}\right) \cdot d\left(f K^{\prime}\right)=E \operatorname{am} f K^{\prime}=f H^{\prime}+\text { zn } f K^{\prime},
$$

where zn is Jacobi's Zeta Function, and $H, H^{\prime}$ the complete E. I. II to modulus $\kappa, \kappa^{\prime}$, defined by,

$$
\begin{aligned}
& H=\int_{0}^{\frac{\pi}{2}} \Delta(\phi, \kappa) d \phi=\int_{0}^{\mathrm{I}} \mathrm{dn}^{2}(e K) \cdot d(e K) \\
& H^{\prime}=\int_{0}^{\frac{\pi}{2}} \Delta\left(\phi, \kappa^{\prime}\right) d \phi=\int_{0}^{\mathrm{I}} \mathrm{dn}^{2}\left(f K^{\prime}\right) \cdot d\left(f K^{\prime}\right) .
\end{aligned}
$$

The function zn $u$ is derived by logarithmic differentiation of $\theta u$, zn $u=\frac{d \log \Theta u}{d u}$, or concisely,

$$
\Theta u=\exp \iint \mathrm{zn} u \cdot d u
$$

and a function zs $u$ is derived similarly from

$$
\text { zs } \begin{aligned}
u & =\frac{d \log H u}{d u} \\
& =\frac{d \log \theta u}{d u}+\frac{d \log \operatorname{sn} u}{d u} \\
& =\operatorname{zn} u+\frac{\operatorname{cn} u \operatorname{dn} u}{\operatorname{sn} u}
\end{aligned}
$$

For the incomplete E. I. II in the regions,

and

$$
\infty>s>s_{1}>s_{2}>s>s_{3}
$$

$$
\operatorname{sn}^{2} e K=\frac{s_{1}-s_{3}}{s-s_{3}} \text { or } \frac{s-s_{3}}{s_{2}-s_{3}}
$$




$$
\begin{aligned}
& \int_{s}^{s_{1}} \frac{s-s_{1}}{\sqrt{s_{1}-s_{3}}} \frac{d s}{\sqrt{S}}=\int_{s}^{s_{2}} \frac{s_{2}-s}{s-s_{3}} \frac{\sqrt{s-s_{3}}}{\sqrt{S}} d s=-(\mathrm{I}-e) H+\mathrm{zs} e K \\
& \int \frac{s-s_{2}}{\sqrt{s_{1}-s_{3}}} \frac{d s}{\sqrt{S}}=\kappa^{2} \int \frac{s_{1}-s}{s-s_{3}} \frac{\sqrt{s_{1}-s_{3}}}{\sqrt{S}} d s=-(\mathrm{I}-e)\left(H-\kappa^{\prime 2} K\right)+\mathrm{zs} e K \\
& \int \frac{s-s_{3}}{\sqrt{s_{1}-s_{3}}} \frac{d s}{\sqrt{S}}=\int \frac{s_{2}-s_{3}}{s-s_{3}} \frac{\sqrt{s_{1}-s_{3}}}{\sqrt{S}} d s=(\mathrm{I}-e)(K-H)+\mathrm{zs} e K
\end{aligned}
$$

the integrals being $\infty$ at the upper limit, $s=\infty$, or at the lower limit, $s=s_{3}$ where $e=0$ and $\mathrm{zs} e K=\infty$.

So also,

$$
\begin{aligned}
& \int_{s, s_{1}}^{\infty, s} \frac{s-s_{2}}{s-s_{3}} \frac{\sqrt{s_{1}-s_{3}}}{\sqrt{S}} d s=\int_{s_{3}, s}^{s_{1} s_{2}} \frac{s_{1}-s}{\sqrt{s_{1}-s_{3}}} \frac{d s}{\sqrt{S}}=\begin{array}{l}
e H+\mathrm{zn} e K \\
(\mathrm{I}-e) H-\mathrm{zn} e K
\end{array} \\
& \int \frac{s-s_{1}}{s-s_{3}} \frac{\sqrt{s_{1}-s_{3}}}{\sqrt{S}} d s=\int \frac{s_{2}-s}{\sqrt{s_{1}-s_{3}}} \frac{d s}{\sqrt{S}}=\begin{array}{l}
e\left(H-\kappa^{\prime 2} K\right)+\mathrm{zn} e K \\
(\mathrm{I}-e)\left(H-\kappa^{\prime 2} K\right)-\mathrm{zn} e K
\end{array} \\
& \int \frac{s_{2}-s_{3}}{s-s_{3}} \frac{\sqrt{s_{1}-s_{3}}}{\sqrt{S}} d s=\int \frac{s-s_{3}}{\sqrt{s_{1}-s_{3}}} \frac{d s}{\sqrt{S}}=\begin{array}{l}
e(K-H)-\mathrm{zn} e K \\
\left(\mathrm{I}-e^{\prime} / K-H\right)+\mathrm{zn} e K
\end{array}
\end{aligned}
$$

Similarly, for the variable $\sigma$ in the regions

$\Sigma$ negative, and

$$
s_{1}>\sigma>s_{2}>s_{3}>\sigma>-\infty
$$

$$
\mathrm{sn}^{2} f K^{\prime}=\frac{s_{1}-\sigma}{s_{1}-s_{2}} \text { or } \frac{s_{1}-s_{3}}{s_{1}-\sigma}
$$

$\int_{\sigma, s_{2}}^{s_{1}, \sigma} \frac{s_{1}-\sigma}{\sqrt{s_{1}-s_{3}}} \frac{d \sigma}{\sqrt{-\Sigma}}=\int_{-\infty, \sigma}^{\sigma, s_{3}} \frac{s_{1}-s_{2}}{s_{1}-\sigma} \frac{\sqrt{s_{1}-s_{3}}}{\sqrt{-\Sigma}} d \sigma=\begin{aligned} & f\left(K^{\prime}-H^{\prime}\right)-\operatorname{zn} f K^{\prime} \\ & (\mathrm{I}-f)\left(K^{\prime}-H^{\prime}\right)+\mathrm{zn} f K^{\prime}\end{aligned}$

$$
\int \frac{\sigma-s_{2}}{\sqrt{s_{1}-s_{3}}} \frac{d \sigma}{\sqrt{-\Sigma}}=\int \frac{s_{3}-\sigma}{s_{1}-\sigma} \frac{\sqrt{s_{1}-s_{3}}}{\sqrt{-\Sigma}} d \sigma=\begin{aligned}
& f\left(H^{\prime}-\kappa^{\prime 2} K^{\prime}\right)+\operatorname{zn} f K^{\prime} \\
& (\mathrm{I}-f)\left(H^{\prime}-\kappa^{\prime 2} K^{\prime}\right)-\operatorname{zn} f K^{\prime}
\end{aligned}
$$$$
\int \frac{\sigma-s_{3}}{\sqrt{s_{1}-s_{3}}} \frac{d \sigma}{\sqrt{-\Sigma}}=\int \frac{s_{2}-\sigma}{s_{1}-\sigma} \frac{\sqrt{s_{1}-s_{3}}}{\sqrt{-\Sigma}} d \sigma=\begin{aligned}
& f H^{\prime}+\mathrm{zn} f K^{\prime} \\
& (\mathrm{I}-f) H^{\prime}-\mathrm{zn} f K^{\prime}
\end{aligned}
$$

$$
\int_{s_{2}}^{\sigma} \frac{s_{1}-s_{2}}{s_{1}-\sigma} \frac{\sqrt{s_{1}-s_{3}}}{\sqrt{-\Sigma}} d \sigma=\int_{\sigma}^{s_{3}} \frac{s_{1}-\sigma}{\sqrt{s_{1}-s_{3}}} \frac{d \sigma}{\sqrt{-\Sigma}}=(\mathrm{I}-f)\left(K^{\prime}-H^{\prime}\right)+\mathrm{zs} f K^{\prime}
$$

$\kappa^{\prime 2} \int \frac{s_{3}-\sigma}{s_{1}-\sigma} \frac{\sqrt{s_{1}-s_{3}}}{\sqrt{-\Sigma}} d \sigma=\int \frac{s_{2}-\sigma}{\sqrt{s_{1}-s_{3}}} \frac{d \sigma}{\sqrt{-\Sigma}}=-(\mathrm{I}-f)\left(H^{\prime}-\kappa^{2} K^{\prime}\right)+\mathrm{zS} f K^{\prime}$

$$
\int \frac{s_{2}-\sigma}{s_{1}-\sigma} \frac{\sqrt{s_{1}-s_{3}}}{\sqrt{-\Sigma}} d \sigma=\int \frac{s_{3}-\sigma}{\sqrt{s_{1}-s_{3}}} \frac{d \sigma}{\sqrt{-\Sigma}}=-(\mathrm{I}-f) H^{\prime}+\mathrm{zs} f K^{\prime}
$$

these last three integrals being infinite at the upper limit, $\sigma=s_{1}$, or lower limit $\sigma=-\infty$, where $f=0$, zs $f K^{\prime}=\infty$.

Putting $e=\mathrm{I}$ or $f=\mathrm{I}$ any of these forms will give the complete E. I. II, noticing that $\mathrm{zn} K^{\prime}$ and $\mathrm{zs} K^{\prime}$ are zero. 
11.9. In dealing practically with an E. I. III it is advisable to study it frst in the algebraical form of Weierstrass,

$$
\int \frac{\frac{1}{2} \sqrt{\Sigma} d s}{(s-\sigma) \sqrt{S}}
$$

where $S=4 \cdot s-s_{1} \cdot s-s_{2} \cdot s-s_{3}, \Sigma$ the same function of $\sigma$, and begin by examining the sequence of the quantities $s, \sigma, s_{1}, s_{2}, s_{3}$

Then in the region

$$
s>s_{1}>s_{2}>\sigma>s_{3},
$$

put

$$
\begin{gathered}
s-s_{3}=\frac{s_{1}-s_{3}}{\operatorname{sn}^{2} u}, \sigma-s_{3}=\left(s_{2}-s_{3}\right) \operatorname{sn}^{2} v, \kappa^{2}=\frac{s_{2}-s_{3}}{s_{1}-s_{3}}, \\
s-\sigma=\frac{s_{1}-s_{3}}{\operatorname{sn}^{2} u}\left(\mathrm{I}-\kappa^{2} \operatorname{sn}^{2} u \mathrm{sn}^{2} v\right), \frac{\sqrt{s_{1}-s_{3}} d s}{\sqrt{S}}=d u, \\
\sqrt{\Sigma}=\sqrt{s_{1}-s_{3}}\left(s_{2}-s_{3}\right) \operatorname{sn} v \operatorname{cn} v \operatorname{dn} v, \text { making } \\
\int \frac{\frac{1}{2} \sqrt{\Sigma}}{s-\sigma} \frac{d s}{\sqrt{S}}=\int \frac{\kappa^{2} \operatorname{sn} v \operatorname{cn} v \operatorname{dn} v \operatorname{sn}^{2} u}{\mathrm{I}-\kappa^{2} \operatorname{sn}^{2} u \mathrm{sn}^{2} v} d u=\Pi(u, v) .
\end{gathered}
$$

But in the region,

$$
\begin{gathered}
\sigma>s_{1}>s_{2}>s>s_{3} \\
s-s_{3}=\left(s_{2}-s_{3}\right) \mathrm{sn}^{2} u, \sigma-s_{3}=\frac{s_{1}-s_{3}}{\operatorname{sn}^{2} v}, \frac{\mathrm{I}}{2} \sqrt{\Sigma}=\left(s_{1}-s_{3}\right)^{\frac{3}{2}} \frac{\mathrm{cn} v \mathrm{dn} v}{\mathrm{sn}^{3} v} \\
\sigma-s=\frac{s_{1}-s_{3}}{\mathrm{sn}^{2} v}\left(\mathrm{I}-\kappa^{2} \operatorname{sn}^{2} u \mathrm{sn}^{2} v\right)
\end{gathered}
$$

making,

$$
\int \frac{\frac{1}{2} \sqrt{\Sigma}}{\sigma-s} \frac{d s}{\sqrt{S}}=\int \frac{\frac{\operatorname{cn} v d n v}{\operatorname{sn} v} d u}{I-\kappa^{2} \operatorname{sn}^{2} u \operatorname{sn}^{2} v}=\Pi_{1}=\Pi(u, v)+u \frac{\operatorname{cn} v \operatorname{dn} v}{\operatorname{sn} v} .
$$

In a dynamical application the sequence is usually

$$
s>s_{1}>\sigma>s_{2}>s>s_{3}
$$

or

$$
s>s_{1}>s_{2}>s>s_{3}>\sigma,
$$

making $\Sigma$ negative, and the E. I. III is then called circular; the parameter $v$ is then imaginary, and the expression by the Theta function is illusory.

The complete E. I. III, however, was shown by Legendre to be tractable and falls into four classes, lettered $\left(l^{\prime}\right)\left(m^{\prime}\right)$, p. I3 $8,\left(i^{\prime}\right),\left(k^{\prime}\right)$, pp. I33, I34 (Fonctions elliptiques, I).

$$
\begin{aligned}
s_{1}>\sigma>s_{2} & \operatorname{sn}^{2} f K^{\prime}=\frac{s_{1}-\sigma}{s_{1}-s_{2}} \\
\operatorname{cn}^{2} f K^{\prime} & =\frac{\sigma-s_{2}}{s_{1}-s_{2}} \\
\operatorname{dn}^{2} f K^{\prime} & =\frac{\sigma-s_{3}}{s_{1}-s_{3}}
\end{aligned}
$$


A.

$$
\infty>s>s_{1} \int_{s_{1}}^{\infty} \frac{\frac{1}{2} \sqrt{-\Sigma}}{s-\sigma} \frac{d s}{\sqrt{S}}=A\left(f K^{\prime}\right)=\frac{1}{2} \pi(\mathrm{I}-f)-K \operatorname{zn} f K^{\prime}
$$

B.

$$
\begin{gathered}
s_{2}>s>s_{3} \int_{s_{3}}^{s_{2} \frac{1}{2} \sqrt{-\Sigma}} \frac{d s}{\sigma-s}=B\left(f K^{\prime}\right)=\frac{1}{2} \pi f+K \operatorname{zn} f K^{\prime} \\
\begin{aligned}
s_{3}>\sigma>-\infty & =\frac{1}{2} \pi . \\
\operatorname{sn}^{2} f K^{\prime} & =\frac{s_{1}-s_{3}}{s_{1}-\sigma} \\
\mathrm{cn}^{2} f K^{\prime} & =\frac{s_{3}-\sigma}{s_{1}-\sigma} \\
\mathrm{dn}^{2} f K^{\prime} & =\frac{s_{2}-\sigma}{s_{1}-\sigma} \\
\infty>s>s_{1} \int_{s_{1}}^{\infty} \frac{\frac{1}{2} \sqrt{-\Sigma}}{s-\sigma} \frac{d s}{\sqrt{S}} & =C\left(f K^{\prime}\right)=K \mathrm{zS} f K^{\prime}-\frac{1}{2} \pi(\mathrm{I}-f) \\
s_{2}>s>s_{3} \int_{s_{3}}^{s_{2}} \frac{\frac{1}{2} \sqrt{-\Sigma}}{s-\sigma} \frac{d s}{\sqrt{S}} & =D\left(f K^{\prime}\right)=K \mathrm{zs} f K^{\prime}+\frac{1}{2} \pi f \\
D-C & =\frac{1}{2} \pi .
\end{aligned}
\end{gathered}
$$

C.

D. 


\section{TABLES OF ELLIPTIC FUNCTIONS}

By Col. R. L. Hippisley 


\begin{tabular}{|c|c|c|c|c|c|c|}
\hline $\mathbf{r}$ & $\mathrm{F} \phi$ & $\phi$ & & $E(r)$ & $\mathbf{D}(\mathbf{r})$ & $\mathbf{A}(\mathbf{r})$ \\
\hline o & 0.0000000000 & $0^{\circ}$ & $\mathbf{o}^{\prime}$ & 0.0000000000 & I. .00000 00000 & 0.0000000000 \\
\hline I & o.oI748 65792 & I & 0 & 0.0000664649 & I. 0000005812 & o.01745 23906 \\
\hline 2 & 0.0349731585 & 2 & 0 & 0.0001328485 & I.00000 23240 & 0.0348994650 \\
\hline 3 & 0.0524597377 & 3 & o & 0.0001990699 & I. .0000052264 & 0.0523359088 \\
\hline 4 & 0.0699463169 & 4 & o & 0.0002650480 & I.00000 92847 & 0.0697564107 \\
\hline 5 & 0.0874328962 & 5 & I & 0.0003307023 & I.0000I 44942 & 0.0871556642 \\
\hline 6 & o. 1049I 94754 & 6 & I & 0.0003959525 & I. 0000208483 & o. 1045283693 \\
\hline 7 & o.12240 60546 & 7 & I & 0.0004607190 & I.00002 83393 & 0.1218692343 \\
\hline 8 & o. 1398926338 & 8 & $\mathbf{I}$ & 0.0005249226 & I. $00003695^{82}$ & o.13917 29770 \\
\hline 9 & o. I573792I3I & 9 & $\mathbf{I}$ & $0.0005^{8} \quad 84849$ & I.00004 66945 & o. I5643 43264 \\
\hline ro & o.17486 57923 & IO & I & $0.00065 \quad 13283$ & I.00005 75362 & o.1736480247 \\
\hline I I & o. 19235 23716 & I I & $\mathbf{I}$ & 0.0007 I 33760 & I.00006 94702 & o. 1908088283 \\
\hline 12 & 0.2098389508 & 12 & I & $0.00077455^{23}$ & I. 0000824819 & $0.2079 \mathrm{I}$ I $5 \mathrm{IOI}$ \\
\hline 13 & 0.2273255300 & I3 & I & 0.0008347824 & I.00009 65555 & 0.2249508603 \\
\hline I4 & $0.2448 \mathrm{I} 21092$ & I4 & 2 & 0.0008939929 & I.000II I 6738 & 0.24192 I6887 \\
\hline I5 & 0.2622986885 & I5 & 2 & 0.000952 III 4 & I.00012 78184 & $0.25^{88}$ I 88257 \\
\hline 16 & $0.27978 \quad 52677$ & 16 & 2 & 0.0010090670 & I. 0001449696 & 0.2756371244 \\
\hline I7 & $0.29727 \quad 18469$ & 17 & 2 & 0.0010647903 & I.00016 31066 & $0.29237 \quad 14618$ \\
\hline I 8 & 0.3147584262 & I 8 & 2 & 0.001 II 92 I 32 & 1.00018 22072 & $0.30901 \quad 67404$ \\
\hline 19 & 0.3322450054 & I9 & 2 & 0.00II7 22694 & I. 0002022482 & 0.3255678900 \\
\hline 20 & 0.34973 I5846 & 20 & 2 & $0.001223^{8941}$ & I.00022 3205 I & 0.3420198690 \\
\hline 21 & 0.3672181639 & $2 \mathrm{I}$ & 2 & 0.0012740244 & I. .0002450525 & $0.35^{836} 7665^{8}$ \\
\hline 22 & $0.384704743 \mathrm{I}$ & 22 & 2 & 0.0013225992 & I. 0002677636 & 0.3746063009 \\
\hline 23 & 0.4021913223 & 23 & 2 & 0.0013695594 & I.00029 I3109 & 0.3907308277 \\
\hline 24 & 0.4196779016 & 24 & 2 & $0.00 I_{4} \mathrm{I} \quad 48476$ & I. 0003 I 56657 & 0.4067363347 \\
\hline 25 & 0.4371644808 & 25 & 3 & 0.0014584087 & I .00034 07982 & $0.42261 \quad 79464$ \\
\hline 26 & 0.4546510600 & 26 & 3 & 0.0015001897 & I.00036 66779 & $0.438370825 I$ \\
\hline 27 & 0.4721376393 & 27 & 3 & 0.00154 OI 398 & I.00039 32731 & 0.45399 o1723 \\
\hline 28 & $0.48962 \quad 42185$ & 28 & 3 & 0.0015782103 & I. .00042055 I 6 & $0.46947 \quad 12303$ \\
\hline 29 & 0.507 II 07977 & 29 & 3 & 0.0016 I 43549 & I. .0004484801 & $0.48480 \quad 92833$ \\
\hline 30 & 0.5245973770 & 30 & 3 & 0.0016485297 & I.00047 70246 & o.4999996593 \\
\hline $3 \mathrm{I}$ & 0.5420839562 & $3 I$ & 3 & 0.0016806931 & I.00050 6I502 & 0.51503773 II \\
\hline 32 & $0.55957 \quad 05354$ & 32 & 3 & 0.0017108062 & I. $000535^{82 \text { I } 5}$ & 0.5299189180 \\
\hline 33 & 0.577057 II 47 & 33 & 3 & 0.0017388322 & I.00056 60024 & 0.5446386870 \\
\hline 34 & 0.5945436939 & 34 & 3 & 0.0017647373 & I. 000596656 I & 0.5591925543 \\
\hline 35 & 0.6120302731 & 35 & 3 & 0.0017884901 & I.00062 7745I & 0.5735760867 \\
\hline 36 & 0.6295168524 & 36 & 3 & 0.0018100617 & I. 0006592318 & o. 5877849028 \\
\hline 37 & 0.6470034316 & 37 & 3 & 0.0018294261 & I.00069 10776 & $0.60181 \quad 46744$ \\
\hline 38 & 0.6644900108 & 38 & 3 & 0.0018465599 & I. $000723243^{8}$ & 0.61566 II 280 \\
\hline 39 & 0.6819765900 & 39 & 3 & $0.00186 \quad 14423$ & I. 0007556912 & $0.629320045^{8}$ \\
\hline 40 & 0.6994631693 & 40 & 3 & 0.0018740556 & I. .0007883803 & 0.6427872670 \\
\hline $4 I$ & 0.7169497485 & $4 I$ & 4 & 0.0018843845 & I. 00082 I2712 & 0.6560586895 \\
\hline 42 & 0.7344363278 & 42 & 4 & 0.0018924166 & I. 0008543239 & 0.6691302706 \\
\hline 43 & 0.7519229070 & 43 & 4 & 0.0018981424 & I. $000887498 \mathrm{I}$ & 0.6819980287 \\
\hline 44 & 0.7694094862 & 44 & 4 & 0.00190 I $555^{2}$ & I .00092 07533 & 0.6946580439 \\
\hline 45 & 7868960655 & 45 & 4 & $0.00190 \quad 26510$ & I. $0009540492^{\circ}$ & 0.7071064600 \\
\hline $90^{\circ} \mathrm{r}$ & $\mathrm{F} \psi$ & $\psi$ & & $\mathbf{G}(\mathbf{r})$ & $\mathbf{C}(\mathbf{r})$ & $\mathbf{B}(\mathbf{r})$ \\
\hline
\end{tabular}




\begin{tabular}{|c|c|c|c|c|c|}
\hline $\mathbf{B}(\mathbf{r})$ & $\mathrm{C}(\mathrm{r})$ & $\mathbf{G}(\mathbf{r})$ & $\psi$ & $\mathrm{F} \psi$ & $90^{\circ}-r$ \\
\hline I. .0000000000 & I.00I90 80984 & 0.0000000000 & $90^{\circ}$ & I.57379 21309 & 90 \\
\hline 0.9998476949 & I.00I90 75172 & 0.0000663384 & 89 & I. $55^{6} 30 \quad 555^{17}$ & 89 \\
\hline 0.9993908259 & I.00190 57743 & $0.000132596 \mathrm{I}$ & 88 & I. 5388 I 89724 & 88 \\
\hline 0.9986295323 & I.00190 28720 & 0.0001986928 & 87 & I. 52 I 3323932 & 87 \\
\hline $0.997564045^{8}$ & I.0018988136 & $0.000264548 \mathrm{I}$ & 86 & I. 5038458140 & 86 \\
\hline 0.9961946912 & I. 0018936042 & 0.0003300820 & 85 & I. 4863592347 & 85 \\
\hline $0.9945^{2} \quad \mathrm{I} 8855$ & I.00I88 72501 & 0.0003952149 & 84 & I. 4688726555 & 84 \\
\hline 0.9925461382 & I.00I8797590 & 0.0004598676 & 83 & I. 4513860763 & 83 \\
\hline 0.9902680513 & I. .00187 II 401 & 0.0005239616 & 82 & I. 433899497 I & 82 \\
\hline 0.9876883186 & I. $.00186 \quad 14039$ & $0.0005^{8} 74190$ & $8 I$ & I. $4164 I 29 I 78$ & $8 \mathrm{I}$ \\
\hline 0.9848077260 & I .00I 850562 I & 0.0006501626 & 80 & I.39892 63386 & 80 \\
\hline 0.9816271510 & I. 0018386282 & 0.0007121163 & 79 & I.3814397593 & 79 \\
\hline 0.9781475623 & I.00182 56165 & 0,0007732046 & 78 & I.36395 3I80I & 78 \\
\hline 0.9743700200 & I. .0018 I 15429 & 0.0008333534 & 77 & I.34646 66009 & 77 \\
\hline 0.9702956747 & I. 0017964246 & 0.0008924894 & 76 & I.32898002I 7 & 76 \\
\hline $0.96592 \quad 57675$ & I. .0017802800 & 0.0009505409 & 75 & I. 3 I I49 34424 & 75 \\
\hline 0.9612616296 & I.00176 31288 & 0.0010074371 & 74 & I. 2940068632 & 74 \\
\hline 0.9563046817 & I .00I74 499 I 8 & 0.0010631089 & 73 & I. 2765202840 & 73 \\
\hline $0.951056433^{8}$ & 1.00172 58912 & 0.001 II 74885 & 72 & I. 2590337047 & 72 \\
\hline 0.9455184846 & I.00170 58502 & 0.0011705097 & 71 & I. 24 I $54 \quad 7$ I 255 & 71 \\
\hline 0.9396925209 & I.00168 48932 & $0.001222108 I$ & 70 & I. 2240605463 & 70 \\
\hline $0.9335^{8} 03176$ & I .00I66 30459 & 0.0012722208 & 69 & I. 2065739670 & 69 \\
\hline $0.92718 \quad 37364$ & I.00I64 03347 & 0.0013207868 & 68 & I. I 890873878 & 68 \\
\hline $0.920504725^{8}$ & I.0016I 67874 & 0.0013677470 & 67 & I. I7I60 08086 & 67 \\
\hline 0.9135453203 & I .00I59 24327 & 0.0014130440 & 66 & I. I54 I I 42293 & 66 \\
\hline 0.9063076400 & I.00156 73002 & 0.0014566228 & 65 & I.1366276501 & 65 \\
\hline 0.8987938894 & I .00I $54 \quad 14205$ & 0.0014984301 & 64 & I. 1191410709 & 64 \\
\hline 0.8910063574 & I.OOI5I $4825^{2}$ & $0.0015384 \mathrm{I} 5 \mathrm{I}$ & 63 & I. 1016544916 & 63 \\
\hline $0.8829474 \mathrm{I} 6 \mathrm{I}$ & I.00148 75467 & 0.0015765289 & 62 & I.08416 79124 & 62 \\
\hline $0.8746 \mathrm{I} 95204$ & I.00I45 96182 & 0.0016127250 & $6 I$ & I. 06668 I 3332 & $6 I$ \\
\hline $0.866025207 \mathrm{I}$ & I.00I43 10738 & 0.0016469592 & 60 & I.04919 47539 & 60 \\
\hline 0.857 I6 7094I & I.00I40 I948I & 0.0016791897 & 59 & I.03170 81747 & 59 \\
\hline 0.8480478798 & I. 0013722768 & 0.0017093771 & $5^{8}$ & I.OI422 I5955 & $5^{8}$ \\
\hline 0.8386703419 & I. 0013420959 & 0.0017374846 & 57 & 0.9967350162 & 57 \\
\hline 0.8290373370 & I.00I3I I 4423 & 0.0017634776 & 56 & 0.9792484370 & 56 \\
\hline $0.81915 \quad 17995$ & I .001 2803532 & $0.00178 \quad 73244$ & 55 & 0.96176 I 8578 & 55 \\
\hline 0.8090167404 & I .00I24 88666 & $0.001808995^{8}$ & 54 & 0.9442752785 & 54 \\
\hline 0.7986352473 & I.0012I 70208 & $0.001828465 \mathrm{I}$ & 53 & 0.9267886993 & 53 \\
\hline 0.7880104823 & I.00II 848546 & 0.0018457085 & 52 & 0.9093021201 & 52 \\
\hline 0.7771456818 & I.0OII5 24072 & 0.0018607047 & $5^{I}$ & 0.89 I 8 I 55409 & 5 I \\
\hline $0.766044^{1} 556$ & I. O0III 9718 I & 0.0018734353 & 50 & 0.8743289616 & 50 \\
\hline $0.754709285 \mathrm{I}$ & 1.00108 68272 & 0.0018838846 & 49 & 0.8568423824 & 49 \\
\hline 0.7431445232 & I.00105 37745 & 0.0018920395 & $4^{8}$ & $0.839355^{803 \mathrm{I}}$ & 48 \\
\hline 0.7313533926 & I. .0010206003 & 0.0018978900 & 47 & 0.8218692239 & 47 \\
\hline 0.7193394850 & I. 0009873450 & $0.00190 \quad 14287$ & 46 & $0.80438 \quad 26447$ & 46 \\
\hline 0.7071064600 & I .00095 40492 & $0.00190 \quad 26510$ & 45 & 0.7868960655 & 45 \\
\hline$A(r)$ & $\mathbf{D}(\mathbf{r})$ & $\mathbf{E}(\mathbf{r})$ & $\phi$ & $\mathrm{F} \phi$ & $\mathbf{r}$ \\
\hline
\end{tabular}


$\mathrm{K}=1.5828428043, \mathrm{~K}^{\prime}=3.153385252, \mathrm{E}=1.5588871966, \mathrm{E}^{\prime}=1.040114396$

\begin{tabular}{|c|c|c|c|c|c|c|}
\hline $\mathbf{r}$ & $\mathbf{F} \phi$ & $\phi$ & & $\mathbf{E}(\mathbf{r})$ & $\mathbf{D}(\mathbf{r})$ & $\cdot A(\mathbf{r})$ \\
\hline 0 & 0.0000000000 & $0^{\circ}$ & $\mathrm{o}^{\prime}$ & 0.0000000000 & I. .0000000000 & 0.0000000000 \\
\hline I & $0.0175^{8} 71423$ & $\mathbf{I}$ & 0 & 0.0002661187 & I. 0000023404 & 0.0174521509 \\
\hline 2 & 0.0351742845 & 2 & $\mathbf{I}$ & $0.00053 \quad 19095$ & I .00000 $935^{87}$ & $0.034898986 \mathrm{I}$ \\
\hline 3 & $0.05276 \quad 14268$ & 3 & I & 0.0007970448 & I. $.00002 \quad 10463$ & 0.0523351918 \\
\hline 4 & $0.070348569 \mathrm{I}$ & 4 & 2 & 0.00106 II979 & I.00003 73890 & 0.0697554570 \\
\hline 5 & 0.0879357 II 3 & 5 & 2 & 0.0013240433 & I.00005 83670 & $0.087154475^{8}$ \\
\hline 6 & o. $1055^{2} 28536$ & 6 & 3 & $0.0015^{8} \quad 5^{2573}$ & I.00008 39546 & o. $1045^{2} 69489$ \\
\hline 7 & 0.12310 99959 & 7 & 3 & 0.0018445182 & I.000II 4I206 & o.12186 75849 \\
\hline 8 & 0.1406971382 & 8 & 4 & 0.00210 I5066 & I.000I4 88284 & 0.13917 I I019 \\
\hline 9 & 0.1582842804 & 9 & 4 & 0.0023559064 & I.000I 880356 & o. 1564322298 \\
\hline I0 & o.17587 I4227 & I0 & 5 & 0.0026074044 & I.00023 I6945 & o. 1736457109 \\
\hline I I & o. $193458565^{\circ}$ & II & 5 & 0.0028556913 & I. $00027975 \mathrm{r} 8$ & 0.1908063023 \\
\hline 12 & 0.2110457072 & 12 & 5 & 0.0031004619 & I.000332 I 49I & $0.207908777 \mathrm{I}$ \\
\hline 13 & 0.2286328495 & I3 & 6 & 0.00334 I4I53 & I. .0003888224 & $0.224947926 \mathrm{I}$ \\
\hline 14 & 0.2462 I 99918 & I4 & 6 & 0.0035782555 & I.00044 97028 & 0.2419185595 \\
\hline I 5 & 0.2638071340 & I 5 & 7 & 0.0038106920 & 1.0005147160 & $0.2588 \mathrm{I} \quad 55080$ \\
\hline I6 & 0.2813942763 & 16 & 7 & 0.0040384394 & I. $.0005^{8} 37829$ & $0.27563 \quad 3625^{2}$ \\
\hline 17 & $0.29898 \quad$ I 4186 & 17 & 7 & 0.00426 12186 & I. 0006568 I93 & 0.2923677883 \\
\hline 18 & 0.3165685609 & I 8 & 8 & 0.0044787567 & I. .0007337362 & 0.3090129003 \\
\hline 19 & $0.334 \mathrm{I} 55703 \mathrm{I}$ & 19 & 8 & 0.0046907873 & I .0008I 44399 & 0.3255638912 \\
\hline 20 & $0.35^{174} 28454$ & 20 & 8 & 0.00489705 II & I .0008988322 & $0.34201 \quad 57197$ \\
\hline $2 I$ & 0.3693299877 & 21 & 9 & 0.0050972961 & I. 0009868100 & $0.35^{8} 3633745$ \\
\hline 22 & 0.3869171299 & 22 & 9 & 0.0052912778 & I.00107 82664 & 0.37460 I 8764 \\
\hline 23 & $0.40450 \quad 42722$ & 23 & 9 & 0.0054787596 & I.00II7 30898 & 0.3907262791 \\
\hline 24 & 0.42209 14145 & 24 & I0 & 0.0056595 I $3 I$ & I.00127 II647 & 0.40673 I67II \\
\hline 25 & 0.4396785568 & 25 & IO & 0.0058333185 & I.00137 237 I 7 & $0.422613177 \mathrm{I}$ \\
\hline 26 & 0.4572656990 & 26 & IO & 0.0059999643 & I. 0014765874 & 0.4383659597 \\
\hline 27 & 0.4748528413 & 27 & I I & 0.0061592485 & I. 0015836848 & 0.4539852206 \\
\hline 28 & 0.4924399836 & 28 & II & 0.0063109780 & I.00169 35336 & 0.4694662019 \\
\hline 29 & 0.5100271258 & 29 & I I & 0.0064549693 & I. .0018059998 & $0.484804188 \mathrm{I}$ \\
\hline 30 & $0.5276 \mathrm{I} \quad 4268 \mathrm{I}$ & 30 & I I & 0.0065910484 & I.00I92 09464 & 999945073 \\
\hline 31 & $0.54520 \quad 14104$ & $3 I$ & $\mathrm{I}$ & 671905 & 03823 & 32532 I \\
\hline 32 & 0.5627885526 & 32 & 12 & 0683882 & 1577178 & 299136820 \\
\hline 33 & 0.5803756949 & 33 & 12 & 0.0069502232 & I. .0022792542 & $0.54463 \quad 34239$ \\
\hline 34 & 0.5979628372 & 34 & 12 & 0.0070531150 & I .00240 26944 & 0.5591872740 \\
\hline 35 & 5499795 & 35 & 12 & 0.0071473769 & 25278880 & 5707990 \\
\hline 36 & 0.6331371217 & 36 & 13 & 0.0072328968 & I. .0026546826 & $0.5^{8777} 96173$ \\
\hline 37 & 0.6507242640 & 37 & I3 & 0.0073095735 & 1.00278 29236 & 0.6018094008 \\
\hline 38 & $0.66831 \quad 14063$ & 38 & 13 & 0.0073773166 & 1.0029124548 & $0.615655^{8756}$ \\
\hline 39 & 0.6858985485 & 39 & 13 & 0.0074360469 & 1.0030431183 & 0.6293148239 \\
\hline 40 & $0.70348 \quad 56908$ & 40 & 13 & $0.00748 \quad 56962$ & I.003I 74755 I & $0.64278 \quad 20847$ \\
\hline $4 I$ & 0.7210728331 & $4 \mathrm{I}$ & I3 & $0.0075^{2} 62073$ & I.00330 72046 & 0.6560535555 \\
\hline 42 & 0.7386599754 & 42 & 13 & 0.0075575345 & I. .0034403056 & 0.6691251936 \\
\hline 43 & 0.756247 II76 & 43 & 13 & 0.0075796433 & I.00357 38959 & 0.6819930169 \\
\hline 44 & $0.77383 \quad 42599$ & 44 & I3 & 0.0075925102 & I. 0037078127 & 0.6946531055 \\
\hline 45 & $0.79142 \quad 14022$ & 45 & 13 & 0.0075961235 & I. $.00384 \quad$ I 8928 & 0.7071016026 \\
\hline $90-r$ & $\mathrm{~F} \psi$ & & $\mathrm{G}(\mathbf{r})$ & $\mathrm{C}(\mathbf{r})$ & $\mathbf{B}(\mathbf{r})$ \\
\hline
\end{tabular}


TABLE $\theta=10^{\circ}$

$q=0.00191359459017$, Ө $0=0.9961728108, \mathrm{HK}=0.418305976553$

\begin{tabular}{|c|c|c|c|c|c|}
\hline $\mathbf{B}(\mathbf{r})$ & $\mathbf{C}(\mathbf{r})$ & $\mathbf{G}(\mathbf{r})$ & $\psi$ & $\mathbf{F} \psi$ & $90-r$ \\
\hline I. 0000000000 & I. $00768 \quad 37857$ & 0.0000000000 & $90^{\circ}$ & I. 5828428043 & 90 \\
\hline 0.9998476907 & I. 00768 I 4453 & 0.0002640908 & 89 & I. $565255662 I$ & 89 \\
\hline 0.9993908092 & I. 0076744270 & 0.0005278635 & 88 & I. 5476685198 & 88 \\
\hline 0.9986294947 & I.00766 27394 & 0.0007910004 & 87 & I. $53008 \quad$ I 3775 & 87 \\
\hline 0.9975639792 & I.00764 63966 & 0.0010531846 & 86 & I. 5 I249 42353 & 86 \\
\hline 0.996 I9 $45^{873}$ & I. .0076254187 & 0.0013141001 & $85^{\circ}$ & I.49490 70930 & 85 \\
\hline $0.9945^{2}$ I 7362 & I .00759 983 II & 0.0015734327 & 84 & I.47731 99507 & 84 \\
\hline o.99254 59357 & I.00756 $9665^{\circ}$ & 0.0018308697 & 83 & I. 4597328084 & 83 \\
\hline 0.9902677878 & I .00753 49572 & 0.0020861008 & 82 & I. 4421456662 & 82 \\
\hline 0.9876879866 & I.00749 57500 & 0.0023388 I 83 & $8 I$ & I. 4244585239 & $8 \mathrm{I}$ \\
\hline $0.9848073 \mathrm{I} 8 \mathrm{I}$ & I.00745 20912 & $0.0025^{8} 87173$ & 80 & I.40697 I3816 & 80 \\
\hline 0.9816266600 & I. $.00740 \quad 40338$ & 0.0028354962 & 79 & I.38938 42394 & 79 \\
\hline $0.978 I_{4} 69814$ & I.00735 I6366 & 0.0030788572 & 78 & I.37I79 7097I & 78 \\
\hline 0.9743693426 & I. .0072949632 & 0.0033185063 & 77 & I. 3542099548 & 77 \\
\hline 0.9702948945 & I. .0072340828 & 0.0035541538 & 76 & I. $33662 \quad 28125$ & 76 \\
\hline 0.9659248785 & I.007I6 90696 & $0.0037855^{150}$ & 75 & I.31903 56703 & 75 \\
\hline 0.9612606262 & I.00710 00027 & 0.0040123098 & 74 & I. 3014485280 & 74 \\
\hline $0.95^{6} 30355^{86}$ & I. .0070269663 & 0.0042342636 & 73 & I. 28386 I3857 & 73 \\
\hline $0.951055^{186 I}$ & I.00695 00494 & 0.00445 I 1077 & 72 & I. 2662742435 & 72 \\
\hline $0.9455^{1} 71076$ & I. 0068693457 & 0.0046625790 & $7 I$ & I.2486871012 & $7 \mathrm{I}$ \\
\hline 0.93969 I0107 & I. .0067849535 & 0.0048684209 & 70 & I.23I09 $995^{89}$ & 70 \\
\hline 0.9335786703 & I.00669 69756 & 0.0050683836 & 69 & I. 2135 I 28167 & 69 \\
\hline $0.92718 \quad 19488$ & I .00660 $55^{192}$ & 0.0052622237 & 68 & I. I9592 56744 & 68 \\
\hline 0.9205027950 & I .0065I $0695^{8}$ & 0.0054497055 & 67 & I. I78338532 I & 67 \\
\hline 0.9135432440 & I. 0064126209 & 0.0056306006 & 66 & I. I6075 I3898 & 66 \\
\hline 0.9063054160 & $1.00631 \quad 14139$ & $0.00580 \quad 46884$ & Io & I. 1431642476 & 65 \\
\hline 0.89879 I 5164 & I.00620 71982 & 0.00597 I756I & IO & I. I2557 71053 & 64 \\
\hline $0.89100 \quad 38343$ & I.00610 01007 & 0.006 I3 I 5997 & 63 & I . I079899630 & 63 \\
\hline 0.8829447424 & I .0059902520 & 0.0062840232 & 62 & I. 0904028208 & 62 \\
\hline $0.8746 \mathrm{I} 6696 \mathrm{I}$ & I. $005^{87} 77^{8} 5^{8}$ & 0.0064288398 & $6 I$ & I.0728I 56785 & $6 I$ \\
\hline $0.86602 \quad 22325$ & I. .0057628392 & $0.0065^{6} 5^{8716}$ & 60 & I. $055^{22} 85362$ & 60 \\
\hline 0.857 I6 39703 & I .00564 $555^{22}$ & 0.0066949498 & 12 & I .03764 I3940 & 59 \\
\hline 0.8480446080 & I. $0055^{2} 60678$ & 0.0068 I 59 I 54 & 12 & I. 02005425 I 7 & $5^{8}$ \\
\hline 0.8386669240 & I. $.00540 \quad 45314$ & 0.0069286187 & 12 & I.00246 71094 & 57 \\
\hline 0.8290337754 & I.00528 I0912 & 0.0070329201 & 56 & $0.984879967 \mathbf{I}$ & 56 \\
\hline 0.8191480969 & I.005I5 $5^{8975}$ & 0.0071286900 & 12 & 0.9672928249 & 55 \\
\hline 0.8090129003 & I.00502 91030 & $0.00721 \quad 5^{8089}$ & 54 & 0.9497056826 & 54 \\
\hline $0.79863 \quad 12733$ & I. .0049008620 & 0.0072941679 & 53 & 0.932 I I 85403 & 53 \\
\hline 0.7880063786 & I. $.00477 \quad 13308$ & 0.0073636683 & I3 & 0.91453 I 3981 & $5^{2}$ \\
\hline $0.77714 \quad 14532$ & I. .0046406672 & $0.00742 \quad 42224$ & I3 & $0.896944255^{8}$ & $5^{I}$ \\
\hline 0.7660398071 & I.00450 90305 & 0.0074757531 & 50 & 0.879357 II35 & 50 \\
\hline $0.75470 \quad 48222$ & I .00437 65809 & 0.0075 I 8I94I & 49 & 0.8617699712 & 49 \\
\hline 0.7431399518 & I. 0042434799 & $0.00755 \quad 14902$ & 48 & 0.8441828290 & 48 \\
\hline 0,7313487191 & I. 0041098897 & o.00757 55973 & 47 & 0.8265956867 & 47 \\
\hline 0.7193347160 & I.00397 59729 & 0.0075904823 & 46 & o. 8090085444 & 46 \\
\hline $0.70710 \quad 16026$ & I. $00384 \quad 18928$ & 0.0075961235 & $45 \quad 13$ & $0.79142 \quad 14022$ & 45 \\
\hline$A(r)$ & $\mathbf{D}(\mathbf{r})$ & $\mathbf{E}(\mathbf{r})$ & $\phi$ & $\mathbf{F} \phi$ & $\mathbf{r}$ \\
\hline
\end{tabular}


$K=1.5981420021, \quad K^{\prime}=K \sqrt{3}=2.7680631454, \quad E=1.5141501939, \quad E^{\prime}=1.076405113$

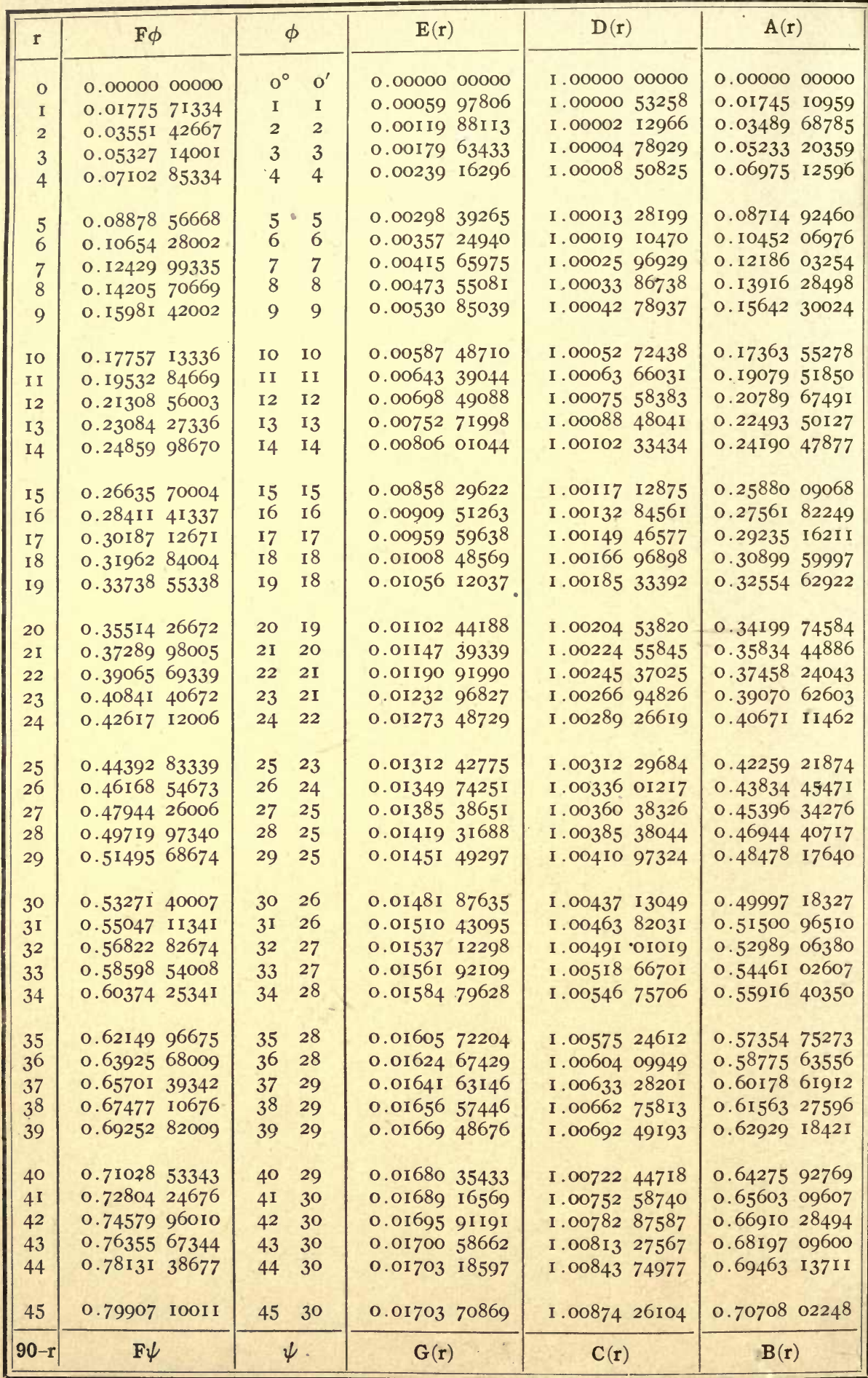

SMITHSONIAN TABLES 




TABLE $\theta=15^{\circ}$

$q=0.004333420509983, \quad \theta 0=0.9913331597, \quad$ HK $=0.5131518035$

\begin{tabular}{|c|c|c|c|c|c|}
\hline $\mathbf{B}(\mathbf{r})$ & $\mathbf{C}(\mathbf{r})$ & $\mathbf{G}(\mathbf{r})$ & $\psi$ & $\mathrm{F} \psi$ & $90-r$ \\
\hline I.00000 00000 & I.OI748 52237 & 0.0000000000 & $90^{\circ}$ & I.598I4 2002 I & 90 \\
\hline 0.9998476723 & I. OI747 98979 & 0.0005894801 & 89 & I. $58038 \quad 48688$ & 89 \\
\hline $0.999390735^{6}$ & I.01746 39271 & 0.0011782606 & 88 & I. 5626277354 & 88 \\
\hline 0.9986293293 & I.01743 73307 & 0.0017656424 & 87 & I . 5448706021 & 87 \\
\hline $0.9975^{6} 36857$ & I. OI740 OI4I2 & $0.002350928 \mathrm{I}$ & 86 & I. 527 I I 34687 & 86 \\
\hline 0.9961941297 & I.0I735 24037 & 0.0029334228 & 85 & I.50935 63353 & 85 \\
\hline $0.9945^{2} 10792$ & I.01729 41766 & 0.0035 I $2434^{2}$ & 84 & I. 4915992020 & 84 \\
\hline 0.9925450444 & I.OI722 55307 & 0.0040872741 & 83 & I . 4738420686 & 83 \\
\hline 0.9902666280 & I. OI7I4 65496 & $0.00465 .725^{89}$ & 82 & I. 4560849353 & 82 \\
\hline 0.9876865251 & I.01705 73297 & $0.00522 \quad 17102$ & $8 \mathrm{I}$ & I. 4383278019 & $8 I$ \\
\hline $0.98480 \quad 55^{225}$ & I.01695 79795 & o.0057799557 & 80 & I. 4205706685 & 80 \\
\hline 0.9816244990 & I.0168486202 & $0.00633 \mathrm{I} 3300$ & 79 & I. $402813535^{2}$ & 79 \\
\hline 0.9781444248 & I. oI672 93849 & 0.0068751750 & 78 & I. 3850564019 & 78 \\
\hline 0.97436 636I3 & I. 0166004190 & $0.0074 \mathrm{I} \quad 08412$ & 77 & I.3672992685 & 77 \\
\hline 0.9702914608 & I.01646 I8796 & 0.0079376880 & 76 & I. 3495421352 & 76 \\
\hline $0.965920966 \mathrm{I}$ & I.oI63I 39354 & $0.00845 \quad 50845$ & 75 & I. 3317850018 & 75 \\
\hline 0.9612562102 & I.0I6I5 67668 & 0.0089624102 & 74 & I.3I 40278684 & 74 \\
\hline $0.95^{629} 8615^{8}$ & I.oI599 0565I & 0.0094590560 & 73 & I. 296270735 I & 73 \\
\hline 0.9510496947 & I.OI58I 55329 & 0.0099444245 & 72 & I. $2785 \mathrm{I} 36017$ & 72 \\
\hline $0.9455^{\mathrm{I}} 10478$ & I. . 1563 I 8834 & $0.0104 \mathrm{I} \quad 79308$ & 71 & I. 2607564684 & $7 \mathrm{I}$ \\
\hline 0.9396843642 & I.oI 54398405 & o.0108790033 & 70 & I. 2429993350 & 70 \\
\hline $0.93357 \quad 14207$ & I.oI523 96380 & 0.0113270844 & 69 & I. 2252422016 & 69 \\
\hline 0.9271740815 & I.OI 503 I 5198 & 0.01176 16310 & 68 & I. $20748 \quad 50683$ & 68 \\
\hline o.92049 42975 & I. OI 48I 57396 & 0.0121821151 & 67 & I. I8972 79349 & 67 \\
\hline 0.9135341057 & I.01459 25602 & $0.0125^{8} 80246$ & 66 & I. 1719708016 & 66 \\
\hline 0.9062956284 & I. 0143622536 & 0.0129788640 & 65 & I. I 542 I 36682 & 65 \\
\hline 0.89878 10728 & I.01412 51003 & 0.013354 I547 & 23 & I. 13645 $6534^{8}$ & 64 \\
\hline 0.8909927303 & I. oI388 I3892 & o.0137 I 34359 & 24 & I. I I 869940 I 5 & 63 \\
\hline $0.882932975^{6}$ & I.01363 I4I74 & 0.0140562649 & 62 & I. 100942268 I & 62 \\
\hline $0.874604266 \mathrm{I}$ & I.OI337 54893 & $0.0143^{8} 22180$ & 25 & I. .0831851348 & $6 \mathrm{I}$ \\
\hline 0.8660091414 & I. OI3II 39167 & 0.0146908906 & 26 & I. 0654280014 & 60 \\
\hline 0.8571502219 & I. 0128470184 & 0.01498 I 8982 & 59 & I. 0476708681 & 59 \\
\hline 0.8480302085 & I. .01 2575 I I95 & 0.0152548767 & $5^{8}$ & I. 0299 I 37347 & $5^{8}$ \\
\hline 0.83865 I 8817 & I. 0122985512 & o.01550 94825 & 57 & I.012I5 660I4 & 57 \\
\hline 0.8290181005 & I.O1201 76507 & o.or574 53939 & $\begin{array}{ll}56 & 28\end{array}$ & 0.9943994680 & 56 \\
\hline 0.8191318020 & I. OII73 27599 & 0.0159623105 & 55 & 0.9766423346 & 55 \\
\hline 0.8089959997 & I. OI I $44 \quad 42262$ & 0.0I6I5 99545 & 54 & $0.95^{888} 52013$ & 54 \\
\hline $0.79861 \quad 37836$ & I. OIII5 24009 & 0.0163380704 & 53 & 0.9411280679 & 53 \\
\hline 0.7879883184 & I. 0108576397 & $0.016496425^{8}$ & $5^{2}$ & 0.9233709346 & 52 \\
\hline 0.7771228430 & I. 01056 030I 7 & 0.0166348119 & $5 I$ & 0.9056138012 & $5^{\mathrm{I}}$ \\
\hline 0.7660206691 & I.01026 0749I & 0.0167530432 & 50 & 0.8878566678 & 50 \\
\hline $0.75468 \quad 51808$ & I.0099593468 & 0.0168509584 & 49 & 0.8700995345 & 49 \\
\hline 0.743 I I 98330 & I. 0096564622 & 0.0169284205 & 48 & 0.852342401 I & 48 \\
\hline 0.7313281506 & I .00935 24642 & 0.0169853170 & 47 & $0.8345^{8} \quad 5^{2678}$ & 47 \\
\hline 0.7193137274 & 1. 0090477232 & $0.01702 \quad 15600$ & 46 & 0.8168281344 & 46 \\
\hline 0.7070802248 & I. 0087426104 & 0.0170370869 & $45 \quad 30$ & 0.79907 IOOII & 45 \\
\hline $\mathbf{A}(\mathbf{r})$ & $\mathbf{D}(\mathbf{r})$ & $\mathbf{E}(\mathbf{r})$ & $\phi$ & $\mathbf{F} \phi$ & $\mathbf{r}$ \\
\hline
\end{tabular}


$\mathrm{K}=1.6200258991, \quad \mathrm{~K}^{\prime}=2.5045500790, \quad \mathrm{E}=1.5237992053, \quad \mathrm{E}^{\prime}=1.118377738$

\begin{tabular}{|c|c|c|c|c|c|c|c|c|c|c|}
\hline \multirow{2}{*}{$\frac{\mathbf{r}}{0 .}$} & \multicolumn{2}{|l|}{$\mathbf{F} \phi$} & \multicolumn{2}{|c|}{$\phi$} & \multicolumn{2}{|l|}{$\mathbf{E}(\mathbf{r})$} & \multicolumn{2}{|l|}{$\mathbf{D}(\mathbf{r})$} & \multicolumn{2}{|c|}{$A(r)$} \\
\hline & 0.00000 & o0000் & & $\mathrm{o}^{\prime}$ & 0.00000 & 00000 & I. .00000 & 00000 & 0.00000 & 00000 \\
\hline I & 0.01800 & 02878 & I & 2 & 0.001068 & $895^{8 \mathrm{I}}$ & I . 00000 & 962 I 8 & 0.01744 & 81883 \\
\hline 2 & 0.03600 & 05755 & 2 & 4 & 0.002136 & $655^{22}$ & I. 00003 & 84757 & 0.03489 & 10694 \\
\hline 3 & 0.05400 & 08633 & 3 & 6 & 0.00320 & 14202 & I . 00008 & 65263 & 0.05232 & 33377 \\
\hline 4 & 0.07200 & II 5 I I & 4 & 7 & 0.004262 & 22042 & I.000I 5 & 37152 & 0.06973 & 96909 \\
\hline 5 & 0.09000 & I 4388 & 5 & 9 & 0.005317 & $755^{19}$ & I . 00023 & 99605 & 0.08713 & 48313 \\
\hline 6 & 0. 10800 & 17266 & 6 & II & 0.00636 & 61189 & I .00034 & $5^{1} 572$ & 0.10450 & 34678 \\
\hline 7 & 0.12600 & 20144 & 7 & I3 & 0.007406 & 65708 & I .00046 & 91770 & o. 12184 & 03169 \\
\hline 8 & 0.14400 & 2302 I & 8 & 15 & 0.008437 & $75^{848}$ & I .0006I & I 8689 & O.I39I4 & $0105 \mathrm{I}$ \\
\hline 9 & 0.16200 & 25899 & 9 & I7 & 0.009457 & 78515 & I. .00077 & 30591 & o. I 5639 & 75697 \\
\hline I0 & o. I 8000 & 28777 & 10 & I9 & 0.010466 & 60772 & I.00095 & & o. 17360 & 74610 \\
\hline I I & I9800 ? & 31655 & I I & 20 & 0.011460 & & I.00II5 & & o. 19076 & 45434 \\
\hline I 2 & 21600 & 34532 & 12 & 22 & 0.01244 I & I 3 I 88 & I.00I36 & $543^{8}$ & 0.20786 & 5973 \\
\hline I3 & 23400 & 37410 & I3 & 24 & 0.013405 & $5^{8406}$ & I.00I59 & & 0.22489 & 94205 \\
\hline 14 & $0.25^{200}$ & 40288 & I4 & 25 & 0.014353 & 33370 & I. .00184 & & 0.24186 & 68298 \\
\hline I 5 & .27000 & 43165 & 15 & 27 & 0.015282 & 26 I 80 & I.002II & 61200 & $0.25^{876}$ & 06626 \\
\hline 16 & 28800 & 46043 & 16 & 28 & 0.016192 & 97 & I .00240 & 00704 & 0.27557 & 786 \\
\hline I 7 & 30600 & $4892 \mathrm{I}$ & 17 & 30 & $0.01708 \mathrm{I}$ & & I. .00270 & 03405 & 230 & 609 \\
\hline I 8 & 32400 & 517 & I 8 & 32 & o.017949 & 96683 & I.0030I & 65642 & 894 & 182 \\
\hline I9 & 0.34200 & 54676 & 19 & 33 & 0.018794 & 47304 & I. .00334 & 83565 & 0.32549 & 77855 \\
\hline 20 & 0.36000 & 575 & 20 & 35 & o.org6I 6 & 60466 & I . .00369 & & 0.34194 & 71266 \\
\hline $2 I$ & 37800 & 60431 & 21 & 36 & 0.020412 & 26046 & I.00405 & I 2 & 829 & 24349 \\
\hline 22 & 0.39600 & 63309 & 22 & 37 & 0.021183 & & I. 00443 & OI & $745^{2}$ & 87349 \\
\hline 23 & 0.41400 & 66187 & 23 & 39 & 0.021927 & 75 & I. .00482 & 28518 & 9065 & 10844 \\
\hline 24 & 0.43200 & 69064 & 24 & 40 & 0.022644 & $4 I 32 I$ & I .00522 & 60614 & 0.40665 & 45753 \\
\hline 25 & 5000 & & 25 & $4 I$ & 0.02333 & 22426 & I. .00564 & 21475 & 0.42253 & \\
\hline 26 & 0.46800 & 74820 & 26 & 42 & 0.02399 & 10740 & I . 00607 & 060 & 828 & $55^{296}$ \\
\hline 27 & 0.48600 & 77697 & 27 & 44 & 0.024619 & 98378 & I .0065I & 09067 & 390 & 33618 \\
\hline 28 & 0.50400 & 80575 & 28 & 45 & 0.025217 & 77862 & I . 00696 & 25213 & 0.46938 & $3076 I$ \\
\hline 29 & 0.52200 & 83453 & 29 & 46 & 0.02578 & 42130 & I. 00742 & 48968 & $0.4847 \mathrm{I}$ & $995^{82}$ \\
\hline 30 & & & 30 & 46 & 0.026318 & & I. .00789 & & 90 & \\
\hline $3 I$ & $0.55^{800}$ & & 31 & 47 & 0.02681 & 98888 & I . 00837 & & 94 & $65^{8} 5^{8}$ \\
\hline 32 & 0.57600 & 92086 & 32 & 48 & 0.02728 & 79396 & I . 00887 & 08946 & 982 & \\
\hline 33 & 0.59400 & 94963 & 33 & 49 & 0.02772 & 20732 & I .00937 & 05 & 0.54454 & 64 I $8 I$ \\
\hline 34 & 0.61200 & $9784 \mathrm{I}$ & 34 & 50 & 0.02812 & I 8009 & I . .00987 & 80525 & 0.55909 & 99835 \\
\hline 35 & 0.63001 & 00719 & 35 & 50 & 0.02848 & 66791 & 1.01039 & & $0.5734^{8}$ & \\
\hline 36 & $0.6480 \mathrm{I}$ & 03597 & 36 & 5 I & 0.0288 I 6 & 63091 & I.01091 & $4037 \mathrm{I}$ & $0.5^{8769}$ & 22416 \\
\hline 37 & $0.6660 \mathrm{r}$ & 06474 & 37 & $5 \mathrm{I}$ & 0.029 II 0 & 03382 & I.0I I 44 & 12669 & 0.60172 & 22208 \\
\hline $3^{8}$ & $0.6840 \mathrm{I}$ & $0935^{2}$ & 38 & 52 & 0.029368 & $8459 \mathrm{I}$ & I.0II 97 & 38011 & 0.6 I 556 & 90470 \\
\hline 39 & 0.70201 & 12230 & 39 & $5^{2}$ & 0.02959 & 04103 & I.0I25I & 09908 & 0.62922 & 84994 \\
\hline 40 & 0.72001 & I5I07 & 40 & 53 & 0.029775 & 59763 & I.01305 & 2 I 8 I 5 & 0.64269 & 64140 \\
\hline $4^{I}$ & 0.73801 & I 7985 & $4 I$ & 53 & 0.02992 & 49874 & I .01359 & $6713^{8}$ & 0.65596 & 86845 \\
\hline 42 & $0.7560 \mathrm{I}$ & 20863 & 42 & 53 & 0.030037 & 73198 & I.0I4I 4 & & 0.66904 & 12642 \\
\hline 43 & 0.77401 & 23740 & 43 & 53 & 0.03011 & 28953 & I . OI 469 & 31466 & $0.68 \mathrm{IgI}$ & oI 665 \\
\hline 44 & 0.79201 & 26618 & 44 & 53 & 0.03015 & I68 I I & I.01524 & 37112 & 0.69457 & 14668 \\
\hline 45 & $0.8 \mathrm{I} 00 \mathrm{I}$ & 29496 & 45 & 53 & 0.030153 & 36896 & I . OI 579 & 49474 & 0.70702 & 13033 \\
\hline$\overline{90-r}$ & $\mathbf{F} \psi$ & & & & $\mathbf{G}(\mathbf{r})$ & & $C(r)$ & & $B(r)$ & \\
\hline
\end{tabular}

SMITHSONIAN TABLES 


\begin{tabular}{|c|c|c|c|c|c|}
\hline $\mathbf{B}(\mathbf{r})$ & $C(r)$ & $G(r)$ & $\psi$ & $\mathbf{F} \psi$ & $90-r$ \\
\hline I.00000 00000 & I.03158 99246 & 0.0000000000 & $90^{\circ}$ & I. 6200258991 & 90 \\
\hline 0.99984762 I5 & I.03I58 03027 & 0.0010362474 & 89 & I. 6020256113 & 89 \\
\hline 0.9993905327 & I.03I $55 \quad$ I 4488 & 0.0020712902 & 88 & I. 5840253236 & 88 \\
\hline 0.9986288734 & I.03I50 33980 & 0.0031039250 & 87 & I. 5660250358 & 87 \\
\hline 0.9975628767 & I.03I43 62088 & 0.0041329509 & 86 & I. 5480247480 & 86 \\
\hline 0.9961928686 & I.03134 99632 & 0.0051571704 & 85 & I.53002 44603 & 85 \\
\hline $0.9945^{1} 92682$ & I.03124 4766I & 0.006 I 7 53910 & 84 & I.51202 41725 & 84 \\
\hline $0.9925425^{876}$ & I.0311207458 & 0.0071864259 & 83 & I.49402 38847 & 83 \\
\hline 0.99026343 I 5 & I.03097 80534 & 0.008 I 8.90957 & 82 & I. 4760235970 & 82 \\
\hline 0.9876824970 & I.03081 68627 & 0.0091822293 & $8 I$ & I. $45^{802} 33092$ & 81 \\
\hline $0.98480 \quad 05736$ & I.03063 7370I & o.010i6 4665I & 80 & I. 4400230214 & 80 \\
\hline 0.98 I6I 85429 & I.0304397942 & o.oIII3 52523 & 79 & I. $42202 \quad 27337$ & 79 \\
\hline $0.978 \mathrm{I} 37378 \mathrm{I}$ & I.03022 43759 & 0.0120928519 & 78 & I. $40402 \quad 24459$ & 78 \\
\hline 0.9743581442 & I .02999 I3775 & 0.0130363381 & 77 & I. 386022 I 58 I & 77 \\
\hline $0.97028 \quad 19968$ & I. $02974 \quad 10829$ & 0.0139645994 & 76 & I. $36802 \quad 18704$ & 76 \\
\hline 0.96591 oI 827 & I.02947 37972 & 0.0148765396 & 75. 27 & I. 35002 I5826 & 75 \\
\hline 0.9612440390 & I .029I $89845^{8}$ & 0.01577 10793 & $74 \quad 28$ & I .33202 12948 & 74 \\
\hline 0.9562849924 & I . 0288895748 & 0.0166471568 & 73 & I. 3140210070 & 73 \\
\hline 0.95 I03 45595 & I. 0285733501 & $0.01750 \quad 37292$ & 72 & I. 2960207193 & 72 \\
\hline 0.9454943456 & I. 02824 I 5568 & o.or833 97739 & $7 \mathrm{I}$ & I. $27802043 \mathrm{I} 5$ & 71 \\
\hline 0.9396660449 & I. 0278945992 & o.oI9I5 42895 & 70 & I. 26002 oI 437 & 70 \\
\hline 0.93355 I 439 I & I. $02753 \quad 28994$ & 0.0199462967 & 69 & I. $2420 \mathrm{I} 98560$ & 69 \\
\hline 0.9271523977 & I.027I5 69001 & $0.02071 \quad 48399$ & 68 & I. 2240195682 & 68 \\
\hline 0.9204708768 & I.02676 70574 & 0.02 I 458988 I & 67 & I. $20601 \quad 92804$ & 67 \\
\hline 0.9135089187 & I. 0263638468 & 0.0221778360 & 40 & I. I 88 or 89927 & 66 \\
\hline 0.9062686515 & I. 0259477596 & $0.02287 \quad 05049$ & 65 & I.I700I 87049 & 65 \\
\hline 0.8987522880 & I. $0255^{\text {I }} 93029$ & $0.023536144^{2}$ & 64 & I. I520I $8417 \mathrm{I}$ & 64 \\
\hline $0.890962125^{2}$ & I. 0250789985 & 0.0241739320 & 63 & I.1340I 8I294 & 63 \\
\hline 0.8829005436 & I.02462 73829 & $0.02478 \quad 30767$ & 62 & I. I I60I 784 I6 & 62 \\
\hline 0.8745700067 & I. 0241650064 & 0.0253628172 & 45 & I. $098017553^{8}$ & $6 I$ \\
\hline 0.8659730595 & I .0236924323 & 0.0259 I 24248 & 60 & I.0800I 7266I & 60 \\
\hline 0.857 I I 23285 & I. $.02321 \quad 02363$ & $0.02643 \quad 12037$ & 59 & I.0620I 69783 & 59 \\
\hline 0.8479905205 & I.0227 I 90060 & 0.0269184920 & $5^{8}$ & I.0440I 66905 & $5^{8}$ \\
\hline $0.83861 \quad 04218$ & I .0222 I 93398 & 0.0273736626 & 57 & I.02601 64028 & 57 \\
\hline 0.8289748973 & I.02I $71 \quad 18465$ & 0.0277961243 & 56 & I. 0080161150 & 56 \\
\hline 0.8190868896 & I.02II9 7I444 & $0.02818 \quad 53227$ & 55 & $0.99001 \quad 5^{8272}$ & 55 \\
\hline 0.8089494182 & I. 0206758606 & 0.0285407409 & 54 & 0.9720155395 & 54 \\
\hline 0.7985655784 & I. 0201486302 & 0.02886 I 9001 & 53 & 0.9540152517 & 53 \\
\hline 0.7879385407 & I.oI96I. 60955 & 0.029 I4 836II & $5^{2}$ & 0.9360149639 & $5^{2}$ \\
\hline 0.77707 I 5491 & I.0I907 89054 & 0.0293997245 & $5 \mathrm{I}$ & 0.9180146761 & $5 \mathrm{I}$ \\
\hline 0.7659679209 & I.oI 85377 I 43 & 0.0296 I 56313 & 50 & $0.90001 \quad 43884$ & 50 \\
\hline $0.75463 \quad$ I0450 & I. 0179931816 & 0.0297957642 & 49 & 0.88201 41006 & 49 \\
\hline $0.74306 \quad 43814$ & I.OI 74459707 & 0.0299398477 & 48 & $0.86401 \quad 38129$ & 48 \\
\hline $0.73127 \quad 14598$ & I.0I689 67484 & 0.0300476489 & 47 & $0.8460 \mathrm{I} 3525 \mathrm{I}$ & 47 \\
\hline 0.7192558784 & I.0I634 6I 837 & o.030II 89783 & 46 & $0.82801 \quad 32373$ & 46 \\
\hline $0.70702 \quad 13033$ & I.OI579 49474 & 0.0301536896 & $45 \quad 53$ & 0.8 1001 29496 & 45 \\
\hline $\mathbf{A}(\mathbf{r})$ & $\mathbf{D}(\mathbf{r})$ & $\mathbf{E}(\mathbf{r})$ & $\phi$ & $\mathrm{F} \phi$ & $\mathbf{r}$ \\
\hline
\end{tabular}


$K=1.6489952185, \quad K^{\prime}=2.3087867982, \quad E=1.4981149284, \quad E^{\prime}=1.1638279645$

\begin{tabular}{|c|c|c|c|c|c|c|}
\hline $\mathbf{r}$ & $\mathrm{F} \phi$ & $\phi$ & $\phi$ & $\mathbf{E}(\mathbf{r})$ & $\mathbf{D}(\mathbf{r})$ & $\mathbf{A}(\mathbf{r})$ \\
\hline o & 0.0000000000 & & $\mathrm{o}^{\prime}$ & 0.0000000000 & I. 0000000000 & 0.0000000000 \\
\hline I & 0.0183221691 & I & 3 & 0.00167608 I 5 & I.OOOOI 53565 & 0.01744 I859I \\
\hline 2 & 0.0366443382 & 2 & 6 & o.0033499667 & I.00006 14074 & 0.0348784245 \\
\hline 3 & 0.0549665073 & 3 & 9 & o.00501 94629 & I.000I3 80964 & 0.0523044041 \\
\hline 4 & 0.0732886764 & 4 & 12 & 0.0066823842 & I.00024 53303 & o.0697I 45088 \\
\hline 5 & 0.0916108455 & 5 & I 5 & $0.008336555^{I}$ & I.00038 29783 & 0.0871034544 \\
\hline 6 & o. 1099330145 & 6 & I 8 & o.0099798I39 & I .00055 08728 & o. 1044659627 \\
\hline 7 & 0.1282551836 & 7 & 21 & 0.0116100163 & I. .0007488092 & o.12179 67635 \\
\hline 8 & o. 1465773527 & 8 & 24 & 0.0132250382 & I .00097 65463 & o.1390905958 \\
\hline 9 & o. 1648995218 & 9 & 26 & o.01482 27797 & I.00123 38067 & o.15634 22095 \\
\hline IO & o. $18322 \quad 16909$ & Io & 29 & $0.01640 \quad 11677$ & I.00I5202770 & o.17354 63669 \\
\hline I I & 0.2015438600 & I I & 32 & 0.0179581596 & I. .001835608 I & o. 1906978446 \\
\hline 12 & 0.2198660291 & 12 & 35 & $0.019491745^{8}$ & I.002I 794 I 59 & 0.20779 I 4345 \\
\hline 13 & 0.2381881982 & I3 & 37 & 0.0209999533 & I. .0025512815 & 0.22482 I9454 \\
\hline 14 & $0.25651 \quad 03673$ & 14 & 40 & 0.0224808485 & I .00295 07519 & $0.241784205^{2}$ \\
\hline 15 & 0.2748325364 & I5 & 43 & 0.0239325396 & I .00337 73404 & 0.25867306 I 5 \\
\hline 16 & 0.2931547055 & 16 & 45 & 0.0253531798 & I. .0038305272 & $0.2754^{8} 33^{8} 3^{8}$ \\
\hline I 7 & 0.3114768746 & 17 & 48 & 0.0267409700 & I .00430 97603 & 0.2922100649 \\
\hline 18 & 0.3297990437 & I 8 & 50 & 0.0280941609 & I .0048I 44557 & $0.308848022 \mathrm{I}$ \\
\hline 19 & $0.34812 \quad 12128$ & I9 & 53 & $0.0294 \mathrm{I} \quad 10555$ & I .00534 39986 & 0.3253921991 \\
\hline 20 & 0.3664433819 & 20 & 56 & 0.0306900118 & I. $.005^{89} 7743^{8}$ & 418375673 \\
\hline $2 \mathrm{I}$ & 0.38476555 10 & $2 \mathrm{I}$ & 57 & 0.0319294445 & I. .0064750167 & 0.3581791274 \\
\hline 22 & 0.4030877201 & 22 & 59 & 0.0331278272 & I. .007075 II 40 & $0.3744 \mathrm{I} 19107$ \\
\hline 23 & 0.4214098892 & 24 & I & $0.03428 \quad 36945$ & I. .0076973046 & 0.3905309808 \\
\hline 24 & $0.43973285^{82}$ & 25 & 3 & 0.0353956434 & I. $00834 \quad 08304$ & 0.40653143 .52 \\
\hline 25 & $0.45^{805} 42273$ & 26 & 5 & $0.036462335^{2}$ & I .00900 49074 & 24084064 \\
\hline 26 & 0.4763763964 & 27 & 7 & 0.0374824970 & I .00968 87266 & 0.438 I5 70635 \\
\hline 27 & 0.4946985655 & 28 & 9 & 0.0384549232 & I. OIO39 I 4548 & $0.4537726 \mathrm{I} 4 \mathrm{O}$ \\
\hline 28 & 0.5130207346 & 29 & I I & 0.0393784764 & I .OI I I I $2235^{8}$ & 0.4692503045 \\
\hline 29 & 0.5313429037 & 30 & 12 & 0.0402520886 & I.0II 85 0I9I6 & $0.4845^{8} \quad 54231$ \\
\hline 30 & 0.5496650728 & $3 \mathrm{I}$ & 14 & 0.0410747627 & I .01260 44231 & o.49977 32999 \\
\hline $3 I$ & 0.5679872419 & 32 & I5 & 0.0418455726 & I.0I33740II3 & $0.5^{14} 40 \quad 93092$ \\
\hline 32 & 0.5863094110 & 33 & 16 & 0.0425636643 & I.0I4I5 80186 & 0.5296888703 \\
\hline 33 & 0.60463 I 5801 & 34 & I 8 & 0.0432282564 & I. 0149554899 & 0.5444074492 \\
\hline 34 & 0.6229537492 & 35 & 19 & 0.0438386406 & I. OI576 54535 & 0.5589605600 \\
\hline 35 & 0.6412759183 & 36 & $2 C$ & 0.0443941821 & I.oI658 69227 & 0.5733437662 \\
\hline 36 & 0.6595980874 & 37 & 2 & 0.04489431 & I.0I74I 88967 & o. 5875526819 \\
\hline 37 & 0.6779202565 & 38 & 22 & 0.04533856 & I.01826 036I 7 & $0.6015^{8} 29737$ \\
\hline 38 & 0.6962424256 & 39 & 23 & 0.04572650 & I.0I9I I 02927 & 0.6 I543 036II \\
\hline 39 & 0.7145645947 & 40 & 23 & 0.0460578000 & I.01996 76540 & 0.6290906189 \\
\hline 40 & 32886763 & $4 I$ & 23 & 0.046332 I 809 & I. $02083 \mathrm{I}_{4} \mathrm{OI} 3$ & 425595777 \\
\hline $4 \mathrm{I}$ & 0.7512089328 & 42 & 24 & 0.0465494543 & I. .0217004820 & $0.655^{83} 31255$ \\
\hline 42 & 0.76953 IIOI & 43 & 24 & 0.04670949 & I. $02257 \quad 38374$ & 0.6689072089 \\
\hline 43 & 0.7878532710 & 44 & 24 & 0.0468 I 22622 & I. $02345 \quad 04035$ & 0.6817778347 \\
\hline 44 & 0.806 I 754401 & 45 & 24 & 0.0468577678 & I. 02432 91122 & $0.69444 \quad 10704$ \\
\hline 45 & 0.8244976092 & 46 & 24 & 0.0468461065 & I .02520 88930 & 0.7068930463 \\
\hline $90-r$ & $\mathrm{~F} \psi$ & & & $\mathbf{G}(\mathbf{r})$ & $\mathbf{C}(\mathbf{r})$ & $\mathbf{B}(\mathbf{r})$ \\
\hline
\end{tabular}

SMITHSONIAN TABLES 
TABLE $\theta=25^{\circ}$

$q=0.012294560527181, \quad \theta 0=0.975410924642, \quad \mathrm{HK}=0.666076159327$

\begin{tabular}{|c|c|c|c|c|c|}
\hline $\mathbf{B}(\mathbf{r})$ & $\mathrm{C}(\mathrm{r})$ & $\mathbf{G}(\mathbf{r})$ & $\psi$ & $\mathrm{F} \psi$ & $90-r \mid$ \\
\hline I.00000 00000 & I.0504I 79735 & 0.0000000000 & $90^{\circ}$ & I .64899 52 I 85 & 90 \\
\hline O.99984 75 III I & I. $05040 \quad 26167$ & 0.0015957045 & 89 & I. 6306730494 & 89 \\
\hline 0.99939 009I2 & I. 0503565652 & 0.0031896046 & 88 & I. 6123508803 & 88 \\
\hline $0.99862788 \mathrm{I} 2$ & $1.050279875^{\circ}$ & 0.0047798977 & 87 & I.5940287112 & 87 \\
\hline 0.99756 I I I 58 & I.050I7 26395 & 0.0063647840 & 12 & I. 575706542 I & 86 \\
\hline 0.99619 oI235 & I . 0500349895 & 0.0079424686 & 85 & I. $55738 \quad 43730$ & 85 \\
\hline 0.9945 I 53263 & I. 0498670926 & 0.0095 I II 627 & 84 & I. 5390622039 & 84 \\
\hline 0.9925372400 & I .04966 91533 & 0.01 I06 90855 & 83 & I. 5207400348 & 83 \\
\hline 0.9902564734 & I. $04944 \quad$ I 4129 & 0.01261 .44653 & 82 & I. $5024 \mathrm{I} \quad 78657$ & 82 \\
\hline 0.9876737287 & I. 0491841489 & o.014I4 554I6 & $8 \mathrm{I}$ & I. 4840956966 & $8 I$ \\
\hline o.98478 98010 & I.04889 76746 & 0.0156605663 & 29. & I. 4657735275 & 80 \\
\hline 0.9816055779 & I. $0485^{8} 23391$ & 0.0171578054 & $3 I$ & I . 44745 I 3584 & 79 \\
\hline 0.978 I2 20395 & I. 0482385265 & 0.0186355407 & 78 & I.42912 91893 & 78 \\
\hline 0.9743402576 & I. 0478666559 & 0.0200920712 & 77 & I. 4108070202 & 77 \\
\hline 0.97026 I3962 & I. 0474671802 & $0.0215^{2} \quad 57149$ & 76 & I. 39248485 I I & 76 \\
\hline o.96588 67IоI & I.04704 05862 & $0.0229348 \mathrm{IO} 2$ & 75 & I. 3741626821 & 75 \\
\hline $0.961217545^{2}$ & I. 0465873936 & $0.02431 \quad 77177$ & 44 & I. $355^{84}$ O5I30 & 74 \\
\hline 0.9562553377 & I.04610 81546 & 0.0256728218 & 73 & I.3375I 83439 & 73 \\
\hline $0.95100 \quad 16139$ & I . 0456034530 & 0.0269985322 & 72 & I.31919 61748 & 72 \\
\hline 0.9454579893 & I. 0450739038 & 0.0282932857 & 52 & I. 3008740057 & 71 \\
\hline 0.939626 I686 & I. $0445^{2}$ or 522 & o.02955 55477 & 54 & I . $28255 \cdot$ I 8366 & 70 \\
\hline 0.9335079444 & I. 0439428728 & 0.0307838140 & 56 & I. 2642296675 & 69 \\
\hline 0.9271051976 & I . 0433427690 & 0.0319766123 & $5^{8}$ & I . 2459074984 & 68 \\
\hline $0.920419895^{8}$ & I. 0427205719 & 0.0331325038 & 68 & I . $2275^{8} 53293$ & 67 \\
\hline 0.9134540932 & I. 0420770396 & 0.0342500853 & 67 & I. 2092631602 & 66 \\
\hline 0.9062099299 & I.04I4I 2956I & $0.03532 \quad 79902$ & 66 & I . I9094 099 I I & 65 \\
\hline 0.8986896309 & I .04072 9I305 & 0.0363648907 & 65 & I. I7261 88220 & 64 \\
\hline $0.8908955^{05^{8}}$ & I.04002 63960 & o.0373594992 & 64 & I. I5429 66529 & 63 \\
\hline 0.8828299477 & I .03930 56088 & 0.0383105700 & 63 & I. I3597 44838 & 62 \\
\hline o. 8744954326 & I . 0385676470 & 0.0392169009 & 62 & I. II 76523 I 47 & $6 I$ \\
\hline $0.865^{89} \quad 45^{1} 84$ & I. .0378 I 34098 & 0.0400773349 & $6 I$ & I. 09933 oI 456 & 60 \\
\hline 0.8570298444 & I. $037043^{8161}$ & 0.04089076 & 60 & I. .0810079765 & 59 \\
\hline $0.847904 \mathrm{I} 300$ & I. .0362598035 & 0.0416561200 & 59 & I. 0626858075 & 58 \\
\hline 0.83852 oI744 & I. $03546 \quad 23272$ & 0.0423723976 & $5^{8}$ & I .04436 36384 & 57 \\
\hline 0.8288808549 & I. 0346523588 & 0.0430386345 & 57 & I .02604 I4693 & 56 \\
\hline 0.8189891269 & I. 0338308852 & 0.0436539236 & 56 & I.0077I 93002 & 55 \\
\hline $0.808848022 \mathrm{I}$ & I.0329989073 & 0.0442 I 74127 & 55 & 0.989397 I3 II & 54 \\
\hline 0.7984606482 & I.032I5 $743^{86}$ & 0.0447283056 & 54 & 0.9710749620 & 53 \\
\hline 0.78783 oI 874 & I.03I30 75044 & 0.0451858637 & 53 & 0.9527527929 & $5^{2}$ \\
\hline 0.7769598956 & I. 03045 OI 401 & $0.0455^{8} 94076$ & $5^{2}$ & 0.9344306238 & $5 \mathrm{I}$ \\
\hline 0.76585 3I0I & I. $0295^{8} 63905$ & 0.0459383183 & $5 \mathrm{I}$ & 0.9161084547 & 50 \\
\hline $0.7545 \mathrm{I} \quad 32053$ & I. 0287 I 73077 & 0.0462320386 & 50 & 0.8977862856 & 49 \\
\hline 0.7429436775 & I .02784 39507 & 0.0464700744 & 49 & 0.8794641165 & 48 \\
\hline 0.73 II 480583 & I. .0269673835 & 0.04665 I 9961 & 48 & 0.86 II4 I9474 & 47 \\
\hline 0.7191299561 & I. 026088674 I & 0.0467774393 & 47 & $0.84281 \quad 97783$ & 46 \\
\hline 0.7068930463 & I. 0252088930 & 0.0468461065 & 46 & 0.8244976092 & 45 \\
\hline$A(r)$ & $D(r)$ & $\mathbf{E}(\mathbf{r})$ & $\phi$ & $\mathrm{F} \phi$ & r \\
\hline
\end{tabular}

SMITHSONIAN TABLES 
$K=1.6857503548, \quad K^{\prime}=2.1565156175, \quad E=1.4674622093 \quad E^{\prime}=1.211056028$

\begin{tabular}{|c|c|c|c|c|c|c|}
\hline $\mathbf{r}$ & $\mathbf{F} \phi$ & G & $b$ & $\mathbf{E}(\mathbf{r})$ & $\mathbf{D}(\mathbf{r})$ & $\mathbf{A}(\mathbf{r})$ \\
\hline 0 & 0.0000000000 & $0^{\circ}$ & $\mathrm{o}^{\prime}$ & 0.0000000000 & I.00000 00000 & 0.0000000000 \\
\hline I & 0.0187305595 & $\mathbf{I}$ & 4 & 0.0024248763 & I.00002 27I 25 & 0.0174298716 \\
\hline 2 & 0.03746 III 90 & 2 & 9 & 0.0048464683 & I.00009 08222 & 0.034854475 I \\
\hline 3 & $0.05619 \quad 16785$ & 3 & I3 & 0.00726 I 4977 & I. 0002042462 & $0.05^{226} 8543^{8}$ \\
\hline 4 & 0.0749222380 & 4 & I 8 & 0.0096666975 & I.00036 28463 & 0.0696668140 \\
\hline 5 & 0.0936527975 & 5 & 22 & 0.0120588 I78 & I.00056 64294 & 0.0870440267 \\
\hline 6 & o.II238 33570 & 6 & 26 & 0.0144346319 & I. 0008 I 47472 & o. 1043949285 \\
\hline 7 & o.I3III 39 I65 & 7 & 30 & 0.0167909412 & I.OOI IO 74975 & 0.1217142736 \\
\hline 8 & o.14984 44760 & 8 & 35 & 0.0I9I2 $45^{8} \mathrm{I} 3$ & I.00I 4443235 & o.13899 68254 \\
\hline 9 & 0.1685750355 & 9 & 39 & 0.0214324269 & I. .0018248148 & o. 1562373574 \\
\hline IO & o.I8730 $5595^{\circ}$ & Io & 43 & 0.0237 I I3976 & I. .0022485079 & o. I7343 0655 I \\
\hline I I & 0.206036 I545 & II & 47 & 0.0259584626 & I. .0027I 48868 & o. 19057 I5I 75 \\
\hline 12 & 0.2247667140 & 12 & $5 I$ & 0.0281706459 & I.00322 33830 & $0.20765475^{84}$ \\
\hline 13 & 0.2434972734 & I3 & 55 & 0.0303450312 & I.00377 33773 & $0.224675208 \mathrm{I}$ \\
\hline I4 & 0.2622278329 & I4 & 59 & 0.0324787664 & I. 0043641996 & 0.2416277146 \\
\hline I5 & 0.2809583924 & 16 & 3 & 0.0345690685 & I.004995 51300 & $0.25^{8} 5^{0} \quad 71454$ \\
\hline 16 & 0.2996889519 & I7 & 6 & 0.0366132272 & I. .0056654000 & 0.2753083886 \\
\hline I7 & $0.3184 \mathrm{I} 95 \mathrm{II} 4$ & I 8 & IO & 0.0386086097 & I. 0063741929 & 0.2920263549 \\
\hline I 8 & 0.337 I5 00709 & 19 & I 4 & 0.0405526642 & I. 007 I 206453 & 0.3086559785 \\
\hline 19 & $0.355^{88} \quad 06304$ & 20 & I7 & 0.0424429236 & I. $00790 \quad 38477$ & 0.3251922190 \\
\hline 20 & 0.3746 I I I 899 & $2 \mathrm{I}$ & 20 & 0.0442770092 & I . 008722846 I & 0.3416300625 \\
\hline $2 I$ & $0.39334 \quad 17494$ & 22 & 23 & 0.0460526335 & I.00957 66426 & $0.3579645^{236}$ \\
\hline 22 & 0.4120723089 & 23 & 27 & 0.0477676034 & I.01046 4197I & 0.3741906461 \\
\hline 23 & $0.43080 \quad 28684$ & 24 & 30 & o.0494I 98229 & I. OII $38 \quad 44282$ & $0.390303505 \mathrm{I}$ \\
\hline 24 & 0.4495334279 & 25 & 33 & $0.051007295^{8}$ & I. 0123362150 & 0.4062982084 \\
\hline 25 & 0.4682639874 & 26 & 36 & $0.05^{252} 81275$ & I.oI33I 83978 & 0.4221698975 \\
\hline 26 & 0.4869945469 & 27 & 38 & 0.0539805273 & I. 0143297800 & 0.4379 I 37495 \\
\hline 27 & 0.5057251064 & 28 & $4 \mathrm{I}$ & 0.0553628100 & I.01536 91295 & $0.4535^{2} 49782$ \\
\hline 28 & 0.5244556659 & 29 & 43 & 0.0566733976 & I.0I643 5I800 & $0.468998835^{8}$ \\
\hline 29 & 0.5431862254 & 30 & 46 & $0.0579 \mathrm{I} \quad 08204$ & I.0I752 66329 & $0.484330614^{2}$ \\
\hline 30 & 0.56 I9I 67849 & $3 \mathrm{I}$ & 48 & 0.0590737 I 8 I & I. OI $864215^{83}$ & 0.4995 I 56464 \\
\hline 3 I & $0.5^{8064} 73444$ & 32 & 50 & 0.0601608407 & I. 0197803972 & 5493080 \\
\hline 32 & 0.5993779039 & 33 & 52 & 0.0611710486 & I. 0209399629 & 4270185 \\
\hline 33 & 0.6181084634 & 34 & 54 & 0.0621033138 & I . 022 I I 94428 & 1442428 \\
\hline 34 & 0.6368390229 & 35 & 55 & 0.0629567191 & I :0233 I 73997 & $0.55^{869} 64925$ \\
\hline 35 & $.6555^{6} 95^{824}$ & 36 & 56 & $0.06373045^{87}$ & 45323 & 0793 \\
\hline 36 & 0.67430 oI 419 & 37 & $5^{8}$ & $0.0644^{2} 383$ & 57628 & 2883566 \\
\hline 37 & 0.69303070 & 38 & 59 & 0.0650362710 & I. .0270074 & 3192403 \\
\hline 38 & $0.71176 \quad 12609$ & 40 & 0 & 0.0655672843 & I. 0282645087 & o.61516 76907 \\
\hline 39 & 0.73049 I 8204 & $4 \mathrm{I}$ & I & 0.0660165 II 2 & I.02953257I4 & $0.628829473^{8}$ \\
\hline 40 & 2223 & 42 & 2 & $0.0663^{8} 3693^{8}$ & I.0308I 00797 & 3004 \\
\hline $4 I$ & 0.76795293 & 43 & 3 & 0.0666686806 & I .03209 5477 I & 0.6555763772 \\
\hline 42 & $0.78668 \quad 34989$ & 44 & 3 & $0.06687 \quad$ I 4255 & I.03338 71976 & 0.6686533089 \\
\hline 43 & $0.8054 \mathrm{I} \quad 40584$ & 45 & 3 & 0.06699 I9865 & I.03468 36674 & 0.681527 I 988 \\
\hline 44 & $0.82414 \quad 46179$ & 46 & 4 & $0.06703 \quad 05237$ & I . $03598 \quad 33070$ & 0.6941941003 \\
\hline 45 & 0.8428751774 & 47 & 3 & 0.0669872981 & I. 0372845330 & 0.70665 o1 282 \\
\hline $90-r$ & $\mathbf{F} \psi$ & & & $\mathbf{G}(\mathbf{r})$ & $\mathbf{C}(\mathbf{r})$ & $\mathrm{B}(\mathbf{r})$ \\
\hline
\end{tabular}

SMITHSONIAN TABLES 
TABLE $\theta=30^{\circ}$

$q=0.017972387008967, \quad \theta 0=0.9640554346, \quad$ HK $=0.7325237222$

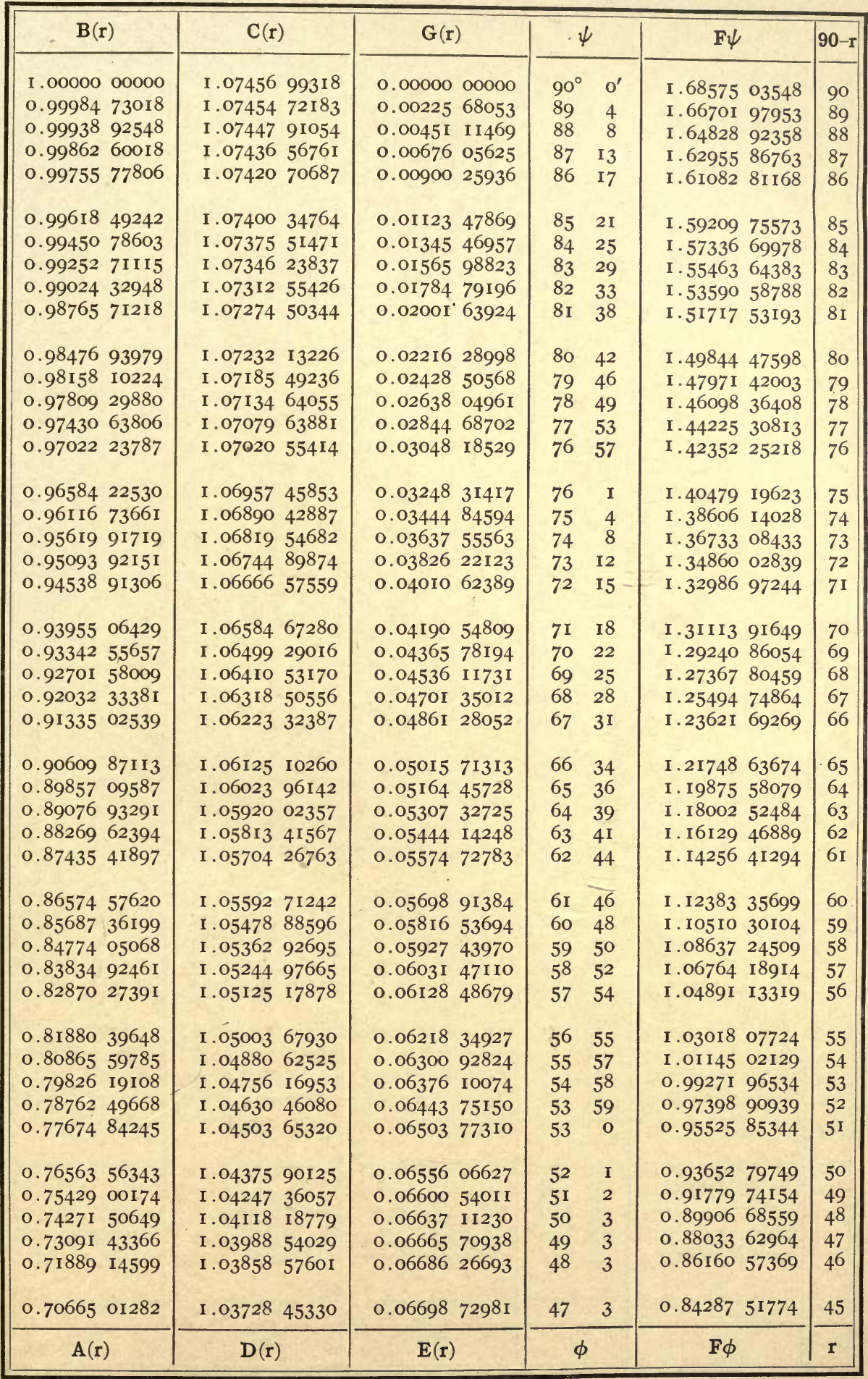


$K=1.7312451757, \quad K^{\prime}=2.0347153122, \quad E=1.4322909693, \quad E^{\prime}=1.2586796248$,

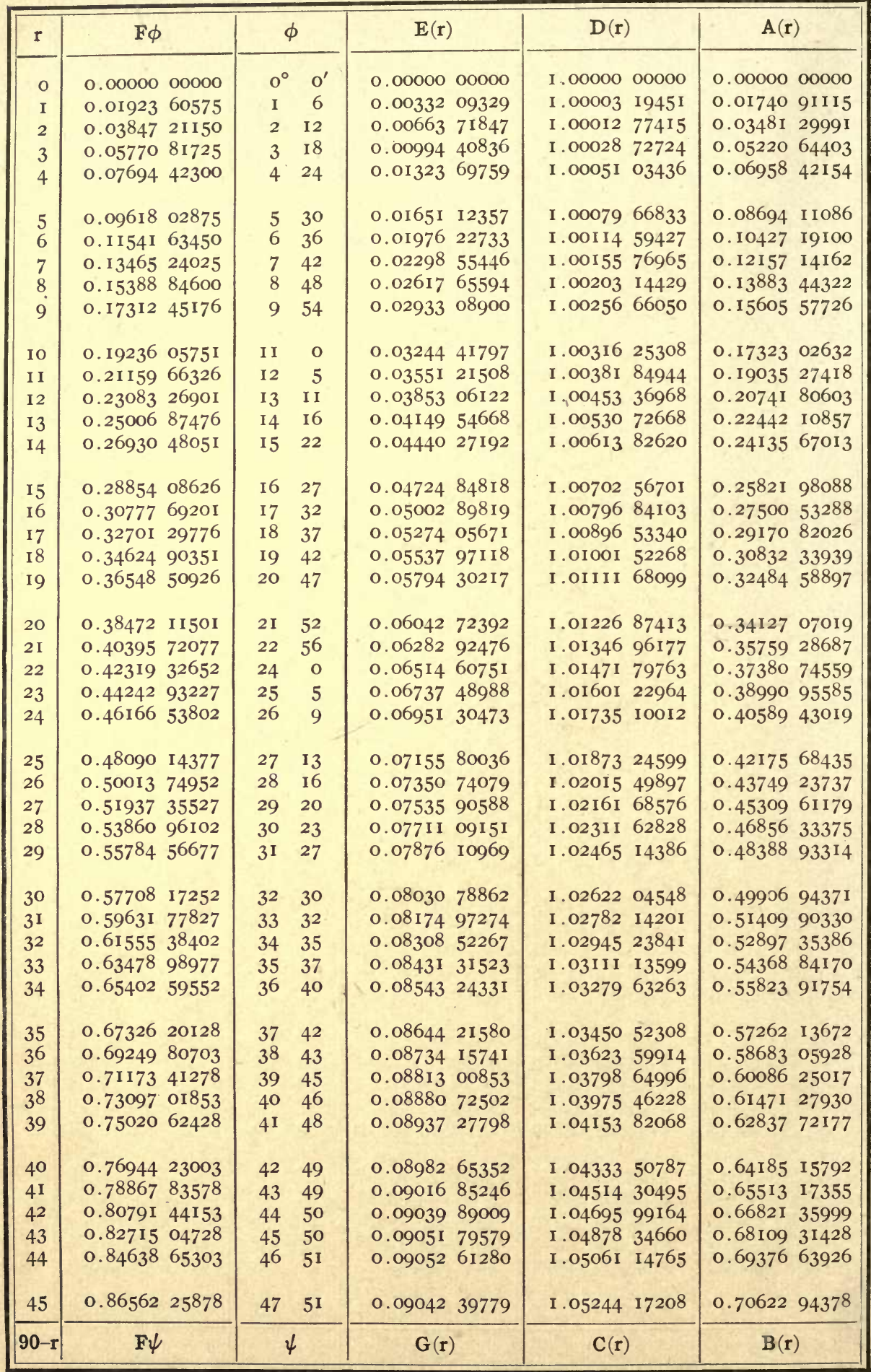


TABLE $\theta=35^{\circ}$

$q=0.024915062523981, \quad \theta 0=0.9501706456, \quad$ HK $=0.7950876364$

\begin{tabular}{|c|c|c|c|c|c|}
\hline $\mathbf{B}(\mathbf{r})$ & $\mathbf{C}(\mathbf{r})$ & $\mathbf{G}(\mathbf{r})$ & $\psi$ & $\mathrm{F} \psi$ & $90-r \mid$ \\
\hline I .00000 00000 & I. 1048866859 & 0.0000000000 & $90^{\circ} \quad \mathrm{o}^{\prime}$ & I. 7312451757 & 90 \\
\hline 0.9998469394 & I. 1048547369 & 0.0030062320 & 89 & I.71200 9II 81 & 89 \\
\hline 0.9993878065 & I. 1047589287 & 0.00600932 I 8 & 88 & I. 6927730606 & 88 \\
\hline 0.998622747 I & I. IO4599378I & 0.0090061288 & 87 & I. 67353.70031 & 87 \\
\hline 0.9975520048 & I. 1043762795 & 0.0119935 I 56 & 23 & I. 6543009456 & 86 \\
\hline 0.9961759200 & I. 1040899048 & o.0I49683495 & 85 & I. 635064888 I & 85 \\
\hline o.99449 49305 & I. 1037406029 & o.01792 75043 & 84 & I. 6 I 58288306 & 84 \\
\hline 0.9925095707 & I. 1033287996 & 0.0208678620 & 83 & I. 596592773 I & 83 \\
\hline 0.9902204719 & I. IO285 49965 & 0.0237863 I 4 I & 82 & I. 5773567156 & 82 \\
\hline 0.98762836 I5 & I. IO23I 977 II & 0.0266797640 & $8 I$ & I. $55^{8} \mathrm{I} 20658 \mathrm{I}$ & $8 \mathbf{I}$ \\
\hline 0.9847340633 & I. IOI $723775^{6}$ & $0.02954 \quad 51279$ & 80 & I. 5388846006 & 80 \\
\hline 0.98 I 5384966 & I. I0106 77362 & 0.0323793372 & 80 & I. 5 I964 8543I & 79 \\
\hline $0.97804 \quad 26763$ & I. 1003524524 & 0.0351793404 & 79 & I. $5004 \mathrm{I} 24856$ & 78 \\
\hline 0.9742477 II 7 & I .09957 87957 & 0.0379421046 & 78 & I. 48 II 76428 I & 77 \\
\hline 0.9701548073 & I.09874 77089 & 0.0406646178 & 77 & I. 46 I94 03706 & 76 \\
\hline 0.9657652612 & I. 0978602047 & $0.04334 \quad 38907$ & 76 & I. 4427043130 & 75 \\
\hline 0.96 I08 04649 & I.09691 73646 & 0.0459769592 & 75 & I. 4234682555 & 74 \\
\hline $0.95610 \quad 19028$ & I. 0959203375 & 0.048560886 I & 74 & I. 4042321980 & 73 \\
\hline 0.95083 I I 5 I 6 & I. 0948703382 & 0.0510927637 & 38 & I.38499 61 405 & 72 \\
\hline 0.9452698796 & I. 0937686463 & 0.0535697 I 6 I & 72 & I. 3657600830 & $7 \mathrm{I}$ \\
\hline $0.9394 I \quad 9846 I$ & I.0926I 66042 & $0.05598 .890 I_{4}$ & 71 & I. $34652 \quad 40255$ & 70 \\
\hline $0.93328 \quad 29005$ & I.0914I 56156 & $0.0583475^{1} 47$ & 70 & I $: 3272879680$ & 69 \\
\hline 0.92686 098I7 & I. 0901671440 & 0.0606427902 & 69 & I. 3080519105 & 68 \\
\hline 0.9201561173 & I. 0888727107 & 0.0628720041 & 69 & I. 2888 I 58530 & 67 \\
\hline 0.9131704228 & I . $087533^{8930}$ & 0.0650324775 & 68 & I. 2695797955 & 66 \\
\hline 0.9059061007 & I.086I5 2322 I & 0.067 I 2 I 5792 & 67 & I. $25034373^{80}$ & 65 \\
\hline 0.8983654396 & I. 08472968 I 5 & 0.0691367285 & 66 & I.23IIO 76805 & 64 \\
\hline 0.8905508 I 35 & I.08326 77048 & 0.0710753988 & 65 & I. $21187 \quad 16230$ & 63 \\
\hline 0.8824646805 & I. 0817681732 & 0.0729351200 & 64 & I. I9263 55655 & 62 \\
\hline $0.8741095^{823}$ & I. 0802329140 & 0.0747 I 34824 & 63 & I. I733995080 & $6 I$ \\
\hline 0.8654881427 & I . 0786637978 & 0.0764081398 & 62 & I. I 54 I 634504 & 60 \\
\hline 0.8566030670 & I. $07706 \quad 27365$ & 0.0780168127 & 29 & I. 13492 73929 & 59 \\
\hline 0.8474571408 & I. 07543 I6809 & 0.0795372924 & 60 & I. II569 I3354 & $5^{8}$ \\
\hline 0.8380532290 & I $.07377 \quad 26184$ & 0.0809674440 & 34 & I. $09645 \quad 52779$ & 57 \\
\hline 0.8283942745 & I. 0720875705 & $0.08230 \quad 52102$ & $5^{8}$ & I.07721 92204 & 56 \\
\hline $0.81848 \quad 32973$ & I. 0703785902 & 0.0835486152 & 57 & I. $05798 \quad 31629$ & 55 \\
\hline 0.8083233933 & I. 0686477599 & 0.0846957684 & 56 & I. 0387471054 & 54 \\
\hline $0.79791 \quad 77333$ & I.066897I884 & 0.0857448680 & 43 & I.0I95I 10479 & 53 \\
\hline 0.7872695615 & I.06512 90086 & 0.0866942053 & 44 & I. .0002749904 & 52 \\
\hline 0.7763821945. & I .06334 53750 & 0.0875421680 & 53 & 0.9810389329 & $5 \mathrm{I}$ \\
\hline 0.7652590201 & I.06I5484606 & $0.08828 \quad 72448$ & $5^{2}$ & 0.96 I $80 \quad 28754$ & 50 \\
\hline 0.753903496 I & I. $059740454^{8}$ & 0.0889280287 & $5^{I}$ & 0.9425668 I 79 & 49 \\
\hline 0.74231 91490 & I. 0579235605 & 0.0894632214 & 50 & 0.9233307604 & 48 \\
\hline 0.7305095727 & I.05609 99913 & 0.08989 I6370 & 49 & 0.9040947028 & 47 \\
\hline 0.7184784273 & I.05427 I9690 & 0.0902122056 & 48 & 0.8848586453 & 46 \\
\hline 0.7062294378 & I. $05244 \quad 17208$ & 0.0904239779 & $47 \quad 5 I$ & $0.855^{62} 25^{8} 7^{8}$ & 45 \\
\hline $\mathbf{A}(\mathbf{r})$ & $\mathbf{D}(\mathbf{r})$ & $\mathbf{E}(\mathbf{r})$ & $\phi$ & $\mathbf{F} \phi$ & $\mathbf{r}$ \\
\hline
\end{tabular}


$K=1.7867691349, \quad K^{\prime}=1.9355810960, \quad E=1.3931402485, \quad E^{\prime}=1.3055390943$,

\begin{tabular}{|c|c|c|c|c|c|c|c|c|c|}
\hline \multirow{2}{*}{$\frac{r}{0}$} & \multicolumn{2}{|l|}{$\mathbf{F} \phi$} & \multicolumn{2}{|c|}{$\phi$} & \multicolumn{2}{|l|}{$E(r)$} & $\mathbf{D}(\mathbf{r})$ & \multicolumn{2}{|c|}{$A(r)$} \\
\hline & 0.00000 & 00000 & $0^{\circ}$ & $\mathrm{o}^{\prime}$ & 0.00000 & 00000 & I. .0000000000 & 0.00000 & 00000 \\
\hline I & o.01985 & 29904 & I & 8 & 0.00437 & 25767 & I.00004 34107 & 0.01737 & $5^{2657}$ \\
\hline 2 & 0.03970 & 59807 & 2 & I6 & 0.00873 & 86910 & I.00017 35897 & 0.03474 & 53796 \\
\hline 3 & 0.05955 & 89712 & 3 & 24 & 0.01309 & I 8945 & I. .0003903787 & 0.05210 & 51913 \\
\hline 4 & $0.0794 \mathrm{I}$ & I96I5 & 4 & 32 & 0.01742 & $5768 \mathrm{I}$ & I. $0006935^{1} 36$ & 0.06944 & 95525 \\
\hline 5 & 0.09926 & 49519 & 5 & $4 I$ & 0.02173 & $3935^{I}$ & I.00108 26253 & 0.08677 & 33185 \\
\hline 6 & o. II9I I & 79423 & 6 & 49 & 0.02601 & 00761 & I.00155 72398 & 0.10407 & I 3496 \\
\hline 7 & o. 1 3897 & 09327 & 7 & 57 & 0.03024 & 79420 & I.002II 67791 & 0.12133 & 85 I 17 \\
\hline 8 & o. I $5^{882}$ & 39231 & 9 & 5 & 0.03444 & I 3683 & I. .0027605620 & o. 13856 & 96780 \\
\hline 9 & o. 17867 & 69 I35 & Io & 13 & $0.0385^{8}$ & 42875 & I. $00348 \quad 78042$ & o. I 5575 & 97300 \\
\hline Io & o. 19852 & 99039 & I I & 21 & 0.04267 & 07422 & I.00429 76203 & 0.17290 & $355^{87}$ \\
\hline I I & 0.21838 & 28943 & 12 & 28 & 0.04669 & 48973 & I.005I 890239 & o. 18999 & 60657 \\
\hline 12 & 0.23823 & 58847 & I3 & 36 & 0.05065 & 10519 & I.006I6 09295 & 0.20703 & 21648 \\
\hline 13 & 0.25808 & $8875^{\mathrm{I}}$ & I4 & 43 & 0.05453 & 36499 & I. 0072 I 2I 534 & 0.22400 & 67828 \\
\hline 14 & 0.27794 & I 8655 & I 5 & $5^{I}$ & $0.05^{8} 33$ & 72913 & I. .00834 I4I54 & $0.2409 \mathrm{I}$ & 48609 \\
\hline 15 & 0.29779 & $4855^{8}$ & I6 & $5^{8}$ & 0.06205 & 67422 & I. 0095473402 & 0.25775 & I 3559 \\
\hline I6 & 0.31764 & 78462 & I 8 & 5 & 0.06568 & 69435 & I .01082 84592 & $0.2745 \mathrm{I}$ & 12417 \\
\hline 17 & 0.33750 & 08366 & 19 & 12 & 0.06922 & 30203 & I. $01218 \quad 32120$ & 0.29118 & 95099 \\
\hline 18 & 0.35735 & 38270 & 20 & I8 & 0.07266 & 02895 & I .oI360 99487 & 0.30778 & I 718 \\
\hline 19 & 0.37720 & 68 I 74 & $2 \mathrm{I}$ & 25 & 0.07599 & 42673 & I.oI510 69318 & 0.32428 & 12593 \\
\hline 20 & 0.39705 & 98078 & 22 & $3 I$ & 0.07922 & 06754 & I.01667 23379 & 0.34068 & 48260 \\
\hline 21 & $0.4169 \mathrm{I}$ & 27981 & 23 & 37 & 0.08233 & 54475 & I.01830 42606 & o. 35698 & 69491 \\
\hline 22 & 0.43676 & 57885 & 24 & 42 & 0.08533 & 47336 & I. 0200007123 & 0.37318 & 27300 \\
\hline 23 & $0.4566 \mathrm{I}$ & 87789 & 25 & 48 & 0.08821 & 49046 & I.02I75 96267 & 0.38926 & 72959 \\
\hline 24 & 0.47647 & 17693 & 26 & 53 & 0.09097 & 25564 & 1. 0235788616 & 0.40523 & 58014 \\
\hline 25 & 0.49632 & 47597 & $27^{\circ}$ & 59 & 0.09360 & $45^{123}$ & I.02545 62012 & 0.42108 & 34293 \\
\hline 26 & 0.51617 & $775 \mathrm{OI}$ & 29 & 4 & 0.09610 & 78252 & I. $.0273^{8} 935^{89}$ & 0.43680 & 53924 \\
\hline 27 & 0.53603 & 07405 & 30 & 8 & 0.09847 & 97792 & I. 0293759801 & $0.45^{239}$ & 69344 \\
\hline 28 & o. $555^{88}$ & 37309 & 31 & 13 & $0.1007 \mathrm{I}$ & 78905 & I.03I4I 36450 & 0.46785 & 33318 \\
\hline 29 & 0.57573 & 67212 & 32 & I 7 & 0.10281 & 99075 & I.03349 98717 & 0.48316 & 98948 \\
\hline 30 & $.0 .5955^{8}$ & 97116 & 33 & 22 & o. 10478 & $38 \mathrm{IOI}$ & I. 0356321191 & o. 49834 & 19688 \\
\hline $3 I$ & 0.6 I 544 & 27020 & 34 & 25 & 0.10660 & 78092 & I. $03780 \quad 77899$ & 0.51336 & 49360 \\
\hline 32 & 0.63529 & 56924 & 35 & 28 & 0.10829 & 03444 & I. 0400242340 & 0.52823 & 42166 \\
\hline 33 & $0.655^{14}$ & 86828 & 36 & 31 & 0.10983 & 00821 & I.04227 87515 & o. 54294 & 52702 \\
\hline 34 & 0.67500 & I 6732 & 37 & 34 & 0.11122 & 59132 & I. 044568596 I & 0.55749 & 35973 \\
\hline 35 & 0.69485 & 46636 & 38 & 37 & o. 11247 & 69491 & I. .0468909786 & 0.57187 & 47405 \\
\hline 36 & 0.71470 & 76540 & 39 & 39 & o. $1135^{8}$ & 25187 & I.04924 30699 & $0.5^{8608}$ & 42864 \\
\hline 37 & 0.73456 & 06443 & 40 & $4 I$ & o. I I 454 & 21645 & I. 0516220047 & $0.6001 \mathrm{I}$ & 78665 \\
\hline 38 & $0.7544 \mathrm{I}$ & 36347 & $4 \mathrm{I}$ & 42 & o. I I 535 & 56375 & I. 054024885 I & 0.61397 & I 1590 \\
\hline 39 & 0.77426 & $6625^{I}$ & 42 & 44 & 0.11602 & 28932 & I. 0564487839 & $0.6276_{3}$ & 98902 \\
\hline 40 & 0.794 I I & 96155 & 43 & 46 & o. 11654 & $4086 I$ & I.05889 0748 I & 0.64 III & 98356 \\
\hline $4 I$ & 0.81397 & 26059 & 44 & 46 & 0.11691 & 95649 & I.06134 78029 & 0.65440 & 68220 \\
\hline 42 & 0.83382 & 55963 & 45 & 47 & o. II 7 I 4 & 98662 & I.06381 $6955^{\circ}$ & 0.66749 & 67282 \\
\hline 43 & 0.85367 & 85867 & 46 & 47 & o. 11723 & 57096 & 1.0662951962 & 0.68038 & 54871 \\
\hline 44 & o. 87353 & I $577 \mathrm{I}$ & 47 & 48 & 0.11717 & 79914 & I. 0687795074 & 0.69306 & 90869 \\
\hline 45 & 0.89338 & 45674 & 48 & 48 & o. 11697 & 77784 & I. 0712668617 & 0.70554 & 35725 \\
\hline $90-r$ & $\mathbf{F} \psi$ & & $\psi$ & & $\mathrm{G}(\mathbf{r})$ & & $\mathrm{C}(\mathbf{r})$ & $\mathrm{B}(\mathbf{r})$ & \\
\hline
\end{tabular}


TABLE $\theta=40^{\circ}$

$q=0.033265256695577, \quad \theta 0=0.9334719356, \quad \mathrm{HK}=0.8550825245$

\begin{tabular}{|c|c|c|c|c|c|}
\hline $\mathrm{B}(\mathbf{r})$ & $\mathrm{C}(\mathbf{r})$ & $\mathbf{G}(\mathbf{r})$ & $\psi$ & $\mathrm{F} \psi$ & $90-r$ \\
\hline I. 0000000000 & I. I 425442 I 77 & 0.0000000000 & $90^{\circ} \quad 0^{\prime}$ & I.78676 9I349 & 90 \\
\hline 0.9998463487 & I . I 425007942 & 0.0038284907 & 89 & I. 7669 I 61445 & 89 \\
\hline $0.9993^{8} 5445^{1}$ & I. I 423705769 & 0.0076531872 & 88 & I.74706 3I 54 I & 88 \\
\hline 0.9986 I 74408 & I. I 421537243 & 0.0114702963 & 87 & I. 72721 oI 637 & 87 \\
\hline $0.997542588 \mathrm{I}$ & I . I 4 I 8505008 & 0.0152760269 & 30 & I.70735 71733 & 86 \\
\hline $0.996 \mathrm{I} 6 \mathrm{I} 240 \mathrm{I}$ & I. $14146 \quad 12760$ & 0.0190665913 & $3^{8}$ & I. $68750 \quad 41829$ & 85 \\
\hline $0.99447 \quad 38506$ & I. I 409865243 & 0.0228382057 & 46 & I . 66765 I 1926 & 84 \\
\hline 0.9924809734 & I. 1404268243 & $0.02658 \quad 7.0918$ & 83 & I. 6477982022 & 83 \\
\hline 0.9901832628 & I. I $3978 \quad 28584$ & 0.0303094781 & 83 & I. 6279452118 & 82 \\
\hline $0.98758 \quad 14726$ & I. I3905 54 II 3 & $0.03400 \quad 16009$ & 82 & I. 60809222 I 4 & $8 I$ \\
\hline 0.9846764560 & I. I3824 53698 & 0.0376597054 & I6 & I. 5882392310 & 80 \\
\hline 0.9814691652 & I. I3735 37211 & 0.0412800477 & 23 & I. $56838 \quad 62406$ & 79 \\
\hline 0.9779606509 & I. I $363^{8}$ I $55^{2} \mathrm{I}$ & $0.044^{8} 58895^{8}$ & 30 & I. $54853 \quad 32502$ & 78 \\
\hline 0.974 I 5206 I 6 & I. I 353300476 & 0.0483925314 & 37 & I. $\mathbf{5 2 8 6 8} 02598$ & 77 \\
\hline 0.9700446432 & I. I 342004893 & 0.0518772514 & 44 & I. $50882 \quad 72694$ & 76 \\
\hline 0.9656397386 & I. I3299 42539 & $0.05530 \quad 93702$ & 76 & I.48897 4279I & 75 \\
\hline 0.9609387866 & I. 1317128116 & 0.0586852206 & 75 & I. $46912 \quad 12887$ & 74 \\
\hline $0.95594332 \mathrm{I} 3$ & I. I3035 77242 & 0.06200 I I 573 & 75 & I. 4492682983 & 73 \\
\hline 0.95065497 I6 & I. I 289306433 & 0.0652535577 & 74 & I. $42941 \quad 53079$ & 72 \\
\hline 0.9450754604 & I. 1274333082 & $0.068438825 \mathrm{I}$ & 73 & I. 4095623175 & 71 \\
\hline 0.9392066032 & I. $125^{86} 7543^{8}$ & 0.0715533910 & 23 & I. $38970 \quad 93271$ & 70 \\
\hline 0.9330503082 & I. $12423 \quad 52584$ & $0.07459 \quad 37177$ & 29 & I. 3698563367 & 69 \\
\hline 0.9266085744 & I. I 2253844 I 4 & o.07755 630II & 34 & I. 3500033463 & 68 \\
\hline 0.9198834913 & I. 1207791607 & 0.0804376736 & 40 & I. 3301503560 & 67 \\
\hline 0.9128772377 & I. II895 95604 & 0.0832344077 & 45 & I.31029 73656 & 66 \\
\hline 0.9055920807 & I. II $708 \quad 18582$ & 0.085943 II 88 & 67 & I. 2904443752 & 65 \\
\hline 0.8980303745 & I. II5I4 83422 & 0.0885604692 & 56 & I. 2705913848 & 64 \\
\hline o.890I9 45598 & I. I I3I6 I3690 & 0.09108317 I4 & 66 & I. 2507383944 & 63 \\
\hline 0.882087 I 6 I 8 & I. I I I I 233599 & 0.0935079923 & 65 & I. 2308854040 & 62 \\
\hline 0.8737 I 0790 I & I. 1090367986 & $0.095^{8} 3$ I 7573 & 64 & I. 2110324136 & $6 I$ \\
\hline 0.8650681367 & I. $10690 \quad 42279$ & 0.09805 I3545 & 63 & I. I9II7 94233 & 60 \\
\hline 0.856 I6 I975I & I. I0472 82465 & o. 10016 3739I & 62 & I. I7I32 64329 & 59 \\
\hline 0.846995 I593 & I. 1025 I I 506I & o.10216 $593^{8} 3$ & $6 I$ & I. I5147 34425 & $5^{8}$ \\
\hline $0.83757 \quad 06220$ & I. 1002567080 & o. 1040550557 & 60 & I. I3162 04521 & 57 \\
\hline 0.82789 I3739 & I.09796 65999 & o. 1058282770 & 59 & I. I I 76 746I 7 & 56 \\
\hline 0.8179605020 & I. 0956439724 & o. $10748 \quad 28746$ & $5^{8}$ & I.0919I 447 I3 & 55 \\
\hline 0.80778 I I 684 & I. 09329 I 6556 & 0.1090162132 & 57 & I. $07206 \quad 14809$ & 54 \\
\hline $0.797356609 \mathrm{I}$ & I. $.0909 \mathrm{I} \quad 25 \mathrm{I} 60$ & O.I 1042 57553 & 56 & I. 0522084905 & 53 \\
\hline 0.78669 oI322 & I .08850 94525 & 0. III70 90668 & 55 & I.03235 5500 I & 52 \\
\hline $0.77578 \quad 5$ II 73 & I. 0860853932 & o. I1286 38228 & 54 & I. OI250 25098 & $5^{I}$ \\
\hline 0.7646450133 & I. 0836432917 & o.11388 78137 & 53 & 0.9926495194 & 50 \\
\hline 0.7532733376 & I. .08 II 8 6I 237 & O. I I 477895 I I & $5^{2}$ & 0.9727965290 & 49 \\
\hline 0.7416736742 & I. 0787 I 68830 & o. II553 52736 & $5^{I}$ & 0.9529435386 & 48 \\
\hline 0.7298496728 & I. 0762385782 & o. II6I5 49535 & 50 & 0.9330905482 & 47 \\
\hline $0.71780 \quad 50468$ & I. $07375 \quad 42288$ & o.II663 63025 & $49 \quad 47$ & 0.9132375578 & 46 \\
\hline 0.7055435725 & I. 07126686 I 7 & o. IÍ697 77784 & $4^{8} \quad 4^{8}$ & $0.8933^{8} \quad 45674$ & 45 \\
\hline$A(\mathbf{r})$ & $\mathbf{D}(\mathbf{r})$ & $\mathrm{E}(\mathbf{r})$ & $\phi$ & $\mathrm{F} \phi$ & $\mathbf{r}$ \\
\hline
\end{tabular}

SMITHSONIAN TABLES 
$\mathrm{K}=\mathrm{K}^{\prime}=1.8540746773, \quad \mathrm{E}=\mathrm{E}^{\prime}=1.3506438810$

\begin{tabular}{|c|c|c|c|c|c|c|c|c|c|c|}
\hline $\mathbf{r}$ & \multicolumn{2}{|l|}{$\mathbf{F} \phi$} & \multicolumn{2}{|c|}{$\phi$} & \multicolumn{2}{|c|}{$\mathbf{E}(\mathbf{r})$} & \multicolumn{2}{|l|}{$\mathbf{D}(\mathbf{r})$} & \multicolumn{2}{|c|}{$A(r)$} \\
\hline o & 0.00000 & 00000 & $0^{\circ}$ & $\mathrm{o}^{\prime}$ & 0.00000 & 00000 & I .00000 & 00000 & 0.00000 & 00000 \\
\hline I & 0.02060 & 08297 & I & I I & 0.00559 & 22 I 85 & I. 00005 & 76114 & 0.01732 & 23240 \\
\hline 2 & 0.04120 & I6595 & 2 & 22 & 0.01117 & 56998 & I. .00023 & $0375^{2}$ & 0.03463 & 96092 \\
\hline 3 & 0.06180 & 24892 & 3 & 32 & 0.01674 & 17286 & $1.0005 \mathrm{I}$ & $808 I_{4}$ & 0.05194 & 68175 \\
\hline 4 & 0.08240 & 33190 & 4 & 43 & O 02228 & 16343 & I .00092 & 03796 & 0.06923 & 89126 \\
\hline 5 & 0.10300 & 41487 & 5 & 54 & 0.02778 & 68124 & I .00I 43 & 67802 & $0.0865 \mathrm{I}$ & o86 I I \\
\hline 6 & 0.12360 & 49785 & 7 & 4 & 0.03324 & 87460 & I . 00206 & 66547 & o. 10375 & 76329 \\
\hline 7 & o. 14420 & 58082 & 8 & I 5 & 0.03865 & 90273 & I .00280 & 92364 & o. 12097 & 42023 \\
\hline 8 & 0.16480 & 66380 & 9 & 25 & 0.04400 & 93780 & I .00366 & 36213 & 0.13815 & 55494 \\
\hline 9 & o. I 8540 & 74677 & Io & 36 & 0.04929 & 16689 & I. 00462 & 87696 & o. 15529 & 66598 \\
\hline 10 & 0.20600 & 82975 & I I & 46 & 0.05449 & 79400 & I .00570 & 35065 & o. 17239 & 25270 \\
\hline I I & 0.22660 & 91272 & 12 & 56 & 0.05962 & 04166 & I .00688 & 65237 & o. I 8943 & 81524 \\
\hline 12 & 0.24720 & 99570 & I4 & 6 & 0.06465 & I 5306 & 1.00817 & 63813 & 0.20642 & 85463 \\
\hline I3 & 0.26781 & 07867 & I 5 & I 5 & $0.0695^{8}$ & 39334 & I. 00957 & I 509 I & 0.22335 & 87294 \\
\hline I4 & $0.2884 \mathrm{I}$ & I6165 & 16 & 25 & $0.0744 \mathrm{I}$ & 05129 & I.OIIO7 & 02088 & 0.24022 & 37330 \\
\hline I 5 & 0.30901 & 24462 & I7 & 34 & 0.07912 & 44078 & I.01267 & 06562 & $0.2570 \mathrm{I}$ & 86008 \\
\hline 16 & 0.32961 & 32760 & I 8 & 43 & 0.08371 & 90207 & I. 01437 & 09030 & 0.27373 & 83893 \\
\hline 17 & $0.3502 \mathrm{I}$ & 41057 & 19 & 52 & 0.08818 & 80301 & I.0I6I6 & 88793 & 0.29037 & 81691 \\
\hline I 8 & 0.37081 & 49355 & 21 & I & 0.09252 & 54012 & I. 01806 & 23965 & 0.30693 & 30262 \\
\hline I9 & $0.39 \mathrm{I} 4 \mathrm{I}$ & 57652 & 22 & 9 & 0.09672 & 53955 & I. 02004 & 91494 & 0.32339 & 80622 \\
\hline 20 & $0.4 \mathrm{I} 20 \mathrm{I}$ & 65950 & 23 & 17 & o. 10078 & 25794 & 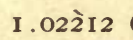 & 67193 & 0.33976 & 83967 \\
\hline $2 \mathrm{I}$ & $0.4326 \mathrm{I}$ & 74247 & 24 & 25 & 0.10469 & 18308 & I. .02429 & 25769 & 0.35603 & $9167 \mathrm{I}$ \\
\hline 22 & $0.4532 \mathrm{I}$ & 82545 & 25 & 33 & o. 10844 & 83455 & I. 02654 & 40853 & 0.37220 & 55308 \\
\hline 23 & $0.473^{81}$ & 90842 & 26 & 40 & o. I1 204 & 76417 & I. 02887 & 85035 & 0.38826 & .26656 \\
\hline 24 & $0.4944^{\mathrm{I}}$ & 99139 & 27 & 47 & o. I I $54^{8}$ & 55630 & I. 03129 & 29893 & .0 .40420 & 57714 \\
\hline 25 & 0.51502 & 07437 & 28 & 54 & o. I I 875 & 82813 & I. 03378 & 46028 & 0.42003 & 007 I I \\
\hline 26 & 0.53562 & I 5734 & 30 & 0 & o. I 2 I 86 & 22978 & I. 03635 & 03103 & 0.43573 & 08120 \\
\hline 27 & 0.55622 & 24032 & 3 I & 6 & o. 12479 & 44425 & I. 03898 & 69880 & 0.45130 & 32670 \\
\hline 28 & 0.57682 & 32329 & 32 & 12 & o. 12755 & 18736 & I. 04169 & 14251 & 0.46674 & 27359 \\
\hline 29 & $0.5974^{2}$ & 40627 & 33 & 17 & 0.13013 & 20757 & I . 04446 & 03288 & 0.48204 & 45468 \\
\hline 30 & $0.6 \mathrm{I} 802$ & 48924 & 34 & 22 & 0.13253 & 2856 I & I . 04729 & 03271 & 0.49720 & 40572 \\
\hline 31 & 0.63862 & 57222 & 35 & 27 & o.I3475 & 234 I 3 & I.05017 & 79739 & 0.51221 & 66556 \\
\hline 32 & 0.65922 & 65519 & 36 & 32 & o. 13678 & 89725 & I .053II & 97528 & 0.52707 & 77628 \\
\hline 33 & 0.67982 & 73817 & 37 & 36 & o.13864 & 14993 & I. 056 I I & 20812 & 0.54178 & 28334 \\
\hline 34 & 0.70042 & 82 I I 4 & 38 & 39 & 0.14030 & 89744 & I. 05915 & I3I 49 & $0.55^{6} 3^{2}$ & 73569 \\
\hline 35 & 0.72102 & 90412 & 39 & 43 & o. I4I 79 & 07457 & I. 06223 & $375^{24}$ & 0.57070 & 68597 \\
\hline 36 & 0.74162 & 98709 & 40 & 46 & o.1 4308 & 64509 & I . 06535 & 56397 & $0.5^{849}$ I & 69061 \\
\hline 37 & 0.76223 & 07007 & $4 I$ & 48 & 0.14419 & 60059 & I .0685 I & 31742 & o. 59895 & 31001 \\
\hline 38 & 0.78283 & 15304 & 42 & $5 I$ & o. I 45 I I & 96000 & I. 07170 & 25103 & 0.61281 & 10868 \\
\hline 39 & 0.80343 & 23602 & 43 & 54 & o. $145^{8} 5$ & 76849 & I .0749I & 97630 & 0.62648 & 65539 \\
\hline 40 & 0.82403 & 31899 & 44 & 54 & $0.1464 I$ & 0967 I & I. 078 I 6 & 10137 & 0.63997 & 52334 \\
\hline $4 I$ & 0.84463 & 40197 & 45 & 55 & 0.14678 & 03964 & I. 08142 & 23139 & 0.65327 & 29030 \\
\hline 42 & 0.86523 & 48494 & 46 & 56 & 0.14696 & 71583 & I. 08469 & 96910 & 0.66637 & 53880 \\
\hline 43 & 0.88583 & 56792 & 47 & 57 & 0.14697 & 26631 & I. 08798 & 91523 & 0.67927 & 85625 \\
\hline 44 & 0.90643 & 65089 & 48 & 57 & 0.14679 & 85365 & I. 09128 & 66907 & 0.69197 & 83514 \\
\hline 45 & 0.92703 & 73387 & 49 & 57 & o. 14644 & 66094 & I. $.0945^{8}$ & 82886 & 0.70447 & 07318 \\
\hline $90-r$ & $\mathbf{F} \psi$ & & & & $G(r)$ & & $\mathrm{C}(\mathrm{r})$ & & $\mathbf{B}(\mathbf{r})$ & \\
\hline
\end{tabular}


TABLE $\theta=45^{\circ}$

$q=\mathrm{e}^{-\pi}=0.04321391826377, \quad \theta 0=0.9135791382, \quad \mathrm{HK}_{-}=0.9135791382$

\begin{tabular}{|c|c|c|c|c|c|}
\hline $\mathbf{B}(\mathbf{r})$ & $\mathrm{C}(\mathbf{r})$ & $\mathbf{G}(\mathbf{r})$ & $\psi$ & $\mathrm{F} \psi$ & $90-r$ \\
\hline I. 0000000000 & I. I 89207 I I 50 & 0.0000000000 & $90^{\circ} \quad 0^{\prime}$ & I. $85407 \quad 46773$ & 90 \\
\hline 0.9998454246 & I. I89 I 494665 & 0.0047060108 & $89 \quad 10$ & I . 8334738476 & 89 \\
\hline $0.9993^{8}$ I75I4 & I. 1889765912 & 0.0094076502 & $88 \quad 20$ & I. 8 I 28730178 & 88 \\
\hline 0.9986091406 & I. I8868 87000 & 0.0141005467 & 30 & I. 792272188 I & 87 \\
\hline $0.9975^{2} \quad 785^{84}$ & I. I 88286 I 440 & 0.0187803289 & 40 & I. $77167 \quad 13583$ & 86 \\
\hline 0.996 I3 82775 & I. I 877694140 & 0.0234426255 & 85 & I.75107 05286 & 85 \\
\hline 0.9944408767 & I. I87I3 91403 & 0.0280830653 & 59 & I.7304696988 & 84 \\
\hline 0.9924362407 & I. I8639609I 4 & 0.0326972774 & 84 & I.70986 8869I & 83 \\
\hline 0.9901250593 & I. I 8554 II 736 & 0.0372808916 & 83 & I . 6892680393 & 82 \\
\hline 0.9875081276 & I. 1845754293 & 0.0418295382 & 82 & I.6686672096 & $8 I$ \\
\hline $0.9845^{8} 6345^{\circ}$ & I. I $8350 \quad 00363$ & 0.0463388487 & $8 I$ & I. 6480663798 & 80 \\
\hline 0.9813607151 & I.I823I 63059 & 0.0508044575 & 80 & I. $627465550 \mathrm{I}$ & 79 \\
\hline 0.9778323446 & I. I 810256817 & 0.05522 I9994 & 79 & I . 6068647203 & 78 \\
\hline 0.9740024430 & I. I7962 97376 & $0.0595^{8} 7$ II39 & 79 & I. 5862638906 & 77 \\
\hline 0.9698723216 & I. I $78130175^{6}$ & 0.0638954439 & 78 & I. 5656630608 & 76 \\
\hline 0.9654433929 & I. 1765288244 & 0.0681426379 & 23 & I. 54506223 I I & 75 \\
\hline 0.9607171696 & I. I7482 76366 & 0.0723243506 & 32 & I. 52446 I 40 I 3 & 74 \\
\hline 0.9556952639 & I. I7302 86866 & 0.0764362449 & 40 & I. 50386057 I 6 & 73 \\
\hline 0.9503793863 & I. I 7 I I3 4I 680 & 0.0804739933 & 48 & I. 48325974 I 8 & 72 \\
\hline $0.94477 \quad$ I3447 & I. 1691463907 & 0.0844332799 & 57 & I . 4626589121 & 71 \\
\hline 0.9388730433 & I. 1670677783 & $0.08830 \quad 98027$ & 73 & I. 4420580823 & 70 \\
\hline $0.93268648 \mathrm{I} 4$ & I. I 649008653 & 0.0920992756 & 72 & I. 42145.72526 & 69 \\
\hline 0.9262137526 & I. I626482937 & 0.0957974315 & 20 & I. 4008564228 & 68 \\
\hline 0.9194570430 & I. 1603128097 & 0.0994000252 & 27 & I. 380255593 I & 67 \\
\hline 0.9124186305 & I. I5789 72608 & o. $10290 \quad 28362$ & 34 & I. 3596547634 & 66 \\
\hline 0.9051008831 & I. I 554045920 & $0.10630 \quad 16727$ & 68 & I.33905 39336 & 65 \\
\hline $0.89750 \quad 62579$ & I. I 528378419 & o. $109592375^{2}$ & 48 & I. 3184531039 & 64 \\
\hline 0.8896372995 & I. I 5020 01398 & 0.1127708206 & 54 & I . 297852274 I & 63 \\
\hline 0.8814966386 & I. I 4749470 I I & o.II58329266 & 66 & I. $27725 \quad$ I 4444 & 62 \\
\hline 0.8730869906 & I. I 447248239 & o.II $877 \quad 46567$ & 65 & I. 2566506146 & $6 r$ \\
\hline 0.8644 I II 542 & I. I 4 I 8938846 & o.12I5920252 & 64 & I. 2360497849 & 60 \\
\hline 0.8554720099 & I . I 390053339 & o. 12428 I 1025 & 63 & I. 2 I 5448955 I & 59 \\
\hline 0.8462725182 & I. I3606 26928 & o. I2683 802 I I & 21 & I. I9484 8I254 & $5^{8}$ \\
\hline $0.83681 \quad 57$ I 84 & I . I3306 95480 & o.12925 898I5 & 26 & I. 1742472956 & 57 \\
\hline $0.82710 \quad 47269$ & I. I3002 95477 & o. I3I54 02588 & 30 & I. I 536464659 & 56 \\
\hline 0.8171427355 & I . 1269463970 & o.1336782099 & 59 & I. I3304 5636I & 55 \\
\hline 0.8069330099 & I. I $2382 \quad 3^{8} 537$ & o. I 356692789 & 38 & I . I I $244 \quad 48064$ & 54 \\
\hline $0.796478888 \mathrm{I}$ & I. I 206657231 & 0.1375100077 & 42 & I.0918439766 & 53 \\
\hline $0.78578 \quad 37785$ & I. I I 74758542 & o.13919 70407 & 45 & I. 0712431469 & $5^{2}$ \\
\hline 0.77485 II 587 & I. I I 42581342 & 0.1407271344 & 47 & I. 0506423 I 7 I & $5 \mathrm{I}$ \\
\hline 0.7636845735 & I. I IIOI 64844 & o.14209 71663 & 50 & I.03004 I4874 & 50 \\
\hline 0.7522876332 & I. I0775 48548 & o. I 4330 4I 4I 5 & 53 & I. .0094406576 & 49 \\
\hline 0.7406640121 & I . $10447 \quad 72199$ & o.14434 52037 & 53 & 0.9888398279 & 48 \\
\hline 0.7288174469 & I. IOI I 875735 & o.1 452 I 76436 & $5 \mathrm{I}$ & $0.968238998 \mathrm{I}$ & 47 \\
\hline 0.7167517348 & I.09788 99237 & o.I459I 89078 & 50 56 & 0.9476381684 & 46 \\
\hline 0.7044707318 & I. $0945^{8} 82886^{\circ}$ & o.I4644 66094 & $49 \quad 57$ & 0.9270373387 & 45 \\
\hline$A(r)$ & $\mathbf{D}(\mathbf{r})$ & $\mathbf{E}(\mathbf{r})$ & $\phi$ & $\mathrm{F} \phi$ & $\mathbf{r}$ \\
\hline
\end{tabular}


$K=1.9355810960, \quad K^{\prime}=1.7867691349, \quad E=1.3055390943, \quad E^{\prime}=1.3931402485$,

\begin{tabular}{|c|c|c|c|c|c|c|}
\hline $\mathbf{r}$ & $\mathbf{F} \phi$ & c & & $\mathbf{E}(\mathbf{r})$ & $\mathbf{D}(\mathbf{r})$ & $\mathbf{A}(\mathbf{r})$ \\
\hline o & 0.0000000000 & & $o^{\prime}$ & 0.0000000000 & I. .00000 00000 & 0.0000000000 \\
\hline I & 0.0215064566 & $\mathbf{I}$ & 14 & o.00699852 I 2 & I. 0000752700 & 0.01724 I783I \\
\hline 2 & 0.0430129132 & 2 & 28 & o.or398 53763 & I .00030 09884 & 0.0344786990 \\
\hline 3 & 0.0645 I 93699 & 3 & $4 \mathrm{I}$ & o.0209489334 & I. 0006768809 & 0.0517058810 \\
\hline 4 & 0.0860258265 & 4 & 55 & 0.0278776288 & I. OO120 24903 & 0.0689184630 \\
\hline 5 & o.10753 22831 & 6 & 9 & 0.0347600006 & I.00I 8771775 & 0.086 II I 5805 \\
\hline 6 & o.12903 87397 & 7 & 22 & $0.0415^{8} \quad 42717$ & I. .00270 or 222 & 0.1032803705 \\
\hline 7 & o.15054 51963 & 8 & 36 & 0.0483406320 & I. 0036703237 & 0.1204199725 \\
\hline 8 & o.17205 I6530 & 9 & 49 & $0.05501 \quad 67694$ & I. 0047866023 & o. 13752 $55^{28} 3$ \\
\hline 9 & o. I9355 81096 & I I & 3 & 0.0616024003 & I.00604 76005 & o. I 54592183 I \\
\hline Io & 0.2150645662 & 12 & I6 & $0.06808 \quad 70479$ & I . $00745 \quad$ I 7850 & 0.1716150856 \\
\hline I I & $0.23657 \quad 10228$ & 13 & 28 & 0.0744605194 & I. 0089974482 & o.1 $885^{8} 93888$ \\
\hline I2 & 0.2580774795 & 14 & $4 \mathrm{I}$ & 0.0807 I 293 & 0106827105 & 55102505 \\
\hline 13 & $0.2795^{8} 3936 \mathrm{I}$ & 15 & 53 & 0.0868347367 & I . O1250 55225 & $0.22237 \quad 28335$ \\
\hline I4 & 0.3010903927 & 17 & 6 & $0.09281 \quad 67403$ & I. 0144636673 & 0.2391723067 \\
\hline I 5 & 0.3225968493 & I 8 & I 8 & 5 or 256 & I.oI655 47635 & o.25590 38457 \\
\hline 16 & 41033059 & 19 & 29 & o. 10432646 & 37762678 & 25626330 \\
\hline I 7 & 0.3656097626 & 20 & 40 & o. 1098377593 & I. 0211254784 & $9 I 43859 \mathrm{I}$ \\
\hline I 8 & 0.387 I I 62192 & $2 \mathrm{I}$ & $5 \mathrm{I}$ & o.II5I7 64068 & I.02359 95379 & 56427234 \\
\hline 19 & $0.408622675^{8}$ & 23 & 2 & o. 1203352604 & I. 0261954370 & 0.3220544344 \\
\hline 20 & 0.4301291324 & 24 & I3 & o.12530 76I46 & I. 0289I OOI79 & $0.33^{8} 37 \quad 42110$ \\
\hline $2 \mathrm{I}$ & $0.45^{163} 55^{8}$ & 25 & 22 & 0.13008721 & I.0317399787 & 0.3545972832 \\
\hline 22 & 0.47314204 & 26 & 3 I & o.13466 82799 & I.03468 I 8764 & 0.3707188930 \\
\hline 23 & 0.4946485023 & 27 & $4 I$ & o.13904 54724 & I. 0377321323 & 0.3867342953 \\
\hline 24 & $0.51615495^{89}$ & 28 & 50 & o.I 432139340 & I. $040887035^{2}$ & $0.40263875^{89}$ \\
\hline 25 & $766 \quad 141$ & 29 & 59 & o. I4716 92687 & I.044I 427466 & 0.4184275678 \\
\hline 26 & 0.5591678722 & $3 I$ & 6 & o.15090 75443 & I. 0474953052 & 0.4340960218 \\
\hline 27 & o. 5806743288 & 32 & I4 & o.15442 528 & I. 0509406315 & 49639438 I \\
\hline 28 & 0.6021807854 & 33 & $2 \mathrm{I}$ & o. I577 I 9487 I & I. 0544745329 & 0.4650531522 \\
\hline 29 & 0.6236872420 & 34 & 29 & 0.1607875703 & .I .05809 27090 & $0.480332519 \mathrm{I}$ \\
\hline 30 & 51936 & 35 & 36 & 36274123 & I.06I 790756 I & 0.4954729148 \\
\hline $3 \mathrm{I}$ & 6670 oI & 36 & $4 I$ & o. 1662373 & I. $06556 \quad 41737$ & 4697376 \\
\hline 32 & 0.68820661 & 37 & 46 & 0.16861601 & I .06940 83686 & 253 I 8409 I \\
\hline 33 & 0.7097 I 30685 & 38 & $5 \mathrm{I}$ & o.17076 2634I & I.0733I 866I7 & $400 I 4376 I$ \\
\hline 34 & $0.731219525 I$ & 39 & 56 & o. I7267 67142 & I.0772902929 & 0.5545531119 \\
\hline 35 & 72598 & $4 \mathrm{I}$ & I & o.I7435817 & I. 0813184270 & 6893 o1 \\
\hline 36 & $0.77423 \quad 243^{84}$ & 42 & 4 & o. I7580 72936 & I. 085398 I 601 & $0.5^{83} 1409242$ \\
\hline 37 & o.7957388950 & 43 & 7 & o.17702 47258 & I. $0895^{2} 45^{247}$ & 0.59718 10935 \\
\hline $3^{8}$ & 0.8172453516 & 44 & 9 & o. I780I I 4536 & I. 0936924965 & 0.6110462201 \\
\hline 39 & $0.83875 \quad 18083$ & 45 & 12 & o. 1787687890 & I. 0978970001 & $0.62473 \quad 19335$ \\
\hline 40 & o. 8602582649 & 46 & 15 & o.I7929 83 & I. 1021329153 & 382338991 \\
\hline $4 I$ & 0.88 I $76 \quad 47215$ & 47 & 15 & 0.17960206 & I. 1063950831 & 5478204 \\
\hline 42 & 0.90327 I I 78 I & 48 & 16 & o.17968 212 & I. I I06783124 & 6694406 \\
\hline 43 & 0.9247776347 & 49 & I6 & o. I7954 09878 & I. I I 4977386 I & 0.6775945449 \\
\hline 44 & 0.9462840914 & 50 & I 7 & o.179I 8 1364I & I. I1928 70673 & 0.6903189618 \\
\hline 45 & 0.9677905480 & $5 \mathrm{I}$ & 17 & o.17860 61952 & I. $123602105^{8}$ & $0.702838565^{2}$ \\
\hline $90-\mathrm{r}$ & $\mathbf{F} \psi$ & & & $\mathbf{G}(\mathbf{r})$ & $\mathbf{C}(\mathbf{r})$ & $\mathrm{B}(\mathbf{r})$ \\
\hline
\end{tabular}


TABLE $\theta=50^{\circ}$

$q=0.055019933698829, \quad \Theta 0=0.8899784604, \quad$ HK $=0.9715669451$

\begin{tabular}{|c|c|c|c|c|c|}
\hline $\mathbf{B}(\mathbf{r})$ & $\mathbf{C}(\mathbf{r})$ & $\mathbf{G}(\mathbf{r})$ & $\psi$ & $\mathrm{F} \psi$ & $90-r$ \\
\hline I.00000 00000 & I. $24728 \quad 65857$ & 0.0000000000 & $90^{\circ} \quad 0^{\prime}$ & I. $9355^{8}$ I0960 & 90 \\
\hline 0.9998440186 & I. 2472 I I2 I 54 & 0.0056 I 92362 & $89 \quad 12$ & I. $91407 \quad 46394$ & 89 \\
\hline 0.9993761319 & I. $24698 \quad 51964$ & o.01I23 36482 & 25 & I . 8925681828 & 88 \\
\hline 0.9985965127 & I. 2466088048 & 0.0168384106 & 37 & I.87106 17261 & 87 \\
\hline $0.99750 \quad 54487$ & I. 2460824999 & 0.0224289646 & 50 & I. 8495552695 & 86 \\
\hline 0.9961033424 & I. $24540 \quad 69243$ & o.0279996670 & 86 & I. 8280488129 & 85 \\
\hline 0.9943907108 & I. $2445^{8} 29027$ & 0.0335464884 & 14 & I. 8065423563 & 84 \\
\hline 0.992368 I 849 & I. 24361 I 4410 & 0.0390643123 & 26 & I.78503 58997 & 83 \\
\hline 0.9900365093 & I. 2424937250 & 0.0445482835 & 39 & I. 7635294430 & 82 \\
\hline 0.9873965416 & I.24123 III92 & 0.0499935367 & $5 I$ & I. 7420229864 & 81 \\
\hline 0.9844492517 & I. 2398251648 & 0.055395 I96I & 82 & I.7205I 65298 & 80 \\
\hline 0.9811957210 & I. 2382775779 & 0.0607483740 & $8 \mathbf{I}$ & I. 6990100732 & 79 \\
\hline 0.9776371417 & I. 2365902476 & 0.0660481700 & 26 & I. $67750 \quad 36165$ & 78 \\
\hline 0.9737748160 & I. $23476 \quad 52334$ & 0.0712896708 & 37 & I.655997I599 & 77 \\
\hline 0.96961 or 546 & I. $23280 \quad 47629$ & 0.0764679497 & 49 & I. 63449 o7033 & 76 \\
\hline 0.9651446762 & I.23071 12287 & 0.0815780662 & 78 & I. 6129842467 & 75 \\
\hline 0.9603800059 & I. $22848 \quad 7$ I 860 & 0.0866 I 50665 & 77 & I. 5914777901 & 74 \\
\hline 0.9553 I 78745 & I.226I3 5349I & 0.0915739836 & $2 I$ & I. 56997 I 3334 & 73 \\
\hline 0.94996 oI I 67 & I. 2236585882 & 0.0964498379 & $3 I$ & I. 5484648768 & 72 \\
\hline 0.9443086698 & I.2210599257 & 0. I0123 76383 & 74 & I. 5269584202 & 71 \\
\hline 0.9383655727 & I. 2183425328 & o. 1059323833 & 52 & I.50545 I9636 & 70 \\
\hline 0.9321329639 & I. 2155097252 & o.1105290627 & 73 I & I.48394 55069 & 69 \\
\hline 0.9256130802 & I. 2125649596 & o.II502 26595 & $72 \quad$ I I & I. 4624390503 & 68 \\
\hline $0.918808255^{2}$ & I. $2095 \mathrm{I} \quad 18289$ & o. I I940 81521 & 20 & I. 4409325937 & 67 \\
\hline 0.9117209173 & I. 2063540582 & 0.1236805174 & 30 & I. 419426137 I & 66 \\
\hline $0.9043535^{883}$ & I .20309 54999 & o.12783 47335 & 69 & I 3979 I 96805 & 65 \\
\hline 0.89670888 I 5 & I. 19974 OI 294 & o.I3I86 57834 & 47 & I. $37641 \quad 32238$ & 64 \\
\hline 0.8887894998 & I. 1962920396 & o. I3576 86595 & 55 & I. $35490 \quad 67672$ & 63 \\
\hline 0.8805982341 & I. I9275 54368 & o. 1395383674 & 67 & I. 3334003106 & 62 \\
\hline 0.8721379612 & I. I 89 I3 46345 & 0.1431699314 & 10 & I.31189 38540 & $6 I$ \\
\hline 0.86341 I 6420 & I. I 854340490 & o.14665 83999 & 65 & I .29038 73973 & 60 \\
\hline $0.85442 \quad 23195$ & I. I8I65 8I935 & o. I499988516 & 24 & I. 2688809407 & 59 \\
\hline 0.8451731166 & I. I 778 I I6727 & o.15318 64017 & 30 & I .24737 4484I & $5^{8}$ \\
\hline 0.8356672345 & I. I738991774 & o.I5621 62095 & 36 & I. $225^{86} 80275$ & 57 \\
\hline 0.8259079506 & I. I6992 54783 & o. I5908 34859 & 42 & I. $20436 \quad$ I 5709 & $5^{6}$ \\
\hline $0.8 \mathrm{I} 58986 \mathbf{I} 6 \mathbf{I}$ & I. I6589 54205 & 0.1617835017 & 48 & Í. 182855 II 42 & 55 \\
\hline 0.8056426543 & I. I6I8I 39175 & o. I643I I 5964 & 59 & I. I6I34 86576 & 54 \\
\hline $0.79514355^{83}$ & I. I 576859453 & o. I6666 31878 & $5^{8}$ & I. 1398422010 & 53 \\
\hline $0.784404889 \mathrm{I}$ & I. I 535 I 6536 I & o. 1688337818 & $5^{8}$ & I. II 83357444 & $5^{2}$ \\
\hline 0.7734302735 & I. I 4931 07723 & o.I7081 89832 & 57 & I. $09682 \quad 92877$ & $5^{I}$ \\
\hline 0.7622234019 & I. 1450737802 & o.1726r 45069 & $5^{6}$ & I.07532 283 II & 50 \\
\hline 0.7507880264 & I. I 408107240 & o.I742I 61892 & 55 & I.0538I 63745 & 49 \\
\hline 0.7391279584 & I. I3652 67992 & 0.1756200006 & 54 & I .03230 99179 & 48 \\
\hline 0.7272470671 & I. I3222 72263 & o. 1768220583 & 53 & I. $01080 \quad 34613$ & 47 \\
\hline 0.7151492767 & I. I2791 72446 & o.I778I 86395 & I5 & 0.9892970046 & 46 \\
\hline 0.7028385652 & I. $123602105^{8}$ & o. I7860 61952 & $\begin{array}{ll}51 & 17\end{array}$ & 0.9677905480 & 45 \\
\hline$A(r)$ & $\mathbf{D}(\mathbf{r})$ & $\mathbf{E}(\mathbf{r})$ & $\phi$ & $\mathbf{F} \phi$ & $\mathbf{r}$ \\
\hline
\end{tabular}


$K=2.0347153122, \quad K^{\prime}=1.7312451757, \quad E=1.2586796248, \quad E^{\prime}=1.4322909693$,

\begin{tabular}{|c|c|c|c|c|c|c|}
\hline $\mathbf{r}$ & $\mathrm{F} \phi$ & & & $\mathbf{E}(\mathbf{r})$ & $\mathbf{D}(\mathbf{r})$ & $A(r)$ \\
\hline 0 & 0.0000000000 & & $o^{\prime}$ & 0.0000000000 & I. 0000000000 & 0.0000000000 \\
\hline I & 0.0226079479 & I & I 8 & 0.0086200346 & I.00009 74600 & $0.01712 \quad 13223$ \\
\hline 2 & $0.045^{21} 5^{895^{8}}$ & 2 & 35 & o.01722 45749 & I. 0003897217 & 0.0342380342 \\
\hline 3 & $0.06782 \quad 38437$ & 3 & 53 & 0.025798 I 795 & I. 0008764305 & 0.0513455249 \\
\hline 4 & 0.0904317916 & 5 & 10 & $0.0343^{2} 55^{123}$ & I.00I55 69957 & 0.0684391832 \\
\hline 5 & o. II30397395 & 6 & 28 & 0.04279 I 3942 & I.0024305914 & 0.0855 I 4397 I \\
\hline 6 & o.13564 76875 & 7 & 45 & 0.0511808539 & I.003496I575 & o. 1025665538 \\
\hline 7 & o.15825 56354 & 9 & 2 & o.05947 91769 & I. 0047524006 & o. 11959 10390 \\
\hline 8 & o. I $808635^{833}$ & Io & 19 & $0.06767 \quad 19530$ & I.006I9 77962 & o. $1365^{8} \quad 32373$ \\
\hline 9 & $0.20347 \quad 15312$ & I I & 36 & 0.0757451216 & I.00783 05901 & o. I 5353853 I 8 \\
\hline 10 & 0.2260794791 & 12 & 52 & 0.0836850144 & I. .0096488003 & o. 1704523039 \\
\hline I I & 0.2486874270 & I4 & 9 & o.0914783960 & I. 0116502201 & o.18731 99332 \\
\hline I 2 & 0.2712953749 & 15 & 25 & o.099II 25013 & I.01383 24199 & 0.2041367975 \\
\hline I3 & 0.2939033229 & 16 & 40 & o. 1065750694 & I.01619 27508 & 0.2208982730 \\
\hline 14 & $0.31651 \quad 12708$ & I 7 & 56 & o.II385 43755 & I.01872 83473 & 0.2375997340 \\
\hline I 5 & 0.339 II 92187 & 19 & I I & o. 1209392580 & I.02I43 6I3II & 0.2542365532 \\
\hline 16 & 0.3617271666 & 20 & 25 & 0.12781 91435 & I. $02431 \quad 28147$ & 0.2708041017 \\
\hline I 7 & 0.384335 I I 45 & $2 \mathrm{I}$ & 40 & o.13448 40670 & I. 0273549050 & 0.2872977496 \\
\hline I 8 & 0.4069430624 & 22 & 54 & o.14092 46901 & I. 0305587080 & 0.3037128656 \\
\hline I9 & 0.42955 10103 & 24 & 7 & o. 1471323140 & I .03392 03331 & 0.3200448178 \\
\hline 20 & $0.45^{215} 89583$ & 25 & 20 & o.1530988906 & I. $03743 \quad 56974$ & o. 3362889743 \\
\hline $2 \mathrm{I}$ & 0.4747669062 & 26 & 33 & o.1588I 70288 & I. 0411005314 & 0.3524407031 \\
\hline 22 & 0.4973748541 & 27 & 45 & o.1642799989 & I. $04491 \quad 03831$ & 0.3684953729 \\
\hline 23 & 0.5199828020 & 28 & 56 & o. $16948 \quad 17327$ & I. 0488606244 & 0.3844483538 \\
\hline 24 & 0.5425907499 & 30 & 8 & o.1744I 68208 & I. $052946455^{8}$ & 0.4002950181 \\
\hline 25 & 0.5651986978 & $3 I$ & 18 & o.17908 05075 & 1.05716 29130 & 0.4160307408 \\
\hline 26 & 0.5878066457 & 32 & 28 & o. I8346 86827 & I.06I50 48720 & 0.4316509003 \\
\hline 27 & 0.6104145937 & 33 & 38 & o.18757 78710 & I.06596 70560 & 0.4471508801 \\
\hline 28 & 0.6330225418 & 34 & 46 & o.19I40 52 I 88 & I. $07054404 \mathbf{I}$ & 0.4625360691 \\
\hline 29 & 0.6556304895 & 35 & 55 & o. 1949484794 & I. .0752302647 & $0.47777 \quad 18627$ \\
\hline 30 & 0.6782384374 & 37 & 3 & o.19820 59959 & I. 0800200285 & $0.49288 \quad 36645$ \\
\hline 31 & 0.7008463853 & 38 & 10 & 0.2011766827 & I. $08490 \quad 75092$ & 0.5078568872 \\
\hline 32 & $0.723454333^{2}$ & 39 & 16 & 0.2038600053 & I. $08988 \quad 67634$ & $0.522686954 \mathrm{I}$ \\
\hline 33 & 0.7460622811 & 40 & 23 & 0.2062559591 & I. $09495 \quad 17358$ & 0.5373693004 \\
\hline 34 & 0.7686702290 & $4 \mathrm{I}$ & 28 & 0.2083650468 & I. 1000962656 & 0.5518993747 \\
\hline 35 & 0.7912781769 & 42 & 33 & 0.2101882554 & I. 1053140947 & o. 5662726408 \\
\hline 36 & 0.8138861249 & 43 & 38 & 0.2117270324 & I . I I059 88749 & $0.58048 \quad 45794$ \\
\hline 37 & 0.8364940728 & 44 & $4 I$ & 0.21298326 I I & I. II 5944 I 760 & 0.5945306894 \\
\hline 38 & $0.85910 \quad 20207$ & 45 & 45 & 0.2139592364 & I. I2I34 34929 & 0.6084064905 \\
\hline 39 & 0.8817099686 & 46 & 48 & 0.2146576400 & I. 1267902542 & 0.6221075244 \\
\hline 40 & 0.9043179165 & 47 & 50 & $0.21508 \quad 15155$ & I. 1322778297 & $0.635629357 \mathrm{I}$ \\
\hline $4 I$ & 0.9269258644 & 48 & $5 \mathrm{I}$ & 0.2152342440 & I. 1377995386 & 0.6489675812 \\
\hline 42 & $0.94953 \quad 38123$ & 49 & 53 & 0.2151195200 & I. I 433486579 & 0.6621178175 \\
\hline 43 & 0.9721417602 & 50 & 53 & 0.2147413276 & I. I4891 84299 & $0.67507 \quad 57$ I 77 \\
\hline 44 & 0.994749708 I & 51 & 53 & 0.2141039170 & I. I 5450207 I I & 0.6878369663 \\
\hline 45 & I.0I735 7656 I & 52 & 52 & $0.21321 \quad 17818$ & 1. 1600927802 & 0.7003972833 \\
\hline $90-r$ & $\mathrm{~F} \psi$ & & & $\mathbf{G}(\mathbf{r})$ & $C(r)$ & $\mathbf{B}(\mathbf{r})$ \\
\hline
\end{tabular}






TABLE $\theta=55^{\circ}$

$q=0.069042299609032, \quad \theta 0=0.8619608462, \quad \mathrm{HK}=1.0300875730$

\begin{tabular}{|c|c|c|c|c|c|}
\hline $\mathbf{B}(\mathbf{r})$ & $\mathrm{C}(\mathrm{r})$ & $\mathbf{G}(\mathbf{r})$ & $\psi$ & $\mathrm{F} \psi$ & $90-r$ \\
\hline I.00000 00000 & I.3203964540 & 0.0000000000 & $90^{\circ} \quad \mathrm{o}^{\prime}$ & .2 .03471 53122 & 90 \\
\hline o.99984 I9I55 & I.320298737 I & 0.0065466917 & $89 \quad 15$ & 2.0121073643 & 89 \\
\hline 0.9993677261 & I.32000 57060 & 0.0I308 82806 & $3 I$ & I. 9894994164 & 88 \\
\hline 0.9985776238 & I.3195I 77192 & 0.0196I 96606 & 46 & I. 96689 I 4685 & 87 \\
\hline $0.99747 \quad 19280$ & I. 3188353734 & 0.0261357182 & 87 & I. $94428 \quad 35205$ & 86 \\
\hline 0.9960510861 & I.3179595033 & $0.03263 \quad 13295$ & 86 & I. 9216755726 & 85 \\
\hline 0.9943156720 & I. 31689 II 801 & 0.0391013564 & 32 & I. 8990676247 & 84 \\
\hline 0.9922663864 & I. 3156317106 & $0.04554 \quad 06434$ & 47 & I. 8764596768 & 83 \\
\hline 0.9899040553 & I. $31418 \quad 26349$ & 0.0519440144 & 84 & I. $85385 \quad 17289$ & 82 \\
\hline 0.9872296302 & I. 3125457253 & 0.0583062693 & 83 & I. 8312437810 & $8 \mathrm{I}$ \\
\hline $0.984244186 I$ & I. 3107229838 & 0.0646221812 & 82 & I. 8086358331 & 80 \\
\hline 0.9809489213 & I.3087I 66392 & 0.0708864934 & 46 & I. $786027885 \mathrm{I}$ & 79 \\
\hline $0.977345155^{8}$ & I. 3065291.449 & 0.0770939167 & $8 I$ & I.76341 99372 & 78 \\
\hline 0.9734343300 & I.304I6 31759 & 0.0832391270 & 80 & I.74081 19893 & 77 \\
\hline 0.9692180039 & 1.3016216250 & 0.0893167629 & 79 & I. $71820 \quad 40414$ & 76 \\
\hline 0.9646978546 & I . 2989075994 & $0.09532 \quad 14240$ & 78 & I.6955960935 & 75 \\
\hline $0.959875675^{8}$ & I. $29602 \quad 44173$ & 0.1012476688 & 77 & I. 6729881456 & 74 \\
\hline 0.9547533753 & I. 2929756032 & 0.1070900133 & ro & I. 65038 oI977 & 73 \\
\hline 0.9493329736 & I. 2897648840 & O.II284 2930I & 23 & I. 6277722497 & 72 \\
\hline 0.9436166021 & I. 2863961840 & o. I I 85008473 & 75 & I. 6051643018 & 71 \\
\hline 0.9376065006 & I. 2828736204 & 0.1240581487 & 74 & I. 5825563539 & 70 \\
\hline 0.9313050161 & I. $27920 \quad 14980$ & 0.1295091731 & 74 & I. 5599484060 & 69 \\
\hline 0.9247145998 & I. $27538 \quad 4304$ I & o.1348482153 & 73 & I. 537340458 I & 68 \\
\hline 0.9178378055 & I.27142 67027 & 0.1400695267 & 23 & I. 5147325102 & 67 \\
\hline 0.9106772870 & I .26733 3529I & 0.1451673172 & 35 & I. 4921245623 & 66 \\
\hline $0.903235796 \mathrm{I}$ & I. 2631097835 & o.15013 57566 & 70 & I. 4695 I 66 I 44 & 65 \\
\hline $0.89551 \quad 61797$ & I. 2587606253 & o.1549689777 & 69 & I. 4469086665 & 64 \\
\hline $0.8875^{2} \quad \mathrm{I} 377^{8}$ & I. $25429 \quad 13663$ & o. I5966 10790 & 69 & I. 4243007185 & 63 \\
\hline 0.8792544206 & I. 2497074646 & 0.1642061290 & 68 & I. 4016927706 & 62 \\
\hline 0.8707184265 & I. $24501 \quad 45176$ & o.168598170I & 6726 & I. $37908 \quad 48227$ & $6 I$ \\
\hline 0.86 I9I 65988 & I. $240218255^{2}$ & o. I7283 I2244 & 66 & I. 3564768748 & 60 \\
\hline 0.8528522237 & I. 2353245329 & o.176899299I & 65 & I. 3338689269 & 59 \\
\hline $0.8435^{2} 86672$ & I. 2303393242 & o. I8079 63935 & 64 & I. 3112609790 & $5^{8}$ \\
\hline 0.8339493726 & I. 2252687137 & 0.1845 I 65064 & 63 & I. 28865303 II & 57 \\
\hline 0.824 II 78578 & I.220II 88895 & o. 1880536444 & $63^{\circ}$ & I. 2660450832 & 56 \\
\hline 0.8140377126 & I. 2148961356 & o. 19140 I 8312 & $62 \quad 12$ & I. 243437 I 353 & 55 \\
\hline 0.8037125960 & I. 2096068240 & o. 1945551177 & $6 I, 19$ & I. 2208291873 & 54 \\
\hline 0.7931462334 & I . 2042574072 & o. 1975075927 & $60 \quad 24$ & I. $19822 \quad 12394$ & 53 \\
\hline 0.7823424136 & I. $1988544 \mathrm{IO2}$ & 0.2002533955 & 59 & I. $17561 \quad 32915$ & $5^{2}$ \\
\hline 0.7713049868 & I. $19340 \quad 44225$ & 0.2027867279 & 58 & I. I 530053436 & $5 I$ \\
\hline 0.7600378612 & I.I879I 40899 & $0.20510 \quad 18688$ & 57 & I. I303973957 & 50 \\
\hline 0.7485450007 & I. 1823901066 & 0.2071931885 & 56 & I. 1077894478 & 49 \\
\hline 0.7368304220 & I. I 768392068 & $0.209055^{1650}$ & 55 & I. 085 I $8 \quad$ I 4999 & 48 \\
\hline 0.7248981922 & I. I7 12681567 & 0.2106824001 & 54 & I. 06257355 I9 & 47 \\
\hline 0.7127524260 & I. 1656837461 & 0.2120696376 & 53 & I.03996 56041 & 46 \\
\hline 0.7003972833 & I. 1600927802 & $0.21321 \quad 17818$ & $5^{2} \quad 5^{2}$ & I.0I735 7656I & 45 \\
\hline $\mathbf{A}(\mathbf{r})$ & $\mathbf{D}(\mathbf{r})$ & $\mathbf{E}(\mathbf{r})$ & $\phi$ & $\mathbf{F} \phi$ & $\mathbf{r}$ \\
\hline
\end{tabular}


$K=2.1565156475, \quad K^{\prime}=1.6857503548, \quad E=1.211056028, \quad E^{\prime}=1.4674622093$,

\begin{tabular}{|c|c|c|c|c|c|}
\hline $\mathbf{r}$ & $\mathbf{F} \phi$ & $\phi$ & $\mathbf{E}(\mathbf{r})$ & $\mathbf{D}(\mathbf{r})$ & $\mathbf{A}(\mathbf{r})$ \\
\hline o & 0.0000000000 & $0^{\circ} \quad 0^{\prime}$ & 0.0000000000 & I .00000 00000 & 0.0000000000 \\
\hline $\mathbf{I}$ & $0.02396 \quad 12850$ & I 22 & 0.0105021636 & I. $000125845^{2}$ & 0.0169424822 \\
\hline 2 & 0.0479225699 & 45 & $0.02098 \quad 36904$ & I. $00050 \quad 32288$ & $0.033880735^{I}$ \\
\hline 3 & 0.07 I $88 \quad 38549$ & 7 & 0.0314240274 & I. .OOI I.3 I6945 & 0.0508105279 \\
\hline 4 & $0.095845^{1} 399$ & 29 & 0.0418027880 & I. .00201 .04822 & $0.06772 \quad 76275$ \\
\hline 5 & o.II980 64248 & $5^{I}$ & 0.0520998337 & I.003I3 85295 & 0.0846277970 \\
\hline 6 & o. 1437677098 & I3 & o.06229 53533 & I.0045I 44723 & o. 10150 67944 \\
\hline 7 & o.16772 89948 & 35 & 0.0723699392 & I. 0061366468 & o. II 836037 I 7 \\
\hline 8 & o.1916902798 & 56 & 0.0823046606 & I. 00800309 II & o.I 35 I $8 \quad 42734$ \\
\hline 9 & 0.21565 I 5647 & I 7 & 0.09208 II 326 & I.OIOI I I 5480 & o. I5197 42358 \\
\hline I0 & 0.2396 I 28497 & 38 & o. 10168 I 5801 & I. OI245 94672 & o. I6872 59855 \\
\hline I I & 0.2635741347 & $5^{8}$ & o. I II I08 88976 & I. 0150440088 & o. 1854352386 \\
\hline 12 & 0.2875354197 & I 8 & o.12028 67034 & I.OI786 20463 & 0.2020976999 \\
\hline 13 & 0.3114967046 & $3^{8}$ & o.12925 93879 & I.0209I OI70I & 0.2187090619 \\
\hline 14 & 0.3354579896 & 57 & o. 13799 21563 & I.024I8 46923 & 0.2352650037 \\
\hline I5 & 0.3594192746 & I6 & o. $14647 \quad 10652$ & I.02768 I6504 & 0.25176 II9II \\
\hline I6 & $0.3^{8} 33^{8} \quad 05595$ & $2 \mathrm{I}$ & o. I5468 30530 & I.03I3968I20 & 0.268 I9 $3275^{\circ}$ \\
\hline I 7 & $0.40734 \quad 18445$ & 53 & o.1626I 59647 & I. 0353256803 & 0.2845568916 \\
\hline I 8 & $0.43130 \quad 31295$ & IO & o.17025 85702 & I. 0394634991 & 0.3008476617 \\
\hline I9 & $0.45526 \quad 44145$ & 25 & 0.1776005773 & I. $04380 \quad 525^{8} 3$ & 0.31706 II 903 \\
\hline 20 & 0.4792256994 & 42 & o. 1846326382 & I. 0483457003 & 0.3331930665 \\
\hline $2 \mathrm{I}$ & 0.5031869844 & 27 & o.19134 63517 & I. 0530793260 & o. 3492388634 \\
\hline 22 & 0.5271482694 & 29 & o. 1977342593 & I. 05800 04010 & $0.365 I 94 I 38 I$ \\
\hline 23 & 0.55 II0 95544 & 30 & $0.203789837 \mathbf{I}$ & I.06310 29632 & $0.3^{8105} 44318$ \\
\hline 24 & 0.5750708393 & $3 I$ & 0.2095074827 & I . 068380829 I & o.3968I 52701 \\
\hline 25 & 0.5990321243 & 32 & $0.21488 \quad 24988$ & I.07382 76019 & 0.4124721633 \\
\hline 26 & 0.6229934093 & 34 & $0.21991 \quad 10718$ & I. 0794366784 & 0.4280206069 \\
\hline 27 & 0.6469546942 & 35 & 0.2245902484 & I . $08520 \quad$ I 2575 & 0.4434560826 \\
\hline 28 & 0.6709159792 & 29 & $0.22891 \quad 79082$ & I. 09 I I I 43480 & $0.45^{877} 40585$ \\
\hline 29 & 0.6948772642 & 37 & 0.2328927342 & I.09716 8777I & 0.4739699905 \\
\hline 30 & 0.7188385492 & 38 & $0.2365 I \quad 4 I 807$ & I. 1033571989 & 0.4890393230 \\
\hline $3 I$ & o. 7427998341 & 39 & 0.2397824399 & I. 1096721031 & 0.5039774905 \\
\hline 32 & 0.76676 II I9I & $4 \mathrm{I}$ & 0.2426984060 & I. I 61058243 & 0.5187799184 \\
\hline 33 & 0.7907224041 & 42 & $0.24526 \quad 36394$ & I. I 2265055 IO & 0.5334420249 \\
\hline 34 & 0.8146836890 & 43 & $0.24748 \quad 03283$ & I. I 292983350 & 0.5479592224 \\
\hline 35 & 0.8386449740 & 44 & 0.24935 I2513 & I. 13604 IIOIO & 0.5623269191 \\
\hline 36 & 0.8626062590 & 45 & 0.2508797387 & I. 1428706563 & $0.57654 \quad 05212$ \\
\hline 37 & 0.8865675440 & 46 & 0.2520696336 & I. I 497787007 & 0.5905954347 \\
\hline 38 & 0.9105288289 & 47 & 0.2529252540 & I. I5675 68364 & 0.6044870673 \\
\hline 39 & 0.93449 ol 139 & $48 \quad 4^{2}$ & $0.25345 \quad$ I 3545 & I. I637965783 & 0.6182108313 \\
\hline 40 & 0.95845 I 3989 & 49 & 0.2536530884 & I. 1708893642 & $0.631762145 I$ \\
\hline $4 I$ & $0.98241 \quad 26838$ & 45 & 0.25353597 I 3 & I. I 780265652 & 0.6451364364 \\
\hline 42 & I. 0063739688 & 46 & $0.253105845^{0}$ & I. I 851994959 & 0.6583291446 \\
\hline 43 & I. 0303352538 & 52 & 0.2523688429 & I. 1923994253 & 0.6713357232 \\
\hline 44 & I.05429 65388 & $53 \quad 45$ & $0.25133 \quad 1355^{8}$ & I. 1996175873 & $0.68415 \quad 16433$ \\
\hline 45 & I. $07825 \quad 78237$ & $54 \quad 44$ & 0.2500000000 & I. 2068451910 & 0.6967723959 \\
\hline $90-\mathrm{r}$ & $\mathbf{F} \psi$ & $\psi$ & $\mathrm{G}(\mathbf{r})$ & $\mathbf{C}(\mathbf{r})$ & $\mathrm{B}(\mathrm{r})$ \\
\hline
\end{tabular}




\begin{tabular}{|c|c|c|c|c|c|}
\hline $\mathbf{B}(\mathbf{r})$ & $\mathrm{C}(\mathbf{r})$ & $\mathrm{G}(\mathrm{r})$ & $\psi$ & $\mathbf{F} \psi$ & $90-r$ \\
\hline I.00000 00000 & I. 4 I 42 I 35624 & 0.0000000000 & $90^{\circ} \quad \mathrm{o}^{\prime}$ & $2.15651 \quad 56475$ & 90 \\
\hline 0.9998387925 & I.4I 40870799 & 0.0074645017 & 8919 & 2.13255 43625 & 89 \\
\hline $0.99935 \quad 52434$ & I : 4137077878 & o.01492 38646 & 38 & 2.1085930775 & 88 \\
\hline 0.9985495732 & I. 41307615 I 5 & 0.0223729430 & 57 & 2.08463 I7926 & 87 \\
\hline 0.9974221491 & I. 4121929466 & 0.0298065777 & 87 & 2.0606705076 & 86 \\
\hline 0.9959734843 & I.4IIO5 92570 & 0.0372195889 & 86 & 2.0367092226 & 85 \\
\hline $0.99420 \quad 42378$ & I. 4096764744 & 0.0446067701 & 85 & 2.0127479377 & 84 \\
\hline 0.9921152135 & I. $408046295^{8}$ & 0.05196 288I5 & 85 & I. 9887866527 & 83 \\
\hline 0.9897073588 & I. 4061707222 & $0.05928^{\circ} 26440$ & 29 & I. 9648253677 & 82 \\
\hline 0.98698 I 764I & I. $404052055^{I}$ & 0.0665607336 & 47 & I. 9408640827 & $8 \mathrm{I}$ \\
\hline 0.9839396610 & I. 4016928947 & $0.07379 \quad 17757$ & 83 & I.91690 27978 & 80 \\
\hline 0.9805824210 & I.39909 6I356 & 0.08097 0340I & 82 & I. 89294 I5I 28 & 79 \\
\hline 0.9769 I I 554 I & I. 3962649639 & 0.0880909364 & $4 I$ & I. 8689802278 & 78 \\
\hline 0.9729287065 & I. 393202853 I & 0.095 I4 80095 & $5^{8}$ & I. $84501 \quad 89429$ & 77 \\
\hline 0.9686356591 & I.3899I 35592 & 0. I02I3 59353 & 15 & I. 8210576579 & 76 \\
\hline $0.964034325^{\circ}$ & I $\cdot 38640$ I I I 69 & o.1090490I75 & 32 & I.79709 63729 & 75 \\
\hline 0.9591267478 & I.38266 98339 & o. II 588 I 4840 & 78 & I. 7731350879 & 74 \\
\hline 0.9539 I 50985 & I. $37872 \quad 42853$ & o.12262 74837 & 78 & I. 7491738030 & 73 \\
\hline $0.94840 \quad 16738$ & I. 3745693090 & o. I2928 I0844 & 77 & I. 7252125180 & 72 \\
\hline 0.9425888926 & I. 3702099983 & o. 1358362697 & 37 & I. $70125 \quad 12330$ & $7 \mathrm{I}$ \\
\hline $0.936479294 \mathrm{I}$ & I. 36565 I 6965 & o. 1422869378 & 75 & I. 6772899480 & 70 \\
\hline 0.9300755342 & I.3608999899 & o. 1486268991 & 75 & I. 6533286631 & 69 \\
\hline 0.9233803829 & I. 3559607006 & o. I5484 98749 & 74 & I. 6293673781 & 68 \\
\hline 0.9163967210 & I. 3508398797 & o. I6094 94967 & 73 & I. 6054060931 & 67 \\
\hline 0.9091275372 & I. 3455437995 & o. I669I 93054 & 72 & I. 58 I 4448082 & 66 \\
\hline 0.90I57 59245 & I.34007 89457 & o. I7275 27505 & 72 & I. $5574^{8} 35232$ & 65 \\
\hline $0.893745077 \mathrm{I}$ & I. 3344520094 & o.17844 31913 & 71 & I. $5335^{2} 22382$ & 64 \\
\hline o. 8856382868 & I. 3286698789 & o. 1839838964 & 70 & I. 5095609532 & 63 \\
\hline 0.8772589396 & I. 3227396308 & o. I8936 80462 & 69 & I.48559 96683 & 62 \\
\hline 0.8686 I 05122 & I.3I666 85215 & o. $1945^{8} 8734^{\circ}$ & $68 \quad 54$ & I. $46 \mathrm{I} 6383833$ & $6 I$ \\
\hline 0.8596965682 & I.31046 39783 & o. I99638969I & 68 & I. 4376770983 & 60 \\
\hline 0.8505207549 & I. $3041335^{898}$ & $0.2045 \mathrm{I} \quad \mathrm{I} 6802$ & 67 & I. $4 \mathrm{I} 37 \mathrm{I} \quad 58 \mathrm{I} 34$ & 59 \\
\hline 0.8410867990 & I. 29768,50969 & 0.2091997204 & 66 & I. 3897545284 & $5^{8}$ \\
\hline 0.8313985036 & I. 2911263832 & 0.2136958722 & 65 & I. 3657932434 & 57 \\
\hline 0.8214597438 & I. 2844654650 & 0.2179928546 & 45 & I. 34 I $83 \quad 19584$ & 56 \\
\hline 0.8112744636 & I.2777I 04815 & $0.22208333 \mathrm{I} 3$ & 63 & I. 3178706735 & 55 \\
\hline 0.8008466719 & I.27086 96850 & 0.2259599196 & 63 & I. 2939093885 & 54 \\
\hline 0.7901804386 & I. $26395 \quad 14305$ & 0.2296152018 & 62 & I. 269948 IO35 & 53 \\
\hline 0.7792798915 & I. 2569641655 & $0.23304 \quad 17372$ & 61 & I. $24598 \quad 68185$ & $5^{2}$ \\
\hline 0.7681492120 & I. 2499164194 & $0.236232076 \mathrm{I}$ & $2 \mathrm{I}$ & I. 2220255336 & $5^{I}$ \\
\hline 0.7567926317 & I. $24281 \quad 67937$ & $0.239178775^{8}$ & 59 & I. 1980642486 & 50 \\
\hline 0.7452 I 44290 & I. 2356739504 & 0.2418744177 & $5^{8}$ & I. I7410 29636 & 49 \\
\hline $0.73341 \quad 89253$ & I. 2284966025 & $0.24431 \quad 16265$ & 36 & I. I5OI4 I6787 & 48 \\
\hline 0.7214104816 & I.22I 2935025 & 0.2464830908 & 56 & I. I 26 I 803937 & 47 \\
\hline $0.709193495^{2}$ & I. 2140734320 & 0.24838 I5864 & 55 & I. 1022191087 & 46 \\
\hline 0.6967723959 & I. 206845 I910 & 0.2500000000 & $54 \quad 44$ & I. 0782578237 & 45 \\
\hline$A(r)$ & $\mathbf{D}(\mathbf{r})$ & $\mathbf{E}(\mathbf{r})$ & $\phi$ & $\mathbf{F} \phi$ & $\mathbf{r}$ \\
\hline
\end{tabular}

SMITHSONIAN TABLES 
$K=2.3087867982, \quad K^{\prime}=1.6489952185, \quad E=1.1638279645, \quad E^{\prime}=1.4981149284$,

\begin{tabular}{|c|c|c|c|c|c|c|}
\hline $\mathbf{r}$ & $\mathbf{F} \phi$ & & & $\mathbf{E}(\mathbf{r})$ & $\mathbf{D}(\mathbf{r})$ & $A(r)$ \\
\hline 0 & 0.0000000000 & $0^{\circ}$ & $o^{\prime}$ & 0.0000000000 & I .00000 00000 & 0.0000000000 \\
\hline $\mathbf{I}$ & 0.0256531866 & I & 28 & 0.0127171437 & I.00016 31607 & o.or667 62945 \\
\hline 2 & 0.0513063733 & 2 & 56 & 0.0254065870 & I. 0006524464 & 0.0333489266 \\
\hline 3 & 0.0769595599 & 4 & 24 & 0.0380407622 & I.00146 72698 & $0.05001 \quad 42309$ \\
\hline 4 & 0.1026I 27466 & 5 & $5^{2}$ & $0.050592365 \mathrm{I}$ & I. 0026066524 & 0.0666685367 \\
\hline 5 & o.12826 59332 & 7 & 20 & 0.0630344839 & I .00406 92257 & 0.0833081651 \\
\hline 6 & o.1539I 9I I99 & 8 & 47 & 0.0753407235 & I. .0058532333 & 0.0999294260 \\
\hline 7 & o. I7957 23085 & Io & 14 & 0.0874853252 & I.00795 65320 & o. I I652 86I 59 \\
\hline 8 & 0.2052254932 & I I & $4 I$ & 0.0994432800 & I. 0103765954 & o. 1331020150 \\
\hline 9 & 0.2308786798 & 13 & 8 & O. I I I I9 0434 I & I.013II 05159 & o. 1496458850 \\
\hline IO & o. 25653 I 8665 & 14 & 34 & o. $1227035^{875}$ & I.0I6I5 50083 & o.16615 64662 \\
\hline I I & 0.282 I 850531 & 16 & o & o. 1339605824 & I.01950 64139 & o.1826299754 \\
\hline 12 & 0.3078382398 & 17 & 25 & o. I 449403827 & I.02316 07042 & o. 1990626038 \\
\hline I3 & $0.33349 \quad 14264$ & I 8 & 50 & o. I 556231436 & I. .027 I I 34860 & 0.2 I545 05144 \\
\hline 14 & 0.3591446131 & 20 & 14 & o.1659902705 & I. 0313600060 & 0.2317898405 \\
\hline 15 & 0.3847977997 & $2 \mathrm{I}$ & 38 & o. I7602 44678 & I. $03589 \quad 51569$ & 0.2480766833 \\
\hline 16 & 0.4104509864 & 23 & I & o. 1857097766 & I. 0407 I 34825 & 0.2643071105 \\
\hline I7 & 0.4361041730 & 24 & 23 & o. 1950316024 & I .04580 9I 848 & $0.28047 \quad 71545$ \\
\hline I 8 & 0.4617573596 & 25 & 44 & 0.2039767323 & I. 0511761304 & 0.2965828 II \\
\hline 19 & $0.48741 \quad 05463$ & 27 & 4 & 0.2125333427 & I.05680 78572 & 0.3126000376 \\
\hline 20 & $0.5^{1306} 37329$ & 28 & 24 & 0.2206909968 & I. 0626975825 & o. 3285847528 \\
\hline $2 I$ & 0.5387169196 & 29 & 43 & 0.2284406338 & I. 0688382109 & 0.3444728350 \\
\hline 22 & 0.5643701062 & $3 I$ & 1 & 0.2357745496 & I. 0752223418 & 0.3602801217 \\
\hline 23 & $0.59002 \quad 32929$ & 32 & 19 & 0.2426863696 & I. 0818422789 & 0.3760024088 \\
\hline 24 & 0.6156764795 & 33 & 36 & 0.24917 IOI5I & I . 0886900386 & 0.3916354503 \\
\hline 25 & 0.6413296662 & 34 & 52 & $0.255^{22} 46626$ & I. 0957573598 & 0.4071749584 \\
\hline 26 & 0.6669828528 & 36 & 7 & 0.2608446988 & I. 1030357129 & $0.4226 \mathrm{I} 66028$ \\
\hline 27 & 0.6926360395 & 37 & $2 I$ & 0.2660296698 & I. I105I 63106 & 0.4379560117 \\
\hline 28 & 0.7182892261 & 38 & 34 & 0.2707792271 & I. II8I9 oII75 & $0.45318 \quad 87717$ \\
\hline 29 & 0.7439424127 & 39 & 46 & 0.2750940704 & I. 1260478613 & $0.46831 \quad 04285$ \\
\hline 30 & 0.7695955994 & 40 & $5^{8}$ & 0.2789758872 & I . I3408 00433 & $0.48331 \quad 64880$ \\
\hline $3 I$ & 0.7952487860 & 42 & 9 & 0.2824272920 & I . I 4227 69496 & $0.49820 \quad 24170$ \\
\hline 32 & $0.82090 \quad 19727$ & 43 & I 8 & $0.28545 \quad$ I 7629 & I. I 506286634 & 0.5129636449 \\
\hline 33 & 0.8465551593 & 44 & 26 & 0.2880535786 & I. $159125075^{2}$ & 0.5275955647 \\
\hline 34 & 0.8722083460 & 45 & 34 & $0.290237755^{1}$ & I. $16775 \quad 58964$ & 0.5420935352 \\
\hline 35 & $0.89786 \quad 15326$ & 46 & $4 \mathrm{I}$ & 0.2920099830 & I. $17651 \quad 06705$ & o. $55645 \quad 28823$ \\
\hline 36 & $0.9235 \mathrm{I} \quad 47 \mathrm{I} 93$ & 47 & 47 & 0.2933765659 & I. I 853787860 & 0.5706689018 \\
\hline 37 & 0.9491679059 & 48 & $5^{2}$ & 0.2943443597 & I. I 943494887 & $0.58473686 \mathrm{I}_{4}$ \\
\hline 38 & $0.97482 \quad 10926$ & 49 & 56 & 0.2949207 I 41 & I .2034 I I 895 I & 0.5986520033 \\
\hline $39^{\circ}$ & I. 0004742792 & 50 & 59 & 0.295 II 34159 & I. 2125550050 & 0.6124095465 \\
\hline 40 & 1. $02612 \quad 74659$ & 52 & I & 0.2949306347 & I. 2217677148 & $0.62600 \quad 46907$ \\
\hline 41 & I. 0517806525 & 53 & 2 & 0.2943808705 & 1. 2310388308 & 0.6394326185 \\
\hline 42 & I. $07743 \quad 38392$ & 54 & 2 & 0.2934729047 & I. 2403570830 & 0.6526884992 \\
\hline 43 & I. $103087025^{8}$ & 55 & I & 0.2922 I 57532 & I . 2497 I I I383 & 0.6657674922 \\
\hline 44 & I. I2874 02 I 25 & 56 & o & 0.2906186227 & I. 2590896 I 45 & 0.6786647507 \\
\hline 45 & I. I 54393399 I & 56 & $5^{8}$ & 0.288690869 I & I. $26848 \quad 10938$ & 0.6913754254 \\
\hline $90-r$ & $\mathbf{F} \psi$ & & & $\mathbf{G}(\mathbf{r})$ & $C(\mathbf{r})$ & $\mathbf{B}(\mathbf{r})$ \\
\hline
\end{tabular}

SMITHSONIAN TABLES 
TABLE $\theta=65^{\circ}$

$q=0.106054020185994, \quad \theta 0=0.7881449667, \quad \mathrm{HK}=1.1541701350$

\begin{tabular}{|c|c|c|c|c|c|}
\hline $\mathrm{B}(\mathrm{r})$ & $\mathrm{C}(\mathrm{r})$ & $\mathbf{G}(\mathbf{r})$ & $\psi$ & $\mathrm{F} \psi$ & $90-r$ \\
\hline I. 0000000000 & I. 5382462687 & 0.0000000000 & $90^{\circ} \quad 0^{\prime}$ & 2.3087867982 & 90 \\
\hline 0.9998341412 & I. $53^{808}$ I $544^{\circ}$ & 0.0083487781 & 88 & 2.283 I3 36II5 & 89 \\
\hline 0.9993366526 & I. $5375^{8} 75740$ & 0.0166926008 & 46 & 2.2574804249 & 88 \\
\hline 0.9985077970 & I. 5367649688 & $0.025026504 I$ & 88 & $2.23182 \quad 72382$ & 87 \\
\hline 0.9973480125 & I. 5356 I 47447 & 0.0333455075 & 32 & 2.2061740516 & 86 \\
\hline $0.995^{85} 79109$ & I. 534 I3 83232 & 0.0416446052 & 86 & 2. I805208649 & 85 \\
\hline 0.9940382778 & I. 532337528 I & 0.0499187582 & 86 & 2.1548676783 & 84 \\
\hline 0.9918900707 & I. 5302 I $45^{843}$ & $0.05^{816} 28855$ & 38 & 2. I2921 44916 & 83 \\
\hline 0.9894 I 44 I 82 & I. 5277721140 & $0.06637 \quad 18564$ & 85 & 2.1035613050 & 82 \\
\hline 0.9866 I 26176 & I. $52501 \quad 31340$ & 0.0745404819 & 84 & 2.0779081184 & $8 I$ \\
\hline $0.9834^{8} 61339$ & I.52I94 I05I 4 & 0.0826635068 & 44 & 2.0522549317 & 80 \\
\hline 0.9800365970 & I.5I855 96596 & 0.0907356016 & 83 & $2.02660 \mathrm{I} 745 \mathrm{I}$ & 79 \\
\hline 0.9762657996 & I.5I487 3I329 & o.09875 I3547 & 27 & $2.00094855^{84}$ & 78 \\
\hline 0.9721756947 & I. $5 \mathrm{IO} 88602 \mathrm{I} 8$ & 0.1067052642 & 48 & I. 97529537 I 8 & 77 \\
\hline 0.9677683924 & I.50660 32466 & o. I I 459 I 7308 & $8 \mathrm{I}$ & I.94964 2 I85I & 76 \\
\hline 0.9630461576 & I. 5020300916 & 0.1224050500 & 30 & I.92398 89985 & 75 \\
\hline $0.95^{801} 14060$ & I.49717 21977 & o.13013 94047 & 50 & I. 8983358 I I 8 & 74 \\
\hline 0.9526667013 & I.49203 55559 & O.I 377888583 & 10 & I. 872682625 I & 73 \\
\hline $0.9470 \mathrm{I} 475 \mathrm{II}$ & I. 4866264993 & o.14534 73477 & 30 & I $.84702 \quad 94385$ & 72 \\
\hline 0.9410584035 & I. $48095 \quad$ I 6947 & o.15280 86769 & 49 & I. 8213762519 & 71 \\
\hline $0.93480 \quad 06429$ & I. 4750 I 8 I 348 & o.16016 65105 & 77 & I.79572 $3065^{2}$ & 70 \\
\hline 0.9282445859 & I. 4688331288 & $0.16741 \quad 43683$ & 26 & I.77006 98786 & 69 \\
\hline 0.9213934772 & I. $46240 \quad 42933$ & o.17454 56190 & 44 & I.7444I 66919 & 68 \\
\hline $0.914250685 \mathrm{I}$ & I . 4557395424 & o. I 8I 5534763 & 75 & I.71876 35053 & 67 \\
\hline 0.9068 I 96968 & I. 448847078 I & o. I 884309933 & 74 & I. 693 II 03186 & 66 \\
\hline 0.899104 I I 40 & I . 44 I 7353793 & o.195I 7 I0594 & 36 & I.66745 71320 & 65 \\
\hline o.89110 76479 & I.4344I 3I9I6 & 0.2017663966 & 72 & I. 6418039453 & 64 \\
\hline 0.882834 II 44 & I. 4268895 I 62 & o.20820 95570 & 72 & I.6I6I5 $075^{87}$ & 63 \\
\hline $0.87428 \quad 74294$ & I. $41917359^{8 I}$ & 0.2 I449 292 I I & 71 & I. 590497572 I & 62 \\
\hline $0.86547 \quad \mathrm{I} 6034$ & I. 4 II27 49 I 49 & 0.2206086968 & 37 & I. $56484 \quad 43854$ & $6 I$ \\
\hline 0.8563907366 & I. $40320 \quad 31647$ & 0.2265489197 & 69 & I. 53919 I1988 & 60 \\
\hline 0.8470490138 & I. 394968254 I & 0.2323054536 & 69 & I. 5 I353 80I 2 I & 59 \\
\hline 0.8374506991 & I. 3865802852 & 0.2378699932 & 17 & I. $48788 \quad 48255$ & $5^{8}$ \\
\hline 0.82760 oI 310 & I. 3780495440 & 0.2432340676 & 29 & I. $46223 \quad$ I6388 & 57 \\
\hline 0.81750 I7 168 & I. 3693864865 & 0.2483890447 & $4 I$ & I. 4365784522 & 56 \\
\hline 0.8071599276 & I. $36060 \quad$ I726I & 0.2533261379 & 65 & I.41092 52655 & 55 \\
\hline 0.7965792934 & I. 3517060205 & $0.25^{803} 64133$ & 65 & I. $3^{8527} 20789$ & 54 \\
\hline 0.7857643973 & I. 3427 I $025^{82}$ & $0.2625 \mathrm{I} 0800 \mathrm{I}$ & 64 & I. 3596 I 88922 & 53 \\
\hline 0.7747 I 98708 & I. $33362 \quad 54449$ & 0.26674 01012 & 63 & I. 3339657055 & 52 \\
\hline 0.7634503889 & I. 3244626900 & $0.2707 \mathrm{I} 50065$ & 62 & I. $30831 \quad 25189$ & $5 I$ \\
\hline 0.7519606646 & I.31523 31927 & 0.2744261086 & $6 I$ & I. 2826593322 & 50 \\
\hline 0.7402554443 & I. 3059482284 & 0.2778639198 & 60 & I. 2570061456 & 49 \\
\hline 0.7283395027 & I.29661 91348 & 0.28 IOI 88920 & 46 & I. 23 I35 $295^{89}$ & 48 \\
\hline $0.71621 \quad 76383$ & I. 2872572976 & $0.28388 \quad \mathbf{1} 43^{88}$ & $5^{8}$ & I. 2056997723 & 47 \\
\hline 0.7038946686 & I. 2778741372 & $0.28644 \quad 19600$ & 57 & I. I 800465856 & 46 \\
\hline 0.6913754254 & I. $26848 \quad 10938$ & 0.2886908691 & $5^{6} \quad 5^{8}$ & I. I 54393399 I & 45 \\
\hline $\mathbf{A}(\mathbf{r})$ & $\mathbf{D}(\mathbf{r})$ & $\mathbf{E}(\mathbf{r})$ & $\phi$ & $\mathbf{F} \phi$ & $\mathbf{r}$ \\
\hline
\end{tabular}

SMITHSONIAN TABLES 
$\mathrm{K}=2.5045500790, \quad \mathrm{~K}^{\prime}=1.6200258991, \quad \mathrm{E}=1.1183777380, \quad \mathrm{E}^{\prime}=1.5237992053$

\begin{tabular}{|c|c|c|c|c|c|c|}
\hline $\mathbf{r}$ & $\mathrm{F} \phi$ & & $p$ & $E(r)$ & $\mathbf{D}(\mathbf{r})$ & $A(r)$ \\
\hline o & 0.0000000000 & $0^{\circ}$ & $\mathrm{o}^{\prime}$ & 0.0000000000 & I. .0000000000 & 0.0000000000 \\
\hline I & 0.0278283342 & I & 36 & o.or539 55735 & I. $00021 \quad 42837$ & 0.0162742346 \\
\hline 2 & 0.0556566684 & 3. & I I & 0.0307531429 & I. 0008568806 & 0.0325456619 \\
\hline 3 & $0.0834^{8} 50026$ & 4 & 47 & 0.0460349252 & I.00192 70294 & $0.0488 \mathrm{I} \quad \mathrm{I} 4698$ \\
\hline 4 & o.III3I 33368 & 6 & 22 & 0.0612035769 & I .00342 34614 & $0.065068835^{8}$ \\
\hline 5 & o.13914 I6710 & 7 & 57 & $0.07622 \quad 24069$ & I. .0053444028 & 0.0813149227 \\
\hline 6 & o. I6697 00053 & 9 & 32 & $0.0910555^{815}$ & I. $.00768 \quad 75763$ & 0.0975468734 \\
\hline 7 & o.1947983395 & II & 6 & o. 1056683193 & I. 0104502032 & o. I1 $376 \quad 18057$ \\
\hline 8 & 0.2226266737 & 12 & 40 & o.12002 70732 & I. 0136290072 & o. I2995 68083 \\
\hline 9 & 0.2504550079 & 14 & I3 & o.1340996984 & I.01722 02172 & o.14612 89355 \\
\hline IO & $0.278283342 \mathrm{I}$ & 15 & 46 & o. I 478556040 & I.02121 95717 & o.16227 52029 \\
\hline I I & 0.306 II I6763 & 17 & I 8 & 0.1612658874 & I. $02562 \quad 23237$ & o. 1783925828 \\
\hline 12 & 0.33394 00105 & 18 & $5^{\circ}$ & o. 1743034501 & I. $03042 \quad 32454$ & o. 1944780006 \\
\hline I3 & 0.3617683447 & 20 & 20 & o. 1869430948 & I. 0356 I 6634 I & 0.2105283297 \\
\hline 14 & 0.3895966790 & $2 \mathrm{I}$ & 50 & $0.19916 \quad 16028$ & I.04119 63185 & 0.2265403885 \\
\hline I5 & $0.41742 \quad 50132$ & 23 & 20 & 0.2109377918 & I. 047 I5 56657 & 0.2425109363 \\
\hline 16 & 0.4452533474 & 24 & 48 & 0.2222525549 & I . $0534875^{877}$ & 0.2584366697 \\
\hline 17 & 0.47308 I6816 & 26 & I6 & 0.2330888806 & I. 0601845500 & 0.2743142196 \\
\hline 18 & 0.5009100158 & 27 & 42 & $0.24343 \quad 18557$ & I. 0672385795 & 0.2901401480 \\
\hline 19 & 0.5287383500 & 29 & 8 & 0.2532686498 & I. $.07464 \quad 12734$ & 0.3059109453 \\
\hline 20 & $0.55^{6} 5^{6} 66842$ & 30 & 32 & $0.2625^{8} 84862$ & I .08238 38086 & 0.3216230277 \\
\hline $2 \mathrm{I}$ & $0.5^{8439} 50184$ & 31 & 56 & 0.2713825968 & I .09045 69513 & 0.3372727349 \\
\hline 22 & 0.6122233526 & 33 & I 8 & 0.2796441653 & I . $09885 \quad 10673$ & $0.35^{285} 63285$ \\
\hline 23 & 0.6400516869 & 34 & 40 & $0.287368258 \mathrm{I}$ & I. 1075561330 & 0.3683699898 \\
\hline 24 & 0.66788002 II & 36 & o & $0.29455 \quad 17462$ & I. $11656 \quad 17464$ & 0.3838098186 \\
\hline 25 & o.69570 83553 & 37 & 19 & 0.3011932185 & I. 1258571388 & $0.39917 \quad 18323$ \\
\hline 26 & 0.7235366895 & 38 & 37 & 0.3072928884 & I. I 3543 I I 869 & 0.4144519649 \\
\hline 27 & $0.75^{136} 50237$ & 39 & 54 & 0.3128524953 & I. I 452724256 & 0.4296460668 \\
\hline 28 & 0.7791933579 & $4 I$ & 10 & Q.31787 52022 & I. I5536 90607 & 0.4447499043 \\
\hline 29 & $0.80702 \quad 16921$ & 42 & 24 & 0.32236549 I I & I. I6570 89825 & 0.4597591601 \\
\hline 30 & 0.8348500263 & $43^{\circ}$ & 38 & 0.3263290569 & I. I762797795 & o.4746694339 \\
\hline $3 I$ & 0.8626783605 & 44 & 50 & 0.3297727014 & I. I 870687529 & 0.4894762428 \\
\hline 32 & $0.8905^{\circ} 66948$ & 46 & 1 & $0.33270 \quad 42283$ & I. 1980629307 & 0.5041750229 \\
\hline 33 & 0.9183350290 & 47 & I I & 0.3351323398 & I. 2092490830 & $0.51876 \quad 11309$ \\
\hline 34 & 0.9461633632 & 48 & 20 & 0.3370665364 & I .2206I 37375 & 0.5332298456 \\
\hline 35 & $0.97399 \quad 16974$ & 49 & 27 & $0.3385 \mathrm{I} 701$ & I. 2321431946 & 0.5475763701 \\
\hline 36 & I.001 8200316 & 50 & 34 & 0.3394945975 & I .2438235438 & 6179.58348 \\
\hline 37 & I .02964 $8365^{8}$ & $5 I$ & 39 & 0.3400105978 & I. 2556406798 & $0.575^{88} \quad 32996$ \\
\hline 38 & I. 0574767000 & $5^{2}$ & 43 & 0.3400767814 & I. $2675^{8}$ o3194 & $0.5^{8983} 37576$ \\
\hline 39 & I. 0853050342 & 53 & 46 & $0.33970 \quad 52640$ & 1. 2796280178 & $0.603642138 I$ \\
\hline 40 & I. II3I3 33684 & $\dot{5} 4$ & 48 & 0.3389084414 & I. 2917691861 & $0.61730 \quad 33109$ \\
\hline 41 & I. I 4096 I 7027 & 55 & 49 & 0.3376989203 & I. 3039891085 & 0.6308 I 20897 \\
\hline 42 & I. I687900369 & 56 & 48 & 0.3360894543 & I. 3162729599 & 0.6441632373 \\
\hline 43 & I. I966I 837 II & 57 & 47 & $0.334092885 \mathrm{I}$ & I. $32860 \quad 58237$ & 0.6573514695 \\
\hline 44 & I. 2244467053 & $5^{8}$ & 44 & 0.3317220892 & I. 3409727096 & $0.67037 \quad 14605$ \\
\hline 45 & I. 2522750395 & 59 & $4 \mathrm{I}$ & 0.3289899283 & I. 3533585717 & $0.68321 \quad 78479$ \\
\hline $90-r$ & $\mathbf{F} \psi$ & & $\mathbf{G}(\mathbf{r})$ & $\mathrm{C}(\mathbf{r})$ & $\mathbf{B}(\mathbf{r})$ \\
\hline
\end{tabular}

SMITHSONIAN TABLES 


\begin{tabular}{|c|c|c|c|c|c|}
\hline $\mathrm{B}(\mathbf{r})$ & $\mathrm{C}(\mathrm{r})$ & $\mathrm{G}(\mathbf{r})$ & $\psi$ & $\mathbf{F} \psi$ & $90-r$ \\
\hline I. 0000000000 & I. 7099 I 3565 I & 0.0000000000 & $90^{\circ} \quad \mathrm{o}^{\prime}$ & 2.5045500790 & 90 \\
\hline 0.9998271058 & I .70969 53883 & 0.0091703805 & $89 \quad 27$ & $2.47672 \quad 17448$ & 89 \\
\hline 0.9993085325 & I .70904 I I 308 & 0.0183363062 & 55 & 2.4488934106 & 88 \\
\hline 0.9984446074 & 1.70795 I6110 & 0.0274933119 & 22 & 2.4210650764 & 87 \\
\hline $0.997235^{8755}$ & I.70642 81917 & 0.0366369110 & 49 & 2.3932367422 & 86 \\
\hline 0.9956830984 & I.70447 27784 & $0.0457625^{853}$ & 16 & 2.3654084079 & 85 \\
\hline $0.99378 \quad 72533$ & 1.7020878163 & 0.0548657745 & 43 & $2.3375^{8} 00737$ & 84 \\
\hline 0.9915495309 & I. 6992762875 & 0.06394 I 8650 & 86 & $2.30975 \quad 17395$ & 83 \\
\hline 0.98897 I 3334 & I. $69604 \quad 17067$ & $0.07298 \quad 6$ I 798 & 36 & 2.2819234053 & 82 \\
\hline 0.9860542725 & I. 6923881168 & 0.0819939678 & 85 & 2.2540950711 & $8 \mathrm{I}$ \\
\hline 0.98280 or66I & I.6883200831 & 0.0909603928 & 84 & 2.2262667369 & 80 \\
\hline $0.97921 \quad 1035^{6}$ & I. 6838426872 & $\begin{array}{lll}0.09988 & 05231\end{array}$ & 83 & 2. 1984384027 & 79 \\
\hline 0.9752891023 & I. 67896 I5207 & o.1087493206 & 83 & 2.I706I 00685 & 78 \\
\hline 0.9710367835 & I. 673682677 I & o. II756 I6303 & 82 & $2.14278 \quad 17343$ & 77 \\
\hline 0.9664566885 & I. $66801 \quad 27439$ & 0.1263121691 & 82 & 2. I1 49534000 & 76 \\
\hline 0.96 I 55 I6I 44 & I.66I9587940 & o.I3499 55I 58 & $8 \mathbf{I}$ & 2.0871250658 & 75 \\
\hline 0.9563245409 & I. 655528376 I & o. 1436060995 & $8 \mathbf{I}$ & 2.0592967316 & 74 \\
\hline 0.9507786259 & I. 6487295046 & o. I 52138 I 898 & 80 & 2.0314683974 & 73 \\
\hline 0.9449 I 7I996 & I.64I57 0649I & o.16058 58855 & 79 & 2.0036400632 & 72 \\
\hline 0.9387437597 & I. 6340607230 & o.16894 31044 & 79 & I. 9758 I I 7290 & $7 \mathrm{I}$ \\
\hline $0.93226 \quad 19647$ & I. 6262090720 & o.17720 35729 & 78 & I.94798 33948 & 70 \\
\hline $0.92547 \quad 56289$ & I. 6180254615 & o. 18536 08 I 58 & 77 & I.920I5 50606 & 69 \\
\hline 0.9183887 I55 & I. 6095200637 & $0.1934^{0} 8 \mathrm{I} 46 \mathrm{I}$ & 77 & I. 8923267264 & 68 \\
\hline 0.9110053304 & I. $60070 \quad 34445$ & $0.20 \mathrm{I} 338655 \mathrm{I}$ & 76 & I. 864498392 I & 67 \\
\hline 0.9033297156 & I. 59 I 5865494 & 0.2091452034 & 76 & I. 8366700579 & 66 \\
\hline 0.8953662423 & I. 582 I 8 0689I & 0.2168204110 & 75 & I. 80884 I7237 & 65 \\
\hline 0.887 I I 94043 & I. 5724975252 & 0.2243566494 & 74 & I .78101 33895 & 64 \\
\hline 0.8785938 I06 & I . 5625490544 & 0.2317460328 & 74 & I. 753 I 850553 & 63 \\
\hline 0.8697941783 & I. 5523475933 & 0.23898 O4 I I I & 73 & I. 72535672 I I & 62 \\
\hline 0.8607253257 & I. $54190 \quad 57623$ & $0.24605 \quad$ I 3624 & $\begin{array}{ll}72 & 39\end{array}$ & I. 6975283869 & $6 I$ \\
\hline 0.85 I39 21644 & I. 5312364694 & 0.25295 or 875 & $5^{6}$ & I. 6697000527 & 60 \\
\hline 0.8417996923 & I. 5203528933 & 0.2596679043 & I3 & I. $64187 \quad$ I 7 I 85 & 59 \\
\hline 0.83 I95 2986I & I. 5092684668 & 0.2661952443 & 29 & I. 6140433842 & 58 \\
\hline 0.8218571938 & I.49799 68595 & $0.2725^{2} 26492$ & 69 & I.5862I 50500 & 57 \\
\hline 0.8 II $^{1} \mathrm{I} \quad 75^{269}$ & I. 48655 I960I & 0.2786402697 & 59 & I. $55^{8} 3^{8} 6715^{8}$ & 56 \\
\hline 0.8009392537 & I.47494 78592 & 0.2845379654 & 68 & I. 53055838 I6 & 55 \\
\hline 0.7901276914 & 1. 4631988308 & 0.2902053069 & 67 & I. 5027300474 & 54 \\
\hline 0.7790881986 & I. $45^{1} 3^{1} 93$ I 48 & 0.29563 I 5786 & 66 & I. 47490 I 7 I 32 & 53 \\
\hline 0.7678261683 & I. $43932 \quad 38985$ & 0.3008057852 & 65 & I. 4470733790 & 52 \\
\hline 0.7563470207 & I. 4272272983 & 0.3057 I 66593 & 64 & I. 4192450448 & $5 I$ \\
\hline 0.7446561957 & I. 4 I 504434 I 3 & 0.3103526720 & 64 & I.3914I 67106 & 50 \\
\hline 0.7327591466 & I . 4027899470 & $0.31470 \quad 20462$ & 63 & I. $3635^{8} 83763$ & 49 \\
\hline $0.72066 \quad 13327$ & I. 3904791083 & $0.31875 \quad 27727$ & 62 & I. 335760042 I & 48 \\
\hline 0.7083682126 & I. 378 I 268735 & 0.3224926298 & $6 I$ & I. $30793 \quad 17079$ & 47 \\
\hline $0.695^{88} \quad 5^{2382}$ & I. 365748327 I & 0.3259092064 & 60 & I. 2801033737 & 46 \\
\hline $0.68321 \quad 78479$ & I. 35335857 I 7 & o. 3289899283 & $59 \quad 4 \mathrm{I}$ & I. 2522750395 & 45 \\
\hline$A(r)$ & $\mathbf{D}(\mathbf{r})$ & $\mathbf{E}(\mathbf{r})$ & $\phi$ & $\mathbf{F} \phi$ & $\mathbf{r}$ \\
\hline
\end{tabular}


$K=2.7680631454=K^{\prime} \sqrt{3}, \quad K^{\prime}=1.5981420021, \quad E=1.076405113, \quad E^{\prime}=1.5441504969$

\begin{tabular}{|c|c|c|c|c|c|c|c|}
\hline $\mathbf{r}$ & $\mathbf{F} \phi$ & 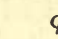 & $b$ & $\mathbf{E}(\mathbf{r})$ & $\mathbf{D}(\mathbf{r})$ & $\mathbf{A}(\mathbf{r}$ & \\
\hline o & 0.0000000000 & $0^{\circ}$ & $o^{\prime}$ & 0.0000000000 & I.00000 00000 & 0.00000 & 00000 \\
\hline I & 0.0307562572 & I & 46 & 0.0187871553 & 1.0002890226 & 0.01564 & 67728 \\
\hline 2 & 0.0615125143 & 3 & 37 & 0.03752 O120I & I.00II5 57568 & 0.03129 & 207 I I \\
\hline 3 & 0.0922687715 & 5 & 17 & 0.056 I 450985 & I.00259 92025 & 0.04693 & 44040 \\
\hline 4 & 0.1230250287 & 7 & 2 & 0.0746090790 & I.00461 76935 & 0.06257 & 22754 \\
\hline 5 & o. I $5378 \quad 12859$ & 8 & 47 & 0.0928602109 & I.00720 88997 & 0.07820 & $4155^{8}$ \\
\hline 6 & o. 1845375430 & 10 & 31 & o.11084 81632 & I.01036 98288 & 0.09382 & 84843 \\
\hline 7 & 0.2152938002 & 12 & I5 & o. 1285244620 & I. 0140968295 & 0.10944 & 36574 \\
\hline 8 & 0.2460500574 & 13 & $5^{8}$ & o. $145^{84} 27986$ & I.01838 55946 & o. 12504 & 80220 \\
\hline 9 & 0.2768063 I 45 & I5 & 40 & 0.1627593073 & I. .02323 II $65^{8}$ & 0.14063 & 98665 \\
\hline 10 & 0.30756257 I 7 & I 7 & 22 & o. I7923 28093 & I.02862 79374 & o. I 562 I & 74137 \\
\hline I I & $0.33^{83} 188289$ & 19 & 3 & o. 1952250184 & I.03456 96626 & 0.17177 & 88130 \\
\hline 12 & 0.3690750860 & 20 & 43 & $0.21070 \quad 07095$ & I.0410494593 & o. 18732 & 21327 \\
\hline 13 & $0.39983 \quad 13432$ & 22 & 22 & $0.22562 \quad 78479$ & I.04805 98163 & 0.20284 & 53538 \\
\hline 14 & $0.4305^{8} 76004$ & 23 & 59 & 0.2399776797 & I.05559 26010 & 0.21834 & 63622 \\
\hline I 5 & 0.46 I34 38576 & 25 & 36 & 0.2537247838 & I. 0636390673 & 0.23382 & 29430 \\
\hline 16 & 0.492 I0 01147 & 27 & 12 & 0.2668470884 & I. 0721898642 & 0.24927 & 27739 \\
\hline 17 & 0.5228563719 & 28 & 46 & 0.2793258519 & I. .0812350446 & 0.26469 & 34194 \\
\hline I 8 & $0.5536 \mathrm{I} 2629 \mathrm{I}$ & 30 & 19 & 0.2911456129 & I. .0907640755 & 0.28008 & 23255 \\
\hline 19 & 0.5843688862 & 31 & 50 & 0.3022941110 & I. I0076 58484 & 0.29543 & 68145 \\
\hline 20 & $0.61512 \quad 51434$ & 33 & $2 \mathrm{I}$ & 0.3127621816 & I. I I I22 86903 & 0.31075 & 40803 \\
\hline 21 & 0.64588 I 4006 & 34 & 50 & 0.3225436297 & 1. 1221403756 & 0.32603 & I 1842 \\
\hline 22 & 0.6766376577 & 36 & 17 & 0.3316350828 & I. $13348 \quad 81382$ & 0.34126 & 50509 \\
\hline 23 & 0.7073939149 & 37 & 43 & 0.3400358309 & I. 1452586847 & 0.35645 & 24653 \\
\hline 24 & $0.73^{815}$ oI72I & 39 & 8 & 0.3477476532 & I. I5743 82078 & 0.37159 & 00694 \\
\hline 25 & $0.76890 \quad 64293$ & 40 & $3 I$ & $0.35477 \quad 46364$ & I. I 7001 24008 & 0.38667 & 43599 \\
\hline 26 & 0.7996626864 & 41 & 52 & 0.36 II 2988 I & I. I 829664722 & 0.40170 & 16862 \\
\hline 27 & $0.8304 \mathrm{I} 89436$ & 43 & 12 & 0.3668008467 & I. 1962851612 & 0.41666 & 82489 \\
\hline 28 & 0.86 II7 52008 & 44 & $3 \mathrm{I}$ & 0.37 I 8 I 809 I 8 & I. $209952753^{8}$ & 0.43157 & 00988 \\
\hline 29 & $0.89193 \quad 14579$ & 45 & 48 & $0.37618 \quad 61563$ & I .22395 30995 & 0.44640 & $3136 I$ \\
\hline 30 & 0.92268 .77151 & 47 & 3 & 0.3799178428 & I. 2382696285 & 0.46116 & 3 IIIO \\
\hline 31 & 0.9534439723 & 48 & 18 & 0.3830271460 & I. $25288 \quad 53692$ & $0.475^{84}$ & 56238 \\
\hline 32 & $0.98420 \quad 02294$ & 49 & 30 & $0.3855^{2} 90817$ & I. $26778 \quad 29672$ & 0.49044 & 61259 \\
\hline 33 & I. 0149564866 & 50 & $4 \mathrm{I}$ & 0.3874395246 & I . 2829447038 & 0.50495 & 99214 \\
\hline 34 & I.0457I $2743^{8}$ & $5 \mathrm{I}$ & $5^{I}$ & $0.388775055^{2}$ & I. 2983525154 & $0.5193^{8}$ & 21695 \\
\hline 35 & 1.0764690010 & $5^{2}$ & 59 & $0.38955 \quad 28159$ & I. 3139880140 & 0.53370 & 78866 \\
\hline 36 & I. $10722525^{81}$ & 54 & 5 & 0.3897903785 & I. 3298325072 & 0.54793 & 19494 \\
\hline 37 & I. $13798 \quad 15153$ & 55 & 10 & 0.3895056204 & I. $345^{86} 70195$ & 0.56204 & 90989 \\
\hline 38 & I. 1687377725 & 56 & 14 & 0.3887166125 & I. 3620723140 & 0.57605 & 39442 \\
\hline 39 & I. 1994940296 & 57 & 16 & $0.3^{8744} \quad$ I5I 7 I & I. 3784289138 & 0.58994 & 09669 \\
\hline 40 & I . 2302502868 & $5^{8}$ & I 7 & 0.3856984955 & I.3949I 7I25I & 0.60370 & 45267 \\
\hline $4 \mathrm{I}$ & I. 2610065440 & 59 & 17 & $0.38350 \quad 56260$ & I. 4 II 5I 70596 & 0.61733 & 88663 \\
\hline 42 & I.29I76 280II & 60 & I5 & 0.3808808305 & I. 4282086579 & 0.63083 & 81179 \\
\hline 43 & I. 3225 I $905^{8} 3$ & $6 I$ & 12 & $0.37784 \quad 18107$ & I. 44497 I 7 1 32 & 0.64419 & 63092 \\
\hline 44 & I. $35327 \quad 53155$ & 62 & 8 & 0.3744059923 & I. $46178 \quad 5^{8952}$ & 0.65740 & 73705 \\
\hline 45 & I. $38403 \quad 15727$ & 63 & 2 & 0.3705904774 & I. 4786307744 & 0.67046 & 51423 \\
\hline $90-r$ & $\mathbf{F} \psi$ & \multicolumn{2}{|c|}{$\psi$} & $\mathbf{G}(\mathbf{r})$ & $\mathbf{C}(\mathbf{r})$ & \multicolumn{2}{|l|}{$\mathbf{B}(\mathbf{r})$} \\
\hline
\end{tabular}




\begin{tabular}{|c|c|c|c|c|c|}
\hline $\mathbf{B}(\mathbf{r})$ & $\mathbf{C}(\mathbf{r})$ & $\mathbf{G}(\mathbf{r})$ & $\psi$ & $\mathbf{F} \psi$ & $90-r$ \\
\hline I. 0000000000 & I. 9656305108 & 0.0000000000 & $90^{\circ} \quad o^{\prime}$ & $2.76806 \quad 31454$ & 90 \\
\hline 0.9998 I 60886 & I.96533 I 295I & 0.0098991720 & $89 \quad 33$ & 2.7373068882 & 89 \\
\hline 0.9992644975 & I. $96443 \quad 40309$ & 0.0197947043 & 89 & 2.7065506310 & 88 \\
\hline $0.998345655^{2}$ & I.9629398674 & 0.0296829453 & 88 & 2.6757943738 & 87 \\
\hline 0.9970602753 & I.96085 07176 & 0.0395602195 & Io & 2.6450381167 & 86 \\
\hline 0.9954093546 & I.958I6 9256I & $0.04942 \quad 281_{54}$ & 87 & $2.6 \mathrm{I} 428 \mathrm{I} 8595$ & 85 \\
\hline 0.9933941714 & I. 9548989147 & 0.0592669738 & 87 & $2.5^{8} 35^{2} 56023$ & 84 \\
\hline 0.9910162829 & I.95104 38778 & $0.069088875^{2}$ & 86 & 2.5527693451 & 83 \\
\hline 0.9882775221 & I. 9466090763 & $0.07888 \quad 46278$ & 86 & 2.5220130880 & 82 \\
\hline 0.9851799940 & I. 94160 01 803 & 0.0886502550 & 85 & 2.4912568308 & $8 I$ \\
\hline 0.9817260720 & I.93602 35909 & 0.09838 I6828 & 85 & $2.46050 \quad 05736$ & 80 \\
\hline $0.9779 \mathrm{I} 83923$ & I. 9298864309 & o. 1080747268 & 84 & 2.4297443165 & 79 \\
\hline 0.9737598498 & I.92319 65349 & o.II772 50798 & 84 & 2.3989880593 & 78 \\
\hline 0.9692535914 & I.9I596 24373 & o.1273282981 & 83 & 2.3682318021 & 77 \\
\hline 0.9644030106 & I.90819 33609 & o.1368797883 & 83 & 2.3374755450 & 76 \\
\hline $0.95921 \quad$ I7405 & I.8998992030 & o. 1463747936 & 82 & 2.3067192878 & 75 \\
\hline 0.9536836468 & I. 8910905214 & o. 1558083802 & 82 & 2.2759630306 & 74 \\
\hline $0.94782 \quad 28200$ & I. 8817785195 & o.16517 54225 & $8 I$ & 2.2452067734 & 73 \\
\hline 0.9416335686 & I.87I97 5030I & o. I7447 05894 & $8 \mathrm{I}$ & -2.2144505163 & 72 \\
\hline $0.935^{12} \quad 04092$ & I. 8616924991 & o. 1836883293 & 80 & 2.18369 4259I & $7 I$ \\
\hline 0.9282880593 & I. 8509439670 & o. 1928228550 & 80 & 2.15293800I9 & 70 \\
\hline $0.92114 \quad 14274$ & I. 8397430516 & 0.2018681293 & 79 & 2.1221 8 I 7448 & 69 \\
\hline 0.9136856040 & I. 828 10 39279 & $0.21081 \quad 78488$ & 79 & 2.0914254876 & 68 \\
\hline $0.905925^{8} 5^{2 I}$ & I. 8160413089 & 0.21966 .54291 & 78 & 2.0606692304 & 67 \\
\hline 0.8978675972 & I. 8035704247 & $0.22840 \quad 39887$ & 78 & 2.0299129733 & 66 \\
\hline $0.8895 \mathrm{I} \quad 64174$ & I. 7907070015 & 0.2370263334 & 77 & I.999I5 67I6I & 65 \\
\hline 0.8808780328 & I.77746 72401 & $0.2455^{2} 49406$ & 76 & I . $96840 \quad 04589$ & 64 \\
\hline 0.8719582952 & I .76386 77929 & $0.25389 \quad 19433$ & 76 & I .93764 42017 & 63 \\
\hline $0.86276 \quad 31773$ & I.74992 57419 & 0.2621 I 91147 & 75 & I .90688 79446 & 62 \\
\hline 0.8532987622 & I. 7356585746 & 0.2701978524 & 75 & I. 876 I 3 I 6874 & $6 I$ \\
\hline $0.84357 \quad 12322$ & I. $72108 \quad 41609$ & 0.2781191636 & 74 & I. 8453754302 & 60 \\
\hline $0.8335^{8} 685^{80}$ & I.7062207286 & $0.285^{87} 36500$ & 73 & I. 81461 9I73I & 59 \\
\hline 0.82335 I9876 & I. 6910868389 & 0.2934514936 & 73 & I. 7838629159 & 58 \\
\hline 0.8128730353 & I. 67570 I 3618 & 0.3008424433 & 72 & I.75310 66587 & 57 \\
\hline 0.8021564710 & I. 6600834507 & 0.3080358026 & 71 & I. 7223504016 & 56 \\
\hline 0.7912088085 & I. 6442525 I 75 & 0.3150204176 & 71 & I.69I59 4I444 & 55 \\
\hline 0.7800365955 & I. 6282282065 & 0.3217846673 & 70 & I . 6608378872 & 54 \\
\hline 0.7686464021 & I. 6120303692 & 0.3283164547 & 69 & I . 63008 I6300 & 53 \\
\hline 0.7570448103 & I. 5956790385 & 0.3346032006 & 68 & I. 5993253729 & 52 \\
\hline $0.745^{23} 84036$ & I. 5791944025 & $0.34063 \quad 18384$ & 68 & I. 56856 9I I 57 & $5 I$ \\
\hline 0.7332337566 & I. 5625967789 & 0.3463888130 & 67 & I. 5378 I 28585 & 50 \\
\hline 0.7210374248 & I. 5459065890 & 0.35 I 8600808 & 66 & I. 50705 660I 4 & 49 \\
\hline 0.7086559347 & I. 5291443320 & 0.35703 I I I 48 & 65 & I. $476300344^{2}$ & 48 \\
\hline 0.6960957739 & I. $51233055^{88}$ & 0.36 I 88 69II5 & 64 & I. 4455440870 & 47 \\
\hline 0.6833633823 & I. 4954858469 & 0.3664120039 & 55 & I. 4147878299 & 46 \\
\hline 0.6704651423 & I. $47863 \quad 07744$ & 0.3705904774 & 63 & I. $38403 \quad$ I 5727 & 45 \\
\hline $\mathbf{A}(\mathbf{r})$ & $\mathbf{D}(\mathbf{r})$ & $\mathbf{E}(\mathbf{r})$ & $\phi$ & $\mathbf{F} \phi$ & $\mathbf{r}$ \\
\hline
\end{tabular}


$K=3.1533852519, \quad K^{\prime}=1.5828428043, \quad E=1.0401143957, \quad E^{\prime}=1.5588871966$,

\begin{tabular}{|c|c|c|c|c|c|c|}
\hline $\mathbf{r}$ & $\mathbf{F} \phi$ & $\phi$ & $b$ & $\mathbf{E}(\mathbf{r})$ & $\mathbf{D}(\mathbf{r})$ & $A(r)$ \\
\hline 0 & 0.0000000000 & $0^{\circ}$ & $\mathbf{o}^{\prime}$ & 0.0000000000 & I. 0000000000 & 0.0000000000 \\
\hline $\mathbf{I}$ & 0.0350376139 & 2 & o & 0.0234668886 & $1.00041 \quad 13182$ & 0.0146006854 \\
\hline 2 & 0.0700752278 & 4 & I & 0.0468505457 & I.00I64 48264 & 0.0292020956 \\
\hline 3 & 0.105 II 28417 & 6 & $\mathbf{I}$ & $0.07006854 \mathrm{I} 7$ & I.00369 91860 & $0.04380 \quad 49412$ \\
\hline 4 & o.14015 04556 & 8 & o & 0.0930400333 & I. 0065721668 & 0.0584099043 \\
\hline 5 & o.1751 880695 & 9 & 59 & o. I I568 65I73 & I.01026 06485 & $0.073017625 \mathrm{I}$ \\
\hline 6 & $0.21022 \quad 56835$ & I I & $5^{8}$ & o. 1379325365 & I.01476 06225 & 0.0876286871 \\
\hline 7 & 0.2452632974 & I3 & 55 & o. I5970 63263 & I. 0200671948 & o. 1022436040 \\
\hline 8 & 0.2803009113 & I5 & 52 & o. I 80940390 I & I.026I7 $45^{886}$ & o. I I686 2806 I \\
\hline 9 & 0.3153385252 & 17 & 47 & o.20I 57,19949 & I. 0330761484 & o.13148 66263 \\
\hline 10 & 0.3503761391 & I9 & $4 \mathrm{I}$ & 0.22 I $5435^{8}$ I 3 & I. 0407643440 & o. I 46I I 52882 \\
\hline I I & $0.3^{8} 54 \mathrm{I} \quad 37530$ & $2 \mathrm{I}$ & 34 & $0.24080 \quad 30831$ & 1.0492307759 & o.1607488922 \\
\hline 12 & $0.42045 \quad 13669$ & 23 & 26 & 0.2593041559 & I .05846 61 800 & 0.1753874040 \\
\hline I3 & o. $4554^{8} 89808$ & 25 & 16 & $0.27700 \quad 63163$ & I .06846 04345 & 0.1900306422 \\
\hline I 4 & 0.4905265947 & 27 & 4 & 0.2938749943 & I. .0792025667 & 0.2046782669 \\
\hline I5 & $0.5255^{6} 42086$ & 28 & $5 \mathrm{I}$ & $0.30988 \quad$ I 5035 & I. 0906807598 & 0.2193297686 \\
\hline I 6 & $0.56060 \quad$ I 8226 & 30 & 36 & 0.3250029380 & I. 1028823622 & $0.23398 \quad 44577$ \\
\hline 17 & 0.5956394365 & 32 & 20 & $0.33922 \quad 20017$ & I. I 157938955 & $0.24864 \quad 14540$ \\
\hline 18 & 0.6306770504 & 34 & I & $0.35^{252} 67798$ & I . $12940 \quad 10647$ & 0.2632996779 \\
\hline 19 & 0.6657146643 & 35 & $4 I$ & 0.3649104618 & I. I 436887684 & 0.2779578408 \\
\hline 20 & 0.7007522782 & 37 & 18 & $0.37637 \quad 10249$ & I. I5864 I I IOI & 0.2926 I 44375 \\
\hline 21 & $0.735789892 \mathrm{I}$ & $3^{8}$ & 54 & $0.38691 \quad 08879$ & I. 17424 I4105 & 0.3072677376 \\
\hline 22 & 0.7708275060 & 40 & 28 & 0.3965365430 & I. 1904722196 & 219157797 \\
\hline 23 & 0.8058651199 & $4 I$ & 59 & 0.4052581757 & I. 2073153312 & 0.3365563638 \\
\hline 24 & $0.840902733^{8}$ & 43 & 29 & 0.4130892784 & I .22475 I7970 & 0.3511870467 \\
\hline 25 & 0.8759403477 & 44 & 56 & 0.42004626 & I. $24276 \quad 19$ & $5805^{1}$ \\
\hline 26 & 0.9109779617 & 46 & 22 & 0.42614809 & 32538 & 4076 \\
\hline 27 & 0.9460155756 & 47 & 45 & 0.43 I 4I 59095 & I. $28042 \quad 10369$ & 49915050 \\
\hline 28 & 0.981053 I 895 & 49 & 7 & 0.4358726721 & I. 3000271557 & 0.4095531244 \\
\hline 29 & I.0160908034 & 50 & 26 & 0.4395428505 & I.32012 I3294 & 0.4240888287 \\
\hline 30 & I.05112 84173 & $5 \mathrm{I}$ & 44 & $0.442452 \mathrm{IC}$ & I.34068 05 & 0.4385946375 \\
\hline $3 \mathrm{I}$ & I.086I6 603I2 & 52 & 59 & 0.4446269813 & I. 36 I68 10 & 0663090 \\
\hline 32 & I. 121203645 I & 54 & I.2 & 0.4460946931 & I. 3830986893 & 0.4674993405 \\
\hline 33 & I. I 562412590 & 55 & 24 & $0.44688 \quad 28394$ & I. 4049086089 & 0.48 I88 89699 \\
\hline 34 & I. I9I27 88729 & 56 & 33 & 0.4470192128 & I. 4270854443 & 0.49623 or 775 \\
\hline 35 & I.2263I 64868 & 57 & $4 I$ & 0.44653160 & I. $44960 \quad 33094$ & 5 I 76900 \\
\hline 36 & I. 2613541008 & $5^{8}$ & 47 & 0.4454476404 & I. $472435824 \mathrm{I}$ & 247459832 \\
\hline 37 & I. 29639 I7I 47 & 59 & $5 \mathrm{I}$ & 0.4437946284 & I.49555 61410 & 389092878 \\
\hline 38 & I. 3314293286 & 60 & 53 & 0.4415994403 & I.5I8936973I & $0.55300 \quad 15938$ \\
\hline 39 & I.36646 69425 & $6 I$ & 54 & $0.43888 \quad 84024$ & I. 5425506233 & 0.5670166575 \\
\hline 40 & I. $40150 \quad 45564$ & 62 & 53 & 0.4356872080 & I. $566369013^{8}$ & 0.5809480084 \\
\hline $4 I$ & I . 4365421703 & 63 & 50 & 0.4320208450 & I. 5903637173 & o. 5947889567 \\
\hline 42 & I. 47 I 5797842 & 64 & 45 & 0.4279 I 3538 I & I. 6 I $450 \quad 59885$ & 0.6085326019 \\
\hline 43 & I. 5066 I 7398I & 65 & 39 & 0.4233887053 & I. 6387667967 & $0.62217 \quad 18423$ \\
\hline 44 & I. 5416550120 & 66 & 32 & 0.4184689243 & I. 663 II 68595 & 0.6356993846 \\
\hline 45 & I. 5766926259 & 67 & 23 & 0.4131759112 & I. $6875^{2} 66770$ & $0.649107754^{8}$ \\
\hline $90-\mathbf{r}$ & $\mathrm{F} \psi$ & \multicolumn{2}{|c|}{$\psi$} & $\mathbf{G}(\mathbf{r})$ & $C(\mathbf{r})$ & $\mathbf{B}(\mathbf{r})$ \\
\hline
\end{tabular}


$K=3.2553029421, \quad K^{\prime}=1.5805409339, \quad E=1.033789462, \quad E^{\prime}=1.5611417453$,

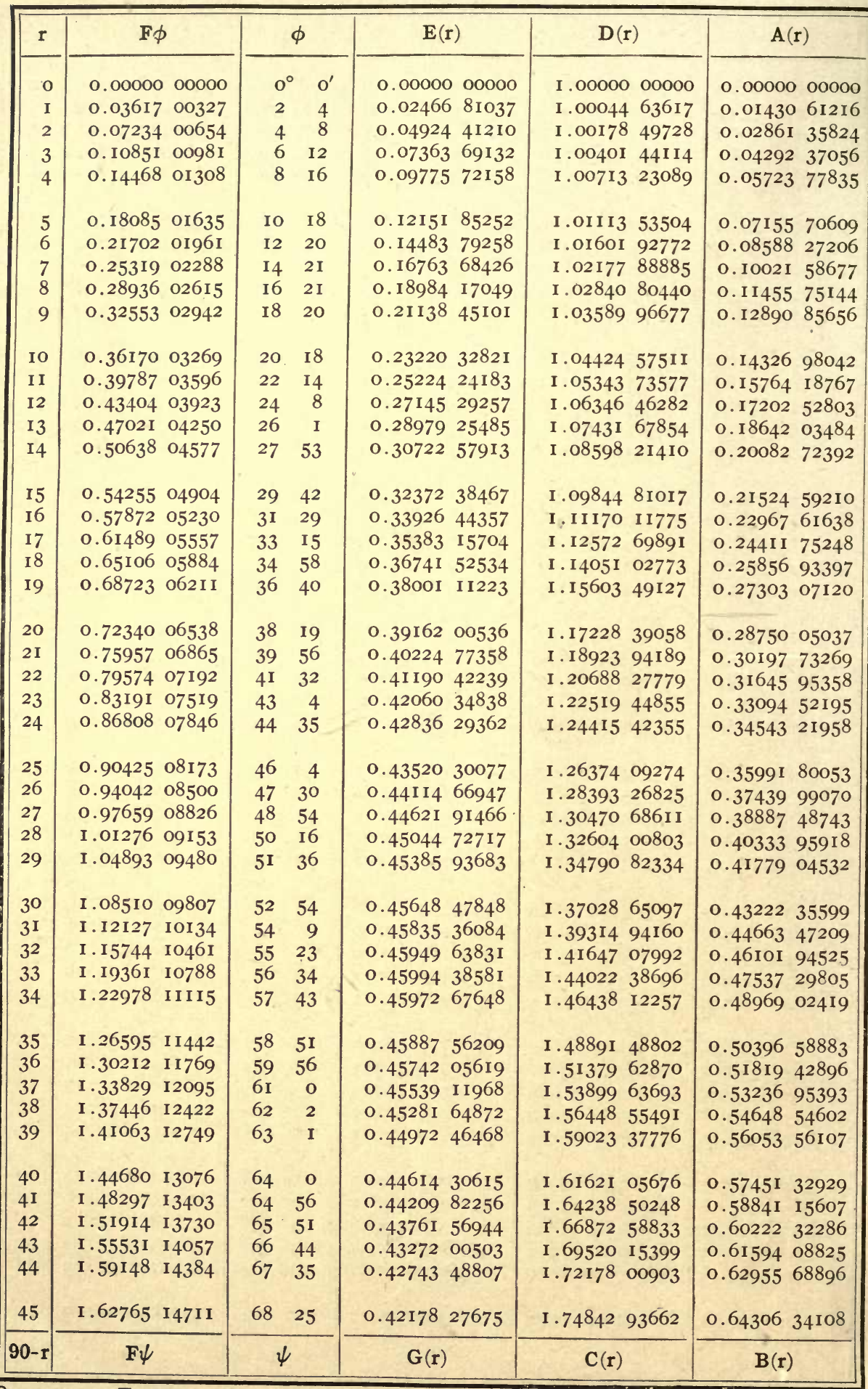




\begin{tabular}{|c|c|c|c|c|c|}
\hline $\mathrm{B}(\mathrm{r})$ & $\mathbf{C}(\mathbf{r})$ & $\mathbf{G}(\mathbf{r})$ & $\psi$ & $\mathrm{F} \psi$ & $90-r$ \\
\hline I.00000 00000 & 2.52833 oI $25 \mathrm{I}$ & 0.0000000000 & $90^{\circ} \quad 0^{\prime}$ & $3.25530 \quad 2942$ I & 90 \\
\hline 0.9997922836 & 2.5278454320 & 0.0106010292 & $89 \quad 4 I$ & 3.2191329095 & 89 \\
\hline 0.9991693515 & 2.5263920136 & o.0211997963 & $2 \mathrm{I}$ & 3. I8296 28768 & 88 \\
\hline 0.998 I3 I8540 & $2.52397 \quad 18509$ & 0.0317940278 & 89 & $3.146792844 \mathrm{I}$ & 87 \\
\hline $0.99668 \quad 08734$ & $2.5^{20} 5^{8} 82420$ & $0.04238 \quad 14278$ & 42 & 3.11062 28II4 & 86 \\
\hline $0.9948 \mathrm{I} \quad 792 \mathrm{I} 3$ & 2.5162457960 & 0.0529596662 & 88 & 3.0744527787 & 85 \\
\hline 0.9925449353 & $2.51095 \quad 04254$ & $0.0635^{2} 63677$ & 88 & 3.0382827460 & 84 \\
\hline 0.9898642745 & 2.5047093354 & 0.0740790993 & 42 & 3.0021127133 & 83 \\
\hline 0.9867787 I39 & 2.49753 IOI 20 & 0.0846 I 53590 & 22 & 2.9659426806 & 82 \\
\hline 0.98329 I 4382 & $2.48942 \quad 52067$ & 0.0951325631 & 87 & 2.9297726479 & $8 I$ \\
\hline 0.9794060344 & $2.48040 \quad 29203$ & o. 1056280337 & 86 & 2.8936026152 & 80 \\
\hline 0.9751264836 & $2.47047 \quad 63835$ & o. II60989854 & 20 & $2.8574325^{825}$ & 79 \\
\hline 0.9704571520 & 2.4596590364 & o. 1265425123 & 85 & 2.8212625499 & 78 \\
\hline 0.9654027806 & $2.447965505 \mathrm{I}$ & o.I3695 55734 & $3^{8}$ & 2.7850925172 & 77 \\
\hline 0.9599684748 & 2.4354 I I 5773 & o. I4733 49785 & 85 & 2.7489224845 & 76 \\
\hline 0.9541596925 & 2.4220141749 & o. I5767 73727 & 84 & 2.7127524518 & 75 \\
\hline 0.9479822318 & $2.40779 \quad 13262$ & o. I679792208 & 84 & $2.6765^{8} 24191$ & 74 \\
\hline 0.94 I 4422 I 8 I & 2.3927621349 & o.17823 67907 & 84 & 2.6404 I 23864 & 73 \\
\hline 0.9345460898 & 2.3769467487 & o.I8844 61360 & 83 & 2.6042423537 & 72 \\
\hline $0.92730 \quad 05843$ & 2.3603663252 & o. 1986030778 & 8321 & 2.5680723210 & $7 \mathrm{I}$ \\
\hline 0.9197127230 & 2.3430429976 & 0.2087031860 & 82 & $2.53190 \quad 22883$ & 70 \\
\hline 0.9117897950 & 2.3249998377 & $0.21874 \quad 17592$ & 32 & 2.4957322556 & 69 \\
\hline 0.9035393417 & 2.3062608184 & $0.2287 \mathrm{I} \quad 3^{8038}$ & 82 & $2.4595^{6} 22230$ & 68 \\
\hline o.894969I397 & 2.2868507750 & 0.2386 I 40125 & $4 I$ & 2.4233921903 & 67 \\
\hline 0.8860871836 & 2.2667953647 & 0.2484367407 & I4 & 2.3872221576 & 66 \\
\hline 0.87690 I669o & $2.24612 \quad 10260$ & $0.25^{817} 59833$ & 80 & 2.3510521249 & 65 \\
\hline 0.8674209743 & 2.2248549364 & 0.2678253494 & I9 & 2.3148820922 & 64 \\
\hline 0.8576536425 & $2.20302 \quad 49697$ & $0.277378035^{8}$ & 79 & 2.2787 I 20595 & 63 \\
\hline 0.8476083633 & 2.1806596524 & 0.2868268004 & 20 & 2.2425420268 & 62 \\
\hline $0.837293954 \mathrm{I}$ & 2.157788 II97 & 0.2961639332 & 78 & 2.20637 I994I & $6 I$ \\
\hline 0.8267 I 934 I6 & 2. 1344400706 & $0.30538 \quad 12272$ & 78 & $2.17020 \quad 19614$ & 60 \\
\hline $0.815^{89} 354^{29}$ & 2.1106457227 & 0.3144699478 & 47 & 2.13403 19287 & 59 \\
\hline 0.8048256467 & 2.0864357672 & $0.3234^{2} 08014$ & I4 & 2.09786 I 8960 & $5^{8}$ \\
\hline $0.7935^{2} 47945$ & $2.06184 \quad 13229$ & 0.3322239026 & 40 & $2.06 \mathrm{I} 69 \mathrm{I} 8634$ & 57 \\
\hline 0.7820001623 & 2.0368938902 & 0.3408687415 & 76 & $2.0255^{2} \quad 18307$ & 56 \\
\hline 0.77026094 I I & 2.0116253056 & 0.3493441494 & 75 & I. $98935 \quad$ I 7980 & 55 \\
\hline $0.75831 \quad 63194$ & I.98606 $7695^{8}$ & 0.3576382644 & 74 & I. 953 I 8 I 7653 & 54 \\
\hline 0.746 I7 54642 & I . 9602534320 & 0.365738497 I & 74. & I.91701 I7326 & 53 \\
\hline 0.7338475039 & I. $93421 \quad 50843$ & 0.37363 I 4953 & 73 & I. 88084 I6999 & $5^{2}$ \\
\hline $0.72134 \quad 15096$ & I. 907985377 I & 0.3813031100 & $72 \quad 55$ & I. $84467 \quad 16672$ & $5^{I}$ \\
\hline 0.7086664787 & I.88I597 I433 & 0.38873836 I6 & 72 & I. $80850 \quad$ I 6345 & 50 \\
\hline 0.69583 I3178 & I. $85508 \quad 32817$ & 0.3959214068 & 71 & I. 77233 I601 8 & 49 \\
\hline 0.6828448256 & I. 8284767 II 7 & 0.4028355079 & 70 & I. 736 I6 I569I & 48 \\
\hline 0.6697 I 5678 I & I. 8018103311 & 0.4094630040 & 70 & I .69999 I 5365 & 47 \\
\hline 0.6564524120 & I.775II 69734 & 0.4157852846 & 69 & I. 66382 I 5038 & 46 \\
\hline 0.6430634108 & I. 7484293662 & $0.42178 \quad 27675$ & $68 \quad 25$ & I. 62765 I 47 I I & 45 \\
\hline $\mathbf{A}(\mathbf{r})$ & $\mathbf{D}(\mathbf{r})$ & $\mathbf{E}(\mathbf{r})$ & $\phi$ & $\mathbf{F} \phi$ & $\mathbf{r}$ \\
\hline
\end{tabular}


$K=3.3698680267, \quad K^{\prime}=1.5784865777, \quad E=1.027843620, \quad E^{\prime}=1.5629622295$,

\begin{tabular}{|c|c|c|c|c|c|c|c|c|}
\hline $\mathbf{r}$ & $\mathbf{F} \phi$ & \multicolumn{2}{|c|}{$\phi$. } & $\mathbf{E}(\mathbf{r})$ & \multicolumn{2}{|c|}{$\mathbf{D}(\mathbf{r})$} & \multicolumn{2}{|c|}{$\mathbf{A}(\mathbf{r})$} \\
\hline o & 0.0000000000 & & & 0.0000000000 & 1.00000 & 00000 & 0.00000 & 00000 \\
\hline I & 0.0374429781 & 2 & 9 & $0.026005343^{8}$ & I . 00048 & 71379 & 0.01396 & 87846 \\
\hline 2 & $0.074885956 \mathrm{r}$ & 4 & 17 & 0.0519080180 & I . O0I 94 & $8048 \mathrm{I}$ & 0.02793 & $9608 \mathrm{I}$ \\
\hline 3 & o. I 1232 89342 & 6 & 26 & $0.07760 \quad 64875$ & I. .00438 & 12208 & 0.04191 & 44920 \\
\hline 4 & o.14977 I9123 & 8 & 35 & 0.1030014601 & I . 00778 & 41400 & $0.055^{8} g$ & 54231 \\
\hline 5 & o.I872I 48904 & 10 & 40 & o.12799694I6 & I. OI 215 & 32844 & 0.06988 & 43359 \\
\hline 6 & 0.2246578684 & 12 & 46 & o. $15250 \quad 12188$ & I.01748 & 41292 & 0.08388 & 30956 \\
\hline 7 & 0.2621008465 & r 4 & $5^{I}$ & o.17642 77402 & I. 02377 & I 1470 & 0.09789 & 34813 \\
\hline 8 & 0.2995438246 & 16 & 55 & o.19969 $5^{8914}$ & I. $03 \mathrm{roo}$ & 78103 & o. II I9I & 71690 \\
\hline 9 & 0.3369868027 & I 8 & $5^{8}$ & $0.22223 \quad 16400$ & I.03918 & $6594 I$ & o. 12595 & 57152 \\
\hline 10 & $0.3744^{2} 97807$ & 20 & 59 & $0.243968048 \mathrm{I}$ & I. 04829 & $8978 \mathrm{I}$ & 0.14001 & 05412 \\
\hline II & 0.4118727588 & 22 & $5^{8}$ & 0.2648456468 & I . 05833 & 54510 & 0.15408 & 29167 \\
\hline 12 & 0.4493157369 & 24 & 56 & $0.2848 \mathrm{r} 26740$ & I . 06928 & $55 \times 35$ & $0.168 \mathrm{I} 7$ & $3945^{I}$ \\
\hline I3 & 0.4867587 I50 & 26 & 52 & $0.30382 \quad 51779$ & I . 08 II 3 & 76835 & 0.18228 & 45483 \\
\hline 14 & $0.52420 \quad 16930$ & 28 & 46 & 0.3218469961 & I .09387 & 95005 & o. I964I & 54524 \\
\hline I5 & 0.56 I64 467II & 30 & 38 & 0.3388496193 & I. 10749 & & 0.21056 & 71740 \\
\hline 16 & 0.5990876492 & 32 & 28 & $0.3548 \mathrm{I}$ I 9530 & I. 12197 & 73762 & 474 & 071 \\
\hline 17 & 0.6365306273 & 34 & 16 & 0.3697199918 & I . 13730 & 36763 & 0.23893 & 40100 \\
\hline 18 & 0.6739736053 & 36 & 2 & $0.38356 \quad 64197$ & I . I 5346 & 01207 & 0.25314 & 89941 \\
\hline I9 & 0.7114165834 & 37 & 46 & 0.39635 or539 & I. I 7042 & 94549 & 0.26738 & $45^{123}$ \\
\hline 20 & 0.7488595615 & 39 & 27 & Bo7 $5845^{\circ}$ & I. 18 & 34902 & 0.2 & 98484 \\
\hline 21 & 0.7863025396 & $4 \mathrm{I}$ & 6 & 0.4187533497 & I . 20673 & 31139 & 0.2959 & 40077 \\
\hline 22 & 0.8237455176 & 42 & 42 & 0.4283971871 & I . 22602 & 82998 & $0.3102 c$ & 57076 \\
\hline 23 & o. 86 I I 884957 & 44 & 16 & 0.4370259916 & I . 24605 & 81209 & $0.3245^{1}$ & 33701 \\
\hline 24 & 0.8986314738 & 45 & 48 & $0.44466 \quad 19725$ & I. 26680 & 07616 & 0.33883 & 51142 \\
\hline 25 & 0.9360744519 & 47 & I 8 & $0.45^{1} 3303888$ & I. 28823 & & 0.353 I 6 & 87494 \\
\hline 26 & 0.9735 I 74299 & 48 & 45 & 0.4570590462 & I. 31033 & 28836 & 0.3675 & 17704 \\
\hline 27 & I.01096 04080 & 50 & 10 & 0.4618778212 & I . 33307 & 44242 & 0.38186 & I 3526 \\
\hline 28 & I. $04840 \quad 3386$ I & $5 \mathrm{I}$ & 32 & $0.465818218 \mathrm{I}$ & I . 35643 & 29365 & 0.39621 & 43484 \\
\hline 29 & I. 085846364 I & 52 & $5^{2}$ & 0.4689 I 29597 & I $\cdot 38038$ & 23962 & 0.41056 & 72843 \\
\hline 30 & I. 1232893422 & 54 & 10 & $0.471195614^{8}$ & I. .40489 & 59917 & 0.42491 & 63594 \\
\hline $3^{1}$ & I. 1607323203 & 55 & 26 & 0.4727002620 & I . 42994 & 61457 & 0.43925 & $7444^{8}$ \\
\hline 32 & I. I9817 52984 & 56 & 39 & 0.47346 I I 908 & I .45550 & 45373 & $0.4535^{8}$ & 60835 \\
\hline 33 & I. 2356182764 & 57 & 50 & $0.47351 \quad 26377$ & I. 4 & & 0.46789 & 74917 \\
\hline 34 & I. $27306 \quad 12545$ & 59 & o & 0.4728885574 & I . 50802 & 91764 & 0.48218 & 65611 \\
\hline 35 & 05042326 & 60 & 7 & $0.47 \times 6224256$ & & & 0.49644 & \\
\hline 36 & 479472107 & 61 & 12 & 0.4697470729 & & 941 & 0 . & 56480 \\
\hline 37 & I. 38539 or 887 & 62 & 15 & 0.46729454 & I . 58987 & 98960 & 52486 & 38600 \\
\hline 38 & I. 4228331668 & 63 & 16 & 0.464295996 & I. 61785 & 45092 & 0.53900 & 61335 \\
\hline 39 & I. 4602761449 & 64 & 15 & 0.46078 I 5892 & I. 64612 & 04680 & 0.55309 & $5^{8052}$ \\
\hline 40 & I.49771 91230 & 65 & 12 & $0.456780433^{8}$ & & & 0.56712 & \\
\hline $4 I$ & I. 5351621010 & 66 & 7 & $0.45^{232} 05363$ & 1.7 & & $0.5^{8108}$ & 92454 \\
\hline 42 & I. 5726050791 & 67 & I & 0.4474287637 & 1. .73233 & 10960 & 0.59497 & 82708 \\
\hline 43 & I. 6100480572 & 67 & 53 & 0.4421308242 & I. 76142 & 48657 & 0.60878 & 52287 \\
\hline 44 & I. $64749 \quad 10353$ & 68 & 44 & 0.4364512599 & 1. 79063 & 90777 & 0.62250 & 21016 \\
\hline 45 & I. 6849340133 & 69 & 32 & 0.4304134495 & I . 81993 & 84164 & 0.63612 & 06349 \\
\hline $90-r$ & $\mathbf{F} \psi$ & & & $\mathbf{G}(\mathbf{r})$ & & & & \\
\hline
\end{tabular}

SMITHSONIAN TABLES 
TABLE $\theta=82^{\circ}$

$q=0.229567159881194, \quad \theta 0=0.5464169465, \quad \mathrm{HK}=1.4575481002$

\begin{tabular}{|c|c|c|c|c|c|}
\hline $\mathbf{B}(\mathbf{r})$ & $\mathbf{C}(\mathbf{r})$ & $\mathbf{G}(\mathbf{r})$ & $\psi$ & $\mathbf{F} \psi$ & $90-r$ \\
\hline I. 0000000000 & 2.6805403437 & 0.0000000000 & $90^{\circ} \quad \mathrm{o}^{\prime}$ & 3.3698680267 & 90 \\
\hline 0.9997862 II 2 & $2.68000 \quad 36787$ & 0.0106949135 & $89 \quad 42$ & $3 \cdot 3324250486$ & 89 \\
\hline 0.9991450809 & 2.6783944283 & 0.0213878301 & $89 \quad 24$ & 3.2949820705 & 88 \\
\hline 0.9980773170 & 2.6757 I 48255 & 0.0320767423 & 89 & 3.2575390925 & 87 \\
\hline 0.9965840972 & 2.6719685860 & 0.0427596209 & 48 & 3.2200961144 & 86 \\
\hline 0.9946670666 & 2.6671609043 & 0.0534344040 & 88 & 3.1826531363 & 85 \\
\hline 0.9923283334 & 2.6612984418 & 0.0640989867 & 88 & 3.14521 oI 582 & 84 \\
\hline 0.9895704645 & 2.6543893 I 56 & $0.07475 \quad 12085$ & 53 & 3.1077671802 & 83 \\
\hline 0.9863964786 & 2.6464430842 & $0.08538 \quad 88428$ & 35 & 3.0703242021 & 82 \\
\hline 0.9828098400 & 2.6374707296 & 0.0960095847 & 87 & $3.03288 \quad 12240$ & $8 \mathrm{I}$ \\
\hline 0.9788 I 44497 & 2.6274846381 & O. I066I I0385 & 86 & 2.9954382459 & 80 \\
\hline $0.9744 \mathrm{I} \quad 46367$ & 2.6164985778 & 0.II7I9 07054 & 86 & 2.9579952679 & 79 \\
\hline 0.9696 I 51474 & $2.6045^{2} 76741$ & o.I2774 $5970 \mathrm{I}$ & 86 & 2.9205522898 & 78 \\
\hline 0.96442 I I 348 & $2.5915^{8} \quad 83828$ & o.13827 40870 & 85 & 2.8831093117 & 77 \\
\hline 0.9588381466 & 2.5776984606 & o. 1487721662 & 85 & 2.8456663336 & 76 \\
\hline 0.952872 III 7 & $2.56287 \quad 69342$ & o. I5923 7 I 580 & 85 & 2.8082233556 & 75 \\
\hline 0.9465293269 & $2.54714 \quad 40664$ & o.16966 58376 & 56 & 2.7707803775 & 74 \\
\hline 0.9398164421 & 2.53052 I 3208 & o. I8005 47885 & 35 & 2.7333373994 & 73 \\
\hline 0.9327404449 & 2.5130313248 & o. 1904003849 & 84 & $2.695^{89} 44213$ & 72 \\
\hline 0.9253086446 & 2.4946978294 & 0.2006987739 & $5^{I}$ & $2.65845 \quad 14433$ & 71 \\
\hline 0.9175286553 & 2.4755456695 & $0.210945^{8} 55^{6}$ & 83 & 2.6210084652 & 70 \\
\hline 0.9094083786 & $2.45560 \quad 07207$ & 0.2211372633 & 83 & $2.5^{8} 35^{6} 54^{87} \mathrm{I}$ & 69 \\
\hline 0.9009559853 & $2.434889855^{6}$ & 0.2312683422 & 82 & $2.54612 \quad 25090$ & 68 \\
\hline 0.8921798975 & 2.4134408985 & 0.2413341265 & I6 & 2.5086795310 & 67 \\
\hline 0.8830887690 & $2.39128 \quad 25787$ & 0.2513293157 & 51 & 2.4712365529 & 66 \\
\hline 0.87369 I 4660 & 2.3684444831 & 0.2612482501 & $8 \mathbf{I}$ & 2.4337935748 & 65 \\
\hline 0.8639970475 & 2.3449570070 & 0.2710848837 & 59 & 2.3963505967 & 64 \\
\hline o. $85401 \quad 4745^{2}$ & $2.32085 \quad 13053$ & 0.2808327574 & 32 & $2.35^{890} 76187$ & 63 \\
\hline 0.8437539427 & 2.2961592414 & 0.2904849692 & 80 & 2.3214646406 & 62 \\
\hline 0.8332241555 & 2.2709133365 & $0.30003 \quad 41444$ & 35 & $2.28402 \quad 16625$ & $6 I$ \\
\hline 0.8224350100 & $2.24514 \quad 67182$ & $0.309472403 \mathrm{I}$ & 79 & 2.2465786844 & 60 \\
\hline 0.8113962227 & 2.2188930687 & 0.3187913276 & 78 & 2.2091357064 & 59 \\
\hline 0.800 I I 75795 & 2. 1921865719 & 0.3279819272 & 78 & 2.1716927283 & $5^{8}$ \\
\hline 0.7886089149 & 2. 16506 I 8621 & 0.3370346027 & 77 & 2.1342497502 & 57 \\
\hline 0.7768800911 & 2. 1375539706 & 0.3459391087 & 76 & 2.0968067721 & 56 \\
\hline 0.7649409778 & 2. 1096982742 & $0.35468 \quad 45^{1} 5^{2}$ & 76 & $2.059363794 I$ & 55 \\
\hline $0.75280 \quad 14315$ & 2.0815304423 & 0.3632591686 & 75 & 2.0219208160 & 54 \\
\hline $0.74047 \quad 12755$ & 2.0530863856 & 0.37 I65 06505 & 75 & I. $98447 \quad 78379$ & 53 \\
\hline 0.7279602805 & $2.02440 \quad 22044$ & o. 3798457377 & 74 & I. 9470348599 & 52 \\
\hline 0.7152781443 & I. $9955^{I} \quad 4$ I 373 & 0.3878303601 & 73 & I.90959 I8818 & $5 \mathrm{I}$ \\
\hline 0.7024344736 & I. 9664585 II 5 & $0.3955^{8} 95596$ & 73 & I. 8721489037 & 50 \\
\hline 0.6894387648 & I.93727 I6923 & 0.4031074491 & 72 & I . $83470 \quad 59256$ & 49 \\
\hline 0.6763003866 & I. 9079900345 & 0.4103671725 & $7 \mathrm{I}$ & I.79726 29476 & 48 \\
\hline 0.6630285617 & I. 8786498345 & 0.4173508655 & 71 & I.7598I 99695 & 47 \\
\hline 0.6496323506 & I. $84928 \quad 72824$ & 0.4240396200 & 70 & I. 7223769914 & 46 \\
\hline 0.6361206349 & I. 8 I993 84 I64 & 0.4304 I 34495 & 69 & I. 6849340133 & 45 \\
\hline $\mathbf{A}(\mathbf{r})$ & $\mathbf{D}(\mathbf{r})$ & $\mathbf{E}(\mathbf{r})$ & $\phi$ & F $\phi$ & $\mathbf{r}$ \\
\hline
\end{tabular}


$\mathrm{K}=3.5004224992, \quad \mathrm{~K}^{\prime}=1.5766779816, \quad \mathrm{E}=1.022312588, \quad \mathrm{E}^{\prime}=1.5649475630$

\begin{tabular}{|c|c|c|c|c|c|c|c|c|c|c|}
\hline $\mathbf{r}$ & \multicolumn{2}{|c|}{$\mathrm{F} \phi$} & \multicolumn{2}{|c|}{$\phi$} & \multicolumn{2}{|l|}{$\mathbf{E}(\mathbf{r})$} & \multicolumn{2}{|l|}{$\mathrm{D}(\mathbf{r})$} & \multicolumn{2}{|l|}{$A(r)$} \\
\hline o & 0.00000 & 00000 & $0^{\circ}$ & $\mathrm{o}^{\prime}$ & 0.00000 & 00000 & I. .00000 & 00000 & 0.000000 & 00000 \\
\hline I & 0.03889 & $35^{8} 33$ & 2 & I 4 & $0.0275 \mathrm{I}$ & 52459 & I.00053 & 54142 & o.013578 & 81428 \\
\hline 2 & 0.07778 & 71666 & 4 & 27 & $0.0549 \mathrm{I}$ & 49 I 7 I & I. $.002 \mathrm{I} 4$ & I 1230 & 0.027159 & 91294 \\
\hline 3 & o. I I 668 & 07500 & 6. & 40 & 0.08208 & 48196 & I. 004815 & 55243 & 0.040745 & 57840 \\
\hline 4 & o. I 5557 & 43333 & 8 & 53 & 0.10891 & 34862 & I. .00855 & 59486 & 0.054340 & 08922 \\
\hline 5 & o. 19446 & 79166 & I I & 4 & o.135293 & $3453 \mathrm{I}$ & I.0I335 & 86590 & 0.067947 & 71815 \\
\hline 6 & o.. 23336 & I 4999 & I3 & I 5 & o. I6II2 2 & 24388 & I.OI92I & 88518 & 0.081567 & 73027 \\
\hline 7 & 0.27225 & 50833 & I 5 & 25 & o. 18630 & 43989 & I .026I 3 & 06577 & 0.095203 & 38101 \\
\hline 8 & 0.3 I I I 4 & 86666 & I 7 & 33 & 0.21075 & 04315 & I. 03408 & 71422 & o. 108859 & 91438 \\
\hline 9 & 0.35004 & 22499 & I9 & 40 & 0.23437 & $95^{2} 37$ & I. 04308 & 03072 & o. 122535 & 56 I I I \\
\hline I0 & 0.38893 & 58332 & $2 \mathrm{I}$ & 45 & 0.257 II & 91248 & I. 05310 & 10924 & 0.136235 & $5368 \mathrm{I}$ \\
\hline I I & 0.42782 & 94166 & 23 & 48 & 0.27890 & 55463 & I. .06413 & 93774 & o. I 4996 & 04030 \\
\hline 12 & 0.46672 & 29999 & 25 & 50 & 0.29968 & 41874 & I. 076 I 8 & 39836 & 0.163712 & 25182 \\
\hline I3 & $0.5056 \mathrm{I}$ & 65832 & 27 & 50 & 0.31940 & 95974 & I . 08922 & 26769 & o.I77493 & 33 I 4 I \\
\hline I 4 & 0.5445 I & or 665 & 29 & 47 & 0.33804 & $53^{8} 36$ & I. 10324 & 21710 & 0.191304 & 41733 \\
\hline I5 & $0.5^{8} 34^{\circ}$ & 37499 & $3 I$ & 42 & 0.355563 & 39822 & I. II 822 & 81308 & 0.205146 & 62446 \\
\hline I6 & 0.62229 & $7333^{2}$ & 33 & 35 & 0.37 I 94 & 63079 & I . I 34 I 6 & 51764 & 0.21902 & 04287 \\
\hline I 7 & 0.66119 & 09165 & 35 & 26 & 0.38718 & 13038 & I . I $5 \mathrm{IO}_{3}$ & 68883 & 0.232927 & 73637 \\
\hline I 8 & 0.70008 & 44998 & 37 & I 4 & 0.401265 & 54102 & I. I 6882 & 58124 & 0.246867 & 74120 \\
\hline I9 & $0.73^{897}$ & 80832 & $3^{8}$ & 59 & 0.41420 & 19722 & I. I $875 \mathrm{I}$ & 34668 & 0.26084 & 06476 \\
\hline 20 & 0.77787 & I 6665 & 40 & 42 & 0.42600 & 06064 & I .20708 & 03483 & 0.274846 & 68440 \\
\hline $2 \mathrm{I}$ & 0.81676 & $5249^{8}$ & 42 & 23 & 0.43667 & 65427 & I $.2275^{\circ}$ & 59404 & 0.288885 & 54637 \\
\hline 22 & $0.855^{6} 5$ & $8833 \mathbf{I}$ & 44 & I & 0.44624 & $9958 \mathrm{I}$ & I. 24876 & 87226 & 0.30295 & 56475 \\
\hline 23 & 0.89455 & 24165 & 45 & 37 & 0.45474 & 53170 & I . 27084 & 61798 & 0.31705 & 62057 \\
\hline 24 & 0.93344 & 59998 & 47 & IO & 0.46219 & $0728 I$ & I . $2937 \mathrm{I}$ & $4^{81} 35$ & 0.331185 & 56095 \\
\hline 25 & 0.97233 & $95^{8} 3 \mathrm{I}$ & 48 & 40 & 0.468617 & 73287 & I. 3 I 735 & oI 537 & 0.34534 & 19839 \\
\hline 26 & I.OI 123 & 31664 & 50 & 8 & 0.47405 & 87042 & I. 34172 & 67728 & $0.3595^{2} 3$ & 31012 \\
\hline 27 & I. 05012 & $6749^{8}$ & $5 \mathrm{I}$ & 33 & 0.47855 & 03463 & I. 3668 I & 82994 & 0.37372 & 63757 \\
\hline 28 & I . 08902 & 03331 & $5^{2}$ & 56 & 0.48212 & 91569 & I. 39259 & $7434^{8}$ & o.387948 & 88593 \\
\hline 29 & I . I 279I & 39164 & 54 & I7 & 0.48483 & 29959 & I. 41903 & 59703 & 0.40218 & 72381 \\
\hline 30 & I . I6680 & 74997 & 55 & 35 & 0.48670 & 02770 & I . 446 Io & 48057 & 0.416437 & 78306 \\
\hline $3 I$ & I . 20570 & 10830 & 56 & 50 & 0.48776 & 96093 & I . 47377 & 39701 & 0.43069 & $65^{86} \mathrm{I}$ \\
\hline 32 & I . 24459 & 46664 & $5^{8}$ & 4 & 0.48807 & 94838 & I .50201 & 26433 & 0.44495 & 90849 \\
\hline 33 & I. 28348 & 82497 & 59 & I 4 & 0.48766 & 80032 & I . 53078 & 91792 & 0.45922 & 05390 \\
\hline 34 & I $\cdot 3^{22} 3^{8}$ & I 8330 & 60 & 23 & 0.48657 & 26520 & I. 56007 & I1317 & 0.47347 & $5794^{8}$ \\
\hline 35 & I. 36127 & 54163 & 6I & 30 & 0.48483 & o1039 & I. $5^{8982}$ & 52804 & 0.48771 & 93356 \\
\hline 36 & I. 40016 & 89997 & 62 & 34 & 0.48247 & 60647 & I . 62001 & 76598 & 0.50194 & 52865 \\
\hline 37 & I.43906 & $25^{8} 30$ & 63 & 36 & 0.47954 & $5^{1} 45^{6}$ & I . $6506 \mathrm{I}$ & $35^{895}$ & 0.51614 & 74196 \\
\hline $3^{8}$ & I. 47795 & 61663 & 64 & 36 & 0.47607 & 07644 & I . 681 57 & $7705^{8}$ & 0.53031 & 91603 \\
\hline 39 & I. 5 I 684 & 97496 & 65 & 35 & 0.47208 & 50753 & I . 71287 & 39955 & 0.54445 & 35952 \\
\hline 40 & I . 55574 & 33330 & 66 & $3 I$ & $0.4676 \mathrm{I}$ & 89121 & I . 74446 & 58318 & $0.55^{8} 54$ & 34803 \\
\hline $4 I$ & I . 59463 & 69163 & 67 & 25 & 0.46270 & 17621 & I. 77631 & 60110 & $0.5725^{8}$ & I 25 I I \\
\hline 42 & I. 63353 & 04996 & 68 & 18 & 0.45736 & I 7475 & I . 80838 & 67918 & $0.5^{8655}$ & 90333 \\
\hline 43 & I. 67242 & 40829 & 69 & 9 & 0.45162 & 56249 & I. 84063 & 99362 & 0.60046 & 86540 \\
\hline 44 & I. 7 I I 3I & 76663 & 69 & $5^{8}$ & $0.4455^{\mathrm{I}}$ & 87962 & I . 87303 & 67513 & 0.61430 & 16549 \\
\hline 45 & I . 75021 & 12496 & 70 & 45 & 0.43906 & 53283 & I . 90553 & 81344 & 0.62804 & 93057 \\
\hline $90-r$ & $F \psi$ & & & $\psi$ & $\mathbf{G}(\mathbf{r})$ & & $\mathbf{C}(\mathbf{r})$ & & $\mathbf{B}(\mathbf{r})$ & \\
\hline
\end{tabular}








\begin{tabular}{|c|c|c|c|c|c|}
\hline $\mathbf{B}(\mathbf{r})$ & $C(r)$ & $\mathrm{G}(\mathbf{r})$ & $\psi$ & $\mathrm{F} \psi$ & $90-r$ \\
\hline I .00000 00000 & $2.8645^{2} \quad 59727$ & 0.0000000000 & $90^{\circ}$ & $3 \cdot 50042 \quad 24992$ & 90 \\
\hline o: 99977 91249 & $2.86392545^{80}$ & $0.01078 \quad 10889$ & $89 \quad 44$ & $3 \cdot 4615^{2} 8915^{8}$ & 89 \\
\hline 0.999 I I $675^{83}$ & 2.8621247652 & 0.0215604536 & 89 & 3.4226353325 & 88 \\
\hline 0.9980 I 36755 & $2.859 \mathrm{I} 26446 \mathrm{I}$ & 0.0323363597 & 89 & $3.3^{8} 374 \quad$ I 7492 & 87 \\
\hline 0.99647 II670 & 2.8549347485 & 0.0431070526 & 55 & 3.3448481659 & 86 \\
\hline 0.99449 I0345 & $2.84955 \quad 56077$ & 0.053870747 I & 88 & $3.3059545^{826}$ & 85 \\
\hline $0.9920755^{874}$ & 2.8429966356 & 0.0646256168 & 88 & 3.2670609992 & 84 \\
\hline 0.9892276367 & 2.8352671062 & 0.0753697836 & 88 & 3.228 I 674 I59 & 83 \\
\hline 0.9859504884 & 2.8263779377 & 0.08610 .13069 & 87 & 3.1892738326 & 82 \\
\hline $0.982247935^{\circ}$ & $2.81634 \quad 16722$ & 0.0968 I 8 I7I 8 & 30 & 3.1503802493 & $8 \mathrm{I}$ \\
\hline $0.97812 \quad 42473$ & 2.8051724517 & o.IO75I 82779 & 87 & 3.11 I48 66659 & 80 \\
\hline $0.9735^{8} 4 \mathrm{I} 628$ & 2.7928859919 & o. II8I9 94268 & 86 & 3.0725930826 & 79 \\
\hline 0.9686328755 & $2.77949955^{23}$ & o. I2885 93097 & 37 & 3.0336994993 & 78 \\
\hline 0.9632760226 & 2.7650319042 & o. I3949 $5493^{8}$ & 86 & $2.99480 \quad 59160$ & 77 \\
\hline 0.9575 I 967 II & $2.74950 \quad 32957$ & o. I5010 54088 & 86 & 2.9559 I 23326 & 76 \\
\hline 0.9513703036 & 2.7329354142 & o. 16068 63318 & 85 & 2.9170187493 & 75 \\
\hline 0.9448348022 & $2.71535 \quad 13465$ & o.17123 53724 & 85 & $2.87812 \quad 51660$ & 74 \\
\hline 0.9379204329 & 2.6967755363 & o.18I7494560 & 85 & 2.83923 I 5827 & 73 \\
\hline 0.9306348276 & 2.6772337397 & o. 1922253067 & 84 & 2.8003379993 & 72 \\
\hline 0.9229859663 & 2.6567529786 & 0.2026594294 & 84 & $2.76 \mathrm{I} 4444 \mathrm{I} 6 \mathrm{o}$ & 71 \\
\hline $0.9149^{8} 215^{85}$ & 2.63536 I 492 I & 0.2130480901 & 84 & $2.72255 \quad 08327$ & 70 \\
\hline 0.9066320234 & $2.613088685^{8}$ & 0.2233872956 & 83 & 2.6836572494 & 69 \\
\hline o. 8979444698 & 2.5899650797 & 0.2336727719 & 83 & 2.6447636660 & 68 \\
\hline 0.8889286753 & $2.56602 \quad 22548$ & 0.2438999414 & 82 & $2.605^{87} 00827$ & 67 \\
\hline 0.8795940653 & 2.5412927973 & $0.254063898 \mathbf{I}$ & $3 I$ & 2.5669764994 & 66 \\
\hline 0.8699502909 & 2.5 I 58 I 02430 & 0.264 I5 93822 & 82 & $2.528082916 I$ & 65 \\
\hline 0.8600072069 & 2.4896090190 & $0.27418075^{25}$ & $8 \mathrm{I}$ & $2.48918 \quad 93327$ & 64 \\
\hline 0.8497748495 & 2.4627243859 & $0.28412 \quad 19576$ & $8 \mathrm{I}$ & 2.4502957494 & 63 \\
\hline $0.83926 \quad 34$ I 34 & 2.4351923782 & 0.2939765053 & 80 & $2.4 \mathrm{I} 1402 \mathrm{I} 66 \mathrm{I}$ & 62 \\
\hline $0.82848 \quad 32287$ & 2.4070497447 & 0.3037374301 & 23 & 2.3725085828 & $6 I$ \\
\hline $0.81744 \quad 47382$ & $2.37833 \quad 38874$ & 0.3133972593 & 79 & 2.3336 I 49994 & 60 \\
\hline 0.806 I5 $8473^{8}$ & 2.34908 280I 5 & 0.3229479773 & 79 & 2.29472 I 4I6I & 59 \\
\hline 0.7946350337 & 2.3193350143 & 0.3323809873 & 78 & $2.255^{82} 78328$ & $5^{8}$ \\
\hline 0.7828850590 & 2.2891295239 & $0.34168 \quad 70724$ & 78 & $2.21693 \quad 42495$ & 57 \\
\hline 0.7709192109 & $2.25^{8} 5^{0} \quad 573^{8} 3$ & 0.3508563539 & 77 & 2. I7804 06662 & $5^{6}$ \\
\hline $0.75^{874} 81476$ & 2.2275034 I5I & 0.3598782486 & 77 & 2.1391470828 & 55 \\
\hline 0.7463825018 & 2. I96I6 26008 & $0.36874 \quad 14237$ & 76 & 2.10025 34995 & 54 \\
\hline $0.733^{8} 3285^{87}$ & 2.1645235708 & $0.37743 \quad 37507$ & 76 & 2.0613599162 & 53 \\
\hline 0.72 I I0 97334 & 2. 13262 67708 & $0.3^{8594} 22578$ & 75 & 2.0224663329 & 52 \\
\hline 0.7082235503 & 2. $1005 \mathrm{I} 27578$ & 0.3942530813 & 74 & I. $98357 \quad 27495$ & $5^{\mathrm{I}}$ \\
\hline 0.69518462 Io & $2.06822 \quad 21426$ & $0.40235 \quad 14155$ & 74 & I. 9446791662 & 50 \\
\hline $0.68200 \quad 31247$ & $2.03579 \dot{9} 5533 \mathrm{I}$ & 0.4102214630 & 73 & I. $90578 \quad 55^{829}$ & 49 \\
\hline 0.6686890878 & 2.0032734790 & 0.4178463843 & 72 & I. 86689 I9996 & 48 \\
\hline $0.655^{2} 523646$ & I.97069 64I70 & 0.4252082479 & 72 & I . 8279984 I 62 & 47 \\
\hline $0.64170 \quad 26188$ & I.938I0 46I79 & $0.43228 \quad 79822$ & $3 I$ & I. $78910 \quad 48329$ & 46 \\
\hline 0.6280493057 & I. 905538 I 344 & 0.4390653283 & $70 \quad 45$ & I. $75021 \quad 12496$ & 45 \\
\hline $\mathbf{A}(\mathbf{r})$ & $\mathbf{D}(\mathbf{r})$ & $\mathbf{E}(\mathbf{r})$ & $\phi$ & $\mathbf{F} \phi$ & $\mathbf{r}$ \\
\hline
\end{tabular}

SMITHSONIAN TABLES 
$\mathrm{K}=3.6518559695, \quad \mathrm{~K}^{\prime}=1.5751136078, \quad \mathrm{E}=1.017236918, \quad \mathrm{E}^{\prime}=1.5664967878$,

\begin{tabular}{|c|c|c|c|c|c|c|c|c|c|c|}
\hline \multirow{2}{*}{$\frac{r}{0}$} & \multicolumn{2}{|l|}{$F \phi$} & \multicolumn{2}{|c|}{$\phi$} & \multicolumn{2}{|c|}{$\mathbf{E}(\mathbf{r})$} & \multicolumn{2}{|c|}{$\mathbf{D}(\mathbf{r})$} & \multicolumn{2}{|c|}{$A(r)$} \\
\hline & 0.00000 & 00000 & $0^{\circ}$ & $\mathrm{o}^{\prime}$ & 0.00000 & 00000 & I. . 00000 & 00000 & 0.00000 & 00000 \\
\hline I & 0.04057 & 61774 & 2 & $\mathbf{I}$ & 0.02925 & I 5342 & I .00059 & 38572 & 0.0I3II & 92586 \\
\hline 2 & 0.08 II 5 & 23549 & 4 & 29 & 0.05837 & 13484 & I .00237 & 4864 I & 0.02624 & 22974 \\
\hline 3 & 0.12172 & 85323 & 6 & 55 & 0.08722 & $943^{80}$ & I. 00534 & 13262 & 0.03937 & 28749 \\
\hline 4 & 0.16230 & 47098 & 9 & I6 & o. I I 569 & 91812 & I .00949 & 04192 & $0.0525 \mathrm{I}$ & 47063 \\
\hline 5 & 0.20288 & 08872 & I I & 33 & o. 14365 & 89152 & I . OI 48 I & 81886 & 0.06567 & 14426 \\
\hline 6 & 0.24345 & 70646 & I3 & 49 & o. I7099 & 33783 & I. .02 I $3 \mathrm{I}$ & 95491 & 0.07884 & 66485 \\
\hline 7 & 0.28403 & $3242 \mathrm{I}$ & I6 & 4 & o. I9759 & 49853 & I. 02898 & $8284 \mathrm{I}$ & 0.09204 & 37819 \\
\hline 8 & 0.32460 & 94195 & I 8 & I 7 & 0.22336 & 49075 & I. 0378 I & $7045^{\circ}$ & o. 10526 & 61731 \\
\hline 9 & 0.36518 & 55969 & 20 & 29 & $0.2482 \mathrm{I}$ & $3938 I$ & I .04779 & 73504 & 0. I I 85 I & 70041 \\
\hline IO & 0.40576 & I 7744 & 22 & 39 & 0.27206 & 3 I 34 I & I .0589I & $95^{8} 57$ & 0.I3I79 & 92889 \\
\hline II & 0.44633 & 79518 & 24 & 46 & 0.29484 & 42309 & 1.07117 & 30024 & 0.145 I I & 58534 \\
\hline I 2 & $0.4869 \mathrm{I}$ & 41293 & 26 & $5^{2}$ & 0.31649 & 98365 & I. 08454 & 57174 & o. I 5846 & 93168 \\
\hline 13 & 0.52749 & 03067 & 28 & 56 & 0.33698 & $34 \mathrm{I} 75$ & I. 09902 & 47131 & 0.17186 & 20726 \\
\hline I 4 & 0.56805 & $6484 I$ & 30 & $5^{8}$ & 0.35625 & 90959 & I . I I 459 & $5^{8374}$ & 0.18529 & 627 III \\
\hline I 5 & 0.60864 & 266 I6 & 32 & 55 & $0.3743^{\circ}$ & 12782 & I. I3I 24 & 38038 & o. 19877 & 38016 \\
\hline 16 & 0.64921 & 88390 & 34 & 51 & 0.39109 & 41430 & I . I 4895 & 21925 & 0.21229 & $6275^{8}$ \\
\hline 17 & 0.68979 & 50165 & 36 & 44 & 0.40663 & 10147 & I. I6770 & 345 I 4 & $0.225^{86}$ & 50123 \\
\hline I 8 & 0.73037 & I 1939 & 38 & 36 & $0.4209 \mathrm{I}$ & $3648 \mathrm{I}$ & I . I 8747 & 88983 & 0.23948 & IO2 I I \\
\hline I9 & 0.77094 & 73713 & 40 & 24 & 0.43395 & I 4533 & I . 20825 & 87235 & 0.25314 & 49894 \\
\hline 20 & 0.81152 & 35488 & 42 & 9 & 0.44576 & 06829 & I. 23002 & I 9929 & 0.26685 & 72683 \\
\hline $2 \mathrm{I}$ & 0.85209 & 97262 & 43 & $5 I$ & 0.45636 & 36044 & I . 25274 & 66524 & $0.2806 \mathrm{I}$ & 78600 \\
\hline 22 & 0.89267 & 59037 & 45 & 31 & 0.46578 & 76783 & I . 27640 & 95335 & 0.29442 & 64067 \\
\hline 23 & 0.93325 & 208 I I & 47 & 8 & 0.47406 & 47564 & I $\cdot 30098$ & 63590 & 0.30828 & 21794 \\
\hline 24 & $0.973^{82}$ & $825^{8} 5$ & 48 & 42 & 0.48123 & 03147 & I. 32645 & 17509 & 0.32218 & 40690 \\
\hline 25 & I. 01440 & 44360 & 50 & I3 & 0.48732 & 27312 & I $\cdot 35^{277}$ & 92393 & 0.33613 & 05773 \\
\hline 26 & I .05498 & 06I34 & $5 I$ & 42 & 0.49238 & 26159 & I. 37994 & $1272 \mathrm{I}$ & 0.350 I I & 98097 \\
\hline 27 & I .09555 & 67908 & 53 & 8 & 0.49645 & 21966 & I. 40790 & 92268 & 0.36414 & 94689 \\
\hline 28 & I. 136 I3 & 29683 & 54 & $3 I$ & 0.49957 & 47663 & I .43665 & 34239 & 0.37821 & 68497 \\
\hline 29 & I. I 7670 & 91457 & 55 & $5 \mathrm{I}$ & 0.50179 & 41897 & I. 46614 & $314 I 2$ & 0.39231 & $8835^{\circ}$ \\
\hline 30 & 1.21728 & 53232 & 57 & 9 & 0.50315 & $4470 \mathrm{I}$ & I . 49634 & 66307 & 0.40645 & I 8927 \\
\hline 31 & I. 25786 & I 5006 & $5^{8}$ & 25 & 0.50369 & 93739 & I. 52723 & I I369 & 0.42061 & 20743 \\
\hline 32 & I . 298437 & 76780 & 59 & 38 & 0.50347 & $2 \mathrm{IIO}_{4}$ & I $\cdot 55^{876}$ & 29167 & 0.43479 & 50141 \\
\hline 33 & I.33901 & 38555 & 60 & 48 & $0.5025 \mathrm{I}$ & 50624 & I . 59090 & 72622 & 0.44899 & 59303 \\
\hline 34 & I.37959 & 00329 & $6 I$ & 56 & 0.50086 & $9565 \mathrm{I}$ & I. 62362 & $8524 I$ & 0.46320 & 96265 \\
\hline 35 & I. 42016 & 62104 & 63 & 2 & 0.49857 & 57270 & I . 65689 & OI387 & 0.47743 & 04952 \\
\hline 36 & I. 46074 & 23878 & 64 & 5 & 0.49567 & 22903 & I . 69065 & $4655^{8}$ & 0.49165 & 252 I 8 \\
\hline 37 & 1.50131 & 85652 & 65 & 7 & 0.49219 & 65260 & I .72488 & 37696 & 0.50586 & 92908 \\
\hline 38 & I. 54 I 89 & 47427 & 66 & 6 & 0.48818 & 41583 & I. 75953 & $835 \mathrm{I} 4$ & 0.52007 & 39919 \\
\hline 39 & I .58247 & 09201 & 67 & 3 & 0.48366 & 93168 & I. 79457 & 84847 & $0.534^{25}$ & 94285 \\
\hline 40 & I. 62304 & 70975 & 67 & $5^{8}$ & 0.47868 & 45099 & I . 82996 & 35024 & $0.5484 \mathrm{I}$ & 80268 \\
\hline $4 I$ & I. 66362 & $3275^{\circ}$ & 68 & $5 I$ & 0.47326 & 06 I 89 & I . 86565 & 20265 & 0.56254 & I 846 I \\
\hline 42 & 1.70419 & $945^{24}$ & 69 & 42 & $0.4674^{2}$ & $6907 \mathrm{I}$ & I. 90160 & 20099 & 0.57662 & 25903 \\
\hline 43 & I. 74477 & 56299 & 70 & $3 I$ & 0.46 I 21 & 10428 & I. 93777 & 07807 & 0.59065 & 16209 \\
\hline 44 & I. 78535 & I 8073 & $7 \mathrm{I}$ & I9 & 0.45463 & 91336 & I. 974 I I & $5088 \mathrm{I}$ & $0.6046 \mathrm{I}$ & 99704 \\
\hline 45 & I. 82592 & 79847 & 72 & 5 & 0.44773 & 57684 & 2.01059 & I 1517 & $0.6185 \mathrm{I}$ & 83573 \\
\hline $90-r$ & $\mathbf{F} \psi$ & & $\psi$ & & $\mathbf{G}(\mathbf{r})$ & & $C(r)$ & & $\mathbf{B}(\mathbf{r})$ & \\
\hline
\end{tabular}


TABLE $\theta=84^{\circ}$

$q=0.257940195766337, \quad \theta 0=0.4929628191, \quad$ HK $=1.5205617314$

\begin{tabular}{|c|c|c|c|c|c|}
\hline $\mathbf{B}(\mathbf{r})$ & $\mathbf{C}(\mathbf{r})$ & $\mathbf{G}(\mathbf{r})$ & $\psi$ & $\mathbf{F} \psi$ & $90-\mathrm{r}$ \\
\hline I.00000 00000 & $3.09301992 \mathrm{I} 3$ & 0.0000000000 & $90^{\circ} \quad 0^{\prime}$ & 3.65 I 8559695 & 90 \\
\hline 0.9997707150 & 3.0923385676 & 0.0108590483 & $89 \quad 45$ & 3.6112797920 & 89 \\
\hline $0.999083145^{8}$ & 3.0902954977 & 0.0217166503 & $3 I$ & $3.57070 \quad 36146$ & 88 \\
\hline 0.9979381489 & 3.0868936827 & $0.03257 \quad 13506$ & 16 & 3.5301274372 & 87 \\
\hline 0.9963371496 & 3.0821380679 & $0.04342 \quad 16747$ & 89 & $3.48955 \quad 12597$ & 86 \\
\hline 0.9942821381 & 3.0760355627 & 0.0542661204 & 88 & 3.4489750823 & 85 \\
\hline 0.9917756649 & 3.0685950269 & 0.0651031473 & 32 & 3.4083989048 & 84 \\
\hline 0.9888208340 & $3.05982 \quad 72527$ & 0.07593 I 1673 & 88 & $3 \cdot 36782 \quad 27274$ & 83 \\
\hline $0.98542 \quad$ I 2955 & $3.049744943 \mathrm{I}$ & 0.08674 .85345 & 88 & $3.32724 \cdot 65500$ & 82 \\
\hline $0.98158 \quad 12363$ & 3.0383626866 & 0.0975535344 & 46 & 3.2866703725 & $8 \mathrm{I}$ \\
\hline $0.97730 \quad 53698$ & 3.0256969280 & o. 108344373 I & 30 & 3.2460941951 & 80 \\
\hline 0.9725989240 & $3.011765935^{8}$ & o.II9II 9I660 & I 4 & $3.2055 \mathrm{I} \quad 80177$ & 79 \\
\hline 0.9674676286 & $2.9965^{8} 97659$ & o. I 298759255 & $5^{8}$ & 3.16494 I 8402 & 78 \\
\hline $0.96191 \quad 77007$ & 2.9801902223 & o. I 406 I 25487 & 42 & 3.1243656628 & 77 \\
\hline 0.9559558299 & 2.9625908137 & 0.1513268040 & 25 & $3.0837894^{8} 53$ & 76 \\
\hline $0.9495^{8} 91609$ & $2.943^{81} \quad 67083$ & 0.1620163172 & 86 & 3.0432133079 & 75 \\
\hline 0.9428252769 & 2.9238946843 & o. 1726785562 & 50 & 3.0026371305 & 74 \\
\hline 0.9356721802 & 2.9028530783 & 0.1833108161 & 32 & 2.9620609530 & 73 \\
\hline 0.9281382732 & 2.88072 I 7308 & o.1939I 020I3 & 85 & $2.9214^{8} 4775^{6}$ & 72 \\
\hline 0.9202323376 & 2.85753 I9293 & 0.2044736088 & 55 & $2.880908598 \mathrm{I}$ & 71 \\
\hline 0.9119635133 & 2.8333163492 & 0.2149977081 & 84 & $2.84033 \cdot 24207$ & 70 \\
\hline $0.90334 \quad 12763$ & 2.8081089917 & 0.2254789218 & 16 & 2.7997562433 & 69 \\
\hline $0.8943754 \mathrm{I} 54$ & 2.7819451210 & $0.23591 \quad 34034$ & 55 & $2.759180065^{8}$ & 68 \\
\hline 0.8850760096 & 2.75486 I1988 & 0.2462970143 & 34 & $2.71860 \quad 38884$ & 67 \\
\hline 0.8754534034 & 2.7268948173 & 0.2566252995 & I3 & 2.6780277109 & 66 \\
\hline 0.8655 I 8 I 826 & $2.69808 \quad 463$ I 3 & 0.2668934606 & 82 & $2.63745 \quad 15335$ & 65 \\
\hline 0.85528 I I 49 I & 2.6684702880 & 0.2770963287 & 28 & 2.596875356 I & 64 \\
\hline $0.844753295^{8}$ & 2.6380923575 & 0.2872283335 & 82 & 2.5562991786 & 63 \\
\hline o.83394 57809 & 2.6069922604 & $0.29728 \quad 34722$ & 39 & 2.5157230012 & 62 \\
\hline 0.8228699019 & 2.5752121966 & 0.3072552753 & 14 & $2.475146823^{8}$ & $6 I$ \\
\hline 0.8115370701 & 2.5427950725 & 0.3171367705 & 48 & $2.43457 \quad 06463$ & 60 \\
\hline 0.7999587840 & $2.509784428 \mathrm{I}$ & 0.3269204449 & 80 & 2.3939944689 & 59 \\
\hline 0.7881466036 & $2.47622 \quad 43648$ & 0.3365982039 & 53 & 2.3534 I 829 I 4 & $5^{8}$ \\
\hline 0.776 II 21247 & 2.4421594723 & $0.34616 \quad 13287$ & 24 & 2.3128421140 & 57 \\
\hline 0.7638669524 & 2.4076347564 & $0.35560 \quad 04313$ & 78 & 2.2722659366 & 56 \\
\hline $0.75142 \quad 26764$ & 2.372695567 I & $0.36490 \quad 54063$ & 78 & $2.231689759 I$ & 55 \\
\hline 0.7387908451 & 2.3373875276 & 0.3740653814 & 77 & 2.I9III $35^{8} \mathrm{I} 7$ & 54 \\
\hline 0.7259829409 & 2.3017564635 & 0.383068665 I & I 8 & 2.1505374042 & 53 \\
\hline 0.7130103561 & $2.265^{84} 83337$ & 0.3919026919 & 76 & 2.1099612268 & $5^{2}$ \\
\hline $0.69988 \quad 43682$ & 2.2297091619 & 0.4005539659 & 76 & 2.0693850494 & $5 \mathrm{I}$ \\
\hline 0.6866 I 61172 & 2.1933849695 & 0.4090080023 & 75 & 2.02880887 I9 & 50 \\
\hline $0.67321 \quad 65825$ & 2.15692 I 7102 & 0.4172492673 & 74 & I. 9882326945 & 49 \\
\hline 0.6596965607 & 2.12036 52053 & 0.42526 I I I65 & 74 & I. 9476565 I 7 I & 48 \\
\hline 0.6460666446 & 2.0837610820 & $0.43302 \quad 57335$ & 73 & I. 9070803396 & 47 \\
\hline 0.6323372022 & 2.047 I5 47 II7 & 0.4405240667 & 49 & I. 8665041622 & 46 \\
\hline 0.6 I 85 I 83573 & 2.01059 II5I7 & 0.4477357684 & 72 & I. $82592 \quad 79847$ & 45 \\
\hline$A(r)$ & $\mathbf{D}(\mathbf{r})$ & $\mathbf{E}(\mathbf{r})$ & $\phi$ & $\mathbf{F} \phi$ & $\mathbf{r}$ \\
\hline
\end{tabular}

SMITHSONIAN TABLES 
$\mathrm{K}=3.8317419998, \quad \mathrm{~K}^{\prime}=1.5737921309, \quad \mathrm{E}=1.0126635062, \quad \mathrm{E}^{\prime}=1.5678090740$,

\begin{tabular}{|c|c|c|c|c|c|c|c|c|c|c|}
\hline \multirow{2}{*}{$\frac{\mathbf{r}}{0}$} & \multicolumn{2}{|l|}{$\mathbf{F} \phi$} & \multicolumn{2}{|c|}{$\phi$} & \multicolumn{2}{|l|}{$\mathbf{E}(\mathbf{r})$} & \multicolumn{2}{|l|}{$\mathbf{D}(\mathbf{r})$} & \multicolumn{2}{|l|}{$\mathbf{A}(\mathbf{r})$} \\
\hline & 0.00000 & 00000 & & $0^{\prime}$ & 0.00000 & 00000 & I. 000000 & 00000 & 0.000000 & 00000 \\
\hline I & 0.04257 & 49 I I I & 2 & 26 & 0.031297 & $75^{84} \mathrm{I}$ & I. 000666 & 67396 & o.012569 & 98450 \\
\hline 2 & 0.08514 & 98222 & 4 & 52 & 0.062442 & 25476 & I.00266 6 & 63652 & 0.025144 & 45765 \\
\hline 3 & o. 12772 & 47333 & 7 & I 8 & 0.093284 & 44601 & I. 005997 & 70974 & 0.037729 & 90570 \\
\hline 4 & 0. I 7029 & 96444 & 9 & 43 & 0.123677 & 72052 & I.01065 5 & 59692 & 0.050328 & 81006 \\
\hline 5 & 0.21287 & 45555 & 12 & 6 & o. I $534^{8}$ & 09749 & 1. 016638 & 88247 & 0.062946 & 64495 \\
\hline 6 & 0.25544 & 94667 & I4 & 29 & o. 182564 & 40780 & I. 023940 & 03165 & 0.075588 & 87497 \\
\hline 7 & 0.29802 & 43778 & 16 & 50 & 0.210804 & $45 \mathrm{I} 54$ & I. 032553 & 39030 & 0.088259 & $9528 \mathrm{I}$ \\
\hline 8 & 0.34059 & 92889 & I9 & 9 & 0.23809 & I 2866 & I. 04247 I & 18453 & 0.100963 & 31685 \\
\hline 9 & 0.38317 & 42000 & 2 I & 26 & 0.264325 & 54039 & I.053685 & 52030 & o. II 3703 & 38895 \\
\hline Io & 0.42574 & 9I I I I & 23 & 42 & 0.28942 & 06026 & I.066I 83 & 38299 & o.12648 5 & $572 \mathrm{r}_{4}$ \\
\hline I I & 0.46832 & 40222 & 25 & 55 & 0.313303 & 37505 & I.07995 6 & 63700 & o.1393I 2 & 24846 \\
\hline I 2 & 0.51089 & 89333 & 28 & 5 & 0.33591 & 49667 & I .09499 o & 02519 & o. 152187 & 77682 \\
\hline I3 & 0.55347 & 38444 & 30 & I3 & 0.357207 & 74739 & I. 17127 & 16844 & 0.165 II 4 & 49087 \\
\hline I4 & 0.59604 & 87555 & 32 & I 8 & o 377147 & 72117 & I. I 28785 & $565 \mathrm{I} 3$ & o.17809 6 & 69700 \\
\hline I5 & 0.63862 & 36666 & 34 & $2 I$ & 0.395712 & 22464 & I. I $475 \mathrm{I} 5$ & 59063 & 0.191136 & 67239 \\
\hline 16 & 0.68119 & 85777 & 36 & 20 & 0.412892 & 20138 & I. I6744 & 49685 & 0.20423 & 663 I 5 \\
\hline 17 & 0.72377 & 34889 & 38 & I 7 & 0.428686 & 64336 & I. I 88554 & $4 I I 78$ & 0.217398 & 88246 \\
\hline I 8 & 0.76634 & 84000 & 40 & I I & 0.443104 & 49337 & I. 210823 & 33907 & 0.230625 & 50891 \\
\hline I9 & 0.80892 & 33 III & 42 & I & 0.456165 & 54173 & I. 23423 & 15771 & 0.24391 & 68485 \\
\hline 20 & 0.85149 & 82222 & 43 & 49 & 0.467893 & 32075 & I. 258756 & 62174 & 0.257275 & 51484 \\
\hline $2 \mathrm{I}$ & 0.89407 & 31333 & 45 & 33 & 0.47831 & $9995^{2}$ & I . 284373 & 36007 & 0.27070 & 06428 \\
\hline 22 & 0.93664 & 80444 & 47 & I 5 & 0.48748 & 28142 & I.3IIO58 & 87634 & 0.284193 & 35800 \\
\hline 23 & 0.97922 & 29555 & 48 & 53 & 0.495423 & 30625 & I. 338785 & 54900 & 0.297753 & 37910 \\
\hline 24 & I. 02179 & 78666 & 50 & 28 & 0.502185 & $55^{8} 4^{2}$ & I. 367526 & 63142 & 0.31138 & 06778 \\
\hline 25 & I. 06437 & 27777 & $5^{2}$ & 0 & $0.5078 \mathrm{I}$ & 78217 & I. 397252 & 25218 & 0.325073 & 32040 \\
\hline 26 & I. 10694 & 76888 & 53 & 29 & 0.512369 & 90454 & I. 427934 & 41552 & 0.33882 & 98857 \\
\hline 27 & I. I 4952 & 25999 & 54 & 56 & 0.515889 & 96635 & I. 45954 & 00195 & 0.352648 & 87839 \\
\hline 28 & I. I9209 & $75 \mathrm{IIO}$ & 56 & 19 & 0.51843 & 06138 & I . 492037 & 76904 & 0.366527 & 74982 \\
\hline 29 & I . 23467 & 24222 & 57 & 39 & 0.52004 & $2833^{8}$ & I .525393 & $35^{243}$ & 0.38046 & 31619 \\
\hline 30 & I. 27724 & 73333 & $5^{8}$ & 59 & 0.520776 & 68087 & I .55957 & 26706 & 0.39445 & \\
\hline $3 I$ & I. 3 I982 & 22444 & 60 & 12 & 0.52068 & 21896 & I. 59453 & 90851 & 0.40849 & 15164 \\
\hline 32 & I.36239 & 71555 & $6 I$ & 24 & 0.51980 & 74799 & I. 630255 & 554 & 0.42257 & 61140 \\
\hline 33 & I. 40497 & 20666 & 62 & 34 & 0.51819 & 978 I I & I. 66668 & $368 \mathrm{I} 4$ & 0.43670 & I 4735 \\
\hline 34 & I. 44754 & 69777 & 63 & $4 I$ & 0.51590 & 45944 & I . 70378 & 39728 & 0.45086 & $2365^{8}$ \\
\hline 35 & I. 49012 & I 8888 & 64 & 46 & 0.51296 & 56697 & I.74I5I 5 & 57980 & 0.46505 & 30926 \\
\hline 36 & I . 53269 & 67999 & 65 & 48 & 0.50942 & 48984 & I. 77983 & 74487 & 0.47926 & 74909 \\
\hline 37 & I. 57527 & I 7110 & 66 & 48 & 0.50532 & 2242 I & I. 81870 & 61627 & 0.49349 & 89386 \\
\hline 38 & I. 61784 & 6622 I & 67 & 46 & 0.50069 & 56936 & I. 858078 & 81564 & 0.50774 & 03615 \\
\hline 39 & I . 66042 & I 5332 & 68 & $4 I$ & $0.4955^{8}$ & 12646 & I . 897908 & 86607 & 0.52198 & 42419 \\
\hline 40 & I.70299 & 64444 & 69 & 35 & 0.49001 & 29952 & I. 938 I 5 & I9599 & 0.53622 & \\
\hline $4 I$ & I.74557 & I 3555 & 70 & 26 & 0.48402 & 29824 & I. 97876 & I 433 I & 0.55044 & 71457 \\
\hline 42 & I. 78814 & 62666 & 71 & I6 & 0.47764 & I 4227 & 2.01968 & 95998 & 0.56464 & 90099 \\
\hline 43 & I. 83072 & I 1777 & 72 & 3 & 0.47089 & 66670 & 2.060888 & 81669 & $0.5788 \mathrm{I}$ & 90394 \\
\hline 44 & I. 87329 & 60888 & 72 & 49 & $0.4638 \mathrm{I}$ & 52836 & 2.10230 & 80805 & 0.59294 & 76712 \\
\hline 45 & I.9I 587 & 09999 & 73 & 33 & 0.45642 & 21286 & 2.14389 & 95792 & 0.60702 & 49768 \\
\hline $30-r$ & $F \psi$ & & & $\psi$ & $\mathrm{G}(\mathbf{r})$ & & $\mathbf{C}(\mathbf{r})$ & & & \\
\hline
\end{tabular}


TABLE $\theta=85^{\circ}$

$q=0.275179804873563, \quad \theta 0=0.4610905222, \quad \mathrm{HK}=1.5588714533$

\begin{tabular}{|c|c|c|c|c|c|}
\hline $\mathbf{B}(\mathbf{r})$ & $\mathbf{C}(\mathbf{r})$ & $\mathbf{G}(\mathbf{r})$ & $\psi$ & $\mathrm{F} \psi$ & $90-r$ \\
\hline I. .00000 00000 & 3.3872870037 & 0.0000000000 & $90^{\circ}$ & $3.83174 \quad 19998$ & 90 \\
\hline б.99976 0504I & $3 \cdot 3864990904$ & 0.0109282185 & $89 \quad 47$ & 3.7891670887 & 89 \\
\hline 0.9990423353 & $3.3^{84} 1365337$ & 0.0218552713 & $89 \quad 34$ & 3.7465921776 & 88 \\
\hline 0.9978464504 & $3 \cdot 38020$ 288I 5 & o.0327799847 & 89 & $3.70401 \quad 72665$ & 87 \\
\hline 0.9961744409 & $3.37470 \quad 40379$ & 0.04370 II 679 & 89 & $3.66 \mathrm{I} 4423554$ & 86 \\
\hline 0.9940285290 & 3.3676482512 & 0.0546176051 & 88 & $3.6 \mathrm{I} 88674443$ & 85 \\
\hline $0.9914 I$ I 5622 & $3.359046096 \mathrm{I}$ & $0.0655^{2} 80467$ & 43 & $3.576292533 \mathrm{I}$ & 84 \\
\hline $0.988327005^{8}$ & 3.3489104507 & 0.07643 I2000 & 29 & $3.5337 \mathrm{I} 76220$ & 83 \\
\hline 0.9847789335 & 3.3372564694 & 0.0873257205 & 16 & 3.4911427109 & 82 \\
\hline 0.9807720177 & $3.32410 \quad 15504$ & 0.0982102023 & 88 & 3.4485677998 & $8 \mathbf{I}$ \\
\hline $0.9763 \mathrm{I}$ I5168 & 3.3094652989 & o. 1090831677 & 87 & 3.4059928887 & 80 \\
\hline $0.97140 \quad 32619$ & 3.2933694854 & o. II994 30573 & 35 & 3.3634 I 79776 & 79 \\
\hline 0.9660536420 & 3.2758379999 & o.13078 82183 & 20 & $3 \cdot 3208430665$ & 78 \\
\hline $0.9602695^{874}$ & 3.2568968018 & o.I4I6I 68937 & 87 & 3.278268 I 554 & 77 \\
\hline 0.9540585520 & $3.23657 \quad 38654$ & o.I5242 72092 & $5 I$ & 3.2356932443 & 76 \\
\hline 0.9474284947 & 3.2148991220 & 0.1632171605 & 86 & 3.1931183332 & 75 \\
\hline 0.9403878585 & $3.19190 \quad 43978$ & o. I7398 45990 & 20 & 3. I 50543422 I & 74 \\
\hline 0.9329455499 & 3.1676233486 & o. I 847272171 & 86 & 3.1079685109 & 73 \\
\hline 0.925 II 09158 & 3.1420913909 & o. 195442532 I & 48 & 3.0653935998 & 72 \\
\hline 0.9168937204 & 3.II534 56304 & 0.2061278689 & $3 I$ & $3.02281 \quad 86887$ & 71 \\
\hline $0.90830 \quad 41205$ & $3.08742 \quad 47870$ & 0.2167803419 & 85 & 2.9802437776 & 70 \\
\hline 0.8993526403 & $3.05^{8} 36$ 9II77 & 0.2273968349 & 55 & 2.9376688665 & 69 \\
\hline 0.89005 or 452 & $\begin{array}{lll}3.02822 & 03368\end{array}$ & o.23797 39802 & 37 & 2.8950939554 & 68 \\
\hline 0.8804078152 & $2.99702 \quad 15345$ & 0.248508 I 357 & I 8 & $2.85251 \quad 90443$ & 67 \\
\hline 0.8704371170 & $2.96481 \quad 70925$ & 0.2589953603 & $5^{8}$ & 2.8099441332 & 66 \\
\hline 0.8601497763 & 2.9316525995 & 0.26943 I3876 & 38 & 2.7673692221 & 65 \\
\hline $0.849557749 \mathrm{I}$ & 2.8975747641 & $0.27981 \quad$ I 5977 & 17 & 2.7247943 IIO & 64 \\
\hline 0.8386731932 & 2.8626313272 & 0.2901309871 & 55 & 2.6822193999 & 63 \\
\hline $0.8275^{\circ} 843^{8} 3$ & 2.8268709732 & 0.3003841353 & 33 & 2.6396444888 & 62 \\
\hline 0.8160759576 & 2.7903432412 & 0.3105651708 & 10 & 2.5970695776 & $6 I$ \\
\hline $0.8043^{8} 83372$ & $2.753098435 \mathrm{I}$ & 0.3206677330 & 46 & 2.5544946665 & 60 \\
\hline 0.7924582474 & 2.7151875345 & 0.3306849323 & 21 & 2.5 II9I 97554 & 59 \\
\hline 0.7802984129 & 2.6766621047 & 0.3406093073 & 55 & 2.4693448443 & $5^{8}$ \\
\hline 0.76792 I 5834 & $2.637574208 \mathrm{I}$ & 0.3504327789 & 28 & 2.4267699332 & $\cdot 57$ \\
\hline 0.7553405043 & 2.5979763158 & 0.3601466018 & 80 & 2.3841950221 & 56 \\
\hline o. 7425678883 & $2.55792 \quad 12198$ & 0.36974 I3I24 & 79 & 2.34162 oIIIO & 55 \\
\hline 0.7296163864 & 2.51746 I947 I & 0.3792066740 & 79 & 2.2990451999 & 54 \\
\hline 0.7164985603 & 2.47665 I6742 & 0.38853 I6I 85 & 78 & $2.25647 \quad 02888$ & 53 \\
\hline 0.7032268545 & $2.435543643^{8}$ & $0.39770 \quad 41848$ & $5^{8}$ & 2.2138953777 & $5^{2}$ \\
\hline $0.68981 \quad 35699$ & $2.39419 \quad 10827$ & 0.40671 I 4546 & 77 & 2.1713204666 & $5^{I}$ \\
\hline 0.6762708370 & 2.3526471220 & 0.4155394843 & 76 & 2.12874 55554 & $5^{\circ}$ \\
\hline 0.6626105910 & 2.3109647190 & 0.4241732345 & 76 & 2.0861706443 & 49 \\
\hline 0.6488445467 & 2.2691965819 & 0.4325964967 & 75 & 2.0435957332 & 48 \\
\hline $0.6349^{8} 41750$ & 2.2273950955 & 0.44079 I 8172 & 74 & $2.001020822 \mathrm{I}$ & 47 \\
\hline 0.6210406800 & 2. 1856122515 & $0.44874 \quad 04204$ & $74 \quad 16$ & I.95844 59110 & 46 \\
\hline 0.6070249768 & 2.1438995792 & $0.45642 \quad 21286$ & $73 \quad 33$ & I.9I58709999 & 45 \\
\hline $\mathbf{A}(\mathbf{r})$ & $\mathbf{D}(\mathbf{r})$ & $\mathbf{E}(\mathbf{r})$ & $\phi$ & $\mathrm{F} \phi$ & $\mathbf{r}$ \\
\hline
\end{tabular}


$\mathrm{K}=4.0527581695, \quad \mathrm{~K}^{\prime}=1.5727124350, \quad \mathrm{E}=1.0086479569, \quad \mathrm{E}^{\prime}=1.5688837196$,

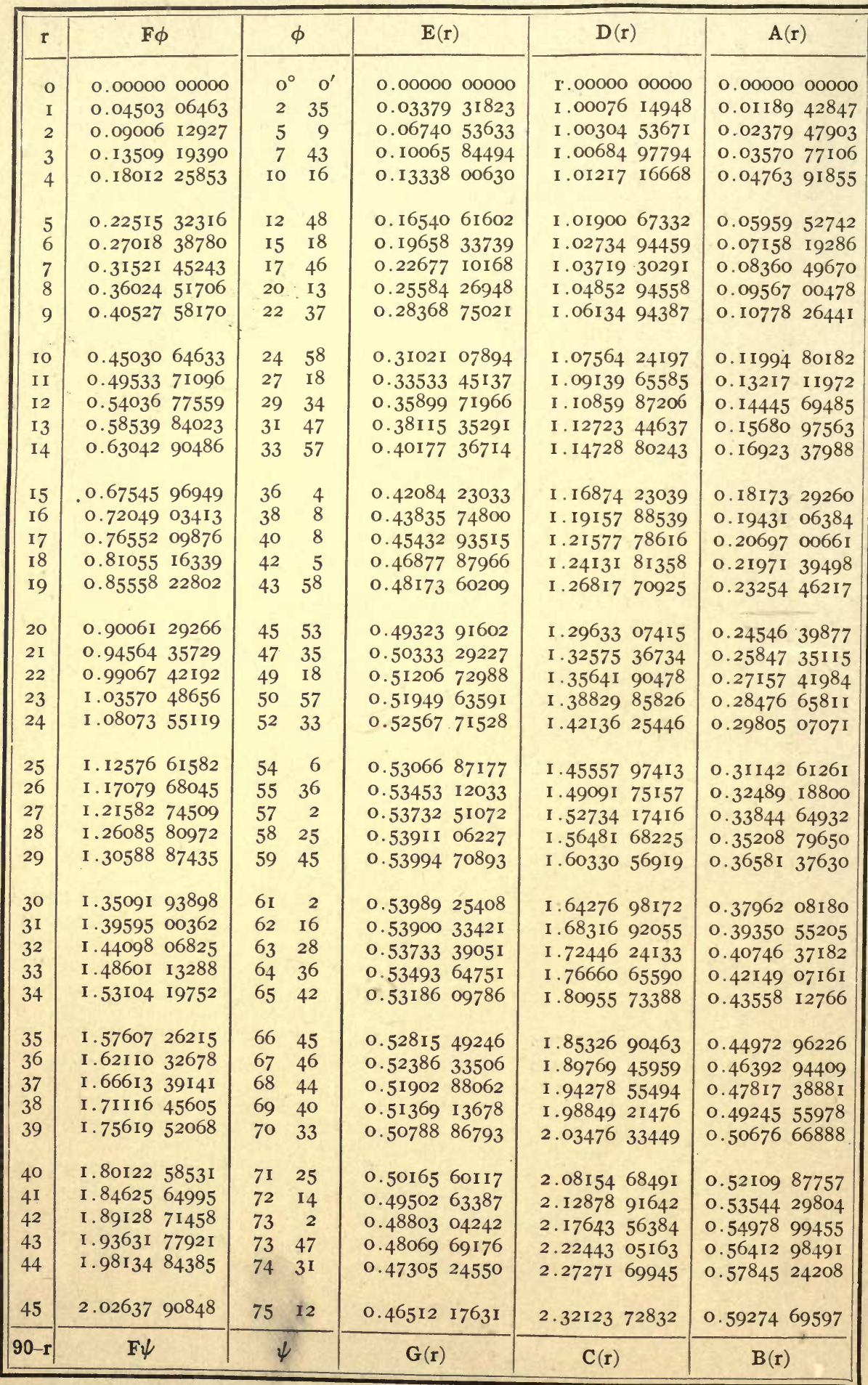


TABLE $\theta=86^{\circ}$

$q=0.295488385558687, \quad \theta 0=0.4242361430, \quad \mathrm{HK}=1.6043008048$

\begin{tabular}{|c|c|c|c|c|c|}
\hline $\mathbf{B}(\mathbf{r})$ & $\mathbf{C}(\mathbf{r})$ & $\mathbf{G}(\mathbf{r})$ & $\psi$ & $\mathbf{F} \psi$ & $90-r$ \\
\hline I.CO000 00000 & 3.7862365254 & 0.0000000000 & $90^{\circ} \quad 0^{\prime}$ & 4.0527581695 & 90 \\
\hline 0.9997476964 & 3.7852999318 & o.01098 79345 & $89 \quad 49$ & 4.0077275232 & 89 \\
\hline 0.99899 I I 477 & $3.78249 \quad 16163$ & 0.0219749829 & 38 & 3.9626968769 & 88 \\
\hline $0.99773 \quad 14382$ & $3.7778 \mathrm{I} \quad 597 \mathrm{I} 4$ & 0.0329602520 & 28 & 3.9176662306 & 87 \\
\hline 0.99597 o3726 & $3.77128 \quad 03065$ & 0.0439428343 & 89 & $3.8726355^{842}$ & 86 \\
\hline 0.9937104703 & 3.7628948312 & 0.05492 1 8007 & 89 & $3.82760 \quad 49379$ & 85 \\
\hline $0.99095495^{88}$ & 3.7526726317 & 0.0658961931 & 88 & 3.7825742916 & 84 \\
\hline 0.9877077652 & 3.7406296405 & 0.0768650165 & 88 & $3.737543645^{2}$ & 83 \\
\hline $0.9839735^{058}$ & $3 \cdot 7267846000$ & $0.08782^{\circ} 72314$ & 88 & $3.6925 \mathrm{I} \quad 29989$ & 82 \\
\hline 0.9797574732 & 3.7III5 90I9I & $0.09878 \quad 1745^{2}$ & 88 & $3.64748 \quad 23526$ & $8 I$ \\
\hline 0.9750656227 & $3.69377 \quad 71248$ & o.10972 74034 & 88 & $3.60245 \quad 17063$ & 80 \\
\hline $0.969904555^{8}$ & $3.674665806 \mathrm{r}$ & o. 1206629807 & 56 & $3.55742 \quad 10599$ & 79 \\
\hline 0.96428 I5032 & 3.6538545535 & o.13158 71709 & 44 & $3 \cdot 5$ I239 04I36 & 78 \\
\hline $0.95^{820} 43054$ & 3.6313753926 & o. 1424985767 & 32 & 3.4673597673 & 77 \\
\hline 0.95 I 68 I39I4 & $3.60726 \quad 28 \mathrm{II} 4$ & o. I5339 56986 & 19 & 3.4223291209 & 76 \\
\hline o. 94472 I 7573 & $3 \cdot 5^{8} \mathrm{I} 5536840$ & o.16427 69227 & 87 & 3.3772984746 & 75 \\
\hline 0.9373349419 & 3.554287 I 880 & o. I75I4 05085 & 86 & 3.3322678283 & 74 \\
\hline 0.92953 IOOI7 & $3 \cdot 5^{2} 55^{\circ} \quad 47 \mathrm{r} 84$ & o. I 859845746 & 86 & 3.2872371820 & 73 \\
\hline $0.9^{2} \mathrm{I}^{2} \quad 04850$ & 3.4952497967 & 0.1968070842 & 86 & 3.2422065356 & 72 \\
\hline 0.9127144039 & 3.4635679762 & $0.20760 \quad 58292$ & 86 & 3.197I 758893 & 71 \\
\hline 0.9037242062 & $3.43050 \quad 67437$ & 0.2183784126 & 85 & 3.1521452430 & 70 \\
\hline 0.89436 I 7453 & 3.396 I I 54 I 78 & 0.2291222300 & 38 & 3. I07II 45967 & 69 \\
\hline 0.8846392502 & $3 \cdot 3604450445$ & 0.2398344495 & 22 & 3.0620839503 & 68 \\
\hline 0.8745692937 & 3.3235482896 & $0.25051 \quad 19896$ & 85 & 3.0170533040 & 67 \\
\hline 0.86416476 Io & 3.2854793300 & 0.26 II5 I 4957 & 48 & $2.97202 \quad 26577$ & 66 \\
\hline 0.8534388 I67 & $3.24629 \quad 37417$ & 0.2717493142 & 84 & 2.9269920113 & 65 \\
\hline 0.8424048716 & 3.2060483874 & $0.28230 \quad 14649$ & II & 2.8819613650 & 64 \\
\hline 0.83107 .65499 & $3.16480 \quad 13024$ & 0.2928036106 & $5^{2}$ & 2.8369307187 & 63 \\
\hline 0.8194676545 & 3.1226 I I 5798 & $0.30325 \quad 10250$ & 83 & 2.7919000724 & 62 \\
\hline 0.8075921336 & $3.079539255 \mathrm{I}$ & 0.3136385568 & II & 2.7468694260 & $6 I$ \\
\hline o. 7954640466 & 3.0356451912 & 0.3239605923 & 82 & 2.7018387797 & 60 \\
\hline 0.7830975297 & 2.9909909630 & $0.33421 \quad$ IOI35 & 26 & $2.656808 \mathrm{I} 334$ & 59 \\
\hline $0.77050 \quad 67624$ & $2.945^{6} 38743^{2}$ & $0.34438 \quad 31544$ & 82 & 2.6 II 7774870 & $5^{8}$ \\
\hline o. $75770 \quad 59335$ & 2.89965 I I 884 & 0.3544697527 & $8 \mathrm{I}$ & $2.56674 \quad 68407$ & 57 \\
\hline 0.7447092077 & 2.85309 I3269 & $0.36446 \quad 28984$ & I3 & 2.5217161944 & $5^{6}$ \\
\hline 0.7315306927 & $2.80602 \quad 24483$ & 0.3743539786 & 80 & 2.4766855480 & 55 \\
\hline 0.7 I8I8 44065 & $2.75^{8} 5^{\circ} 79940$ & 0.3841336176 & 80 & 2.4316549017 & 54 \\
\hline $0.70468 \quad 42455$ & $2.71061 \quad 14508$ & 0.39379 I6I 42 & 79 & $2.38662 \quad 42554$ & 53 \\
\hline 0.6910439537 & 2.6623962465 & 0.4033168729 & 20 & 2.3415936091 & $5^{2}$ \\
\hline 0.6772770914 & $2.613925648 \mathrm{I}$ & 0.4126973321 & 49 & 2.2965629627 & 51 \\
\hline $0.663397006 \mathrm{I}$ & 2.5652626633 & 0.4219198869 & 78 & $2.25 \mathrm{I} 5323 \mathrm{I} 64$ & 50 \\
\hline $0.6494 \mathrm{I} \quad 68038$ & 2.5164699446 & 0.4309703076 & 77 & 2.20650 I670I & 49 \\
\hline 0.6353493209 & $2.467609697 \mathrm{I}$ & 0.4398331542 & 77 & 2. I6I $47 \quad 10238$ & 48 \\
\hline 0.6212070978 & $2.4187435^{896}$ & $0.44849 \quad 16855$ & 76 & 2. I I644 03774 & 47 \\
\hline 0.6070023531 & 2.3699326700 & $0.456927765 \mathrm{I}$ & $75 \quad 5^{2}$ & $2.07 \mathrm{I} 40973 \mathrm{II}$ & 46 \\
\hline 0.5927469597 & 2.3212372832 & $0.465 \mathrm{I} 2 \quad \mathrm{I} 763 \mathrm{I}$ & $75 \quad 12$ & 2.0263790848 & 45 \\
\hline $\mathbf{A}(\mathbf{r})$ & $\mathrm{D}(\mathbf{r})$ & $\mathbf{E}(\mathbf{r})$ & $\phi$ & $\mathbf{F} \phi$ & $\mathbf{r}$ \\
\hline
\end{tabular}

SMITHSONIAN TABLES 
$\mathrm{K}=4.3386539760, \quad \mathrm{~K}^{\prime}=1.5718736105, \quad \mathrm{E}=1.0052585872, \quad \mathrm{E}^{\prime}=1.5697201504$,

\begin{tabular}{|c|c|c|c|c|c|c|c|}
\hline $\mathbf{r}$ & $\mathbf{F} \phi$ & & $\phi$ & $\mathbf{E}(\mathbf{r})$ & $\mathbf{D}(\mathbf{r})$ & $\mathbf{A}(\mathbf{r})$ & \\
\hline 0 & 0.0000000000 & $0^{\circ}$ & $\mathrm{o}^{\prime}$ & 0.0000000000 & I.00000 00000 & 0.00000 & 00000 \\
\hline I & 0.0482072664 & 2 & 46 & $0.03700 \quad 05198$ & I. 0008926934 & 0.01102 & $97 \times 5^{8}$ \\
\hline 2 & $0.0964 \mathrm{I} \quad 45328$ & 5 & $3 I$ & 0.0737786246 & I.00357 01695 & 0.02206 & 73089 \\
\hline 3 & 0.14462 I 7992 & 8 & 15 & O. I IOI I 59944 & I . 0080306 I 4 I & 0.03312 & 06260 \\
\hline 4 & o.1928290656 & 10 & 59 & o. 1458023384 & I. 0142709982 & 0.04419 & $7454 \mathrm{I}$ \\
\hline 5 & 0.2410363320 & I3 & $4 I$ & o.1806390239 & I.02228 70707 & 0.05530 & 54893 \\
\hline 6 & 0.2892435984 & I6 & $2 \mathrm{I}$ & 0.2144422668 & I.032073347 I & 0.06645 & $2308 \mathrm{I}$ \\
\hline 7 & 0.3374508648 & 18 & 59 & 0.2470457854 & I. 0436230963 & 0.07764 & 53371 \\
\hline 8 & 0.3856581312 & $2 \mathrm{I}$ & 34 & 0.2783028485 & I. 0569283239 & 0.08889 & I 8239 \\
\hline 9 & 0.4338653976 & 24 & 7 & 0.3080876822 & I.07I97 9753 I & 0.10019 & 88085 \\
\hline I0 & 0.4820726640 & 26 & 37 & 0.3362962369 & I. 0887668032 & o. I I I 57 & 30946 \\
\hline I I & o.5302799304 & 29 & 3 & 0.3628463422 & I. $107277565^{2}$ & 0.12302 & 12218 \\
\hline 12 & 0.5784871968 & $3 I$ & 27 & 0.3876773064 & I. I274987762 & o. 13454 & 94383 \\
\hline I3 & 0.6266944632 & 33 & 46 & 0.4107490335 & I. I 494I 57909 & 0.14616 & 36738 \\
\hline I4 & 0.67490 I 7296 & 36 & 2 & 0.4320407437 & I. I7301 25520 & o. I 5786 & 95 I39 \\
\hline 15 & 0.7231089960 & 38 & I 4 & 0.45 I54 93887 & I. I9827 I559I & o. 16967 & 21746 \\
\hline 16 & 0.7713162624 & 40 & 23 & $0.46928 \quad 78534$ & I.22517 38362 & o. I 8 I 57 & 64776 \\
\hline I7 & $0.8195^{2} 35^{288}$ & 42 & 27 & $0.48528 \quad 30289$ & I. 2536988987 & o. $1935^{8}$ & 68272 \\
\hline 18 & 0.8677307952 & 44 & 28 & 0.4995738349 & I. $28382 \quad 47$ I 93 & 0.20570 & 71870 \\
\hline 19 & 0.91593806 I6 & 46 & 24 & 0.5122092565 & I.31552 76945 & 0.21794 & 10587 \\
\hline 20 & 0.9641453280 & 48 & 16 & 0.5232464512 & I. 3487826100 & 0.23029 & 14612 \\
\hline $2 \mathrm{I}$ & I.OI235 25944 & 50 & 5 & 0.5327489656 & I. 3835626077 & 0.24276 & O9 I I I \\
\hline 22 & I.06055 98608 & $5^{I}$ & 50 & 0.5407850933 & I. 4198391529 & 0.25535 & I 4044 \\
\hline 23 & I. 1087671272 & 53 & 30 & 0.5474263924 & I. $4575^{8} 2002 \mathrm{I}$ & 0.26806 & 43994 \\
\hline 24 & I. I5697 43936 & 55 & 7 & 0.5527463730 & I.4967591734 & 0.28090 & 08008 \\
\hline 25 & I. $20518 \quad 16600$ & 56 & 40 & 0.5568 I 93566 & I.53733 69I75 & 0.29386 & $0945^{2}$ \\
\hline 26 & I. 2533889264 & 58 & Io & 0.5597195044 & I.5792796919 & 0.30694 & $45^{879}$ \\
\hline 27 & I.30I59 6I928 & 59 & 36 & $0.5^{61} 5^{2} 00057$ & I. 6225501370 & 2015 & 08913 \\
\hline 28 & I. $34980 \quad 34592$ & 60 & $5^{8}$ & 0.5622924153 & I.667I0 $9055^{1}$ & 0.33347 & 84147 \\
\hline 29 & I. 3980107256 & 62 & I7 & 0.5621061265 & I.7129I 53925 & 0.34692 & 51057 \\
\hline 30 & I.4462I 79920 & 63 & 33 & $0.561027965^{8}$ & I. 7599262260 & 0.36048 & 82928 \\
\hline $3 I$ & I. $4944^{2} \quad 5^{2} 5^{84}$ & 64 & 46 & $0.55912 \quad 18929$ & I. 8080967519 & 0.37416 & 46804 \\
\hline 32 & I. 5426325248 & 65 & 55 & 0.5564487947 & I .8573802804 & 0.38795 & 03444 \\
\hline 33 & I. 5908397912 & 67 & 2 & $0.553066356 \mathrm{I}$ & I. 9077282336 & 0.40184 & 07305 \\
\hline 34 & I. 6390470676 & 68 & 6 & o. 5490289975 & I. 9590901488 & 0.41583 & 06538 \\
\hline 35 & I. 6872543240 & 69 & 7 & $0.5443^{8} 7866$ I & $2.0114 I \quad 36867$ & $0.4299 \mathrm{I}$ & \\
\hline 36 & I. 73546 I 5904 & 70 & 5 & 0.5391908711 & 2.0646446451 & 0.44408 & 52267 \\
\hline 37 & I. 7836688568 & 71 & I & $0.5334^{8} \quad 27539$ & 2. II872 69773 & $0.45^{833}$ & 63730 \\
\hline $3^{8}$ & I. 8318761232 & 71 & 54 & 0.5273051847 & 2. $17360 \quad 28173$ & 0.47266 & 00609 \\
\hline 39 & I. 8800833896 & 72 & 45 & 0.5206968791 & $2.22921 \quad 25107$ & 0.48704 & 80065 \\
\hline 40 & I. 9282906560 & 73 & 34 & 0.5136937297 & 2.2854946508 & 0.50149 & \\
\hline $4 \mathrm{I}$ & I.97649 79224 & 74 & 20 & 0.5063289466 & 2.3423861220 & 0.51598 & 05665 \\
\hline 42 & $2.02470 \quad 51888$ & 75 & 5 & $0.49863 \quad 32034$ & $2.39982 \quad 21493$ & 0.53050 & 56822 \\
\hline 43 & $2.07291 \quad 24552$ & 75 & 47 & 0.4906347860 & 2.4577363538 & $0: 54505$ & 60878 \\
\hline 44 & 2.121II 972 I6 & 76 & $5^{8}$ & 0.48235974 II & 2.5160608149 & 0.55962 & 06569 \\
\hline 45 & 2.16932 69880 & 77 & 7 & 0.4738320219 & 2.574726 I 393 & 0.57418 & $7745 \mathrm{I}$ \\
\hline $90-r$ & $\mathrm{~F} \psi$ & \multicolumn{2}{|c|}{$\psi$} & $\mathbf{G}(\mathbf{r})$ & $C(r)$ & \multicolumn{2}{|l|}{$\mathbf{B}(\mathbf{r})$} \\
\hline
\end{tabular}

SMITHSONIAN TABLES 
TABLE, $\theta=87^{\circ}$

$q=0.320400337134867, \quad \theta 0=0.3802048484, \quad$ HK $=1.6608093153$

\begin{tabular}{|c|c|c|c|c|c|}
\hline $\mathrm{B}(\mathrm{r})$ & $C(r)$ & $\mathbf{G}(\mathbf{r})$ & $\psi$ & F $\psi$ & $90-r$ \\
\hline I. . & 4.37 II9 23556 & 0.0000000000 & $90^{\circ} \quad 0^{\prime}$ & $4 \cdot 3386539760$ & 90 \\
\hline 0.9997308085 & $4.3700295^{871}$ & 0.0110373956 & $89 \quad 5 I$ & $4 \cdot 2904467096$ & 89 \\
\hline 0.9989236540 & $4 \cdot 3665432014$ & 0.0220741777 & 43 & 4.2422394432 & 88 \\
\hline 0.9975797949 & 4.3607389539 & 0.0331097273 & 89 & 4.1940321768 & 87 \\
\hline $0.99570 \quad 13248$ & $4 \cdot 35^{262} 64203$ & 0.0441434137 & 25 & $4.14582 \quad 49104$ & 86 \\
\hline 0.99329 II666 & $4.342218973 I$ & $0.055^{17} 45^{893}$ & I6 & 4.0976 I 76440 & 85 \\
\hline 0.9903530638 & $4 \cdot 32953 \quad 3747$ I & 0.0662025830 & 89 & 4.0494103776 & 84 \\
\hline 0.98689 I 5704 & $4 \cdot 31459$ I5972 & 0.0772266944 & 88 & 4.0012031112 & 83 \\
\hline 0.9829120378 & $4.29741 \quad 70454$ & 0.0882461873 & 88 & 3.9529958448 & 82 \\
\hline 0.9784205999 & 4.2780382196 & 0.0992602826 & 39 & 3.9047885784 & 81 \\
\hline 0.9734241557 & $4.25648 \quad 67836$ & O.IIO26 8I515 & 88 & $3.85658 \quad 13120$ & 80 \\
\hline 0.9679303503 & 4.2327978580 & o.1212689076 & 88 & 3.8083740456 & 79 \\
\hline 0.9619475529 & 4.2070099336 & o. I3226 I5989 & 88 & 3.7601667792 & 78 \\
\hline $0.95548 \quad 48341$ & 4.179 I6 47765 & 0.1432451989 & 88 & $3.71195 \quad 95128$ & 77 \\
\hline 0.9485519406 & 4. I4930 73254 & o.I542I 85972 & 49 & $3.66375 \quad 22464$ & 76 \\
\hline 0.9411592676 & $4.1174^{8} 55^{826}$ & o. 1651805896 & $3^{8}$ & 3.6155449800 & 75 \\
\hline 0.9333178308 & 4.083750497 I & o.17612 98666 & 87 & $3 \cdot 5673377136$ & 74 \\
\hline 0.9250392359 & 4.0481558427 & o. I8706 50017 & 87 & 3.5191304472 & 73 \\
\hline 0.9163356463 & $4.010758089 \mathrm{I}$ & o. 1979844386 & 87 & 3.4709231808 & 72 \\
\hline 0.9072197509 & 3.9716162682 & 0.2088864763 & $5 \mathrm{I}$ & $3.42271 \quad 59144$ & 71 \\
\hline o. $89770 \quad 47288$ & $3.93079 \quad 18356$ & 0.2197692546 & 86 & $3.3745^{0} 86480$ & 70 \\
\hline $0.88780 \quad 42140$ & 3.8883485274 & 0.2306307363 & 86 & 3.3263013816 & 69 \\
\hline 0.8775322590 & 3.8443522135 & 0.2414686896 & 86 & 3.278094 II 52 & 68 \\
\hline 0.866903297 I & $3.79887 \quad 07472$ & 0.2522806673 & 85 & $3.22988 \quad 68488$ & 67 \\
\hline 0.8559321039 & $3.75197 \quad 38123$ & 0.2630639853 & 85 & $3.1816795^{824}$ & 66 \\
\hline $0.84463375^{89}$ & 3.7037327678 & $0.27381 \quad 56982$ & 85 & 3.1334723160 & 65 \\
\hline 0.8330236055 & 3.6542204910 & 0.2845325731 & 85 & 3.0852650496 & 64 \\
\hline 0.8211172113 & $3.6035 \mathrm{I} \quad \mathrm{I} 2 \mathrm{I} 93$ & 0.2952110610 & 54 & 3.0370577832 & 63 \\
\hline $0.808930328 I$ & 3.55168 03915 & 0.3058472655 & 84 & 2.9888505168 & 62 \\
\hline 0.79647885 I6 & $3.49880 \quad 4489$ I & 0.316436908 I & 84 & 2.9406432504 & $6 \mathrm{I}$ \\
\hline 0.7837787810 & 3.4449608773 & 0.3269752911 & 84 & 2.8924359840 & 60 \\
\hline 0.7708461787 & $3 \cdot 390227648 I$ & 0.3374572566 & 40 & 2.8442287176 & 59 \\
\hline 0.7576971307 & 3.3346834641 & 0.3478771421 & 19 & $2.79602 \quad 14512$ & $5^{8}$ \\
\hline 0.7443477069 & 3.2784074042 & $0.35^{822} 87319$ & 57 & $2.74781 \quad 4 I 848$ & 57 \\
\hline 0.7308139218 & 3.2214788 II 8 & $0.36850 \quad 52042$ & 35 & 2.6996069184 & 56 \\
\hline 0.717 II 16962 & 3.1639771463 & 0.3786990740 & 82 & 2.6513996520 & 55 \\
\hline 0.7032568193 & $3.10598 \quad 18371$ & $0.38880 \quad 21304$ & $8 I$ & 2.6031923856 & 54 \\
\hline 0.6892649116 & 3.0475721420 & $0.39880 \quad 53693$ & $8 I$ & 2.5549851192 & 53 \\
\hline $0.67515 \quad$ I3887 & 2.9888270090 & 0.4086989202 & 80 & 2.5067778528 & $5^{2}$ \\
\hline $0.66093 \quad 14267$ & 2.9298249435 & $0.4 \mathrm{I} 847 \quad 19672$ & 80 & $2.45^{857} \quad 05^{864}$ & $5^{I}$ \\
\hline 0.6466 I 99275 & 2.8706438790 & 0.428 I I 26638 & 79 & 2.4103633200 & 50 \\
\hline $0.63223 \quad 14865$ & $2.81136 \quad 10542$ & 0.4376080415 & 79 & 2.3621560536 & 49 \\
\hline 0.6177803606 & $2.75^{205} 28945$ & 0.4469439 I I I & 78 & 2.3139487872 & 48 \\
\hline 0.6032804384 & 2.6927948995 & $0.45^{610} 475^{83}$ & 78 & $2.26574 \quad 15208$ & 47 \\
\hline 0.5887452110 & $2.63366 \quad 15364$ & 0.4650736311 & 77 & $2.21753 \quad 42544$ & 46 \\
\hline 0.5741877451 & 2.5747261393 & 0.4738320219 & 77 & 2.16932 69880 & 45 \\
\hline $\mathbf{A}(\mathbf{r})$ & $\mathbf{D}(\mathbf{r})$ & $\mathbf{E}(\mathbf{r})$ & $\phi$ & $\mathbf{F} \phi$ & r \\
\hline
\end{tabular}


$\mathrm{K}=4.7427172653, \quad \mathrm{~K}^{\prime}=1.5712749524, \quad \mathrm{E}=1.0025840855, \quad \mathrm{E}^{\prime}=1.5703179199$,

\begin{tabular}{|c|c|c|c|c|c|c|}
\hline $\mathbf{r}$ & $\mathbf{F} \phi$ & $\phi$ & $p$ & $\mathbf{E}(\mathbf{r})$ & $\mathbf{D}(\mathbf{r})$ & $A(r)$ \\
\hline o & 0.0000000000 & & $\mathbf{o}^{\prime}$ & 0.0000000000 & I.00000 00000 & 0.0000000000 \\
\hline I & $0.05^{269} 685^{85}$ & 3 & I & o.04I50 83698 & I.00109 49202 & 0.0098461866 \\
\hline 2 & o. 1053937 I 70 & 6 & 2 & 0.0827260369 & I.0043791719 & o.01970 23988 \\
\hline 3 & o. I5809 05755 & 9 & I & o.12336 86879 & I. $.00985 \quad 12249$ & 0.0295786287 \\
\hline 4 & 0.2107874340 & I I & 59 & o.16316 44916 & I.OI750 85180 & $0.03948,48012$ \\
\hline 5 & 0.2634842925 & 14 & 56 & 0.2018596235 & I.02734 74434 & 0.0494307415 \\
\hline 6 & 0.3161811510 & I 7 & 49 & $0.23922 \quad 29917$ & I. 0393633238 & 0.0594261408 \\
\hline 7 & 0.3688780095 & 20 & 40 & 0.2750499964 & I. 0535503843 & 0.0694805245 \\
\hline 8 & 0.4215748680 & 23 & 28 & 0.3091652198 & I .06990 I7180 & 0.0796032187 \\
\hline 9 & $0.47427 \quad 17265$ & 26 & 13 & 0.3414240166 & I. $.088409245^{8}$ & $0.08980 \quad 33181$ \\
\hline IO & $0.5^{269685^{850}}$ & 28 & 53 & 0.3717130376 & I. 1090636709 & o. 1000896542 \\
\hline I I & 0.5796654435 & 31 & 30 & 0.3999497772 & I. I3185 44282 & 0.1104707636 \\
\hline I2 & 0.6323623020 & 34 & 2 & $0.42608 \quad 127$ & I. I5676 96284 & o. I $2095 \quad 48573$ \\
\hline I3 & 0.6850591605 & 36 & 30 & 0.4500821300 & I. I8379 59985 & o.1315497896 \\
\hline 14 & 0.7377560190 & $3^{8}$ & 53 & $0.47195 \quad 19964$ & I. 2129188175 & o. 1422630292 \\
\hline I5 & 0.7904528775 & $4 I$ & 12 & 0.4917127 & I. 244 I $2 \quad 18489$ & o. I5310 I6293 \\
\hline 16 & 0.84314 .97360 & 43 & 26 & 94053 & I. 2773872698 & $07: 21$ \\
\hline I7 & o. 8958465946 & 45 & 35 & 508697 & I. 3126955975 & 51808788 \\
\hline 18 & 0.9485434531 & 47 & 40 & 88277072 & I. $35002 \quad 56142$ & $643 \quad 33074$ \\
\hline I9 & 1.0012403116 & 49 & 40 & 0.5507078595 & I. 3893542896 & o. $19783 \quad 46027$ \\
\hline 20 & 3937 I 701 & $5 I$ & 34 & 0.5608 I 52531 & I. 4306567027 & $0.2093^{8} 9333^{8}$ \\
\hline $2 \mathrm{I}$ & I. 1066340286 & 53 & 25 & 92428378 & I. $47390 \quad 59$ & 0.2211014976 \\
\hline 22 & I. I59330887 I & 55 & I I & 0.5760865921 & I. 5190731 & 2974497 I \\
\hline 23 & I. 2120277456 & 56 & $5^{2}$ & $3144 \quad 37172$ & I. 56612715 & 0.2450 I I I 93 \\
\hline 24 & I. $264724604 \mathrm{I}$ & $5^{8}$ & 29 & 0.5854 I III 88 & I. 6150347485 & $0.25721 \quad 35159$ \\
\hline 25 & I. $31742 \quad 14626$ & 60 & 2 & 0.5880841618 & I . 6657603865 & $0.2695^{8} 31846$ \\
\hline 26 & I.370I I 832 I I & $6 I$ & $3 I$ & $8955 \quad 56773$ & 1. 71826617 & 0.2821209517 \\
\hline 27 & I . 4228 I 51796 & 62 & 55 & $0.58991 \quad 51945$ & I. $77251 \quad 18082$ & 0.2948269565 \\
\hline 28 & I. $4755 \mathrm{I} \quad 2038 \mathrm{I}$ & 64 & 16 & 0.5892483721 & I. 8284544989 & $0.30770 \quad 06377$ \\
\hline 29 & I. 5282088966 & 65 & 33 & 0.5876366017 & I. 8860489185 & 0.3207407202 \\
\hline 30 & I. $58090 \quad 5755$ I & 66 & 46 & $0.5^{8} 515675$ & I. 9 & 0.3339452050 \\
\hline $3 I$ & I. 6336026136 & 67 & 56 & I $88 \quad 10$ & 9859 & $4731 \quad 13599$ \\
\hline 32 & I . 686299472 I & 69 & 3 & 77877036 & 2.0682500238 & 3608357125 \\
\hline 33 & I.73899 63306 & 70 & 6 & 0.573207601 & 2.1319454360 & 0.3745 I 40449 \\
\hline 34 & I.79I693189I & 71 & 7 & 0.56793 I I 188 & 2. 1970260925 & $0.38834 \quad 13902$ \\
\hline 35 & I. 84439 oo & 7 & 4 & 0.5 & 2.2634304764 & $0.40231 \quad 20314$ \\
\hline 36 & I. 897086906 & 72 & 59 & 576876 & 2.3310942822 & 0.4164195021 \\
\hline 37 & I .94978 37646 & 73 & 51 & o. 54897850 & 2.3999504116 & 0.4306565890 \\
\hline 38 & 2480623 & 74 & 41 & 0.5417728388 & 2.4699289791 & 0.445015337 I \\
\hline 39 & $2.05517748 \mathrm{I} 6$ & 75 & 28 & $0.534190285 \mathrm{I}$ & 2.5409573266 & $0.45948 \quad 70563$ \\
\hline 40 & $787 \quad 434$ & 76 & 12 & 0.52626606 & 2.6129600482 & 0.4740623311 \\
\hline $4 I$ & 2. I6057 II986 & 76 & 55 & 0.5180323296 & $2.685^{8} 590$ & 0.4887310316 \\
\hline 42 & $2.213268057 \mathrm{I}$ & 77 & 35 & 0.5095 I 83887 & 2.75957347 & $0.5034^{8} .23272$ \\
\hline 43 & 2.2659649156 & 78 & 14 & $0.500750924 \mathrm{I}$ & 2.8340199954 & $0.51830 \quad 47025$ \\
\hline 44 & 2.31866 I 7741 & 78 & 50 & $0.49175 \quad 41985$ & 2.9091126530 & $0.533^{18} .5975^{\circ}$ \\
\hline 45 & 2.3713586326 & 79 & 25 & 0.4825502516 & $2.98476 \quad 30422$ & 0.548 I I 33 I 55 \\
\hline $20-r$ & $\mathbf{F} \psi$ & & & $\mathbf{G}(\mathbf{r})$ & $\mathbf{C}(\mathbf{r})$ & $\mathbf{B}(\mathbf{r})$ \\
\hline
\end{tabular}




\begin{tabular}{|c|c|c|c|c|c|}
\hline $\mathbf{B}(\mathbf{r})$ & $\mathbf{C}(\mathbf{r})$ & $G(r)$ & $\psi$ & $\mathrm{F} \psi$ & $90-r$ \\
\hline I. 0000000000 & $5 \cdot 35^{29} \mathrm{I} \quad 5^{8734}$ & 0.0000000000 & $90^{\circ} \quad o^{\prime}$ & 4.7427 I 72653 & 90 \\
\hline 0.9997065254 & 5.3513539870 & 0.01107 $55^{804}$ & $89 \quad 54$ & 4.6900204068 & 89 \\
\hline 0.9988266090 & $5 \cdot 34667$ III20 & 0.0221508037 & 47 & $4.63732 \quad 35483$ & 88 \\
\hline 0.99736 I77 II & $5 \cdot 3388755928$ & 0.0332253090 & $4 \mathrm{I}$ & $4 \cdot 5^{8462} 66898$ & 87 \\
\hline 0.9953 I 45401 & 5.32798 I3106 & 0.0442987274 & 35 & 4.5319298313 & 86 \\
\hline 0.9926884456 & 5.3140076445 & 0.0553706778 & 89 & 4.4792329728 & 85 \\
\hline 0.9894880069 & 5.2969794165 & 0.0664407630 & $2 \mathrm{I}$ & 4.42653 6II 43 & 84 \\
\hline 0.9857187199 & 5.2769268222 & $0.0775^{\circ} 85650$ & 89 & $4.373839255^{8}$ & 83 \\
\hline $0.9813^{8} 70401$ & $5.25388 \quad 53459$ & $0.08857 \cdot 36405$ & 89 & $4 \cdot 32$ II 423973 & 82 \\
\hline $0.97650 \quad 03636$ & 5.2278956618 & 0.0996355161 & 89 & 4.2684455388 & $8 \mathbf{I}$ \\
\hline 0.9710670046 & $5.1990035^{203}$ & o. I1069 36828 & 88 & 4.2157486803 & 80 \\
\hline 0.9650961704 & 5.1672596214 & o.12174 75905 & 88 & 4. 16305 I 8218 & 79 \\
\hline $0.95^{8} 5979343$ & 5.1327 I 94744 & o.13279 66420 & 88 & 4. I I035 49633 & 78 \\
\hline $0.9515^{8} 32050$ & $5.09544 \quad 32457$ & o. I 4384 or 862 & 88 & 4.0576581048 & 77 \\
\hline 0.9440636948 & 5.0554955939 & o.15487 75II2 & 23 & $4.00496 \quad 12463$ & 76 \\
\hline 0.93605 I 8846 & 5.0129454947 & o.16590 7836I & 88 & $3.95226 \quad 43878$ & 75 \\
\hline 0.9275609875 & $4.967866053^{8}$ & 0.1769303026 & 88 & 3.8995675293 & 74 \\
\hline 0.9186049094 & 4.9203343119 & o. 1879439654 & 87 & 3.8468706707 & 73 \\
\hline 0.9091982095 & $4.87043 \quad 10392$ & o.19894 77822 & 87 & 3.7941738122 & 72 \\
\hline 0.8993560570 & 4.8182405226 & 0.20994 06015 & 39 & 3.7414769537 & 71 \\
\hline 0.8890941880 & 4.7638503454 & 0.22092 I I 507 & 87 & $3.688780095^{2}$ & 70 \\
\hline 0.8784288604 & 4.70735 I 1607 & 0.2318880216 & 18 & $3.63608 \quad 32367$ & 69 \\
\hline $0.867376807 \mathrm{I}$ & 4.6488364589 & 0.2428396552 & 87 & $3.5^{8} 33^{8} \quad 63782$ & 68 \\
\hline $0.85595 \quad 51894$ & $4.58840 \quad 23314$ & 0.2537743247 & 86 & 3.5306895197 & 67 \\
\hline 0.844 I 8 I 548 I & 4.5261472300 & 0.26469 oI I 66 & 45 & 3.4779926612 & 66 \\
\hline $0.832073755^{2}$ & $4.46217 \quad 17234$ & $0.2755^{8} 49098$ & 86 & 3.4252958027 & 65 \\
\hline 0.8196499644 & $4 \cdot 3965782526$ & 0.2864563526 & 86 & 3.3725989442 & 64 \\
\hline 0.8069285610 & $4 \cdot 3294708849$ & $0.29730 \quad$ I 8370 & 86 & 3.3199020857 & 63 \\
\hline 0.793928 I 128 & 4.2609550677 & 0.308 I I 847 I I & $5^{2}$ & $3.26720 \quad 52272$ & 62 \\
\hline 0.7806673195 & 4. I9II3 73836 & 0.3189030470 & $85 \quad 37$ & $3.21450 \quad 83687$ & $6 I$ \\
\hline 0.767 I6 49636 & 4.12012 53075 & 0.3296520072 & 85 & $3.16 \mathrm{I} 8 \mathrm{I} \quad 15 \mathrm{IO} 2$ & 60 \\
\hline 0.7534398604 & 4.0480269653 & $0.34036 \quad 14062$ & 85 & 3.1091146517 & 59 \\
\hline 0.7395 I 08099 & 3.9749508972 & $0.351026868 \mathrm{I}$ & 48 & 3.0564177932 & $5^{8}$ \\
\hline 0.7253965478 & 3.9010058247 & 0.3616435409 & 29 & 3.0037209347 & 57 \\
\hline 0.7 IIIII 56987 & $3.82630 \quad 04227$ & 0.3722060448 & 10 & 2.9510240762 & 56 \\
\hline 0.69668672 )I & 3.7509430973 & 0.3827084160 & 83 & $2.89832 \quad 72177$ & 55 \\
\hline 0.6821279026 & $3.67504 \quad 17706$ & 0.3931440446 & 30 & 2.8456303592 & 54 \\
\hline 0.6674572351 & $3.59870 \quad 36716$ & 0.4035056060 & 83 & 2.7929335007 & 53 \\
\hline 0.6526924519 & 3.5220351359 & 0.4137849862 & 44 & 2.7402366422 & $5^{2}$ \\
\hline 0.6378509470 & 3.445 I 4 I4I 33 & 0.4239731992 & 20 & 2.6875397837 & $5^{I}$ \\
\hline 0.6229497425 & $3 \cdot 36812 \quad 64840$ & 0.4340602965 & $8 \mathrm{I}$ & 2.6348429252 & 50 \\
\hline 0.6080054504 & 3.2910928843 & 0.4440352686 & 28 & 2.5821460667 & 49 \\
\hline 0.5930342368 & $3.21414 \quad \mathrm{I} 542 \mathrm{I}$ & 0.4538859368 & 80 & $2 \cdot 529449208 \mathrm{I}$ & $4^{8}$ \\
\hline $0.57805 \quad 17864$ & $3.13737 \quad 16225$ & 0.4635988357 & 80 & $2.47675 \quad 23496$ & 47 \\
\hline 0.5630732704 & $3.06088 \quad 03834$ & $0.473159085 \mathrm{I}$ & 79 & 2.4240554911 & 46 \\
\hline 0.548 I I 33 I 55 & $2.98476 \quad 30422$ & $0.48255025 \mathrm{I} 6$ & $79 \quad 25$ & 2.3713586326 & 45 \\
\hline $\mathbf{A}(\mathbf{r})$ & $\mathbf{D}(\mathbf{r})$ & $\mathbf{E}(\mathbf{r})$ & $\phi$ & $\mathbf{F} \phi$ & $\mathbf{r}$ \\
\hline
\end{tabular}

SMITHSONIAN TABLES 
$K=5.4349098296, \quad K^{\prime}=1.5709159581, \quad E=1.0007515777, \quad E^{\prime}=1.5706767091$,

\begin{tabular}{|c|c|c|c|c|c|}
\hline $\mathbf{r}$ & $\mathbf{F} \phi$ & $\phi$ & $\mathbf{E}(\mathbf{r})$ & $\mathbf{D}(\mathbf{r})$ & $\mathbf{A}(\mathbf{r})$ \\
\hline 0 & 0.0000000000 & $0^{\circ} \quad 0^{\prime}$ & 0.0000000000 & I. .0000000000 & 0.0000000000 \\
\hline I & 0.0603878870 & 327 & 0.0491951488 & I.00I48 76066 & 0.0079798676 \\
\hline 2 & o.12077 57740 & 54 & 0.0979531901 & I .00595 04088 & o.01597 27570 \\
\hline 3 & 0.1811636610 & I9 & o.1458495983 & I.0I338 83449 & $0.02399 \quad 16544$ \\
\hline 4 & $0.24 I 55 \quad 15480$ & 13 & o. 1924842494 & I. $.02380 \quad 12862$ & 0.0320494760 \\
\hline 5 & $0.301939435^{\circ}$ & I 7 & o.23749 I7959. & 1.037I $8 \quad 89963$ & 0.0401590322 \\
\hline 6 & 0.3623273220 & 20 & 0.2805500559 & I. $05355 \quad 10766$ & 0.0483329925 \\
\hline 7 & 0.4227152090 & 23 & $0.3^{21} 3^{8} 60670$ & I.07288 68948 & $0.0565^{8} 3^{8508}$ \\
\hline 8 & 0.4831030960 & 40 & 0.3597796610 & I.09519 55002 & 0.0649238899 \\
\hline 9 & 0.5434909830 & 43 & 0.3955646136 & I. 1204755228 & 0.0733651472 \\
\hline I0 & 0.6038788700 & 40 & 0.4286275917 & I. $14872 \quad 50597$ & 0.08 I9I 93794 \\
\hline I I & 0.6642667569 & 35 & $0.45^{890} 5^{245^{0}}$ & I. I 7994 I 5472 & 0.0905980283 \\
\hline 12 & 0.7246546439 & 18 & 0.4863798590 & 1.21412 16208 & $0.09941 \quad 21860$ \\
\hline 13 & 0.7850425309 & $5^{8}$ & 0.5110740138 & I. 25 I26 09628 & o. 1083725614 \\
\hline 14 & 0.8454304179 & 43 & $0.53304 \quad 46717$ & I.29I35 4I39I & o. I I 74894454 \\
\hline I 5 & $0.90581 \quad 83049$ & 45 & $0.55^{237} 70723$ & I.33439 $44^{250}$ & o.12677 26784 \\
\hline 16 & 0.9662061919 & $4^{8}$ & 0.5691787466 & 1.3803736227 & o. 1362316162 \\
\hline 17 & I. .0265940789 & 50 & $0.5^{8357} 3^{8857}$ & I. $42928 \quad$ I 8693 & o. I $45^{87} 50978$ \\
\hline I 8 & I.08698 I9659 & 44 & 0.5956982320 & I . 48 I I0 74384 & 0.1557 I 14129 \\
\hline 19 & I. 1473698529 & 54 & $0.6056945^{8} 5 \mathrm{I}$ & I. 5358365353 & o.1657482707 \\
\hline 20 & I. .2077577399 & 56 & 0.61370897 I 5 & I. 5934530865 & o. 1759927682 \\
\hline $2 I$ & I. 268 I 456269 & $5^{8}$ & 0.6198874725 & I. 6539385266 & o. I 8645 I3603 \\
\hline 22 & I. 3285335 I 39 & 60 & 0.6243736797 & I.7I727 I5815 & o.19712 98307 \\
\hline 23 & 1. $38892 \quad 14009$ & 62 & $0.62730 \cdot 67243$ & I. 7834280514 & 0.2080332624 \\
\hline 24 & I.44930 92879 & 63 & $0.6288 \mathrm{I} \quad 98 \mathrm{I} 44$ & I. 8523805926 & 0.2191660113 \\
\hline 25 & I.50969 71749 & 65 & 0.6290392100 & I. 9240985022 & $0.23053 \quad 16788$ \\
\hline 26 & I. $57008 \quad 50619$ & 66 & 0.6280835657 & I. 9985475042 & 0.2421330872 \\
\hline 27 & I. 6304729489 & 67 & 0.6260635735 & 2.0756895405 & 0.2539722556 \\
\hline 28 & I. 6908608359 & 69 & $0.62308 \quad 18462$ & $2: 1554825676$ & 0.2660503772 \\
\hline 29 & I. 7512487229 & 70 & 0.6192329878 & 2.2378803597 & 0.2783677989 \\
\hline 30 & I. 8 I I63 66099 & 71 & 0.6146038040 & $2.32283 \quad 23203$ & 0.2909240017 \\
\hline $3 \mathrm{I}$ & I. 8720244969 & 72 & 0.6092736149 & 2.4102833038 & $0.3037175^{832}$ \\
\hline 32 & I.9324I 23839 & 73 & 0.6033146378 & 2.5001734479 & 0.3167462424 \\
\hline 33 & I. 9928002709 & 74 & 0.5967924144 & 2.5924380185 & 0.3300067656 \\
\hline 34 & $2.05318 \quad 81579$ & 75 & 0.5897662623 & 2.6870072681 & 0.3434950157 \\
\hline 35 & 2. II357 60449 & 76 & $0.582289734^{I}$ & $2.78380 \quad 63098$ & 0.3572059222 \\
\hline 36 & 2.17396 39318 & 77 & $0.57441 \quad 10737$ & $2.88275 \quad 50068$ & 0.3711334754 \\
\hline 37 & $2.23435 \quad 18188$ & 77 & o. 566 I 736598 & 2.9837678796 & 0.38527072 I I \\
\hline 38 & $2.294739705^{8}$ & 78 & $0.5576 \mathrm{I} 643 \mathrm{I} 5$ & 3.0867540315 & o.39960 97596 \\
\hline 39 & $2.355^{12} 75928$ & 79 II & 0.5487742910 & 3.1916170942 & $0.41414 \quad 1746 \mathrm{I}$ \\
\hline 40 & $2.41551 \quad 54798$ & 79 & 0.5396784809 & 3.2982551932 & 0.4288568946 \\
\hline $4 \mathrm{I}$ & 2.4759033668 & 80 & 0.5303569362 & 3.4065609346 & 0.4437444843 \\
\hline 42 & $2.53629 \quad 12538$ & 80 & 0.5208346089 & $3.51642 \quad 14148$ & $0.45^{879} 28694$ \\
\hline 43 & 2.5966791408 & 30 & 0.5 I I I 337664 & 3.6277182525 & 0.4739894906 \\
\hline 44 & 2.6570670278 & 82 & 0.5012742646 & $3 \cdot 7403276441$ & 0.4893208915 \\
\hline 45 & $2.71745 \quad 49148$ & $82 \quad 28$ & 0.4912737968 & 3.8541204436 & 0.5047727366 \\
\hline $90-r$ & $\mathbf{F} \psi$ & . $\psi$ & $\mathbf{G}(\mathbf{r})$ & $C(r)$ & $\mathbf{B}(\mathbf{r})$ \\
\hline
\end{tabular}


TABLE $\theta=89^{\circ}$

$q=0.403309306338378, \quad \theta 0=0.2457332317, \quad$ HK $=1.8599580878$

\begin{tabular}{|c|c|c|c|c|c|}
\hline $\mathrm{B}(\mathrm{r})$ & $C(r)$ & $\mathbf{G}(\mathbf{r})$ & $\psi$ & $\mathrm{F} \psi$ & $90-r$ \\
\hline I. .0000000000 & $7 \cdot 5^{6958} 97180$ & 0.0000000000 & $90^{\circ} \quad \mathrm{o}^{\prime}$ & 5.4349098296 & 90 \\
\hline 0.9996643156 & $7 \cdot 5670529325$ & $0.01110 \quad 10463$ & $89 \quad 56$ & $5 \cdot 3745^{2} \quad 19426$ & 89 \\
\hline 0.9986579343 & $7 \cdot 5594477064$ & $0.02220 \quad 19579$ & 53 & 5.3141340556 & 88 \\
\hline 0.9969828696 & $7 \cdot 5467894142$ & $0.03330 \quad 25985$ & 49 & 5.2537461686 & 87 \\
\hline 0.9946424694 & $7.52910 \quad 36233$ & $0.04440 \quad 28272$ & 45 & 5.1933582816 & 86 \\
\hline 0.9916414052 & $7 \cdot 50642$ 6o102 & $0.0555^{\circ} 24979$ & 89 & 5.1329703946 & 85 \\
\hline $0.98798 \quad 56557$ & $7 \cdot 47880 \quad 22428$ & $0.06660 \quad 14556$ & $3^{8}$ & $5.07258 \quad 25077$ & 84 \\
\hline $0.98368 \quad 24869$ & $7 \cdot 4462878301$ & 0.0776995354 & 89 & 5.0121946207 & 83 \\
\hline $0.97874 \quad 04272$ & 7.4089479407 & 0.0887965593 & 30 & $4.95180 \quad 67337$ & 82 \\
\hline 0.9731692390 & $7 \cdot 3668571893$ & $0.09989^{\circ} 23340$ & 89 & 4.8914188467 & $8 \mathrm{I}$ \\
\hline 0.9669798856 & $7 \cdot 3200993943$ & o.II098 6648I & 89 & 4.8310309597 & 80 \\
\hline o.960I 844944 & $7.26876 \quad 73054$ & o.1220792686 & 89 & 4.7706430727 & 79 \\
\hline $0.95^{279} 63165$ & 7.2129623044 & o.1331699380 & 89 & 4.7102551857 & 78 \\
\hline 0.9448296828 & 7.1527940797 & 0.1442583704 & 89 & 4.6498672987 & 77 \\
\hline 0.9362999559 & $7.0883^{8} \quad 02759$ & o. I5534 42469 & 89 & $4 \cdot 5^{8947} 94$ II & 76 \\
\hline $0.92722 \quad 34802$ & 7.0198461207 & 0.1664272118 & $5^{8}$ & $4.52909 \quad 15247$ & 75 \\
\hline 0.9176175278 & 6.9473240301 & o. I7750 68667 & 53 & $4.46870 \quad 36377$ & 74 \\
\hline $0.90750 \quad 02426$ & 6.8709531948 & o. I $885^{8} 2764^{8}$ & 47 & $4.40831 \quad 57507$ & 73 \\
\hline 0.8968905812 & 6.7908791481 & o. 1996544048 & $4 I$ & $4 \cdot 3479278637$ & 72 \\
\hline $0.88580 \quad 82522$ & 6.7072533191 & $0.21072 \quad 12232$ & 35 & 4.2875399767 & 71 \\
\hline $0.874273^{6} 532$ & 6.6202325717 & $0.2217^{8} \quad 25^{86} 3$ & 88 & 4.2271520897 & 70 \\
\hline 0.8623078063 & 6.5299787323 & 0.2328377807 & 88 & 4.1667642027 & 69 \\
\hline 0.849932292 I & 6.4366581080 & 0.2438860035 & 88 & 4. 1063763157 & 68 \\
\hline 0.8371691826 & 6.3404409975 & 0.2549263501 & 88 & $4.04598 \quad 84287$ & 67 \\
\hline 0.8240409732 & 6.24150 I 1966 & 0.2659578012 & 59 & 3.9856005417 & 66 \\
\hline 0.8105705141 & 6.1400155012 & 0.2769792084 & 87 & $3.92521 \quad 26547$ & 65 \\
\hline 0.7967809414 & $6.03616 \quad 32083$ & 0.2879892768 & 87 & $3.86482 \quad 47677$ & 64 \\
\hline 0.7826956083 & $5.93012 \quad 56192$ & $0.298986547 \mathrm{I}$ & 87 & 3.8044368807 & 63 \\
\hline 0.7683380165 & $5.822085545^{2}$ & 0.3099693739 & 87 & 3.7440489937 & 62 \\
\hline $0.75373 \quad 17477$ & $5.71222 \quad 68183$ & 0.3209359022 & 87 & 3.68366 I 1067 & $6 I$ \\
\hline $0.73890 \quad 03962$ & 5.6007338100 & 0.3318840408 & 87 & 3.6232732197 & 60 \\
\hline $0.72386 \quad 75024$ & 5.4877909576 & $0.34281 \quad 14317$ & 86 & $3.56288 \quad 53328$ & 59 \\
\hline 0.7086564877 & $5 \cdot 3735^{8} 23026$ & $0.35371 \quad 54168$ & 86 & $3 \cdot 502497445^{8}$ & $5^{8}$ \\
\hline 0.6932905904 & 5.25829 I04I 3 & 0.3645929992 & 86 & 3.4421095588 & 57 \\
\hline 0.6777928032 & 5.1420990885 & 0.3754408012 & 86 & $3.38172 \quad 16718$ & $5^{6}$ \\
\hline $0.662185^{8136}$ & $5.02518 \quad 66588$ & $0.3^{8625} 50154$ & 85 & $3.32133 \quad 37848$ & 55 \\
\hline $0.646491944^{8}$ & $4.90773 \mathrm{I} 8631$ & $0.39703 \quad 13507$ & 85 & $3.260945^{8978}$ & 54 \\
\hline 0.6307330999 & $4.78991 \quad 03252$ & 0.4077649715 & 85 & 3.2005580108 & 53 \\
\hline 0.6149307081 & $4.67189 \quad 48167$ & 0.4184504298 & 85 & 3.1401701238 & 52 \\
\hline 0.5991056732 & $4.553^{8} 549133$ & 0.42908 I5883 & 47 & $3.07978 \quad 22368$ & $5^{I}$ \\
\hline $0.5^{8327} 83254$ & 4.4359566732 & $0.43965 \quad 15347$ & 84 & 3.0193943498 & 50 \\
\hline $0.567468375^{0}$ & $4 \cdot 318362337 I$ & 0.4501524856 & 84 & 2.9590064628 & 49 \\
\hline 0.5516948696 & $4.201230052 \mathrm{I}$ & 0.4605756791 & 44 & 2.8986 I $8575^{8}$ & $4^{8}$ \\
\hline 0.5359761539 & 4.0847 I 36196 & $0.47091 \quad 12546$ & 83 & 2.8382306888 & 47 \\
\hline 0.5203298326 & 3.9689622668 & 0.48 II 8 II89 & 55 & 2.7778428018 & 46 \\
\hline 0.5047727366 & 3.8541204436 & 0.4912737968 & $82 \quad 28$ & 2.7174549148 & 45 \\
\hline $\mathbf{A}(\mathbf{r})$ & $\mathbf{D}(\mathbf{r})$ & $\mathbf{E}(\mathbf{r})$ & $\phi$ & $\mathrm{F} \phi$ & $\mathbf{r}$ \\
\hline
\end{tabular}









\section{INDEX}

The numbers refer to pages.

A

Addition formulas, Elliptic Functions 250

Algebraic equations............. 2

Algebraic identities............. I

Alternating series............... I

Archimedes, spiral of ........... 52

Area of polygon................ 36

Arithmetical progressions.......... $\quad 26$

Asymptotes to plane curves........ 40

Axial vector............... 95

\section{B}

Ber and Bei functions............ 204

Bernoullian numbers............. 25

polynomial................ I40

Bessel functions.............. I96

addition formula............ 199

multiplication formula......... 199

references................. 2 I3

Bessel-Clifford differential equation .. 205

Beta functions............... I I $_{32}$

Binomial coefficients............ I9

Binormal.................. 59

Biquadratic equations............ Io

Bromwich's expansion theorem...... 212

\section{C}

Cassinoid................... 53

Catenary................... 52

Cauchy's test............... I09

Center of curvature, plane curves.... 39

surfaces................. ${ }_{5}^{6}$

Change of variables in multiple integrals.................. I7

Characteristic of surface.......... $\quad 5_{56}$

Chord of curvature, plane curves.... 39

Circle of curvature............ 39

Circular functions, see Trigonometry

Cissoid................... 53

Clairaut's differential equation...... $\mathbf{\text { I66 }}$

Coefficients, binomial........... I9

Combinations............... I 7

Comparison test.............. I०9
PAGE

. Complementary function......... I67

Concavity and convexity of plane

curves ............... 38,42

Conchoid................. 53

Conditional convergence.......... rog

Confluent hypergeometric function.... $\quad$ I85

Conical coordinates............ I04

Consistency of linear equations...... I I

Convergence of binomial series...... I I 7

tests for infinite series......... rog

Covariant property............. r7

Cubic equations............... 9

Curl..................... 93

Curvature, plane curves.......... 38

space curves............... 58

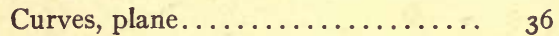

space.................... 57

Curvilinear coordinates.......... 99

Curvilinear coordinates, surfaces of revolution............... I06

Cycloid.................. 5 I

Cylindrical coordinates......... 32, 102

Cylinder functions, see Bessel functions 197

D

d'Alembert's Test............ rog

Definite integrals, computation by difference functions........... 225

Simpson's method............ 22 I

expressed as infinite series........ 134

de Moivre's theorem............. 66

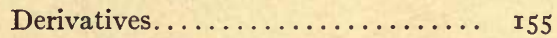

of definite integrals.......... I56

of implicit functions. . ......... I6I

Descartes' rule of signs........... 5

Determinants................. II

Difference functions............ 222

Differential equations............ I6 $6_{2}$

numerical solution............ 220

Differentiation of determinants...... I3

Discriminant of biquadratic equation .................. II

Divergence................ 93

Double periodicity of elliptic functions 250 
Ellipse..................... $4_{46}$

Ellipsoidal coordinates........... 102

Elliptic cylinder coordinates........ IO IO4

Elliptic integrals, first kind........ 245

second kind................ 248

third kind................ 25I

Elliptic integral expansions..... I I55, I95

Envelope................... 40

Envelope of surfaces............ $5^{6}$

Epicycloid.................. 52

Equations, algebraic............ 2

transcendental, roots of........ 84

Equiangular spiral............. 53

Eta functions................ 25 I

Euler's constant............... 27

summation formula........... 25

transformation formula.......... II 3

theorem for homogeneous functions. I 57

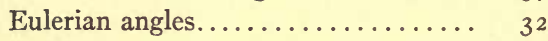

Evolute................... 39

Exact differential equations...... I63, I77

Expansion of determinants........ I3

Expansion theorem, Bromwich's..... 212

Heaviside's.............. $2 \mathrm{I} 2$

\section{F}

Finite differences and sums.

20

Finite products of circular functions. . 84

Finite series, special. ............ 26

Fourier's series............... I36

Fresnel's integrals............. I34

Functional determinants......... I6

\section{G}

Gamma function............. I3I

Gauss's II function ............. I33

theorem................. 95

Geometrical progressions.......... 26

Gradient of vector............. 93

Graeffe's method.............. 8

Green's theorem............... 95

Gregory's series............... 122

Gudermannian.............. $\quad 76$

\section{$\mathrm{H}$}

Harmonical progressions.......... 26

Harmonics, zonal .............. I9I

Heaviside's operational methods..... 210 expansion theorem............ 212

Helical coordinates............. I0 6

Hessian.

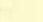

Homogeneous differential equations

Homogeneous linear equations...... I5

Horner's method............. 7

l'Hospital's rule.............. I45

Hyperbola................ 48

Hyperbolic functions............ 7 I

spiral................ 52

Hypergeometric differential equation 209 series................. 209

Hypergeometric function, confluent... $\quad$ I85

Hypocycloid................ 52

\section{I}

Identities, algebraic............ I

Implicit functions, derivatives of.... . I6I

Indeterminate forms........... $\mathbf{I 4 5}$

Indicial equation. ............ I 74

Infinite products............. I

series................. Iо9

Integrating factors............. I6

Interpolation formula, Newton's..... 22

Intrinsic equation of plane curves.... 44

Involute of plane curves......... 39

$\mathrm{J}$

Jacobian.................. I6

$\mathrm{K}$

Ker and Kei functions........... 205

Kummer's transformation......... II4

L

Lagrange's theorem............. II 2

Laplace's integrals............. I93

Latus rectum, ellipse........... 48

hyperbola ............... 49

parabola................. 46

Leclert's transformation........... II 5

Legendre's equation............ I9I

Leibnitz's theorem............. ${ }_{157}$

Lemniscate................ 53

Limiting values of products........ ${ }_{152}$

sums..................... I5I

Linear equations.............. I5

Linear vector function. . . . . . . 96

Lituus.................. 53

Logarithmic spiral............ 53

M

Maclaurin's theorem........... II

Markoff's transformation formula.. . . I II3 
PAGE

I 52

I93

14

I 20

I2

5

N

Neoid

Neumann's expansion, zonal harmonics................ I94

Newton's interpolation formula..... 22 method for roots of equations...... theorem on roots of algebraic equations.

Normal to plane curves...........

Numbers, Bernoulli's. . . . . . . . . . .

Euler's.

Numerical series. ..............

Numerical solution of differential equations.

\section{O}

Oblate spheroidal coordinates........ Operational methods............. Orthogonal curvilinear coordinates....

\section{$\mathbf{P}$}

II function, Gauss's ........... I I3

Parabola.................. 45

Parabolic coordinates............ I07

Parabolic cylinder coordinates....... I05

Parabolic spiral.............. 53

Parallelepipedon, volume of....... 92

Partial fractions................ 20

Particular integral............ I67

Pedal curves................ 10

Pendulum................ 247

Permutations and combinations..... I 7

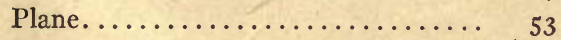

Plane curves................ 36

polar coordinates............ $4 \mathrm{I}$

Plane geometry.............. 34

Points of inflexion.......... 39,42

Polar coordinates........... 32, IoI

Plane curves.............. 4I

Polar subtangent............ 37

subnormal............... 37

normal................. 37

tangent................. 37

Polar vector............... 95
Polynomial.

PAGE

Bernoullian.

series.

Principal normal to space curves.... 58

Products, finite of circular functions. . 84

limiting values of ............ ${ }_{5} 5^{2}$

of two series............... I Io

Progressions................. 26

Prolate spheroidal coordinates....... I07

\section{Q}

Quadratic equations..............

Quadriplanar coordinates...........

$\mathrm{R}$

Raabe's test............... I09

Radius of curvature, plane curves. . . 38, 42 space curves............... $\quad 5^{8}$

surfaces................ 55

Radius of torsion............. 59

Reciprocal determinants.......... I4

Resolution into partial fractions...... 20

Reversion of series............... I 16

Rodrigues' formula ............. I93

Roots of algebraic equations....... 2

transcendental equations....... 84

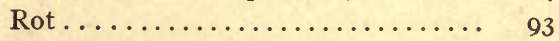

Routh's rule................ 6

Scảlar product................

Schlomilch's expansion, Bessel functions................. $20 \mathrm{I}$

Series, finite, circular functions...... $8 \mathrm{I}$

infinite................ rog

special finite............... 26

numerical................. I 40

of Bessel functions........... $20 \mathrm{I}$

hypergeometric............. 209

of zonal harmonics............ I94

Simpson's method............. 22 I

Singular points.............. 4I

Skew determinants............. I4

Skew-symmetrical determinants...... I5

Solid geometry............... 53

Space curves............... 57

Spherical polar coordinates........ IOI

Spherical triangles............. 78

Spheroidal coordinates........... I?7

Spiral of Archimedes........... 52

Stirling's formula............. 28 
PAGE

Stokes's theorem............. 95

Sturm's theorem............... 6

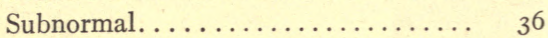

Subtangent................. 36

Sums, limiting values of........... I5 I

Summation formula, Euler's........ 25

Surfaces................... 55

Symbolic form of infinite series...... II 2

Symbolic methods in differential equations................ I73

Symmetrical determinants......... I4

Symmetric functions of roots of algebraic equations............ 2

\section{$\mathrm{T}$}

Tables, binomial coefficients....... 20

hyperbolic functions.......... 72

trigonometric functions........ $6_{2}$

Tangent to plane curves.......... 36

Taylor's theorem.............. II I

Theta function............ 248, 25 I

Toroidal coordinates............ I08

Tractrix................... 53

Transcendental equations, roots of.... 84

Transformation of coordinates...... 29

determinants............... I2

equations.................. 4

infinite series................. II 3

Triangles, solution of plane....... 77

spherical................................ 78

Trigonometry............... 6r

Trilinear coordinates........... 33

Trochoid................

\section{$\mathrm{U}$}

Uniform convergence........... I

Unit vector................ 92

\section{V}

Variation of parameters.......... I80

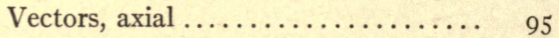

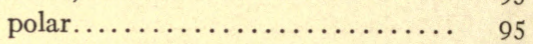

functions, linear.................................. 96

Vector product...............

W

Witch of Agnesi.............. 53

\section{Z}

Zeta function................ 255

Zonal harmonics............. I9I 


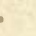




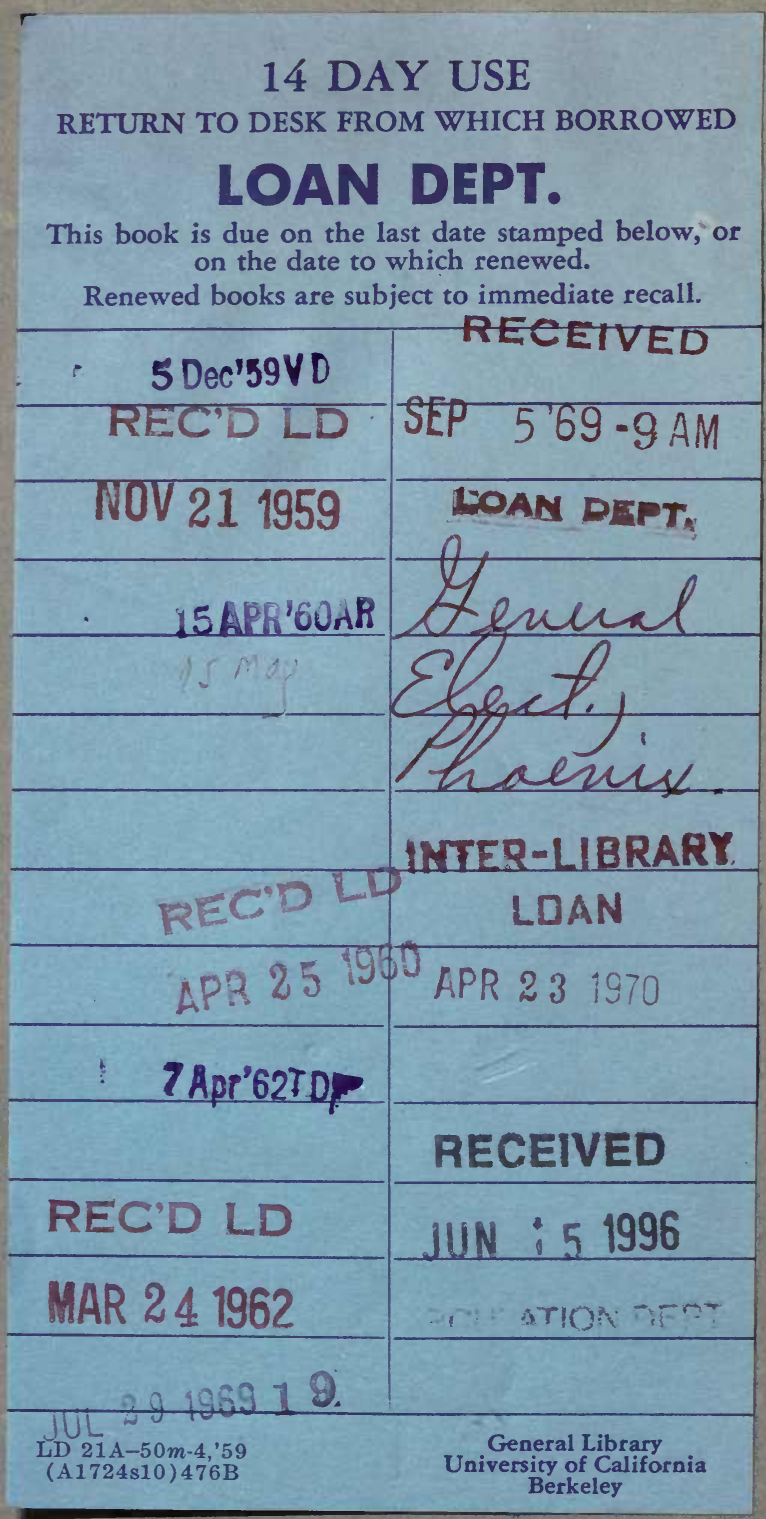


G.

GENERAL LIBRARY - U.C. BERKELEY

IIIII

B000570358 
